Skin Microbiome in Cutaneous T-Cell Lymphoma by $16 \mathrm{~S}$ and Whole-Genome Shotgun Sequencing

\title{
Salava, Alexander
}

2020-11-01

Salava , A, Deptula , P , Lyyski , A, Laine , P , Paulin , L, Väkevä , L , Ranki , A, Auvinen , P \& Lauerma , A 2020 , ' Skin Microbiome in Cutaneous T-Cell Lymphoma by 16 S and Whole-Genome Shotgun Sequencing ' , Journal of Investigative Dermatology, vol. 140 , no. 11 , pp. 2304-+ . https://doi.org/10.1016/j.jid.2020.03.951, https://doi.org/10.1016/j.jid.2020.03.951, https://doi.org/10.1016/j.jid.2020.03.951

http://hdl.handle.net/10138/329450

https://doi.org/10.1016/j.jid.2020.03.951

cc_by_nc_nd

acceptedVersion

Downloaded from Helda, University of Helsinki institutional repository.

This is an electronic reprint of the original article.

This reprint may differ from the original in pagination and typographic detail.

Please cite the original version. 


\section{Journal Pre-proof}

Skin microbiome in cutaneous $\mathrm{T}$ cell lymphoma by $16 \mathrm{~S}$ and whole genome shotgun sequencing

Alexander Salava, Paulina Deptula, Annina Lyyski, Pia Laine, Lars Paulin, Liisa Väkevä, Annamari Ranki, Petri Auvinen, Antti Lauerma

PII: S0022-202X(20)31356-7

DOI: https://doi.org/10.1016/j.jid.2020.03.951

Reference: JID 2393

To appear in: The Journal of Investigative Dermatology

Received Date: 19 September 2019

Revised Date: 5 March 2020

Accepted Date: 5 March 2020

Please cite this article as: Salava A, Deptula P, Lyyski A, Laine P, Paulin L, Väkevä L, Ranki A, Auvinen P, Lauerma A, Skin microbiome in cutaneous T cell lymphoma by $16 \mathrm{~S}$ and whole genome shotgun sequencing, The Journal of Investigative Dermatology (2020), doi: https://doi.org/10.1016/ j.jid.2020.03.951.

This is a PDF file of an article that has undergone enhancements after acceptance, such as the addition of a cover page and metadata, and formatting for readability, but it is not yet the definitive version of record. This version will undergo additional copyediting, typesetting and review before it is published in its final form, but we are providing this version to give early visibility of the article. Please note that, during the production process, errors may be discovered which could affect the content, and all legal disclaimers that apply to the journal pertain.

(C) 2020 The Authors. Published by Elsevier, Inc. on behalf of the Society for Investigative Dermatology. 


\section{Title page}

\section{Skin microbiome in cutaneous T cell lymphoma by 165 and whole genome shotgun sequencing}

Alexander Salava ${ }^{1}$, Paulina Deptula ${ }^{2 *}$, Annina Lyyski ${ }^{2}$, Pia Laine ${ }^{2}$, Lars Paulin ${ }^{2}$, Liisa Väkevä $^{1}$, Annamari Ranki ${ }^{1}$, Petri Auvinen ${ }^{2}$, Antti Lauerma ${ }^{1}$

${ }^{1}$ Department of Dermatology and Allergology, University of Helsinki, and Helsinki University Hospital, Helsinki, Finland

${ }^{2}$ Institute of Biotechnology, DNA Sequencing and Genomics Laboratory, University of Helsinki, Helsinki, Finland

${ }^{*}$ Current address: Department of Food Science, University of Copenhagen, Frederiksberg,

Denmark

\section{Correspondence:}

Alexander Salava, Helsinki University Hospital, Department of Dermatology and Allergology, Meilahdentie 2, 00250 Helsinki, Finland, Tel: +358 9471 86362, Fax: +358

9471 86474, Email: alexander.salava@hus.fi

Short title: Skin microbiome in CTCL

Word count: (body text): 745

Figures: 1 and 2

Supplementary material: Figures S1, S2, S3; Tables S1, S2, S3, S4, S5, S6;

Supplementary Text 1: Supplementary Outline to Methods, Supplementary Text 2:

Supplementary Outline to Discussion 
E-mail addresses and ORCIDs:

Alexander Salava alexander.salava@hus.fi / 0000-0001-5471-5894

Paulina Deptula deptula@food.ku.dk / 0000-0002-8854-3573

Annina Lyyski annina.lyyski@helsinki.fi / 0000-0002-3778-5118

Pia Laine pia.k.laine@helsinki.fi / 0000-0002-3727-8749

Lars Paulin lars.paulin@helsinki.fi / 0000-0003-0923-1254

Liisa Väkevä liisa-väkevä@hus.fi / 0000-0003-4828-1553

Annamari Ranki annamari.ranki@hus.fi / 0000-0003-4335-0396

Petri Auvinen petri.auvinen@helsinki.fi / 0000-0002-3947-4778

Antti Lauerma antti.lauerma@hus.fi / 0000-0002-5078-3547

Abbreviations: CTCL: Cutaneous T-cell lymphoma, WGS: Whole genome shotgun sequencing, OTU: Operational Taxonomic Unit, NMDS: Non-metric multidimensional scaling 


\section{Letter}

\section{To the Editor}

The development of DNA sequencing methods have widened our conceptions of cutaneous microbial communities (Byrd et al., 2018, Eyerich et al., 2018, Fyhrqvist et al., 2016). Recently, we investigated the cutaneous microbiome in parapsoriasis, but did not find significant differences between healthy skin and diseased skin with the $16 \mathrm{~S}$ method (Salava et al., 2017). In vitro studies have demonstrated associations between staphylococcal enterotoxins and cutaneous T cell lymphoma (CTCL) (Krejsgaard et al., 2014, Willerslev-Olsen et al., 2013, Blümel et al., 2019). We were thus interested in microbiome characteristics of CTCL and if they could add supplementary information to traditional diagnostics. Patients $(n=20)$ with mycosis fungoides stages IA-IIB at sampling were included. All patients had cutaneous CTCL lesions and 11 patients were on bexarotene maintenance therapy (Supplementary Table S1). Skin swab samples were obtained from active CTCL lesions and from contralateral healthy-looking skin noninvasively using sterile gloves (Supplementary Figure S3). The extracted DNA from both lesional and non-lesional skin was analyzed by $16 \mathrm{~S}$ and whole genome shotgun (WGS) sequencing. The sampling method, DNA extraction and sequence data analysis are outlined in more detail in the supplementary material (Supplementary Text 1) (Kong et al., 2017, Lu et al., 2017).

The $16 \mathrm{~S}$ data (relative abundances) represented a total of 410 genera, 39 classes and 21 phyla. Four phyla: Actinobacteria (class Actinobacteria, $59.37 \%$ ), Firmicutes (predominantly the classes Clostridia and Bacilli, $15.02 \%$ ), Proteobacteria (mostly Alpha-, Gamma-, and Betaproteobacteria, 12.46\%) and Bacteroidetes (mainly Bacteroidia, 2.16 $\%$ represented $89 \%$ of all classified sequences and $10.19 \%$ remained unclassified at the phylum level. The most common genera were Staphylococcus (30,0\%), Corynebacterium 
(22,3\%) Propionibacterium (5,0\%) and Streptococcus (3,0\%) (Figure 1b, Supplementary Table S4). We applied oligotyping of the potential Staphylococcus sequences and the three most common oligotypes were identified as S. epidermidis $(48,1 \%)$, S. aureus $(33,8$ $\%$ ) and S. capitis (18,0\%) and the rest were unidentified species (Supplementary Table S5).

DESeq2 (Love et al., 2014) was used to analyze the 16 S sequencing data for differences between the sample types (lymphoma-affected vs. healthy-appearing skin) (Grogan et al., 2019). Comparisons based on a model corrected for the subject-specific variation but no significant differences in bacterial taxa (Figure 2, Supplementary Table S4) or in the Staphylococcus oligotypes (data not shown) were observed (Lindahl et al., 2019). WGS data analyzed with BRACKEN (Lu et al., 2017) represented 1312 identified organisms at genus level, with Cutibacterium (member of the former genus Propionibacterium) (25\%), Corynebacterium (19\%) and Staphylococcus (18\%) as the most abundant genera (Supplementary Figure S2, Supplementary Table S2). We observed no significant differences at the genus level or in microbial diversity (Figure 2). $16 S$ sequencing does not capture propionibacteria very well when compared with WGS (Meisel et al., 2016), therefore explaining the differences in abundances (5\% vs. $25 \%$ ). On the species level most abundant were Cutibacterium acnes (24\%), Staphylococcus epidermidis (7\%), Staphylococcus aureus (7\%) and Corynebacterium kroppenstedtii (6 \%) (Figure 1a). WGS data was then analyzed with DESeq2 to test for different abundances of bacterial taxa by comparing sample types (lymphoma vs. healthy skin) (Supplementary Table S2).

The microbiome varied substantially based on the investigated body site and individual patient (Oh et al., 2016). WGS data at species level showed that Staphylococcus argenteus was more abundant in lymphoma skin. However, after removal of reads identified by BRACKEN as additionally mapping to human genome (and previously not 
identified by BWA), S. argenteus was no longer identified as more abundant in lymphomaaffected skin (adjusted p-value 0.683 ). Interestingly, S. argenteus has been recently shown to express 4-6 fold higher levels of the alpha-hemolysin exotoxin compared to $S$. aureus (Johansson et al., 2019), and thus, the pathogenic role of the $S$. argenteus exotoxin may be more importance than the relative abundance of $S$. argenteus. This possibility needs to be addressed in future studies. The following 10 bacterial species were identified as more abundant in healthy-appearing skin samples (adjusted p-values $<0.05$ ), regardless of additional reads mapping to human genome: Streptomyces sp. SM17, Bordetella pertussis, Streptomyces sp. PVA 94-07, Methylobacterium oryzae, Serratia sp. LS-1, Burkholderia mallei, Enterobacteriaceae bacterium, Achromobacter ruhlandii, Pseudomonas sp. A214, Pseudomonas sp. st29 (Supplementary Table S3). The known associations of these species to human diseases or skin disorders are summarized in Supplementary Table S6 and outlined in the supplementary material (Supplementary Text 2). The role and significance of the relative reduction of these opportunistic pathogens in MF skin remains to be elucidated.

\section{Conflict of interest}

All authors: no conflicts of interest

\section{Data availability}

The data analysed during this study are included in this published article and its supplementary information files. The raw sequences generated during this study have been uploaded to the European Nucleotide Archive (https://www.ebi.ac.uk), accession number: PRJEB36953. 


\section{Acknowledgements}

We would like to thank Leila Jeskanen, M.D. for the histopathological investigations. We thank the personnel of the DNA Sequencing and Genomics Laboratory for performing the laboratory procedures and sequencing for the samples.

The study was approved by the Ethics Committee of Medicine of the Hospital District of Helsinki and Uusimaa (HUS), Finland, (approval number 12/13/03/01/2012), and all patients provided their written informed consent.

This work was supported by Helsinki-Uusimaa Hospital District Research Funds, TYH 2012207 and Helsinki University Hospital Research Funds (Department of Dermatology).

\section{Author contributions:}

Conceptualization: AS, LV, AR, AL

Data Curation: AS, LV

Formal Analysis: PD, ALY, PL, LP, PA

Funding Acquisition: AS, AR, AL

Investigation: AS, ALY, PL, LP, PA, AR, AL

Methodology: AS, PD, ALY, PL, LP, PA

Project Administration: AS, LV, AL

Resources: AS, AR, AL

Software: AS, PD, PL, PA 


\section{References}

Blümel E, Willerslev-Olsen A, Gluud M, Lindahl LM, Fredholm S, Nastasi C et al. Staphylococcal alpha-toxin tilts the balance between malignant and non-malignant CD4+ T cells in cutaneous T-cell lymphoma. Oncoimmunology 2019; 8(11):e1641387.

Byrd AL, Belkaid Y, Segre JA. The human skin microbiome. Nat Rev Microbiol 2018; 16(3): 143-155.

Eyerich S, Eyerich K, Traidl-Hoffmann C, Biedermann T. Cutaneous Barriers and Skin Immunity: Differentiating A Connected Network. Trends Immunol 2018; 39(4): 315-327.

Fyhrquist N, Salava A, Auvinen P, Lauerma A. Skin Biomes. Curr Allergy Asthma Rep 2016; 16: 40.

Grogan MD, Bartow-McKenney C, Flowers L, Knight SAB, Uberoi A, Grice EA. Research Techniques Made Simple: Profiling the Skin Microbiota. J Invest Dermatol 2019; 139(4): 747-752.

Johansson C, Rautelin H, Kaden R. Staphylococcus argenteus and Staphylococcus schweitzeri are cytotoxic to human cells in vitro due to high expression of alpha-hemolysin Hla. Virulence 2019; 10(1):502-510.

Kong HH, Andersson B, Clavel T, Common JE, Jackson SA, Olson ND et al. Performing Skin Microbiome Research: A Method to the Madness. J Invest Dermatol 2017; 137(3): 561-568. 
Krejsgaard T, Willerslev-Olsen A, Lindahl LM, Bonefeld CM, Koralov, SB., Geisler, C. et al. Staphylococcal enterotoxins stimulate lymphoma-associated immune dysregulation. Blood 2014; 124: 761-770.

Lindahl LM, Willerslev-Olsen A, Gjerdrum LMR, Nielsen PR, Blümel E, Rittig AH et al. Antibiotics inhibit tumor and disease activity in cutaneous T cell lymphoma. Blood 2019; pii: blood.2018888107.

Love MI, Huber W, Anders S. Moderated estimation of fold change and dispersion for RNA-seq data with DESeq2. Genome biology 2014; 5(12):550.

Lu J, Breitwieser FP, Thielen P, Salzberg SL. Bracken: estimating species abundance in metagenomics data. PeerJ Computer Science 2017; 2;3:e104.

Meisel JS, Hannigan GD, Tyldsley AS, SanMiguel AJ, Hodkinson BP, Zheng Q et al. Skin Microbiome Surveys Are Strongly Influenced by Experimental Design. J Invest Dermatol 2016; 136(5):947-956.

Oh J, Byrd AL, Park M, NISC Comparative Sequencing Program, Kong HH, Segre JA. Temporal Stability of the Human Skin Microbiome. Cell 2016; 165: 854-866.

Salava, A., Pereira, P., Aho, V., Väkevä, L., Paulin, L., Auvinen, P. et al. Skin Microbiome in Small- and Large-plaque Parapsoriasis. Acta Derm Venereol. 2017; 97(6): 685-691. 
Willerslev-Olsen A, Krejsgaard T, Lindahl LM, Bonefeld CM, Wasik MA, Koralov SB. et al. Bacterial toxins fuel disease progression in cutaneous T-cell lymphoma. Toxins 2013; 5: $1402-1421$.

\section{Tables}

Table S1: Patient characteristics

Table S2: Differential abundance comparisons of species and genera in the WGS sequencing data

Table S3: P-values and species that were significantly more abundant in control samples (healthy appearing skin); adjusted p-values $<0.05$

Table S4: Results of the 16 sequencing data

Table S5: Oligotyping results of the Staphylococcus sequences, 16S sequencing data Table S6: Summary of identified species that were significantly more abundant in healthyappearing skin samples (non-lesional): names, genera, earlier descriptions in human diseases, earlier descriptions in skin disorders

\section{Figure legends}

Figure 1: Microbiome data (WGS and 16S), grouped by subject and sample; naming of the samples: first number corresponds to patient identifier followed by a sample number, where odd numbers indicate lesional samples, while even numbers indicate non-lesional samples.

Figure 1a: Relative abundances of the 25 most common species (histogram; different species represented by different colors) in the WGS sequencing data, grouped by subject 
and sample type; for each patient (p1-p20) there were 2 samples: from left to right, first lesional and then healthy skin

Figure 1b: Relative abundances of the 25 most abundant genera (histogram; different genera represented by different colors) in the $16 \mathrm{~S}$ sequencing data, grouped by subject and sample type; for each patient (p1-p20) there were 2 samples: from left to right, first lesional and then healthy skin

Figure 2: Comparisons of the WGS and 16S microbiome sequencing data

Figure 2a: Alpha diversity comparisons of the WGS (left) and 16S (right) sequencing data; box plot comparisons of Shannon indices and inverse Simpson diversity, grouped by sample type. Lower and upper hinge of the box: $1^{\text {st }}$ and $3^{\text {rd }}$ quartile; whiskers: $1.5^{*}$ IQR; line: median, cross: mean; black points: actual values for each sample

Figure 2b: Bray-Curtis-dissimilarity of the WGS (left) and 16S (middle and right) sequencing data, non-metric multidimensional scaling (NMDS) ordination, colors correspond to lesion type or subjects, shapes to sample types. The closer two samples are on the plot, the more similar they are to one another

Figure S1: Pairwise Bray-Curtis dissimilarity, box-blot comparisons of the $16 \mathrm{~S}$ sequencing data; lower and upper hinge of the box: $1^{\text {st }}$ and $3^{\text {rd }}$ quartile; whiskers: $1.5^{*}$ IQR; line: median, cross: mean; black points: actual values for each sample

Figure S2: Relative abundances of the 25 most abundant genera (histogram; different genera represented by different colors) in the WGS sequencing data, grouped by subject and sample type; for each patient (p1-p20) there were 2 samples: from left to right, first lesional and then healthy skin 
Figure S3: Patient with cutaneous T cell lymphoma (mycosis fungoides); swab samples, were obtained from lymphoma affected lesional skin and of healthy appearing skin. The patient is consented to the publication of the image. 
a
WGS
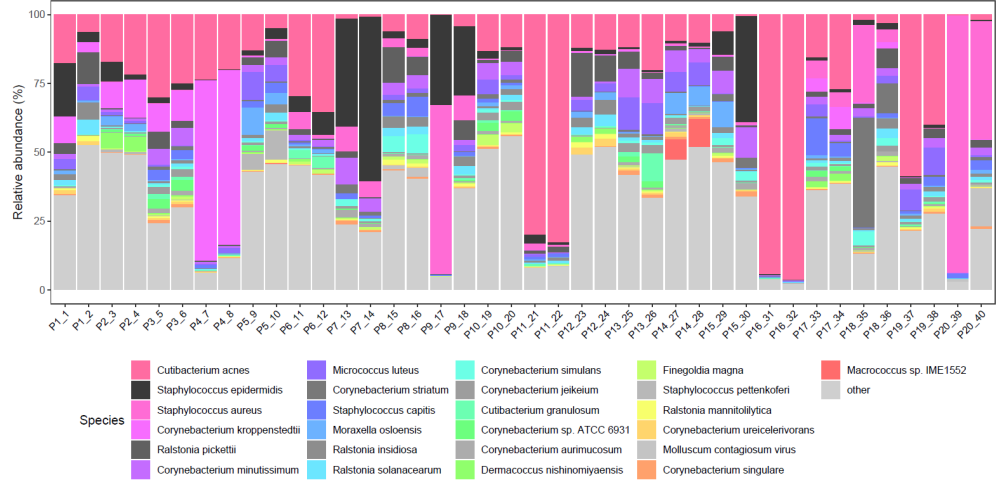

b
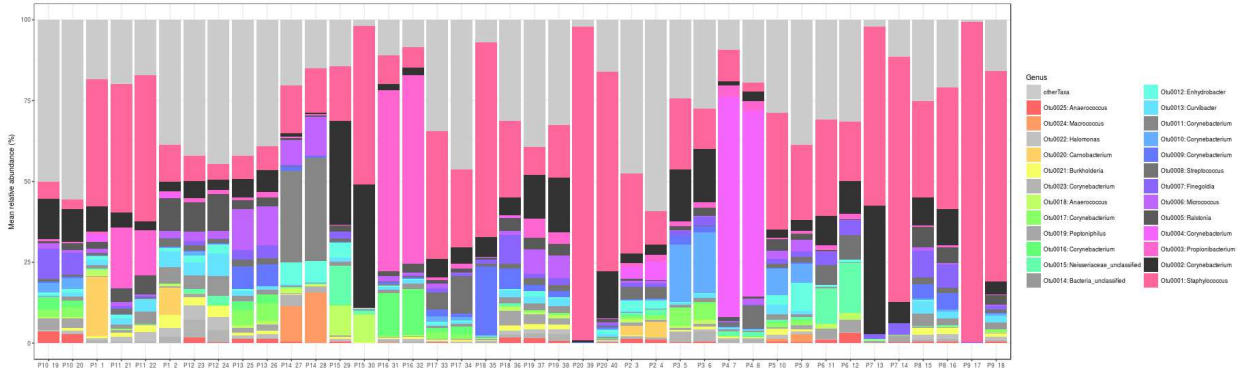
WGS
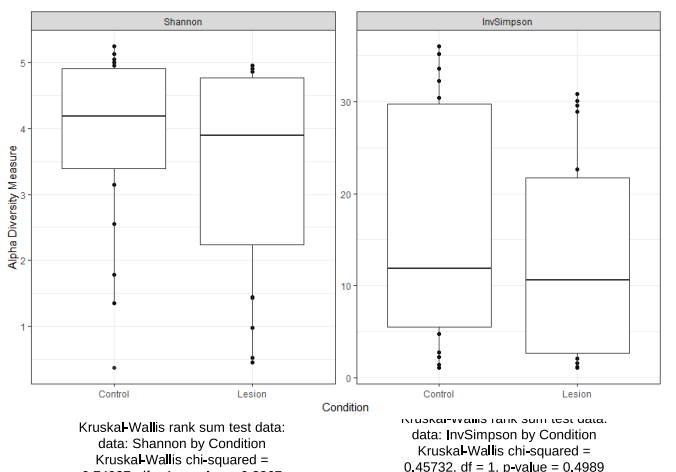

$16 S$
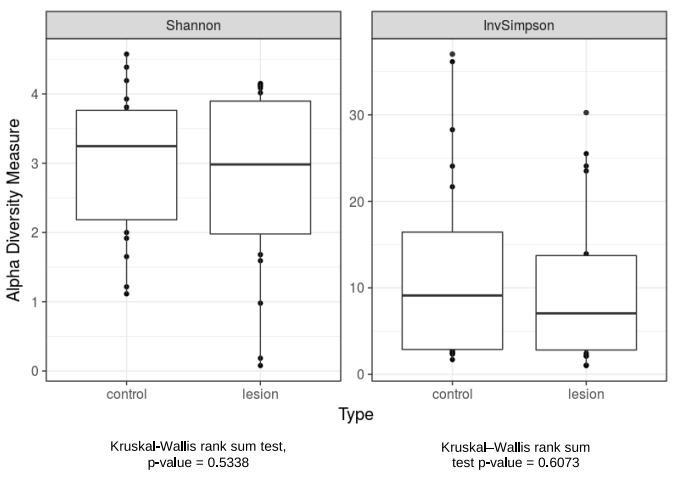

b

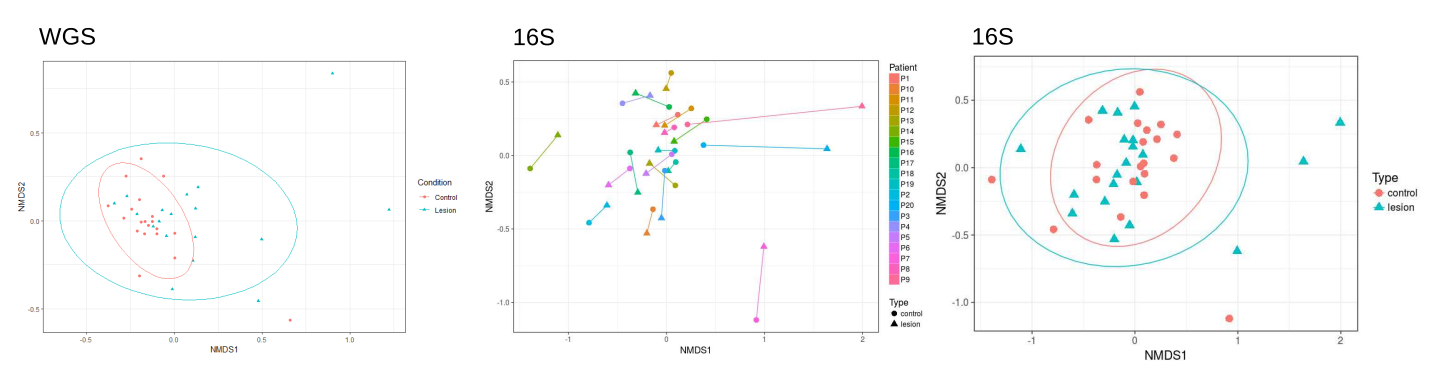




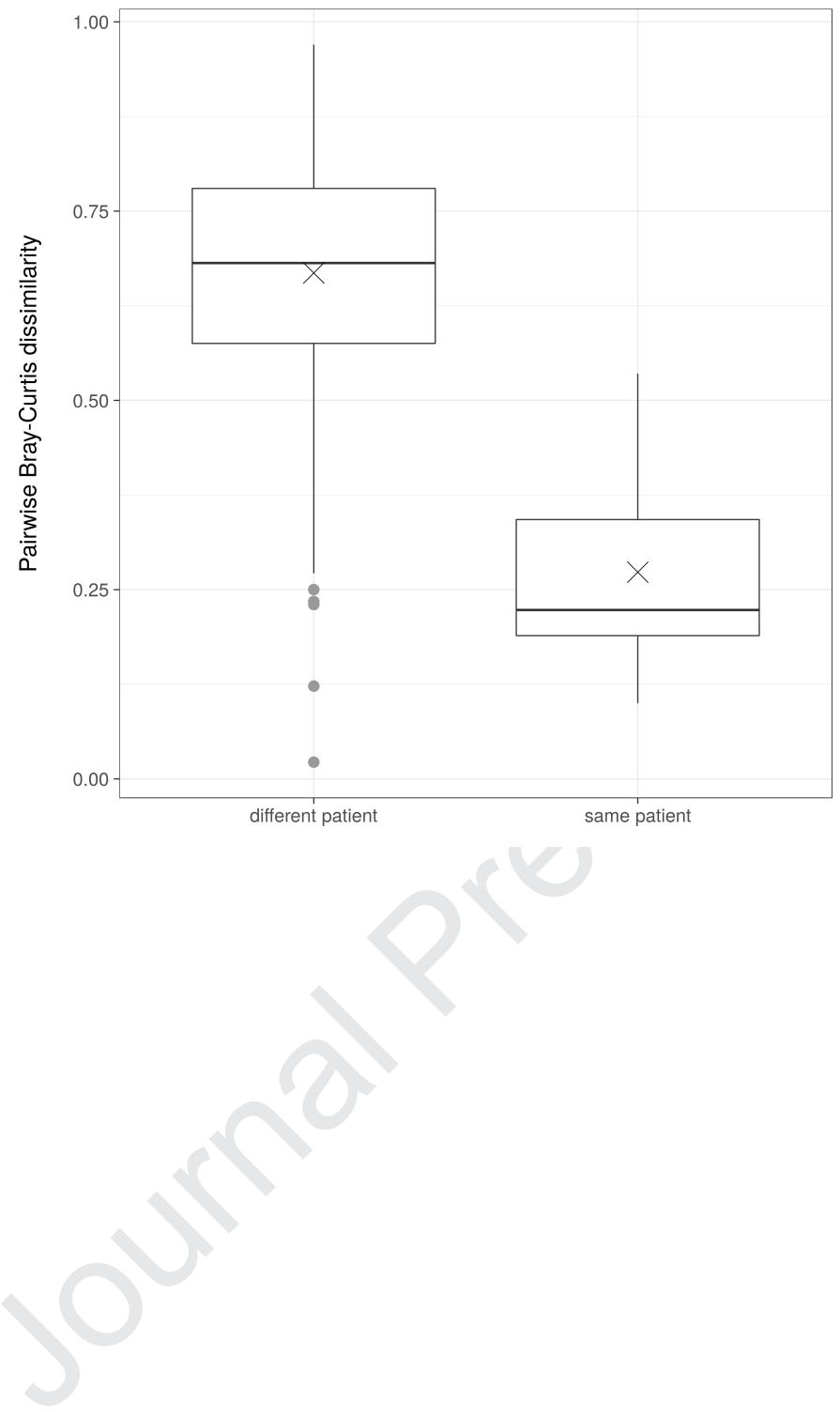




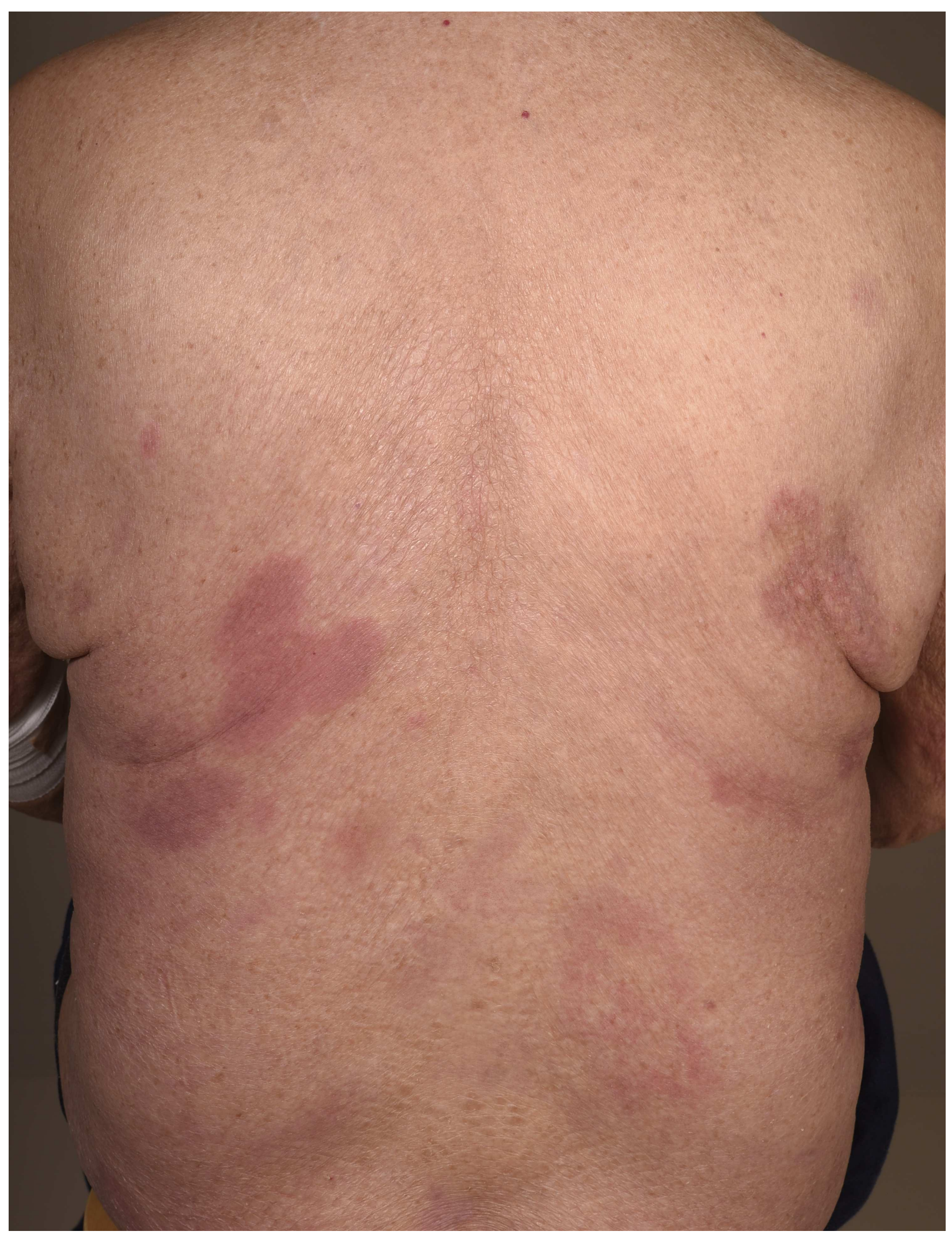




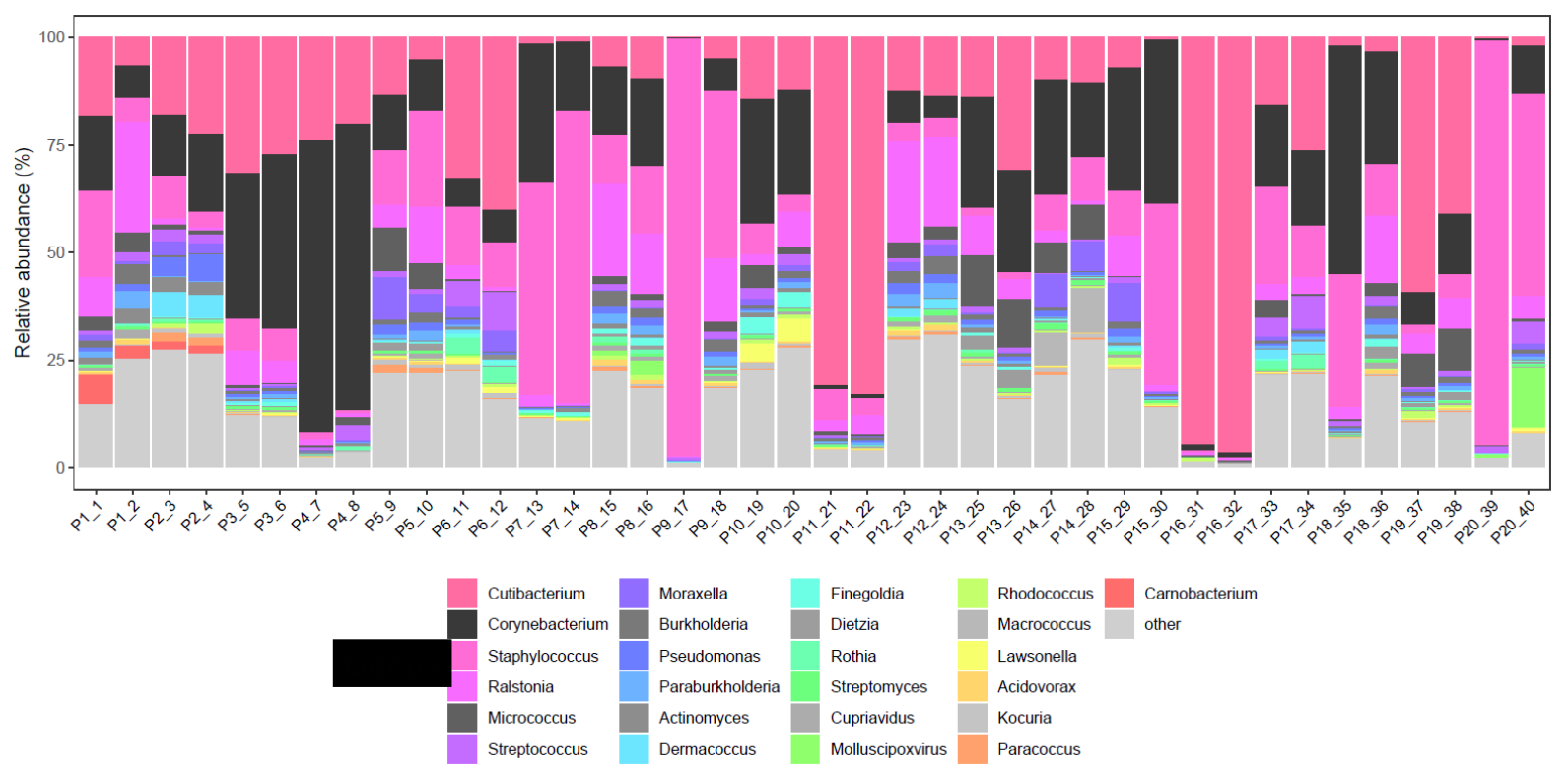




\section{Supplementary Text 1: Supplementary Outline to Methods}

\section{Skin preparatory regimen}

Microbiome sampling and the clinical part of the study was carried out in March-October 2017 in the Department of Dermatology and Allergology, Helsinki University Hospital, Helsinki, Finland.

Twenty consecutive patients with cutaneous T-cell lymphoma (CTCL) were included in the study. The patients had previously been diagnosed with CTCL (confirmed by histopathology, immunohistopathology and T-cell receptor clonality) and were under treatment and follow-up at the university hospital (Figure S3). Time of diagnosis, previous and current treatments at sampling are detailed in Table 1 (patient characteristics, supplementary material). All patients had given an informed consent. None of the patients had received antibiotic treatments during the previous 3 months. The patients were allowed to use topical basic emollient creams. Prior to the sampling procedure, the skin was not manipulated or specifically treated.

\section{Sampling procedure}

Microbiome samples were collected from the skin with a sterile swab (Copan Flocked Swab $^{\circledR}$, Copan Diagnostics Inc., Murrieta, CA, USA) by hands covered in sterile gloves. The swab used for sampling was first submerged gently into a sterile buffer solution $(0.15$ $\mathrm{mol} / \mathrm{l} \mathrm{NaCl}$ with $0.1 \%$ Tween 20 ), and subsequently brushed 20 times in crosswise directions over the sampled skin site (lesional and non-lesional sites, separately, of the same patient) (Salava et al., 2017). Lesional specimens were sampled directly from the 
surface of the CTCL patches and the borders of the different CTCL patches were not crossed. Prior to the sampling, the patients' skin was not manipulated or disinfected. Control samples (non-lesional samples) were collected analogously from clinically healthy appearing skin on the contralateral side of the body (non-lesional samples). We chose to use the patient's own healthy skin as the site for the control sample to avoid interindividual variation in the microbiome (Ursell et al., 2012, Kong et al., 2017, Flores et al., 2014).

To minimize the risk of sample cross-contamination, a new pair of sterile gloves was used for each separate sample. All sample specimens were placed immediately in sterile 1.5-ml Eppendorf ${ }^{\circledR}$ tubes (Eppendorf AG, Hamburg, Germany) and shock frozen in liquid nitrogen. The samples were conserved in an ambient temperature of $-80^{\circ} \mathrm{C}$ until the DNA extraction and sequencing procedures. The average storage time from sampling to analysis was six months (Klymiuk et al., 2016).

\section{DNA extraction methods and library preparation}

The DNA's were extracted as described in our earlier publication (Salava et al., 2016). In addition, the 16S rRNA gene sequencing was performed as described earlier (Aho et al., 2019).

The shotgun metagenomics libraries were constructed from the same DNA used for the 16S rRNA sequencing using Nextera XT library prep kit 1.5. ng as starting material.

\section{Sequencing protocol}




\section{Journal Pre-proof}

The 16S rRNA sequencing was made using Illumina MiSeq and for the shotgun metagenomics data was produced with Illumina NextSeq500 and NovaSeq 6000.

\section{Analysis of sequencing data}

16S paired end reads were first quality checked with fastQC (v0.11.2) (Andrews et al., 2019) followed by quality $(q=30)$, length ( $m=180)$, adapter and primer trimming (cutadapt v.1.8.3) (Martin et al., 2011). Filtered reads were subjected to mothur (1.39.5) following the Standard Operating Procedure (SOP) and additional changes as described in an earlier publication (Aho et al., 2019).

Whole Genome Sequencing reads in fastq format were quality checked with fastQC (v0.11.2) (Andrews et al., 2019) and then subjected to adapter trimming (cutadapt v1.15 and v1.17) (Martin et al., 2011) using the "--nextseq-trim=25" flag retaining only reads longer than 50 nucleotides and the quality score $>25$. Subsequently, the reads mapping to human genome (GCF_000001405.37_GRCh38.p11_genomic.fna database) were identified by the Burrows-Wheeler aligner (BWA-0.7.11) (Li et al., 2009) and removed from further analyses with the aid of samtools (v1.8) (Li, Handsaker et al., 2009). The reads from NextSeq500 and NovaSeq runs were combined and subjected to downstream analysis with BRACKEN (Bayesian Reestimation of Abundance with KrakEN) (v2.0) (Lu et al., 2017) using the KRAKEN2 (v2.0.7-beta) (Wood et al., 2014) taxonomic classification system (database GLIBCXX_3.4.21, 10.01.2019). BRACKEN analysis resulted in classification between $7.99-94.1 \%$ reads per sample. However, 0.04 to $37.11 \%$ of reads additionally mapped to human genome and were removed from the analysis, bringing the final fraction of classified reads to $31.18-94.13 \%$. The species and genera identifications ranged from 148 to 4116 and 88 to 1248 , respectively and were subjected to estimations 
of alpha and beta diversities with R! packages Phyloseq (McMurdie et al., 2013) and DESeq2 (Love et al., 2014).

Figure S3: The patient is consented to the publication of the image.

\section{References}

Aho VTE, Pereira PAB, Voutilainen S, Paulin L, Pekkonen E, Auvinen P et al. Gut microbiota in Parkinson's disease: Temporal stability and relations to disease progression. EBioMedicine 2019; 44:691-707.

Andrews S. Fast QC. A Quality Control tool for High Throughput Sequence Data, accessed 27.01.2020; http://www.bioinformatics.babraham.ac.uk/projects/fastqc/

Flores GE, Caporaso JG, Henley JB, Rideout JR, Domogala D, Chase J et al. Temporal variability is a personalized feature of the human microbiome. Genome Biol 2014; 15(12):531 .

Klymiuk I, Bambach I, Patra V, Trajanoski S, Wolf P. 16S Based Microbiome Analysis from Healthy Subjects' Skin Swabs Stored for Different Storage Periods Reveal Phylum to Genus Level Changes. Front Microbiol 2016; 7:2012.

Kong HH, Andersson B, Clavel T, Common JE, Jackson SA, Olson ND et al. Performing Skin Microbiome Research: A Method to the Madness. J Invest Dermatol 2017; 137(3):561-568. 
Li H, Durbin R. Fast and accurate short read alignment with Burrows-Wheeler transform. Bioinformatics 2009; 25(14):1754-1760.

Li H, Handsaker B, Wysoker A, Fennell T, Ruan J, Homer N et al. The sequence alignment/map format and SAMtools. Bioinformatics 2009; 25(16):2078-2079.

Love MI, Huber W, Anders S. Moderated estimation of fold change and dispersion for RNA-seq data with DESeq2. Genome biology 2014; 15(12):550.

Lu J, Breitwieser FP, Thielen P, Salzberg SL. Bracken: estimating species abundance in metagenomics data. PeerJ Computer Science 2017; 2;3:e104.

Martin M. Cutadapt removes adapter sequences from high-throughput sequencing reads. EMBnet journal 2011; 17(1):10-12.

McMurdie PJ, Holmes S. phyloseq: an R package for reproducible interactive analysis and graphics of microbiome census data. PloS one 2013; 8(4):e61217.

Salava A, Aho V, Pereira P, Koskinen K, Paulin L, Auvinen P et al. Skin microbiome in melanomas and melanocytic nevi. Eur J Dermatol 2016; 26:49-55.

Salava A, Pereira P, Aho V, Väkevä L, Paulin L, Auvinen P, Ranki A et al. Skin Microbiome in Small- and Large-plaque Parapsoriasis. Acta Derm Venereol 2017; 97(6):685-691. 
Ursell LK, Clemente JC, Rideout JR, Gevers D, Caporaso JG, Knight R. The interpersonal and intrapersonal diversity of human-associated microbiota in key body sites. J Allergy Clin Immunol 2012; 129(5):1204-1208.

Wood DE, Salzberg SL. Kraken: ultrafast metagenomic sequence classification using exact alignments. Genome biology 2014; 15(3):R46. 


\begin{tabular}{|c|c|c|c|c|c|c|c|c|}
\hline $\begin{array}{l}\text { Patient } \\
\text { nr. }\end{array}$ & $\begin{array}{l}\text { Stage } \\
\text { At the } \\
\text { time of }\end{array}$ & Sex & $\begin{array}{c}\text { Age } \\
(y)\end{array}$ & $\begin{array}{l}\text { Diagnosis } \\
\text { time } \\
\text { (years }\end{array}$ & $\begin{array}{l}\text { Sample site } \\
\text { Joury }\end{array}$ & $\begin{array}{l}\text { Therapy at sampling } \\
\text { (year of treatment } \\
\text { started) }\end{array}$ & $\begin{array}{l}\text { Previous therapies } \\
\text { (year of treatment) }\end{array}$ & Concomitant diseases \\
\hline & $\begin{array}{l}\text { sampling } \\
\text { (stage at } \\
\text { diagnosis) }\end{array}$ & & & $\begin{array}{c}\text { prior } \\
\text { sampling) }\end{array}$ & & & & \\
\hline 1 & $\mathrm{IA}(\mathrm{IA})$ & M & 96 & $2008(8)$ & right flank & MTX (since 2011) & EB (2014) & $\begin{array}{l}\text { hypothyreosis, asthma, } \\
\text { prostatic hyperplasia }\end{array}$ \\
\hline 2 & $\mathrm{IA}(\mathrm{IA})$ & $\mathrm{F}$ & 91 & $2012(4)$ & right flank & Bexarotene (since 2016) & $\begin{array}{l}\text { NB-UVB (2015, 2014, } \\
\text { 2013), PUVA (2015, } \\
\text { 2017) }\end{array}$ & $\begin{array}{l}\text { breast cancer (2010), } \\
\text { asthma, hypertension }\end{array}$ \\
\hline 3 & IIB (IIB) & M & 82 & 1987 (29) & right shin & Bexarotene (since 2005) & $\begin{array}{l}\text { oral/bath PUVA (2001, } \\
\text { 2002, 2004, } 2005, \\
\text { 2010), NB-UVB (2004, } \\
\text { 2005), IFNa (2017), }\end{array}$ & $\begin{array}{l}\text { atrial fibrillation, } \\
\text { hypertension, renal } \\
\text { insufficiency, benign } \\
\text { prostatic hyperplasia }\end{array}$ \\
\hline 4 & $\mathrm{IA}(\mathrm{IA})$ & M & 70 & 2002 & left flank & none & $\begin{array}{c}\text { oral/bath PUVA } \\
\text { (annually 2005 } 2009, \\
2011,2014) ; \text { EB }(2005, \\
\text { 2008) }\end{array}$ & $\begin{array}{c}\text { type II diabetes, } \\
\text { hypertension, } \\
\text { hypercholesterolemia }\end{array}$ \\
\hline 5 & IIB (IIB) & M & 76 & 2006 & $\begin{array}{l}\text { right } \\
\text { forearm }\end{array}$ & Bexarotene since 2007 & oral PUVA 2006, 2007 & $\begin{array}{c}\text { prostate carcinoma, } \\
\text { dyslipidemia, } \\
\text { hypothyreosis, anemia }\end{array}$ \\
\hline 6 & IIB (IIB) & M & 75 & 2011 & $\begin{array}{l}\text { left side of } \\
\text { the neck }\end{array}$ & Bexarotene since 2011 & $\begin{array}{c}\text { local PUVA 2015; EB } \\
(2012,2014,2015, \\
2016), \text { IFNa 2016-2017 }\end{array}$ & $\begin{array}{l}\text { type II diabetes, } \\
\text { hypercholesterolemia, }\end{array}$ \\
\hline 7 & IB (IIB) & M & 82 & 2008 & $\begin{array}{l}\text { left inguinal } \\
\text { fold }\end{array}$ & Bexarotene since 2014 & $\begin{array}{c}\text { NB-UVB }(2009,2011) \\
\text { bath PUVA }(2012, \\
2013) ; \text { EB }(2015,2017)\end{array}$ & $\begin{array}{c}\text { glaucoma, coronary } \\
\text { heart disease, renal } \\
\text { insufficiency }\end{array}$ \\
\hline 8 & IIB (IIB) & M & 53 & 2010 & $\begin{array}{l}\text { right } \\
\text { forearm }\end{array}$ & Bexarotene since 2010 & $\begin{array}{l}\text { IFNa 2013-2014, oral } \\
\text { PUVA 2015, 2016; EB } \\
\quad(2015-2016)\end{array}$ & $\begin{array}{l}\text { hypercholesterolemia, } \\
\text { hypertension, type II } \\
\text { diabetes, renal } \\
\text { insufficiency }\end{array}$ \\
\hline 9 & IA (IIA) & M & 52 & 2013 & right flank & Bexarotene since 2014 & $\begin{array}{l}\text { NB-UVB 2013, oral } \\
\text { PUVA } 2013\end{array}$ & $\begin{array}{c}\text { asthma, allergic } \\
\text { rhinoconjunctivitis, atopic } \\
\text { dermatitis }\end{array}$ \\
\hline 10 & IB (IB) & M & 74 & 2012 & left thigh & Bexarotene since 2013 & oral PUVA 2012, 2013 & $\begin{array}{c}\text { osteoporosis, coronary } \\
\text { heart disease }\end{array}$ \\
\hline 11 & IA (III) & M & 56 & 2016 & right flank & MTX since 2016 & NB-UVB 2016 & $\begin{array}{c}\text { sleep apnea, allergic } \\
\text { rhinoconjunctivitis, atopic } \\
\text { dermatitis }\end{array}$ \\
\hline 12 & IIB (IIB) & $\mathrm{F}$ & 63 & 2016 & right thigh & none & EB 2016 & $\begin{array}{c}\text { arterial hypertension, } \\
\text { diverticulitis, } \\
\text { hypercholesterolemia, } \\
\text { contact allergies }\end{array}$ \\
\hline 13 & $\mathrm{IA}(\mathrm{IA})$ & M & 21 & 2016 & right thigh & none & EB 2016 & none \\
\hline 14 & $\mathrm{IA}(\mathrm{IA})$ & $\mathrm{F}$ & 54 & 2017 & $\begin{array}{l}\text { right upper } \\
\text { arm }\end{array}$ & none & NB-UVB 2017 & $\begin{array}{l}\text { atopic dermatitis, contact } \\
\text { allergies, depression, } \\
\text { heart arrhythmias }\end{array}$ \\
\hline 15 & IB (IB) & $\mathrm{F}$ & 52 & 2010 & $\begin{array}{l}\text { abdomen } \\
\text { right }\end{array}$ & Bexarotene since 2017 & $\begin{array}{l}\text { NB-UVB 2011, } 2012 \text {, } \\
\text { 2016, oral PUVA } 2017\end{array}$ & $\begin{array}{l}\text { hypothyreosis, asthma, } \\
\text { atopic dermatitis }\end{array}$ \\
\hline 16 & $\mathrm{IA}(\mathrm{IA})$ & M & 43 & 2011 & right back & none & $\begin{array}{l}\text { MTX 2011-2012; oral } \\
\text { PUVA (2011, 2014, } \\
\text { 2016) }\end{array}$ & none \\
\hline 17 & IIA (IIA) & M & 63 & 2014 & left thigh & Bexarotene since 2016 & $\begin{array}{c}\text { MTX 2014-2015, } \\
\text { Acitretin 2015, NB-UVB } \\
\text { 2014, oral PUVA 2014, } \\
2015\end{array}$ & $\begin{array}{c}\text { peripheral } \\
\text { atherosclerosis, } \\
\text { hypothyreosis, } \\
\text { dyslipidemia, }\end{array}$ \\
\hline 18 & IIB (IIB) & $\mathrm{F}$ & 69 & 2014 & right thigh & Bexarotene since 2017 & $\begin{array}{c}\text { NB-UVB } 2016,2017, \\
\text { oral PUVA 2017, } \\
\text { Acitretin 2016-2017, } \\
\text { MTX } 2017\end{array}$ & $\begin{array}{l}\text { hypertension, atrial } \\
\text { fibrillation, asthma, } \\
\text { dyslipidemia, renal } \\
\text { insufficiency, coronary } \\
\text { artery disease, } \\
\text { adenocarcinoma of the } \\
\text { colon }\end{array}$ \\
\hline 19 & $\mathrm{IA}(\mathrm{IA})$ & M & 25 & 2016 & left buttock & none & bath PUVA 2016, 2017 & none \\
\hline 20 & IIB (IIB) & $\mathrm{F}$ & 42 & 2014 & $\begin{array}{l}\text { abdomen } \\
\text { left }\end{array}$ & Bexarotene since 2017 & $\begin{array}{c}\text { NB-UVB 2014, oral } \\
\text { PUVA 2016, 2017, MTX } \\
\text { 2014-2015, ECP } 2017\end{array}$ & $\begin{array}{c}\text { atopic dermatitis, } \\
\text { asthma, depression, } \\
\text { dyslipidemia, }\end{array}$ \\
\hline
\end{tabular}


Abbreviations:

F: Female

M: Male

LPP: Large plaque parapsoriasis

SPP: Small plaque parapsoriasis

NB-UVB: Narrow-band ultraviolet B phototherapy

SUP: Selective ultraviolet phototherapy

PUVA: Psoralen ultraviolet A photochemotherapy

ECP: Extracorporal photopheresis

MTX: Methotrexate

IFNa: Interferon-alpha

EB: Electron beam 


\begin{tabular}{|c|c|c|c|c|c|c|}
\hline \multirow[b]{2}{*}{ Streptomyces sp. SM17 } & \multicolumn{3}{|c|}{ baseMean log2FoldCh IfcSE } & stat & \multicolumn{2}{|l|}{ pvalue } \\
\hline & 11.13063 & -23.3284 & 1.95449 & -11.9358 & 7.70E-33 & 2.80E-29 \\
\hline Bordetella pertussis & 175.7464 & -27.0922 & 2.535417 & -10.6855 & 1.19E-26 & 2.17E-23 \\
\hline Streptomyces sp. PVA 94-07 & 13.5985 & -23.6034 & 2.506801 & -9.41573 & 4.70E-21 & 5.70E-18 \\
\hline Methylobacterium oryzae & 5.936652 & -22.4632 & 2.409511 & -9.32273 & 1.13E-20 & 1.03E-17 \\
\hline Serratia sp. LS-1 & 5.682808 & -22.4188 & 2.451911 & -9.14341 & $6.05 E-20$ & 4.40E-17 \\
\hline Burkholderia mallei & 15.64886 & -23.794 & 2.921648 & -8.14405 & $3.82 E-16$ & $2.32 \mathrm{E}-13$ \\
\hline Enterobacteriaceae bacterium ENNII & 15.55911 & -21.9864 & 2.921651 & -7.52535 & $5.26 E-14$ & 2.73E-11 \\
\hline Homo sapiens & 1029155 & 4.356723 & 0.650808 & 6.694328 & 2.17E-11 & 9.86E-09 \\
\hline Achromobacter ruhlandii & 93.17166 & -8.3322 & 1.779652 & -4.68193 & 2.84E-06 & 0.001149 \\
\hline Pseudomonas sp. A214 & 13.08098 & -5.67961 & 1.421667 & -3.99504 & 6.47E-05 & 0.02034 \\
\hline Staphylococcus argenteus & 306.9312 & 2.798081 & 0.7019 & 3.98644 & 6.71E-05 & 0.02034 \\
\hline Pseudomonas sp. St29 & 12.09194 & -5.43832 & 1.358924 & -4.00193 & $6.28 \mathrm{E}-05$ & 0.02034 \\
\hline Pectobacterium polaris & 9.264267 & -5.17848 & 1.42939 & -3.62 & 0.000 & 0.081559 \\
\hline Streptomyces sampsonii & 13.83509 & -5.89603 & 1.6699 & & 0.000 & 7747 \\
\hline Strepton & 2.27656 & -6.65078 & 1.933436 & & & 188 \\
\hline Clost & 86 & 1.293 & 0.380341 & & 0.000 & 514 \\
\hline Geoa & 51 & & & & & 494 \\
\hline Alcani & 38.09102 & -2.10179 & 0.641071 & & 0.001043 & 0.189844 \\
\hline Streptococcus dys & 2144.21 & 3.399 & 1.030 & 05 & 0.000 & 0.189844 \\
\hline Pseudom & 35 & -4.4 & 1.362 & & & 844 \\
\hline Kosakoni & 8.170031 & 189 & & 7448 & 529 & 6343 \\
\hline Rhod & 43 & & & -3.20 & & 343 \\
\hline Xylani & 31 & -0.9 & 0.385 & -2.5 & & 343 \\
\hline Acidir & 24.0 & -1.9 & 0.739527 & -2.695 & 0.007 & 0.196343 \\
\hline Geob & 37.93 & -1.6 & $0.610 t$ & -2.6551 & 0.007 & 0.196343 \\
\hline Burk & & -1.4 & 0.560 & -2.61 & & 343 \\
\hline Halc & & -2. & & & & 343 \\
\hline Bra & & & & & & 343 \\
\hline Xan & 42.16 & -1.39 & 0.5 & -2.54 & & 0.196343 \\
\hline Brac & & -1.1 & 67 & 88 & & 5343 \\
\hline Burkho & 323.0428 & -1.58 & & & & 343 \\
\hline Azoarc & 145.2645 & -1.50924 & 0.524426 & -2.87789 & 0.004004 & 0.196343 \\
\hline Candi & 56.99019 & -1.4227 & 0.534536 & -2.66157 & 0.007778 & 0.196343 \\
\hline Halotal & 38.55573 & -1.71937 & 0.6174 & -2.784 & & 0.196343 \\
\hline Komagataeibacte & 48.50589 & -2.53964 & 0.929082 & -2.73349 & 0.006267 & 0.196343 \\
\hline Fibrella sp. ES10 & & -2.59664 & 0.8973 & & & 0.196343 \\
\hline Hoefle & 24.45562 & -1.96692 & 0.747627 & -2.63089 & 0.008516 & 0.196343 \\
\hline Clostr & 30.6221 & 1.785897 & & 2.532727 & & 0.196343 \\
\hline Herbaspirillum seropedicae & 289.4831 & -1.41694 & 0.489881 & -2.89241 & 0.003823 & 0.196343 \\
\hline Clostridium saccharoperbutylacetoniı & 45.97916 & 2.081401 & 0.666279 & 3.12392 & 0.001785 & 0.196343 \\
\hline Collimonas arenae & 198.5254 & -1.48375 & 0.526266 & -2.81939 & 0.004812 & 0.196343 \\
\hline Bradyrhizobium sp. ORS 3257 & 301.391 & -1.46616 & 0.543122 & -2.69951 & 0.006944 & 0.196343 \\
\hline & 43.91913 & -1.78388 & 0.641396 & -2.78125 & 005415 & 0.196343 \\
\hline Burkholderia oklahomensis & 188.9955 & -1.48524 & 0.536293 & -2.76946 & 0.005615 & 0.196343 \\
\hline Halobacillus halophilus & 14.36545 & -2.52882 & 0.942481 & -2.68315 & 0.007293 & 0.196343 \\
\hline Serratia sp. FGI94 & 15.16026 & -2.59944 & 1.047014 & -2.48272 & 0.013038 & 0.196343 \\
\hline Kitasatospora setae & 113.3681 & -0.94484 & 0.371221 & -2.54522 & 0.010921 & 0.196343 \\
\hline Clostridium beijerinckii & & 1.458743 & & & & 0.196343 \\
\hline Nitrospira defluvii & 16.05457 & -2.46999 & 0.835069 & -2.95782 & 0.003098 & 0.196343 \\
\hline Dehalogenimonas formicexe & 11.84916 & -2.44892 & 0.944753 & -2.59213 & 0.009538 & 0.196343 \\
\hline
\end{tabular}




\begin{tabular}{|c|c|c|c|c|c|c|}
\hline Candidatus Promineofilum breve & 44.76118 & -1.4558 & 1895 & -2.63781 & & \\
\hline Halomonas chromatireducens & 6.37105 & -1.87294 & 0.677506 & -2.76446 & 0.005702 & 196343 \\
\hline Hyphomicrobium nitrativorans & 6.09128 & -2.02927 & 0.676522 & -2.99957 & 0.002704 & 0.196343 \\
\hline Cystobacter fuscus & 160.1816 & -0.99716 & 0.397365 & -2.50942 & 0.012093 & 196343 \\
\hline Rhizobium sp. CIAT894 & 38.12337 & -2.04519 & 0.681291 & -3.00193 & 0.002683 & 0.196343 \\
\hline Burkholderia seminalis & 228.542 & -1.38849 & 0.533723 & -2.60151 & 0.009281 & 0.196343 \\
\hline Borrelia miyamotoi & 34.58952 & 3.065197 & 0.959851 & 3.19341 & 0.001406 & 0.196343 \\
\hline Komagataeibacter eur & 4.05994 & -2.45152 & 0.856957 & -2.86073 & 0.004227 & 0.196343 \\
\hline Bradyrhizobiacea & 125.8117 & -1.578 & 0.530001 & -2.97736 & 0.002907 & 0.196343 \\
\hline Cloacibacillus porcorum & 13.28384 & -2.47327 & 0.841255 & -2.93997 & 0.003282 & 0.196343 \\
\hline ohataei & 11.15167 & -2.86186 & 0.996847 & -2.87091 & 0.004093 & 0.19 \\
\hline Desulfococcus mul & 16.9677 & -2.25732 & 0.908319 & -2.48516 & 0.012949 & 0.196343 \\
\hline Bordetella & 98.28672 & -1.36623 & 0.541886 & -2.52126 & 0.011693 & 0.196343 \\
\hline Sphae & 52.84899 & -1.11288 & 0.443506 & -2.50928 & 0.012098 & 6343 \\
\hline Agrobac & 42.34258 & -1.65882 & 0.558751 & -2.9688 & 0.00299 & 0.196343 \\
\hline Staph & 24.5651 & 1.604676 & 0.585072 & 2.742698 & 5094 & 196343 \\
\hline Echinicola strongylocentr & 10.8961 & -2.78248 & 1.111078 & -2.5043 & 0.012269 & 0.196343 \\
\hline Flavis & 353 & -1.55495 & 0.58524 & -2.6 & 885 & 343 \\
\hline Methylo & 9.542653 & -3.05097 & 1.04558 & -2.91797 & 0.003523 & 0.196343 \\
\hline Burkr & 346.9632 & -1.56201 & 0.583128 & -2.67867 & 0.007392 & 0.196343 \\
\hline Xant & 100.0469 & -1.29568 & 0.495599 & -2.61437 & 0.008939 & 0.196343 \\
\hline Haloa & 4.56142 & -4.30136 & 1.615647 & -2.66232 & 0.00776 & 0.196343 \\
\hline Paraburkł & 872.7312 & -1.33425 & 0.529802 & -2.5184 & 0.011789 & 0.196343 \\
\hline avidus pauculus & 600.1976 & -1.52855 & 0.536495 & -2.84915 & 0.004384 & 0.196343 \\
\hline Caulob & 64.59854 & -1.35925 & 0.541338 & -2.51091 & 0.012042 & 0.196343 \\
\hline Limnc & 56.01389 & -1.46264 & 0.571732 & -2.55826 & 0.01052 & 0.196343 \\
\hline Bacil & 15.79563 & -1.99788 & 0.795964 & -2.51001 & 0.012073 & 0.196343 \\
\hline Pand & 231.1461 & -1.39123 & 0.508649 & -2.73515 & 0.006235 & 0.196343 \\
\hline Phaeo & 5.04048 & -4.4424 & 1.63178 & -2.72242 & 0.006481 & 0.196343 \\
\hline Caul & 128.5123 & -1.39775 & 0.537736 & -2.59933 & 0.009341 & 0.196343 \\
\hline aera limnophila & 58.24474 & -1.64176 & 0.619919 & -2.64835 & 0.008089 & 0.196343 \\
\hline Bordetella genomosp. 13 & 171.8408 & -1.42804 & 0.511764 & -2.79043 & 0.005264 & 0.196343 \\
\hline Libanicoccus massiliensis & 34.76263 & -1.68336 & 0.6549 & -2.57041 & 0.010158 & 0.196343 \\
\hline Desulfuromonas sp. DDH964 & 33.46485 & -1.49592 & 0.559601 & -2.67318 & 0.007513 & 0.196343 \\
\hline Anae & 112. & -1.30889 & 0.482851 & -2.71076 & 0.006713 & 0.196 \\
\hline Alcanivor & 29.99751 & -1.68782 & 0.66568 & -2.53548 & 0.011229 & 0.196343 \\
\hline Grar & 60.71829 & -1.367 & 0.524337 & -2.6071 & 0.009131 & 0.196343 \\
\hline Geob & 20.31273 & -1.79568 & 0.717458 & -2.50284 & 0.01232 & 0.196343 \\
\hline Cyanobium sp. NIES-981 & 62.82027 & -1.64926 & 0.590786 & -2.79164 & 0.005244 & 0.196343 \\
\hline Collimon & 303.25 & -1.20633 & 0.466325 & -2.58689 & 0.009685 & 0.196343 \\
\hline Streptomyces venezuelae & 173.3353 & -1.00105 & 0.352431 & -2.84042 & 0.004505 & 0.196343 \\
\hline Sphingobium sp. RACO3 & 83.40695 & -1.57962 & 0.617487 & -2.55815 & 0.010523 & 0.196343 \\
\hline Thauera hydrothermalis & 230.7644 & -1.52682 & 0.59012 & -2.5873 & 0.009673 & 0.196343 \\
\hline Hermi & 81.576 & -1.661 & 0.594453 & -2.79416 & 0.005203 & 0.196343 \\
\hline Rhizobium sp. NXC14 & 32.35091 & -1.50045 & 0.567353 & -2.64466 & 0.008177 & 0.196343 \\
\hline Acidianus brierleyi & 3.810802 & 5.873655 & 2.362252 & 2.486464 & 0.012902 & 0.196343 \\
\hline Afipia sp. GAS231 & 268.1899 & -1.25968 & 0.48572 & -2.59343 & 0.009502 & 0.196343 \\
\hline Methylomicrobium album & 31.13957 & -1.79704 & 0.554142 & -3.24293 & 0.001183 & 0.196343 \\
\hline & 1671.054 & -1.30836 & 0.521105 & -2.51074 & 0.012048 & 0.196343 \\
\hline Desulfomicrobium baculatum & 18.5654 & -1.65919 & 0.649077 & -2.55623 & 0.010581 & 0.196343 \\
\hline
\end{tabular}


Enterobacteriaceae bacterium w6

Zoogloeaceae bacteirum Par-f-2

Synechococcus sp. KORDI-49

Aquabacterium olei

Lactobacillus paracollinoides

Nitratireductor basaltis

Burkholderia glumae

Methylococcus capsulatus

Ectothiorhodospira sp. BSL-9

Verrucomicrobium sp. GAS474

Sulfurimonas autotrophica

Limnohabitans sp. 63ED37-2

Pseudomonas cichorii

Ralstonia solanacearum

Klebsiella quasivariicola

Rhodobacteraceae bacterium BAR1

Tolumonas auensis

Campylobacter curvus

Phreatobacter cathodiphilus

Desulfomicrobium orale

Sulfuriferula sp. AH1

Bradyrhizobium diazoefficiens

Yoonia vestfoldensis

Staphylococcus simiae

Achromobacter denitrificans

Hyphomicrobium denitrificans

Bradyrhizobium lablabi

Pseudomonas alcaliphila

Burkholderia sp. MSMB617WGS

Paucibacter sp. KCTC 42545

Rhodopirellula baltica

Nitrosospira lacus

Pandoraea vervacti

Burkholderia sp. KJ006

Cupriavidus sp. USMAA1020

Hyphomonas sp. CACIAM 19H1

Bradyrhizobium sp. WSM471

Streptococcus intermedius

Paraburkholderia terrae

Sutterella megalosphaeroides

Acidobacterium capsulatum

Rhodoferax antarcticus

Helicobacter pylori

endosymbiont of unidentified scaly sı

Orrella dioscoreae

Nitrosomonas sp. AL212

Slackia heliotrinireducens

Woeseia oceani

Mariprofundus ferrinatatus

Bacteroides vulgatus
14.07228

46.89327

9.799147

463.8258

10.40526

17.78089

370.6321

65.39526

33.29973

41.90553

2.525458

224.2197

39.8636

28645.12

9.804078

20.05714

10.49797

7.36574

134.1645

20.82723

21.73259

616.6418

28.90207

130.2543

312.1598

73.60922

778.1702

50.58343

13.44642

830.5104

7.745615

9.527931

194.8922

104.0198

195.8952

25.58665

154.5223

376.4848

672.2825

46.04743

44.4798

147.2561

33.35474

26.98335

167.4957

6.719444

53.89719

13.09536

6.231009

401.5325
$-3.79666$

$-2.133$

$-2.77994$

$-1.50073$

$-2.49749$

$-2.51336$

$-1.30092$

$-1.68324$

$-1.38379$

$-1.42135$

5.674756

$-1.40161$

$-1.75597$

$-1.40229$

$-2.6105$

$-1.96732$

$-2.43148$

$-2.89137$

$-1.31321$

$-1.72157$

$-1.88121$

$-1.45138$

$-1.62121$

1.64278

$-1.21932$

$-1.55237$

$-1.49806$

$-1.77169$

$-3.74286$

$-1.36192$

$-2.67948$

$-1.97099$

$-1.37941$

$-4.89787$

$-2.19893$

$-1.30321$

$-1.94489$

$-1.71327$

$-1.50197$

$-1.55529$

$-1.88733$

$-1.62553$

1.630179

$-1.46161$

$-1.39352$

$-4.85083$

$-1.74528$

$-1.8653$

$-3.56207$

$-1.91495$
1.292994

0.713449

1.064303

0.562262

1.00224

0.854271

0.520039

0.584038

0.541784

0.572616

2.109546

0.557041

0.679745

0.518177

1.034611

0.787748

0.875856

1.157789

0.428734

0.632769

0.712539

0.527659

0.647394

0.532886

0.491065

0.552838

0.577733

0.69637

1.28299

0.516493

1.046617

0.791058

0.511609

1.887057

0.827682

0.490394

0.632814

0.61877

0.552332

0.590295

0.661765

0.523616

0.589567

0.574201

0.523026

1.865537

0.696206

0.727938

1.306778

0.729036

$-2.93633$

$-2.9897$

0.003321

0.196343

$-2.611980 .009002$

$-2.66910 .007606$

$-2.49191$

0.012706

$-2.94211$

0.00326

$-2.50158$

0.012364

$-2.882080 .003951$

$-2.55413$

0.010645

$-2.48221$

0.013057

2.690036

0.007144

$-2.516170 .011864$

$-2.583270 .009787$

$-2.706190 .006806$

$\begin{array}{ll}-2.52317 & 0.01163\end{array}$

-2.4974 0.012511

$\begin{array}{ll}-2.77612 & 0.005501\end{array}$

$-2.497320 .012514$

$-3.06298$

0.002191

$-2.72069$

0.006515

$-2.64015$

0.008287

$-2.7506$

0.005949

$-2.50421$

0.012272

3.082796

0.002051

$\begin{array}{ll}-2.483 & 0.013028\end{array}$

$-2.808$

0.004985

$-2.593$

0.009514

$\begin{array}{ll}-2.54418 & 0.010954\end{array}$

$-2.9173$

0.003531

$-2.63686$

0.008368

$-2.56014$

0.010463

$-2.49159$

0.012717

$-2.69623$

0.007013

$-2.59551$

0.009445

$-2.65673$

0.00789

$-2.65748$

0.007873

$-3.07339$

0.002116

$-2.76883$

0.005626

$-2.71932$

0.006542

$-2.63476$

0.00842

$-2.85196$

0.004345

$-3.10444$

0.001906

2.765046

0.005691

$-2.54546$

0.010913

$-2.664330 .007714$

$-2.60023$

0.009316

$-2.50685$

0.012181

$-2.56244$

0.010394

$-2.725840 .006414$

$-2.626690 .008622$

0.196343

0.196343

0.196343 
Melaminivora sp. SC2-9

Pseudomonas chlororaphis

Natrinema pellirubrum

Aeromonas hydrophila

Prevotella dentalis

Archangium gephyra

Desulfovibrio desulfuricans

Pelobacter carbinolicus

Pseudomonas stutzeri

Bradyrhizobium sp. ORS 285

Burkholderia insecticola

Xanthomonas albilineans

Achromobacter spanius

Halomonas subglaciescola

Phenylobacterium zucineum

Micromonospora auratinigra

Paraburkholderia graminis

Dechloromonas sp. HYN0024

Rhodospirillum rubrum

Sorangium cellulosum

Desulfovibrio magneticus

Azorhizobium caulinodans

Porphyrobacter neustonensis

Comamonas serinivorans

Thioalkalivibrio sulfidiphilus

Polaromonas sp. SP1

Herbaspirillum rubrisubalbicans

Acidiphilium multivorum

Halogeometricum borinquense

Nitrobacter hamburgensis

Candidatus Desulforudis audaxviator

Delftia tsuruhatensis

Myxococcus xanthus

Syntrophobacter fumaroxidans

Avibacterium paragallinarum

Oleiphilus messinensis

Pseudomonas kribbensis

Bradyrhizobium icense

Jeongeupia sp. USM3

Cupriavidus sp. USMAHM13

Gemmatirosa kalamazoonesis

Methylosinus trichosporium

Marinithermus hydrothermalis

Halomonas sp. GFAJ-1

Chitinophaga caeni

Micromonospora siamensis

Parvularcula bermudensis

Azoarcus sp. SY39

Clostridium pasteurianum

Staphylococcus cohnii
530.7087

346.14

8.01346

125.9879

151.5854

139.3558

46.58184

16.30186

864.7782

201.8567

514.9367

32.365

232.616

22.97807

151.7072

86.85877

547.9771

43.92386

54.6124

653.9289

74.54782

103.5418

38.38308

408.0872

57.55934

451.6687

206.71

88.90498

8.73868

83.39907

19.56913

852.7595

78.23294

17.62235

7.670832

4.585674

49.90942

439.9763

81.18859

244.7913

167.5696

62.60254

72.338

4.714566

29.49516

80.69973

6.772203

86.10917

87.59801

246.8407

0.555689

$-2.4889$

0.012814

0.196343

$-1.07389$

0.420959

$-2.55105$

0.01074

0.196343

$-3.30815$

1.062955

$-3.11222$

0.001857

0.196343

$-0.92644$

$-1.47196$

0.363369

$-2.54958$

0.010785

0.196343

$-1.24953$

0.554548

$-2.65434$

0.007946

0.196343

$-1.7721$

0.476581

$-2.62186$

0.008745

0.196343

$-2.39163$

0.573201

$-3.09159$

0.001991

0.196343

$-1.46074$

$-2.90018$

0.003729

0.196343

$-1.30802$

$-2.5736$

0.010065

0.196343

$-1.38841$

0.46873

$-2.79055$

0.005262

0.196343

$-1.97033$

0.688624

$-2.55477$

0.010626

0.196343

$-1.18994$

0.477815

$-2.86126$

0.00422

0.196343

$-1.98003$

0.782171

$-2.49038$

0.012761

0.196343

$-1.4449$

0.575502

$-2.53145$

0.011359

0.196343

$-1.44203$

0.536165

$-2.51068$

0.01205

0.196343

$-1.72889$

0.663914

$-2.68953$

0.007155

0.196343

$-1.60612$

0.64699

$-2.60409$

0.009212

0.196343

$-1.17882$

0.457787

$-2.48245$

0.013048

0.196343

$-1.38218$

0.463073

$-2.57503$

0.010023

0.196343

$-1.25487$

0.461292

$-2.98479$

0.002838

0.196343

$-1.11339$

0.438907

$-2.72034$

0.006521

0.196343

$-2.53673$

0.011189

0.196343

0.516069

$-2.66355$

0.007732

0.196343

$-1.32214$

0.477735

$-2.76751$

0.005649

0.196343

$-1.56289$

0.590131

$-2.6483$

0.008088

0.196343

$-1.39468$

0.474506

$-2.93922$

0.00329

0.196343

2.51995

0.011737

0.196343

$-1.3907$

0.55840

$-2.49048$

0.012757

0.196343

$-2.81975$

1.020782

$-2.76234$

0.005739

0.196343

$-1.54318$

$-2.65333$

0.597267

$-2.58373$

0.009774

0.196343

$-1.43036$

0.906596

$-2.92669$

0.003426

0.196343

$-1.745$

$-2.75617$

0.005848

0.196343

$-2.06723$

$-3.04206$

0.00235

0.196343

$-3.7284$

$-2.54942$

0.01079

0.196343

$-2.86799$

1.12326

$-2.52373$

0.011612

0.196343

$-2.55327$

0.010672

0.196343

$-1.89255$

0.672597

$-2.8138$

0.004896

0.196343

$-1.27757$

0.486048

$-2.62849$

0.008576

0.196343

$-1.4031$

0.495351

$-2.83255$

0.004618

0.196343

$-2.0279$

0.723443

$-2.80312$

0.005061

0.196343 
Bacterioplanes sanyensis

Chlorobaculum parvum

Burkholderiales bacterium GJ-E10

Neisseria weaveri

Maricaulis maris

Acidovorax sp. RAC01

Marinobacter similis

Burkholderia sp. BDU6

Rhizobium jaguaris

Burkholderia pseudomultivorans

Thauera chlorobenzoica

Simiduia agarivorans

Streptomyces pristinaespiralis

Verrucomicrobium spinosum

Pelobacter sp. SFB93

Dickeya chrysanthemi

Rhizobium gallicum

Cupriavidus sp. USMAA2-4

Mesorhizobium loti

Nitrosomonas sp. Is79A3

Phyllobacterium zundukense

Indioceanicola profundi

Geobacter sulfurreducens

Methyloceanibacter caenitepidi

Staphylococcus haemolyticus

Burkholderia lata

Thauera sp. K11

Cronobacter malonaticus

Methyloversatilis sp. RAC08

Methylocaldum marinum

Bradyrhizobium sp. 3 85S1MB

Amycolatopsis mediterranei

Variibacter gotjawalensis

Bacillus thuringiensis

Bosea sp. RAC05

Variovorax sp. PMC12

Herbaspirillum hiltneri

Vogesella sp. LIG4

Bdellovibrio bacteriovorus

Mesorhizobium australicum

Xanthomonas citri

Ralstonia insidiosa

Bordetella genomosp. 9

Pandoraea pnomenusa

Flammeovirgaceae bacterium 311

Novosphingobium tardaugens

Ralstonia pseudosolanacearum

Paenibacillus mucilaginosus

Comamonas kerstersii

Variovorax sp. PAMC 28711

$6.318311-2.72265$

$18.6759-2.39393$

$69.06494-1.53066$

$24.93216-2.61054$

24.08755

$-1.39143$

$499.9973-1.34832$

$15.03429-2.46615$

129.6112

$-1.37208$

41.52251

$-1.51884$

194.4964

$-1.72222$

80.80729

$-1.76771$

15.42822

$-2.40419$

71.37249

32.58723

14.86473

$-1.15036$

$-1.49856$

$-2.89653$

29.53323

$-1.4768$

64.92122

$-1.5053$

2076.938

188.4732

34.09056

$-1.44209$

$-1.39868$

0.879535

$-3.09555$

0.001964

0.196343

0.852885

$-2.80686$

0.005003

0.196343

$\begin{array}{llll}0.598051 & -2.55941 & 0.010485\end{array}$

0.196343

0.882842

$-2.95697$

0.003107

0.196343

0.527174

$-2.63941$

0.008305

0.196343

0.516196

$-2.61204$

0.009

0.196343

0.932603

$-2.64437$

0.008184

0.196343

0.542216

$-2.53051$

0.01139

0.196343

0.554866

$-2.73731$

0.006194

0.196343

0.690316

$-2.49483$

0.012602

0.196343

23.60907

$-2.43768$

$-1.3494$

75.73499

$-1.18126$

78.55701

$-1.87158$

23.13999

$-1.92898$

0.597558

$-2.95822$

0.003094

0.196343

0.962715

$-2.4973$

0.012514

0.196343

0.440128

$-2.61369$

0.008957

0.196343

0.570424

$-2.6271$

0.008612

0.196343

$4880.898 \quad 1.393972$

$-2.77163$

0.005578

0.196343

0.540514

$-2.73222$

0.006291

0.196343

0.557002

$-2.70251$

0.006882

0.196343

0.57161

$-2.52285$

0.011641

0.196343

0.52247

$-2.67706$

0.007427

0.196343

0.776372

$-3.13983$

0.00169

0.196343

$0.538014-2.50811$

0.012138

0.196343

0.464166

$-2.5449$

0.010931

0.196343

0.670111

$-2.79294$

0.005223

0.196343

0.707975

$-2.72465$

0.006437

0.196343

$593.7104-1.45066$

0.529892

2.63067

0.008522

0.196343

$300.7952-1.24063$

0.532045

$-2.72658$

0.006399

0.196343

$5.194957-4.48227$

1.756

$-2.83183$

0.004628

0.196343

$89.54638-1.23714$

0.478967

$-2.55167$

0.010721

0.196343

$61.65105-1.41895$

0.522653

$-2.58293$

0.009796

0.196343

$212.6693-1.45659$

0.532127

$-2.7149$

0.006629

0.196343

$150.3613-0.94555$

0.373936

$-2.7373$

0.006195

0.196343

$66.9483-1.44687$

0.552016

$-2.52865$

0.01145

0.196343

$408.4663 \quad 1.710771$

0.617422

6210

0.008765

0.196343

$202.3757-1.20094$

0.479803

770831

0.005591

0.196343

$783.9175-1.29925$

0.51974

$-2.503$

0.012315

0.196343

$195.0951-1.34192$

0.49114

$-2.4998$

0.012426

0.196343

$95.51681-1.37031$

0.488781

$-2.73226$

0.00629

0.196343

$18.19114-1.76293$

0.619738

$-2.80353$

0.005055

0.196343

$109.2947-1.33432$

0.452162

$-2.84464$

0.004446

0.196343

$160.7346-1.49156$

0.567915

$-2.95098$

0.003168

0.196343

$34006.42-1.28877$

$99.07822-1.28225$

0.511972

$-2.62638$

0.00863

0.196343

0.516904

$-2.51726$

0.011827

0.196343

$457.4967-1.30144$

0.524964

0.013115

0.196399

$11.29662-2.04949$

0.830815

$-2.4791$

0.013171

0.196436

40.0335

$-1.51988$

0.614828

$-2.46685$

0.013631

0.196828

$1307.526-1.52992$

0.617724

$-2.47204$

0.013434

0.196828 
Laribacter hongkongensis

Candidatus Accumulibacter phosphat Magnetospirillum sp. ME-1

Fluviicola taffensis

Rhizobacter gummiphilus

Methylobacterium sp. DM1

Bordetella sp. $\mathrm{H} 567$

Streptomyces sp. CdTB01

Bordetella genomosp. 8

Niabella soli

Chondromyces crocatus

Opitutaceae bacterium TAV5

Paraburkholderia caledonica

Paenibacillus lentus

Methylobacterium nodulans

Gibbsiella quercinecans

Cupriavidus basilensis

Pseudorhodoplanes sinuspersici

Turneriella parva

Lysobacter sp. TY2-98

Granulibacter bethesdensis

Serpentinomonas raichei

Rubrivivax gelatinosus

Bordetella holmesii

Methylocystis rosea

Herbaspirillum robiniae

Spiribacter curvatus

Acaryochloris marina

Rubinisphaera brasiliensis

Granulicella tundricola

Mycoplasma mycoides

Tsukamurella tyrosinosolvens

Thermaerobacter marianensis

Streptomyces sp. fd1-xmd

Hydrogenophaga crassostreae

Halomonas sp. SF2003

Micavibrio aeruginosavorus

Acetobacter ascendens

Streptomyces sp. Sge12

Cupriavidus pinatubonensis

Flavonifractor plautii

Roseateles depolymerans

Terriglobus roseus

Deinococcus irradiatisoli

Burkholderia stagnalis

Acidiferrobacter sp. SPIII_3

Bradyrhizobium sp. CCGE-LA001

Melaminivora sp. SC2-7

Halomonas aestuarii

Haloplanus aerogenes

72.32607

$-1.14238$

0.463195

$-2.46631$

0.013651

0.196828

98.84152

$-1.28656$

0.521839

$-2.46544$

0.013684

0.196828

45.13294

10.44445

$-1.30371$

0.52775

$-2.47031$

0.0135

0.196828

635.6745

0.948142

$-2.45492$

0.014092

0.197584

106.4633

51093

$-2.46063$

0.013869

0.197584

111.598

$-0.88674$

0.36079

$-2.45777$

0.01398

0.197584

98.73597

$-1.14131$

0.466009

$-2.44912$

0.01432

0.197584

107.2699

$-1.0769$

0.439436

$-2.45063$

0.014261

50.39957

0.604748

$-2.45791$

0.013975

0.197584

157.1191

0.704378

$-2.45362$

0.014143

0.197584

41.03438

$-1.2391$

$-2.4623$

0.013805

0.197584

822.5195

$-1.39786$

0.57078

$-2.44903$

0.014324

0.197584

8.076414

0.532091

$-2.44878$

0.014334

0.197584

177.6302

$-1.0607$

0.906282

$-2.44903$

0.014324

0.197584

36.95672

$-1.43357$

921.8826

$-1.30855$

0.433593

$-2.44631$

0.014433

0.197584

80.24367

$-1.21164$

11.45061

$-2.05169$

0.587465

$-2.44027$

0.014676

0.19819

99.51492

$-1.44371$

0.535887

$-2.44184$

0.014612

0.198812

55.69695

$-1.06865$

159.3291

$-1.30642$

892.5631

36.26807

$-1.24384$

0.498316

$-2.43147$

0.015038

0.198812

0.841585

$-2.43789$

0.014773

0.198812

0.59348

$-2.43262$

0.01499

0.198812

25.11555

$-1.33958$

0.438209

$-2.43868$

0.014741

0.198812

$0.537164-2.43208$

0.015013

0.198812

$313.6006-1.20622$

$-1.71369$

$-2.43531$

0.014879

0.198812

0.549257

$-2.43889$

0.014732

0.198812

0.705082

$-2.43048$

0.015079

0.198812

10.55038

$-2.10457$

17.17174

$-2.31005$

0.495185

$-2.43589$

0.014855

0.198812

12.27126

$-2.0772$

$-2.42827$

0.015171

0.198812

38.61704

$-1.41282$

0.86127

$-2.42586$

0.015272

0.199305

11.69433

3.515472

0.586031

$-2.41178$

0.015875

0.199911

312.9574

$-0.97728$

1.466213

$-2.41083$

0.015916

0.202292

74.04929

$-1.09508$

0.405718

2.397654

0.0165

0.202292

88.29179

$-1.18403$

0.453653

$-2.40876$

0.016007

0.202292

271.8213

$-1.26423$

0.495045

$-2.41391$

0.015782

0.202292

$-2.39176$

0.016768

0.202292

34.25502

$-1.60146$

0.525751

$-2.40462$

0.016189

0.202292

13.32489

$-2.36073$

0.668175

$-2.39677$

0.01654

0.202292

12.16727

$-2.43305$

0.986411

$-2.39325$

0.0167

0.202292

38.12095

$-2.09667$

1.019783

$-2.38585$

0.01704

0.202292

587.6781

$-1.16552$

878112

$-2.38771$

0.016954

0.202292

140.3928

$-1.56587$

0.484356

$-2.40634$

0.016113

0.202292

$727.0714-1.18944$

0.649592

$-2.41054$

0.015929

0.202292

92.70462

$-1.13419$

$-2.40158$ 
Sulfuricaulis limicola

Methanoculleus marisnigri

Prosthecochloris sp. GSB1

Burkholderia sp. LA-2-3-30-S1-D2

Myxococcus stipitatus

Burkholderia cepacia

Burkholderia sp. OLGA172

Meiothermus ruber

Symbiobacterium thermophilum

Massilia oculi

Stenotrophomonas sp. ZAC14D2_NAI

Rhizobium favelukesii

Allochromatium vinosum

[Pseudomonas] mesoacidophila

Caldisphaera lagunensis

Komagataeibacter saccharivorans

Paraburkholderia sp. SOS3

Aeromonas sp. CA23

Ensifer sojae

Geobacter anodireducens

Paraburkholderia caffeinilytica

Brachyspira pilosicoli

Candidatus Solibacter usitatus

Acidovorax cattleyae

Rhodoferax ferrireducens

Arcobacter skirrowii

Acidithiobacillus ferrooxidans

Hydrogenophaga sp. RAC07

Nocardiopsis alba

Burkholderia latens

Bradyrhizobium japonicum

Methanobrevibacter millerae

Streptomyces sp. CMB-StM0423

Dyadobacter fermentans

Leuconostoc lactis

Stenotrophomonas sp. SAU14A_NAIN

Pandoraea oxalativorans

Pseudomonas sp. K2W31S-8

Vulgatibacter incomptus

Marinobacterium aestuarii

Spiroplasma diminutum

Halobiforma lacisalsi

Pandoraea norimbergensis

Alicycliphilus denitrificans

Bosea sp. PAMC 26642

Acidovorax avenae

Myxococcus hansupus

Fusobacterium varium

Streptomyces bingchenggensis

Frankia casuarinae

\begin{tabular}{|c|c|c|c|c|c|}
\hline & & & & & \\
\hline 50425 & -2.31261 & 970022 & -2.38408 & 0.017122 & 0.202292 \\
\hline & -2.45337 & 1.023023 & -2.39815 & 0.016478 & 0.202292 \\
\hline 5363 & -1.35791 & 0.566951 & -2.39511 & 0.016615 & 0.202292 \\
\hline .9556 & -1.3199 & 0.552116 & -2.39061 & 0.01682 & 0.202292 \\
\hline 00.831 & -1.27235 & 0.529857 & -2.4013 & 0.016337 & 0.202292 \\
\hline 7.943 & -1.28067 & 0.5352 & -2.39288 & 0.016717 & 0.202292 \\
\hline 2.76638 & -1.36185 & 0.568575 & -2.39519 & 0.016612 & 0.202292 \\
\hline 9.65952 & -1.3765 & 0.577803 & -2.38229 & 0.017205 & 0.20262 \\
\hline 5.1598 & -1.22347 & 0.517694 & -2.36331 & 0.018112 & 0.202794 \\
\hline .89313 & -2.74758 & 1.165615 & -2.35719 & 0.018414 & 0.202794 \\
\hline 0.84777 & -1.52434 & 0.64236 & -2.37303 & 0.017643 & 0.202794 \\
\hline 4.25316 & -1.20139 & 0.507746 & -2.36613 & 0.017975 & 0.202794 \\
\hline 66.3604 & -1.32274 & 0.561459 & -2.3559 & 0.018478 & 0.202794 \\
\hline 200222 & 5.708873 & 2.414693 & 2.364224 & 0.018068 & 0.202794 \\
\hline 16.95182 & -2.22765 & 0.939903 & -2.37009 & 0.017784 & 0.202794 \\
\hline 896.3952 & -1.32098 & 0.559599 & -2.36059 & 0.018246 & 0.202794 \\
\hline 23.10963 & -1.69039 & 0.716875 & -2.358 & 0.018374 & 0.202794 \\
\hline 53.27381 & -1.56081 & 0.660339 & -2.36364 & 0.018096 & 0.202794 \\
\hline 14.57521 & -2.06471 & 0.875236 & -2.35904 & 0.018322 & 0.202794 \\
\hline 1408.339 & -1.29361 & 0.547206 & -2.36403 & 0.018077 & 0.202794 \\
\hline 20.42504 & 2.281033 & 0.968418 & 2.355423 & 0.018502 & 0.202794 \\
\hline 72.20712 & -1.17658 & 0.498647 & -2.35955 & 0.018297 & 0.202794 \\
\hline 307.7678 & -1.33006 & 0.559122 & -2.37884 & 0.017367 & 0.202794 \\
\hline 238.1661 & -1.27238 & 0.53884 & -2.36134 & 0.018209 & 0.202794 \\
\hline 6.906604 & 4.352584 & 1.834955 & 2.372039 & 0.01769 & 0.202794 \\
\hline 11.79577 & -2.27788 & 0.966027 & -2.35799 & 0.018374 & 0.202794 \\
\hline 538.9125 & -1.16306 & 0.492589 & -2.36112 & 0.01822 & 0.202794 \\
\hline 93.10457 & -0.8891 & 0.374926 & -2.3714 & 0.017721 & 0.202794 \\
\hline 91.47003 & -1.84321 & 0.781502 & -2.35855 & 0.018346 & 0.202794 \\
\hline 561.6976 & -1.2269 & 0.518545 & -2.36603 & 0.01798 & 0.202794 \\
\hline 4.577509 & 4.715655 & 1.989541 & 2.370223 & 0.017777 & 0.202794 \\
\hline 75.64777 & -1.38736 & 0.58964 & -2.35289 & 0.018628 & 0.202956 \\
\hline 41.77275 & -1.36773 & 0.581105 & -2.35366 & 0.018589 & 0.202956 \\
\hline 8.483363 & -4.15686 & 1.768478 & -2.35053 & 0.018747 & 0.203641 \\
\hline 103.0302 & -1.40888 & 0.600373 & -2.34668 & 0.018941 & 0.203722 \\
\hline 159.694 & -1.36717 & 0.582173 & -2.3484 & 0.018854 & 0.203722 \\
\hline 76.13582 & -1.18352 & 0.504495 & -2.34596 & 0.018978 & 0.203722 \\
\hline 92.70183 & -1.36437 & 0.581285 & -2.34716 & 0.018917 & 0.203722 \\
\hline 32.02492 & -1.42719 & 0.612372 & -2.33059 & 0.019775 & 0.204157 \\
\hline 5.144539 & 5.815593 & 2.488535 & 2.336955 & 0.019442 & 0.204157 \\
\hline 13.41586 & -1.60807 & 0.689792 & -2.33124 & 0.019741 & 0.204157 \\
\hline 215.7293 & -1.25139 & 0.536348 & -2.33316 & 0.01964 & 0.204157 \\
\hline 1485.121 & -1.18972 & 0.510265 & -2.33157 & 0.019723 & 0.204157 \\
\hline 126.1238 & -1.14966 & 0.492794 & -2.33295 & 0.019651 & 0.204157 \\
\hline 1126.936 & -1.27986 & 0.547823 & -2.33626 & 0.019478 & 0.204157 \\
\hline 83.00739 & -1.28108 & 0.54952 & -2.33128 & 0.019739 & 0.204157 \\
\hline 36.61048 & 1.412095 & 0.60604 & 2.330037 & 0.019804 & 0.204157 \\
\hline 119.8143 & -1.07916 & 0.460452 & -2.3437 & 0.019094 & 0.204157 \\
\hline 46.0501 & -1.19787 & 0.51174 & -2.34078 & 0.019243 & 0.204157 \\
\hline
\end{tabular}




\begin{tabular}{|c|c|c|c|c|c|c|}
\hline Desulfurivibrio alkaliphilus & 17.08433 & -1.9051 & 0.816943 & -2.33198 & 0.019702 & \\
\hline Streptomyces niveus & 143.765 & -1.10662 & 0.472943 & -2.33986 & 0.019291 & 0.204157 \\
\hline Massilia violaceinigra & 214.8186 & -1.10891 & 0.474167 & -2.33865 & 0.019353 & 0.204157 \\
\hline Bradyrhizobium oligotrophicum & 11.7548 & -1.15966 & 0.498163 & -2.32787 & 0.019919 & 0.204184 \\
\hline Pseudomonas citronellolis & 186.3447 & -0.99809 & 0.428657 & -2.32841 & 0.01989 & 0.204184 \\
\hline Rhodospirillum centenum & 87.50121 & -1.22613 & 0.527862 & -2.32282 & 0.020189 & 0.204793 \\
\hline Streptomyces albireticuli & 102.0943 & -0.95925 & 0.413017 & -2.32255 & 0.020204 & 0.204793 \\
\hline Hyphomonas sp. Mor2 & 18.84229 & -2.01654 & 0.867317 & -2.32504 & 0.02007 & 0.204793 \\
\hline Bradyrhizobium sp. 2 39S1MB & 213.4903 & -1.27725 & 0.549916 & -2.32262 & 0.0202 & 0.204793 \\
\hline Pseudoflavitalea sp. 5GH32-13 & 85.21835 & -1.09877 & 0.473898 & -2.31857 & 0.020418 & 205672 \\
\hline Marinobacter sp. LQ44 & 15.00942 & -1.64446 & 0.709176 & -2.31884 & 0.020404 & 0.205672 \\
\hline Acidisp & 68.75947 & -1.23907 & 0.534587 & -2.31781 & 0.02046 & 0.205672 \\
\hline Bordet & 52.90601 & -1.30097 & 0.576447 & -2.25687 & 0.024016 & 0.207049 \\
\hline Burkhol & 491.8471 & -1.26383 & 0.549408 & -2.30034 & 0.021429 & 0.207049 \\
\hline Burkholderia diffusa & 154.9107 & -1.39859 & 0.621253 & -2.25124 & 0.02437 & 0.207049 \\
\hline Dyella jiaı & 95.54478 & -1.18005 & 0.513527 & -2.29793 & 0.021566 & 0.207049 \\
\hline Streptomyces sp. TLI_053 & 89.22485 & -0.92391 & 0.406807 & -2.27112 & 0.023139 & 0.207049 \\
\hline Pseud & 44.155 & -1.36808 & 0.596146 & -2.29488 & & 049 \\
\hline chaeum sulfurireducens & 7.345483 & -2.55 & 1.118746 & -2.27933 & 0.022647 & 0.207049 \\
\hline Sand & 189.9124 & -1.04098 & 0.456313 & -2.28128 & 0.022532 & 0.207049 \\
\hline Niast & 147.1089 & -1.22531 & 0.541976 & -2.26082 & 0.023771 & 0.207049 \\
\hline Paraburkholderia rhizoxinica & 120.9353 & -1.37083 & 0.609761 & -2.24814 & 0.024567 & 0.207049 \\
\hline Streptomyces spongiicola & 48.2067 & -1.11222 & 0.488226 & -2.27808 & 0.022722 & 0.207049 \\
\hline Cupr & 434.609 & -1.15679 & 0.514664 & -2.24766 & 0.024598 & 0.207049 \\
\hline Pseud & 24.86022 & -1.45961 & 39922 & -2.28091 & 0.022554 & 7049 \\
\hline Paral & 4662.431 & -1.30712 & 0.579675 & -2.25492 & 0.024138 & 0.207049 \\
\hline Pand & 182.6276 & -1.07912 & 0.48022 & -2.24714 & 0.024631 & 7049 \\
\hline Tistr & 138.577 & -1.2071 & 0.527561 & -2.28807 & 0.022134 & 0.207049 \\
\hline Vibric & 5.493461 & -3.45237 & 1.515487 & -2.27806 & 0.022723 & 0.207049 \\
\hline Pseudom & 21.58523 & -1.32009 & 0.571588 & -2.3095 & 0.020916 & 0.207049 \\
\hline Streptomyces sp. Z022 & 69.60653 & -1.19521 & 0.523767 & -2.28195 & 0.022492 & 0.207049 \\
\hline Methylorubrum extorquens & 351.4212 & -0.82498 & 0.364623 & -2.26255 & 0.023663 & 0.207049 \\
\hline Azospirillum sp. TSH58 & 106.3105 & -1.28316 & 0.572437 & -2.24157 & 0.024989 & 0.207049 \\
\hline Clostridium pe & 93.05474 & 1.087757 & 0.483845 & 2.248152 & 0.024566 & 0.207049 \\
\hline gamı & 17.99443 & -1.52144 & 0.671886 & -2.26443 & 0.02 & 049 \\
\hline Acidovor & 874.9685 & -1.20444 & 0.537329 & -2.24153 & 0.024992 & 0.207049 \\
\hline Pseu & 62.03114 & -1.31282 & 0.569292 & -2.30606 & 0.021107 & 0.207049 \\
\hline Phyci & 56.21903 & -1.32056 & 0.570884 & -2.31319 & 0.020712 & 0.207049 \\
\hline Dokdonella koreensis & 227.9516 & -1.2239 & 0.538304 & -2.27362 & 0.022989 & 0.207049 \\
\hline Actinop & 163.6303 & -1.00946 & 0.448933 & -2.24857 & 0.02454 & 0.207049 \\
\hline Blastochloris viridis & 81.45372 & -1.31423 & 0.575012 & -2.28557 & 0.022279 & 0.207049 \\
\hline Klebsiella quasipn & 26.00474 & -2.88798 & 1.272666 & -2.26924 & 0.023254 & 0.207049 \\
\hline Gordonia terrae & 446.0852 & -1.14564 & 0.510345 & -2.24483 & 0.024779 & 0.207049 \\
\hline Bordetella petrii & 194.2214 & -1.24826 & 0.541911 & -2.30344 & 0.021254 & 0.207049 \\
\hline Variovorax paradoxus & 2160.775 & -1.10443 & 0.486914 & -2.26822 & 0.023316 & 0.207049 \\
\hline Synechococcus sp. KORDI-52 & 12.75959 & -2.57546 & 1.138921 & -2.26132 & 0.02374 & 0.207049 \\
\hline Halomonas elongata & 36.63961 & -1.17173 & 0.514515 & -2.27734 & 0.022766 & 0.207049 \\
\hline Thiomonas sp. X19 & 164.4631 & -1.30196 & 0.570676 & -2.28144 & 0.022522 & 0.207049 \\
\hline Cupriavidus taiwane & 2661.351 & -1.14565 & 0.504767 & -2.26965 & 0.023229 & 0.207049 \\
\hline Frankineae bacterium MT45 & 72.29022 & -0.95977 & 0.426573 & -2.24995 & 0.024452 & 0.207049 \\
\hline
\end{tabular}


Magnetospira sp. QH-2

Microbulbifer thermotolerans

Rubrobacter indicoceani

Clostridium kluyveri

Gemmata obscuriglobus

Planococcus sp. PAMC 21323

Blastomonas fulva

Devosia sp. 1507

Streptomyces glaucescens

Gluconacetobacter diazotrophicus

Verminephrobacter eiseniae

Burkholderiales bacterium JOSHI_001

Rhodoplanes sp. Z2-YC6860

Cupriavidus metallidurans

Methylocystis sp. SC2

Pseudomonas brenneri

Streptomyces dengpaensis

Mycolicibacterium vaccae

Azoarcus sp. CIB

Burkholderia sp. CCGE1002

Staphylococcus kloosii

Burkholderia gladioli

Thioflavicoccus mobilis

Sulfuricella denitrificans

Nitrosospira briensis

Dyella japonica

Nitratireductor sp. OM-1

Ketobacter alkanivorans

Paraburkholderia aromaticivorans

Azospirillum brasilense

Nitrospira japonica

Fuerstia marisgermanicae

Herbaspirillum sp. meg3

Croceicoccus marinus

Klebsiella michiganensis

Pseudomonas sp. MT-1

Vitreoscilla filiformis

Arachidicoccus sp. KIS59-12

Clostridium butyricum

Pseudomonas sp. M30-35

Chitinophaga sp. MD30

Advenella mimigardefordensis

Xanthomonas vesicatoria

Paraburkholderia xenovorans

Variovorax sp. HW608

Streptomyces roseochromogenus

Ferriphaselus amnicola

Mycobacterium paragordonae

Paraburkholderia terricola

Pseudonocardia sp. HH130629-09
21.54949

10.06234

29.28155

49.92195

77.84845

12.47075

40.86759

42.61403

60.41927

79.87048

478.1433

615.5616

158.9039

1025.429

27.23241

35.91105

64.87857

241.5921

98.07296

995.4892

108.7361

1218.353

38.09932

41.94941

5.311836

138.7635

43.59432

7.076831

1501.028

286.403

25.01463

14.94313

118.6768

67.61156

46.22921

24.8451

132.945

53.90642

46.29946

16.04039

39.16005

23.49383

41.28131

1579.447

815.9538

62.8318

38.47835

202.2399

1155.138

218.6384
$-1.32597$

$-1.73026$

$-1.5244$

1.590392

$-1.26214$

$-2.50195$

$-2.00232$

$-1.5554$

$-1.06671$

$-1.16249$

$-1.2803$

$-1.18443$

$-1.14249$

$-1.28131$

$-1.52642$

$-1.34936$

$-1.27882$

$-0.99088$

$-1.32566$

$-1.24921$

1.333606

$-1.17298$

$-1.12509$

$-1.21972$

$-2.81338$

$-1.11279$

$-1.36691$

$-2.79016$

$-1.28671$

$-1.25411$

$-1.49415$

$-1.7711$

$-1.34741$

$-0.96149$

$-1.71237$

$-1.74812$

$-1.08561$

$-1.36371$

1.527253

$-1.45676$

$-1.32676$

$-1.4026$

$-1.30469$

$-1.16082$

$-1.1465$

$-1.02467$

$-1.29158$

$-0.8754$

$-1.21166$

$-0.99206$
0.584928

0.767933

0.673058

0.696327

0.557029

1.105961

0.892355

0.673716

0.469389

0.516166

0.558737

0.518698

0.508234

0.5542

0.662891

0.600479

0.560374

0.438341

0.588647

0.540537

0.594879

0.518937

0.500035

0.53713

1.220311

0.484147

0.600849

1.237851

0.574203

0.553107

0.653947

0.788822

0.590625

0.419203

0.748262

0.778618

0.475794

0.599962

0.678369

0.64711

0.593686

0.62712

0.583791

0.519192

0.512794

0.458586

0.579321

0.393076

0.544266

0.445912

$-2.26689$

0.023397

0.207049

$-2.25314$

0.02425

0.207049

$-2.26489$

0.02352

0.207049

2.283972

0.022373

0.207049

$-2.26584$

0.023461

0.207049

$-2.26224$

0.023683

0.207049

$-2.24386$

0.024841

0.207049

$\begin{array}{ll}-2.30868 & 0.020961\end{array}$

0.207049

$-2.27256$

0.023053

0.207049

$-2.25217$

0.024312

0.207049

$-2.291420 .021939$

0.207049

$-2.283470 .022403$

0.207049

$-2.247970 .024578$

0.207049

$-2.31199$

0.020778

0.207049

$-2.30267$

0.021297

0.207049

$\begin{array}{ll}-2.24714 & 0.024631\end{array}$

0.207049

$-2.28209$

0.022484

0.207049

$-2.26052$

0.023789

0.207049

$-2.25205$

0.024319

0.207049

$-2.31106$

0.02083

0.207049

2.241811

0.024974

0.207049

$-2.26035$

0.023799

0.207049

$-2.25002$

0.024448

0.207049

$-2.27081$

0.023159

0.207049

$-2.30546$

0.021141

0.207049

$-2.29845$

0.021536

0.207049

$-2.27497$

0.022908

0.207049

$-2.25404$

0.024194

0.207049

$-2.24087$

0.025035

0.207049

$-2.26739$

0.023367

0.207049

$-2.28482$

0.022323

0.207049

$-2.24525$ 
Paraburkholderia caribensis

Enterococcus casseliflavus

Gloeobacter violaceus

Pandoraea sputorum

Polaromonas sp. JS666

Dyella thiooxydans

Nocardiopsis dassonvillei

Moorella thermoacetica

Rhodopseudomonas palustris

Conexibacter woesei

Tsukamurella paurometabola

Burkholderia ambifaria

Pseudomonas sp. GR 6-02

Pseudomonas sp. CC6-YY-74

Mycobacterium sp. EPa45

Rhizobium sp. N324

Nonlabens spongiae

Streptomyces albus

Neisseria meningitidis

Marichromatium purpuratum

Mitsuaria sp. 7

Bradyrhizobium sp.

Halomonas beimenensis

Spiroplasma corruscae

[Polyangium] brachysporum

Simplicispira suum

Methylomonas methanica

Ottowia sp. oral taxon 894

Kushneria konosiri

Burkholderia sp. CCGE1003

Sinorhizobium medicae

Desulfovibrio sp. FW1012B

Pseudonocardia sp. AL041005-10

Pseudomonas sp. DY-1

Pseudogulbenkiania sp. NH8B

Roseomonas gilardii

Pseudomonas sp. LTJR-52

Mycobacterium shigaense

Burkholderia sp. PAMC 26561

Rhodoferax koreense

Paraburkholderia phymatum

Pseudomonas furukawaii

Leadbetterella byssophila

Agrobacterium rhizogenes

Epibacterium mobile

Martelella mediterranea

Massilia sp. NR 4-1

Burkholderia sp. BDU8

Filimonas lacunae

Curvibacter sp. AEP1-3

$1432.258-1.14455$

12.2771

$-3.12669$

0.514793

$-2.22332$

0.026194

0.211148

74.51494

$-1.14632$

1.407654

$-2.22121$

0.026337

0.211148

150.0011

$-1.3528$

506.7319

$-1.21965$

0.5161

$-2.22112$

0.026343

0.211148

170.3471

$-1.13883$

0.608616

$-2.22275$

0.026233

0.211148

178.7999

$-0.91683$

549332

$-2.22025$

0.026402

0.211159

24.06321

$-1.42302$

1080.474

$-0.98865$

203.52

158.2708

$-1.04625$

0.513751

$-2.21669$

0.026644

0.212057

357.9768

$-0.89855$

0.413727

$-2.21603$

0.026689

0.212057

12.22931

$-1.25813$

0.642075

$-2.21629$

0.026672

0.212057

36.8397

$-2.70062$

0.446584

$-2.2138$

0.026842

0.212346

131.876

$-1.2552$

0.472426

$-2.21462$

0.026786

0.212346

6.643865

$-1.09187$

0.40666

$-2.20958$

0.027135

0.214193

10.88234

$-4.82893$

94.05979

$-2.02242$

0.570271

$-2.20621$

0.02737

0.214252

396.882

$-0.91791$

61.30216

$-1.42425$

1.222985

$-2.20822$

0.027229

0.214252

0.568589

$-2.20757$

0.027274

0.214252

0.494936

$-2.20609$

0.027378

0.214252

$-2.204560 .027485$

0.214633

$1177.453-1.09457$

$-1.00385$

0.917913

$-2.20328$

0.027575

0.214805

0.416748

$-2.20257$

0.027625

0.214805

0.647363

$-2.20007$

0.027802

0.215714

0.456696

$-2.19807$

0.027944

0.216357

284.1972

$-1.16957$

93.99516

$-1.22456$

0.499454

$-2.19152$

0.028414

0.217565

5.516335

420.1768

4.647944

0.55848

$-2.18843$

0.028638

0.217565

$-2.192660 .028332$

0.217565

$-1.20371$

2.122794

2.189541

0.028558

0.217565

$401.5206-1.08327$

0.549846

$-2.18917$

0.028584

0.217565

$9.418541-2.63202$

0.494885

$-2.18894$

0.028601

0.217565

118.6465

$-1.16531$

1.200651

$-2.19216$

0.028368

0.217565

26.15004

$-1.25064$

(50974

$-2.1946$

0.028187

0.217565

791.1143

$-1.25826$

$-2.18862$

0.028624

0.217565

$37.16478-1.41755$

0.575288

$-2.18718$

0.028729

0.217577

$41.8837-1.22765$

$-2.18615$

0.028805

0.217577

$268.1204-0.82524$

$-2.18278$

0.029052

0.217577

60.12014

$-1.42285$

$-2.18563$

0.028842

0.217577

106.4323

$-1.0661$

651563

$-2.18375$

0.028981

0.217577

$244.4759-1.09708$

0.488432

$-2.1827$

0.029058

0.217577

$39.49161-1.37652$

0.50239

$-2.18371$

0.028983

0.217577

107.4006

$-0.9245$

0.631514

$-2.17972$

0.029278

0.218328

360.7089

$-1.27965$

0.424069

$-2.18006$

0.029253

0.218328

952.406

$-1.18646$

0.588253

$-2.17533$

0.029605

0.218786

921.1521

$-1.20986$

0.54516

$-2.17635$

0.029529

0.218786

74.31384

$-1.09008$

0.556619

$-2.17358$

0.029737

0.218786

8.171851

$-1.9925$

$-2.17007$

0.030001

0.218786

49.09142

$-1.36312$

0.918088

$-2.17027$ 
Porphyrobacter sp. CACIAM 03H1

Oxalobacter formigenes

Serratia rubidaea

Brevibacterium siliguriense

Sodalis glossinidius

Stappia sp. ES.058

Azoarcus sp. BH72

Burkholderia sp. YI23

Mycolicibacterium rhodesiae

Bifidobacterium scardovii

Bradyrhizobium ottawaense

Frankia inefficax

Bosea vaviloviae

Actinoplanes derwentensis

Cyanobium gracile

Methanosaeta harundinacea

Ahniella affigens

Lysobacter maris

Serpentinomonas mccroryi

Propionibacterium freudenreichii

Pseudomonas knackmussii

Bosea sp. AS-1

Methylobacillus flagellatus

Yangia pacifica

Microbulbifer sp. A4B17

Serratia sp. FDAARGOS_506

Streptomyces reticuli

Burkholderia sp. Bp7605

Bacillus glycinifermentans

Pseudarthrobacter chlorophenolicus

Cedecea neteri

Methylibium petroleiphilum

Azotobacter chroococcum

Rhizobium sp. ACO-34A

Geobacter sp. DSM 9736

Pelodictyon luteolum

Mesorhizobium opportunistum

Luteibacter rhizovicinus

Pseudomonas resinovorans

Thauera humireducens

Xanthomonas arboricola

Paraburkholderia phytofirmans

Lysobacter enzymogenes

Burkholderia sp. JP2-270

Delftia acidovorans

Shewanella loihica

Streptomyces pratensis

Breoghania sp. L-A4

Atopobium parvulum

Elizabethkingia miricola
52.88924

11.03401

23.11728

251.8477

9.535456

50.63894

60.55231

232.2497

142.1426

44.67497

705.1545

120.9766

158.6424

158.0644

54.66859

4.251252

17.93602

109.1053

202.1545

480.2236

126.708

166.4367

15.28666

77.55608

9.880218

9.014885

66.62839

283.5671

13.85688

128.6262

48.42009

721.4686

123.5132

71.30616

16.26264

11.24908

108.2081

84.45217

133.9931

101.6069

33.08294

3818.717

265.9291

214.435

645.0632

21.78584

21.91969

59.97377

154.3651

6.285831
1.141510 .528216

$-1.91277$

$-2.14959$

0.88419

$-1.0039$

$-2.0307$

0.991558

0.463157

0.938719

$-1.06851$

0.493379

$-2.10991$

0.975983

$-1.31852$

0.610036

0.416217

$-1.14039$

417692

$-1.04994$

0.527931

$-1.15156$

0.48646

0.533924

$-0.84365$

0.391194

$-1.06875$

0.496019

$-3.37834$

1.56913

$-1.359$

0.631735

$-1.07897$

0.501684

$-1.05566$

0.490616

$-0.8299$

0.385444

$-0.99629$

0.464528

$-1.07156$

$-1.5215$

$-1.05126$

0.499414

0.708304

0.490091

$-1.66382$

0.775412

$-3.16417$

1.475276

$-1.17346$

0.546426

$-1.13564$

0.529694

$-2.0228$

0.94394

$-0.79499$

0.371332

$-1.12856$

0.527909

$-1.16035$

0.543339

$-1.12838$

0.528374

0.490202

$-1.04642$

0.841416

$-1.79071$

0.855906

0.547531

$-1.16607$

$-0.94394$

0.443163

$-1.16943$

0.54939

$-1.23789$

0.581333

0.668293

$-1.42246$

0.54984

0.450103

$-0.95679$

$-1.15583$

0.544537

$-1.30553$

0.614701

0.590557

$-1.25475$

1.424157

0.638998

$-1.35435$

$-1.33642$

0.6313

$-3.71969$

1.757861

$-2.16106$

$-2.1633$

0.03069

0.218985

-2.16790 .030167
0.16

0.218985

$-2.167510 .030196$

0.218985

$-2.16327 \quad 0.03052$

218985

$\begin{array}{lll}-2.16569 & 0.030335 & 0.218985\end{array}$

$\begin{array}{lll}-2.16183 & 0.030632 & 0.218985\end{array}$

$\begin{array}{llll}-2.16138 & 0.030666 & 0.218985\end{array}$

$\begin{array}{llll}-2.16225 & 0.030599 & 0.218985\end{array}$

$\begin{array}{llll}-2.1658 & 0.030327 & 0.218985\end{array}$

$\begin{array}{lll}-2.16011 & 0.030764 & 0.219082\end{array}$

$\begin{array}{llll}-2.15833 & 0.030902 & 0.219637\end{array}$

$\begin{array}{llll}-2.15679 & 0.031022 & 0.219732\end{array}$

$\begin{array}{lll}-2.1566 & 0.031037 & 0.219732\end{array}$

$\begin{array}{lll}-2.15465 & 0.031189 & 0.220383\end{array}$

$\begin{array}{lll}-2.153 & 0.031319 & 0.22044\end{array}$

$\begin{array}{lll}-2.15123 & 0.031458 & 0.22044\end{array}$

$\begin{array}{lll}-2.1507 & 0.0315 & 0.22044\end{array}$

$\begin{array}{lll}-2.15169 & 0.031422 & 0.22044\end{array}$

$\begin{array}{lll}-2.1531 & 0.031311 & 0.22044\end{array}$

$\begin{array}{llll}-2.14473 & 0.031974 & 0.220786\end{array}$

$-2.145630 .031902$

0.220786

$\begin{array}{ll}-2.14809 & 0.031707\end{array}$

$-2.145020 .031951$

0.220786

$-2.145720 .031895$

220786

$-2.14480 .031969$

0.220786

$-2.14753$

0.031751

0.220786

$-2.14396$

0.032036

0.220786

$-2.14293$

0.032119

0.220796

$-2.14093$

$-2.1378$

0.03228

0.220946

$-2.13559$

0.032533

0.221635

$-2.13556$

0.032712

0.222954

$-2.13467$

0.032715

0.223359

$-2.1282$

0.032788

223359

0.033319

$-2.1311$

0.03308

0.224119

$-2.129690 .033198$

0.224119

$\begin{array}{ll}-2.13 & 0.033171\end{array}$

$\begin{array}{ll}-2.1286 & 0.033287\end{array}$

224119

0.033221

0.224119

$-2.128490 .033296$

0.224119

$\begin{array}{ll}-2.1266 & 0.033453\end{array}$

0.224119

$-2.125720 .033526$

$-2.1226$

0.033788

0.224682

$-2.12385$

0.033683

0.224778

$-2.12469$

0.033612

0.224778

$-2.12304$

0.033751

0.224778

$-2.11948 \quad 0.03405$

$-2.116930 .034266$ 
Burkholderia pseudomallei

Mesorhizobium oceanicum

Rhodovulum sp. P5

Frateuria aurantia

Streptomyces cyaneogriseus

Desulfobulbus sp. ORNL

Burkholderia plantarii

Bernardetia litoralis

Anaeromyxobacter dehalogenans

Janibacter indicus

Geobacter bemidjiensis

Brachyspira intermedia

Hydrogenophaga sp. PBC

Aromatoleum aromaticum

Salipiger profundus

Synechococcus sp. PCC 7336

Massilia sp. WG5

Paraburkholderia phenoliruptrix

Aureimonas sp. AU20

Chelatococcus sp. CO-6

Burkholderia sp. NRF60-BP8

Mesorhizobium ciceri

Myxococcus macrosporus

Fimbriimonas ginsengisoli

Nitrosomonas ureae

Rhodobaca barguzinensis

Mycobacterium marseillense

Brenneria goodwinii

Sinorhizobium americanum

Cupriavidus nantongensis

Sphingomonas wittichii

Chromobacterium rhizoryzae

Citrobacter amalonaticus

Bradyrhizobium sp. SK17

Kitasatospora albolonga

Methylomonas koyamae

Marinobacter salarius

Streptosporangium roseum

Methyloceanibacter sp. wino2

Acidovorax citrulli

Halioglobus pacificus

Comamonas aquatica

Serratia proteamaculans

Paenibacillus ihbetae

Ramlibacter tataouinensis

Streptomyces sp. Mg1

Natronobacterium gregoryi

Streptomyces albulus

Myxococcus fulvus

Natronococcus occultus

\begin{tabular}{|c|c|c|c|c|c|}
\hline & & & & & \\
\hline 9.34601 & -0.94463 & 0.4465 & -2.11563 & 0.034376 & 0.22621 \\
\hline 1.04714 & -1.16542 & 0.550281 & -2.11787 & 0.034186 & 0.22621 \\
\hline 4894 & -1.14994 & 0.544106 & -2.11345 & 0.034562 & 0.226966 \\
\hline .2256 & -1.56561 & 0.741103 & -2.11254 & 0.03464 & 0.226966 \\
\hline 8.73754 & -1.16258 & 0.550438 & -2.1121 & 0.034678 & 0.226966 \\
\hline 577.506 & -1.1521 & 0.546565 & -2.10789 & 0.035041 & 0.228929 \\
\hline 21.53614 & 1.500661 & 0.712719 & 2.105544 & 0.035244 & 0.229844 \\
\hline 2.5812 & -1.06349 & 0.50534 & -2.10451 & 0.035334 & 0.230017 \\
\hline 30.9402 & -1.14109 & 0.542654 & -2.1028 & 0.035483 & 0.230217 \\
\hline .23624 & -1.60027 & 0.761049 & -2.10271 & 0.035491 & 0.230217 \\
\hline 12.79653 & 2.73547 & 1.302069 & 2.100863 & 0.035653 & 0.230856 \\
\hline 11.0746 & -1.12595 & 0.5373 & -2.09557 & 0.03612 & 0.231005 \\
\hline 1.06852 & -1.12017 & 0.534435 & -2.09599 & 0.036083 & 0.231005 \\
\hline 95.44949 & -1.097 & 0.522838 & -2.09816 & 0.035891 & 0.231005 \\
\hline 15.2538 & -1.56941 & 0.748293 & -2.09732 & 0.035965 & 0.231005 \\
\hline 477.4718 & -1.18905 & 0.566752 & -2.09801 & 0.035904 & 0.231005 \\
\hline 51.5109 & -1.31062 & 0.625385 & -2.0957 & 0.036109 & 0.231005 \\
\hline 92.95594 & -1.22047 & 0.581441 & -2.09905 & 0.035812 & 0.231005 \\
\hline 129.1411 & -1.12118 & 0.53595 & -2.09196 & 0.036442 & 0.232656 \\
\hline 149.2112 & -1.21078 & 0.57973 & -2.08852 & 0.036751 & 0.233488 \\
\hline 369.3732 & -1.09379 & 0.52359 & -2.08902 & 0.036706 & 0.233488 \\
\hline 164.3908 & -1.03841 & 0.498185 & -2.08438 & 0.037125 & 0.233488 \\
\hline 32.55406 & -1.29783 & 0.622331 & -2.08544 & 0.03703 & 0.233488 \\
\hline 35.81294 & -1.4793 & 0.709018 & -2.08641 & 0.036941 & 0.233488 \\
\hline 16.47096 & -1.6726 & 0.801937 & -2.0857 & 0.037006 & 0.233488 \\
\hline 76.54196 & -0.84344 & 0.404821 & -2.08348 & 0.037207 & 0.233488 \\
\hline 13.26664 & -1.48973 & 0.714721 & -2.08435 & 0.037128 & 0.233488 \\
\hline 34.80947 & -1.26861 & 0.608678 & -2.08421 & 0.037141 & 0.233488 \\
\hline 556.8998 & -1.32801 & 0.637422 & -2.0834 & 0.037214 & 0.233488 \\
\hline 226.7654 & -1.01956 & 0.489842 & -2.08142 & 0.037396 & 0.23384 \\
\hline 68.86433 & -0.99904 & 0.479988 & -2.08138 & 0.037399 & 0.23384 \\
\hline 30.39007 & -2.36014 & 1.134738 & -2.0799 & 0.037535 & 0.234289 \\
\hline 565.1861 & -1.04453 & 0.50282 & -2.07734 & 0.03777 & 0.234935 \\
\hline 65.62484 & -1.16019 & 0.558679 & -2.07667 & 0.037832 & 0.234935 \\
\hline 4.797689 & -3.20679 & 1.543685 & -2.07736 & 0.037768 & 0.234935 \\
\hline 21.03759 & -1.46485 & 0.706105 & -2.07456 & 0.038028 & 0.235746 \\
\hline 121.6563 & -0.7852 & 0.378981 & -2.07186 & 0.038279 & 0.236495 \\
\hline 44.47985 & -1.11289 & 0.537027 & -2.07232 & 0.038236 & 0.236495 \\
\hline 435.5654 & -1.10755 & 0.536584 & -2.06408 & 0.03901 & 0.237387 \\
\hline 13.07153 & -2.00849 & 0.972131 & -2.06607 & 0.038822 & 0.237387 \\
\hline 420.7361 & -1.0108 & 0.489084 & -2.06672 & 0.038761 & 0.237387 \\
\hline 112.7917 & 1.733449 & 0.838675 & 2.06689 & 0.038745 & 0.237387 \\
\hline 8.167303 & -2.04866 & 0.99086 & -2.06756 & 0.038681 & 0.237387 \\
\hline 1432.045 & -1.08327 & 0.524593 & -2.06496 & 0.038926 & 0.237387 \\
\hline 53.94508 & -1.26978 & 0.613999 & -2.06805 & 0.038636 & 0.237387 \\
\hline 5.850195 & -3.22118 & 1.557061 & -2.06876 & 0.038569 & 0.237387 \\
\hline 87.61917 & -0.81826 & 0.396328 & -2.06459 & 0.038962 & 0.237387 \\
\hline 137.5792 & -0.93507 & 0.453916 & -2.06001 & 0.039398 & 0.238493 \\
\hline 14.35782 & -1.72378 & 0.837492 & -2.05827 & 0.039564 & 0.238493 \\
\hline
\end{tabular}


Sinorhizobium fredii

Candidatus Thiodictyon syntrophicun

Staphylococcus virus Sextaec

Pseudomonas balearica

Polaromonas naphthalenivorans

Ottowia oryzae

Janthinobacterium sp. 1_2014MBL_^

Sulfolobus islandicus

Pseudomonas alcaligenes

Cryobacterium sp. LW097

Pseudomonas savastanoi

Mycolicibacterium litorale

Ethanoligenens harbinense

Paraburkholderia hospita

Burkholderia stabilis

Rhodococcus sp. PBTS 2

Leptothrix cholodnii

Erythrobacter sp. Alg231-14

Halomonas sp. K0116

Frankia symbiont of Datisca glomerat

Streptomyces globisporus

Chelatococcus daeguensis

Methanosarcina barkeri

Burkholderia cenocepacia

Bradyrhizobium sp. ORS 278

Dickeya dianthicola

Olsenella sp. GAM18

Serratia marcescens

Paludisphaera borealis

Chlorobium chlorochromatii

Synechococcus sp. KORDI-100

Actinomyces sp. oral taxon 414

Thermomicrobium roseum

Staphylococcus sp. AntiMn-1

Rhodanobacter denitrificans

Sphingomonas sp. MM-1

Streptomyces sp. NEAU-S7GS2

Opitutus sp. GAS368

Streptococcus pluranimalium

Castellaniella defragrans

Xylella taiwanensis

Kurthia sp. $11 \mathrm{kri321}$

Streptomyces sp. Tu6071

Pontimonas salivibrio

Paenibacillus glucanolyticus

Leptolyngbya sp. 0-77

Zobellella denitrificans

Salinivirga cyanobacteriivorans

Diaphorobacter polyhydroxybutyrati

Halomonas sp. JS92-SW72
291.1773

83.65761

347.4298

70.89283

511.0684

443.7861

209.4687

5.760535

84.79765

105.6044

14.65847

121.5805

26.41157

1056.761

619.7888

394.3179

700.4511

12.5706

14.49601

80.44359

82.752

86.417

19.46776

1991.342

196.7948

25.59627

22.37628

247.4995

97.23897

4.612109

5.325063

955.4657

12.84396

28.36465

179.4494

159.783

109.745

66.98513

27.95895

196.1157

8.981168

17.89909

15.35421

22.95856

19.55473

24.4972

63.59105

5.799261

1064.767

155.606

$-0.93342$

$-1.0585$

3.6624

0.453314

$-2.0591$

0.039485

0.238493

$-1.02319$

0.514256

$-2.0583$

0.039561

0.238493

$-1.05234$

0.4971

2.060337

0.039366

0.238493

$-1.1979$

$-2.05805$

0.039585

0.238493

$-1.1979$

0.511849

$-2.05595$

0.039788

0.238922

$-1.10137$

0.582641

$-2.05599$

0.039784

0.238922

4.392774

0.536462

$-2.05303$

0.040069

0.239179

$-1.03199$

2.139956

2.05274

0.040098

0.239179

$-1.05771$

0.502788

$-2.05254$

0.040117

0.239179

$-2.87331$

0.515775

$-2.05072$

0.040294

0.239179

$-0.77024$

0.375699

$-2.04894$

0.040468

0.239179

$-1.58659$

0.774423

$-2.05015$

0.04035

0.239179

$-1.16261$

0.566522

$-2.04874$

0.040488

0.239179

$-1.06871$

0.521037

$-2.05218$

0.040152

0.239179

1.323119

0.645438

$-2.05111$

0.040256

0.239179

$-1.12997$

0.551827

2.049955

0.040369

0.239179

$-1.92635$

0.941134

$-2.04768$

0.040591

0.239403

$-2.31958$

1.134028

0.040673

0.239499

$-0.80764$

0.394996

$-2.04544$

0.040812

0.239927

$-1.11973$

0.548137

2.04467

0.040887

0.23998

$-1.34106$

0.657414

$-2.0428$

0.041073

0.240681

2.382938

1.168071

$-2.0399$

0.04136

0.241588

$-1.12429$

0.551492

2.040063

0.041344

0.241588

$-0.96936$

0.476132

2.03863

0.041487

0.241943

$-1.96241$

0.966125

$-2.0359$

0.041761

0.243147

$-1.33039$

0.655899

$-2.03122$

0.042233

0.244516

$-0.84991$

0.419033

$-2.02835$

0.042524

0.244516

$-0.90212$

0.444578

$-2.02826$

0.042533

0.244516

$-2.48388$

1.223638

$-2.02915$

0.042443

0.244516

$-2.51171$

1.236859

$-2.02991$

0.042365

0.244516

$-1.1035$

0.543996

$-2.03072$

0.042284

0.244516

$-1.80652$

0.889768

$-2.0285$

0.042509

0.244516

$-2.31664$

1.143665

$-2.03033$

0.042323

0.244516

$-1.04294$

0.515124

$-2.02563$

0.042803

0.245676

$-1.10934$

0.548436

$-2.02463$

0.042905

0.245876

$-0.91827$

0.454233

$-2.02274$

0.0431

0.245935

$-1.09842$

0.543626

$-2.02158$

0.04322

0.245935

$-1.707$

0.844562

$-2.02055$

0.043327

0.245935

$-1.10419$

0.546642 
Xenorhabdus nematophila

Thermogutta terrifontis

Alkalilimnicola ehrlichii

Paenibacillus swuensis

Streptomyces sp. CB09001

Rhizobium tropici

Nocardia terpenica

Hyphomonas neptunium

Mycobacterium colombiense

Melittangium boletus

Pseudomonas xanthomarina

Cellulomonas fimi

Pantoea stewartii

Lysobacter antibioticus

Nocardiopsis gilva

Methanosarcina horonobensis

Pseudoalteromonas arctica

Thiomonas intermedia

Burkholderia ubonensis

Xanthomonas phaseoli

Leuconostoc garlicum

Mycobacterium dioxanotrophicus

Burkholderia vietnamiensis

Desulfovibrio fairfieldensis

Kiritimatiella glycovorans

Kosakonia cowanii

Arcobacter mytili

Burkholderia multivorans

Arcobacter sp. L

Cronobacter muytjensii

Aeromicrobium sp. A1-2

Luteitalea pratensis

Achromobacter xylosoxidans

Mycolicibacterium thermoresistibile

Sphingomonas sanxanigenens

Mycolicibacterium smegmatis

Staphylococcus succinus

Roseobacter litoralis

Plantibacter flavus

Natronomonas moolapensis

Sphingomonas koreensis

Streptomyces alboflavus

Crenobacter sp. K1W11S-77

Halobacterium sp. DL1

Prochlorococcus marinus

Pseudomonas sabulinigri

Pirellula staleyi

Enterococcus thailandicus

Limnochorda pilosa

Luteimonas sp. JM171
5.423185

6.218

4

9.604103

32.96034

50.71224

158.8668

17.07061

103.6358

89.88569

28.16909

240.5768

16.84162

109.8964

83.85189

4.545018

7.854462

239.1885

1187.458

152.425

5.292133

156.8124

831.1656

21.55392

28.00106

44.82605

9.585912

1164.836

10.96466

11.36795

121.8505

124.7263

968.5862

134.6462

162.4081

197.6459

393.9425

17.28542

136.5367

9.427167

179.2796

69.41862

97.36991

24.02772

67.37285

37.11542

13.64207

7.306036

93.57684

77.16791
$-3.33506$

$-1.84437$

$-1.04679$

$-1.7897$

$-1.32934$

$-1.1871$

$-0.96817$

$-1.74441$

$-0.79903$

$-0.94745$

$-1.20404$

$-0.90847$

$-1.50496$

$-0.9961$

$-0.84938$

$-3.59984$

$-4.02243$

$-0.98756$

$-1.05915$

$-1.44286$

$-2.97553$

$-0.60138$

$-1.26368$

$-1.51227$

$-1.35355$

$-1.50232$

2.999916

$-1.06409$

3.490724

$-2.12782$

$-1.04051$

$-0.92506$

$-0.94377$

$-0.72823$

$-0.83124$

$-0.67363$

$-1.8722$

$-1.64375$

$-0.94965$

$-2.15077$

$-0.95135$

$-0.96568$

$-1.17902$

$-1.58345$

0.890928

$-1.04883$

$-1.4667$

$-2.18758$

$-1.02664$

$-1.08492$
1.660084

0.918655

0.522004

0.891834

0.662808

0.592837

0.482363

0.870874

0.399141

0.473262

0.600792

0.453225

0.752059

0.497945

0.424905

1.801447

2.013603

0.495813

0.532655

0.724894

1.49558

0.301632

0.637727

0.762278

0.682059

0.756662

1.512253

0.536719

1.761755

1.072836

0.524894

0.466029

0.473721

0.366275

0.418533

0.337762

0.93773

0.825455

0.47938

1.080643

0.479851

0.48412

0.594221

0.799657

0.450351

0.530607

0.742271

1.108987

0.520531

0.549786
$-2.00897$

$-2.00769$

$-2.00532$

$-2.00677$

$-2.00562$

$-2.00241$

$-2.00715$

$-2.00306$

$-2.00188$

$-2.00197$

$-2.00409$

$-2.00445$

$-2.00112$

$-2.00042$

$-1.999$

$-1.9983$

$-1.99763$

$-1.9918$

$-1.98844$

$-1.99045$

$-1.98955$

$-1.99374$

$-1.98153$

$-1.98388$

$-1.98451$

$-1.98545$

1.983739

$-1.98258$

1.98139

$-1.98336$

$-1.98232$

$-1.98497$

$-1.99225$

$-1.9882$

$-1.98608$

$-1.99438$

$-1.99652$

$-1.99132$

$-1.981$

$-1.99027$

$-1.9826$

$-1.99471$

$-1.98415$

$-1.98016$

1.978296

$-1.97666$

$-1.97597$

$-1.97259$

$-1.9723$

$-1.97334$

0.248975

$0.044677 \quad 0.248999$

0.0449280 .248999

0.0447750 .248999

0.044897

0.248999

0.045240 .248999

$0.044734 \quad 0.248999$

0.0451710 .248999

0.045297

0.248999

0.045288

0.248999

0.04506

0.248999

0.045022

0.248999

0.04538

0.249076

0.045454

0.24911

0.045609

0.249578

0.045684

0.249615

0.045757

0.249641

0.046393

0.249907

0.046763

0.249907

0.046542

0.249907

$\begin{array}{ll}0.046641 & 0.249907\end{array}$ 
Chromobacterium vaccinii

Alicyclobacillus acidocaldarius

Mycobacterium kansasii

Clostridium novyi

Robiginitalea biformata

Sulfurifustis variabilis

Methanococcus maripaludis

Aminomonas paucivorans

Salinarchaeum sp. Harcht-Bsk1

Acidaminococcus fermentans

Megasphaera hexanoica

Bifidobacterium choerinum

Pseudomonas saudiphocaensis

Sphingomonas sp. YZ-8

Sphingopyxis sp. FD7

Thiolapillus brandeum

Mycolicibacterium vanbaalenii

Polaribacter sp. SA4-10

Caulobacter vibrioides

Nocardia nova

Clostridium saccharobutylicum

Aeromonas sp. ASNIH3

Planctomyces sp. SH-PL14

Rhizobium phaseoli

Stenotrophomonas sp. YAU14D1_LEI

Chelativorans sp. BNC1

Methylorubrum populi

Collimonas pratensis

Pseudohongiella spirulinae

Streptomyces rubrolavendulae

Chromobacterium sp. IIBBL 112-1

Frankia sp. EAN1pec

Burkholderia sp. MSMB0266

Bifidobacterium actinocoloniiforme

Chlorobaculum limnaeum

Thauera sp. MZ1T

Neorhizobium sp. NCHU2750

Azoarcus olearius

Caulobacter sp. K31

Sphingopyxis macrogoltabida

Stenotrophomonas sp. ZAC14D2_NAI Halothiobacillus neapolitanus

Campylobacter gracilis

Azospirillum sp. CFH 70021

Aminobacter sp. MSH1

Bordetella pseudohinzii

Streptomyces olivoreticuli

Delftia sp. Cs1-4

Clostridium sp. DL-VIII

Novosphingobium aromaticivorans
110.1633

121.5655

102.1599

36.31267

15.01258

64.71086

13.84507

11.18092

13.03434

13.85675

12.86881

30.79644

23.91252

32.76592

53.19022

11.23128

214.3754

9.933151

209.6005

138.6893

34.21906

294.7209

63.35783

135.8227

32.56497

58.31467

288.3158

197.3293

3.368594

70.68203

35.14998

129.2096

143.3401

18.58387

21.29006

147.329

51.76257

144.7262

184.5092

153.2917

40.38628

20.29646

128.4254

186.6039

104.9791

92.54898

88.19951

539.0331

31.44299

53.92098
0.93876

$-1.0408$

$-0.70758$

1.157645

$-1.61312$

$-0.95914$

1.947176

$-1.39719$

$-1.70289$

$-1.60056$

$-1.76563$

$-1.06622$

$-1.35126$

$-1.31688$

$-1.32581$

$-1.67178$

$-0.82117$

2.672502

$-0.9305$

$-0.77063$

1.455208

$-1.28103$

$-0.87201$

$-1.22768$

$-2.16492$

$-0.83353$

$-0.78576$

$-1.00977$

$-3.09551$

$-0.92068$

$-3.0053$

$-0.75594$

$-1.44444$

$-1.1698$

$-1.71699$

$-0.97967$

$-1.09219$

$-1.26022$

$-1.02372$

$-1.09894$

$-1.75963$

$-1.37258$

$-1.3618$

$-0.96862$

$-0.97249$

$-1.24846$

$-0.97508$

$-1.09497$

1.031522

$-0.93907$

0.476201

$-1.97135$

0.048683

0.252723

0.528169

$-1.97058$

0.048772

0.252824

0.360298

$-1.96388$

0.049544

0.254046

0.589457

1.963918

0.04954

0.254046

0.820661

$-1.96563$

0.049341

0.254046

0.488424

$-1.96374$

0.04956

0.254046

$\begin{array}{lll}0.989989 & 1.966866 & 0.049199\end{array}$

0.254046

0.711351

$-1.96413$

0.049515

0.254046

0.867191

0.81376

$-1.96369$

0.049567

0.254046

0.899763

$-1.96686$

0.049199

0.254046

0.543805

$-1.96233$

0.049724

0.254497

0.689282

$-1.96066$

0.049919

0.254937

0.672099

$-1.96039$

0.049951

0.254937

0.677097

$-1.95935$

0.050072

0.2552

0.853694

$-1.95807$

0.050221

0.255245

0.419621

$-1.95829$

0.050196

0.255245

1.366123

$-1.95694$

0.050355

0.255566

0.47587

1.956268

0.050434

0.25561

0.394256

$-1.95536$

0.05054

0.255794

0.744783

$-1.95465$

0.050625

0.255866

0.655885

1.953867

0.050717

0.255976

0.446858

$-1.95312$

0.050805

0.256065

0.630708

$-1.95143$

0.051006

0.256723

$-1.94652$

0.051593

0.257039

1.112169

$-1.94658$

0.051585

0.257039

0.427981

$-1.94759$

0.051465

0.257039

0.403724

$-1.94629$

0.05162

0.257039

0.518849

$-1.94617$

0.051634

0.257039

$-1.9484$

0.051367

0.257039

0.473009

$-1.94643$

0.051604

0.257039

1.542432

$-1.94842$

0.051365

0.257039

0.388752

$-1.94454$

0.05183

0.257313

0.742798

$-1.9446$

0.051823

0.257313

0.601778

$-1.9439$

0.051908

0.257346

0.883869

$-1.94259$

0.052066

0.257358

0.504585

$-1.94154$

0.052193

0.257358

0.562446 
Deinococcus sp. NW-56

Arcobacter ellisii

Paenibacillus sp. FSL R5-0345

Azospirillum thiophilum

Microbacterium sp. BH-3-3-3

Betaproteobacteria bacterium GR16

Oceanimonas sp. GK1

Bordetella flabilis

Pannonibacter phragmitetus

Pigmentiphaga sp. $\mathrm{H} 8$

Sphingobium hydrophobicum

Novosphingobium sp. PP1Y

Rhodococcus sp. WB1

Bacillus weihaiensis

Isoptericola variabilis

Deinococcus maricopensis

Candidatus Nitrospira inopinata

Auraticoccus monumenti

Rubrobacter xylanophilus

Steroidobacter denitrificans

Burkholderia sp. KK1

Desulfovibrio africanus

Pseudomonas sp. CMR12a

Burkholderia sp. CCGE1001

Actinomyces sp. 2129

Desulfococcus oleovorans

\begin{tabular}{rrrrrr}
19.35353 & -1.33791 & 0.693112 & -1.93029 & 0.053571 & 0.259563 \\
10.62353 & -1.74161 & 0.902185 & -1.93043 & 0.053553 & 0.259563 \\
64.59791 & -1.03837 & 0.538686 & -1.9276 & 0.053905 & 0.260226 \\
279.8687 & -1.03757 & 0.538464 & -1.92692 & 0.05399 & 0.260226 \\
178.9702 & -0.91426 & 0.474438 & -1.92703 & 0.053976 & 0.260226 \\
16.26143 & -1.13015 & 0.587393 & -1.924 & 0.054354 & 0.260599 \\
75.88099 & -0.90937 & 0.472473 & -1.92469 & 0.054268 & 0.260599 \\
171.2138 & -0.84318 & 0.438084 & -1.92471 & 0.054266 & 0.260599 \\
86.18106 & -0.89211 & 0.463613 & -1.92425 & 0.054323 & 0.260599 \\
94.25543 & -1.04788 & 0.546231 & -1.91838 & 0.055063 & 0.260945 \\
47.43696 & -1.07971 & 0.563006 & -1.91776 & 0.055141 & 0.260945 \\
197.5481 & -1.1483 & 0.597422 & -1.92209 & 0.054595 & 0.260945 \\
93.40702 & -0.86892 & 0.452485 & -1.92033 & 0.054817 & 0.260945 \\
17.72753 & -1.42178 & 0.740631 & -1.91968 & 0.054898 & 0.260945 \\
130.0102 & -0.94198 & 0.489929 & -1.92269 & 0.054519 & 0.260945 \\
4.469906 & -2.65982 & 1.385265 & -1.92008 & 0.054848 & 0.260945 \\
119.6457 & -0.90868 & 0.473829 & -1.91775 & 0.055143 & 0.260945 \\
10.95524 & -1.4019 & 0.730779 & -1.91837 & 0.055065 & 0.260945 \\
121.8177 & -1.01924 & 0.530852 & -1.92 & 0.054857 & 0.260945 \\
34.85993 & -0.9563 & 0.499255 & -1.91545 & 0.055435 & 0.261359 \\
90.98278 & -0.9476 & 0.494881 & -1.9148 & 0.055518 & 0.261359 \\
592.3551 & -0.9876 & 0.515564 & -1.91557 & 0.05542 & 0.261359 \\
70.05624 & -0.95894 & 0.500669 & -1.91531 & 0.055453 & 0.261359 \\
67.37473 & -0.89394 & 0.467602 & -1.91176 & 0.055907 & 0.261496 \\
79.76599 & -0.89906 & 0.470205 & -1.91206 & 0.055869 & 0.261496 \\
9.882002 & 2.901871 & 1.517434 & 1.912354 & 0.055831 & 0.261496 \\
5.340178 & -2.35102 & 1.228869 & -1.91315 & 0.055728 & 0.261496 \\
122.2045 & -0.87011 & 0.454928 & -1.91264 & 0.055794 & 0.261496 \\
150.1222 & -0.907 & 0.474975 & -1.90957 & 0.056189 & 0.261636 \\
\hline 30.37438 & -1.15957 & 0.611673 & -1.89574 & 0.057994 & 0.265128 \\
56.18875 & -1.31702 & 0.695319 & -1.89413 & 0.058208 & 0.265438 \\
853.5653 & -1.24503 & 0.657143 & -1.8946 & 0.058145 & 0.265438 \\
136.8403 & -0.67783 & 0.358447 & -1.89103 & 0.05862 & 0.26698 \\
9.411646 & -1.43772 & 0.760511 & -1.89047 & 0.058695 & 0.266989
\end{tabular}

Geobacter uraniireducens

Paenibacillus sp. FSL R7-0331

Janthinobacterium sp. Marseille

Achromobacter sp. AONIH1

Microbacterium paludicola

Ferrimonas balearica

Sagittula sp. P11

Caulobacter flavus

Streptomyces atratus

Micromonospora echinaurantiaca

Sulfitobacter sp. AM1-D1

Sulfuritalea hydrogenivorans

Kitasatospora aureofaciens

Shewanella algae

Sphingobium cloacae

Endozoicomonas montiporae

Beutenbergia cavernae

Cellvibrio sp. PSBB006

Bordetella sp. $\mathrm{N}$

Pseudomonas palleroniana

Streptomyces sp. P3

Cupriavidus gilardii

Sphingomonas indica$$
1
$$

\section{Streptomyces actuosus}


Polynucleobacter asymbioticus

Marinobacter hydrocarbonoclasticus

Acidovorax sp. T1

Alteromonas australica

Ereboglobus luteus

Brenneria sp. EniD312

Massilia armeniaca

Saccharothrix espanaensis

Niabella ginsenosidivorans

Enterococcus sp. FDAARGOS_553

Lactobacillus jensenii

Streptacidiphilus sp. DSM 106435

Streptomyces cattleya

Mycoplasma hominis

Nitrospira moscoviensis

Kozakia baliensis

Streptococcus agalactiae

Pediococcus damnosus

Chryseobacterium shandongense

Streptomyces fulvissimus

Thermodesulfovibrio yellowstonii

Nocardia farcinica

Frankia sp. QA3

Pseudomonas fulva

Paenibacillus crassostreae

Tateyamaria omphalii

Mycobacterium sp. djl-10

Burkholderia sp. MSMB0856

Mycolicibacterium hassiacum

Ralstonia mannitolilytica

Pseudomonas litoralis

Candidatus Babela massiliensis

Coriobacteriaceae bacterium 68-1-3

Gammapapillomavirus 9

Pseudomonas sp. 7SR1

Devosia sp. A16

Mesorhizobium amorphae

Sphingopyxis sp. QXT-31

Geobacter lovleyi

Rhizobium leguminosarum

Devriesea agamarum

Polynucleobacter difficilis

Bordetella bronchialis

Planococcus rifietoensis

Erythrobacter sp. HL-111

Pseudomonas yamanorum

Parvibaculum lavamentivorans

Vagococcus penaei

Pseudomonas simiae

Streptomyces sp. Go-475
14.49204

28.93262

989.8993

7.378944

31.71416

18.22818

208.1486

165.9831

45.69594

9.348087

44.37833

95.78908

121.92

5.994334

27.33773

11.35536

671.2474

5.229412

102.4112

56.02158

6.400683

246.6189

79.9495

283.5268

4.552297

25.29429

196.1424

163.5694

165.8259

11733.71

54.34602

3.444598

44.53416

5.71564

50.81208

86.95073

158.8102

73.328

11.23002

390.9798

49.27329

9.777026

150.7775

10.85947

60.19816

97.89893

54.10709

8.91445

4.434456

79.07617

$-2.284341 .208694$

$-1.88992$

$-0.99148$

0.52489

$-1.88892$

0.058768

0.266989

$-1.00552$

0.532528

$-1.8882$

0.058902

0.26712

$-2.62611$

1.3909

$-1.88806$

0.059

0.26712

$-1.34086$

0.710719

$-1.88663$

0.059017

0.26712

$-1.28458$

0.681995

$-1.88356$

0.05921

0.267659

$-0.94277$

0.500459

$-1.8838$

0.059624

0.268863

$-0.80783$

0.429207

$-1.88214$

0.059591

0.268863

$-1.11503$

0.592309

$-1.88252$

0.059817

0.269065

$-2.47738$

1.321136

$-1.87519$

0.059765

0.269065

1.41904

0.757669

1.872902

0.060767

0.273002

$-0.72868$

0.390108

$-1.86789$

0.061082

0.274078

$-0.8638$

0.462616

$-1.8672$

0.061778

0.274248

2.751179

1.472099

1.868882

0.061873

0.274248

$-1.11294$

0.595047

.

0.061639

0.274248

$-1.43397$

0.766131

$-1.8717$

0.061437

0.274248

0.993852

0.531502

1.869895

0.061249

0.274248

$-2.75914$

1.47506

$-1.87053$

0.061498

0.274248

$-1.08737$

0.582204

$-1.86768$

0.061411

0.274248

$-0.95117$

0.509014

$-1.86866$

0.061806

0.274248

$-2.4475$

1.308776

$-1.87007$

0.06167

0.274248

$-0.64638$

0.34686

$-1.86352$

0.061475

0.274248

$-1.01238$

0.543185

$-1.86378$

0.06239

0.275863

$-1.02799$

0.553107

$-1.85857$

0.062353

0.275863

$-2.27748$

1.225156

$-1.85893$

0.276614

$-1.3847$

0.744572

$-1.85972$

0.063037

0.276614

1.86129

0.062925

0.276614

1.86109

0.062703

0.276614

0.607931

$-1.85801$

0.062732

0.276614

$-0.67989$

0.365925

$-1.85824$

0.063167

0.276614

$-0.94346$

0.507719

$-1.85972$

0.063135

0.276614

$-1.06871$

0.574664

1.8

1.856084

0.062926

0.276614

0.589035

$-1.8562$

0.063442

0.277148

2.824982

$-1.85437$

0.063425

0.277148

$-5.23857$

0.494865

$-1.85119$

0.277881

$-0.91609$

0.527632

$-1.8508$

0.064142

0.279447

$-0.97654$

0.504728

$-1.84791$

0.064198

0.279447

0.531591

$-1.84493$

0.064616

0.279559

$-1.2746$

0.689517

$-1.84854$

0.065048

0.279559

$-0.74716$

0.404176

$-1.8486$

0.064525

0.279559 


\begin{tabular}{|c|c|c|c|c|c|c|}
\hline Acidobacteriaceae bacterium SBC82 & 966 & & & & & \\
\hline Thermus oshimai & & & 0.676628 & -1.84136 & 0.065568 & \\
\hline Jeotgalibaca sp. PTS2502 & 12.16754 & -1.62557 & 0.880206 & -1.8468 & 0.064776 & 0.279559 \\
\hline lood disease bacterium A2-HR MAR & 297.173 & -1.45003 & 0.78458 & -1.84816 & 0.064579 & 279559 \\
\hline Oligella urethralis & 8.684264 & -2.10793 & 1.145773 & -1.83975 & 0.065805 & 0.279594 \\
\hline omamonas testosteroni & 564.7262 & -0.97583 & 0.53039 & -1.83983 & 0.065794 & 279594 \\
\hline Pandoraea pulmonicola & 244.9978 & -0.98819 & 0.537216 & -1.83947 & 0.065846 & .279594 \\
\hline Burkholderia anthina & 164.9668 & -1.26816 & 0.689948 & -1.83804 & 0.066056 & 0.279663 \\
\hline MB1588 & 118.8795 & -1.812 & 0.985911 & -1.8379 & 0.066078 & 279663 \\
\hline Bacillus sp. FJAT-45348 & 20.05495 & 2.373065 & 1.291255 & 1.837797 & 0.066092 & 0.279663 \\
\hline Raoultella planticola & 23.51323 & -1.67437 & 0.911565 & -1.83681 & 0.066238 & 79953 \\
\hline ter mangrovi & 24.83404 & -1.07331 & 0.584722 & -1.83559 & 0.066419 & 0.280392 \\
\hline 52074 & 4.421065 & -2.38093 & 1.301459 & -1.82943 & 0.067335 & 0.281442 \\
\hline Micro & 61.14 & -0.94957 & 0.518533 & -1.83125 & 0.067063 & 281442 \\
\hline Zymobacter palmae & 16.2009 & -1.21635 & 0.664416 & -1.8307 & 0.067145 & 0.281442 \\
\hline Brevu & 119.5441 & -0.89352 & 0.487555 & -1.83265 & 854 & 1442 \\
\hline Pseudomonas sp. 09C 129 & 13.24948 & -2.51429 & 1.373791 & -1.83018 & 0.067222 & 0.281442 \\
\hline Vario & 890.9 & -0.9 & 0.515696 & -1.8 & & \\
\hline arcticus & 8.854693 & -1.36133 & 0.743504 & -1.83096 & 0.067106 & 0.281442 \\
\hline Thioa & 36.85399 & -0.897 & 0.490365 & -1.82924 & 0.067364 & 1442 \\
\hline Halor & 51.23869 & -0.9958 & 0.544268 & -1.82961 & 0.067308 & 1442 \\
\hline r kilaueensis & 76.14071 & -1.17145 & 0.641028 & -1.82746 & 0.067631 & 0.282236 \\
\hline Pedob & 15.78615 & -1.6 & 0.892586 & -1.82694 & 0.067709 & 0.282237 \\
\hline Halor & 24.8887 & -1.34405 & 0.735906 & -1.82638 & 7793 & 2262 \\
\hline Lacto & & -1.21868 & 0.668132 & -1.82401 & 8151 & 8343 \\
\hline Polyn & 5.259361 & -1.83932 & 1.008935 & -1.82303 & 0.068298 & 83719 \\
\hline Paenil & 10.35453 & -1.69963 & 0.932848 & -1.82198 & 3457 & 4055 \\
\hline Strep & 60.63847 & -0.87562 & 0.482533 & -1.8 & 958 & 5457 \\
\hline Myco & 269.172 & -2.88867 & 1.588954 & -1.81797 & 0.069068 & 0.285457 \\
\hline Filomicrobium marinum & 17.33187 & -1.3873 & 0.763922 & -1.81602 & 0.069367 & 0.285457 \\
\hline Acidovorax sp. 1608163 & 613.6447 & -1.04867 & 0.577641 & -1.81543 & 0.069458 & 0.285457 \\
\hline Xanthomonas gardneri & 26.90717 & -2.13043 & 1.171516 & -1.81852 & 0.068984 & 0.285457 \\
\hline eimia varigena & 7.882171 & -2.04963 & 1.129484 & -1.81466 & 0.069576 & 5457 \\
\hline Hyme & 80.81134 & -0.90059 & 0.496167 & -1.81509 & 0.06951 & 5457 \\
\hline Oligc & 78.76 & $-0.8 s$ & 0.494 & -1.8 & 169 & 5457 \\
\hline Celer & 29.01756 & -1.34895 & 0.74242 & -1.81696 & 0.069224 & 0.285457 \\
\hline Agrol & 409.9235 & -0.75741 & 0.41688 & -1.81686 & 0.069238 & 35457 \\
\hline Rivul & 15.03808 & 1.920754 & 1.059223 & 1.813362 & 0.069776 & 0.28594 \\
\hline Aliivit & 3.945662 & -2.80769 & 1.54957 & -1.81191 & 0.07 & 0.28641 \\
\hline Bifid & 21.09944 & -1.3347 & 0.736752 & -1.8116 & 0.070048 & 0.28641 \\
\hline Neisseria elongata & 444.8412 & -1.0242 & 0.566168 & -1.80901 & 0.070449 & 0.287558 \\
\hline Streptomyces sp. CFMR 7 & 42.67636 & -1.68935 & 0.933978 & -1.80877 & 0.070487 & 0.287558 \\
\hline Advenella kashmirensis & 31.63713 & -1.43615 & 0.795113 & -1.80623 & 0.070883 & 0.288526 \\
\hline Rhizo & 28.40621 & -2.29957 & 1.273034 & -1.80637 & 0.07086 & 0.288526 \\
\hline & 155.307 & -0.74052 & 0.410193 & -1.80529 & 0.07103 & 0.288801 \\
\hline Thioalkalivibrio versutus & 32.32296 & -1.14531 & 0.634768 & -1.80429 & 0.071186 & 0.289114 \\
\hline & 65.8343 & -1.00276 & 0.556038 & -1.80339 & 0.071326 & 0.289361 \\
\hline Nitrospirillum amazonense & 175.3716 & -0.94843 & 0.52612 & -1.80269 & 0.071438 & 0.28949 \\
\hline & 114.0495 & -0.80344 & 0.447297 & -1.79622 & 0.07246 & 0.290078 \\
\hline Staphylococcus stepanovicii & 89.01579 & 1.318014 & 0.733418 & 1.797085 & 0.072322 & 0.290078 \\
\hline
\end{tabular}


Pedobacter steynii

Staphylococcus lutrae

Verrucomicrobia bacterium IMCC261

Serratia sp. YD25

Thauera aromatica

Pseudomonas syringae

Muricauda ruestringensis

Saccharomonospora marina

Halorubrum lacusprofundi

Parvimonas micra

Burkholderia sp. HB1

Nostoc sp. 'Peltigera membranacea c

Burkholderia contaminans

Micromonospora purpureochromoge

Nocardia sp. CFHSO054

Nitrosospira multiformis

Aquitalea magnusonii

Streptomyces globosus

Martelella endophytica

Candidatus Phaeomarinobacter ecto

Asticcacaulis excentricus

Orientia tsutsugamushi

Mycolicibacterium gilvum

Burkholderia sp. IDO3

Streptomyces anulatus

Kushneria marisflavi

Stenotrophomonas sp. SAU14A_NAIN

Bradyrhizobium sp. S23321

Micromonospora coriariae

Streptomyces lunaelactis

Micromonospora echinofusca

Prauserella marina

Neisseria sp. 10023

Brachyspira murdochii

Neorhizobium sp. SOG26

Sedimenticola thiotaurini

Buchnera aphidicola

Providencia sp. WCHPr000369

Paenibacillus naphthalenovorans

Desulfovibrio vulgaris

Staphylococcus capitis

Spirosoma aerolatum

Altererythrobacter atlanticus

Ochrobactrum sp. A44

Corynebacterium resistens

Agrobacterium sp. RAC06

Pseudomonas oryzae

Sphingobium sp. TKS

Rhodobiaceae bacterium

Halothiobacillus sp. LS2
22.23062

36.65092

33.93547

15.97898

97.84397

331.5797

6.786159

112.8639

20.10912

254.8848

621.1341

8.160661

433.4966

84.76969

171.4699

10.58747

58.03234

76.00159

43.73371

14.87942

34.79609

5.051183

308.8538

325.9053

67.0561

12.25585

4.956979

295.6863

61.66198

58.95126

78.54304

80.73686

44.90315

12.33745

38.77721

17.49793

78.41897

5.874327

8.94792

93.22025

44191.08

20.87942

27.75844

42.63014

1932.214

70.5098

116.0565

67.97201

5.957214

23.99147

\subsection{4}

0.963955

1.733717

$-0.98635$

0.548671

$-1.49561$

$-1.01976$

0.830338

0.567195

$-0.83324$

0.463862

$-2.13742$

1.187948

$-0.82804$

$-1.56281$

$-0.94032$

$-1.13995$

0.460321

0.86982

0.524033

0.635564

3.013437

$-0.97826$

$-0.75611$

$-0.55479$

1.681176

0.545793

0.422159

0.309791

$-1.60339$

0.89697

$-0.88725$

0.496223

$-0.75583$

$-1.01457$

$-1.53015$

0.422609

0.567743

$-1.28293$

0.856628

3.64096

$-0.81531$

$-1.03955$

$-0.76344$

$-1.6257$

$-4.40232$

$-0.89457$

$-0.92212$

0.718651

2.041057

0.457166

0.583623

0.428751

0.914337

2.479627

0.503934

0.51927

$-0.88645$

0.500102

$-0.76711$

0.432951

$-0.57324$

$-1.17468$

0.323541

2.21203

0.662337

1.247809

$-0.89903$

0.507807

$-1.39514$

0.78796

0.528525

0.935458

1.393698

0.900004

$-0.8438$

1.099543

0.477503

0.622621

$-1.31125$

$-1.08489$

0.743431

0.615406

$-1.11084$

0.630888

$-0.76435$

0.433944

$-0.98621$

0.559891

$-1.05032$

0.596438

$-0.95829$

0.544533

$-1.7035$

0.968792

$-1.1344$

0.64552

$-1.79763$

0.072235

0.290078

1.798546

0.072091

0.290078

$\begin{array}{ll}-1.79771 & 0.072224\end{array}$

0.290078

$\begin{array}{ll}-1.80121 & 0.07167\end{array}$

0.290078

$-1.79790 .072193$

0.290078

$-1.79630 .072446$

0.290078

$\begin{array}{ll}-1.79925 & 0.071979\end{array}$

0.290078

$-1.798840 .072045$

0.290078

$\begin{array}{ll}-1.79671 & 0.072382\end{array}$

0.290078

$-1.79439$

0.072751

0.290923

$-1.79360 .072877$

0.291107

1.7924580 .07306

0.291253

$\begin{array}{ll}-1.79237 & 0.073073\end{array}$

0.291253

$-1.79106$

0.073284

0.29159

$-1.79085$

0.073318

0.29159

$\begin{array}{ll}-1.78756 & 0.073847\end{array}$

0.292734

$\begin{array}{ll}-1.788 & 0.073777\end{array}$

0.292734

$-1.788490 .073698$

0.292734

$-1.78703$

0.073932

0.292753

$-1.78625$

0.074059

0.292937

$-1.78520 .074229$

0.29329

1.78386

0.074446

0.29381

$-1.78339$

0.074522

0.29381

$-1.78121$

0.074878

0.294894

$-1.780620 .074975$

0.294957

$-1.778010 .075403$

0.296318

$-1.77540 .075832$

0.297191

$-1.775170 .07587$

0.297191

$-1.77579$

0.075767

0.297191

$-1.772530 .076306$

0.297795

$-1.77181$

0.076426

0.297795

$-1.77177$

0.076433

0.297795

$-1.77353 \quad 0.07614$

0.297795

1.772731

0.076273

0.297795

$\begin{array}{ll}-1.77042 & 0.076657\end{array}$

0.298021

-1.77057 0.076632

0.298021

1.769942

0.076737

0.298021 
Achromobacter sp. MFA1 R4

Plesiomonas shigelloides

Amycolatopsis albispora

Sphingopyxis fribergensis

Gammaproteobacteria bacterium ESI

Chryseobacterium sp. 6424

Dinoroseobacter shibae

Bacillus sp. OxB-1

Pandoraea faecigallinarum

Mycobacterium sp. MS1601

Streptomyces sp. SM18

Acidovorax sp. KKS102

Microchaete diplosiphon

Chitinophaga pinensis

Immundisolibacter cernigliae

Pseudanabaena sp. PCC 7367

Burkholderiales bacterium YL45

Gemmobacter sp. HYN0069

Altererythrobacter ishigakiensis

Thermosediminibacter oceani

Streptococcus uberis

Agrococcus carbonis

Mesorhizobium sp. WSM1497

Lysobacter capsici

Vibrio coralliilyticus

Microbulbifer agarilyticus

Desulfuromonas soudanensis

Lelliottia nimipressuralis

Amycolatopsis orientalis

Massilia sp. YMA4

Achromobacter insolitus

Saccharomonospora cyanea

Pseudomonas soli

Pseudomonas monteilii

Singulisphaera acidiphila

Staphylococcus pseudintermedius

Burkholderia sp. MSMB0852

Streptococcus himalayensis

Synechococcus sp. SynAce01

Microbacterium sp. PAMC 28756

Arcobacter nitrofigilis

Brevibacillus brevis

Shewanella amazonensis

Rhodobacter sp. CZR27

Neorhizobium galegae

Nonomuraea sp. ATCC 55076

Euzebyella marina

Porphyrobacter sp. LM 6

Streptococcus macedonicus

Desulfatibacillum alkenivorans

144.3747

17.09034

171.3577

63.89562

$-0.94119$

0.536906

$-1.75299$

0.079603

0.304561

6.172502

18.76948

62.31015

3.832427

175.5017

162.1313

1.33092

0.759408

$-1.75257$

0.079676

0.304561

66.65319

1361.291

9.078717

52.43415

99.15992

0.64395

0.368011

$-1.7498$

0.080153

0.305997

$-0.9796$

0.559958

$-2.58187$

1.477468

$-1.54234$

0.88325

$-0.96288$

0.552459

$-2.27144$

1.303867

$-0.90034$

0.517417

$-0.57923$

0.332797

$-0.81762$

0.470721

$-0.84449$

0.486013

3.48249

2.004719

$-0.98664$

0.568553

$-0.80346$

0.463463

6.988811

$-1.8427$

1.063637

5.672349

$-2.71571$

1.567303

42.86132

7.388458

$-0.96563$

0.557403

4.152153

$-1.82147$

1.052067

$-2.05628$

1.188147

17.03308

$-1.39134$

0.804481

137.9268

$-0.74856$

0.433605

38.00845

164.8923

$-2.37208$

1.374296

19.8287

$-1.12582$

0.652301

$-1.3278$

0.769302

20.60297

$-0.98908$

50.90021

$-0.97585$

0.572985

5.501194

75.93968

$-2.11234$

0.565797

261.5821

$-0.68671$

1.225323

$-1.74941$

0.08022

0.305997

$-1.7475$

0.080551

0.306938

$-1.74621$

0.080775

0.307468

$-1.7429$

0.081351

0.309339

$-1.74208$

0.081494

0.309558

$-1.74007$

0.081847

0.310251

$-1.74048$

0.081775

0.310251

$-1.73696$

0.082394

0.311352

$-1.7376$

0.082282

0.311352

1.737146

0.082361

0.311352

$-1.73536$

0.082677

0.312097

$-1.73361$

0.082988

0.312805

$-1.73245$

0.083193

0.312805

$-1.73273$

0.083144

0.312805

$-1.73237$

0.083208

0.312805

$-1.73133$

0.083393

0.313176

$-1.73066$

0.083512

0.313298

$-1.72948$

0.083723

0.313766

$-1.72636$

0.084282

0.314546

$-1.72603$

0.084342

0.314546

$-1.72591$

0.084363

0.314546

$-1.72598$

0.084351

0.314546

$-1.72619$

0.084314

0.314546

$-1.72473$

0.084576

0.315018

$-1.72391$

0.084725

0.315066

$-1.7237$

0.084762

0.315066

121.3855

$-0.84529$

0.490672

$-1.72272$

0.084939

0.315081

111.8757

$-0.78684$

0.456645

$-1.72309$

0.084873

0.315081

3.183112

0.526976

$-1.72042$

0.085355

0.316302

223.5688

$-3.76456$

2.191133

$-1.71809$

0.08578

0.31665

63.79171

0.558847

$-1.71866$

0.085676

0.31665

300.8995

33.26022

$-0.86654$

$-1.71804$

0.08579

0.31665

1.194031

0.695014

1.717995

0.085797

0.31665

14.47554

0.991893

$-1.7158$

0.086199

0.317168

15.31498

0.914711

$-1.71614$

0.086136

0.317168

179.1562

0.720689

$-1.71594$

0.086172

0.317168

11.29236

$-0.83603$

$-1.71464$

0.086412

0.317308

25.59911

5.119629

2.499792

1.457664

1.71493

0.086358

0.317308

$-1.71342$ 
Microbacterium sp. TPU 3598

Pelagibacterium halotolerans

Halomonas sp. N3-2A

Geminocystis herdmanii

Vibrio gazogenes

Idiomarina sp. OT37-5b

Massilia putida

Aeromicrobium erythreum

Sphingobacterium sp. 21

Rhodobacter sphaeroides

Magnetospirillum gryphiswaldense

Starkeya novella

Streptomyces lydicus

Francisella noatunensis

Kosakonia oryzae

Acidovorax sp. JS42

Pseudomonas sp. R2A2

Rhodoferax saidenbachensis

Staphylococcus pasteuri

Eggerthella lenta

Bradyrhizobium sp. BTAi1

Desulfovibrio gigas

Arcobacter halophilus

Pseudomonas sp. HLS-6

Azospirillum humicireducens

Altererythrobacter namhicola

Coxiella burnetii

Pelobacter acetylenicus

Akkermansia muciniphila

Runella sp. HYN0085

Lysobacter gummosus

Agrobacterium fabrum

Oblitimonas alkaliphila

Brevirhabdus pacifica

Azospirillum lipoferum

Corynebacterium maris

Arcobacter suis

Lactobacillus brevis

Delftia sp. HK171

Mycobacteroides saopaulense

Streptomyces sp. Tue 6075

Streptomyces vietnamensis

Thermobacillus composti

Cellvibrio japonicus

Rummeliibacillus stabekisii

Corynebacterium humireducens

Leptospira santarosai

Pseudomonas guangdongensis

Pantoea sp. PSNIH1

Pedobacter sp. eg
165.6648

38.82378

3.109329

9.388917

7.095905

5.379733

430.5439

180.0977

34.72206

322.3975

413.5128

111.0314

178.4668

1.773597

138.165

1391.588

22.32542

282.0151

7090.308

50.01482

293.8172

26.10715

10.36311

28.75913

64.38383

42.27711

8.969604

12.85882

79.06022

10.53749

91.58467

57.44537

6.627171

53.4755

184.7914

906.4084

6.425009

13.96127

192.754

74.129

42.11233

81.24047

48.91641

14.51258

6.483205

941.3176

38.15851

42.49735

124.8972

35.8162

$-0.74753$

$-0.84239$

$-3.7307$

0.43796

$-1.70685$

$-1.70561$

0.08785

0.319368

1.902258

0.493892

$-1.69806$

0.088081

0.319888

$-2.14608$

1.119722

1.698866

0.089497

0.323852

$-2.40815$

1.263969

$-1.69789$

089344

0.323852

$-0.84418$

1.417958

$-1.69832$

0.089529

0.323852

$-0.75337$

$-1.03576$

0.497366

$-1.69731$

0.089447

0.323852

$-0.84643$

$-1.69469$

0.089639

0.323927

$-1.02793$

611746

$-1.69311$

0.090135

0.325399

$-0.79451$

500285

$-1.69189$

.090434

0.326153

$-0.53785$

0.607428

$-1.69226$

0.090666

0.326345

4.902936

0.318491

$-1.68862$

0.090596

0.326345

$-0.87381$

2.902244

$-1.68873$

0.091292

0.327175

$-0.99813$

0.517555

1.68936

0.327175

$-1.30249$

0.591072

$-1.68834$

0.327175

$-0.95221$

0.772709

$-1.68868$

0.091347

0.327175

$-0.82662$

0.564706

$-1.68561$

0.091281

0.327175

$-0.71306$

0.490401

$-1.6862$

0.091871

0.327866

$-0.88727$

0.423066

$-1.6855$

0.327866

$-1.68546$

0.327866

$-0.99628$

0.527092

$-1.68333$

0.0919

0.327866

$-1.68403$

0.092312

0.327968

2.596703

1

$-0.93144$

0.553881

1.682072

0.092175

0.327968

$-0.92702$

0.550719

$-1.68167$

0.092555

0.327968

$-0.86938$

0.516184

$-1.68329$

0.092634

0.327968

$-1.68425$

0.092319

0.327968

$-1.68209$

0.092133

0.327968

$-2.14754$

1.27671

$-1.68159$

0.092552

0.327968

0.697862

$-1.67795$

0.092649

0.327968

1.56503

$-1.67873$

0.093357

0.328186

$-1.64198$

0.978107

$-1.67701$

0.093205

0.328186

$-1.6791$

0.09354

0.328186

$-0.88434$

0.526672

$-1.67941$

0.093132

0.328186

$-1.6791$

0.093073

0.328186

90676

$-1.67782$

0.09313

0.328186

$-0.77319$

0.460828

$-1.67618$

0.093382

0.328186

$-0.49009$

0.292387

1.

$-1.79444$

1.070476

1.676801

0.093703

0.328186

$-1.00729$

0.600816

$-1.6763$

0.093581

0.328186

$-0.92789$

0.553764

$-1.67653$

0.093679

0.328186

$-1.6756$

0.093634

0.328186

$-1.24831$ 


\begin{tabular}{|c|c|c|c|c|c|c|}
\hline Beggiatoa leptomitoformis & & & & & & \\
\hline Methylotenera mobilis & 8.508418 & -1.39469 & 0.835207 & -1.66987 & & \\
\hline Chromatiaceae bacterium 2141T.STB & 40.83716 & -0.73216 & 0.43908 & -1.66749 & 0.095417 & 329636 \\
\hline Hyphomicrobium sp. MC1 & 33.51745 & -1.39111 & 0.833065 & -1.66987 & 0.094945 & 636 \\
\hline Actinomyces sp. Z16 & 202.7395 & -0.6901 & 0.414231 & -1.66598 & 0.095717 & 156 \\
\hline Thiobacillus denitrificans & 64.00899 & -0.88256 & 0.530258 & -1.66439 & 0.096035 & 0.330938 \\
\hline Bacillus lehensis & 12.92377 & -1.40962 & 0.847443 & -1.66338 & 0.096237 & 331322 \\
\hline seudoalteromonas spongiae & 14.57554 & 2.125557 & 1.27848 & 1.662566 & 0.096399 & 0.331566 \\
\hline Lachnospiraceae bacterium GAM79 & 145.8845 & -1.19625 & 0.72043 & -1.66047 & 0.09682 & 0.332698 \\
\hline Novosphingobium resinovorum & 158.1872 & -0.79636 & 0.479962 & -1.65922 & 0.097072 & 0.33325 \\
\hline Pelagibaca abyssi & 61.68843 & -0.8569 & 0.516606 & -1.65871 & 0.097174 & 0.333285 \\
\hline Aggregati & 149.1823 & -1.065 & 0.642714 & -1.65703 & 0.097514 & 4137 \\
\hline Actinom & 481.8617 & -0.82732 & 0.49953 & -1.6562 & 0.097681 & 0.334394 \\
\hline Mycoli & 1500.936 & -0.92273 & 0.557984 & -1.65369 & .09819 & 563 \\
\hline Microb & 13.47201 & -1.4802 & 0.894849 & 5413 & 0.0981 & 1563 \\
\hline Protein & 17.96967 & -1.49408 & 0.903392 & -1.65386 & 0.098156 & 0.334563 \\
\hline Mycoba & 171.7884 & -0.61111 & 0.36948 & -1.65396 & 0.098135 & 1563 \\
\hline Tanne & 163.2043 & -1.02957 & 0.6221 & -1.65499 & 0.097926 & 0.334563 \\
\hline Sulfito & 25.72218 & -1.20372 & 0.728658 & -1.65197 & 854 & 5441 \\
\hline Pseudo & 55.44427 & -0.88017 & 0.533057 & -1.65117 & 0.098704 & 0.33568 \\
\hline Pseud & 34.69003 & -1.09465 & 0.663493 & -1.64982 & & 33568 \\
\hline Brach & 5.114669 & 2.775493 & 1.681608 & 1.6505 & 0.098841 & 0.33568 \\
\hline Bacillus & 26.81687 & 1.194287 & 0.723833 & 1.649948 & 98954 & 3568 \\
\hline Vibric & 4.009242 & -2.8469 & 1.728309 & -1.64722 & 0.099513 & 0.337176 \\
\hline Serrat & 126.638 & -1.087 & 0.661677 & -1.64279 & 0.100427 & 0.338898 \\
\hline Thern & 4.336488 & -2.93936 & 1.788056 & -1.64389 & 199 & 3898 \\
\hline Sphing & 48.9315 & -0.83981 & 0.510956 & -1.64361 & 0.100257 & 0.338898 \\
\hline Kitasat & 117.9886 & -0.72774 & 0.442939 & -1.64299 & 0.100385 & 0.338898 \\
\hline Pectol & 3.66832 & -0.95394 & 0.580788 & -1.6425 & 487 & 8898 \\
\hline Xanth & 809.8201 & 0.813884 & 0.495842 & 1.641419 & 0.100711 & 0.339338 \\
\hline Micro & 117.2732 & -0.7913 & 0.482934 & -1.63852 & 0.101313 & 0.341054 \\
\hline Brevu & 336.4668 & -0.80275 & 0.490105 & -1.63792 & 1439 & 1162 \\
\hline Eiken & 92.01282 & -0.86159 & 0.526242 & -1.6 & 578 & 1312 \\
\hline Eryth & 21.49538 & -1.07367 & 0.656375 & -1.6 & 892 & 1736 \\
\hline Cronc & 16.9015 & -1.84631 & 1.128542 & -1.63601 & 0.101837 & 0.341736 \\
\hline Parac & 80.719 & -0.79355 & 0.485432 & -1.63473 & 0.102106 & 0.34214 \\
\hline Actinc & 194.9928 & -0.70371 & 0.431302 & -1.6316 & 0.102764 & 0.343649 \\
\hline Arcok & 2.07987 & 1.870137 & 1.146628 & 1.630988 & 0.102893 & 649 \\
\hline Janthi & 348.5473 & -0.77697 & 0.476438 & -1.63079 & 0.102934 & 3649 \\
\hline Sphing & 149.228 & -0.82837 & 0.507627 & -1.63184 & 0.102713 & 3649 \\
\hline Fluoriba & 3.178485 & -3.2516 & 1.995196 & -1.62971 & 0.103162 & 0.344094 \\
\hline Geoba & 9.38246 & -1.4474 & 0.888495 & -1.62904 & 0.103304 & 0.344251 \\
\hline Salinic & 77.26757 & -0.84574 & 0.519407 & -1.62827 & 0.103467 & 0.344481 \\
\hline Spiroplasma helicoides & 4.922059 & 3.585434 & 2.203313 & 1.627292 & 0.103675 & 0.344857 \\
\hline Amycc & 32.1488 & -0.66336 & 0.407824 & -1.62658 & 0.103826 & 0.345043 \\
\hline Reinekea forsetii & 5.30261 & -2.14015 & 1.316518 & -1.62561 & 0.104032 & 0.345097 \\
\hline Alcanivorax pacificus & 47.02199 & -1.16503 & 0.71666 & -1.62564 & 0.104027 & 0.345097 \\
\hline Pseudodesulfovibrio indicus & 50.69483 & -0.84585 & 0.520777 & -1.6242 & 0.104332 & 0.345465 \\
\hline Maritalea myrionectae & 11.62095 & -1.48116 & 0.911921 & -1.62422 & 0.104328 & 0.345465 \\
\hline Parageobacillus genomosp. 1 & 4.349983 & -2.36573 & 1.457062 & -1.62363 & 0.104455 & 0.345555 \\
\hline
\end{tabular}


Elizabethkingia anophelis

Sinorhizobium sp. CCBAU 05631

Phaeobacter piscinae

Terriglobus saanensis

Algoriphagus sp. M8-2

Pseudomonas trivialis

Mycolicibacterium fortuitum

Saccharomonospora viridis

Erythrobacter flavus

Streptomyces sp. SCSIO 03032

Natronolimnobius aegyptiacus

Rhizobium sp. NXC24

Anaerolinea thermophila

Phaeobacter gallaeciensis

Mycolicibacterium chubuense

Synechococcus sp. NIES-970

Actinoalloteichus sp. GBA129-24

Croceicoccus naphthovorans

Pasteurella multocida

Chthonomonas calidirosea

Stackebrandtia nassauensis

Streptococcus mitis

Bifidobacterium animalis

Pseudomonas aeruginosa

Synechococcus sp. RCC307

Clostridium taeniosporum

Escherichia coli

Streptomyces autolyticus

Komagataeibacter medellinensis

Aggregatibacter segnis

Ensifer adhaerens

Flavobacterium pallidum

Bifidobacterium angulatum

Pseudomonas sp. LH1G9

Streptomyces sp. 3214.6

Streptomyces lincolnensis

Nocardioides sp. JS614

Sphingomonas sp. NIC1

Pectobacterium atrosepticum

Victivallales bacterium CCUG 44730

Erythrobacter litoralis

Deinococcus sp. S14-83

Aeromicrobium marinum

Kyrpidia tusciae

Bordetella hinzii

Magnetospirillum magneticum

Sphingobium yanoikuyae

Arachidicoccus sp. BS20

Herminiimonas arsenitoxidans

Sphingobium sp. EP60837
58.455

6.441557

23.58488

38.17582

4.39735

88.90743

23.62939

73.17759

40.40057

103.2082

20.89905

41.58413

5.216669

67.98729

203.0172

6.471517

28.34508

92.9579

500.4636

11.37317

110.0361

3687.344

69.74894

3541.15

16.60396

20.95509

617.7633

38.34041

16.84699

148.7299

153.7402

3.930511

41.31118

21.61554

72.99508

73.23615

278.6914

165.0226

15.47644

23.04644

104.5257

18.67232

127.553

16.16646

137.5905

78.93685

353.2958

39.09386

59.52639

26.7508

0.80503

$-2.37793$

0.496357

1.621876

0.10483

0.346167

$-1.28402$

$1.465982-1.62208$

0.104787

0.346167

$-1.06809$

0.791927

$-1.62138$

0.104935

0.346201

$-2.2437$

$-1.62026$

0.105176

0.346681

$-0.82187$

1.386143

$-1.61867$

0.105519

0.347497

$-1.40358$

0.507986

$-1.61789$

0.105686

0.347713

$-0.70994$

0.867763

$-1.61747$

0.105776

0.347713

$-1.02032$

0.63197

$-1.61678$

0.105925

0.347888

$-0.75594$

0.468186

$-1.61451$

0.106418

0.348877

$-1.50963$

0.935527

$-1.61461$

0.106394

0.348877

$-0.94971$

0.588764

$-1.61367$

0.1066

0.349161

$-1.621$

$-1.61306$

0.106731

0.349274

$-0.79419$

$-1.61106$

0.107166

0.349755

$-0.87527$

$-1.61111$

0.107155

0.349755

$-1.8946$

$-1.61175$

0.107017

0.349755

$-3.47983$

2.162246

$-1.60902$

0.107612

0.350581

$-1.01683$

0.632826

$-1.60936$

0.107538

0.350581

$-0.81053$

0.504669

$-1.6068$

0.108097

0.351848

$-1.6873$

1.051197

$-1.60606$

0.10826

0.352062

$-0.52057$

0.324707

$-1.60512$

0.108467

0.352421

$-0.87245$

0.544057

$-1.60319$

0.108893

0.352934

$-0.7257$

0.452794

$-1.6036$

0.108803

0.352934

$-0.80567$

0.50271

$-1.60271$

0.108999

0.352934

$-1.51611$

0.948121

$-1.60265$

0.109013

0.352934

1.423886

0.890628

$-1.59906$

0.109806

0.354403

0.673029

0.420936

1.598744

0.109878

0.354403

$-1.8211$

1.138974

1.598885

0.109846

0.354403

$-1.44511$

0.904099

$-1.5989$

0.109844

0.354403

$-0.95717$

0.59983

$-1.5984$

0.109954

0.354403

$-0.68589$

0.429984

$-1.59573$

0.110549

0.355163

$-2.74515$

1.71965

$-1.59516$

0.110677

0.355163

$-1.59634$

0.110412

0.355163

$-1.14507$

0.717756

$-1.59534$

0.110636

0.355163

$-1.6087$

1.007796

$-1.59626$

0.110431

0.355163

$-0.79817$

0.500655

$-1.59425$

0.110879

0.355497

$-0.8969$

0.562946

$-1.59322$

0.11111

0.35561

$-0.78925$

0.49533

$-1.59339$

0.111073

0.35561

$-0.9083$

0.57089

$-1.59103$

0.111604

0.356876

$-1.63741$

1.029547

$-1.59042$

0.111741

0.357002

$-0.97067$

0.611235 
Micromonospora inositola

Haloferax mediterranei

Acidithiobacillus caldus

Akkermansia glycaniphila

Truepera radiovictrix

Methylophilus sp. TWE2

Micropruina glycogenica

Pimelobacter simplex

Cytophagales bacterium TFI 002

Caulobacter segnis

Thermoanaerobacter kivui

Nocardioides sp. CF8

Streptomyces katrae

Celeribacter indicus

Streptomyces hygroscopicus

Wenzhouxiangella marina

Pseudomonas azotoformans

Plantibacter sp. PA-3-X8

Pseudoalteromonas piscicida

Thermobifida fusca

Xylella fastidiosa

Lachnospiraceae bacterium Choco86

Streptomyces clavuligerus

Mycobacterium avium

Komagataeibacter nataicola

Micromonospora krabiensis

Nostocales cyanobacterium HT-58-2

Pandoravirus dulcis

Campylobacter lari

Candidatus Methylopumilus turicensi

Pseudomonas poae

Leifsonia sp. 21MFCrub1.1

Plautia stali symbiont

Runella slithyformis

Blattabacterium punctulatus

Pseudomonas sp. PONIH3

Geobacillus thermodenitrificans

Micromonospora narathiwatensis

Brachyspira hyodysenteriae

Bordetella sp. HZ2O

Macrococcus canis

Beijerinckia indica

Thermobaculum terrenum

Deferribacter desulfuricans

Paenibacillus graminis

Vagococcus teuberi

Catenulispora acidiphila

Halopiger xanaduensis

Methylobacterium sp. 4-46

Denitrobacterium detoxificans

\begin{tabular}{|c|c|c|c|c|c|}
\hline & & & & & \\
\hline 2.761317 & -2.717 & 1.720564 & -1.57913 & 0.114306 & 0.361075 \\
\hline 29265 & -1.05294 & 0.667361 & -1.57776 & 0.11462 & \\
\hline 15.80469 & -1.53837 & 0.975535 & -1.57695 & 0.114808 & 0.361406 \\
\hline .99921 & -0.86725 & 0.549885 & -1.57714 & 0.114762 & 0.361 \\
\hline 18.05416 & -1.61464 & 1.023298 & -1.57788 & 0.114594 & 0.361406 \\
\hline 73.0943 & -0.60771 & 0.385713 & -1.57555 & 0.115129 & 0.362105 \\
\hline 241.5847 & -0.67929 & 0.432216 & -1.57164 & 0.116035 & 0.363643 \\
\hline .379138 & -1.7065 & 1.086193 & -1.57109 & 0.116162 & 0.363643 \\
\hline 164.6597 & -0.8796 & 0.55995 & -1.57085 & 0.116218 & 0.363643 \\
\hline 6.618002 & -2.78027 & 1.769712 & -1.57103 & 0.116176 & 0.363643 \\
\hline 288.9303 & -0.81788 & 0.520503 & -1.57132 & 0.116108 & 0.363643 \\
\hline 63.87104 & -0.75045 & 0.477297 & -1.57229 & 0.115883 & 0.363643 \\
\hline 67.74218 & -0.74884 & 0.476898 & -1.57022 & 0.116364 & 0.363786 \\
\hline 303.663 & -0.74132 & 0.472627 & -1.5 & 676 & 4713 \\
\hline 40.62756 & -0.74727 & 0.476728 & -1.5675 & 0.116999 & 0.365144 \\
\hline 144.7901 & -0.97727 & 0.623704 & -1.56688 & 0.117143 & 5282 \\
\hline 179.5749 & -0.81724 & 0.522428 & -1.56432 & 0.117743 & 0.366839 \\
\hline 5.407991 & 2.356633 & 1.507365 & 1.563413 & 0.117956 & 0.366906 \\
\hline 55.54363 & -0.65516 & 0.419073 & -1.56337 & 0.117967 & 0.366906 \\
\hline 12.78716 & -1.2358 & 0.791026 & -1.56227 & 0.118224 & 0.367393 \\
\hline 61.20806 & -1.142 & 0.731273 & -1.56166 & 0.118369 & 36753 \\
\hline 80.61255 & -0.75659 & 0.485207 & -1.5593 & 0.118925 & 0.368372 \\
\hline 262.2296 & -0.7009 & 0.449352 & -1.55981 & 0.118805 & 0.368372 \\
\hline 31.56152 & -1.23801 & 0.793994 & -1.55922 & 0.118944 & 0.368372 \\
\hline 119.3975 & -0.77314 & 0.49628 & & 263 & \\
\hline 16.47553 & 1.626874 & 1.0449 & 1.556966 & 0.119478 & 0.369399 \\
\hline 2.228853 & -3.25453 & 2.091444 & -1.55612 & 0.11968 & 0.369709 \\
\hline 19.47639 & 1.565442 & 1.00634 & 1.555579 & 0.119808 & 0.369719 \\
\hline 2.967508 & -2.50888 & 1.613168 & -1.55525 & 0.119887 & 0.36971 \\
\hline 68.04628 & -1.14969 & 0.739864 & -1.55392 & 0.120202 & 0.370378 \\
\hline 223.095 & -0.83652 & 0.539094 & -1.55172 & 0.120729 & 0.371564 \\
\hline 48.08774 & -1.16255 & 0.749327 & -1.55 & 0.120791 & 0.371564 \\
\hline 12.88022 & -1.4602 & 0.94241 & -1.54943 & 0.121279 & 0.372433 \\
\hline 3.766786 & -4.00668 & 2.585432 & -1.54971 & 0.12121 & 0.37243 \\
\hline 29.03209 & -2.10021 & 1.356808 & -1.54791 & 0.121644 & 0.37287 \\
\hline 3.682104 & -3.97383 & 2.567818 & -1.54755 & 0.12173 & 0.37287 \\
\hline 58.36008 & -0.82041 & 0.53003 & -1.54785 & 0.121658 & 0.372875 \\
\hline 15.25195 & 1.885969 & 1.219794 & 1.546137 & 0.122071 & 0.373606 \\
\hline 18.4753 & -0.93504 & 0.605063 & -1.54535 & 0.122261 & 0.373873 \\
\hline 20.11054 & 1.637789 & 1.060321 & 1.544616 & 0.122439 & 0.373922 \\
\hline 20.15567 & -1.07822 & 0.698134 & -1.54444 & 0.122483 & 0.373922 \\
\hline 5.651263 & -1.85943 & 1.204353 & -1.54392 & 0.122607 & 0.37398 \\
\hline 8.057834 & 2.351633 & 1.5267 & 1.540338 & 0.123478 & 0.3747 \\
\hline 4.494498 & -2.28747 & 1.486661 & -1.53866 & 0.123887 & 0.37476 \\
\hline 10.0315 & -1.39698 & 0.907945 & -1.53862 & 0.123898 & 0.3747 \\
\hline 123.2827 & -0.72047 & 0.467761 & -1.54025 & 0.123498 & 0.3747 \\
\hline 22.23698 & -1.31399 & 0.853891 & -1.53883 & 0.123847 & 0.3747 \\
\hline 178.3471 & -0.7426 & 0.482765 & -1.53823 & 0.123993 & 0.3747 \\
\hline 14.58725 & -1.52551 & 0.990791 & -1.53969 & 0.123636 & 0.3747 \\
\hline
\end{tabular}


Sulfitobacter sp. JL08

Jonquetella anthropi

Streptomyces sp. 2323.1

Arcobacter butzleri

Azoarcus communis

Actinoplanes sp. N902-109

Anderseniella sp. Alg231-50

Klebsiella sp. FDAARGOS_511

Mycolicibacterium rutilum

Streptomyces scabiei

Blastomonas sp. RAC04

Gardnerella vaginalis

Amycolatopsis methanolica

Streptococcus sp. NPS 308

Clostridium argentinense

Mycolicibacter terrae

Neisseria polysaccharea

Cellulophaga lytica

Aeromicrobium sp. 592

Gemmatimonas aurantiaca

Defluviimonas alba

Weissella jogaejeotgali

Kutzneria albida

Leptospira interrogans

Desulfitobacterium hafniense

Muribaculum intestinale

Microbacterium chocolatum

Pantoea sp. PSNIH2

Streptococcus oralis

Cronobacter dublinensis

Nonlabens marinus

Corynebacterium glyciniphilum

Legionella hackeliae

Cryobacterium sp. GCJ02

Chloracidobacterium thermophilum

Methylocella silvestris

Desulfurobacterium thermolithotrop

Caldilinea aerophila

Thioalkalivibrio sp. K90mix

Streptomyces malaysiensis

Rhodothermaceae bacterium RA

Neisseria zoodegmatis

Salinimonas sp. N102

Thermomonospora curvata

Streptomyces sp. HNM0039

Actinobacillus porcitonsillarum

Roseibacterium elongatum

Bacillus infantis

Mycolicibacter sinensis

Arsenicicoccus sp. oral taxon 190
11.62513

49.2022

75.79492

15.65526

161.1398

147.7083

16.21596

6.921577

169.645

76.99777

34.57287

389.2049

143.3455

716.512

30.78503

76.15183

38.26301

12.55926

223.0555

54.58886

114.1973

6.270503

171.5595

13.05474

6.172999

22.11401

162.8819

4.376535

4674.86

15.02026

3.779116

473.0626

4.214733

93.32907

13.31807

52.90454

3.81459

18.12036

25.93694

34.7346

41.80933

34.40045

3.182952

104.9473

49.02471

9.928051

40.08803

9.733232

101.5284

223.3571
$-1.69913$

$-1.28658$

$-0.73566$

1.896554

$-0.79777$

$-0.69197$

$-0.86381$

$-1.47089$

$-0.52879$

$-0.63725$

$-1.13872$

0.973934

$-0.60721$

$-0.85424$

1.021335

$-0.57406$

$-1.215$

1.666224

$-0.77609$

$-0.76169$

$-0.87874$

$-1.92542$

$-0.50503$

2.044741

$-1.86518$

$-1.51704$

$-0.8273$

$-4.21879$

$-0.8217$

$-1.24641$

$-2.40203$

$-0.71422$

$-2.2646$

$-0.84233$

$-1.22876$

$-0.95617$

$-2.39137$

$-1.01597$

$-0.92854$

$-1.41779$

$-1.43491$

$-1.00546$

$-2.57885$

$-0.71059$

$-0.71829$

$-1.71656$

$-0.88123$

$-1.41629$

$-0.66214$

$-0.63082$

1.103338

0.836292

0.477209

$-1.53999$

0.123562

0.123943

$-1.53843$

0.123175

1.230151

$-1.54158$

0.12314

0.5189

1.541725

0.124188

0.124331

0.124246

$-1.53719$

0.12463

$-1.53563$

0.124943

0.344632

$-1.53435$

$-1.5341$

0.125006

$-1.53267$

0.125356

0.742963

0.6359811 .531389

0.125673

$-1.52829$

0.126441

$-1.52837$

0.126422

0.668409

1.528009

0.12651

0.375331

$-1.52947$

0.126147

0.794784

$-1.52872$

0.126334

$\begin{array}{ll}1.091194 & 1.526973\end{array}$

0.126768

0.508758

$-1.52545$

0.127147

$-1.52349$

0.127636

0.576449

$-1.52441$

0.127407

$-1.5237$

0.127583

1.263642

0.331406

$-1.52389$

0.127536

1.342754

1.522796

0.12781

1.225463

$-1.52202$

0.128003

0.997002

$-1.5216$

0.128109

0.543915

$-1.52102$

0.128256

2.776235

$-1.51961$

0.12861

$-1.51917$

0.128719

0.37476

0.37476

0.37476

0.37476

0.540889

$-1.51704$

0.129256

1.583078

$-1.51732$

0.129186

0.470881

$-1.51678$

0.129321

1.493944

$-1.51585$

0.129556

0.556469

$-1.5137$

0.130103

0.374846

0.374846

0.374846

0.375436

0.375948

0.375948

0.376689

0.377331

0.378283

0.378283

0.378283

0.812637

$-1.51207$

0.130516

0.378283

0.378283

0.378742

0.379564

0.379779

0.379779

0.379779

0.379779

0.379983

0.380248

0.380252

0.380377

0.381116

0.38113

0.381981

0.381981

0.381981

0.382364

0.383666

0.631985

$-1.51296$

0.13029

0.383683

1.581227

$-1.51235$

0.130445

0.383683

0.671931

$-1.51201$ 
Olsenella uli

Bacteroidales bacterium CF

Peptoclostridium acidaminophilum

Pseudomonas syringae group genom

Janthinobacterium svalbardensis

Paenibacillus sp. RUD330

Coriobacterium glomerans

Arcanobacterium haemolyticum

Acidovorax ebreus

Aggregatibacter actinomycetemcomi

Microcystis aeruginosa

Bacillus amyloliquefaciens

Phaeobacter porticola

Pseudovibrio sp. FO-BEG1

Shewanella sp. ANA-3

Luteimonas sp. 100111

Methylomonas denitrificans

Weeksella virosa

Streptomyces sp. 3211

Oceanithermus profundus

Hirschia baltica

Halomonas hydrothermalis

Pseudopedobacter saltans

Fibrella aestuarina

Mesoplasma florum

Corynebacterium halotolerans

Mannheimia haemolytica

Pseudomonas sp. MYb193

Austwickia chelonae

Rhodocyclaceae bacterium

Altererythrobacter sp. B11

Nitrobacter winogradskyi

Pelodictyon phaeoclathratiforme

Celeribacter manganoxidans

Corynebacterium xerosis

Chloroflexus aurantiacus

Leisingera methylohalidivorans

Sphaerochaeta pleomorpha

Sphingomonas panacis

Desulfarculus baarsii

Marinovum algicola

Streptomyces puniciscabiei

Pseudomonas psychrophila

Rhodococcus sp. YL-1

Trichodesmium erythraeum

Jeotgalibaca dankookensis

Pseudomonas sp. ATCC 13867

Paenibacillus sabinae

Streptococcus pseudopneumoniae

Actinobacteria bacterium IMCC2625€
63.9443

4.196043

6.286656

71.51348

120.2751

36.19845

21.48826

65.43776

937.0497

15.09915

5.382946

6.126276

8.923607

8.22878

4.757375

87.7219

18.83825

19.02097

37.84734

67.85183

1.768145

40.18397

27.28402

44.74934

14.41245

1275.92

5.634772

88.42196

64.83328

53.96172

53.44701

44.35457

2.633016

43.0842

783.149

8.014163

34.58833

3.598214

170.3358

45.60488

60.5268

65.99402

83.5117

133.4787

17.17001

6.656069

93.63538

18.21746

546.1563

4.424699
$-0.87659$

$-2.22614$

$-1.90945$

$-1.5022$

$-0.75157$

$-0.85855$

$-1.13875$

$-0.70376$

$-0.96455$

$-1.60766$

$-2.51902$

$-2.35835$

$-1.67276$

$-1.26647$

$-2.24971$

$-0.85017$

$-1.18683$

$-1.89648$

$-0.76547$

$-0.72527$

$-2.92289$

$-0.92398$

$-1.13883$

$-1.07619$

2.307859

$-0.38086$

$-1.69902$

$-0.92204$

$-0.66042$

$-1.0227$

$-0.79576$

$-0.93027$

$-2.55835$

$-0.86822$

$-0.58503$

$-1.61451$

$-0.85774$

$-1.92067$

$-0.85149$

$-0.84361$

$-0.84396$

$-0.55676$

$-0.85222$

$-1.08153$

1.391566

$-1.62736$

$-0.6672$

$-1.29551$

$-0.89829$

$-2.02415$

0.585234

$-1.49784$

0.134175

1.486859

$-1.49721$

0.134338

0.390298

$\begin{array}{llll}1.277676 & -1.49447 & 0.135053\end{array}$

0.39046

1.005053

$-1.49465$

0.135005

0.390976

0.502848

$-1.49463$

0.135011

0.390976

0.574256

$-1.49506$

0.134899

0.390976

$0.761479-1.49545$

0.134797

0.390976

0.471156

$-1.49368$

0.135259

0.390976

0.646164

$-1.49273$

0.135509

0.391263

1.077255

$-1.49237$

0.135602

0.391321

1.687538

$-1.49272$

0.135511

0.391321

1.580797

$-1.49188$

0.135732

0.391321

1.121746

$-1.49121$

0.135906

0.391385

0.850325

$-1.4894$

0.136383

0.391578

1.511501

$-1.4884$

0.136646

0.392639

0.571461

$-1.48771$

0.136828

0.392999

0.797765

$-1.48769$

0.136832

0.392999

1.275677

$-1.48665$

0.137108

0.392999

0.515472

$-1.48498$

0.137548

0.393484

0.488305

$-1.48527$

0.137472

0.393814

$1.967501-1.48559$

0.137389

0.393814

$0.622684-1.48386$

0.137846

0.393814

$0.767893-1.48305$

0.13806

0.394356

$0.726819-1.48068$

0.138692

0.39466

$1.559075 \quad 1.480274$

0.1388

0.395169

$0.257351-1.47994$

0.13889

0.395169

$1.14742-1.48073$

0.138677

0.395169

0.622791

$-1.48049$

0.138742

0.395169

0.446172

$-1.4802$

0.13882

0.395169

0.691822

$-1.47827$

0.139334

0.395169

0.538252

$-1.47841$

0.139298

0.395814

0.629684

$-1.47735$

0.139581

0.395814

1.733851

$-1.47553$

0.14007

0.396204

0.5888

$-1.47456$

0.140332

0.397283

$0.397273-1.47263$

0.140851

0.397716

$1.097812-1.47066$

0.141383

0.398877

0.583292

$-1.47051$

0.141423

0.39967

1.306245

$-1.47037$

0.14146

0.39967

0.579705

$-1.46883$

0.14188 
Nitrosococcus halophilus

Yangia sp. CCB-MM3

Nitrosomonas eutropha

Magnetospirillum sp. XM-1

Lacinutrix sp. Bg11-31

Caldithrix abyssi

Corynebacterium sp. 2183

Aquimarina sp. BL5

Cellulomonas sp. PSBB021

Corynebacterium ureicelerivorans

Tatumella citrea

Staphylococcus condimenti

Erythrobacter sp. $\mathrm{YH}-07$

Bacillus sonorensis

Yersinia rohdei

Psychrobacter alimentarius

Rhodobacter capsulatus

Flavobacterium crassostreae

Sphingorhabdus flavimaris

Deinococcus puniceus

Streptococcus parasanguinis

Pseudomonas salegens

Propionimicrobium sp. Marseille-P32

Deinococcus wulumuqiensis

Stenotrophomonas acidaminiphila

Cobetia marina

Rhodococcus fascians

Acinetobacter sp. SWBY1

Serratia sp. 3ACOL1

Yersinia similis

Pseudomonas sp. NCO2

Rhizobium sp. 11515TR

Pseudomonas agarici

Streptomyces cavourensis

Pajaroellobacter abortibovis

Thermoanaerobacterium thermosacc

Xanthomonas cassavae

Aeromonas veronii

Paucimonas lemoignei

Wigglesworthia glossinidia

Bacillus paralicheniformis

Aeromonas schubertii

Microvirgula aerodenitrificans

Nocardioides sp. 603

Tumebacillus avium

Sphingopyxis sp. MG

Bifidobacterium pseudolongum

Lactobacillus sakei

Sphingobium sp. SYK-6

Stenotrophomonas sp. Pemsol
16.46689

80.34796

6.386262

69.86906

9.791767

3.592723

1231.099

15.82081

134.3028

11632.75

12.25808

63.07245

24.097

9.487643

4.34842

58.55253

70.4406

3.319957

16.75647

39.90804

1663.424

21.02057

165.8388

589.0279

201.7128

34.81917

443.015

169.0957

2.465467

3.031751

100.3453

71.11979

44.79629

16.1046

2.327978

26.91996

69.39823

63.51096

26.86291

5.183589

16.11031

21.98253

85.92079

227.8075

27.70076

14.24894

44.84731

36.67707

85.82851

37.64758
$-0.9478$

$-0.65661$

$-1.63411$

$-0.77587$

1.731902

$-2.01504$

$-0.38358$

1.342185

$-0.63269$

$-0.79725$

$-1.18052$

0.819656

$-1.10621$

$-1.06388$

$-2.16151$

$-1.38044$

$-0.8603$

$-2.20146$

$-1.09944$

$-0.83862$

$-0.85922$

$-0.93052$

$-0.75312$

$-0.84989$

$-0.69885$

$-0.98778$

0.998147

$-1.32588$

3.607818

$-2.47437$

$-1.07885$

$-0.80987$

$-0.83959$

$-1.5523$

$-3.29899$

1.672927

$-0.86911$

$-0.69977$

$-0.73393$

2.289389

$-1.45597$

$-0.88832$

$-0.8025$

$-0.59822$

$-0.92776$

$-2.57859$

$-0.69044$

$-1.08117$

$-0.55723$

$-0.89729$
0.650183

0.450442

1.121091

0.53241

1.188405

1.38071

0.263334

0.921877

0.434628

0.548038

0.81152

0.564416

0.762074

0.73339

1.491557

0.952769

0.594019

1.521626

0.760223

0.580016

0.594606

0.645319

0.522467

0.589736

0.48597

0.687852

0.694684

0.923531

2.511173

1.723547

0.752765

0.564985

0.585804

1.081781

2.301779

1.166836

0.60667

0.489011

0.513889

1.603086

1.019686

0.622567

0.563469

0.420106

0.651664

1.809961

0.485381

0.76046

0.392213

0.631784

$-1.45775$

0.14491

$-1.4577$

0.144924

$-1.45761$

0.144949

$-1.45727$

0.145041

1.457333

0.145025

$-1.45942$

0.144448

$-1.45661$

0.145223

1.455926

0.145413

$-1.4557$

0.145475

$-1.45473$

0.145743

$-1.45471$

0.14575

1.45222

0.146441

$-1.45157$

0.14662

$-1.45063$

$-1.44917$

0.146883

0.147375

$-1.44828 \quad 0.14754$

$-1.446780 .147959$

$-1.44621$

0.14812

$-1.44586$

0.148217

$-1.44502$

0.148452

$-1.44196$

0.149314

$-1.44147$

0.149452

$-1.44113$

0.149548

$-1.43805$

0.150419

$-1.43603$

0.150993

1.436837

0.150764

$-1.43566$

0.151098

1.436706

0.150802

0.404139

0.404139

0.404139

0.404139

0.404139

0.404139

0.404337

0.404418

0.404418

0.404565

0.404565

0.406172

0.406361

0.406779

0.40752

0.40752

0.407666

0.408515

0.408608

$-1.43563$

0.151109

0.408608

$-1.43318$

0.151805

0.408945

0.411009

0.411032

0.411032

$-1.43344$

0.151731

0.413114

0.413447

0.413447

0.413447 
Salinibacter ruber

Pseudoalteromonas atlantica

Streptomyces sp. 4F

Streptococcus sp. ChDC B345

Zhihengliuella sp. ISTPL4

Nostoc sp. PCC 7107

Cellulomonas flavigena

Corallococcus coralloides

Tessaracoccus sp. T2.5-30

Luteimonas sp. 83-4

Kangiella koreensis

Faecalibacterium prausnitzii

Streptomyces avermitilis

Candidatus Puniceispirillum marinum

Bacillus cereus

Thermus parvatiensis

Paracoccus yeei

Sideroxydans lithotrophicus

Pseudomonas synxantha

Mariniflexile sp. TRM1-10

Marmoricola scoriae

Cylindrospermum stagnale

Roseburia hominis

Blastococcus saxobsidens

Agromyces sp. 30A

Haliangium ochraceum

Shewanella sp. M2

Rhodobacteraceae bacterium

Sphingobium japonicum

Hungateiclostridium clariflavum

Pseudopropionibacterium propionicu

Streptomyces chartreusis

Staphylococcus sciuri

Grimontia hollisae

Nocardia brasiliensis

Gilliamella apicola

Streptomyces davaonensis

Altererythrobacter dongtanensis

Campylobacter hyointestinalis

Microvirga sp. 17 mud 1-3

Clostridium cellulovorans

Halorubrum trapanicum

Martelella sp. AD-3

Streptomyces coelicolor

Micromonospora zamorensis

Corynebacterium testudinoris

Geodermatophilus obscurus

Microvirga ossetica

Shimwellia blattae

Aerococcus christensenii
30.19265

13.05281

57.54497

1277.41

117.126

7.392025

122.3975

124.014

130.1692

99.31658

3.150194

757.7793

50.7999

3.677288

720.8196

2.599109

1046.43

52.1271

180.2328

13.59589

332.8108

3.156269

91.26268

231.9099

93.3398

183.6123

8.042761

7.581533

38.79023

14.98269

353.3056

69.67192

91.83263

3.49451

279.0531

17.12591

68.25913

47.86251

4.640809

60.01372

24.16152

37.91756

66.33866

22.78393

65.16846

988.7515

300.1854

136.6061

901.6882

54.94885
$-0.87517$

$-2.24735$

$-0.79341$

$-0.91447$

$-0.61181$

$-2.25363$

$-0.71311$

$-0.79654$

$-0.61173$

$-0.73803$

$-2.60483$

$-0.73017$

$-0.65664$

$-1.86553$

0.612622

$-3.47393$

$-0.86523$

$-0.8419$

$-0.76615$

1.479103

$-0.59781$

$-2.25005$

$-1.15035$

$-0.67706$

$-0.65401$

$-0.64147$

$-2.01653$

$-1.42688$

$-0.97751$

$-1.12033$

$-0.78466$

$-0.7528$

0.677112

$-2.07823$

$-0.44955$

1.307109

$-0.60248$

$-0.7301$

2.388637

$-0.68828$

0.912341

$-0.96199$

$-0.86113$

$-2.26498$

$-0.59435$

$-0.45759$

$-0.74581$

$-0.7249$

$-1.65677$

$-0.94474$
0.616482

1.583821

0.559344

0.647868

0.433033

1.59258

0.505115

0.564188

0.432642

0.521539

1.841634

0.515482

0.464077

1.318462

0.433952

2.451388

0.610334

0.59524

0.542419

1.04751

0.423896

1.597474

0.816135

0.480707

0.464088

0.455313

1.433398

1.013951

0.694652

0.797683

0.558887

0.536298

0.482916

1.482508

0.321655

0.936812

0.432401

0.523592

1.715444

0.49442

0.654732

0.690874

0.61815

1.627518

0.427499

0.329142

0.536611

0.522434

1.193502

0.68092

$-1.41961$

0.155721

$-1.41894$

0.155917

0.419443

$-1.418470 .156053$

0.41966

$-1.4115$

0.158098

0.419717

$-1.41286$

0.157698

0.41994

$-1.41508$

0.157046

$-1.41178$

0.158016

$-1.41183$

0.157999

$-1.41394$

0.15738

$-1.41511$

0.157037

$-1.41441$

0.157241

$-1.41649$

0.156633

$-1.41494$

0.157086

$-1.41493$

0.15709

1.411727

0.15803

$-1.417130 .156446$

$-1.41763$

0.1563

$-1.41438$

0.15725

$-1.41248$

0.15781

1.412019

0.157944

0.41994

0.41994

0.41994

0.41994

0.41994

0.41994

0.41994

0.41994

0.41994

0.41994

0.41994

0.41994

0.41994

0.41994

0.41994

$-1.41028$

0.158458

0.41994

$-1.40851$

0.158981

0.420475

$-1.40951$

0.158683

0.420475

$-1.40847$

0.158992

0.420475

$-1.40923$

0.158768

0.420475

$-1.40886$

0.158876

0.420475

$-1.40682$

0.159481

0.420475

$-1.40724$

0.159355

0.42085

$-1.4072$

0.159369

0.42085

$-1.40448$

0.160175

0.42085

$-1.40397$ 
Salinispira pacifica

Microbacterium sp. 10M-3C3

Streptomyces albidoflavus

Ketogulonicigenium vulgare

Streptococcus halotolerans

halophilic archaeon DL31

Tumebacillus algifaecis

Corynebacterium sphenisci

Desulfotomaculum nigrificans

Trueperella pyogenes

Gordonibacter urolithinfaciens

Pseudomonas sp. MRSN12121

Corynebacterium mycetoides

Frankia alni

Arthrobacter crystallopoietes

Nocardioides sp. 78

Microbacterium sp. No. 7

Archaeoglobus veneficus

Streptococcus gallolyticus

Zymomonas mobilis

Corynebacterium sp. 2184

Parasaccharibacter apium

Faecalitalea cylindroides

Lactobacillus acetotolerans

Hahella chejuensis

Petrimonas mucosa

Acinetobacter junii

Sulfitobacter sp. SK011

Corynebacterium uterequi

Cnuibacter physcomitrellae

Vibrio scophthalmi

Morganella morganii

Marinobacter sp. es.042

Streptomyces sp. RTd22

Acinetobacter johnsonii

Hoyosella subflava

Actinobacteria bacterium IMCC2500ミ

Leifsonia xyli

Streptomyces gilvosporeus

Lactococcus sp. 1JSPR-7

Arcobacter cryaerophilus

Devosia sp. H5989

Brochothrix thermosphacta

Rhodococcus qingshengii

Streptococcus sp. Z15

Limnobaculum parvum

Methylovorus glucosotrophus

Gammapapillomavirus 15

Pseudonocardia dioxanivorans

Streptomyces nodosus
3.252053

174.7153

13.78214

31.85295

10.72051

3.935413

7.594531

388.8036

6.597362

126.3233

28.08186

26.17022

2103.302

100.3366

151.839

171.9018

271.0968

3.962357

10.01118

16.65407

1481.844

12.80889

51.16706

4.104249

16.58584

18.68028

231.6309

13.95663

810.4508

212.0127

18.32079

25.48427

14.35359

125.1065

434.6131

84.62079

5.845513

284.2904

88.68385

4.066806

10.86165

64.00413

314.3006

111.3314

5.766135

8.893728

10.44418

15.59997

196.1499

64.60014

$-2.37377 \quad 1.711163$

$-1.38723$

0.165372

0.429543

$-0.56127$

0.405343

$-1.38468$

0.16615

0.431142

$-2.4961$

1.803478

$-1.38405$

0.166343

0.431142

$-0.93343$

0.674379

$-1.38413$

0.166319

0.431142

$-1.430571 .034007$

$-1.38352$

0.166506

0.43123

$-2.0277$

1.46623

$-1.38294$

0.166684

0.43123

$-1.31674$

0.952243

$-1.38278$

0.166733

0.43123

$-0.45788$

0.331627

$-1.38072$

0.167366

0.43256

3272

1.111194

$-1.37935$

0.167788

0.432986

$-0.37401$

0.271211

$-1.37902$

0.167888

0.432986

$-1.16994$

0.848189

$-1.37934$

0.16779

0.432986

$-1.48575$

1.078056

$-1.37818$

0.168149

0.433193

$-0.51675$

0.375104

$-1.37761$

0.168325

0.433193

$-0.66929$

0.485726

$-1.37792$

0.168227

0.433193

$-0.50925$

0.370201

$-1.3756$

0.168947

0.434323

$-0.733720 .533452$

$-1.37541$

0.169003

0.434323

$-0.6381$

0.464778

$-1.37291$

0.169781

0.435095

$-1.99837$

1.454767

$-1.37367$

0.169543

0.435095

$-1.56365$

1.138619

$-1.37329$

0.169663

0.435095

$-1.05486$

0.767862

$-1.37376$

0.169515

0.435095

$-0.41134$

0.300117

$-1.37061$

0.170498

0.435916

$-1.39901 \quad 1.021203$

$-1.36996$

0.170698

0.435916

$-1.37056$

0.170511

0.435916

$-2.70171$

1.970349

$-1.37118$

0.170317

0.435916

$-0.87601$

0.639486

$-1.36987$

0.170727

0.435916

$-1.20664$

0.881279

$-1.36919$

0.17094

0.435916

$-1.36944$

0.170861

0.435916

$-1.30003$

0.95093

$-1.36711$

0.171591

0.437269

$-0.40265$

0.294671

$-1.36643$

0.171805

0.437508

$-0.81108$

0.593876

$-1.36575$

0.172018

0.437745

$-1.67606$

1.229104

$-1.36365$

0.172679

0.438092

$-1.36406$

0.172548

0.438092

$-1.16263$

$-1.36263$

0.172998

0.438092

$-0.52785$

$-1.36439$

0.172445

0.438092

$-0.77032$

$-1.36335$

0.172772

0.438092

$-0.64202$

0.471145

$-1.36268$

0.172983

0.438092

$-1.70338$

1.248537

$-1.3643$

0.172473

0.438092

$-0.54676$

0.402034

$-1.35999$

0.173833

0.439902

$-0.52064$

0.382936

$-1.35959$

0.17396

0.439917

$-1.82511$

1.343332

$-1.35864$

0.17426

0.440368

1.794016

1.321436

1.357626

0.174582 


\begin{tabular}{|c|c|c|c|c|c|c|}
\hline Sphingomonas sp. Cra20 & & & & & & \\
\hline Bacillus litoralis & 23.23268 & -1.038 & 0.768418 & -1.35082 & 0.176752 & \\
\hline licrobacterium sp. Y-01 & 09.6727 & -0.68121 & 0.50484 & -1.34936 & .177221 & 0.44333 \\
\hline lostridium sp. JN-1 & 7.00194 & 1.221421 & 0.905433 & 1.348991 & 0.17734 & 0.44333 \\
\hline enotrophomonas sp. ESTM1D_MKC & 7.56057 & -0.70805 & 0.524729 & -1.34936 & 0.177221 & .44333 \\
\hline cetoanaerobium sticklandii & 18.09285 & -0.9433 & 0.699333 & -1.34886 & 0.177381 & 0.44333 \\
\hline bacteria bacteriur & 3.804134 & -2.15701 & 1.600462 & -1.34775 & 0.17774 & 0.443924 \\
\hline revotella enoeca & 125.3038 & -0.78147 & 0.580346 & -1.34656 & 0.178122 & 0.444571 \\
\hline us apis & 11.49473 & 2.469619 & 1.835967 & 1.345132 & 0.178582 & 0.444806 \\
\hline Methylobacterium currus & 138.1093 & -0.62655 & 0.46566 & -1.34551 & 0.178461 & 0.444806 \\
\hline seudomonas thivervalensis & 32.51923 & -1.13914 & 0.846559 & -1.34562 & 0.178426 & 0.444806 \\
\hline nogenes & 73.13039 & -0.76104 & 0.566335 & -1.3438 & 0.179013 & 0.444966 \\
\hline piro & 2.507289 & 3.144742 & 2.339621 & 1.344125 & 0.178908 & 0.444966 \\
\hline trep & 66.22018 & -0.68538 & 0.51003 & -1.34381 & 0.17901 & 1966 \\
\hline Iter & 84.7435 & -0.73327 & 0.546625 & -1.34145 & 0.179775 & 642 \\
\hline ron & 134.0254 & -0.72295 & 0.538777 & -1.34183 & 0.179652 & 0.445642 \\
\hline CJ021 & 274.6223 & 0.653037 & 0.486732 & 1.341675 & 0.179701 & 5642 \\
\hline Cory & 1042.51 & -0.37534 & 0.279658 & -1.34215 & 0.179547 & 0.445642 \\
\hline Ezaki & 577.3077 & 0.668635 & 0.4986 & 1.341024 & 0.179913 & 0.445678 \\
\hline Mett & 2.967855 & -2.15339 & 1.607901 & -1.33926 & 0.180487 & 0.445965 \\
\hline Rufi & 16.06799 & -0.98536 & 0.735271 & -1.34013 & 0.180203 & 0.445965 \\
\hline Stre & 2.801238 & -3.57955 & 2.672286 & -1.33951 & 0.180405 & 0.445965 \\
\hline Ente & 97.28014 & -1.2276 & 0.916696 & -1.33916 & 80518 & 965 \\
\hline Rose & 9.083056 & -1.22599 & 0.917831 & -1.33575 & 0.181632 & 0.446002 \\
\hline Agro & 122.5426 & -0.61812 & 0.462261 & -1.33716 & 0.181172 & 0.446002 \\
\hline $27-7$ & 3.355274 & -3.83892 & 2.873182 & -1.33612 & 0.18151 & 5002 \\
\hline Pedo & 15.06008 & -1.27668 & 0.957369 & -1.33353 & 0.182359 & 0.446002 \\
\hline Strep & 53.8887 & -0.66961 & 0.501887 & -1.33419 & 0.182141 & 0.446002 \\
\hline Stre & 47.9511 & -0.7196 & 0.539348 & -1.3342 & 0.182139 & 5002 \\
\hline Met & 10.12305 & 2.062639 & 1.547162 & 1.333175 & 0.182474 & 0.446002 \\
\hline Muci & 40.38437 & -0.89828 & 0.672359 & -1.33602 & 0.181544 & 0.446002 \\
\hline Planoc & 25.83426 & 1.294851 & 0.970285 & 1.334506 & 0.182038 & 0.446002 \\
\hline Bacill & 6.237793 & -1.60462 & 1.203305 & -1.33351 & 0.182364 & 0.446002 \\
\hline Gord & 266.6298 & -0.63367 & 0.47533 & -1.33311 & 0.182495 & 002 \\
\hline Tess & 166.0348 & -0.46948 & 0.351058 & -1.33732 & 0.181118 & 0.446002 \\
\hline Dein & 168.2842 & 0.872484 & 0.652407 & 1.337331 & 0.181115 & 0.446002 \\
\hline Ente & 10.99994 & -1.47939 & 1.10866 & -1.3344 & 0.182074 & 0.446002 \\
\hline & & -0.67767 & 0.507355 & -1.33569 & 8165 & 002 \\
\hline lorophenolicum & 46.31487 & -0.89571 & 0.670979 & -1.33493 & 0.181898 & 0.446002 \\
\hline & 6199.243 & -0.67515 & & -1.33118 & & 0.44 \\
\hline Des & 7.699173 & -1.3607 & 1.021915 & -1.33152 & 0.183019 & 0.446955 \\
\hline & 14.42284 & -1.0587 & 0.796301 & -1.32952 & 0.183675 & 0.447086 \\
\hline Fusok & 9.774205 & 1.403263 & 1.055171 & 1.329891 & 0.183554 & 0.447086 \\
\hline Oceanisphaera profunda & 4.667748 & -1.76151 & 1.324295 & -1.33015 & 0.18347 & 0.447086 \\
\hline Strep & 907.9504 & -0.86987 & 0.653994 & -1.33009 & 0.183489 & 0.447086 \\
\hline Mycoplasma capricolum & 2.416346 & 3.384139 & 2.547611 & 1.328358 & 0.18406 & 0.447723 \\
\hline & 117.4499 & -1.49723 & 1.127695 & -1.32769 & 0.184281 & 0.447962 \\
\hline Neisseria gonorrhoeae & 34.26475 & -1.26453 & 0.953431 & -1.32629 & 0.184744 & 0.448786 \\
\hline & 161.0853 & -0.56734 & 0.428011 & -1.32553 & 0.184997 & 0.449101 \\
\hline Microbacterium testaceum & 122.1256 & -0.57955 & 0.438065 & -1.32297 & 0.185846 & 0.450862 \\
\hline
\end{tabular}


Paracoccus sp. SC2-6

Xanthomonas translucens

Gramella flava

Erwinia amylovora

Solimonas sp. K1W22B-7

Rhodobacter sp. LPB0142

Aeromonas caviae

Rhodovulum sulfidophilum

Hymenobacter nivis

Pseudomonas sihuiensis

Sphingobium sp. YBL2

Saccharopolyspora erythraea

Corynebacterium aquilae

Octadecabacter arcticus

Vibrio mimicus

Micromonospora viridifaciens

Polaribacter sp. BM10

Microcella alkaliphila

Anoxybacillus flavithermus

Sebaldella termitidis

Propionibacterium virus SKKY

Neisseria sp. KEM232

Corynebacterium riegelii

Psychrobacter sp. DAB_AL43B

Salinispora arenicola

Intrasporangium calvum

Nakamurella sp. s14-144

Natrinema sp. J7-2

Arthrobacter sp. DCT-5

Streptomyces pluripotens

Planctomyces sp. SH-PL62

Deinococcus ficus

Niveispirillum cyanobacteriorum

Burkholderia thailandensis

[Mannheimia] succiniciproducens

Halioglobus japonicus

Halomonas sp. HL-93

Aquiflexum balticum

Brevundimonas sp. GW460-12-10-14

Tabrizicola sp. K13M18

Corynebacterium geronticis

[Enterobacter] lignolyticus

Humibacter sp. BT305

Fusobacterium nucleatum

Cellulosimicrobium cellulans

Streptomyces sp. ETH9427

Klebsiella oxytoca

Shewanella benthica

Amycolatopsis keratiniphila

Staphylococcus aureus

145.698

$121.5775-0.59677$

4.731961

$-1.74395$

0.540579

$-1.32217$

0.186113

$-1.321710 .186266$

$-1.32041$

0.1867

8.108828

126.6999

$-1.46992$

1.320768

$-1.32027$

0.186744

$-0.73973$

1.113349

94.96214

$-0.70752$

0.560204

$-1.32047$

0.186679

$-1.31921$

0.187099

25.69646

$-1.54095$

1.167791

$-1.31954$

0.186989

74.2547

$-0.76134$

67.00852

$-0.73896$

0.577169

$-1.31909$

0.187139

0.560794

$-1.31769$

0.187606

59.03606

$-0.60392$

0.458129

$-1.31823$

0.187426

69.46592

$-0.80313$

0.609427

$-1.31784$

0.187559

157.5128

$-0.47382$

0.360175

$-1.31552$

0.188334

706.0846

8.046924

$-0.44947$

0.341613

$-1.31572$

0.188267

$-1.31574$

0.188262

3.297663

$-2.58276$

79.0711

$-0.57872$

1.967109

$-1.31297$

0.189192

$\begin{array}{ll}-1.3107 & 0.189958\end{array}$

3.597663

3.208513

0.441537

85.25596

$-0.57131$

53.11169

$-1.15594$

0.435876

1.310537

0.190014

$-1.31072$

0.189954

12.94279

1.354688

0.881074

$-1.31197$

0.189532

15.98982

$-1.787$

1.033963

1.310189

0.190132

80.32509

$-0.67484$

1582.47

$-0.31005$

1.363778

$-1.31033$

0.190084

$-1.30948$

0.190373

0.237207

$-1.30709$

0.191183

20.31863

$-1.30363$

0.998172

$-1.30601$

0.191548

40.28316

$-0.70025$

0.535247

$-1.30828$

0.190778

170.3662

$-0.56082$

38.8453

7.689895

149.6073

$-0.72885$

0.428828

$-1.3078$

0.19094

$-1.305910 .191582$

$-1.30693$

0.191235

$-1.30385$

0.192283

59.63513

$-0.56997$

0.437144

$-1.30874$

0.190623

$-0.743$

0.488826

$-1.30405$

0.192216

70.89106

$-0.69622$

$-1.3071$

0.191179

83.65112

$-0.72611$

563.7477

$-0.73057$

.555908

$-1.30617$

0.191495

$-1.3046$

0.19203

4.568427

13.46498

$-2.23066$

1.709354

$-1.30497$

0.191902

$-1.30426$

0.192145

0.45121

0.45128

0.451536

0.451536

0.451536

6.614154

11.3008

2.83998

0.714243

$-1.30569$

0.191659

0.451591

0.451591

0.451591

0.451819

0.451819

0.451819

0.452673

0.452673

0.452673

0.454437

0.454891

0.454891

0.454891

0.454891

0.454891

0.454891

0.455169

0.45525

0.45525

0.45525

0.45525

0.45525

0.45525

0.45525

0.45525

0.45525

0.45525

0.45525

0.45525

0.45525

0.45525

0.45525

332.051

$-1.25241$

0.961127

$-1.30306$

0.192553

0.455591

$-0.69379$

0.532642

$-1.30255$

0.19273

0.455714

52.63045

$-0.71579$

0.550149

$-1.30108$

0.193231

0.456306

551.3027

$-0.47324$

0.363647

$-1.30138$

0.193129

0.456306

21.92834

$-0.83899$

0.645202

$-1.30035$

0.193481

0.456601

236.2321

$-0.69835$

0.538141

$-1.2977$

0.19439

0.457854

500.9623

$-0.59008$

0.454654

$-1.29787$

0.194333 
Shewanella woodyi

Vibrio furnissii

Pseudomonas mendocina

Kineococcus radiotolerans

Enterobacter sp. 638

Parabacteroides distasonis

Propionibacterium phage PAC1

Halorhodospira halochloris

Stanieria cyanosphaera

Bacillus coagulans

Ruegeria sp. AD91A

Aeromonas dhakensis

Alistipes shahii

Thalassospira xiamenensis

Planctopirus limnophila

Pantoea alhagi

Halopenitus persicus

Sinorhizobium meliloti

Gynuella sunshinyii

Haematospirillum jordaniae

Jonesia denitrificans

Pantoea ananatis

Lactobacillus helveticus

Pseudarcicella sp. HME7025

Staphylococcus hominis

Vibrio natriegens

Winogradskyella sp. RHA_55

Streptococcus sp. oral taxon 064

Leclercia adecarboxylata

Phenylobacterium sp. HYN0004

Calothrix parietina

Paenibacillus beijingensis

Corynebacterium vitaeruminis

Gordonia sp. MMS17-SY073

Clavibacter michiganensis

Deinococcus soli Cha et al. 2016

Pseudomonas asplenii

Ketogulonicigenium robustum

Janthinobacterium sp. LM6

Sphingopyxis sp. EG6

Rhizobium etli

Dietzia lutea

Sinomonas atrocyanea

Friedmanniella luteola

Propionibacterium virus PHL171M01

Ornithobacterium rhinotracheale

[Mycobacterium] stephanolepidis

Enterobacteriaceae bacterium w17

Ochrobactrum pituitosum

Acinetobacter sp. WCHA45
4.346635

9.588716

429.4048

131.5607

24.50569

70.78235

18.82994

6.757753

7.190805

14.1243

12.92574

5.178262

96.12636

16.19635

4.292455

21.49226

9.542148

167.7773

4.270634

3.403885

40.64789

38.90208

80.5898

14.30504

7559.36

6.681654

5.951411

785.749

44.53233

75.86408

12.27837

12.31439

1214.055

249.2643

456.8968

38.7032

8.715386

25.89534

218.3958

68.40632

188.5311

1477.374

247.4475

200.6397

9.042595

72.28294

51.07854

2.1704

18.61726

89.60263
$1.85454 \quad 1.432518$

$-1.2541$

$-0.76475$

$-0.5935$

0.969277

$-1.2946$

0.195458

0.195715

0.458589

$-1.43223$

0.591181

$-1.29386$

0.195803

0.458677

$-1.41646$

0.458869

0.195874

0.458677

$-1.95923$

1.108042

0.196158

458677

$-1.4655$

1.097166

$-1.29101$

0.196699

$-1.28943$

0.19725

59047

1.904976

1.13665

$-1.28932$

0.197287

1.291634

0.196484

$-1.28931$

0.19729

$-1.29039$

0.196915

$-1.25689$

0.97404

$-1.28988$

0.197092

$-1.91705$

1.486225

$-1.29082$

0.196765

$-0.82549$

0.640551

$-1.28871$

0.197498

$-1.56746$

1.217505

$-1.28744$

0.197943

$-1.28664$

0.19822

$-1.23131$

0.769123

$-1.28371$

$-0.58039$

0.452126

$-1.28369$

0.199244

$-1.66886$

1.299453

$-1.28428$

199251

$-1.9408$

$-0.61273$

1.512415

$-1.28325$

0.1

$-1.28291$

0.199525

$-0.66875$

0.521854

$-1.28149$

0.20002

$-1.28207$

$-1.28173$

0.199818

0.895123

$-1.2807$

0.199936

0.2003

$-1.27995$

0.200562

$-1.27956$

0.200701

$-1.27865$

0.201022

$-0.75552$

0.590876

$-1.27838$

0.201116

$-1.27844$

0.201094

$-0.74307$

0.581231

$-1.27771$

0.201352

$-0.92264$

$-0.37735$

0.722542

$-1.27694$

0.201624

0.295582

$-1.27662$

0.201736

$-0.62458$

0.489424

$-1.27615$

0.201902

$-1.27482$

0.202373

$-0.55835$

0.437981

$-1.27379$

0.202739

$-1.49918$

1177909

$-1.27275$

0.203108

$-0.73785$

0.57963

$-1.27297$

0.20303

$-1.2723$

0.203266

$-0.71953$

0.61672

$-0.63241$

0.565726

$-1.27187$

0.203419

$-1.2684$

0.204655

$-0.83536$

0.658307

$-1.26896$

0.204456

$-0.59638$

0.470093

$-1.26864$

0.204569

0.204689

2.194199

0.369924

$-1.2683$

0.205154

$-1.452$

1.731803

1.267003

0.205484

$-0.76806$

$-1.26608$

0.205792

$-1.26522$

0.2

$-1.26304$

0.206573

$-1.24567$

1.262527

0.206759

0.459333

0.459333

0.459333

0.459333

0.459333

0.459333

0.459333

0.459333

0.459523

0.460264

0.460615

0.462125

0.462125

0.462125

0.46217

0.46217 
Salinisphaera sp. LB1

Lentibacillus amyloliquefaciens

Thiomicrospira aerophila

Arcobacter bivalviorum

Lentzea guizhouensis

Haemophilus sp. oral taxon 036

Sphingobium sp. YG1

Pragia fontium

Mannheimia sp. USDA-ARS-USMARC-

Streptococcus marmotae

Altererythrobacter epoxidivorans

Kibdelosporangium phytohabitans

Paenibacillus polymyxa

Fibrobacter succinogenes

Shewanella oneidensis

Mucilaginibacter gotjawali

Nakamurella multipartita

Micromonospora tulbaghiae

Spiroplasma cantharicola

Streptomyces sp. GSSD-12

Dietzia sp. oral taxon 368

Streptomyces leeuwenhoekii

Spirosoma rigui

Tamlana sp. UJ94

Pontibacter akesuensis

Vibrio tapetis

Staphylococcus virus SEP9

Shewanella sp. WE21

Pseudomonas psychrotolerans

Corynebacterium argentoratense

Actinosynnema pretiosum

Candidatus Saccharibacteria oral taxc

Streptococcus equi

Neomicrococcus aestuarii

Acetobacter aceti

Streptomyces peucetius

Corynebacterium provencense

[Eubacterium] hallii

Shewanella sp. FDAARGOS_354

Leuconostoc gelidum

Corynebacterium efficiens

Arthrobacter sp. PAMC 25486

Haemophilus haemolyticus

Olsenella sp. Marseille-P2300

Algibacter alginicilyticus

Idiomarina loihiensis

Polaribacter vadi

Comamonas thiooxydans

Halorhodospira halophila

Micromonospora aurantiaca
36.85428

7.652359

6.166325

9.060188

139.306

64.35929

118.1242

2.623248

3.422712

8.912082

21.33498

160.6686

112.1204

7.867617

2.894889

39.34213

179.4181

67.56775

3.517506

84.27062

6009.154

54.358

35.26991

5.362042

25.18509

3.569465

103.7765

13.38922

168.7538

479.0047

173.9739

52.9346

59.72091

97.51132

37.61918

64.48118

302.5847

92.74227

13.27587

94.01237

806.6196

108.9657

199.7911

32.70394

8.888745

6.617331

10.12747

259.9423

26.86063

112.3904
$-0.6699$

$-1.18584$

$-1.91272$

1.58098

$-0.36663$

$-1.01272$

$-0.70915$

$-1.89183$

$-2.31194$

$-1.28589$

$-0.96856$

$-0.50192$

1.018668

$-1.14742$

$-2.96725$

$-0.6075$

$-0.65259$

$-0.75876$

2.86927

$-0.64667$

$-0.79253$

$-0.59131$

$-0.85709$

2.603929

$-0.85565$

$-2.47271$

2.17715

$-1.27595$

$-0.58147$

$-0.47792$

$-0.75253$

$-0.94086$

$-0.74338$

$-0.6107$

$-0.77356$

$-0.47706$

$-0.51426$

$-0.77223$

1.343961

$-0.79863$

$-0.37621$

$-0.42514$

$-0.76464$

$-0.63247$

1.254045

$-1.50175$

1.564494

$-0.85412$

$-0.83478$

$-1.12601$
0.530939

0.939848

1.517428

1.253878

0.290928

0.8031

0.56307

1.502602

1.83646

1.022317

0.771092

0.399647

0.810742

0.912931

2.362749

0.484283

0.520228

0.605165

2.289528

0.516227

0.633596

0.472682

0.684634

2.080993

0.685337

1.980393

1.746549

1.023387

0.467666

0.38431

0.604948

0.756103

0.597901

0.490627

0.622338

0.383443

0.414089

0.621725

1.084768

0.644191

0.303631

0.343005

0.617662

0.51083

1.013583

1.213768

1.26571

0.691445

0.675733

0.911901

$-1.26174$

$-1.26173$

$-1.2605$

1.260872

$-1.26019$

$-1.26101$

$-1.25944$

$-1.25904$

$-1.25891$

$-1.25782$

$-1.25609$

$-1.25592$

1.256464

$-1.25686$

$-1.25585$

$-1.25442$

$-1.25444$

$-1.25381$

1.253215

$-1.25268$

$-1.25084$

$-1.25098$

$-1.2519$

1.251291

$-1.24852$

$-1.2486$

1.246544

$-1.24679$

$-1.24334$

$-1.24358$

$-1.24397$

$-1.24436$

$-1.24332$

$-1.24474$

$-1.24298$

$-1.24415$

$-1.2419$

$-1.24207$

1.238938

$-1.23974$

$-1.23903$

$-1.23945$

$-1.23796$

$-1.23813$

1.23724

$-1.23727$

1.23606

$-1.23526$

$-1.23537$

$-1.23479$

0.207044

0.470311

0.207046

0.470311

0.207488

0.470394

0.207355

0.470394

0.207599

0.470394

0.207305

0.470394

0.207872

0.470565

0.208018

0.470565

0.208062

0.470565

0.208457

0.471165

0.209083

0.471316

0.209147

0.471316

0.208948

0.471316

0.208805

0.471316

0.209172

0.471316

0.209688

0.471896

0.209683

0.471896

0.209913

0.472109

0.210128

0.4723 
Marinobacter sp. Arc7-DN-1

Photobacterium gaetbulicola Lactobacillus amylolyticus

Methylomonas sp. DH-1

Clostridium carboxidivorans

Yersinia aldovae

Streptomyces olivaceus

Oceanobacillus sp. 160

Anaeromyxobacter sp. $\mathrm{K}$

Brevundimonas sp. LM2

Yersinia ruckeri

Mycoplasma fermentans

Pseudoalteromonas agarivorans

Owenweeksia hongkongensis

Treponema brennaborense

Ruegeria pomeroyi

Neisseria mucosa

Pseudonocardia sp. HH130630-07

Candidatus Nucleicultrix amoebiphila

Candidatus Symbiobacter mobilis

Hoeflea sp. IMCC20628

Olsenella umbonata

Actinobacteria bacterium YIM 96077

Bibersteinia trehalosi

Psychrobacter sp. AntiMn-1

Thermanaerovibrio acidaminovorans

Candidatus Bipolaricaulis anaerobius

Xanthobacter autotrophicus

Corynebacterium cystitidis

Campylobacter hominis

Hymenobacter sp. sh-6

Bordetella genomosp. 6

Streptomyces noursei

Corynebacterium choanis

Oscillatoria acuminata

Leclercia sp. LSNIH1

Jatrophihabitans sp. GAS493

Agrococcus jejuensis

Streptococcus pneumoniae

Shewanella japonica

Serratia sp. ATCC 39006

Dickeya dadantii

Thermococcus pacificus

Gammapapillomavirus 11

Streptomyces antibioticus

Carnobacterium sp. CP1

Acidothermus cellulolyticus

Verrucomicrobia bacterium

Dickeya paradisiaca

Corynebacterium stationis
20.81708

$6.63612-1.56865$

3.843271

19.1194

19.05252

3.074069

74.80126

5.970477

70.30998

148.3239

8.720616

2.65424

2.424921

3.755322

5.056428

63.27814

376.0299

220.2813

1.512824

50.84111

30.6534

22.72273

71.41959

8.136686

26.64827

6.582816

4.99455

178.3472

627.1749

16.39872

31.78064

18.89185

51.41079

207.3236

2.74253

11.66124

66.65071

152.3902

2070.195

3.15618

9.244123

48.3805

3.781055

32.58376

71.65414

7.195028

29.89555

60.83269

27.62912

1304.654

$-0.5617$

$-1.32155$

$-1.70873$

$-0.64802$

$-0.52155$

$-2.69158$

$-0.76535$

$-0.84592$

$-0.58655$

$-0.59721$

$-0.36294$

$-1.57517$

$-0.56474$

$-0.64723$

$-1.15637$

$-2.22445$

$-0.81145$
0.695469

$-1.23415$

0.217149

0.217431

$-1.23339$

0.218263

1.853576

$-1.23116$

0.218205

$-1.23131$

1.23154

0.218121

$-1.2313$

0.21821

$-1.23175$

0.218041

$-0.681$

0.552868

$-1.65951$

1.352389

$-1.22709$

0.219787

$-1.22766$

0.219574

$-1.227$

0.219823

0.457784

1.075638

$-1.22862$

0.219214

2.862649

2.332158

1.227468

0.219647

$-1.22829$

0.219339

$-1.22629$

0.22009

$-1.22655$

0.219991

-1.225720 .220306

1.238964

$-0.93928$

0.767658

$-1.22357$

0.221114

0.426471

$-1.22295$

0.22135

$-1.22147$

0.221906

$-1.22096$

0.2221

0.682593

$-1.21939$

0.222696

0.693928

$-1.21903$

0.222834

0.481363

$-1.21853$

0.223023

$-1.21976$

0.222556

$-1.21984$

0.222524

$-1.2185$

0.223034

$-1.21751$

0.223412

1.328931

$-1.21618$

0.223916

0.32644

$-1.21593$

0.224013

0.95674

$-1.21554$

0.224162

$-1.21482$

0.224435

0.491606

$-1.21439$

0.224601

$-0.70724$

$-1.21367$

0.224873

$-1.21367$

0.224874

$-1.21206$

0.225488

$-1.21102$

0.225887

1.30069

$-1.21062$

0.226041

$-1.2087$

0.226779

0.467232

0.53559

$-1.20844$

0.22688

$-1.20884$

0.226723

0.956483

$-1.20898$

0.226669

$-1.2073$

0.227316

0.601533

$-1.20687$

0.227484

1.205655

0.227951

$-1.2059$

0.227856

$-1.20091$

0.229786

$-1.2002$

0.230062

0.676094

$-1.19973$

0.230244

$-1.19812$

0.230872

0.231118

0.478622

0.478954

0.479335

0.479335

0.479335

0.479335

0.479335

0.48102

0.48102

0.48102

0.48102

0.48102

0.48102

0.481025

0.481025

0.481208

0.482683

0.482909

0.483833

0.483965

0.484261

0.484261

0.484261

0.484261

0.484261

0.484261

0.484791

0.485517

0.485517

0.48555

0.485853

0.485923

0.485937

0.485937

0.486975

0.487545

0.487588

0.488241

0.488241

0.488241

0.488241

0.488891

0.488962

0.489388

0.489388

0.493038 
Streptomyces griseorubiginosus

Aerococcus urinaehominis

Agrobacterium sp. H13-3

Ralstonia pickettii

Corynebacterium glutamicum

Caldicellulosiruptor saccharolyticus

Stigmatella aurantiaca

Alteromonas macleodii

Methylobacterium aquaticum

Pseudomonas mucidolens

Pantoea rwandensis

Alcaligenes faecalis

Alteromonas sp. BL110

Planococcus antarcticus

Escherichia albertii

Lacimicrobium alkaliphilum

Erythrobacter atlanticus

Polynucleobacter necessarius

Geminocystis sp. NIES-3708

Acidihalobacter prosperus

Streptococcus phage SpSL1

Ehrlichia ruminantium

Chromobacterium sp. ATCC 53434

Vibrio anguillarum

Pseudolabrys taiwanensis

Alteromonas mediterranea

Micromonospora echinospora

Veillonella rodentium

Burkholderia sp. PAMC 28687

Acidipropionibacterium virtanenii

Acinetobacter indicus

Actinoplanes teichomyceticus

Altererythrobacter marensis

Nocardia seriolae

Lactobacillus zymae

Mycobacteroides immunogenum

Barnesiella viscericola

Weissella cibaria

Cutibacterium granulosum

Haloferax gibbonsii

Porphyromonas gingivalis

Maribacter cobaltidurans

Pseudomonas sp. LAB-08

Lactobacillus ruminis

Lutibacter profundi

Pseudomonas orientalis

Streptomyces sp. 11-1-2

Treponema succinifaciens

Streptomyces xiamenensis

Sulfitobacter sp. D7
70.21219

3.893336

2.642814

76845.62

681.1084

6.754025

85.53332

25.91789

160.5672

33.82596

15.74199

99.24412

6.159315

7.243791

14.69044

16.37825

14.23997

55.54965

14.06948

67.18696

1.660463

3.492061

121.2311

17.00904

116.896

9.015426

89.61349

93.83854

239.8501

102.4762

42.32484

179.5375

30.2539

182.7624

1.824143

81.23211

58.45652

17.04217

14864.27

21.719

231.3332

4.533807

56.61002

12.92102

11.91638

104.4578

28.75652

3.761678

84.33354

22.37244
0.64782

$-1.7573$

0.541202

1.469013

$-3.49051$

2.920482

$-0.59016$

0.494123

$-0.46131$

0.386568

$-1.8821$

1.576973

$-0.60105$

0.504136

$-1.13837$

0.954706

$-0.53574$

$-0.67522$

0.449766

0.566825

$-0.87307$

0.733576

$-0.58315$

0.489949

$-1.50025$

1.263044

$-1.57523$

1.327638

$-1.12935$

0.951841

$-0.85249$

0.719677

$-1.12631$

0.951262

$-0.57066$

1.15697

0.481965

0.978167

$-0.61059$

0.516411

$-2.827$

2.391094

2.949522

2.495337

$-0.56174$

0.47556

$-0.55999$

.945476

0.474877

$-1.69984$

1.442663

$-0.59875$

0.508431

$-0.68233$

0.579609

$-0.76753$

0.652494

$-0.49735$

0.423208

$-0.75198$

0.640035

$-0.5779$

$-0.6512$

0.492214

0.555353

$-0.42046$

0.359306

$-2.08464$

1.781334

$-0.57779$

0.493834

$-0.88559$

0.756487

$-1.2198$

1.047014

$-0.72089$

0.619165

$-0.67966$

0.58374

$-0.85887$

0.738404

$-1.97981$

1.701967

$-0.77117$

0.662859

$-1.53168$

1.318335

1.10165

0.948993

$-0.60167$

0.519866

$-1.18543$

1.024015

1.862987

1.608252

$-0.51856$

0.448001

$-0.83427$

$-1.197$

0.231307

0.2316

0.494841

$-1.19625$

0.2316

0.495178

$-1.19518$

0.232015

0.495774

$-1.19436$

0.232337

0.49617

$-1.19336$

0.232729

0.496425

$-1.19349$

0.232679

0.496425

$-1.19223$

0.23317

0.496782

$-1.19237$

0.233115

$-1.19116$

0.233592

0.496782

$-1.19122$

0.233566

0.4971

$-1.19016$

0.233982

0.4971

$-1.19024$

0.233954

0.497349

$-1.18781$

0.234909

0.497349

$-1.18649$

0.235428

0.499028

$-1.18649$

0.235428

0.499547

$-1.18455$

0.236196

0.499547

$-1.18401$

0.236408

0.50075

0.50075

$-1.18403$

0.236403

0.50075

1.182793

0.236891

0.501262

$-1.18238$

0.237056

0.501262

$-1.18231$

0.237085

0.501262

1.182013

0.2372

0.501262

$-1.18123$

0.237513

0.501631

1.180534

0.237788

0.50192

$-1.17922$

0.238309

0.502728

$-1.17826$

0.238692

0.503244

$-1.17764$

0.23894

0.503475

$-1.17723$

0.239104

0.503529

$-1.1763$

0.239474

0.504016

$-1.1752$

0.239915

0.504614

$-1.1749$

0.240035 
Lactobacillus acidipiscis

Geobacter sp. M18

Achromobacter sp. B7

Spongiibacter sp. IMCC21906

Sphingopyxis alaskensis

Staphylococcus phage StB12

Propionibacterium virus P144

Caulobacteraceae bacterium OTSz_A.

Thiomonas arsenitoxydans

Legionella spiritensis

Methanobrevibacter ruminantium

Paenibacillus stellifer

Pseudomonas lini

Rhodococcus ruber

Halomonas sp. A3H3

Burkholderia dolosa

Macrococcus caseolyticus

Synechococcus sp. CC9902

Rubrobacter radiotolerans

Mucinivorans hirudinis

Streptomyces sp. FR-008

Mycobacterium sp. VKM Ac-1817D

Bacteriovorax stolpii

Arthrobacter sp. U41

Porphyrobacter HT-58-2

Rhodococcus erythropolis

Thalassococcus sp. SH-1

Pusillimonas sp. T7-7

Hymenobacter sp. DG25A

Azoarcus sp. KH32C

Gammapapillomavirus 3

Hungateiclostridium saccincola

Halomonas huangheensis

Auritidibacter sp. NML130574

Corynebacterium falsenii

Roseiflexus castenholzii

Corynebacterium renale

Laceyella sacchari

Pseudomonas sp. phDV1

Streptomyces sp. TN58

Pseudomonas sp. R5-89-07

Pseudomonas parafulva

Methanosphaera stadtmanae

Propionibacterium phage PHL010M0

Microlunatus phosphovorus

Pseudoalteromonas tunicata

Methylobacterium sp. 17Sr1-28

Xenorhabdus hominickii

Heliobacterium modesticaldum

Mycolicibacterium aurum
2.779457

90.54765

120.4422

2.554583

38.33056

74.28254

21.6085

54.28203

101.3228

2.293963

1.488948

10.12649

17.69891

229.3302

36.1619

423.293

30.9879

1.363317

46.97173

4.78116

7.630479

27.29568

5.272975

191.8788

38.70282

699.1942

56.70212

21.57714

28.79956

161.8394

2.368201

11.14649

20.3423

145.6008

741.1009

9.967244

729.4555

2.945505

111.2963

58.57253

49.38014

103.982

5.049627

8.880597

143.3151

12.19202

108.7564

2.654308

28.22495

147.5851
$-2.17136$

$-0.68906$

$-0.61954$

$-1.86101$

$-0.589$

0.902277

1.627749

$-0.7114$

$-0.7552$

$-1.90105$

3.133817

$-0.93984$

$-0.81375$

$-0.34854$

$-0.78904$

$-0.54091$

$-1.02291$

$-2.54711$

$-0.73186$

$-1.50358$

$-2.32009$

$-0.60113$

$-1.71651$

$-0.42543$

$-0.74395$

$-0.63078$

$-0.56344$

$-0.78039$

0.782034

$-0.64216$

$-3.33401$

1.10267

$-0.88026$

$-0.35876$

$-0.43949$

$-0.98233$

$-0.32335$

$-1.70498$

$-0.73089$

$-0.62537$

$-0.71904$

$-0.58493$

2.402586

$-1.85447$

$-0.46169$

$-1.1738$

$-0.58082$

$-1.9743$

$-0.79103$

$-0.5093$
1.873932

0.595656

0.535785

1.611737

0.510963

0.781951

1.411561

0.617141

0.654672

1.650304

2.722116

0.816281

0.707236

0.303281

0.687854

0.471354

0.890773

2.218945

0.638094

1.310508

2.028756

0.524601

1.500495

0.37159

0.650385

0.551398

0.491568

0.681261

0.683902

0.56061

2.920782

0.966295

0.770672

0.314328

0.385308

0.860541

0.283346

1.494685

0.641039

0.548776

0.632075

0.514074

2.112957

1.630169

0.406051

1.032932

0.511354

1.738874

0.697867

0.449305

$-1.15872$

0.246572

0.513587

$\begin{array}{ll}-1.15682 & 0.247348\end{array}$

0.513755

$-1.156320 .247551$

0.513883

$\begin{array}{ll}-1.15466 & 0.248229\end{array}$

0.514998

$-1.152720 .249027$

0.515185

1.1538790 .24855

0.515185

1.153156

0.248846

$-1.15274$

0.249016

0.515185

$-1.15356$

0.248681

0.515185

$-1.15194$

0.249347

0.515185

1.151243

0.249632

0.515553

$-1.15137$

0.24958

0.515557

$-1.15061$

0.249893

0.515557

$-1.14923$

0.250461

0.515802

$-1.1471$

0.251339

0.516681

$-1.14755$

0.251152

0.516869

$-1.14834$

0.250829

0.516869

$-1.14789$

0.251013

0.516869

$-1.14695$

0.251404

0.516869

$-1.14733$

0.251246

0.516869

$-1.1436$

0.25279

0.516869

$-1.14589$

0.251842

0.516892

$-1.14396$

0.252639

0.516892

$-1.14489$

0.252254

0.516892

$-1.14385$

0.252684

0.516892

$-1.14397$

0.252637

0.516892

$-1.1462$

0.251711

0.516892

$-1.1455$

0.252001

0.516892

1.143489

0.252836

0.516892

$-1.14546$

0.252017

0.516892

$-1.14148$

0.253671

0.516892

1.141132

0.253815 
Methylophaga frappieri

Acidihalobacter ferrooxidans

Pseudomonas vancouverensis

Acinetobacter soli

Bordetella bronchiseptica

Nakamurella panacisegetis

Sphingorhabdus sp. YGSMI21

Enterobacter kobei

Stenotrophomonas sp. ZAC14A_NAIA

Vibrio harveyi

Streptomyces parvulus

Rhizobium sp. CCGE532

Francisella tularensis

Pseudomonas brassicacearum

Paracoccus denitrificans

Clostridium chauvoei

Thermoactinomycetaceae bacterium

Mesorhizobium sp. DCY119

Hartmannibacter diazotrophicus

Candidatus Profftella armatura

Calditerrivibrio nitroreducens

Corynebacterium ammoniagenes

Megasphaera elsdenii

Prevotella scopos

Aeromonas media

Corynebacterium crudilactis

Taylorella asinigenitalis

Deinococcus deserti

Anabaenopsis circularis

Rhodococcus pyridinivorans

Chromobacterium sp. IIBBL 274-1

Acinetobacter schindleri

Methanobacterium lacus

Rhodococcus sp. P1Y

Actinobacillus equuli

Gordonibacter pamelaeae

Tenacibaculum maritimum

Erysipelotrichaceae bacterium GAM1

Flavobacterium indicum

Nostoc sp. PCC 7524

Pseudomonas versuta

Mesorhizobium japonicum

Methylobacterium sp. 17Sr1-1

Rhizobium sp. NT-26

Citromicrobium sp. JL477

Staphylococcus phage StB20

Brevundimonas sp. DS20

Glutamicibacter halophytocola

Acetobacter senegalensis

Desulfotalea psychrophila
3.338955

35.52038

53.80825

32.06784

262.3223

101.8539

27.6115

2.963369

21.26677

3.587794

79.84224

34.70849

15.988

38.31337

1941.987

16.46464

16.96631

62.32148

65.54862

1.359321

2.578176

1381.758

25.47842

232.2009

31.36378

322.8855

7.897346

46.36325

47.26199

132.4083

53.04447

161.7815

1.609463

147.8206

35.81616

40.42803

3.887392

52.68847

9.860561

2.937299

47.69739

65.15295

107.0854

62.19321

51.06502

95.91993

362.9909

139.0719

3.059188

1.993738
$-1.60269$

$-0.67719$

$-0.76914$

$-0.90134$

$-0.6672$

$-0.49396$

$-0.71299$

$-2.26254$

$-1.23247$

$-1.62874$

$-0.44901$

$-2.78016$

1.450529

$-0.88266$

$-0.69244$

0.938639

$-0.85075$

$-0.5566$

$-0.60402$

$-2.53623$

2.43698

$-0.32645$

$-0.71467$

$-0.59351$

$-0.70931$

$-0.40026$

$-1.55837$

$-0.61029$

$-0.92416$

$-0.92431$

$-1.38939$

$-0.81462$

3.254977

0.50113

$-2.20238$

$-0.85231$

2.195501

1.065128

1.465631

$-2.22618$

$-0.72659$

$-0.63761$

$-0.54559$

$-0.6837$

$-0.62157$

0.987931

$-0.49824$

$-0.45305$

$-2.37298$

$-1.70082$
1.413225

0.598271

0.679637

0.79608

0.589245

0.436368

0.630369

2.002651

1.091358

1.444285

0.398085

2.46414

1.286666

0.784193

0.616037

0.83477

0.757239

0.495228

0.537661

2.25476

2.170199

0.290884

0.637387

0.529492

0.633432

0.357509

1.391202

0.54651

0.828294

0.828792

1.246073

0.730813

2.924675

0.450028

1.978224

0.766043

1.9744

0.957822

1.319075

2.004403

0.655016

0.57518

0.493977

0.619116

0.562922

0.892799

0.450244

0.409895

2.145769

1.538964

$-1.13406$

0.256768

0.257674

$-1.13191$

0.257765

0.519288

$-1.13169$

0.257541

0.519383

$-1.13222$

0.257513

$-1.13229$

0.257639

0.519383

$-1.13199$

0.258029

519383

$-1.13106$

$-1.12977$

0.258572

$-1.1293$

0.258771

$-1.12772$

0.25944

$-1.12792$

0.259353

$-1.12825$

0.259215

1.127355

0.259592

$-1.12556$

0.26035

0.261

$-1.12403$

1.124428

0.260832

$-1.12349$

0.26123

$-1.12392$

0.261046

$-1.12342$

0.261257

$-1.12483$

0.26066

1.122929

0.261467

$-1.12226$

0.261753

$-1.12126$

0.262179

$-1.12091$

0.262325

$-1.11979$

0.262801

$-1.11957$

0.262895

$-1.12016$

0.262645

$-1.1167$

0.264124

$-1.11574$

0.264531

$-1.11525$

0.264742

$-1.11502$

0.264843

$-1.11467$

0.264992

1.112936

0.265736

1.113552

0.265471

$-1.11331$

0.265574

$-1.11261$

0.265875

1.111984

0.266145

1.112032

0.266125

1.111105

0.266523

$-1.11065$

0.266721

$-1.10927$

0.267312

$-1.10853$

0.267632

$-1.10448$ 


\begin{tabular}{|c|c|c|c|c|c|c|}
\hline Corynebacterium pseudopelargi & 655.004 & -0.39206 & 0.354689 & -1.10537 & & \\
\hline Celeribacter marinus & 9.522279 & -1.23428 & 1.11759 & -1.10441 & 0.269413 & 0.528819 \\
\hline Yersinia frederiksenii & 16.19405 & -1.30338 & 1.179376 & -1.10514 & 0.269097 & 528819 \\
\hline Anaerostipes hadrus & 223.348 & -0.81081 & 0.732041 & -1.1076 & 0.268034 & .528819 \\
\hline Paenibacillus xylanexedens & 18.08912 & -1.17684 & 1.065926 & -1.10406 & 0.269568 & 0.528819 \\
\hline Sphingomonas sp. KC8 & 50.84606 & -0.58472 & 0.530577 & -1.10205 & 0.270439 & 0.528893 \\
\hline hermus sp. CCB_US3_UF1 & 17.75918 & -0.92115 & 0.836087 & -1.10174 & 0.270574 & 0.528893 \\
\hline Vibrio nigripulchritudo & 8.689091 & -1.37301 & 1.246718 & -1.1013 & 0.270767 & 528893 \\
\hline Lachnospiraceae bacterium oral taxo & 38.87317 & -0.68863 & 0.625491 & -1.10095 & 0.27092 & .528893 \\
\hline Pediococcus pentosaceus & 10.09767 & -0.90957 & 0.825427 & -1.10194 & 0.270487 & 0.528893 \\
\hline ella marisflavi & 3.229658 & -1.8997 & 1.724415 & -1.10165 & 0.270615 & 0.528893 \\
\hline has prosekii & 33.14575 & -0.72391 & 0.6577 & -1.10068 & 0.271038 & 0.528893 \\
\hline ticivorans & 5.100471 & -1.2818 & 1.161728 & -1.10336 & 0.269873 & 0.528893 \\
\hline Sphae & 4.788784 & 1.499671 & 1.362562 & 1.100626 & 0.27106 & 0.528893 \\
\hline Prevo & 125.0174 & -0.511 & 0.463375 & -1.10279 & 0.270119 & 0.528893 \\
\hline Klebs & 525.6997 & 0.467763 & 0.425352 & 1.099707 & 0.27146 & 0.529107 \\
\hline Streptococcus equinus & 197.8729 & -0.7292 & 0.663065 & -1.09974 & 0.271444 & 0.529107 \\
\hline Plura & 53.33828 & -0.52344 & 0.476172 & -1.09927 & 0.27165 & 0.529194 \\
\hline Amyc & 100.9777 & -0.53143 & 0.483622 & -1.09886 & 0.271829 & 0.52926 \\
\hline Azot & 140.8811 & -0.57709 & 0.525 & -1.0 & 0.27218 & 529659 \\
\hline Glaes & 7.949431 & -1.27532 & 1.16261 & -1.09694 & 0.272666 & 0.530321 \\
\hline Phyto & 12.54372 & -1.36805 & 1.248432 & -1.09582 & 0.273159 & 0.530406 \\
\hline ormans & 3.415329 & -1.44533 & 1.319027 & -1.09576 & 0.273186 & 0.530406 \\
\hline Rhodococcus sp. WMMA185 & 86.55484 & -0.57165 & 0.52191 & -1.09529 & 0.273388 & 0.530406 \\
\hline Gilvibacter sp. SZ-19 & 1.83077 & -1.95332 & 1.782994 & -1.09553 & 0.273286 & 0.530406 \\
\hline Ferm & 9.200091 & -1.31405 & 1.199847 & -1.09518 & 0.273438 & 0.530406 \\
\hline Lysin & 29.57421 & 0.837358 & 0.765405 & 1.094006 & 0.273952 & .53112 \\
\hline Arth & 141.36 & -0.55748 & 0.510116 & -1.09285 & 0.274458 & 0.531217 \\
\hline Isopt & 152.2442 & -0.48057 & 0.439666 & -1.09304 & 0.274377 & 0.531217 \\
\hline Goro & 452.7702 & -0.53882 & 0.493168 & -1.09256 & 0.274586 & 1217 \\
\hline Yersin & 5.426888 & -1.35332 & 1.238485 & -1.09272 & 0.274517 & 0.531217 \\
\hline Olleya sp. Bg11-27 & 7.591951 & 1.437172 & 1.319377 & 1.089281 & 0.27603 & 0.533726 \\
\hline Actinomyces sp. Chiba101 & 168.272 & -0.47917 & 0.440601 & -1.08753 & 0.276804 & 0.534383 \\
\hline Paraco & 84.87415 & -0.54922 & 0.504845 & -1.08789 & 0.276643 & 0.534383 \\
\hline Sphir & 15.52487 & -3.1542 & 378 & 3751 & 0.27681 & 4383 \\
\hline Saccharomonospora azurea & 71.94424 & -0.38071 & 0.350408 & -1.08647 & 0.277269 & 0.534702 \\
\hline Bacil & 8.215239 & -1.02168 & 0177 & 3668 & 0.277176 & 4702 \\
\hline Cory & 9791.981 & -0.32993 & 0.304711 & -1.08277 & 0.278913 & 0.537586 \\
\hline Halor & 12.39649 & -1.12197 & 1.037401 & -1.08152 & 0.279465 & 0.538366 \\
\hline Sphin & 16.04024 & -0.75907 & 0.702416 & -1.08066 & 0.279848 & 0.538818 \\
\hline Gemmata sp. SH-PL17 & 60.035 & -0.57639 & 0.533811 & -1.07976 & 0.280251 & 0.539308 \\
\hline Dietz & 295.6421 & -0.45062 & 0.417475 & -1.0794 & 0.280409 & 0.539327 \\
\hline Streptomyces fungicidicus & 63.13248 & -0.45005 & 0.417292 & -1.0785 & 0.28081 & 0.539418 \\
\hline Aero & 53.79546 & -0.52318 & 0.485379 & -1.07787 & 0.281091 & 0.539418 \\
\hline Pseudomona & 29.14113 & -0.81651 & 0.757689 & -1.07763 & 0.281197 & 0.539418 \\
\hline Polaribacter sp. $k$ & 11.46493 & 1.528592 & 1.417809 & 1.078136 & 0.280973 & 0.539418 \\
\hline & 111.1694 & -0.7481 & 0.693417 & -1.07886 & 0.28065 & 0.539418 \\
\hline Vibrio rumoiensis & 12.72623 & -1.03303 & 0.960555 & -1.07545 & 0.282171 & 0.540724 \\
\hline & 36.36623 & -0.91882 & 0.854366 & -1.07545 & 0.282175 & 0.540724 \\
\hline Paenibacillus lautus & 3.138213 & -1.9896 & 1.852023 & -1.07429 & 0.282695 & 0.54138 \\
\hline
\end{tabular}


Rhodococcus sp. 008

Propionibacterium virus PHL152M00

Chryseobacterium balustinum

Flammeovirga sp. MY04

Vibrio vulnificus

Streptomyces sp. SAT1

Sphingopyxis sp. 113P3

Porcine type-C oncovirus

Marinobacter sp. LV10R510-11A

Polaribacter reichenbachii

Novosphingobium pentaromativoran

Parabacteroides sp. CT06

alpha proteobacterium HIMB5

Candidatus Ruthia magnifica

Lactobacillus gasseri

Microbacterium foliorum

Eubacterium limosum

Lactococcus virus Bibb29

Corynebacterium ulcerans

Flavobacteriaceae bacterium 3519-1(

Aminobacter aminovorans

Persicobacter sp. JZB09

Pseudomonas plecoglossicida

Clostridium acetobutylicum

beta proteobacterium $\mathrm{CB}$

Labrenzia sp. VG12

Corynebacterium glaucum

Paenarthrobacter aurescens

Alistipes sp. Marseille-P5997

Corynebacterium callunae

Jeotgalicoccus saudimassiliensis

Corynebacterium variabile

Candidatus Sulcia muelleri

Candidatus Nitrosotenuis cloacae

Rhizobium sp. Y9

Mycolicibacterium goodii

Ferroplasma acidarmanus

Fusobacterium sp. oral taxon 203

Hafnia paralvei

Sphingomonas sp. LK11

Bacillus altitudinis

Alteromonas sp. RKMC-009

Georgenia sp. ZLJ0423

Mycobacterium sp. QIA-37

Acetobacterium sp. KB-1

Sphingobium herbicidovorans

Porphyromonas asaccharolytica

Aquaspirillum sp. LM1

Streptomyces sp. S8

Mycobacteroides chelonae
108.8729

18.68585

67.09531

15.05827

36.2618

81.75913

66.14943

4.847069

6.947749

13.2297

64.64741

161.5165

1.805042

1.450857

128.9903

301.5356

43.8314

3.152377

551.9321

10.05996

111.7571

11.86408

99.26476

25.13819

4.528744

32.0401

1604.787

97.52971

44.39913

479.5563

14.7443

464.6741

6.845036

40.23812

69.28895

156.5806

1.913836

6.826747

4.308844

176.8706

14.7632

5.527117

135.6078

28.64242

4.840995

34.4484

1412.78

40.89723

23.87847

604.4024

$1.18526 \quad 1.103576$

$-1.5817$

1.474188

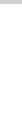

$-1.07402$

0.282815

0.54138

$-0.76915$

0.716642

$-1.07293$

0.283302

0.541643

1.078113

1.005343

$-1.07327$

0.283151

0.541643

$-0.67916$

0.633198

1.072384

0.283548

0.541643

$-0.51289$

0.478953

$-1.07258$

0.28346

0.541643

$-0.68888$

0.643642

$-1.07087$

0.28423

0.542661

2.049136

1.915747

$-1.07029$

0.284491

0.542875

$-1.21904$

1.140723

1.069628

0.284787

0.543155

1.122447

1.050065

$-1.06865$

0.285226

0.543424

$-0.63098$

0.59076

1.068931

0.285101

0.543424

$-1.21755$

1.140685

$-1.06807$

0.28549

0.543642

$-2.24352$

2.102815

$-1.06739$

0.285797

0.54394

$-2.62595$

2.463514

$-1.06691$

0.286011

0.544063

1.085218

1.018863

0.286453

0.544619

$-0.58179$

0.547165

1.065127

0.286818

0.54503

$-0.82512$

0.776761

$-1.06327$

0.287658

0.54634

2.282701

2.149765

$-1.06226$

0.288119

0.546929

$-0.38965$

0.367465

1.061837

0.288309

0.547006

$-1.10948$

1.049661

$-1.06037$

0.288974

0.547982

$-0.58859$

0.556063

$-1.05699$

0.290518

0.548492

$-0.90716$

0.85827

$-1.0585$

0.289826

0.548492

$-0.50137$

0.474117

$-1.05696$

0.290528

0.548492

0.658904

0.623104

0.290292

0.548492

$-1.85816$

1.756129

0.290304

0.548492

$-0.62311$

0.589294

$-1.0581$

0.29001

0.548492

$-0.38242$

0.361859

$-1.05739$

0.290334

0.548492

$-1.05681$

0.2906

0.548492

$-1.06886$

1.010661

$-1.05758$

0.290246

0.548492

$-0.88418$

0.83718

$-1.05614$

0.290902

0.548493

$-0.38579$

0.365263

$-1.0562$

0.290875

0.548493

$-1.02426$

0.971066

$-1.05478$

0.291526

0.548953

$-0.43979$

0.416752

$-1.05527$

0.291302

0.548953

1.370549

1.299971

1.054292

0.291749

0.548953

$-0.73477$

0.696755

$-1.05456$

0.291628

0.548953

$-1.37651$

1.306124

$-1.05389$

0.291932

0.549013

$-0.34772$

0.330347

$-1.05258$

0.292534

0.549861

2.782971

2.645761

1.05186

0.292864

0.550197

$-1.16598$

1.110738

$-1.04973$

0.293842

0.550751

$-1.56039$

1.486005

$-1.05005$

0.293693 
Chromohalobacter salexigens

Staphylococcus virus IPLAC1C

Bacillus krulwichiae

Lactobacillus delbrueckii

Pseudarthrobacter phenanthrenivora

Prosthecochloris sp. CIB 2401

Streptococcus constellatus

Paludibacter propionicigenes

Streptococcus sanguinis

Pseudomonas veronii

Gordonia sp. YC-JH1

Geobacter metallireducens

Bartonella apis

Pseudomonas sp. R3-52-08

Sneathia amnii

Methylobacterium sp. XJLW

Corynebacterium sp. NML98-0116

Serratia plymuthica

Confluentimicrobium sp. EMB200-NS

Pseudomonas sp. URMO17WK12:I11

Rhodococcus sp. PBTS 1

Gemmatimonas phototrophica

Calothrix parasitica

Ruminococcus champanellensis

Nostoc piscinale

Lysinibacillus fusiformis

Desulfobacter postgatei

Citrobacter koseri

Ruminococcus albus

Spirochaeta thermophila

archaeon AArc-SI

Lacinutrix venerupis

Streptococcus acidominimus

cyanobacterium endosymbiont of Epi

Lactobacillus acidophilus

Congregibacter litoralis

Edwardsiella hoshinae

Cellulomonas gilvus

Citrobacter werkmanii

Nostoc flagelliforme

Human alphaherpesvirus 1

Haloarcula taiwanensis

Isosphaera pallida

Lactobacillus rhamnosus

Streptococcus porcinus

Pseudomonas sp. LG1E9

Shewanella sp. Pdp11

Actinopolymorpha singaporensis

Methylovorus sp. MP688

Halothermothrix orenii
31.17292

221.4976

9.90698

46.63198

172.8365

4.996364

52.53185

13.81098

2186.705

35.83389

246.0929

40.92748

16.34091

1.861025

9.719828

60.27152

2608.284

55.9319

44.72249

54.17575

529.9866

32.19833

20.87227

21.94951

5.620581

8.24201

2.289406

28.04444

15.69305

18.89525

6.173191

8.57526

17.25153

3.310266

13.02391

4.350903

18.33571

126.8789

22.2301

10.09987

10.98618

1.948919

12.18421

35.74869

9.559314

68.74451

1.555133

86.78809

7.241994

4.334251

0.496844

0.51851

$-1.96231$

1.884218

1.11012

1.065861

$-0.58113$

$-0.4339$

0.558992

0.417203

$-1.5789$

1.518301

$-0.66805$

0.64244

$-1.05865$

1.01707

$-0.64279$

$-1.08601$

0.618855

0.45233

1.045279

$-0.60587$

$-0.84794$

$-2.98557$

$-1.05852$

0.435718

0.583969

0.818101

2.880004

1.020975

$-1.18961$

1.148837

0.450246

0.435829

0.55979

$-0.60728$

0.541819

0.587277

$-0.69529$

0.672786

0.53423

0.51669

$-0.58997$

0.572244

1.128287

1.094612

$-1.18054$

1.144802

1.822136

1.21894

1.404551

0.62391

1.048252

1.078562

$-0.71929$

0.700224

$-1.10716$

1.078199

1.442368

1.405349

2.511

1.221415

1.251653$$
1 .
$$

1.

1.604221

$-1.04361$

0.296664

$-1.04145$

0.297668

$-1.04152$

0.297634

0.553336

$-1.03961$

0.298522

$-1.04002$

0.29833

$-1.03991$

0.298381

0.554813

$-1.03987$

0.298401

54813

$-1.04088$

0.297929

$-1.03868$

0.298955

$-1.03896$

0.298822

$-1.03812$

0.299215

$-1.0375$

0.299503

$-1.03648$

0.29998

$-1.03665$

0.299897

$-1.03677$

0.299841

0.554813

$-1.03549$

0.300442

1.033079

0.301567

1.033168

0.301525

$-1.03406$

0.30111

$-1.03344$

0.301396

0.554813

0.554813

0.55505

0.55505

0.555249

0.5555

0.555536

0.555536

0.555536

0.556107

0.556774

0.556774

1.033947

0.301161

0.556774

1.03098

0.302552

0.556774

0.556774

1.030765

0.302651

0.557927

$-1.03121$

0.30244

0.557927

$-1.02893$

0.303511

0.557927

$-1.02798$

0.30396

0.558927

$-1.02837$ 
Pasteurella dagmatis

Arcobacter trophiarum

Streptomyces sp. CNQ-509

Haemophilus influenzae

Neisseria sp. 10022

Methanocella conradii

Pseudomonas cremoricolorata

Aeromonas sp. ASNIH1

Campylobacter sputorum

Synechococcus sp. JA-3-3Ab

Rhodobacter blasticus

Veillonella parvula

Eggerthella sp. YY7918

Streptomyces lavendulae

Francisella philomiragia

Oceanicoccus sagamiensis

Kosakonia sacchari

Flavobacterium anhuiense

Lawsonia intracellularis

Gramella sp. SH35

Aurantimicrobium sp. MWH-Mo1

Lactobacillus casei

Aquimarina sp. AD1

Candidatus Aquiluna sp. UB-Maderos

Defluviitoga tunisiensis

Cycloclasticus sp. PY97N

Propionibacterium virus PHL070N00

Actinomyces gaoshouyii

Asaia bogorensis

Lactobacillus paragasseri

Rathayibacter toxicus

Neisseria sicca

Vibrio alginolyticus

Clostridium ljungdahlii

Enterobacter sp. FY-07

Candidatus Gracilibacteria bacterium

Clostridiaceae bacterium 14 S0207

Thermus thermophilus

Plantactinospora sp. BC1

Microcystis sp. MC19

Microlunatus soli

Calothrix sp. NIES-2100

Pseudomonas corrugata

Corynebacterium minutissimum

Haloterrigena daqingensis

Bacillus butanolivorans

Capnocytophaga cynodegmi

Ruminococcaceae bacterium CPB6

Marinifilaceae bacterium SPP2

Olleya aquimaris

\begin{tabular}{|c|c|c|c|c|c|}
\hline & & & & & \\
\hline 7.09 & 1.496624 & 1.476007 & 1.013968 & 0.310598 & 0561 \\
\hline 5.30359 & -0.54268 & 0.535093 & -1.01418 & 0.310497 & \\
\hline & -0.68657 & 0.676368 & -1.01508 & 068 & \\
\hline & -0.8 & & -1.01333 & & \\
\hline & -2.51175 & 2.486527 & -1.01014 & .312427 & \\
\hline 5.6 & -0.53899 & 0.533557 & -1.01019 & .312405 & כ \\
\hline & -2.91949 & 2.889494 & -1.0 & & \\
\hline & 1.1 & & 1.009604 & & \\
\hline 9.74 & -0.76525 & 0.757757 & -1.00988 & .312551 & 0.5 \\
\hline 75.4 & -0.4 & 0.468003 & -1.00808 & 415 & \\
\hline & -0.5 & 0.556297 & -1.00796 & & \\
\hline & -1.1 & 354 & -1. & 681 & \\
\hline 3 & -0.43 & 0.436 & -1.00774 & 578 & \\
\hline & 1.590479 & 1.57696 & 1.008573 & 79 & . \\
\hline & -1.2 & 496 & -1.00464 & .315071 & 0.5004 \\
\hline & -0.9 & 0.907525 & -1.0047 & 0.31504 & \\
\hline 2 & -0.71614 & 0.71318 & -1.00415 & 0.315306 & \\
\hline (2) & & 1.37 & -1.00258 & 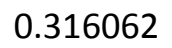 & \\
\hline & -1.2 & & -1.0 & & \\
\hline 5 & -0.61089 & 0.609 & -1.00295 & 0.315 & \\
\hline & -1.09 & & -1.00206 & & \\
\hline & $1.32 \varepsilon$ & 368 & 1.001778 & & 0.00 \\
\hline & -0.8 & 4098 & -1.00012 & 55 & \\
\hline 10070 & 2.415488 & 2.417086 & 0.999339 & 0.317631 & סרחת \\
\hline & -2.9 & 2.91 & -0.99889 & & \\
\hline 2.4 & -1.3 & 358 & -0.99685 & & \\
\hline 326 & -0.36365 & 0.365019 & -0.99625 & 128 & \\
\hline & -0.7 & & -0.99566 & 0.31 & \\
\hline .1 & -2.1 & 2.1 & -0.9 & & \\
\hline 26. & -0.4 & 0.48 & -0.99334 & 0.320 & \\
\hline 07.4594 & -0.77917 & 0.784793 & -0.99284 & 0.320788 & 0.014402 \\
\hline & -1.1 & 1.15 & -0.9 & 0.321 & \\
\hline 1.72 & -2.8 & 2.90 & -0.991 & & \\
\hline & -0.77549 & 0.78 & -0.99076 & & \\
\hline 2.04 & 2.40035 & 2.423695 & 0.990368 & 0.321994 & .575509 \\
\hline 18. & 0.8 & 0.9 & 0.9 & 7 & \\
\hline 65.79469 & -0.5 & 0.592935 & -0.98596 & 52 & \\
\hline & -0.67027 & 0.679754 & -0.98605 & 0.324107 & \\
\hline 2.396422 & -2.18826 & 2.222517 & -0.98458 & 0.3248 & \\
\hline & -0.41711 & 0.423507 & -0.98491 & 0.32467 & \\
\hline & & & 0.984291 & & \\
\hline 36.56537 & -0.81021 & 0.823919 & -0.98337 & 0.325427 & 0.579652 \\
\hline & -0.26313 & 0.267774 & -0.98267 & 0.32577 & \\
\hline רפחב1 14 & -0.95311 & 0.970266 & -0.98231 & 0.325945 & \\
\hline 13.29358 & -0.87624 & 0.892718 & -0.98155 & 0.326323 & \\
\hline 5.425351 & 1.341529 & 1.367984 & 0.980661 & 0.32676 & 0.580605 \\
\hline & -0.78668 & 0.802048 & -0.98084 & 0.326674 & \\
\hline ค & 0.963857 & 0.983432 & 0.980096 & 0.327039 & 0.5000 \\
\hline & 1.450212 & 1.482513 & 0.978212 & 0.327969 & 0.5821 \\
\hline
\end{tabular}


Alcanivorax dieselolei

Streptomyces nigra

Cyanothece sp. ATCC 51142

Bifidobacterium thermophilum

Modestobacter marinus

Dactylococcopsis salina

Rufibacter sp. DG31D

Spiroplasma alleghenense

Micromonospora sp. WMMA2032

Haloterrigena turkmenica

Melissococcus plutonius

[Eubacterium] minutum

Magnetococcus marinus

Prevotella jejuni

Natrialba magadii

Pseudomonas pohangensis

Capnocytophaga ochracea

Chryseobacterium gallinarum

Borrelia hermsii

Corynebacterium casei

Pseudomonas viridiflava

Carnobacterium maltaromaticum

Kingella kingae

Arthrobacter alpinus

Rufibacter sp. DG15C

Actinoalloteichus hoggarensis

Verrucosispora maris

Cytophaga hutchinsonii

Pseudomonas sp. TMW 2.1634

Actinomyces meyeri

Saprospira grandis

Halodesulfurarchaeum formicicum

Parascardovia denticolens

Jiangella sp. DSM 45060

Methylobacterium phyllosphaerae

Rhodothermaceae bacterium

Geobacillus subterraneus

Haemophilus pittmaniae

Pediococcus claussenii

Arthrobacter sp. YC-RL1

Exiguobacterium sp. AT1b

Amphibacillus xylanus

Pseudomonas sp. Leaf58

Propionibacterium phage PHL150M0

Teredinibacter turnerae

Virgibacillus sp. Bac332

Sphingobium sp. MI1205

Sphaerospermopsis kisseleviana

Tessaracoccus flavus

Rhodococcus hoagii
74.74695

63.77807

5.424656

27.73949

257.6073

2.457832

12.25622

2.42321

76.62419

29.23659

12.41026

12.22121

2.095392

439.5349

9.90679

35.99804

55.31321

31.08977

4.656661

1544.238

50.09441

78.88651

16.61741

290.9038

7.898214

112.8712

92.9681

29.30318

22.58609

368.1986

1.942142

2.743146

103.9194

160.9683

69.8064

56.87635

2.302408

49.99471

3.870378

189.6826

52.26777

4.334334

44.93541

9.197266

10.01548

10.53496

28.78013

7.050099

91.07117

214.7415

$-0.57924$

$-0.41551$

0.592734

$-0.97723$

0.328456

0.582713

1.596438

0.425305

$-0.97696$

0.328587

0.582713

0.488249

0.975088

0.329516

0.583225

$-0.47333$

0.975463

0.329331

0.583225

$-2.15384$

$-0.97515$

0.329486

0.583225

$-0.98361$

2.207094

$-0.97587$

0.329128

0.583225

2.323329

2.38867

$-0.97468$

0.32972

0.583302

$-0.50187$

0.516184

0.972644

0.33073

0.584804

$-0.53112$

0.546959

$-0.97228$

0.330913

0.584843

1.186795

1.22302

$-0.97103$

0.331532

0.585653

$\begin{array}{ll}-1.0939 & 1.127257\end{array}$

0.970381

0.331857

0.585658

$-1.83851$

1.895302

$-0.9704$

0.331845

0.585658

$-0.51571$

0.53209

$-0.97004$

0.332028

0.585677

$-0.97278$

1.004395

$-0.96922$

0.332435

0.58611

$-0.66562$

0.688054

$-0.80748$

0.837311

$-0.75987$

0.788321

$-0.96852$

0.332783

0.586439

$\begin{array}{ll}-0.96739 & 0.333349\end{array}$

0.587152

$-0.964370 .33486$

0.589528

2.056907

2.136376

$-0.9639$

0.335094

0.589655

$-0.27373$

0.284693

0.962802

0.335647

0.590343

$-0.96149$

0.336304

0.591212

0.602741

0.627322

0.960815

0.336645

0.591526

$-0.96005$

0.337031

0.591919

$-0.87802$

0.916195

$-0.95833$

0.337894

0.593109

$-0.95801$

0.338058

0.593109

$-0.95773$

0.338198

0.593109

$-1.11025$

1.159252

$-0.95634$

0.3389

0.594054

$-0.95539$

0.33938

0.59461

0.805202

0.953846

0.340162

0.595692

$-0.95156$

0.341322

0.596041

$-0.44981$

$-0.95055$

0.341835

0.596041

$-1.97144$

2.073301

$-0.95087$

0.34167

0.596041

$-1.35985$

1.427467

$-0.95263$

0.340775

0.596041

$-0.60017$

0.63108

$-0.95102$

0.341595

0.596041

$-0.37643$

0.39503

$-0.95291$

0.340634

0.596041

$-0.85792$

0.901829

$-0.95131$

0.341446

0.596041

$-0.632810 .665404$

$-0.95102$

0.341594

0.596041

$-2.2951$

2.413991

$-0.95075$

0.341731

0.596041

$-0.7055$

0.742861

$-0.9497$

0.342263

0.596152

$-1.64659$

1.733315

$-0.94996$

0.34213

0.596152

$-0.3813$

0.401603

$-0.94945$

0.34239

0.596152

$-0.81012$

0.855493

$-0.94697$

0.343655

0.597211

$-0.9478$

0.343231

0.597211

$-0.49796$ 
Streptococcus sp. I-P16

Brucella sp. 09RB8910

Methanosarcina sp. MTP4

Thermodesulfobium narugense

Christensenella sp. Marseille-P3954

Gluconobacter oxydans

Corynebacterium terpenotabidum

Cryobacterium arcticum

Lactococcus virus 712

Thermobispora bispora

Corynebacterium epidermidicanis

Maribacter sp. 1_2014MBL_MicDiv

Cellulosimicrobium sp. TH-20

Corynebacterium urealyticum

Aeromonas rivipollensis

Chondrocystis sp. NIES-4102

Novosphingobium sp. P6W

Clostridiales bacterium CCNA10

Lactobacillus koreensis

Rhodococcus sp. MTM3W5.2

Enterococcus sp. FDAARGOS_375

Alloactinosynnema sp. L-07

Roseovarius sp. TM1035

Pseudomonas extremaustralis

Shewanella putrefaciens

Micromonospora rifamycinica

Bacillus halodurans

Enterobacter sp. SA187

Rhodococcus sp. p52

Clostridium scatologenes

Prevotella ruminicola

Jeotgalibacillus malaysiensis

Methylobacterium sp. C1

Rahnella sp. ERMR1:05

Gryllotalpicola sp. 2DFW10M-5

Segniliparus rotundus

Pseudomonas sp. SWI36

Actinoalloteichus hymeniacidonis

Streptomyces violaceusniger

Bifidobacterium longum

Methylocystis bryophila

Streptococcus respiraculi

Opitutus terrae

Pseudomonas taetrolens

Miniimonas sp. S16

Nostoc punctiforme

Microbacterium sp. CGR1

Mycoplasma bovirhinis

Synechocystis sp. PCC 6714

Xenorhabdus poinarii

\begin{tabular}{|c|c|c|c|c|c|}
\hline & & & & & \\
\hline 5.515648 & -1.70788 & 1.813919 & -0.94154 & 0.346427 & 0.599661 \\
\hline 389043 & 2.02892 & 2.155428 & 0.941307 & 0.346547 & 599661 \\
\hline 419261 & 2.608238 & 2.773024 & 0.940575 & 0.346923 & 0.600024 \\
\hline .26788 & -0.7466 & 0.794116 & -0.94016 & 0.347136 & 0.600099 \\
\hline 9.40281 & -0.70158 & 0.74648 & -0.93985 & 0.347295 & 0.600099 \\
\hline 392.867 & -0.39818 & 0.423953 & -0.9392 & 0.347628 & 0.600389 \\
\hline 105.5593 & -0.44488 & 0.473846 & -0.93888 & 0.347794 & 0.60039 \\
\hline 1.567865 & -2.74136 & 2.922261 & -0.93809 & 0.348196 & 0.600514 \\
\hline 80.90345 & -0.41639 & 0.44377 & -0.93831 & 0.348086 & 0.600514 \\
\hline 726.095 & -0.28729 & 0.306587 & -0.93707 & 0.348722 & 0.601137 \\
\hline 1.567786 & -2.73711 & 2.922274 & -0.93664 & 0.348945 & 0.60123 \\
\hline 173.9058 & -0.46163 & 0.49385 & -0.93475 & 0.349915 & 0.601768 \\
\hline 1687.874 & -0.35294 & 0.377241 & -0.93559 & 0.349487 & 0.601768 \\
\hline 16.1013 & -0.92105 & 0.985206 & -0.93488 & 985 & 0.601768 \\
\hline 21.00528 & -0.74235 & 0.794127 & -0.9348 & 0.349893 & 0.601768 \\
\hline 82.86915 & -0.42852 & 0.459167 & -0.93325 & 0.350693 & 0.6026 \\
\hline 88.18871 & -0.62439 & 0.669102 & -0.93317 & 0.35073 & 0.6026 \\
\hline 1.403435 & -2.55775 & 2.743293 & -0.93237 & 0.351148 & 0.603033 \\
\hline 161.4778 & -0.36939 & 0.396755 & -0.93102 & 0.351842 & 0.6039 \\
\hline 2.560032 & -2.4321 & 2.614927 & -0.93008 & 0.352328 & 0.60449 \\
\hline 124.0243 & -0.39503 & 0.424917 & -0.92967 & 254 & 0.604569 \\
\hline 4.805766 & -1.70338 & 1.836851 & -0.92734 & 0.353751 & 0.6046 \\
\hline 36.13917 & -0.52376 & 0.564714 & -0.92747 & 0.353682 & 0.604 \\
\hline 2.801539 & -2.40064 & 2.583601 & -0.92918 & 0.352794 & 0.604 \\
\hline 54.04763 & -0.45395 & 0.488797 & -0.9 & 038 & \\
\hline 2.132008 & -1.76291 & 1.899942 & -0.92787 & 0.353473 & 0.604 \\
\hline 21.27334 & -0.72967 & 0.785934 & -0.92841 & 0.353195 & 0.604 \\
\hline 148.2449 & -0.8333 & 0.898846 & -0.92707 & 0.353888 & 0.6046 \\
\hline 14.30515 & 1.071601 & 1.154786 & 0.927965 & 0.353426 & 0.6046 \\
\hline 40.79041 & -0.49004 & 0.52895 & -0.92643 & 0.354221 & 0.604681 \\
\hline 5.676057 & -1.11047 & 1.198769 & -0.92634 & 0.354268 & 0.60468 \\
\hline 160.0264 & -0.66232 & 0.716001 & -0.92502 & 0.354954 & 0.605152 \\
\hline 35.26145 & -0.57856 & 0.625463 & -0.92502 & 0.354957 & 0.605152 \\
\hline 106.2372 & -0.41889 & 0.452925 & -0.92485 & 0.355043 & 0.60515 \\
\hline 81.11026 & -0.44388 & 0.480484 & -0.92381 & 0.355585 & 0.60528 \\
\hline 1.522622 & -2.69953 & 2.92239 & -0.92374 & 0.355621 & 0.605287 \\
\hline 75.78246 & -0.4944 & 0.535119 & -0.92391 & 0.355531 & 0.605287 \\
\hline 45.29308 & -0.54888 & 0.595516 & -0.92168 & 0.356695 & 0.60654 \\
\hline 681.6319 & -0.502 & 0.544529 & -0.9219 & 0.356579 & 0.606548 \\
\hline 62.42441 & -0.60575 & 0.657643 & -0.92109 & 0.357002 & 0.60678 \\
\hline 14.21763 & -0.79406 & 0.862746 & -0.92038 & 0.357372 & 0.607132 \\
\hline 40.76503 & -0.46653 & 0.507097 & -0.92 & 0.357571 & 0.60718 \\
\hline 35.52954 & -0.61939 & 0.674389 & -0.91844 & 0.358386 & 0.608287 \\
\hline 155.2263 & -0.42712 & 0.465246 & -0.91805 & 0.35859 & 0.608323 \\
\hline 23.29145 & 1.223093 & 1.332686 & 0.917765 & 0.358742 & 0.60832 \\
\hline 55.27082 & -0.86502 & 0.944782 & -0.91557 & 0.35989 & 0.60998 \\
\hline 2.925487 & 2.552079 & 2.79359 & 0.913548 & 0.360954 & 0.61150 \\
\hline 1.491668 & -2.66648 & 2.922495 & -0.9124 & 0.36156 & 0.61224 \\
\hline 2.070929 & -2.03496 & 2.23156 & -0.9119 & 0.361822 & $0.6124 C$ \\
\hline
\end{tabular}


Clostridium drakei

Dermabacter vaginalis

Acinetobacter equi

Staphylococcus phage BP39

Propionibacterium phage Moyashi

Arthrobacter sp. ATCC 21022

Corynebacterium doosanense

Arthrobacter sp. ZXY-2

Streptomyces sp. MOE7

Kordia sp. SMS9

Arcanobacterium phocae

Erysipelothrix sp. 15TAL0474

Rhodovulum sp. MB263

Lelliottia sp. WB101

Tenericutes bacterium MO-XQ

Gallionella capsiferriformans

Thermus aquaticus

Enterococcus gilvus

Bacillus gobiensis

Paenibacillus odorifer

Propionibacterium virus PHL025MO0

Streptococcus australis

Desulfomonile tiedjei

Tenacibaculum jejuense

Staphylococcus felis

Collinsella aerofaciens

Octadecabacter antarcticus

Betapapillomavirus 2

Deinococcus geothermalis

Bacillus marisflavi

Campylobacter pinnipediorum

Aeromicrobium choanae

Paraoerskovia marina

Mycetocola sp. 449

Lactobacillus crustorum

Staphylococcus virus $\mathrm{PH} 15$

Methanofollis liminatans

Propionibacterium virus Attacne

Halorhabdus utahensis

Methanocella arvoryzae

Hymenobacter sp. PAMC 26554

Paenibacillus sp. CAA11

Nitrososphaera viennensis

Pseudomonas koreensis

Amycolatopsis sp. BJA-103

Aeromonas sp. ASNIH4

Pseudomonas sp. CCOS 191

Candidatus Planktophila limnetica

Paenibacillus sp. 320-W

Corynebacterium singulare

$\begin{array}{lll}10.68261 & 1.343558\end{array}$

1232.017

$-0.35829$

1.47392

18.23494

0.900504

0.394855

0.911554

0.362004

0.612427

1.471158

$-2.64958$

0.990851

$-0.90739$

0.364201

0.612581

10.22334

$-1.65615$

2.922549

0.908819

0.363445

0.612581

110.0737

$-0.71841$

1.823137

0.36462

0.612581

1305.688

$-0.30291$

0.792383

$-0.90841$

0.363663

0.612581

112.562

$-0.70291$

0.332894

$-0.90664$

0.364597

0.612581

23.04392

$-0.92103$

0.774484

$-0.90992$

0.362866

0.612581

7.763178

1.265416

1.013023

$-0.90758$

0.364099

0.612581

31.83363

$-0.49345$

1.389615

0.90919

0.36325

0.612581

2.003288

$-1.73663$

0.542622

0.91062

0.362494

0.612581

68.73372

$-0.52222$

1.908135

13.46635

$-0.74056$

0.575688

$-0.90938$

0.363151

0.612581

7.209333

1.253835

0.814134

$-0.91012$

0.36276

0.612581

24.04221

$-0.51844$

1.381509

0.364342

0.612581

5.449088

$-1.01073$

0.570331

0.363017

0.612581

7.973496

3.323436

$-1.04994$

1.11564

0.907584

0.364098

0.612581

4.940715

$-1.17754$

1.160686

$-0.90902$

0.363341

0.612581

16.56262

$-1.07064$

$-0.90596$

0.364957

0.612864

$-0.90459$

0.365684

0.613785

$-0.90429$

0.365842

0.613785

157.7643

$-1.2316$

1.184422

$-0.90394$

0.366029

0.613815

2.421686

$-0.50557$

1.363387

$-0.90334$

0.366348

0.614067

11.56519

$-1.29237$

0.559912

$-0.90294$

0.366556

0.614133

$-0.90262$

0.36673

0.614142

44.91745

1.179274

.

2261

0.366918

0.614174

321.9694

0.530418

0.588438

0.9014

0.367375

0.614253

13.40356

$-0.58307$

0.646978

$-0.90122$

0.367471

0.614253

22.68281

1.05389

$-0.90183$

0.367147

0.614253

29.58726

$-0.4656$

1.353989

$-0.90038$

0.367917

0.614715

9.752048

$-0.8818$

$-0.89932$

0.368482

0.615377

11.09152

1.044007

0.982213

$-0.89777$

0.369307

0.616189

217.4656

$-0.47494$

1.162655

0.897951

0.369212

0.616189

71.69945

$-0.36425$

0.529439

$-0.89706$

0.369686

0.616539

71.37169

$-0.31717$

406791

$-0.89543$

0.370555

0.617704

5.024966

$-1.19915$

0.354635

$-0.89435$

0.371132

0.618384

167.0586

1.098304

$-0.89396$

0.371345

0.618455

16.4448

$-0.69581$

0.779161

0.893561

0.371557

0.618525

62.39474

1.272234

1.425852

$-0.89303$

0.371842

0.618716

3.168076

$-1.35941$

1.523789

0.892262

0.372252

0.618731

3.211199

$-1.67577$

1.878873

$-0.89212$

0.372327

0.618731

109.2777

$-0.50533$

0.566673

$-0.8919$

0.372445

0.618731

4.686387

$-1.16301$

1.306512

$-0.89174$

0.372531

0.618731 
Oscillibacter valericigenes

Deinococcus gobiensis

Euzebya sp. DY32-46

Frondihabitans sp. PAMC 28766

Betapapillomavirus 3

Bartonella ancashensis

Paracoccus zhejiangensis

Staphylococcus virus IPLA5

Bacillus licheniformis

Sphingobacterium mizutaii

Halobacterium hubeiense

Sporosarcina sp. PTS2304

Friedmanniella sagamiharensis

Nostoc sp. CENA543

Shewanella psychrophila

Corynebacterium deserti

Neisseria lactamica

Sphingobium baderi

Azospira oryzae

Escherichia marmotae

Thioclava nitratireducens

[Clostridium] cellulosi

Mesorhizobium huakuii

Streptococcus sp. FDAARGOS_192

Aeromonas sp. ASNIH2

Pelosinus fermentans

Enterobacter asburiae

Legionella pneumophila

Pseudoalteromonas translucida

Propionibacterium virus P100D

Fusobacterium periodonticum

Sphingopyxis terrae

Syntrophus aciditrophicus

Methylobacterium sp. 17SD2-17

Hymenobacter sp. APR13

Sulfurihydrogenibium sp. YO3AOP1

Bacillus cellulosilyticus

Candidatus Protochlamydia amoebor

Bacteroides fragilis

Prosthecochloris sp. HL-130-GSB

Paenibacillus sp. FSL H7-0357

Desulfobacca acetoxidans

Spirosoma radiotolerans

Pseudomonas tolaasii

Serratia sp. FS14

Rhodococcus phage RRH1

Sulfitobacter sp. SK012

Aerococcaceae bacterium ZY16052

Exiguobacterium sibiricum

Pseudomonas sp. XWY-1
20.83307

72.67843

80.99152

169.5761

57.3316

1.733144

231.3749

112.0623

1.267658

20.1734

14.24599

2.773234

314.1627

11.97487

2.196396

561.6222

75.18637

87.22743

208.175

5.229234

59.2516

6.759717

53.35646

133.5481

1.36569

9.571519

37.04842

44.11284

5.888332

22.26535

164.2814

53.58384

3.608564

145.6556

56.49022

1.831398

6.991013

1.791231

316.6454

2.635969

14.25972

9.806172

21.82047

34.76768

1.335715

1.246904

8.276197

24.99166

13.93188

23.99933
$-0.98049$

$-0.43863$

1.109882

0.497193

$-0.34816$

0.39545

$-0.3753$

0.42737

$-2.0652$

2.349758

$-2.2066$

2.514853

$-0.48104$

0.548048

0.954634

1.087647

2.774224

0.806917

$-0.70794$

$-0.54694$

0.621992

$-1.92441$

2.191435

$-0.4744$

0.541364

$-1.18509$

1.352099

$-1.65703$

1.890995

$-0.27857$

0.318051

$-0.69554$

0.798802

$-0.49236$

0.565924

$-0.54838$

0.629924

$-1.59802$

1.834735

$-0.49742$

0.568902

$-1.2295$

1.413084

$-0.84128$

0.962229

0.75082

0.655365

2.922902

$-2.5445$

1.003524

$-0.87632$

0.778254

$-0.67867$

0.514536

2.11773

2.434488

1.278565

1.47012

$-0.43029$

0.493038

$-0.55602$

0.638466

$-1.07755$

1.232212

$-0.44363$

0.507901

$-0.39991$

0.460068

1.951865

2.247518

$-0.93543$

1.079354

$-1.96996$

2.278538

$-0.584$

0.675378

$-1.50119$

1.739178

$-0.73475$

0.852362

$-0.81815$

0.948967

$-0.59873$

0.694378

$-0.56086$

0.652629

$-2.51115$

2.923019

$-2.40584$

2.797833

$-0.76602$

0.891528

$-0.75933$

0.882834

0.947299

1.108673

$-0.9541$

1.11466

$\begin{array}{ll}-0.88342 & 0.377012\end{array}$

$-0.8822$

0.377666

0.623652

$-0.880410 .378638$

0.624127

$-0.878160 .379859$

0.625449

$\begin{array}{ll}-0.8789 & 0.379455\end{array}$

0.62564

$\begin{array}{ll}-0.87743 & 0.380254\end{array}$

0.62564

$-0.877730 .380088$

$0.877706 \quad 0.380103$

0.62564

0.62564

$-0.87794$

0.379974

0.62564

$-0.87734$

0.380301

0.62564

$-0.87934$

0.379216

0.62564

$-0.87815$

0.379861

0.62564

$-0.8763$

0.380865

0.62564

$-0.876480 .380768$

0.625743

$-0.87628$

0.38088

0.625743

$\begin{array}{ll}-0.87587 & 0.381098\end{array}$

0.625743

$-0.870730 .383901$

0.62582

$-0.87002$

0.384291

0.62626

$-0.87055$

0.384001

0.62626

$-0.87098$

0.383765

0.62626

$-0.87434$

0.381932

0.62626

$-0.87008$

0.384257

0.62626

$-0.87431$

0.381951

0.62626

0.872865

0.382737

0.62626

$-0.87054$

0.384006

0.62626

$-0.87325$

0.382528

0.62626

$-0.87204$

0.383187

0.62626

0.871575

0.38344

0.62626

0.869887

0.384362

0.62626

0.869701

0.384464

0.62626

$-0.87273 \quad 0.38281$

0.62626

$-0.87087$

0.383823

0.62626

$-0.87448$

0.381855

0.62626

$-0.87346$

0.382414

0.62626

$-0.86924$

0.384718

0.62626

0.868454 
Dechloromonas aromatica

Solibacillus sp. R5-41

Providencia alcalifaciens

Lactococcus virus $\mathrm{P} 008$

Syntrophobotulus glycolicus

Oceanobacillus kimchii

Alistipes finegoldii

Halostagnicola larsenii

Paenibacillus physcomitrellae

Methanococcoides methylutens

Pseudoalteromonas carrageenovora

Paenibacillaceae bacterium GAS479

Pseudomonas sp. DR 5-09

Staphylococcus virus IPLA7

Roseiflexus sp. RS-1

Liberibacter crescens

Citrobacter freundii complex sp. CFNI

Hymenobacter swuensis

Thermacetogenium phaeum

Streptococcus ruminantium

Mycobacterium haemophilum

Halobacillus mangrovi

Bacillus atrophaeus

Thioalkalivibrio paradoxus

Tetragenococcus koreensis

Streptosporangium sp. 'caverna'

Xenorhabdus bovienii

Formosa agariphila

Streptococcus parauberis

Phycicoccus dokdonensis

Haloferax volcanii

Finegoldia magna

Flavobacterium arcticum

Arthrobacter sp. ERGS1:01

Spiroplasma apis

Neisseriaceae bacterium DSM 100971

Corynebacterium jeikeium

Fischerella sp. NIES-3754

Paraglaciecola psychrophila

Halalkalicoccus jeotgali

Treponema azotonutricium

Pseudoalteromonas donghaensis

Sinorhizobium sp. RACO2

Gordonia iterans

Cronobacter universalis

Proteus mirabilis

Rhodococcus rhodochrous

Francisella halioticida

Pseudomonas umsongensis

Psychrobacter sp. P11G3
59.20896

11.88477

7.982461

5.500747

1.208913

8.121762

114.5128

11.42217

7.496986

1.681367

1.71556

4.593149

24.60057

152.2537

16.41701

1.926846

3.509445

59.51459

5.157711

11.17325

68.85783

5.801286

2.177068

38.35283

6.114375

97.78072

12.77937

12.41099

14.46649

238.1388

11.75165

7273.933

9.87135

154.7132

5.17317

5.157171

12047.78

15.20903

6.990968

19.9526

2.638406

7.314569

93.42887

294.3666

4.837907

32.18888

151.2529

3.73352

38.21039

34.69336
$-0.36475$

$-0.76791$

$-1.11368$

0.425979

0.900149

1.30575

1.846158

$-2.37073$

2.786397

1.015085

1.183162

$-0.55017$

0.646473

$-0.95015$

1.113228

$-0.91619$

1.071615

$-1.94981$

2.29111

$-2.14439$

2.507065

$-0.99846$

$-0.73746$

1.171845

0.924703

0.859288

1.085753

$-0.64136$

0.751544

$-1.78999$

2.095703

1.82277

2.132583

0.45479

0.532012

$-1.14429$

1.343166

$-0.85373$

1.002458

$-0.36766$

0.430726

$-1.00483$

1.17307

$-2.09538$

2.447578

$-0.54883$

0.646412

$-1.08983$

1.283195

$-0.3644$

0.42964

$-0.77962$

0.918876

0.905743

$-0.68814$

1.068421

0.813173

$-0.42632$

0.504986

$-0.70014$

$-0.32886$

0.828589

0.389678

0.928468

1.099378

$-0.30984$

0.367016

1.567739

1.860078

$-1.08085$

1.283073

$-0.33587$

0.399269

0.97691

$-1.37377$

1.161403

$-0.55368$

1.634469

$-1.18126$

0.659853

$-1.39789$

1.40802

$-0.47698$

1.665396

0.568814

$-0.42767$

0.510825

$-1.4264$

1.703992

0.651057

0.778036

$-0.38842$

0.465351

1.562428

1.871335

$-0.53541$

0.641392

$-0.66536$

$-0.85626$

0.391853

$-0.85309$

0.393607

0.632172

$-0.85291$

0.393711

0.632172

$-0.85627$

0.391847

0.632172

$-0.85082$

0.394869

0.632172

0.857942

0.390924

0.632172

$-0.85103$

0.394754

0.632172

$-0.85351$

0.393376

0.632172

$-0.85496$

0.392571

0.632172

$-0.85103$

0.394752

0.632172

$-0.85534$

0.392363

0.632172

$-0.85204$

0.394192

0.632172

$-0.85822$

0.390773

0.632172

0.85167

0.394397

0.632172

$-0.85339$

0.393443

0.632172

$-0.85412$

0.393037

0.632172

0.854724

0.392704

0.632172

0.85485

0.392634

0.632172

$-0.85193$

0.394252

0.632172

$-0.85164$

0.394414

0.632172

$-0.85358$

0.393338

0.632172

$-0.85658$

0.391676

0.632172

$-0.8561$

0.391941

0.632172

$-0.84904$

0.395857

0.632172

$-0.84931$

0.395708

0.633197

$-0.84814$

0.396359

0.633197

$-0.84845$

0.396188

0.633443

0.84774

0.396583

0.633443

$-0.84624$

0.397417

0.633522

$-0.84422$

0.398546

0.634577

$-0.84498$

0.398123

0.635245

$-0.84393$ 
Spirosoma montaniterrae

Propionibacterium phage QueenBey

Lactobacillus pentosus

Chryseobacterium indoltheticum

Cellvibrio sp. PSBB023

Nostoc sphaeroides

Bacillus clausii

Deinococcus actinosclerus

Sphingopyxis sp. WS5A3p

Gordonia phthalatica

Ilyobacter polytropus

Microbacterium hominis

Flavobacterium commune

Paracoccus contaminans

Candidatus Endolissoclinum faulkneri

Dickeya sp. NCPPB 3274

Allokutzneria albata

Clostridium sp. JN500901

Streptomyces ambofaciens

Agarivorans gilvus

Staphylococcus lugdunensis

Rhodococcus coprophilus

Micromonospora sp. L5

Nitrosococcus oceani

Dichelobacter nodosus

Geminocystis sp. NIES-3709

Pseudomonas oryzihabitans

Clostridioides difficile

Methylovulum psychrotolerans

Corynebacterium kutscheri

Piscirickettsia salmonis

Leptolyngbya sp. NIES-3755

Mariprofundus aestuarium

Desulfohalobium retbaense

Altererythrobacter sp. ZODW24

Desulfitobacterium metallireducens

Exiguobacterium sp. ZWU0009

Klebsiella variicola

Chryseobacterium sp. H3001

Aurantimicrobium minutum

Gramella sp. MAR_2010_147

Paenibacillus sp. JDR-2

Acinetobacter sp. TGL-Y2

Haloarcula marismortui

Psychrobacter sp. PRwf-1

Lactobacillus kunkeei

Pseudomonas libanensis

Mixta gaviniae

Methanobrevibacter sp. AbM4

Streptococcus infantarius
10.98725

5.67296

13.71086

81.80578

16.01061

7.042522

9.198546

87.3922

55.44458

199.6358

6.510066

304.0497

24.64321

551.0446

3.211325

5.312766

151.0935

9.536915

76.5264

2.665162

2286.063

125.2692

1.238018

7.078687

3.609689

14.53007

227.3771

430.682

10.61876

189.4875

9.572768

1.436775

2.38724

3.353673

8.547323

3.990476

9.438092

34.8201

14.47718

15.40946

5.490272

4.020585

15.8775

1.212637

32.78678

2.453916

25.04628

38.82489

4.128045

31.37093
$-0.65652$

$-1.76185$

$-0.68711$

$-0.53142$

$-0.52889$

0.786282

2.109269

0.823859

0.638447

0.63678

1.849179

\subsection{5}

$-0.84788$

1.021108

$-0.37586$

0.452392

0.521595

$-0.33401$

0.403628

1.069605

1.291899

$-0.49392$

$-0.70188$

$-0.42144$

1.673599

0.596765

0.848861

0.510057

2.025641

$-1.40927$

1.707166

$-0.30025$

0.36394

0.925249

$-0.3614$

1.121313

0.438233

$-1.34788$

1.636463

0.430487

$-0.50871$

0.522917

$-2.40569$

0.618106

$-0.89822$

2.923408

$-1.06048$

0.984828

$-0.41526$

0.386081

$-0.87874$

1.092638

1.290259

1.200625

0.506136

0.471265

$-0.30733$

1.074345

0.37597

0.876186

$-1.91892$

1.072352

2.356159

$-1.43623$

1.763867

$-1.15644$

1.418537

$-0.69432$

0.852149

$-1.22053$

1.496141

$-2.33259$

$-0.76857$

2.863901

0.944822

$-0.94037$

1.157949

$-0.73888$

0.91006

$-1.17135$

1.443013

$-0.92648$

0.693345

1.140987

0.854534

$-2.37061$

2.923544

0.984328

1.214747

$-1.43813$

1.77725

0.77016

$-0.45083$

0.951166

0.557209

1.520153

1.880071

$-0.92512$

$-0.83496$

0.403738

$-0.83529$

0.403554

$-0.83402$

0.404271

$-0.83237$

0.405202

$-0.83058$

0.406214

0.405843

0.831231

0.406341

$-0.83035$

0.406072

$-0.83083$

$-0.82764$

0.407875

$-0.82752$

0.407941

0.827932

0.407709

$-0.82766$

0.407863

$-0.82685$

0.408319

$-0.82626$

0.408659

0.826207

0.408686

$\begin{array}{ll}-0.8255 & 0.409088\end{array}$

$-0.825$

0.409373

0.825148

$-0.82467$

0.409288

$-0.82365$

0.409556

0.410137

0.82324

0.410372

$-0.82301$

0.4105

$-0.82291$

0.410561

$-0.82207$

0.411039

$-0.82191$

0.411128

0.8202630 .412066

$-0.82044$

0.411963

0.819243

0.412648

$-0.81793$

0.413395

$-0.81743$

0.413681

0.81707

0.413889

$-0.81443$

0.4154

$-0.81425$

0.415501

$-0.81524$

0.414938

$-0.81478$

0.415196

$-0.81579$

0.414623

$-0.81448$

0.415369

$-0.81345$

0.415958

$-0.8121$

0.416736

$-0.8119$

0.416847

$-0.81174$

0.416941

$-0.812$

0.416792

0.8113720 .417152

$-0.81087$

0.417442

0.417759

0.810316

0.418406

$-0.80919$

0.418112

$-0.8091$

0.41846

0.8085610 .418768 


\begin{tabular}{|c|c|c|c|c|c|c|}
\hline Kosakonia sp. CCTCC M2018092 & 10.27175 & & & & & \\
\hline Veissella koreensis & 5.154469 & -1.18339 & 1.467823 & -0.80622 & 0.420114 & \\
\hline Curtobacterium sp. MR_MD2014 & 162.8535 & -0.40874 & 0.506833 & -0.80647 & 0.419972 & 0.649721 \\
\hline oorosarcina psychrophila & 1.95402 & -0.89572 & 1.112067 & -0.80545 & 0.420558 & 0.649929 \\
\hline ondihabitans sp. 762G35 & 31.6161 & -0.43449 & 0.539491 & -0.80537 & 0.420605 & 9929 \\
\hline ampylobacter hepaticus & 685192 & 1.642727 & 2.044904 & 0.803327 & 0.421786 & 0.651476 \\
\hline Corynebacterium imitans & 664.723 & -0.27526 & 0.34358 & -0.80116 & 0.423038 & 0.652907 \\
\hline acteroides zoogleoformans & 4.64623 & -0.72383 & 0.903543 & -0.8011 & 0.423071 & 0.652907 \\
\hline Candidatus Planktophila dulcis & 19.92264 & -0.68395 & 0.855049 & -0.7999 & 0.423771 & 0.653434 \\
\hline Streptococcus gordonii & 6588.896 & -0.45006 & 0.562456 & -0.80016 & 0.423617 & 0.653434 \\
\hline Streptomyces sp. 769 & 54.91286 & -0.44983 & 0.563155 & -0.79877 & 0.424423 & 0.653742 \\
\hline Arcobacter molluscorum & 2.78883 & 1.054188 & 1.319364 & 0.799012 & 0.424283 & 0.653742 \\
\hline Jeobacillus sp. GHHO1 & 0.409908 & 2.348605 & 2.940827 & 0.798621 & 0.42451 & 0.653742 \\
\hline urinus & 2.39827 & -1.50967 & 1.892396 & -0.79776 & 0.425011 & 236 \\
\hline iguaniorum & 3.566503 & 1.606621 & 2.020886 & 0.795008 & 609 & 587 \\
\hline ter sulfonivorans & 120.72 & -0.28451 & 0.357835 & -0.7 & 0.426561 & 0.655587 \\
\hline thiocyanaticus & 39.98188 & -0.45093 & 0.566608 & -0.79584 & 0.426123 & 5587 \\
\hline Icaliphila & 440.4166 & -0.44221 & 0.55596 & -0.7954 & 0.426379 & 0.655587 \\
\hline p. LBUM920 & 70.78862 & 0.407281 & 0.512821 & 0.794197 & 0.427081 & 0.656035 \\
\hline Micro & 212.5075 & -0.32668 & 0.411582 & -0.79371 & 0.427363 & 0.6561 \\
\hline Pseuc & 33.64115 & -0.78092 & 0.984138 & -0.79351 & 0.427484 & 0.6561 \\
\hline Bacte & 89.90589 & -0.7595 & 0.957784 & -0.79298 & 0.427789 & 0.656292 \\
\hline Neiss & 70.95717 & -0.66025 & 0.833822 & -0.7 & 56 & 7038 \\
\hline sp. JS71 & 108.2019 & -0.64221 & 0.811825 & -0.79107 & 0.428901 & 0.657444 \\
\hline S61001 & 5.942956 & 1.02714 & 1.29976 & 0.790253 & 0.42938 & 0.657507 \\
\hline gans & 11.40297 & -0.92308 & 1.168302 & -0.7901 & 0.429469 & 7507 \\
\hline us saprophyticus & 571.7729 & 0.402909 & 0.509963 & 0.790075 & 0.429484 & 0.657507 \\
\hline Bacill & 6.948055 & -1.03692 & 1.313607 & -0.78937 & 0.429897 & 0.657862 \\
\hline Polyn & 1.915512 & -1.29769 & 1.644823 & -0.78896 & 0.430137 & 0.657953 \\
\hline ndum & 7.971392 & -1.17143 & 1.485424 & -0.78862 & 0.430337 & 0.657981 \\
\hline Desul & 6.918093 & -0.80203 & 1.017675 & -0.7881 & 0.430638 & 0.658165 \\
\hline Acidit & 13.87344 & -0.82673 & 1.049863 & -0.78746 & 0.431011 & 0.658458 \\
\hline sae & 2.685892 & -1.28189 & 1.628706 & -0.78706 & 0.431247 & 0.658544 \\
\hline Candi & 2.591226 & -1.29291 & 1.643882 & -0.7865 & 0.43 & 0.65877 \\
\hline nolyticus & 127.6704 & -0.59429 & 0.757055 & -0.785 & 0.432451 & 0.659828 \\
\hline Rhizo & 1.145974 & -2.29383 & 2.923852 & -0.78452 & 0.432734 & 0.659982 \\
\hline aponinum & 4.844115 & 1.070801 & 1.370997 & 0.781038 & 0.43478 & 0.662126 \\
\hline Micrc & 126.6224 & -0.42662 & 0.546179 & -0.78109 & 0.434748 & 126 \\
\hline tonas crevioricanis & 20.83327 & -0.68818 & 0.881626 & -0.78058 & 0.435049 & 0.662126 \\
\hline orans & 217.9782 & -0.46137 & 0.590176 & -0.78175 & 0.434363 & 0.662126 \\
\hline Oscilli & 95.959 & -0.55146 & 0.706221 & -0.78086 & 0.434882 & 0.662126 \\
\hline Myroides profundi & 0.76939 & 2.284856 & 2.932685 & 0.7791 & 0.435921 & 0.662985 \\
\hline Propionibacterium virus PHL095N00 & 22.74493 & -1.16679 & 1.497803 & -0.779 & 0.435978 & 0.662985 \\
\hline Rahnella sp. Y9602 & 8.38705 & 2.249694 & 2.895901 & 0.776855 & 0.437245 & 0.664634 \\
\hline Pedi & 1.875359 & -1.48373 & 1.914745 & -0.77489 & 0.438402 & 0.665085 \\
\hline Pararhodospirillum photometricum & & & 0.592702 & & 0.438349 & 0.665085 \\
\hline Bacillus sp. FJAT-42376 & 6.350918 & -0.88925 & 1.148331 & -0.77439 & 0.438702 & 0.665085 \\
\hline Glaciecola nitratireducens & 1.683174 & -1.78389 & 2.306253 & -0.7735 & 0.439226 & 0.665085 \\
\hline Wenyingzhuangia fucanilytica & 9.339081 & 0.878222 & 1.136195 & 0.77295 & 0.439552 & 0.665085 \\
\hline Vibrio rotiferianus & 0.928714 & 2.265052 & 2.929029 & 0.773311 & 0.439338 & 0.665085 \\
\hline
\end{tabular}




\begin{tabular}{|c|c|c|c|c|c|c|}
\hline Saccharomonospora glauca & 95.71061 & -0.39193 & & -0.77543 & & \\
\hline Streptomyces collinus & 60.76435 & -0.37795 & 0.48726 & -0.77567 & 0.437943 & \\
\hline Rhodococcus sp. NJ-530 & 59.5397 & -0.68978 & 0.890451 & -0.77465 & 0.438549 & 0.665085 \\
\hline Luteipulveratus mongoliensis & 58.6147 & -0.31706 & 0.409737 & -0.77382 & 0.439039 & 665085 \\
\hline Corynebacterium timonense & 066.647 & -0.32894 & 0.425539 & -0.773 & .43952 & 085 \\
\hline Bifidobacterium catenulatum & 40.2839 & 0.577585 & 0.747673 & 0.772511 & 0.439812 & 0.665202 \\
\hline Candidatus Atelocyanobacterium tha & 3.799913 & 1.437871 & 1.862964 & 0.771819 & 0.440222 & 0.665545 \\
\hline Capnocytophaga haemolytica & 11.17117 & -0.90709 & 1.177257 & -0.77051 & 0.440995 & 0.666161 \\
\hline Thalassolituus oleivorans & 8.142277 & -0.82952 & 1.076471 & -0.77059 & 0.440952 & 0.666161 \\
\hline Propionibacterium virus $\mathrm{P} 100 \mathrm{~A}$ & 8.033207 & -1.43231 & 1.860556 & -0.76983 & 0.441402 & 0.666499 \\
\hline Flexistipes sinusarabici & 2.22763 & -1.58907 & 2.067534 & -0.76858 & 0.442143 & 0.667063 \\
\hline typicum & 940.0356 & -0.27834 & 0.362098 & -0.76869 & 0.442076 & 0.667063 \\
\hline Brevundi & 315.8913 & -0.41977 & 0.546875 & -0.76758 & 0.442736 & 0.667405 \\
\hline Filifac & 81.88294 & -0.47859 & 0.6234 & -0.76771 & 0.442658 & 7405 \\
\hline Mycol & 152.5637 & -0.33348 & 0.434722 & -0.76712 & 011 & 7543 \\
\hline Strep & 72.92124 & -0.29401 & 0.38402 & -0.76562 & 0.443904 & 8335 \\
\hline Xantl & 41.9197 & -0.61262 & 0.800038 & -0.76573 & 0.443835 & 0.668335 \\
\hline Lactob & 4.627313 & 1.347804 & 1.761717 & 0.765052 & 0.444241 & 0.668566 \\
\hline Edwar & 21.40947 & -0.87302 & 1.142588 & -0.76407 & 0.444823 & 0.669061 \\
\hline cterium QY30 & 72.81384 & -0.42748 & 0.559839 & -0.76357 & 0.445122 & 0.669061 \\
\hline & 64.68836 & 1.420713 & & & & 69061 \\
\hline Ente & 364.8547 & -0.37096 & 0.486115 & -0.76311 & 454 & 0.669204 \\
\hline Lact & 2.016285 & -1.7208 & 2.256102 & -0.76273 & 622 & 9261 \\
\hline Myco & 28.46186 & -0.62832 & 0.824461 & -0.7621 & 0.445999 & 0.669551 \\
\hline Myco & 29.41949 & -0.45738 & 0.600616 & -0.76152 & 0.446349 & 9799 \\
\hline Tess & 222.543 & -0.3347 & 0.440046 & -0.7606 & 893 & 0.67034 \\
\hline Coryr & 34999.38 & -0.58379 & 0.770011 & -0.75816 & 0.448357 & 0.672259 \\
\hline Lacto & 15.4149 & -0.79921 & 1.058082 & -0.75534 & 0.450045 & 0.67407 \\
\hline Stre & 74.20755 & -0.31903 & 0.422129 & -0.75576 & 0.449793 & 0.67407 \\
\hline Rothi & 6827.861 & -0.53101 & 0.703415 & -0.7549 & & 0.67407 \\
\hline Calyptogena okutanii thioautotrophic & 2.824137 & -1.25036 & 1.656119 & -0.755 & 0.450251 & 0.67407 \\
\hline Streptomyces pactum & 55.65874 & -0.38296 & 0.50751 & -0.75458 & 0.450502 & 0.674086 \\
\hline Klebs & 43.53047 & -0.43972 & 0.584148 & -0.75276 & 0.451593 & 0.67544 \\
\hline Actin & 7.786188 & -1.0516 & 1.3984 & -0.75201 & 048 & 0.675843 \\
\hline Exigu & 11.84849 & -1.21584 & 1.618433 & -0.75125 & 0.452505 & 0.676248 \\
\hline Clost & 18.82252 & 0.648944 & 0.867635 & 0.747946 & 0.454493 & 0.678122 \\
\hline Anak & 2.762526 & -1.30683 & 1.74542 & -0.74872 & 0.454026 & 0.678122 \\
\hline Lysil & 15.31178 & -0.88437 & 1.181848 & -0.74829 & & 0.678122 \\
\hline Actir & 77.72995 & -0.26113 & 0.349141 & -0.74793 & 0.454504 & 0.678122 \\
\hline Sr1-43 & 154.7247 & -0.37164 & 0.49751 & -0.74699 & 0.455069 & 0.678409 \\
\hline Sphi & 45.81993 & 0.798581 & 1.068688 & 0.747254 & 0.45491 & 0.678409 \\
\hline lamoeba sp. I & 1.393722 & -1.76707 & 2.36764 & -0.74634 & 0.455461 & 0.678475 \\
\hline Mycok & 33.68801 & -0.7711 & 1.033226 & -0.7463 & 0.455486 & 0.678475 \\
\hline Enterococcus faecium & 161.1993 & -0.45214 & 0.60826 & -0.74333 & 0.457284 & 0.680875 \\
\hline & 3.298603 & 1.209548 & 1.635701 & 0.739467 & 0.459623 & 0.683038 \\
\hline Gammapapillomavirus 22 & 1.053148 & -2.16527 & 2.924407 & & & 0.683038 \\
\hline Melioribacter roseus & 2.265137 & -1.31103 & 1.77156 & -0.74004 & 0.459275 & 0.683038 \\
\hline Polymorphum gilvum & 99.18902 & -0.37296 & 0.504425 & -0.73938 & 0.459676 & 0.683038 \\
\hline Shewanella violacea & 1.767353 & -2.04387 & 2.763613 & -0.73956 & 0.459564 & 0.683038 \\
\hline Desulfobacula toluolica & 2.161133 & -1.06516 & 1.442061 & -0.73863 & 0.460129 & 0.683432 \\
\hline
\end{tabular}




\begin{tabular}{|c|c|c|c|c|c|c|}
\hline Bacillus thermoamylovorans & 6.022102 & & 1.084862 & -0.73743 & 0.460862 & \\
\hline Pseudomonas alkylphenolica & 82.62542 & -0.39072 & 0.530898 & -0.73597 & 0.461752 & 0.685283 \\
\hline Lacinutrix sp. 5H-3-7-4 & 3.30361 & 0.792152 & 1.078156 & 0.734728 & 0.462505 & 0.685478 \\
\hline ropionibacterium virus PHL113M01 & 2.25839 & -1.06332 & 1.44588 & -0.73541 & 0.462087 & 0.685478 \\
\hline seudomonas entomophila & 9.95245 & -0.41235 & 0.561393 & -0.73451 & 0.462637 & 5478 \\
\hline ontibacter korlensis & 10.2289 & -0.67832 & 0.923398 & -0.73459 & 0.462587 & 0.685478 \\
\hline /ibrio azureus & 2.396003 & 1.765224 & 2.406406 & 0.733552 & 0.463222 & 0.686066 \\
\hline Tatlockia micdadei & 0.909736 & -1.95582 & 2.669197 & -0.73274 & 0.463718 & 0.686522 \\
\hline Gammapapillomavirus 19 & 1.82535 & 1.993399 & 2.722035 & 0.732319 & 0.463974 & 0.686621 \\
\hline Betapapillomavirus 1 & 407.8851 & -0.87292 & 1.193545 & -0.73137 & 0.464554 & 0.6872 \\
\hline Leuconostoc carnosum & 70.38419 & -0.79943 & 1.093748 & -0.73091 & 0.464835 & 0.687337 \\
\hline Capnocytophaga sp. ChDC OS43 & 91.13369 & -0.48451 & 0.663179 & -0.73059 & 0.465031 & 0.687347 \\
\hline Rhodococcus sp. B7740 & 232.2377 & -0.31333 & 0.429232 & -0.72998 & 0.4654 & 0.687612 \\
\hline s ovatus & 60.8985 & -0.54794 & 0.751149 & -0.72947 & 0.465712 & 0.687795 \\
\hline laga sp. oral taxon 323 & 57.22811 & -0.58755 & 0.807614 & -0.72751 & 66911 & 0.689006 \\
\hline Paeniba & 7.079615 & -0.93953 & 1.290977 & -0.72777 & 0.466757 & 0.689006 \\
\hline Corynek & 2747.963 & -0.21209 & 0.292042 & -0.72624 & 0.467692 & 0.689879 \\
\hline Brach & 304.0947 & -0.37016 & 0.51075 & -0.72473 & 0.468615 & 0.69096 \\
\hline Pseuc & 1385.37 & -0.34141 & 0.471605 & -0.72393 & 0.469107 & 0.691361 \\
\hline Bactero & 38.80631 & -0.6289 & 0.869042 & -0.72367 & 0.469267 & 0.691361 \\
\hline Pseuc & 56.06538 & -0.49721 & 0.688075 & -0.72261 & 46992 & 2044 \\
\hline Strep & 11.90219 & -0.65896 & 0.912719 & -0.72198 & 0.470308 & 0.692334 \\
\hline Leuc & 30.76021 & -0.82266 & 1.141753 & -0.72052 & 0.471205 & 3374 \\
\hline Acet & 1.005213 & -2.10341 & 2.924692 & -0.71919 & 0.472023 & 0.694297 \\
\hline Alterc & 2.028538 & -1.48697 & 2.069077 & -0.71866 & 0.472348 & 0.694495 \\
\hline Cron & 48.67261 & -0.53722 & 0.748251 & -0.71797 & 0.472778 & 1846 \\
\hline Halot & 3.269286 & -1.02979 & 1.435954 & -0.71714 & 0.473285 & 0.69531 \\
\hline Odor & 87.4004 & 0.621014 & 0.868025 & 0.715434 & 0.474341 & 0.695738 \\
\hline Mari & 13.4387 & -0.69048 & 0.964173 & -0.71613 & 0.473908 & 0.695738 \\
\hline Haem & 1347.882 & -0.47977 & 0.670413 & -0.71563 & 0.474219 & 0.695738 \\
\hline Actinom & 226.9808 & -0.2881 & 0.402496 & -0.71578 & 0.474129 & 0.695738 \\
\hline Lactob & 3.54135 & -1.08857 & 1.523829 & -0.71437 & 0.475 & 0.696425 \\
\hline olaceoruber & 49.39765 & -0.3999 & 0.560194 & -0.71387 & 0.47531 & 0.696598 \\
\hline Strep & 9.604099 & -1.29942 & 1.824865 & -0.71206 & 0.476425 & 0.69721 \\
\hline Paeniclc & 27.67157 & 0.481705 & 0.676597 & 0.711953 & 0.476494 & 0.69721 \\
\hline Kytoc & 515.1872 & -0.3243 & 0.455379 & -0.71216 & 0.476368 & 0.69721 \\
\hline Strep & 9.575587 & -1.17304 & 1.647209 & -0.71214 & 0.476381 & 0.69721 \\
\hline Brevi & 284.6662 & -0.29927 & 0.420544 & -0.71163 & 0.476693 & 0.69722 \\
\hline Thern & 3.001126 & 1.678122 & 2.361721 & 0.710551 & 0.477363 & 0.69792 \\
\hline doalcaligenes & 258.7223 & -0.49557 & 0.697764 & -0.71023 & 0.477563 & 0.697931 \\
\hline Polaribacter s| & 11.94907 & 0.916837 & 1.291759 & 0.709759 & 0.477854 & 0.697931 \\
\hline evibacter olleyae & 4.774358 & 1.338237 & 1.886697 & 0.709301 & 0.478137 & 0.697931 \\
\hline Mycobacterium marinur & 76.2301 & 0.412847 & 0.582005 & 0.709354 & 0.478105 & 0.697931 \\
\hline Sediminicola sp. YIK13 & 4.68777 & -0.95807 & 1.351506 & -0.70889 & 0.478393 & 0.698024 \\
\hline & 48.60402 & -0.27745 & 0.391742 & -0.70825 & 0.478788 & 0.698029 \\
\hline Nitrosomonas europaea & 5.417099 & -0.92682 & 1.309147 & -0.70796 & 0.478972 & 0.698029 \\
\hline Simonsiella muelleri & 26.98963 & -0.78951 & 1.114835 & -0.70819 & 0.478828 & 0.698029 \\
\hline Arthrobacter sp. YN & 112.9266 & -0.25439 & 0.359838 & -0.70694 & 0.479601 & 0.698667 \\
\hline Mycobacterium sp. YC-RL4 & 202.2977 & -0.37081 & 0.524967 & -0.70636 & 0.479966 & 0.698918 \\
\hline Clostridium estertheticum & 39.79746 & 0.581722 & 0.824203 & 0.7058 & 0.480313 & 0.699016 \\
\hline
\end{tabular}




\begin{tabular}{|c|c|c|c|c|c|c|}
\hline & & & & & & \\
\hline Rickettsiales endosymbiont of St & 2.720174 & 1.560375 & 2.211658 & 0.705523 & 0.480485 & 0.699016 \\
\hline Virgibacillus sp. Bac330 & 2.02898 & -1.4687 & 2.082381 & -0.7053 & 0.480624 & 699016 \\
\hline Labrenzia aggregata & 14.38336 & -0.83197 & 1.181693 & -0.70405 & 0.481404 & 699332 \\
\hline Neoasaia chiangmaiensis & 27.68192 & -0.41135 & 0.584161 & -0.70418 & 0.481323 & 0.699332 \\
\hline Jiangella alkaliphila & 175.4567 & -0.33594 & 0.477661 & -0.70331 & 0.481862 & 0.699718 \\
\hline Enterococcus sp. CR-Ec1 & 0.973985 & -2.05371 & 2.924929 & -0.70214 & 0.482591 & 0.700498 \\
\hline Chryseobacterium camelliae & 17.55884 & -0.55004 & 0.784132 & -0.70147 & 3012 & 0.700829 \\
\hline Enterobacter roggenkampii & 19.81114 & 0.829007 & 1.186729 & 0.698564 & 0.484824 & 0.702643 \\
\hline Pseudomonas sp. Lz4W & 19.37159 & -0.84592 & 1.210978 & -0.69854 & 0.484839 & 0.702643 \\
\hline ankenforstense & 1530.471 & -0.25396 & 0.363553 & -0.69854 & 0.484841 & 0.702643 \\
\hline Streptc & 164.3046 & 0.335002 & 0.479841 & 0.698152 & 0.485082 & 0.702712 \\
\hline Meth & 4.232578 & -0.93577 & 1.342149 & -0.69722 & 85668 & 0.703281 \\
\hline Paraphotobacterium marinum & 8.662997 & 0.747905 & 1.073331 & 6808 & 0.485923 & 0.703371 \\
\hline Acinet & 92.7 & -0.51088 & 0.734375 & 567 & 637 & 3852 \\
\hline um umeaense & 54.05362 & -0.46886 & 0.673978 & -0.69566 & 0.486642 & 0.703852 \\
\hline dii complex sp. CFNI & 0.958393 & -2.03011 & 2.925045 & -0.69404 & & 5036 \\
\hline Synec & 6.970613 & -1.01293 & 1.462552 & -0.69258 & 0.488576 & 0.705252 \\
\hline Meth & 8.594958 & -0.77024 & 1.111954 & 9269 & 0.488503 & 0.705252 \\
\hline Lact & 2131.945 & -0.44846 & 0.647532 & 9257 & 38579 & 5252 \\
\hline Pseu & 49.64418 & 0.473997 & 0.683973 & 3005 & 8306 & 0.705252 \\
\hline Tatu & 5.216202 & -1.11804 & 1.616476 & 9165 & 155 & 5804 \\
\hline Acin & 136.3566 & 0.440392 & 0.637115 & 0.691229 & 0.489422 & 0.705903 \\
\hline Psel & 0.95 & -2.02102 & 2.92509 & 093 & 612 & 5903 \\
\hline Hym & 46.83717 & -0.35068 & 0.508457 & -0.68969 & 0.490392 & 0.706747 \\
\hline Halis & 25.11901 & 0.347739 & 0.505537 & 3786 & 541 & 7793 \\
\hline Goro & 32.92568 & -0.45473 & 0.661167 & 8776 & 602 & 0793 \\
\hline Osci & 4608 & 0.641752 & 0.934177 & 0.686971 & 2101 & 8369 \\
\hline lamoebae & 2.076727 & -1.55862 & 2.271342 & -0.68621 & 0.49258 & 0.708777 \\
\hline Sphir & 104.4312 & -0.34816 & 0.507927 & -0.68546 & 0.493052 & 0.709177 \\
\hline a sp. JS16-p6b & 265.7111 & -0.29723 & 0.433827 & -0.68514 & 0.493256 & 0.709189 \\
\hline Streptococcus merionis & 13.16407 & -0.515 & 0.752399 & -0.68447 & 676 & 0.709234 \\
\hline Pseudomonas sp. LPH1 & 53.67574 & -0.45432 & & & 3508 & 9234 \\
\hline Rho & 145.5396 & 0.706475 & 1.033304 & 705 & 161 & 0965 \\
\hline Sele & 11.39011 & -0.57881 & 0.848325 & -0.68229 & 5054 & 0.710651 \\
\hline FDAARGOS_380 & 153.1698 & 0.506631 & 0.743041 & 1834 & 344 & 0787 \\
\hline Aerc & 56.47565 & -0.40583 & 936 & 099 & 877 & 0.711272 \\
\hline Aqui & 13.77941 & 0.670383 & 0.985908 & 0.679965 & 0.496527 & 0.711865 \\
\hline Vibr & 25.79309 & -0.59551 & 0.876513 & -0.67941 & 877 & 0.711865 \\
\hline Citrobacter rodentium & 11.06608 & -0.77624 & 1.142008 & -0.67971 & 0.496685 & 0.711865 \\
\hline Pelos & 9.576209 & 0.850184 & 1.253047 & 0.678493 & 0.497459 & 0.712418 \\
\hline Candidatus Sodalis pi & 2.512673 & -1.62526 & 2.40012 & -0.67716 & 0.498306 & 0.71335 \\
\hline HYN0043 & 36.28659 & 0.449408 & 0.664609 & 0.676199 & 0.498914 & 0.71394 \\
\hline $1(2017)$ & 36.84899 & -0.46581 & 0.690076 & -0.67501 & 0.49967 & 0.714261 \\
\hline Aurantimicrobium sp. MWH-Uga1 & 14.73102 & -0.65606 & 0.972054 & -0.67492 & 0.499727 & 0.714261 \\
\hline Pantoea v & 206.2773 & -0.4424 & 0.654968 & -0.67545 & 0.49939 & 0.714261 \\
\hline Cellulopha & 7.554012 & 0.843348 & 1.25088 & 0.674204 & 0.500182 & 0.71463 \\
\hline & 1.533124 & 1.700209 & 2.523082 & 0.673862 & 0.500399 & 0.714659 \\
\hline Ruminococcus bicirculans & 102.2923 & -0.5934 & 0.88208 & -0.67272 & 0.501123 & 0.715137 \\
\hline Tenacibaculum todarodis & 7.073986 & 0.992583 & 1.475481 & 0.672718 & 0.501127 & 0.715137 \\
\hline
\end{tabular}


Streptomyces hundungensis

Lactobacillus amylovorus

Bacillus sp. 1NLA3E

Corynebacterium lactis

Chlorobaculum tepidum

Planococcus sp. MB-3u-03

Frischella perrara

Streptomyces formicae

Pseudodesulfovibrio profundus

Sphingomonas melonis

Selenomonas sp. oral taxon 136

Actinomyces pacaensis

Paenibacillus bovis

Wolinella succinogenes

Mycobacteroides abscessus

Oscillatoriales cyanobacterium JSC-1:

Mahella australiensis

Staphylococcus simulans

Acetobacter ghanensis

[Eubacterium] rectale

Planktothrix agardhii

Pseudodesulfovibrio piezophilus

Gemella sp. oral taxon 928

Lactobacillus alimentarius

Novosphingobium sp. THN1

Curtobacterium pusillum

Paenalcaligenes hominis

Legionella longbeachae

Cryptobacterium curtum

Nocardioides dokdonensis

Candidatus Planktophila versatilis

Saccharospirillum mangrovi

Faecalibaculum rodentium

Pseudomonas sp. CMR5c

Acidaminococcus intestini

Pseudomonas reinekei

Salinicoccus halodurans

Cyanothece sp. PCC 7424

Glutamicibacter arilaitensis

Shewanella frigidimarina

Staphylococcus hyicus

Staphylococcus sp. SDB 2975

Edwardsiella ictaluri

[Bacillus] selenitireducens

Nitrosomonas communis

Idiomarinaceae bacterium HL-53

Stenotrophomonas rhizophila

Terribacillus goriensis

Capnocytophaga sp. oral taxon 864

Xanthomonas hortorum

\begin{tabular}{|c|c|c|c|c|c|}
\hline & & & & & \\
\hline 15.13812 & -0.63252 & 0.942878 & -0.67084 & 0.502322 & 0.716281 \\
\hline 8.21128 & -0.71165 & 1.062057 & -0.67007 & 0.502813 & 0.716701 \\
\hline 730.9379 & -0.26314 & 0.39293 & -0.6697 & 0.50305 & 0.716757 \\
\hline 3.40651 & -0.45975 & 0.687812 & -0.66843 & 503861 & 0.717632 \\
\hline 15.55965 & -0.76893 & 1.151305 & -0.66788 & 0.50421 & 0.717849 \\
\hline 474661 & -0.79273 & 1.187648 & -0.66748 & 0.504467 & 0.717933 \\
\hline 4.90176 & -0.23213 & 0.347983 & -0.66707 & 0.504725 & 0.71802 \\
\hline 7.20698 & -0.54838 & 0.823159 & -0.6662 & 0.505286 & 0.718537 \\
\hline 408.7946 & -0.39629 & 0.596871 & -0.66394 & 0.50673 & 0.718904 \\
\hline 65.04157 & -0.43869 & 0.660451 & -0.66423 & 0.506545 & 0.718904 \\
\hline 162.1109 & -0.32593 & 0.489823 & -0.66541 & 0.505791 & 0.718904 \\
\hline 6.914484 & -0.79749 & 1.200983 & -0.6 & 0.506669 & 0.718904 \\
\hline 1.994592 & -1.50532 & 2.26507 & -0.66458 & 0.50632 & 0.718904 \\
\hline 228.7608 & -0.25551 & 0.384421 & -0.6 & 0.506272 & 718904 \\
\hline 11.31089 & -0.60742 & 0.915743 & -0.66331 & 0.507133 & 0.718916 \\
\hline 2.917087 & -1.06929 & 1.611992 & -0.66334 & 0.507116 & 0.718916 \\
\hline 560.8549 & -0.33944 & 0.512763 & -0.66197 & 0.507988 & 0.719508 \\
\hline 3.49819 & -0.9968 & 1.50635 & -0.66173 & 0.508144 & 0.719508 \\
\hline 401.5116 & 0.497095 & 0.750552 & 0.662306 & 0.507775 & 0.719508 \\
\hline 2.646656 & -1.06403 & 1.611936 & -0.6601 & 0.509192 & 0.720432 \\
\hline 1.53104 & -1.41508 & 2.143211 & -0.66026 & 0.509087 & 0.720432 \\
\hline 3.469306 & -1.25188 & 1.897779 & -0.65966 & 0.509474 & 0.72055 \\
\hline 2.591518 & -1.07438 & 1.631416 & -0.6 & 0.510182 & 0.720643 \\
\hline 74.99233 & -0.31579 & 0.479371 & -0.65876 & 0.510049 & 0.720643 \\
\hline 302.1283 & -0.34126 & 0.518878 & & & \\
\hline 4.013326 & -0.88852 & 1.349079 & -0.65862 & 0.510143 & 0.720643 \\
\hline 1.133498 & -1.60391 & 2.43844 & -0.65776 & 0.510692 & 0.720643 \\
\hline 16.09983 & -0.62349 & 0.947713 & -0.65789 & 0.510612 & 0.720643 \\
\hline 250.1406 & -0.27368 & 0.416304 & 574 & 0.510926 & 0.720643 \\
\hline 18.1091 & -0.47845 & 0.728548 & -0.65671 & 0.511364 & 0.720703 \\
\hline 23.37612 & -0.46621 & 0.709589 & -0.65701 & 0.511174 & 0.720703 \\
\hline 6.100522 & -0.92663 & 1.41 & -0.6 & 0.511705 & 0.720784 \\
\hline 36.26163 & 0.422278 & 0.643707 & 0.656009 & 0.511818 & 0.720784 \\
\hline 6.750365 & 0.90901 & 1.386436 & 0.655645 & 0.512053 & 0.720835 \\
\hline 26.5616 & 0.473407 & 0.72551 & 0.652516 & 0.514068 & 0.722834 \\
\hline 7.121576 & -0.91152 & 1.396684 & -0.65263 & 0.513995 & 0.722834 \\
\hline 9.215098 & 0.860053 & 1.317026 & 0.653027 & 0.513739 & 0.722834 \\
\hline 136.1201 & -0.25824 & 0.397876 & -0.64906 & 0.516301 & 0.724404 \\
\hline 4.436539 & 0.970882 & 1.493466 & 0.650086 & 0.515637 & 0.724404 \\
\hline 21.08586 & 0.57047 & 0.879328 & 0.648757 & 0.516495 & 0.724404 \\
\hline 44.82214 & -0.59031 & 0.910276 & -0.64849 & 0.516665 & 0.724404 \\
\hline 4.202629 & 1.211287 & 1.866797 & 0.648858 & 0.51643 & 0.724404 \\
\hline 1.644212 & -1.37642 & 2.116143 & -0.65044 & 0.515409 & 0.724404 \\
\hline 2.468186 & -0.99135 & 1.529097 & -0.64832 & 0.516777 & 0.724404 \\
\hline 2.190246 & -1.1668 & 1.798008 & -0.64894 & 0.516377 & 0.724404 \\
\hline 256.3934 & -0.3262 & 0.506815 & -0.64362 & 0.519823 & 0.72821 \\
\hline 5.756325 & -0.81728 & 1.270031 & -0.64351 & 0.519893 & 0.72821 \\
\hline 45.33772 & -0.43776 & 0.681231 & -0.64261 & 0.52048 & 0.728752 \\
\hline 114.8468 & -0.52816 & 0.823545 & -0.64133 & 0.521311 & 0.728794 \\
\hline
\end{tabular}


Aerococcus viridans

Treponema sp. OMZ 838

Lachnoclostridium phocaeense

Kocuria turfanensis

Paracoccus aminophilus

Halanaerobium praevalens

Vibrio campbellii

Staphylococcus muscae

Alkaliphilus oremlandii

Pseudomonas sp. R4-34-07

Exiguobacterium sp. N4-1P

Streptococcus pantholopis

Denitrovibrio acetiphilus

Campylobacter jejuni

Legionella anisa

Leptotrichia sp. oral taxon 212

Glaesserella sp. 15-184

Sphingomonas sp. FARSPH

Campylobacter concisus

Pseudonocardia sp. EC080625-04

Syntrophothermus lipocalidus

Hymenobacter sp. PAMC 26628

Gallaecimonas sp. HK-28

Cyanothece sp. PCC 7822

Pedobacter heparinus

Commensalibacter sp. AMU001

Treponema pedis

Arthrobacter sp. Rue61a

Bacteroides coprosuis

Weissella viridescens

Candidatus Thioglobus autotrophicus

Propionibacterium phage PHL067M1

Lactococcus garvieae

Actinobacteria bacterium IMCC2607'

Dehalogenimonas lykanthroporepelle

Ndongobacter massiliensis

Nocardia cyriacigeorgica

Methanosarcina lacustris

Desulfosporosinus meridiei

Winogradskyella sp. PC-19

Staphylococcus schleiferi

Thalassotalea crassostreae

Hydrogenophilus thermoluteolus

Streptomyces sp. PAMC 26508

Mycobacterium canettii

Paenibacillus larvae

Fastidiosipila sanguinis

Mobiluncus curtisii

Shinella sp. HZN7

Nocardia sp. Y48

\begin{tabular}{|c|c|c|c|c|c|}
\hline & & & & & \\
\hline 2.11072 & -0.5 & 0.933971 & -0.6414 & 0.521263 & 0.72 \\
\hline 34.6815 & -0.67228 & 1.046923 & -0.64215 & 0.520778 & 10 \\
\hline 43.763 & -0.28588 & 0.44673 & -0.63995 & 206 & \\
\hline & -0.3 & 0.518913 & -0.63906 & & \\
\hline 87103 & 0.60 & 0.944639 & 0.638557 & 11 & \\
\hline 49169 & 0.81 & 1.284455 & 0.637153 & & \\
\hline & & 0.740845 & 3564 & & \\
\hline & 0.705 & 1.110301 & 0.635207 & .525293 & \\
\hline 0778 & 1.85 & & 0.634503 & & \\
\hline & & & & & \\
\hline & & & & & \\
\hline 763 & -1.2 & 1.9 & & & \\
\hline & 0.41 & & 0.6330 & & \\
\hline & -0.7 & & 3258 & & \\
\hline & & & & & \\
\hline 8459 & -0.6 & 0.985976 & -0.63088 & 0.528117 & \\
\hline & & & -0.6 & & \\
\hline & -0.3 & 0.560998 & -0.63127 & & \\
\hline 6939 & & 2.894 & 0.629281 & & \\
\hline & & & -0.62962 & & \\
\hline & -0.3 & 0.5 & & & \\
\hline & & & & & \\
\hline 10.01121 & 0.888 & 1.419302 & 0.626321 & 0.531105 & \\
\hline & $-0.4 C$ & 0.6511 & -0.62639 & & \\
\hline & 1.3 & & 0.62 & & \\
\hline 4947 & -0.9 & & -0.6 & & \\
\hline & -0.56 & & & & \\
\hline & & & & & \\
\hline & -1 & & -0.6 & & \\
\hline & & & -0.62 & & \\
\hline & & & & & \\
\hline & -0 . & & & & \\
\hline & & & & & \\
\hline & -1.2 & 1.973917 & -0.62316 & & \\
\hline & -0.5 & & & & \\
\hline & -0.2 & & -0.62253 & & \\
\hline & & & & & \\
\hline 32239 & -1.08445 & 1.750631 & -0.61946 & & \\
\hline & & & & & \\
\hline & 0.320722 & & & & \\
\hline & & & & & \\
\hline 5723 & 0.413462 & & 0.6188 & & \\
\hline 3181 & -0.84668 & 1.368158 & -0.61885 & & 0.737 \\
\hline & 0.314637 & 0.509149 & 0.617968 & & \\
\hline 3.552245 & & 1.737017 & -0.61514 & & \\
\hline & & 0.602575 & 0.615208 & 0.538418 & \\
\hline & -0.41636 & 0.675265 & -0.61659 & & \\
\hline & & & -0.61443 & 0.538928 & \\
\hline 161.32 & -0.25273 & 0.411257 & -0.61453 & 0.538864 & 0.7389 \\
\hline
\end{tabular}




\begin{tabular}{|c|c|c|c|c|c|c|}
\hline Desulfurella acetivorans & 3.861191 & 1.082515 & 1.758203 & 0.615694 & 0.538097 & 3945 \\
\hline Agromyces flavus & 111.1853 & -0.261 & 0.424586 & -0.61471 & 0.538748 & 0.738945 \\
\hline Bacillus subtilis & 249.3574 & 0.413314 & 0.672301 & 0.614776 & 0.538703 & 738945 \\
\hline Deinococcus swuensis & 62.83771 & -0.2726 & 0.443964 & -0.61402 & 0.539202 & 0.739042 \\
\hline Plantactinospora sp. KBS50 & 88.71509 & -0.30155 & 0.491803 & -0.61314 & 0.539782 & 0.739558 \\
\hline Treponema denticola & 37.33776 & -0.49527 & 0.810664 & -0.61094 & 0.541238 & 0.741274 \\
\hline Gordonia bronchialis & 743.0208 & -0.34922 & 0.57342 & -0.60902 & 0.542511 & 0.7419 \\
\hline Acinetobacter sp. NCu2D-2 & 21.0708 & -0.48473 & 0.795785 & -0.60912 & 0.542446 & 0.7419 \\
\hline Thiomicrospira cyclica & 2.167078 & -1.01045 & 1.65774 & -0.60954 & 0.542168 & 0.7419 \\
\hline erium taklimakanense & 82.75904 & -0.39884 & 0.654581 & -0.60931 & 0.54232 & 0.7419 \\
\hline Christe & 45.01077 & -0.53012 & 0.871568 & -0.60824 & 0.543027 & 0.742328 \\
\hline Mogiba & 126.7431 & -0.46481 & 0.764606 & & 0.543248 & 235 \\
\hline Janthin & 21.80201 & -0.51093 & 0.841476 & & 0.543732 & 0.742734 \\
\hline Meth & 0.806388 & -1.77535 & 2.92642 & -0.60666 & 0.544075 & 0.742923 \\
\hline Enter & 13.7944 & 0.538862 & 0.889764 & 0.605624 & 1765 & 0.743585 \\
\hline Paen & 5.637258 & -0.66871 & 1.106723 & -0.60423 & 0.545691 & 0.744568 \\
\hline Rhizc & 6.439879 & -1.09922 & 1.82012 & -0.60392 & 0.545894 & 4568 \\
\hline erum thioau & 1.892792 & -1.38258 & 2.290532 & 361 & 5105 & 4577 \\
\hline Cohaesi & 14.46337 & -0.38127 & 0.632415 & -0.60287 & 0.546593 & 0.744963 \\
\hline Erwinia & 2.224806 & -1.45804 & 2.42351 & -0.60163 & 0.547424 & 5816 \\
\hline Barto & 1.588618 & -1.68799 & 2.80828 & -0.60108 & 0.547789 & 0.746034 \\
\hline Strep & 64.52065 & -0.37571 & 0.626573 & 9962 & 8757 & 17073 \\
\hline Meta & 0.432782 & 1.753901 & 2.93782 & 0.59 & 0502 & 48842 \\
\hline Carbc & 1.907581 & -1.14997 & 1.927 & -0 . & 673 & 3842 \\
\hline Sulfu & 2.506593 & 1.227736 & 2.057152 & 6813 & 0632 & 18842 \\
\hline Klebs & 1.530853 & -1.67855 & 2.820514 & 512 & 1762 & 9762 \\
\hline Salm & 4.271365 & -0.96256 & 1.616854 & 533 & 1624 & 9762 \\
\hline Meth & 1.504174 & -1.17276 & 1.975481 & 9366 & 55274 & 5053 \\
\hline Vibri & 32.00065 & -0.5929 & 0.99856 & -0.59376 & 0.552674 & 5053 \\
\hline Vibrio $f$ & 16.97794 & -0.46882 & 0.790442 & -0.59311 & 0.553106 & 0.750747 \\
\hline crobium sp. AMA3305 & 204.8154 & -0.22552 & 0.382817 & -0.58911 & 0.555787 & 0.754105 \\
\hline Bacteroid & 450.4151 & -0.55342 & & -0.58847 & 6219 & 75441 \\
\hline & & & 177 & & 5721 & \\
\hline Pleur & 1.578 & -1.25022 & & & & 0.754887 \\
\hline Synec & 10.10851 & -0.62579 & 1.065684 & -0.58722 & 7059 & 4887 \\
\hline & 18.699 & -0.39929 & 0.680965 & -0.58636 & 0.557633 & 0.755201 \\
\hline Ent & 31.4 & -0.41959 & 0.718432 & 8404 & 9193 & 0.757033 \\
\hline Psychrobacter cryohalolentis & 56.6843 & -0.65828 & 1.127737 & -0.58372 & 0.55941 & 0.757044 \\
\hline Dialister pneumosintes & 28.22709 & -0.54853 & 0.940854 & -0.58301 & 0.559885 & 0.757221 \\
\hline Mycoplasma c & 2.628062 & 1.295062 & 2.221734 & 0.582906 & 0.559957 & 0.757221 \\
\hline Erwin & 118.9145 & 0.452463 & 0.778983 & 0.580837 & 0.56135 & 0.75826 \\
\hline & 12.59256 & -0.37938 & 0.652764 & -0.5812 & 0.561107 & 0.75826 \\
\hline Acine & 85.99955 & 0.355796 & 0.612253 & 0.581125 & 0.561156 & 0.75826 \\
\hline Acinet & 50.65853 & -0.49012 & 0.845222 & -0.57987 & 0.562002 & 0.758578 \\
\hline Chryseobacterium sp. G0162 & 7.898377 & -0.73526 & 1.267761 & -0.57997 & 0.561934 & 0.758578 \\
\hline & 4.794611 & 0.948127 & 1.636873 & & 0.562434 & 0.758803 \\
\hline Tenacibaculum sp. DSM 106434 & 9.337067 & 0.693604 & 1.197924 & 0.579005 & 0.562586 & 0.758803 \\
\hline & 11.30636 & -0.62832 & 1.087391 & -0.57783 & 0.56338 & 0.759593 \\
\hline Enterobacteriaceae bacterium & 7.034816 & -0.70408 & 1.220518 & -0.57687 & 0.564028 & 0.759903 \\
\hline
\end{tabular}




\begin{tabular}{|c|c|c|c|c|c|c|}
\hline Kyrpidia spormannii & & & & & & \\
\hline Sulfurihydrogenibium azorense & 1.775465 & -1.33114 & 2.309062 & -0.57649 & 0.564287 & \\
\hline Pontibacter actiniarum & 2.86696 & -0.38982 & 0.678589 & -0.57445 & 0.565664 & 0.760203 \\
\hline Saccharophagus degradans & 4.926105 & -0.82258 & 1.4307 & -0.57495 & 0.565325 & 0.760203 \\
\hline Acidiphilium cryptum & 5.75437 & -0.56877 & 0.989489 & -0.57481 & & 0203 \\
\hline Raoultella ornithinolytica & 21.0502 & -0.35453 & 0.616498 & -0.57507 & 0.565241 & 0.760203 \\
\hline Propionibacterium phage PHL055NOC & 21.73548 & 0.842576 & 1.466939 & 0.574377 & 0.565713 & 0.760203 \\
\hline Corynebacterium aurimucosum & 789.801 & -0.18045 & 0.313637 & -0.57534 & 0.565064 & 0.760203 \\
\hline [Haemophilus] ducreyi & 22.67466 & -0.37069 & 0.648585 & -0.57154 & 0.567635 & 0.762222 \\
\hline Flavobacteriaceae bacteric & 8.856605 & 0.654012 & 1.144176 & 0.5716 & 0.567593 & 0.762222 \\
\hline Virgibacillus necropolis & 9.128907 & -0.72373 & 1.268148 & -0.5707 & 0.568206 & 0.762708 \\
\hline Sulfuro & 3.115405 & 1.010718 & 1.773707 & 0.569833 & 0.568791 & 0.763211 \\
\hline of Trimyema con & 6.105074 & -0.68292 & 1.20723 & -0.56569 & 0.571603 & 0.766404 \\
\hline Pseuc & 29.75061 & -0.70131 & 1.239707 & -0.5657 & 0.57 & 0.766404 \\
\hline Psych & 55.80148 & -0.3421 & 0.605067 & -0.5654 & 0.57 & 0.766404 \\
\hline Histo & 10.50198 & -0.46834 & 0.831494 & -0.56325 & 0.573262 & 0.767012 \\
\hline secor & 0.732974 & -1.64923 & 2.927081 & -0.56344 & 0.573 & 0.767012 \\
\hline Ichth & 2.152376 & 1.277155 & 2.262921 & 0.564 & 0.572 & 0.767012 \\
\hline Anta & 26.04985 & -0.36975 & 0.656893 & -0.56 & 0.5 & 0.767012 \\
\hline Myco & 4.079462 & -1.29034 & 2.291368 & -0.56313 & 0.573347 & 0.767012 \\
\hline Obes & 4.3028 & 0.862596 & 1.530695 & 0.563532 & 3073 & 0.767012 \\
\hline Syne & 10.54738 & -0.77497 & 1.383995 & -0.55995 & 0.57 & 0.769113 \\
\hline Clost & 9.843327 & 0.524535 & 0.936249 & 0.560252 & 308 & 113 \\
\hline Chlor & 8.335478 & -0.59282 & 1.060366 & -0.55 & $0.57 €$ & 636 \\
\hline Strep & 9.859615 & -0.77081 & 1.381464 & -0.55796 & 0.57 & 0.77 \\
\hline Wei & 1.005312 & -1.53212 & 2.746256 & 579 & 0.57 & 138 \\
\hline Virgi & 3.862828 & -0.69518 & 1.248144 & -0.5 & 0.57 & 0.77 \\
\hline Gram & 2.127829 & -0.99073 & 1.778517 & -0.5 & 0.57 & 0.77 \\
\hline Spiro & 38.39141 & -0.30269 & 0.544495 & -0.55591 & 269 & 0.77 \\
\hline Pseuc & 209.5114 & -0.24104 & 0.433679 & -0.55579 & 0.578352 & 0.770642 \\
\hline Pseud & 107.7111 & -0.33921 & 0.610315 & -0.5558 & 0.578348 & 0.770642 \\
\hline Haloa & 3.081985 & -0.9184 & 1.65349 & -0.55 & 0.5786 & 0.77069 \\
\hline [Clos & 48.36464 & -0.51878 & 0.936397 & -0.5 & 565 & 0.771198 \\
\hline Lons & 5.747201 & -0.75475 & 1.363268 & -0.5 & 829 & 198 \\
\hline Carn & 84.2108 & -0.37302 & 0.673451 & -0.5539 & 0.579648 & 0.771198 \\
\hline Lept & 1.987933 & 1.249949 & 2.254653 & 0.554386 & 0.579315 & 0.771198 \\
\hline Butyr & 13.17293 & 0.570063 & 1.033552 & 0.551557 & 0.581252 & 0.772244 \\
\hline Selen & 48.62629 & -0.47282 & 0.856942 & -0.55 & 0.581 & 0.77 \\
\hline & & -0.67459 & 1.221914 & -0.55207 & 0.580899 & 0.772244 \\
\hline & 16.32752 & -0.44594 & 0.810009 & -0.55054 & 952 & 0.772294 \\
\hline Beta & 12.65821 & -0.94124 & 1.712455 & -0.54965 & 0.582563 & 0.772294 \\
\hline Plano & 2.931789 & -1.12237 & 2.040672 & -0.55 & 0.582318 & 0.772294 \\
\hline Olsenella & 200.6657 & 0.389626 & 0.707856 & 0.550432 & 0.582023 & 0.772294 \\
\hline Sphingobium indicum & 29.34344 & -0.62825 & 1.142439 & -0.54992 & 0.582372 & 0.772294 \\
\hline & 2.16908 & -0.56794 & 1.032066 & -0.5503 & 0.582114 & 0.772294 \\
\hline Chryseobacterium sp. T16E-39 & 16.98599 & 0.414887 & 0.757274 & 0.547869 & 0.583782 & 0.773433 \\
\hline Flavobacterium a & 17.03191 & -0.32659 & 0.596212 & -0.54777 & 0.583847 & 0.773433 \\
\hline Bifidobacterium indicum & 1.257854 & -1.30622 & 2.39077 & -0.54636 & 0.584818 & 0.774437 \\
\hline Stenotrophomonas maltophilia & 1974.673 & -0.29713 & 0.54423 & -0.54596 & 0.585097 & 0.774524 \\
\hline Citrobacter farmeri & 8.673483 & -0.66644 & 1.221912 & -0.54541 & 0.58547 & 0.774737 \\
\hline
\end{tabular}


Thermoanaerobacter wiegelii

Acidipropionibacterium acidipropioni Lactococcus raffinolactis

Calothrix brevissima

Ochrobactrum anthropi

Rhodococcus opacus

Flavobacterium sp. MEBiC07310

Dermacoccus nishinomiyaensis

Prosthecochloris aestuarii

Dermatophilus congolensis

Kocuria sp. BT304

Spiroplasma taiwanense

Propionibacterium virus PHL199M00

Natronomonas pharaonis

Aeromonas sp. ASNIH7

Salmonella enterica

Acinetobacter venetianus

Stenotrophomonas sp. WZN-1

Micrococcus luteus

Clostridiales bacterium 70B-A

Paenibacillus sp. FSL P4-0081

Thermoplasmatales archaeon BRNA1

Bacillus lentus

Rhodococcus jostii

Acholeplasma laidlawii

Rothia mucilaginosa

Polaribacter sp. Hel1_33_78

Bacillus sp. FJAT-22090

Vibrio sp. 2521-89

Thioploca ingrica

Providencia stuartii

Brucella sp. 141012304

Desulfovibrio salexigens

Serratia sp. MYb239

Corynebacterium sp. ATCC 6931

Flavivirga eckloniae

Azospirillum sp. M2T2B2

Methylobacterium brachiatum

Pseudomonas sp. LG1D9

Halanaerobium hydrogeniformans

[Clostridium] sphenoides

Kangiella sediminilitoris

Candidatus Hoaglandella endobia

Butyrivibrio hungatei

Acholeplasma oculi

[Clostridium] saccharolyticum

Fictibacillus arsenicus

Dokdonia sp. PRO95

Staphylococcus warneri

Aerococcus sanguinicola

3.69994

1.37573

$-0.2222$

2.525856

0.544659

0.585988

0.774989

206.0689

$-0.54056$

0.408075

$-0.54452$

0.586087

0.774989

76.88563

14.23004

0.872043

0.993762

$-0.54395$

0.586473

0.775217

136.6243

$-0.3747$

0.543077

0.587077

0.775735

303.7818

$-0.21134$

0.69045

$-0.54269$

0.587344

0.775806

6.725743

$-0.77783$

0.390436

$-0.5413$

0.588301

0.776788

3971.582

0.351185

1.443576

$-0.53882$

0.59001

0.778479

1.347581

$-1.28185$

0.65164

0.538925

0.589939

0.778479

64.05391

$-0.27648$

2.382004

$-0.53814$

0.590482

0.77882

1517.833

$-0.31803$

0.515148

$-0.53669$

0.591479

0.779852

1.233981

1.541584

0.593765

$-0.53562$

0.592222

0.779984

4.565147

6.011339

$-1.00589$

2.877578

0.535723

0.59215

0.779984

$-0.5361$

0.591891

0.779984

2.983916

1.290018

$-0.53395$

0.593373

0.781217

301.1203

$-1.3633$

2.561185

$-0.53229$

0.594522

0.782447

272.6435

0.199955

0.376751

0.530734

0.595603

0.783206

30.44653

52575.43

0.586539

$-0.52991$

0.596172

0.783206

$-0.58853$

1.109227

$-0.53058$

0.595713

0.783206

7.896172

310

$-0.52992$

0.596168

0.783206

16.89855

$-0.68436$

1.29146

$-0.52991$

0.596175

0.783206

$\begin{array}{ll}-0.52867 & 0.597034\end{array}$

0.784052

$-0.5282$

0.597364

0.784202

10.95796

2.927634

$-0.52646$

0.598569

0.784875

$-0.526$

0.598891

0.784875

1.52212

0.457221

$-0.52536$

0.599336

0.784875

3759.433

$-0.30891$

0.587276

$-0.52601$

0.598885

0.784875

6.309228

0.728533

1.386934

0.525283

0.599386

0.784875

7.611616

0.527747

1.004294

0.52549

0.599242

0.784875

2.173273

$-1.40421$

2.672541

$-0.52542$

0.599291

0.784875

2.569972

0.992531

1.892115

0.524562

0.599888

0.785249

9.24547

$-0.59409$

1.133506

$-0.52412$

0.600198

0.785373

1.221996

1.525621

2.919809

0.522507

0.601317

0.786338

4.580803

$-0.65354$

1.250959

$-0.52243$

0.601368

0.786338

0.642373

1.529756

2.931499

0.521834

0.601786

0.786602

8681.033

9.203294

$-0.32735$

0.627855

$-0.52138$

0.602099

0.786728

82.22285

280.3577

0.630607

1.21668

0.518302

0.604248

0.788566

$-0.51812$

0.604372

0.788566

37.55629

0.318008

0.612712

0.519018

0.603748

0.788566

$-0.51857$

0.604061

0.788566

8.28987

0.620526

1.198444

0.517776

0.604615

0.788599

19.938

$-0.51998$

1.004949

$-0.51742$

0.604862

0.78864

1.233782

$-1.1393$

2.205955

$-0.5164$

0.60553

0.789228

0.680223

10.32236

2.930725

0.515489

0.606211

0.789833

1.14942

635001 


\begin{tabular}{|c|c|c|c|c|c|c|}
\hline Arthrobacter sp. PGP41 & 98.56671 & כ & & 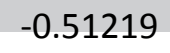 & & \\
\hline Helicobacter cetorum & 1.103785 & -1.49924 & 2.922471 & -0.513 & 0.607948 & 90731 \\
\hline Legionella oakridgensis & 9.042147 & 0.617758 & 1.207982 & 0.511397 & 0.609073 & 790731 \\
\hline Pelobacter propionicus & 37.07028 & -0.2752 & 0.539597 & 0.51001 & 0.610045 & $79092 \varepsilon$ \\
\hline Lactobacillus bombi & 3.924182 & -0.73363 & 1.43899 & -0.50982 & 0.610178 & .790928 \\
\hline Acinetobacter s| & 4.11973 & 0.32857 & 0.644497 & 0.509808 & 0.610186 & 790928 \\
\hline Moraxella catarrhalis & 31.2932 & -0.50637 & 0.9936 & -0.50963 & 0.610312 & .790928 \\
\hline Lactococcus virus blL170 & 5.127352 & 1.093315 & 2.143802 & 0.509989 & 0.610059 & 0.790928 \\
\hline ifaciens & 271.4243 & -0.2714 & 0.534169 & -0.50808 & 0.611401 & 1779 \\
\hline Planococcus plakortidis & 7.619651 & -0.79004 & 1.554977 & -0.50807 & 0.611404 & 0.791779 \\
\hline rosporus & 17.89921 & 0.361911 & 0.715096 & 0.506101 & 2786 & 286 \\
\hline Methy & 142.7882 & -0.34234 & 0.67808 & -0.50487 & 0.613649 & 3604 \\
\hline Sulfurc & 1.318868 & -1.31307 & 2.600793 & -0.50487 & 0.613649 & 3604 \\
\hline Trich & 2.113338 & -1.35233 & 2.678828 & -0.50 & 3685 & 3604 \\
\hline Staph & 200.0751 & -0.29537 & 0.586246 & -0.50384 & 0.614377 & 0.793642 \\
\hline Prev & 873.2539 & -0.23117 & 0.459433 & -0.50316 & 853 & 642 \\
\hline Shewa & 117.4825 & 0.402236 & 0.798078 & 0.504006 & 0.614257 & 0.793642 \\
\hline Geit & 31.5 & -0.35478 & 0.70 & 034 & & \\
\hline p. BJC16-A31 & 28.05238 & -0.35619 & 0.706845 & -0.50391 & 0.614321 & 0.793642 \\
\hline Cand & 4.821915 & -0.68306 & 1.359039 & -0.50261 & 0.615242 & 0.793642 \\
\hline Oleis & 7.85093 & -0.69306 & 1.37876 & -0.50267 & 0.615197 & 3642 \\
\hline 757 & 10.08678 & 0.649715 & 1.29545 & 0.501536 & 0.615994 & 0.794331 \\
\hline um & 33.54657 & -1.0715 & 2.147318 & -0.49899 & 0.617783 & 948 \\
\hline Cam & 497808 & 1.022583 & 2.048748 & 0.499126 & 0.617691 & 0.79 \\
\hline Propi & 20.31135 & 0.862013 & 1.726752 & 0.499211 & 0.617631 & 0.794948 \\
\hline Cap & & -0.37738 & & -0.4 & 515 & \\
\hline Ana & 7.85 & -0.58149 & 1.16 & -0.4 & 7223 & \\
\hline se & 21.3 & 0.374097 & 0.747659 & 0.500358 & 0.616823 & 1948 \\
\hline Mag & 4.95161 & -0.49426 & 0.991806 & -0.49835 & .61824 & 5032 \\
\hline Bifid & 128.5827 & 0.338454 & 0.679242 & 0.498282 & 0.618286 & 0.795032 \\
\hline Cell & 15.14927 & 0.468173 & 0.940736 & 0.497667 & 0.618719 & 0.795098 \\
\hline Cutib & 4212.877 & 0.270352 & 0.544044 & 0.49693 & 0.619239 & 0.795098 \\
\hline Strep & 29.24684 & -0.61312 & 1.23 & -0.49658 & 489 & 098 \\
\hline ABRD_28 & 145.6834 & -0.24004 & 0.483029 & -0.49695 & 0.619225 & 5098 \\
\hline & 19.32807 & 0.759 & 1.52 & 0.49 & & 098 \\
\hline Ocea & 23.8166 & -0.43991 & 0.886293 & -0.49635 & 0.619648 & 0.795098 \\
\hline Lac & 10.9 & -0.82492 & 1.664648 & -0.49555 & 62021 & 5194 \\
\hline Psyc & 098 & -0.46194 & 619 & -0.49531 & 378 & 5194 \\
\hline yangensis & 54.51813 & -0.28238 & 0.569858 & -0.49553 & 0.620223 & 0.795194 \\
\hline & 29 & -0.23207 & 0.47 & -0.49311 & 937 & 6912 \\
\hline um sp. G0186 & 12.99723 & 0.463747 & 0.94211 & 0.492243 & 0.622547 & 0.797132 \\
\hline Stap & 2534. & 0.33917 & 0.688598 & 0.492551 & .62233 & 0.797132 \\
\hline Desulfitobacterium dehalogenans & 2.025979 & -0.93255 & 1.89832 & -0.49125 & 0.623248 & 0.797397 \\
\hline Virgi & 10.23272 & 0.620347 & 1.263234 & 0.491079 & 0.623371 & 0.797397 \\
\hline sp. B006 & 57.58857 & -0.26229 & 0.534263 & -0.49095 & 0.623465 & 0.797397 \\
\hline gofermentans & 3.614526 & -0.74389 & 1.515936 & -0.49071 & 0.623631 & 0.797397 \\
\hline & 397518 & -1.39479 & 2.854124 & -0.48869 & 0.625058 & 0.798413 \\
\hline s mccartyi & 19.61119 & -0.5043 & 1.033314 & -0.48804 & 0.625522 & 0.798413 \\
\hline & 354142 & 0.608715 & 1.244862 & 0.488982 & 0.624855 & 0.798413 \\
\hline Sphingorhabdus sp. Alg231-15 & 5.162121 & -0.62037 & 1.270646 & -0.48824 & 0.625383 & 0.798413 \\
\hline
\end{tabular}


Pedobacter ginsengisoli

Sphingopyxis granuli

Roseovarius mucosus

Thermodesulfobium acidiphilum

Alcaligenes aquatilis

Aerococcus urinaeequi

Pseudoalteromonas luteoviolacea

Rhizorhabdus dicambivorans

Pseudomonas sp. StFLB209

Listeria ivanovii

Pseudomonas antarctica

Prevotella melaninogenica

Gloeocapsa sp. PCC 7428

Pantoea agglomerans

Acinetobacter sp. WCHAc010005

Methylobacterium sp. AMS5

Escherichia fergusonii

Paenisporosarcina sp. K2R23-3

Pseudomonas sp. VLB120

Lachnoclostridium sp. YL32

Mycoplasma californicum

Pseudoalteromonas rubra

Rhizobium sp. IRBG74

Thermanaeromonas toyohensis

Leclercia sp. LSNIH3

Vitreoscilla sp. C1

Staphylococcus xylosus

Nodularia spumigena

Streptococcus thermophilus

Halobacteroides halobius

Raphidiopsis curvata

Leptospirillum ferriphilum

Chlorobium phaeovibrioides

Desulfotomaculum ferrireducens

Calothrix sp. NIES-4101

Propionibacterium virus PHL116M00

Streptococcus cristatus

Maribacter sp. T28

Enterobacteriaceae bacterium ENNIH

Xanthomonas vasicola

Clostridium baratii

Aneurinibacillus soli

Glaciecola sp. THG-3.7

Chryseobacterium nakagawai

Chloroherpeton thalassium

Aureitalea sp. RR4-38

Microbacterium sp. 1.5R

cyanobacterium endosymbiont of $\mathrm{Rh}$

Candidatus Methanomassiliicoccus in

Mucilaginibacter sp. PAMC 26640
26.78294

121.0611

6.965377

1.36465

30.63265

55.01444

10.74196

124.9879

73.54879

14.4178

91.78227

952.0265

4.632895

133.0698

10.49801

55.42099

1.499309

4.744966

0.610326

34.9368

1.018199

1.030726

82.69218

1.542955

82.80999

19.642

162.6876

8.444827

1128.455

7.633674

1.092278

2.191982

2.043763

1.73967

8.186816

12.94259

810.5384

10.40423

2.430076

87.11195

49.47272

2.557044

5.200923

18.29613

2.82806

2.948781

42.1215

3.873213

1.221944

30.05676
$-0.31937$

0.65365

$-0.38439$

0.792191

$-0.69078$

1.424161

$-1.39513$

2.875391

0.39493

0.814805

0.388177

0.801217

0.556691

1.14845

$-0.28165$

0.579258

$-0.28797$

0.594615

$-0.43233$

0.887896

$-0.27643$

0.569921

$-0.22338$

0.462325

$-0.69391$

1.444985

$-0.23617$

0.491783

$-0.56095$

$-0.28085$

1.166713

1.222486

0.584152

2.539518

$-0.65546$

$-1.40535$

1.360751

2.928457

$-0.47007$

0.981188

$-1.31158$

2.738493

$-1.39601$

2.92298

0.507798

1.066863

$-1.10627$

2.323902

$-0.57597$

1.209562

$-0.4707$

0.990968

0.220159

0.464512

$-0.65523$

1.389582

$-0.28207$

0.598562

0.666472

1.417275

$-1.2692$

2.702378

1.250659

2.663094

0.966333

2.06473

$-1.07482$

2.298337

0.564034

1.208995

$-0.83387$

1.787276

$-0.30555$

0.656551

0.588704

1.26682

$-1.0368$

2.233982

$-0.37852$

0.814761

0.276409

0.595247

$-0.87616$

1.889817

$-0.62499$

1.353865

$-0.49151$

1.068233

0.704863

1.531481

$-0.754371 .639865$

$-0.41311$

0.899468

0.934246

2.034361

0.993196

2.162558

0.340449

$-0.4886$

0.625126

0.798413

$-0.485220 .627518$

0.798992

$-0.485040 .627646$

0.798992

$\begin{array}{ll}-0.4852 & 0.627537\end{array}$

0.798992

$-0.4847 \quad 0.627891$

0.798992

0.4844840 .628042

0.798992

0.4847320 .627866

0.798992

$-0.486230 .626806$

0.798992

$\begin{array}{ll}-0.4843 & 0.628172\end{array}$

0.798992

$-0.486920 .626316$

0.798992

$-0.485040 .627651$

0.798992

$-0.483170 .628976$

0.799736

$-0.48022 \quad 0.63107$

0.800719

$-0.48023$

0.631062

0.800719

$-0.4808$

0.630662

0.800719

$\begin{array}{ll}-0.48078 & 0.630672\end{array}$

0.800719

0.481385

0.630243

0.800719

$\begin{array}{ll}-0.48169 & 0.630027\end{array}$

0.800719

$-0.4799$

0.631301

0.800734

$-0.47908$

0.631882

0.801037

$-0.47894$

0.631981

0.801037

$-0.4776$

0.632937

0.80197

0.475973

0.634093

0.802597

$-0.47604$

0.634046

0.802597

$-0.47618$

0.633948

0.802597

$-0.47499$

0.634795

0.803205

0.473958

0.63553

0.803856

$-0.47153$

0.637261

0.805736

$-0.47125$

0.637459

0.805736

0.470249

0.638177

0.806364

$-0.46966$

0.638599

0.806365

0.469626

0.638622

0.806365

0.468019

0.639771

0.807536

$-0.46765$

0.640034

0.807588

0.466532

0.640835

0.808038

$-0.46656$

0.640815

0.808038 
Microbacterium pygmaeum

Paenibacillus yonginensis

Bacillus kochii

Echinicola vietnamensis

Bifidobacterium bifidum

Brachybacterium sp. P6-10-X1

Chryseobacterium sp. $\mathrm{H} 6466$

Lactobacillus fuchuensis

Salegentibacter sp. T436

Clostridium sp. SY8519

Kocuria flava

Polynucleobacter wuianus

Halocynthiibacter arcticus

Streptomyces bacillaris

Sphingobacterium sp. ML3W

Pseudarthrobacter equi

Desulfovibrio piger

Pseudomonas mandelii

Oceanisphaera avium

Yersinia aleksiciae

Buttiauxella sp. 3AFRM03

Lactobacillus coryniformis

Lactobacillus salivarius

Pectobacterium wasabiae

Chryseobacterium lactis

Candidatus Desulfofervidus auxilii

Streptococcus salivarius

Listeria seeligeri

Psychromonas ingrahamii

Polaribacter sp. MED152

Microbacterium aurum

Acinetobacter sp. WCHAc010034

Betapapillomavirus 4

Sphingomonas sp. JJ-A5

Moraxella ovis

Curtobacterium sp. BH-2-1-1

Candidatus Pantoea carbekii

Sporosarcina ureae

Methanoculleus bourgensis

Bacillus beveridgei

Leptolyngbya sp. PCC 7376

Bacteroides cellulosilyticus

Caldicellulosiruptor owensensis

Propionibacterium virus Stormborn

Pseudoalteromonas espejiana

Zunongwangia profunda

Flavobacterium columnare

Roseomonas sp. FDAARGOS_362

Selenomonas sputigena

Nonlabens sp. Hel1_33_55
140.9416

4.830058

7.524256

8.674486

128.8663

326.664

281.2365

4.500786

8.744924

13.15216

639.2663

1.230564

7.157265

52.67331

38.01401

109.4364

21.20824

80.45825

2.326415

1.820069

12.30204

3.915853

58.75663

5.017847

21.25698

2.37507

1499.781

1.642674

9.689619

12.68421

726.443

31.47518

5.47261

54.65138

2.998638

174.6275

0.951279

22.35863

1.363123

2.995799

3.120726

182.6329

2.4083

12.05181

0.563943

11.96061

28.54049

483.3285

82.06401

3.47184

$-0.2418$

0.699331

0.52931

$-0.45683$

0.647793

0.812588

0.501299

1.532456

0.456347

0.648141

0.812744

$-0.47337$

0.455468

0.648773

0.813257

$-0.25374$

0.55819

$-0.45485$

0.649215

0.813502

$-0.21038$

0.464286

$-0.45457$

0.649416

0.813502

0.317459

0.704654

$-0.45312$

0.650463

0.814533

$-0.85549$

1.894455

0.450517

0.652337

0.814716

$-0.59561$

1.320065

$-0.45158$

0.651574

0.814716

$-0.4577$

$-0.4512$

0.651845

0.814716

$-0.21284$

1.015505

$-0.45071$

0.6522

0.814716

1.179528

0.471994

$-0.45094$

0.652032

0.814716

0.460303

2.608968

0.452105

0.651193

0.814716

$-0.42978$

0.450431

0.6524

0.814716

$-0.35493$

$-0.45246$

0.650936

0.814716

$-0.21245$

0.473036

$-0.44971$

0.652919

0.815059

$-0.27235$

0.606076

0.323633

0.721097

$-0.44912$

0.653347

0.815059

$-0.44936$

0.653171

0.815059

$-0.85905$

1.916191

0.448807

0.653571

0.81506

$-1.0298$

2.305949

$-0.44831$

0.65393

0.815229

$-0.5357$

1.200495

$-0.44658$

0.655176

0.816502

$-0.64517$

1.447256

$-0.44623$

0.655428

0.816537

$-0.21953$

0.493291

$-0.44579$

0.655748

0.816655

$-0.65084$

1.463468

$-0.44502$

0.656303

0.817057

$-0.44472$

0.656519

0.817057

0.44319

0.657628

0.817106

$-1.07893$

2.431835

$-0.44367$

0.657281

0.817106

0.286642

0.646877

0.443116

0.657682

0.817106

$-0.93611$

2.112046

$-0.44323$

0.657603

0.817106

$-0.4608$

1.037933

$-0.44396$

0.657073

0.817106

$-0.52565$

1.187147

$-0.44278$

0.657923

0.817127

$-0.25879$

0.587737

$-0.44032$

0.659704

0.817421

$-0.3116$

0.709237

$-0.43935$

0.66041

0.817421

$-0.74872$

1.70618

$-0.43883$

0.660784

0.817421

$-0.2564$

0.584906

$-0.43835$

0.661131

0.817421

1.244877

2.845476

0.437493

0.661754

0.817421

$-0.22769$

0.519146

$-0.43859$

0.66096

0.817421

$-1.21379$

$-0.438$

0.66139

0.817421

$-0.34803$

0.7928

$-0.43899$

0.66067

0.817421

1.24823

2.83618

0.44011

0.659858

0.817421

$-0.62551 .416053$

$-0.44172$

0.658692

0.817421 
Lactococcus phage P680

Nonlabens dokdonensis

Gordonia sp. KTR9

Chryseobacterium sp. F5649

Myroides odoratimimus

Pseudomonas granadensis

Leminorella richardii

Actinomyces sp. oral taxon 897

Brevibacillus formosus

Siansivirga zeaxanthinifaciens

Pseudomonas fragi

Staphylococcus equorum

Nitrosococcus watsonii

Syntrophomonas wolfei

Streptococcus phage EJ-1

Dehalobacterium formicoaceticum

Croceibacter atlanticus

Mogibacterium pumilum

Enterococcus cecorum

Cohnella sp. 18JY8-7

Gramella forsetii

Thermoproteus uzoniensis

Tepidanaerobacter acetatoxydans

Riemerella anatipestifer

Corynebacterium pseudotuberculosis

Moorea producens

Marinomonas primoryensis

Mycobacterium intracellulare

Tenericutes bacterium MZ-XQ

Peptostreptococcaceae bacterium or

Acetomicrobium mobile

Bacillus vallismortis

Nonlabens sp. MB-3u-79

Methanobrevibacter smithii

Prevotella sp. oral taxon 299

Brachybacterium ginsengisoli

Acholeplasma brassicae

Lelliottia amnigena

Pseudoxanthomonas spadix

Acholeplasma palmae

Flavobacterium magnum

Staphylococcus phage 23MRA

Geobacillus sp. WCH70

Psychrobacter sp. P11F6

Paracoccus sp. CBA4604

Flavobacterium kingsejongi

Scytonema sp. HK-05

Staphylococcus phage IME-SA4

Staphylococcus phage StB20-like

Aliivibrio fischeri
12.69789

1.25525

471.1972

259.7272

3.009507

17.4525

10.97545

228.9073

5.693316

4.679154

136.7731

128.8017

0.548643

8.435187

1.233243

8.609729

2.694788

65.84642

12.64709

23.03052

3.184364

5.380654

5.728168

19.05456

315.2054

8.880466

4.113323

146.4717

1.519056

909.5465

1.29682

1.576704

3.696717

22.89059

114.1891

370.9162

8.557255

64.49493

118.5497

9.35182

4.601586

40.36502

6.595148

99.64719

136.0446

8.099727

19.08221

27.75244

10.19833

12.56338
$-0.78212$

$-1.26185$

$-0.2444$

0.30869

1.22379

0.42214

$-0.38514$

$-0.17959$

$-0.58506$

$-0.62519$

$-0.29896$

$-0.24626$

$-1.25901$

0.561371

$-1.13571$

$-0.52795$

$-0.66388$

0.306992

$-0.40925$

$-0.26628$

$-0.65791$

0.704875

$-0.51276$

$-0.32778$

0.16001

0.41302

0.580133

$-0.26447$

$-0.96836$

0.234296

$-0.99608$

$-1.0579$

$-0.72618$

0.484399

$-0.2358$

$-0.21644$

0.478881

$-0.27431$

$-0.23357$

0.471752

$-0.54851$

1.096545

$-0.6206$

0.335151

$-0.23236$

$-0.4393$

$-0.3994$

$-0.37282$

$-0.59242$

0.427797
1.802804

2.910234

0.562913

0.711949

2.823069

0.970956

0.889799

0.415108

1.350899

1.447487

0.692841

0.573672

2.9294

1.302513

2.643274

1.226958

1.544904

0.715228

0.95766

0.623807

1.545726

1.657145

1.205284

0.773582

0.378458

0.982559

1.382586

0.632108

2.322115

0.561916

2.393998

2.543552

1.748194

1.167518

0.568114

0.523184

1.156143

0.662891

0.566438

1.148067

1.337664

2.677862

1.514491

0.820297

0.56969

1.078376

0.981159

0.916568

1.457717

1.054398
$-0.43384$

$-0.43359$

$-0.43416$

0.433585

0.433496

0.434767

$-0.43284$

$-0.43265$

$-0.43309$

$-0.43191$

$-0.4315$

$-0.42927$

$-0.42979$

0.430991

$-0.42966$

$-0.43029$

$-0.42972$

0.429223

$-0.42735$

$-0.42686$

$-0.42563$

0.425355

$-0.42543$

$-0.42372$

0.422795

0.420351

0.4196

$-0.4184$

$-0.41702$

0.416959

$-0.41607$

$-0.41592$

$-0.41539$

0.414897

$-0.41505$

$-0.41371$

0.414205

$-0.4138$

$-0.41234$

0.41091

$-0.41005$

0.409485

$-0.40978$

0.408573

$-0.40786$

$-0.40737$

$-0.40707$

$-0.40676$

$-0.4064$

0.405726

0.664406

0.664586

0.66417

0.66459

0.664654

0.663731

0.665128

0.665273

0.664948

0.665805

0.666106

0.66773

0.667352

0.666475

0.667442

0.666985

0.667399

0.667761

0.669125

0.669483

0.670375

0.670578

0.670523

0.671769

0.672445

0.674229

0.674778

0.675654

0.676667

0.676708

0.677355

0.677472

0.677858

0.678218

0.678103

0.679089

0.678724 


\begin{tabular}{|c|c|c|c|c|c|c|}
\hline Intestinımonas butyrıcıproqucens & & & & & & \\
\hline Propionibacterium virus PHL132N00 & 41.93338 & 0.593389 & 1.468566 & 0.40406 & 0.686169 & 0.831208 \\
\hline esulfovibrio alaskensis & 13.55782 & -0.482 & 1.195618 & -0.40314 & 0.686849 & 0.831208 \\
\hline clobacterium marinum & 3.84702 & -0.38333 & 0.947418 & -0.4046 & 0.685771 & 0.831208 \\
\hline aenibacillus sp. FSL R7-0273 & 165011 & -0.50189 & 1.243716 & -0.40354 & 0.686549 & .831208 \\
\hline etapapillomavirus 6 & 4.056561 & -0.82235 & 2.035765 & -0.40395 & 0.686248 & 0.831208 \\
\hline Kocuria rhizophila & 1715.879 & -0.23878 & 0.592198 & -0.40321 & 0.686795 & 0.831208 \\
\hline cetobacter pasteurianus & 6.43353 & -0.6115 & 1.519549 & -0.40242 & 0.687372 & 0.831288 \\
\hline Celeribacter baekdonensis & 16.20811 & -0.27487 & 0.683011 & -0.40243 & 0.687366 & 0.831288 \\
\hline Marivivens sp. JLT3646 & 4.836552 & -0.5821 & 1.448702 & -0.40181 & 0.687825 & 0.831559 \\
\hline Virgibacillus sp. 6R & 11.54845 & 0.418229 & 1.042949 & 0.401006 & 0.688416 & 0.831997 \\
\hline abiont of Trabutin & 1.903734 & 1.097211 & 2.739836 & 0.400466 & 0.688813 & 0.832202 \\
\hline ccharibacteria bacteriu & 1.035419 & -1.08447 & 2.711458 & -0.39996 & 0.689189 & 0.832379 \\
\hline Anox & 5.251694 & -0.53093 & 1.329496 & -0.39934 & 0.689639 & 0.832647 \\
\hline lafni & 16.0993 & -0.5403 & 1.358867 & -0.39761 & 0.69092 & 3286 \\
\hline Koma & 22.90241 & -0.2959 & 0.744389 & -0.3975 & 0.690995 & 0.83286 \\
\hline Mord & 36.05953 & -0.47118 & 1.182142 & -0.39858 & 0.6902 & 0.83286 \\
\hline Staph & 44.67409 & -0.25052 & 0.630646 & -0.39724 & 0.691189 & 0.83286 \\
\hline Bifidc & 3.2234 & -0.86972 & 2.188397 & -0.39742 & 0.691054 & 0.83286 \\
\hline Helicc & 2.841175 & -0.70582 & 1.775899 & -0.39744 & 0.691041 & 0.83286 \\
\hline Myro & 13.19923 & 0.419046 & 1.063659 & 0.393967 & 505 & 495 \\
\hline Paen & 7.911016 & 0.436931 & 1.11131 & 0.393167 & 0.694196 & 0.835929 \\
\hline Mari & 5.386261 & 0.508589 & 1.294622 & 0.392847 & 432 & 5937 \\
\hline Blaut & 57.64221 & 0.341999 & 0.871719 & 0.39 & 0.65 & 123 \\
\hline Legio & 3.841177 & 0.797097 & 2.034992 & 0.391696 & 5283 & 0.836408 \\
\hline Shew & 5.65624 & -0.60307 & 1.547699 & -0.38965 & 6793 & 7671 \\
\hline Psych & 10.55975 & 0.616918 & 1.583215 & 0.389662 & 0.696787 & 0.837671 \\
\hline Psych & 14.32934 & -0.37824 & 0.97265 & -0.38888 & 0.697366 & 0.838083 \\
\hline Colwe & 2.081857 & -0.8099 & 2.089075 & -0.38768 & 0.698251 & 0.838869 \\
\hline Renib & 55.7969 & -0.17071 & 0.441033 & -0.38707 & 0.698704 & 0.838939 \\
\hline Vibrio owensii & 1.856336 & -1.06039 & 2.740165 & -0.38698 & 0.69877 & 0.838939 \\
\hline Paenibacillus sp. & 0.696667 & 1.129234 & 2.928495 & 0.385602 & 0.699791 & 0.839888 \\
\hline Mari & 6.270861 & -0.47237 & 1.227093 & -0.38495 & 0.700275 & 0.840192 \\
\hline Chrys & 26.04594 & -0.35717 & 0.928667 & -0.38461 & 053 & 4022 \\
\hline NPH82 & 43.7221 & 0.449546 & 1.173058 & 0.383226 & 0.701553 & 0.840959 \\
\hline Anab & 8.690467 & 0.569916 & 1.487448 & 0.38315 & 0.701608 & 0.840959 \\
\hline Bract & 418.2911 & -0.19202 & 0.504539 & -0.38059 & 0.70351 & 0.842961 \\
\hline Flavo & 17.53659 & 0.286758 & 0.754095 & 0.380269 & 0.703746 & 2966 \\
\hline Thermo & 7.936611 & 0.41154 & 1.087948 & 0.378272 & 0.705229 & 0.843385 \\
\hline P91 & 26.32065 & -0.56151 & 1.480364 & & & 3385 \\
\hline rignonense & 30.06788 & -0.34863 & 0.921709 & -0.37824 & 0.705254 & 0.843385 \\
\hline Lactc & 14.82768 & -0.54473 & 1.439448 & -0.37843 & 0.705112 & 0.843385 \\
\hline Thioglobus singularis & 19.65821 & 0.358621 & 0.947752 & 0.378391 & 0.70514 & 0.843385 \\
\hline lus genomosp. 3 & 7.097684 & -0.54532 & 1.4455 & -0.37725 & 0.705984 & 0.843981 \\
\hline & 1.577376 & 0.871181 & 2.311732 & 0.376852 & 0.706284 & 0.844061 \\
\hline Photobacterium damselae & 25.77637 & -0.36781 & 0.978596 & -0.37586 & 0.707024 & 0.844391 \\
\hline Halomicronema hongdechloris & 11.27765 & -0.41489 & 1.102978 & -0.37615 & 0.706804 & 0.844391 \\
\hline Bordetella parapertussis & 4.026741 & -0.9389 & 2.500521 & -0.37548 & 0.707303 & 0.844447 \\
\hline Staphylococcus phage CNPx & 58.47082 & 0.929435 & 2.490052 & 0.373259 & 0.708956 & 0.845865 \\
\hline Bacillus cytotoxicus & 12.72163 & -0.41417 & 1.10875 & -0.37354 & 0.708744 & 0.845865 \\
\hline
\end{tabular}


Cyanothece sp. PCC 7425

Propionibacterium virus PAS50

Brevibacterium sandarakinum

Micromonospora coxensis

Octadecabacter temperatus

Planococcus maritimus

[Eubacterium] eligens

Corynebacterium genitalium

Desulfotomaculum ruminis

Paenibacillus sp. IHBB 10380

Campylobacter fetus

Taylorella equigenitalis

Lelliottia jeotgali

Marivirga tractuosa

Bdellovibrio exovorus

Trichormus azollae

Synechococcus sp. PCC 7502

Natranaerobius thermophilus

Pantoea sp. At-9b

Mycoplasma hyosynoviae

Methanobacterium paludis

Adlercreutzia equolifaciens

Desulfosporosinus youngiae

Clostridium cochlearium

Pseudoalteromonas piratica

Aequorivita sublithincola

[Eubacterium] cellulosolvens

Human papillomavirus

Microbacterium sp. str. 'China'

Acinetobacter radioresistens

Proteus vulgaris

Campylobacter coli

Moraxellaceae bacterium HYN0046

Nostoc sp. NIES-4103

Mycobacterium sp. KMS

Novibacillus thermophilus

Bacillus horikoshii

Lactobacillus amylophilus

Sulfurospirillum sp. JPD-1

Pseudomonas frederiksbergensis

Draconibacterium orientale

Vibrio tubiashii

Jannaschia sp. CCS1

Bacillus mycoides

Arcticibacterium luteifluviistationis

Vibrio crassostreae

Staphylococcus epidermidis

Flavobacteriaceae bacterium MAR_21

Enterobacter bugandensis

Meiothermus silvanus
5.621826

16.46506

279.4017

87.06114

1.949813

1.89675

68.83569

3549.912

2.665672

2.351535

13.97018

2.362239

11.05411

9.984065

1.414271

5.594551

0.962565

6.159975

46.21595

0.261406

0.679451

118.6598

6.872082

13.73673

1.208552

4.078785

10.31791

2.152701

94.83352

134.8986

19.31757

10.42471

6.012056

25.60962

5.59132

5.622224

7.1103

1.846838

2.087111

58.88852

10.48772

20.29988

14.93372

65.07643

6.508474

3.533951

69890.84

3.116995

1.900811

35.30126
$0.51141 \quad 1.375948$

$-0.57458$

$-0.19967$

1.546998

0.17733

0.538788

$-0.70915$

0.48015

1.923958

0.768044

2.093776

$-0.29692$

0.809252

$-0.17562$

0.479763

$-0.60274$

1.646571

$-0.64124$

1.755987

0.431326

1.180366

0.817251

2.253292

$-0.32797$

0.903488

0.354734

0.974052

$-0.93621$

2.583921

0.604975

1.66254

$-0.81974$

2.258941

0.530056

1.463957

0.782853

2.941146

2.928604

$-0.24303$

0.674299

$-0.36063$

1.001706

0.379501

1.052287

2.397968

0.85851

1.758121

$-0.4025$

1.124151

$-0.97052$

2.703859

$-0.32149$

0.89499

0.246209

0.687598

0.341399

0.95751

0.40527

1.135973

$-0.43185$

1.214741

0.314003

0.885203

$-0.94223$

2.654243

$-0.45656$

1.28824

1.16674

0.412758

2.114335

$-0.7961$

2.258245

$-0.19088$

0.542403

0.353583

1.006382

$-0.41588$

1.187923

$-0.25257$

0.726885

$-0.32887$

0.944986

$-0.44874$

1.291119

0.76064

2.184204

0.17901

0.515675

0.605518

1.756172

$-0.94257$

2.733974

$-0.24629$

0.71271

$-0.37168$

0.710132

$-0.37142$

0.710328

$-0.3706$

0.710936

$-0.36931$

0.711894

$-0.36859$

0.712434

0.366822

0.713751

$-0.36691$

0.71369

$-0.36606$

0.714324

$-0.36606$

0.714321

$-0.36517$

0.714981

0.7148

0.365417

0.716835

0.362692

0.716598

0.715721

.364184

0.717112

0.715943

$-0.362890 .71669$

0.717299

0.362071

0.716458

$-0.3632$

0.717877

0.718614

0.360311

.718614

$-0.36042$

0.718533

$-0.36002$

0.718833

0.360643

0.718366

$-0.35802$

0.720331

0.358632

0.71987

$-0.35805$

0.720304

$-0.35894$

0.71964

$-0.35921$

0.719436

0.358071

0.72029

0.356549

0.721429

0.356761

0.721271

$-0.35551$

0.722206

0.354724

0.722796

$-0.35499$

0.722598

$-0.3544$

0.723037

0.35377

0.723511

$-0.35261$

0.724379

$-0.35253$

0.724439

$-0.35192$

0.724896

0.351341

0.725333

$-0.35009$

0.72627

$-0.34747$

0.728238

$-0.34802$

0.727829

$-0.34756$

0.728169

0.348246

0.727655

0.347137

0.728488

0.344794

0.730249

$-0.34476$ 


\begin{tabular}{|c|c|c|c|c|c|c|}
\hline alothrix sp. PCC 7507 & ון & & 499863 & -0.34477 & & \\
\hline Flavobacterium branchiophilum & 14.29872 & 0.306033 & 0.885899 & 0.34545 & & \\
\hline hermoclostridium stercorarium & 4.258979 & -0.522 & 1.521094 & -0.34317 & 0.73147 & 857821 \\
\hline Propionibacterium virus PHL111M01 & 20.33024 & -0.55095 & 1.614088 & -0.34134 & 732848 & 859161 \\
\hline andidatus Fukatsuia symbiotica & 9.103721 & -0.33791 & 0.994018 & -0.33994 & 0.733901 & 0.860118 \\
\hline Shewanella piezotolerans & 2.216228 & -0.55009 & 1.64458 & -0.33448 & 0.738014 & 0.860716 \\
\hline aenibacillus durus & 32.12048 & -0.19061 & 0.563632 & -0.33818 & 0.735229 & 0.860716 \\
\hline Vibrio splendidus & 3.309505 & -0.51231 & 1.542881 & -0.33205 & 0.739852 & 860716 \\
\hline Lactococcus phage 340 & .786294 & 0.723702 & 2.169794 & 0.333535 & 0.738731 & .860716 \\
\hline alothrix sp. 336/3 & 1.082577 & -0.74759 & 2.220463 & -0.33668 & 0.736357 & 0.860716 \\
\hline eohaensis & 20.79795 & 0.194767 & 0.581765 & 0.33 & 0.737786 & 360716 \\
\hline Thermc & 1.87292 & -0.91213 & 2.740859 & -0.33279 & 0.739292 & 0.860716 \\
\hline Lepto & 0.544596 & 0.981934 & 2.930977 & 502 & 0.73761 & 716 \\
\hline Anaer & 915.4101 & 0.147096 & 0.440204 & 0.334156 & 0.738262 & 0716 \\
\hline Auric & 42.27783 & 0.269348 & 0.801966 & 0.335859 & 0.736977 & 0.860716 \\
\hline Mollc & 1966.073 & -0.96311 & 2.885448 & -0.33378 & 0.738545 & 0.860716 \\
\hline Planococc & 2.645774 & -0.68182 & 2.052993 & -0.33211 & 0.739808 & 0.860716 \\
\hline Fusob & 12.80 & 0.275826 & 0.824561 & 0.33 & 0.737993 & 716 \\
\hline Actin & 4.517492 & 0.528501 & 1.56241 & 0.33826 & 0.735167 & 0.860716 \\
\hline Acti & 372 & -0.19849 & 0.587276 & & 378 & 716 \\
\hline Paen & 30.99149 & -0.22392 & 0.673819 & -0.3 & 0.739649 & 0716 \\
\hline ri & 2.149747 & 0.689496 & 2.068585 & 0.333318 & 0.738894 & 0716 \\
\hline als & 2.333994 & 0.89911 & 2.665987 & 0.337252 & 0.735927 & 0716 \\
\hline Salinį & 18.65223 & -0.20397 & 0.601761 & -0.33895 & 0.734649 & 0.860716 \\
\hline um virus Kubed & 9.01114 & 0.705632 & 2.11786 & 0.333181 & 0.738997 & 0.860716 \\
\hline Bact & 65.17688 & -0.34267 & 1.030013 & -0.33268 & 0.739373 & 0.860716 \\
\hline Aren & 9.95459 & -0.38187 & 1.147981 & -0.33264 & 0.739405 & 0716 \\
\hline Stre & 734.89 & -0.19389 & 0.5771 & -0.3 & 0.736892 & 0716 \\
\hline Formc & 0.818433 & -0.96906 & 2.924797 & -0.33133 & 0.740398 & 0.861077 \\
\hline Prop & 16.34438 & -0.4354 & 1.315504 & -0.33097 & 0.740664 & 6111 \\
\hline Selen & 78.55688 & -0.17282 & 0.523655 & -0.33002 & 0.741386 & 0.8614 \\
\hline Basilea psittacipulmonis & 5.642592 & -0.48451 & 1.46705 & -0.33026 & 0.741204 & 0.8614 \\
\hline Lactobacillus reuteri & 29.00835 & -0.25611 & 0.778102 & -0.32915 & 0.742044 & 889 \\
\hline Pepto & 109.602 & 0.187432 & 0.573315 & 0.326927 & 0.743723 & 0.863564 \\
\hline Caldi & 0.87 & -0.94488 & 2.923754 & & 0.746565 & 311 \\
\hline Kosm & 0.681997 & -0.94671 & 2.926963 & -0.32344 & 0.746359 & 0.866311 \\
\hline Lact & 1.894 & -0.62498 & 1.937726 & & 0.7470 & 5595 \\
\hline Stre & 327.4148 & 0.149799 & 0.464952 & 0.322181 & 0.747316 & 0.866629 \\
\hline Lacto & 4.692517 & -0.44298 & 1.376405 & -0.32184 & 0.747573 & 0.866651 \\
\hline Psenc & 6.836623 & 0.443526 & 1.380274 & 0.321331 & 0.747959 & 0.866823 \\
\hline Serini & 187.7579 & -0.13778 & 0.429234 & -0.321 & 0.748213 & 0.86684 \\
\hline Eubs & 796 & -0.49071 & 1.532232 & -0.32026 & 0.74877 & 5721 \\
\hline Deinoc & 32.36419 & -0.25665 & 0.805058 & -0.3188 & 0.74988 & 0.86822 \\
\hline Candidatus Kinetopla & 1.117566 & -0.77315 & 2.438054 & -0.31712 & 0.751154 & 0.868313 \\
\hline Serratia liquefaciens & 54.42773 & 0.222097 & 0.698948 & 0.317759 & 0.750668 & 0.868313 \\
\hline Alkali & 6.361379 & -0.36636 & 1.154809 & -0.31725 & 0.751055 & 0.868313 \\
\hline & 2.558871 & -0.60714 & 1.907881 & -0.31823 & 0.750311 & 0.868313 \\
\hline Rahnella aquatilis & 135.1833 & -0.19656 & 0.619193 & -0.31744 & 0.750908 & 0.868313 \\
\hline & 28.11967 & -0.26707 & 0.844102 & -0.31639 & 0.751704 & 0.868673 \\
\hline Propionibacterium virus P105 & 15.99363 & 0.527325 & 1.685633 & 0.312835 & 0.754406 & 0.871519 \\
\hline
\end{tabular}


Stenotrophomonas sp. LM091

Propionibacterium virus PAD20

Lactococcus virus ASCC191

Providencia sneebia

Moraxella bovis

Geosporobacter ferrireducens

Pedobacter sp. G11

Kocuria palustris

Lactobacillus heilongjiangensis

Staphylococcus phage StB27

Candidatus Planktophila lacus

Listeria monocytogenes

Chryseobacterium sp. StRB126

Spiroplasma turonicum

Candidatus Kuenenia stuttgartiensis

Psychromonas sp. CNPT3

Desulfosporosinus acidiphilus

Winogradskyella sp. PG-2

Hydrogenovibrio crunogenus

Tenacibaculum sp. SZ-18

Candidatus Nitrosoglobus terrae

Cloacibacterium normanense

Streptococcus sp. A12

Halohasta litchfieldiae

Lactobacillus paracasei

Capnocytophaga canimorsus

Bacillus filamentosus

Lutibacter sp. LPB0138

Gammapapillomavirus 13

Candidatus Rhodoluna planktonica

Thermosulfidibacter takaii

Staphylococcus phage SAP-2

Polaribacter sp. ALD11

Pseudomonas sp. s211(2017)

Propionibacterium phage BruceLetha

Aeribacillus pallidus

Bacillus megaterium

Phoenicibacter massiliensis

Tannerella sp. oral taxon HOT-286

Microcoleus sp. PCC 7113

Kribbella flavida

Streptococcus lutetiensis

Leptospira mayottensis

Gammapapillomavirus 18

Dictyoglomus turgidum

Mycobacterium sp. JLS

Flagellimonas sp. HME9304

Labrenzia sp. CP4

Lactobacillus kullabergensis

Treponema putidum

335.7344

335.7344

$-0.16789$

0.539082

$-0.31144$

0.755466

0.872466

17.02172

0.434801

1.403863

0.309717

0.756776

0.873329

5.776468

8.370462

1.408783

$-0.31007$

0.756507

0.873329

10.49999

1.453517

$-0.30951$

0.756933

0.873329

10.49999

30869

1.001002

$-0.30838$

0.757794

0.874045

29.27169

$-0.22755$

0.897264

0.307871

0.75818

0.874083

1301.474

0.838692

$-0.13935$

0.740275

$-0.30739$

0.758548

0.874083

26.49997

$-0.86641$

0.453297

$-0.30742$

0.758523

0.874083

8.071897

248034

(29047

$-0.30625$

0.759411

0.874801

72.88856

$-0.32808$

811642

0.305596

0.759913

0.875102

16.58049

3.20052

0.145185

$-0.30527$

0.760164

0.875115

1.118945

0.573325

0.47663

0.304608

0.760665

0.875414

7.646428

$-0.66525$

$-0.30131$

0.763177

0.877239

1.646428

$-0.3776$

0.301262

0.763215

0.877239

1.393969

$-0.70574$

206863

$-0.30144$

0.763076

0.877239

7.58602

0.355195

1.250431

$-0.30198$

0.762668

0.877239

0.745351

$-0.86815$

2.346262

$-0.30079$

0.763573

0.877373

13.44991

0.26044

0.29971

0.764398

0.877491

3.998032

89473

$-0.29991$

0.764247

0.877491

65.01024

145.4741

0.457153

0.86845

0.29989

0.764261

0.877491

4.469515

0.125859

1.532126

0.298378

0.765415

0.87838

44.98414

0.422752

0.297715

0.765921

0.878684

$17.05439 \quad 0.243912$

$-0.4284$

42071

$-0.29519$

0.767849

0.878859

$-0.29383$

0.768889

0.878859

15.73857

14.11367

1.425743

$-0.22227$

0.987061

$-0.29653$

0.766826

0.878859

12.07164

$-0.36441$

0.294244

0.768571

0.878859

$-0.2972$

0.766317

0.878859

$-0.29399$

0.768765

0.878859

$-0.85726$

$-0.29389$

0.768844

0.878859

3.070635

$-0.22674$

0.76472

$-0.29651$

0.766844

0.878859

0.41249

0.520327

1.759026

0.295804

0.76738

0.878859

5.986388

2.932409

$-0.29407$

0.768704

0.878859

4.243925

464029

1.572921

0.295011

0.767985

0.878859

1.906552

3469

2.160876

$-0.29372$

0.768972

0.878859

8.388508

$-0.66405$

$-0.293$

0.769523

0.879213

77.62391

0.409204

1.401402

0.291996

0.770289

0.879813

8.340904

0.61749

$-0.29072$

0.771268

0.880654

270.525

1.098073

$-0.29003$

0.771795

0.88098

8.590375

16513

0.572317

$-0.28853$

0.772941

0.882011

143.0238

$-0.31662$

1.10015

$-0.28779$

0.773504

0.882376

14.04945

$-0.39914$

0.456637

$-0.28723$

0.773936

0.882592

0.769251

$-0.81929$ 
Psychrobacter sp. G

Acinetobacter oleivorans

Pseudoalteromonas sp. NC201

Chromobacterium violaceum

Propionibacterium virus Pirate

Pseudomonas moraviensis

Solitalea canadensis

Psychroflexus torquis

Capnocytophaga sputigena

Propionibacterium virus PHL179M00

Aliivibrio wodanis

Agarilytica rhodophyticola

Rickettsiales bacterium Ac37b

Streptomyces sp. ICC1

Marinomonas mediterranea

Helicobacter cinaedi

Streptomyces sp. ADI95-16

Lactococcus phage phi7

Brachybacterium saurashtrense

Anaerococcus prevotii

Gordonia virus Wizard

Candidatus Planktophila sulfonica

Bifidobacterium breve

Blautia hansenii

Tenacibaculum dicentrarchi

Plantactinospora sp. BB1

Turicibacter sp. $\mathrm{H} 121$

Scytonema sp. NIES-4073

Caldanaerobacter subterraneus

Macrococcus sp. IME1552

Sulfurovum sp. NBC37-1

Acinetobacter wuhouensis

Acinetobacter sp. WCHAc010052

Virgibacillus sp. SK37

Candidatus Kinetoplastibacterium ble

Serratia fonticola

Candidatus Protochlamydia naegleric

Lactococcus piscium

Propionibacterium phage PHL082MO

Sulfurospirillum sp. UCHOO1

Prevotella denticola

Shewanella denitrificans

Yersinia enterocolitica

Spiroplasma culicicola

Legionella fallonii

Weissella paramesenteroides

Acinetobacter baumannii

Pseudomonas sp. R3-18-08

Bacillus velezensis

Pseudomonas rhodesiae
53.78459

9.543587

1.091319

112.5095

9.806955

110.5513

16.89501

4.738412

144.6081

10.88885

7.348856

4.472106

1.685005

631.9655

3.187238

0.883541

44.91856

8.300431

508.4129

1712.296

1.123393

7.314835

133.6137

78.65005

5.910734

43.01504

12.21761

2.721479

1.626333

60.12743

1.781609

60.58601

13.74822

4.628546

2.8619

43.35822

2.155135

89.26275

36.33028

2.77228

529.3192

1.613512

53.92959

2.931068

4.22809

3.115317

757.6335

1.855267

40.22477

87.31497
$-0.31917 \quad 1.126669$

0.322112

1.132861

2.920254

0.636276

$-0.46665$

1.66243

$-0.17838$

0.635073

$-0.23941$

0.853241

0.438523

1.562528

$-0.1489$

0.527801

$-0.48859$

1.747721

$-0.37159$

1.334584

0.487758

1.767013

0.766311

$-0.2646$

2.786484

0.966426

$-0.39978$

1.462362

0.758082

2.781195

$-0.21492$

0.787287

$-0.49243$

1.805173

$-0.13379$

0.495596

$-0.12242$

0.45181

0.789081

2.919404

$-0.31715$

1.175837

0.119503

0.441221

0.886846

0.371401

1.38517

$-0.23421$

0.873642

$-0.32481$

1.212443

0.699458

2.615821

$-0.56957$

2.135605

$-0.23612$

0.887051

$-0.56172$

2.122502

0.194958

0.740606

$-0.30244$

1.152793

0.457501

1.74621

0.582842

2.241706

$-0.15156$

0.584006

$-0.49152$

1.910141

0.198906

0.772776

0.369008

1.431128

0.46052

1.790155

$-0.14722$

0.573905

$-0.54864$

2.138144

0.134316

0.528978

0.560888

2.227725

0.443281

1.777403

0.357858

1.449631

0.124635

0.505214

$-0.6775$

2.739814

$-0.27251 \quad 1.102702$

$-0.150350 .606449$

$-0.28329$

0.284335

$-0.28256$

0.776956

0.776154

0.777514

$-0.28131$

0.778472

$-0.2807$

0.778937

$-0.28087$

0.778807

$-0.28059$

0.779028

0.28065

0.778979

$-0.2821$

0.777863

$-0.27956$

0.779818

$-0.27843$

0.780679

0.276036

0.782521

0.27501

0.783308

$-0.27379$

0.784242

$-0.27338$

0.784559

0.272574

0.785181

$-0.27299$

0.784858

$-0.27279$

0.785014

$-0.26995$

0.787199

0.786419

$-0.27096$

0.786938

0.270288

0.78737

$-0.26973$

0.786509

0.786705

0.788602

0.268127

0.788636

$-0.2679$

0.788778

0.267395

0.789165

$-0.2667$

0.7897

$-0.26618$

0.790098

$-0.26465$

0.791281

0.263241

0.792365

$-0.26235$

0.793049

0.261997

0.793324

0.26

0.794864

$-0.25952$

0.795232

$-0.25732$

0.796931

0.257391

0.796877

0.257844

0.796527

0.257251

0.796985

$-0.25653$

0.797544

$-0.2566$

0.797489

0.253916

0.799561

0.251776

0.801214

0.249398

0.803053

0.246862

0.805015

0.246698

0.805142

$-0.24728$

0.804691

$-0.247130 .804806$

$-0.24792$ 
Rhodococcus sp. S2-17

Spiroplasma clarkii

Propionibacterium virus Ouroboros

Nitratifractor salsuginis

Pseudomonas sp. SWI44

Vibrio diabolicus

Capnocytophaga stomatis

Acinetobacter lactucae

Blochmannia endosymbiont of Camp

Cyclobacterium amurskyense

Selenomonas sp. oral taxon 126

Propionibacterium sp. oral taxon 193

Propionibacterium virus Wizzo

Propionibacterium virus Solid

Pseudomonas sp. Z003-0.4C(8344-21

[Clostridium] ultunense

Thermocrinis ruber

Mucilaginibacter paludis

Calothrix sp. NIES-2098

Shewanella halifaxensis

Fischerella sp. NIES-4106

Ruminiclostridium cellulolyticum

Desulfotomaculum reducens

Halobacteriovorax marinus

Mesoplasma syrphidae

Candidatus Blochmannia floridanus

Acinetobacter Iwoffii

Desulfosporosinus orientis

Sulfitobacter sp. SK025

Propionibacterium virus PHL041M10

Formosa sp. Hel1_33_131

Lactobacillus allii

Chryseobacterium carnis

Methanosarcina thermophila

Glaciecola sp. 4H-3-7+YE-5

Leuconostoc virus LN03

Ruegeria sp. NKC1-1

Halomonas sp. GT

Monoglobus pectinilyticus

Hungateiclostridium thermocellum

Lactobacillus sanfranciscensis

Desulfobacterium autotrophicum

Crinalium epipsammum

Gramella sp. MAR_2010_102

Cellulophaga algicola

Pseudomonas sp. FGI182

Corynebacterium phocae

Capnocytophaga leadbetteri

Staphylococcus agnetis

Catenovulum sp. CCB-QB4
$164.8666-0.11148$

$\begin{array}{lll}1.946057 & 0.515877\end{array}$

0.453947

4.988039

0.345922

2.101127

10.08068

18.70547

$-0.28036$

1.410766

$-0.24557$

0.806014

1.163526

$-0.37332$

1.149748

10.0724

$-0.70849$

1.537427

2.919138

11.44746

$-0.28903$

1.196679

$-0.26127$

1.092842

0.758318

11.28486

67.76124

$-0.69842$

2.925121

0.250996

1.051993

$-0.12104$

0.50795

$-0.1592$

0.671232

7.617146

22.7918

0.337413

1.42772

$-0.33499$

1.418223

0.176384

0.746558

19.46693

0.20072

0.857824

0.707474

$-0.68182$

2.926028

28.31728

5.834119

$-0.14318$

0.625155

0.43489

1.902137

$-0.32446$

1.428428

6.671652

0.26744

1.183725

6.799959

0.276305

1.23177

2.861364

$-0.36321$

1.6234

6.486107

$-0.31228$

1.400596

0.670536

$-0.64682$

1.057556

0.643667

588.4382

$-0.13394$

2.672512

23.20002

$-0.34937$

$-0.16556$

2.926657

2.920306

0.616648

1.624955

0.773429

13.91921

0.402598

1.879385

5.394505

$-0.35295$

1.662421

2.826125

$-0.3277$

1.541375

21.3288

0.178637

0.840805

2.272949

0.521084

2.448959

4.068463

$-0.33198$

1.56742

1.162436

$-0.61256$

2.919031

1.321255

2.449105

$-0.49732$

2.37193

$-0.46994$

2.23665

20.73177

$-0.27331$

1.30866

4.702029

0.340368

1.636197

1.144477

0.408555

1.996465

6.975836

$-0.23425$

1.143377

$-0.21234$

1.038594

$-0.59811$

2.923503

0.828174

0.211418

1.030982

$-0.35749$

1.759303

6.536868

0.046907

0.232714

$-0.1317$

0.658668

205.6362

0.097556

0.491901

2.193986

1.122209

0.245524

0.245201

0.806051

0.806301

$\begin{array}{ll}-0.24384 & 0.807351\end{array}$

$-0.24282$

0.808145

$-0.24271$

0.808233

$-0.24153$

0.809146

$-0.23907$

0.811049

$-0.23877$

0.811286

0.238591

0.811423

$-0.2383$

0.81165

$-0.23717$

0.812522

$0.23633 \quad 0.813177$

$-0.23621$

0.813273

$0.236264 \quad 0.813228$

0.2339870 .814995

$\begin{array}{ll}-0.23302 & 0.815746\end{array}$

$-0.22902 \quad 0.81885$

0.2286320 .819155

$-0.22714$

0.820312

0.225931

0.821255

0.224316

0.822512

$-0.22374$

0.822962

$-0.22297$

0.823563

$-0.22101$

0.825085

0.220411

0.825551

$-0.21721$

0.828048

$-0.21501$

0.829763

$-0.21406$

0.830499

0.214218

0.830377

$-0.21231$

0.831866

$-0.21261$

0.831635

0.21246

0.831748

0.212778

0.8315

$-0.2118$

0.832264

$-0.20985$

0.833785

$-0.20967$

0.833927

$-0.21011$

0.833581

$-0.20885$

0.834566

0.208024

0.83521

0.837854

0.204639

$-0.20488$

0.837668

$-0.20445$

0.838006

$-0.20459$

0.837894

0.2050650 .837521

0.838979

$-0.2032$

0.840256

0.201566

0.841524

0.1983250 .842791

0.19878

0.842435

0.901974

0.901974

0.901976

0.902874

0.903305

0.903305

0.904048

0.905733

0.905733

0.905733

0.905733

0.906428 
Mycobacterium chimaera

Propionibacterium virus PHL301M00

Propionibacterium phage PHLO30NOC

Cutibacterium acnes

Sphingopyxis sp. LPB0140

Acinetobacter calcoaceticus

Arthrospira platensis

Francisella sp. FDC440

Helicobacter canadensis

Leptolyngbya boryana

Erwinia persicina

Marinomonas sp. MWYL1

Propionibacterium virus $\mathrm{P} 1.1$

Thermoanaerobacterium xylanolytic

Cronobacter turicensis

Marinobacter sp. BSs20148

Gottschalkia acidurici

Helicobacter sp. MIT 01-6242

Negativicoccus massiliensis

Butyrivibrio fibrisolvens

Streptococcus phage SM1

Belliella baltica

Stenotrophomonas sp. G4

Bacillus pseudomycoides

Corynebacterium mustelae

Clostridium aceticum

Photorhabdus asymbiotica

Sedimentisphaera salicampi

Microbacterium sp. PM5

Sphingobacteriaceae bacterium GW4

Weissella soli

Acetobacter persici

Enterobacter ludwigii

Colwellia sp. PAMC 20917

Brevibacterium aurantiacum

Pseudomonas extremorientalis

Dickeya fangzhongdai

Marinobacter sp. CP1

Acetobacterium woodii

Flavobacteriaceae bacterium

Providencia heimbachae

Chryseobacterium arthrosphaerae

Algoriphagus machipongonensis

Candidatus Nanopelagicus limnes

Methanocorpusculum labreanum

Tetragenococcus osmophilus

Pseudothermotoga thermarum

Thalassospira marina

Flavobacterium sp. CJ74

Enterococcus faecalis

148.5829

11.94329

0.119183

11.20651

0.524724

0.600479

0.19848

0.842669

0.929087

290851.9

$-0.39362$

2.65194

0.197864

0.843151

0.929203

3.974258

0.104268

1.996391

$-0.19716$

0.843699

0.929525

39.78422

0.266333

.529878

0.196778

0.844001

0.929576

3.690565

$-0.16423$

$(358799$

0.196006

0.844606

0.929961

2.000327

2.405956

$-0.31811$

0.842177

$-0.195$

0.845391

0.929982

10.56693

0.411325

1.629299

$-0.19525$

0.8452

0.929982

34.03336

0.435713

0.195627

0.844902

0.929982

$-0.18846$

246723

0.193933

0.846229

0.930236

4.56005

$-0.13116$

0.972072

$-0.19388$

0.846274

0.930236

15.09883

$-0.26768$

0.677058

$-0.19373$

0.84639

0.930236

4.118542

$-0.26361$

1.392359

$-0.19225$

0.847548

0.93115

4.107069

$-0.27138$

$-0.19201$

0.847733

0.93115

1.156633

0.357614

1.422965

$-0.19072$

0.848748

0.93159

19.8378

0.555863

1.880264

0.190193

0.849158

0.93159

0.594486

0.138693

2.918576

0.190457

0.848951

0.93159

268.8702

$-0.5532$

0.728282

0.190438

0.848966

0.93159

26.1544

$-0.1104$

$-0.18892$

0.850154

0.932403

3.577398

0.188227

0.588937

$-0.18745$

0.851305

0.933158

11.13254

$-0.38002$

1.004476

0.187388

0.851356

0.933158

43.56978

$-0.20104$

2.034878

$-0.18675$

0.851854

0.933423

33.90907

0.15114

1.085425

$-0.18522$

0.853056

0.934097

342.2789

0.093357

817032

0.184986

0.85324

0.934097

7.361256

0.072731

0.504486

0.185054

0.853187

0.934097

6.398264

$-0.20829$

0.394951

0.184153

0.853894

0.934532

1.068434

$-0.22143$

1.134808

$-0.18355$

0.854368

0.93477

112.1781

$-0.52496$

$-0.18196$

0.855617

0.935855

$-0.17977$

0.857331

0.937166

24.6664

2.366539

0.07806

0.433918

$-0.17989$

0.857241

0.937166

$-0.12122$

0.676196

$-0.17926$

0.857732

0.937323

6.757439

0.368612

2.064603

0.178539

0.8583

0.937554

1.206552

$-0.20549$

1.152249

$-0.17834$

0.858458

0.937554

14.23311

$-0.51667$

2.918451

$-0.17704$

0.859479

0.937906

558.6894

$-0.19825$

120437

$-0.17694$

0.859554

0.937906

43.63968

$-0.08859$

0.49999

$-0.17718$

0.859366

0.937906

1.999349

12.28634

0.751127

0.171046

0.864188

0.937973

5.759189

2.911488

$-0.17295$

0.86269

0.937973

$-0.17041$

0.864686

0.937973

6.542068

$-0.25206$

$-0.17576$

0.860486

0.937973

4.437041

0.243866

1.412757

0.172617

0.862952

0.937973

23.63576

$-0.29939$

1.759333

$-0.17018$

0.864872

0.937973

7.173504 
Bacillus muralis

Corynebacterium camporealensis

Propionibacterium virus PHL060L00

Chryseobacterium piperi

Anabaena sp. WA102

Methylorubrum zatmanii

Bacteroides caecimuris

Streptococcus sobrinus

Shewanella pealeana

bacterium 2013Arg42i

Campylobacter ureolyticus

Sulfurospirillum barnesii

Pectobacterium parmentieri

Moritella yayanosii

Propionibacterium virus $\mathrm{P} 1001$

Sodalis endosymbiont of Henestaris $r$

Lactobacillus kefiranofaciens

Dialister sp. Marseille-P5638

Ignavibacterium album

Colwellia psychrerythraea

Lactobacillus crispatus

Leuconostoc mesenteroides

Erwinia billingiae

Kangiella geojedonensis

Bacillus pseudofirmus

Colwellia sp. Arc7-D

Gillisia sp. Hel1_33_143

Endomicrobium proavitum

Oenococcus oeni

Muricauda lutaonensis

Pseudoalteromonas aliena

Chryseobacterium carnipullorum

Erysipelotrichaceae bacterium SG010

Human polyomavirus 5

Streptomyces sp. ICC4

Enterococcus gallinarum

Spirochaeta africana

Flavobacterium crocinum

Flavobacterium sp. HYN0086

Lachnoclostridium phytofermentans

Tetragenococcus halophilus

Lactobacillus buchneri

Methylomonas clara

Psychrobacter sp. P2G3

Aeromonas sp. CU5

Acinetobacter sp. ADP1

Fusobacterium necrophorum

Dictyoglomus thermophilum

Neisseria subflava

Propionibacterium phage PHL085NOC
4.004267

3741.79

11.24133

14.39492

1.685197

7.673865

72.79392

25.51627

2.825226

1.061429

96.5106

3.252359

17.74033

7.437845

8.138781

0.581574

5.193462

32.227

8.619956

1.54146

50.48906

168.6993

78.66198

1.859181

3.953656

7.803426

5.543672

1.269863

2.631609

4.293425

2.049985

38.24804

10.48894

87.06769

739.0736

1.122953

12.87196

19.62746

29.79099

23.76548

29.63525

4.010687

17.5025

17.20799

7.749173

32.7516

38.40802

1.093265

501.8478

7.029518
$-0.232351$

0.041903

$-0.30015$

0.162446

0.508442

0.310646

$-0.15666$

$-0.15175$

$-0.31783$

0.486156

$-0.13218$

0.393077

$-0.13232$

$-0.18158$

0.288462

$-0.48427$

$-0.23679$

$-0.17595$

$-0.16925$

$-0.4134$

$-0.13496$

$-0.13337$

$-0.09782$

0.336468

$-0.20636$

$-0.23293$

$-0.19302$

$-0.45764$

0.299532

$-0.2456$

0.357904

0.101645

0.175397

$-0.30818$

0.149651

$-0.44792$

0.144703

$-0.1013$

0.148468

0.114724

$-0.13468$

$-0.21314$

0.153998

$-0.22864$

$-0.19538$

0.135031

$-0.10613$

$-0.32322$

0.08791

0.224887

1.339706

0.2444

$-0.17344$

0.862309

0.171454

0.863867

1.765813

0.951038

$-0.16998$

0.865028

0.170809

0.864374

0.17458

0.861409

0.173755

0.862058

$-0.16769$

0.866824

0.934201

0.911699

1.905733

$-0.16645$

0.867806

$-0.16678$

0.867546

2.920147

0.166484

0.867776

0.802473

2.389128

$-0.16471$

0.869171

0.164528

0.869316

0.801387

$-0.16511$

0.868854

1.107626

1.756911

$-0.16393$

0.869783

0.164187

0.869584

2.928458

$-0.16537$

0.868655

1.451904

1.10165

$-0.16309$

0.870445

$-0.15971$

0.873107

$-0.15968$

0.873133

2563606

$-0.16126$

0.871892

0.836723

0.834017

$-0.16129$

0.871863

$-0.15992$

0.872947

0.611506

$-0.15997$

0.872903

0.160747

0.872292

1.296027

$-0.15923$

0.87349

$-0.15685$

0.875365

1.219326

$-0.1583$

0.874217

2.893651

$-0.15815$

0.874337

0.15684

0.875371

$-0.15759$

0.874779

1.558483

2.274076

0.157384

0.874942

0.649654

0.15646

0.875671

1.126558

0.155693

0.876275

1.996462

$-0.15436$

0.877324

0.976448

0.153261

0.878193

2.919308

$-0.15344$

0.878055

0.942737

0.153492

0.87801

$-0.15269$

0.878644

0.97635

0.152064

0.879137

0.756815

0.151587

0.879513

$-0.15124$

0.87979

$-0.15067$

0.88024

0.150323

0.88051

$-0.149620 .881065$

$-0.14927 \quad 0.88134$

$0.14886 \quad 0.881664$

$-0.148360 .882062$

0.883412

$-0.14664$

0.883847

0.937973

0.937973

0.937973

0.937973

0.937973

0.937973

0.93964

0.939864

0.939864

0.939864

0.940327

0.940327

0.940327

0.940327

0.940327

0.940327 
Scardovia inopinata

Exiguobacterium antarcticum

Capnocytophaga gingivalis

Chryseolinea sp. KIS68-18

Lysinibacillus sphaericus

Carnobacterium sp. 17-4

Leuconostoc suionicum

Hippea maritima

Propionibacterium virus Lauchelly

Paraliobacillus sp. X-1125

Actinobacillus suis

Moritella viscosa

Enterobacter cloacae complex sp.

Lactobacillus sp. BHWM-4

Spiroplasma monobiae

Pseudomonas cerasi

Bacillus cohnii

Bacillus pumilus

Streptobacillus moniliformis

Lactococcus virus CB14

Carboxydocella thermautotrophica

Lactobacillus hokkaidonensis

Lactobacillus lindneri

Bacillus sp. Y1

Candidatus Izimaplasma sp. HR1

Propionibacterium virus PHL112NOO

Hydromonas sp. F02

Corynebacterium simulans

Nostoc carneum

Propionibacterium virus ATCC29399E

Candidatus Azobacteroides pseudotri

Bacillus simplex

Staphylococcus virus 37

Chryseobacterium bernardetii

Thermus scotoductus

Pseudomonas sp. Os17

Propionibacterium virus MrAK

Pseudomonas protegens

Vibrio parahaemolyticus

Streptococcus ferus

Pseudomonas rhizosphaerae

Acinetobacter defluvii

Human papillomavirus KC5

Sulfurimonas denitrificans

Clostridium isatidis

Lactobacillus sp. CBA3606

Staphylococcus phage phiRS7

Simkania negevensis

Propionibacterium virus ATCC29399E

Campylobacter cuniculorum
16.06705

4.478952

164.0044

62.07483

32.77632

18.03775

3.864944

0.489219

15.73983

6.570954

3.975317

6.933103

18.88925

0.952547

0.572115

1.688385

14.32187

19.31329

14.2544

6.35364

4.188879

7.080684

3.000482

10.59517

5.661131

30.73272

8.840887

10719.91

0.613572

3.147679

8.060376

32.08908

0.668978

34.35544

36.26347

2.281266

23.75985

207.1108

43.45848

11.17969

62.74324

31.75262

3.381131

0.835584

14.83776

0.617972

2.812828

0.359631

23.65383

0.920229
0.133349

0.196394

0.084522

$-0.0849$

0.099914

$-0.1233$

0.925697

1.374437

0.144053

0.885459

0.142891

0.886377

0.593028

0.142526

0.886664

0.59838

0.706871

$-0.14189$

0.887169

0.141347

0.887596

0.87437

$-0.14102$

0.887856

0.140014

0.888649

0.264008

1.885587

0.140179

0.888518

0.196228

1.409968

0.139172

0.889314

1.329782

0.137582

0.890571

0.137579

0.890573

0.136408

0.891499

1.40084

0.191086

0.892352

$-0.13622$

0.891647

0.336343

2.48339

0.135437

0.892266

$-0.39446$

2.928578

$-0.13469$

0.892855

$-0.13394$

0.893448

0.33743

2.519249

$-0.09809$

0.735303

0.13308

0.894129

$-0.13340 .893875$

$-0.13204$

0.89495

$-0.23407$

$-0.18246$

1.778311

$-0.13163$

0.895281

0.898007

$-0.12818$

0.897484

0.128841

0.897593

0.226477

1.759698

0.128702

1.041422

$-0.12856$

0.897709

0.16789

1.311123

0.128051

0.898109

0.127441

0.898592

1.277621

0.125474

0.900149

0.125385

2.927525

0.124966

0.90055

0.90211

0.902549

0.153023

2.818366

0.122996

$-0.09488$

0.776204

0.122442

0.35819

2.926355

$-0.12223$

0.902713

0.122402

0.902581

$-0.08533$

0.70023

$-0.12186$

0.90301

0.904221

0.07722

0.641738

$-0.12033$

$-0.12105$

0.903654

0.241796

2.908934

0.120842

0.903816

$-0.06643$

0.5518

$-0.12038$

0.904181

$-0.07944$

0.667229

$-0.11907$

0.905224

$-0.11936$

0.904991

$-0.1518$

271808

0.117915

0.906135

$-0.1039$

0.88278

$-0.1177$

0.906306

2.58444

0.117443

0.906509

$-0.29936$

2.545316

$-0.11761$

0.906376

0.117009

0.906853

0.908127

2.927389

0.115402

0.908096

$-0.11544$

0.90867

$-0.1139$

0.909318

$-0.1135$

0.947423

0.948126

0.948155

0.948416

0.948593

0.948593

0.948883

0.948883

0.949315

0.950102

0.950102

0.95069

0.95069

0.951071

0.95142

0.951773

0.951941

0.951941

0.952537

0.95261

0.954224

0.954224

0.954224

0.954224

0.954224

0.954459

0.955629 
Polaribacter sp. KT 15

Clostridium tyrobutyricum

Propionibacterium virus PHL141N00

Microbacterium paraoxydans

Actinotignum schaalii

Fictibacillus phosphorivorans

Staphylococcus auricularis

Nostoc linckia

Bacillus sp. FJAT-18017

Candidatus Planktophila vernalis

Solibacillus silvestris

Acinetobacter bereziniae

Enterobacter sp. Crenshaw

Propionibacterium virus PHL114L00

Salinimonas sp. HMF8227

Propionibacterium virus $\mathrm{P} 104 \mathrm{~A}$

Gammapapillomavirus 24

Gluconobacter albidus

Mycoplasma penetrans

Phaeobacter inhibens

Rhizobium sp. S41

Calothrix sp. NIES-3974

Enterobacter sp. E20

Gloeomargarita lithophora

Flavobacterium faecale

Glutamicibacter nicotianae

Erysipelothrix rhusiopathiae

Sedimentisphaera cyanobacteriorum

Salinibacterium sp. CGMCC 1.16371

Runella sp. SP2

Clostridium sp. BNL1100

Zobellia galactanivorans

Candidatus Methanomethylophilus a

Corynebacterium flavescens

Sphingomonas taxi

Alphapapillomavirus 2

Xenorhabdus doucetiae

Bifidobacterium adolescentis

Rhodococcus sp. 2G

Megasphaera stantonii

Gammapapillomavirus 8

Pseudomonas mediterranea

Thermococcus eurythermalis

Leptotrichia sp. oral taxon 847

Vibrio mediterranei

Thermus brockianus

Propionibacterium virus PHL092M00

Paenibacillus sp. BIHB4019

Staphylococcus piscifermentans

Erwinia tasmaniensis

\begin{tabular}{|c|c|c|c|c|c|}
\hline .455734 & 0.271353 & 2.416522 & 0.112291 & 0.910593 & \\
\hline 9.69631 & -0.09104 & 0.829732 & -0.10973 & 0.912626 & 0.962064 \\
\hline 2973 & -0.1728 & 1.603174 & -0.10778 & 0.914168 & 0.962296 \\
\hline 273 & 0.085927 & 0.795534 & 0.108012 & 3986 & \\
\hline 71.8556 & -0.05524 & 0.507157 & -0.10892 & 0.913262 & 0.962296 \\
\hline 312653 & 0.139015 & .283542 & 0.108306 & 3753 & 0.962296 \\
\hline 962 & -0.06755 & 0.621715 & & & 296 \\
\hline 98 & 0.10 & 1.027871 & 0.1062 & & \\
\hline 8.44677 & -0.11082 & 1.050496 & -0.1055 & 5983 & \\
\hline .579394 & 0.310477 & 2.928284 & 0.106027 & & \\
\hline 39 & 0.10 & 07 & & 022 & \\
\hline 40.5431 & 0.082724 & 0.785242 & 0.105348 & & 0.962937 \\
\hline 0.768553 & -0.30553 & 2.924038 & & 781 & 3374 \\
\hline 791 & -0.16764 & 1.632401 & -0.1027 & 205 & \\
\hline 4.372278 & -0.14931 & .452449 & 028 & 0.918121 & \\
\hline 11.11488 & -0.20261 & 1.983879 & -0.10213 & 0.918656 & \\
\hline 2.04043 & 0.292318 & 2.909087 & 0.100485 & & \\
\hline 6.241471 & -0.11702 & 1.167888 & -0.1002 & 88 & 56 \\
\hline 4.903006 & 0.145914 & 1.481646 & 0.098481 & 155 & 876 \\
\hline 36.51103 & 0.062866 & 0.636991 & & & \\
\hline 140.0008 & 0.119015 & 204928 & 0.098774 & 18 & 376 \\
\hline 5266 & -0.14342 & .452865 & -0.09871 & 366 & \\
\hline 1.03001 & -0.28246 & 2.920252 & & & \\
\hline 5.152177 & & 1.373656 & & & \\
\hline 12.06797 & 0.127044 & 1.30845 & 0.097095 & 51 & 0. \\
\hline 154.392 & -0.03736 & 0.386961 & -0.09654 & 3091 & 0.9 \\
\hline 27.78657 & -0.07124 & 0.748221 & & & \\
\hline 0.841646 & 0.256974 & 2.92303 & & & \\
\hline 81.47531 & -0.04389 & 0.479506 & -0.09154 & 7063 & 0.9 \\
\hline 12.17396 & -0.08823 & 0.954942 & -0.0924 & & \\
\hline 8.957877 & 0.095142 & 1.052271 & & & \\
\hline 5.529271 & 0.10587 & 1.202117 & 0.08807 & 321 & 0.9 \\
\hline 0.480921 & -0.27422 & 2.930922 & & & \\
\hline 2591.012 & -0.02393 & 0.265123 & -0.09026 & 0.928084 & 0.967984 \\
\hline 218.458 & & 0.521129 & & & \\
\hline 5.397106 & 0.22766 & 2.496325 & 0.091198 & 0.927335 & 0.967984 \\
\hline 9.842655 & 0.091028 & 1.001887 & & & \\
\hline 297.7237 & -0.05494 & 0.609919 & -0.09007 & 31 & 0.967 \\
\hline 57.5208 & 0.111009 & 1.252796 & 0.088609 & 393 & 984 \\
\hline 10.4156 & 0.087371 & 0.94693 & 0.092268 & & \\
\hline 2.06058 & 0.241923 & 2.715981 & 0.089074 & 0.929023 & 0.967984 \\
\hline 26.52268 & -0.06853 & 0.756379 & -0.0906 & 0.927813 & 67984 \\
\hline .253701 & -0.26591 & 2.937574 & -0.09052 & 0.927875 & 0.967984 \\
\hline 20.98966 & -0.08194 & 0.923148 & & 0.929272 & \\
\hline 4.254875 & 0.134889 & 1.478979 & 0.091204 & 0.927331 & 0.967984 \\
\hline 2.593819 & -0.15721 & 1.749942 & -0.08984 & 0.928416 & 0.967984 \\
\hline & 0.116094 & 1.328239 & 0.087405 & 0.93035 & 0.968128 \\
\hline 8.286873 & -0.10905 & 1.259221 & -0.0866 & 0.930986 & 0.968513 \\
\hline 16.41086 & 0.068453 & 0.802472 & 0.085303 & 0.932021 & 0.968872 \\
\hline 11.30301 & -0.09393 & 1.10295 & -0.08516 & 0.93213 & 0.968872 \\
\hline
\end{tabular}

12.12973

476.1273

171.8556

5.312653

6.68998

8.44677

0.768553

1.11488

2.04043

6.241471

4.903006

36.51103

140.0008

.265266

1.03001

5.152177

7.78657

81.47531

12.17396

8.957877

0.480921

2591.012

9.842655

297.7237
11.30301 


\begin{tabular}{|c|c|c|c|c|c|c|}
\hline Enterococcus hirae & 7.12692 & -0.09034 & & -0.08567 & 0.93173 & \\
\hline Rhodoluna lacicola & 10.30151 & 0.062777 & 0.741782 & 0.08463 & 0.932556 & 969038 \\
\hline Murdochiella vaginalis & 148.8368 & 0.059178 & 0.703625 & 0.084105 & 0.932973 & 0.969195 \\
\hline Erwinia sp. Ejp617 & 1.269311 & 0.243419 & 2.917152 & 0.083444 & 0.933498 & 0.969464 \\
\hline Christensenella massiliensis & 7.5588 & -0.11374 & 1.388497 & -0.08191 & 0.934715 & 173 \\
\hline Acinetobacter sp. LoGeW2-3 & 27.59901 & 0.067988 & 0.828473 & 0.082065 & 0.934595 & 0.970173 \\
\hline Dickeya solani & 3.099263 & -0.16483 & 2.031176 & -0.08115 & 0.935322 & .970527 \\
\hline Kangiella profundi & 2.473937 & 0.153599 & 1.918058 & 0.080081 & 0.936173 & 0.971133 \\
\hline Bacillus sp & 2.691248 & 0.136967 & 1.748785 & 0.078321 & 0.937573 & 0.972272 \\
\hline Lactobacillus johnsonii & 26.95435 & -0.05554 & 0.711781 & -0.07803 & 0.937805 & 0.972272 \\
\hline Xanthomonas fragariae & 26.14016 & -0.04309 & 0.565784 & -0.07615 & 0.939297 & 0.973541 \\
\hline Desulfocapsa sulfexigens & 1.029351 & -0.18546 & 2.458193 & -0.07545 & 0.939859 & 0.973846 \\
\hline Lysinimonas sp. 2DFWR-13 & 166.9952 & -0.0389 & 0.521486 & -0.0746 & 0.940534 & 0.973991 \\
\hline Fervido & 1.172608 & -0.19858 & 2.660908 & -0.07463 & 0.940511 & 0.973991 \\
\hline mesophilum & 3.929503 & 0.161272 & 2.185528 & 0.073791 & 0.941177 & 4379 \\
\hline Yersin & 0.983462 & -0.21223 & 2.920809 & -0.07266 & 0.942075 & 0.975031 \\
\hline Listeri & 6.940779 & -0.10381 & 1.473818 & -0.07044 & 0.943845 & 0.976356 \\
\hline Bacill & 5.922461 & -0.09221 & 1.310236 & -0.07038 & 0.943891 & 0.976356 \\
\hline Enter & 13.9999 & -0.05985 & 0.860291 & -0.06957 & 0.94 & 0.976744 \\
\hline Citrobact & 69.12152 & -0.06225 & 0.912692 & -0.0682 & 0.945622 & 0.977591 \\
\hline Toba & 3.496666 & 0.159904 & 2.381531 & 0.067144 & 0.946 & 3187 \\
\hline Paste & 5.982181 & -0.0809 & 1.225966 & -0.06599 & 0.947389 & 0.978862 \\
\hline Lacto & 154.3112 & 0.045819 & 0.722937 & 0.063378 & 0.949465 & 0.980449 \\
\hline Serra & 1.457547 & 0.184767 & 2.915028 & 0.063384 & 0.94946 & 0.980449 \\
\hline Fusob & 34.3911 & 0.045382 & 0.734543 & 0.061783 & 0.950736 & 0.980852 \\
\hline Cand & 6.358203 & 0.102928 & 1.673501 & 0.061504 & 0.950 & 0.98 \\
\hline Chlan & 0.442258 & 0.181948 & 2.93242 & 0.062047 & 0.950525 & 0.980852 \\
\hline Lacto & 5.511605 & 0.109202 & 1.784454 & 0.061197 & 0.951203 & 0.980852 \\
\hline Elizał & 3.320574 & -0.12551 & 2.019517 & -0.06215 & 0.950 & 0.980852 \\
\hline Thern & 5.440398 & -0.06429 & 1.114731 & -0.05768 & 0.954007 & 0.981949 \\
\hline Bacillus jeotgali & 7.133047 & 0.074898 & 1.302828 & 0.057489 & 0.954156 & 0.981949 \\
\hline coccus sp. NK55a & 1.616312 & 0.121218 & 2.100157 & 0.057718 & 0.953 & 0.981949 \\
\hline Ius & 16.44283 & -0.06119 & 1.043759 & -0.05862 & 0.953252 & 0.981949 \\
\hline Cand & 3.049867 & -0.11055 & 1.907608 & -0.05795 & 0.953 & 1949 \\
\hline Mora & 25438.99 & 0.033711 & 0.578072 & 0.058316 & 0.953497 & 0.981949 \\
\hline Pseu & 3.396001 & -0.11881 & 2.028112 & -0.05858 & 0.953285 & 0.981949 \\
\hline virus Procrass1 & 5.297636 & -0.08075 & 1.413992 & -0.05711 & 0.954461 & 0.981986 \\
\hline Provi & 15.48054 & 0.049992 & 0.888783 & 0.056248 & 0.955144 & 0.982411 \\
\hline Acin & 49.32213 & -0.04106 & 0.735905 & -0.0558 & 0.955502 & 0.982501 \\
\hline & 0.995594 & 0.159899 & 2.920815 & 0.054745 & 0.956342 & 0.982809 \\
\hline Lysinib & 4.384916 & 0.065329 & 1.187016 & 0.055036 & 0.956109 & 0.982809 \\
\hline us sp. Y412MC10 & 5.207906 & 0.073045 & 1.34785 & 0.054194 & 0.956781 & 0.982983 \\
\hline Vibric & 3.167921 & -0.10884 & 2.027455 & -0.05369 & 0.957186 & 0.98301 \\
\hline Lactobacillus fermentum & 57.20131 & 0.042772 & 0.799732 & 0.053483 & 0.957347 & 0.98301 \\
\hline & 103.371 & 0.03403 & 0.641073 & 0.053083 & 0.957666 & 0.98306 \\
\hline Desulfitobacterium dichloroeliminan! & 5.643478 & -0.08957 & 1.768382 & -0.05065 & 0.959605 & 0.984495 \\
\hline Desulfovibrio hydrothermalis & 1.652632 & 0.09969 & 1.955334 & 0.050983 & 0.959339 & 0.984495 \\
\hline Legionella lansingensis & 6.447443 & 0.074324 & 1.495019 & 0.049714 & 0.96035 & 0.984774 \\
\hline Coraliomargarita akajimensis & 1.131628 & 0.109023 & 2.196798 & 0.049628 & 0.960419 & 0.984774 \\
\hline Serratia sp. P2ACOL2 & 11.98418 & 0.066226 & 1.361927 & 0.048627 & 0.961217 & 0.985315 \\
\hline
\end{tabular}


Photorhabdus laumondii

Leuconostoc kimchii

Winogradskyella sp. J14-2

Chryseobacterium joostei

Rhodococcus sp. BH4

Tessaracoccus sp. Marseille-P5995

Dickeya zeae

Anaerolineaceae bacterium oral taxo

Thermodesulfobacterium geofontis

Enterobacter sp. R4-368

Citrobacter braakii

Colwellia sp. MT41

Bacillus xiamenensis

Salimicrobium jeotgali

Sediminispirochaeta smaragdinae

Bacillus flexus

Acinetobacter pittii

Dokdonia sp. 4H-3-7-5

Entomoplasma luminosum

Gemella morbillorum

Leptotrichia sp. oral taxon 498

Streptococcus mutans

Chryseobacterium glaciei

Thermovibrio ammonificans

Lawsonella clevelandensis

Propionibacterium virus PA6

Chlorobium phaeobacteroides

Chroococcidiopsis thermalis

Shewanella livingstonensis

Snodgrassella alvi

Chamaesiphon minutus

Bifidobacterium pseudocatenulatum

Lactococcus phage jm2

Planococcus versutus

Moraxella bovoculi

Marinobacter salinus

Elizabethkingia meningoseptica

Herbinix luporum

Elizabethkingia ursingii

Seonamhaeicola sp. S2-3

Leptotrichia buccalis

Helicobacter bilis

Exiguobacterium sp. $\mathrm{MH} 3$

Salegentibacter salegens

Propionibacterium virus PHL009M11

Brevundimonas diminuta

Sphingobacterium sp. G1-14

Candidatus Baumannia cicadellinicolá

Propionibacterium phage Enoki

Paenibacillus sp. FSL R5-0912
5.045505

4.808677

2.932742

15.15772

67.74868

416.3434

43.96457

6.7511

0.884575

4.80067

2.337661

2.977952

1.289774

0.654661

0.916883

13.33991

296.8867

0.692705

2.185939

261.2665

41.27761

371.7959

19.75911

0.870763

3725.84

33.65728

1.373599

7.742458

4.103694

30.94269

1.487415

61.65321

7.633806

7.358082

18.55434

10.76016

17.28052

12.21892

3.087138

12.12459

130.8253

4.914937

25.02821

5.739384

16.3902

366.2214

85.61734

2.995057

13.50284

1.713079
0.066351 .374255

$-0.1287$

$-0.08439$

2.69905

$-0.05037$

1.78468

$-0.04665$

1.076054

1.009316

0.027169

0.598575

0.021378

0.475989

$-0.05881$

$-0.12019$

1.376331

2.785944

0.05887

1.370769

$-0.11509$

2.645008

0.071597

1.681313

0.090948

2.171549

$-0.12098$

0.042743

2.265323

0.020814

1.054943

$-0.11451$

0.520064

0.087463

2.925447

2.271854

0.020006

0.025876

0.539033

0.702167

$-0.02582$

$-0.0986$

0.709394

0.902659

2.796009

0.505342

0.017658

1.338504

0.045962

0.079405

2.353006

0.040166

1.211787

$-0.04967$

1.564527

$-0.02563$

0.813336

0.081605

2.567569

0.026916

0.878632

0.045838

1.523255

1.483755

$-0.03321$

1.243898

0.027643

1.106516

0.020422

0.752809

$-0.02637$

1.140058

0.052385

2.046365

0.023963

1.055031

0.013931

0.569009

$-0.04878$

2.176363

$-0.03381$

1.323096

0.029577

1.295469

$-0.05554$

2.469643

0.011077

0.484091

0.912971

1.772752

1.381135

0.035349

2.294109

$-0.04828$

0.961494

$-0.04768$

0.961969

$-0.047290 .962285$

$-0.04681$

0.962668

$-0.04622$

0.963136

0.963797

0.045389

0.964177

0.044912

0.965916

$-0.04314$

0.965589

$-0.04294$

0.965747

$-0.04351$

0.965293

0.042584

0.966033

0.041882

0.966593

$-0.04134$

0.967023

$-0.04107$

0.967238

0.040517

0.967681

0.040022

0.968076

$-0.03914$

0.968776

0.038499

0.96929

0.037114

0.970394

0.036851

0.970604

$-0.0364$

0.970963

$-0.03588$

0.971377

$-0.03527$

0.971868

0.034943

0.972125

0.034338

0.972607

0.033746

0.973079

0.033146

0.973558

$-0.03175$

0.974674

$-0.03151$

0.974861

0.031783

0.974645

0.030634

0.975561

0.030092

0.975994

0.028469

0.977288

$-0.0267$

0.9787

0.024982

0.980069

0.027128

0.978358

$-0.02313$

0.981545

0.025599

0.979577

0.985321

0.985531

0.985577

0.985692

0.985893

0.986293

0.986405

0.986916

0.986916

0.986916

0.986916

0.986916

0.987211

0.987315

0.987315 


\begin{tabular}{|c|c|c|c|c|c|c|}
\hline neurinibacillus sp. $\mathrm{XH} 2$ & 2.45432 & -0.0452 & 1.899461 & -0.0238 & & \\
\hline Bacillus wiedmannii & 2.849854 & -0.04836 & 2.235116 & -0.02164 & 0.982738 & 0.992767 \\
\hline ancisella sp. FSC1006 & 2.232749 & -0.04102 & 1.918752 & -0.02138 & 0.982946 & 0.992767 \\
\hline ubacterium] sulci & 3.66976 & 0.017073 & .83649 & 0.02041 & 983716 & 993118 \\
\hline olwellia sp. PAMC 21821 & .737147 & -0.03544 & 1.779484 & -0.01991 & 0.984112 & 0.993118 \\
\hline legamonas hypermegale & .962252 & 0.021262 & 1.058266 & 0.020091 & 0.983971 & 93118 \\
\hline ifidobacterium kashiwanohense & 51.54783 & -0.0137 & 0.719658 & -0.01903 & 0.984816 & 0.993552 \\
\hline andidatus Nanopelagicus hib & 1.005446 & 0.054208 & 2.92061 & 0.018561 & 985192 & 993656 \\
\hline renivorans & 269.9859 & -0.0062 & 0.355381 & -0.01744 & 0.986087 & 0.994284 \\
\hline Lactob & 3.757794 & -0.02026 & 1.43368 & -0.01413 & 0.988725 & 994335 \\
\hline digens & 4.50697 & -0.01483 & 0.909373 & -0.01631 & 0.986986 & 994335 \\
\hline 208 & 3.793889 & 0.024021 & 1.76539 & 0.013607 & 0.989144 & 0.994335 \\
\hline LOs_7 & & 47 & 377 & & & \\
\hline senc & 2.207399 & -0.03832 & 2.683146 & -0.01428 & 8605 & 994335 \\
\hline lyce & 799243 & 0.034025 & 2.411187 & 0.014111 & 0.988741 & 0.994335 \\
\hline Mycc & 59.11031 & -0.02172 & 1.370528 & -0.01585 & 7354 & 335 \\
\hline Psych & 24.46412 & -0.014 & 0.875988 & -0.01598 & 0.987252 & 0.994335 \\
\hline Acine & 3.34783 & 0.02091 & 1.275749 & 0.01639 & 6923 & 335 \\
\hline rep & 7.243825 & 0.01665 & 1.118864 & 0.014881 & 0.988127 & 0.994335 \\
\hline rysip & 9.774439 & -0.01756 & 1.2018 & -0.01462 & 0.988339 & 4335 \\
\hline Flav & 2706 & -0.00806 & 0.64035 & -0.01259 & 9952 & 0.994873 \\
\hline Chry & 46.7 & -0.00 & 0.813006 & -0.01077 & 9141 & 0.995657 \\
\hline Prop & 14.05821 & 0.018098 & 1.76678 & 0.010244 & 1827 & 0.995657 \\
\hline Salini & 42.92157 & -0.00508 & 0.489356 & -0.01037 & 0.991724 & 0.995657 \\
\hline Strep & 68.09921 & 0.007192 & 0.684907 & 0.010501 & 0.991622 & 0.995657 \\
\hline Meso & 1.60533 & -0.02585 & 2.802152 & -0.00922 & 9264 & 0.996199 \\
\hline Pseuc & 28.2637 & -0.00564 & 0.643619 & -0.00876 & 0.993008 & 0.996293 \\
\hline sel & 51.966 & 0.00369 & 0.518363 & 0.007 & 32 & 335 \\
\hline cetoxidans & 4.516637 & -0.00854 & 1.358617 & -0.00628 & 0.994987 & 0.997428 \\
\hline Gem & 5.290717 & -0.0149 & 2.293333 & -0.0065 & 0.994816 & 0.997428 \\
\hline indologenes & 142.9793 & -0.00356 & 0.617804 & -0.00577 & 0.995397 & 0.997428 \\
\hline Thern & 4.425507 & -0.00771 & 1.369081 & -0.00563 & 0.995509 & 0.997428 \\
\hline us Pelagibacter sp. IMCC906 & 0.989955 & -0.00821 & 2.481651 & -0.00331 & 0.997361 & 0.999008 \\
\hline Clostridium sporogenes & 113.2139 & -0.00014 & 0.950962 & -0.00015 & 0.999878 & 0.999887 \\
\hline & 8.934541 & 0.001464 & 1.115858 & 0.001312 & 0.998953 & 0.999887 \\
\hline ychrophilus & 0.614846 & 0.000413 & 2.927245 & 0.000141 & 0.999887 & 0.999887 \\
\hline & 7.971025 & 0.002148 & 1.167765 & 0.00184 & 0.998532 & 0.999887 \\
\hline sp. R11-23-07 & 2.443808 & 0.002417 & 2.901114 & 0.000833 & 0.999335 & 0.999887 \\
\hline egionella clemsonensis & 2.756605 & -0.00083 & 1.551475 & -0.00054 & 0.999573 & 0.999887 \\
\hline
\end{tabular}




\begin{tabular}{|c|c|c|c|c|c|c|}
\hline \multirow[b]{2}{*}{ Bordetella pertussis } & \multicolumn{3}{|c|}{ baseMean log2FoldCh IfcSE } & stat & \multicolumn{2}{|l|}{ pvalue } \\
\hline & 168.6469 & -27.059 & 2.531905 & -10.6872 & 1.17E-26 & $4.25 E-23$ \\
\hline Streptomyces sp. PVA 94-07 & 13.58082 & -23.6226 & 2.511117 & -9.40722 & 5.09E-21 & 9.27E-18 \\
\hline Methylobacterium oryzae & 5.715724 & -22.4231 & 2.413935 & -9.28901 & $1.56 \mathrm{E}-20$ & 1.89E-17 \\
\hline Serratia sp. LS-1 & 5.553945 & -22.401 & 2.452874 & -9.13255 & 6.69E-20 & $6.08 \mathrm{E}-17$ \\
\hline Enterobacteriaceae bacterium ENNIH2 & 15.22691 & -23.7799 & 2.921655 & -8.13921 & $3.98 E-16$ & 2.72E-13 \\
\hline Burkholderia mallei & 14.79097 & -23.7378 & 2.921668 & -8.12476 & 4.48E-16 & 2.72E-13 \\
\hline Streptomyces sp. SM17 & 7.981915 & -20.9761 & 2.849455 & -7.36144 & $1.82 \mathrm{E}-13$ & 9.46E-11 \\
\hline Achromobacter ruhlandii & 89.1919 & -8.22777 & 1.777764 & -4.62815 & 3.69E-06 & 0.001678 \\
\hline Pseudomonas sp. St29 & 11.43776 & -5.35294 & 1.356342 & -3.9466 & 7.93E-05 & 0.032042 \\
\hline Pseudomonas sp. A214 & 12.16837 & -5.49927 & 1.426912 & -3.85396 & 0.000116 & 0.042281 \\
\hline Pectobacterium polaris & 8.751919 & -5.08193 & 1.427456 & -3.56013 & 0.000371 & 0.122592 \\
\hline Clostridium botulinum & 456.6923 & 1.354896 & 0.383196 & 3.535775 & 0.000407 & 0.123262 \\
\hline Streptomyces sampsonii & 13.51615 & -5.83497 & 1.668742 & -3.49663 & 0.000471 & 0.131858 \\
\hline Streptomyces lividans & 11.87456 & -6.61083 & 1.933129 & -3.41975 & 0.000627 & 0.162872 \\
\hline Streptococcus dysgalactiae & 2222.445 & 3.456461 & 1.035554 & 3.33779 & 0.000844 & 0.188333 \\
\hline alibacter subterraneus & 25.63352 & -1.78476 & 0.536559 & -3.3263 & 0.00088 & 0.188333 \\
\hline Clostridium pasteurianum & 88.3911 & 1.979089 & 0.593534 & 3.334418 & 0.000855 & 0.188333 \\
\hline Alcani & 36.89105 & -2.06229 & 0.63516 & -3.24688 & 0.001167 & 0.203237 \\
\hline Clostridium saccharoperbutylacetonicum & 46.73938 & 2.150106 & 0.66744 & 3.221423 & 0.001276 & 0.203237 \\
\hline Borrelia miyamotoi & 35.47271 & 3.11 & 33 & 3.236509 & 121 & \\
\hline GH32-13 & 115 & & 0.5 & & & \\
\hline Sphaerobacter th & 69.92268 & -1.6 & 0.506493 & -3.28858 & 007 & 03237 \\
\hline Pseudomonas mosselii & 10.40075 & -4.43524 & 1.362083 & -3.25622 & 0.001129 & 0.203237 \\
\hline Rhodomicrobium vannielii & 29.59899 & -2.0752 & 0.649421 & -3.19546 & 0.001396 & 0.206063 \\
\hline Met & 30.12784 & -1.74668 & 0.547316 & -3.19 & & 0.206 \\
\hline Staphyloco & 132.67 & 1.697293 & 0.5361 & 3.165999 & 0.001546 & 0.216253 \\
\hline Kosakc & 7.82992 & -5.04277 & 1.85056 & -2.725 & 643 & 0.224 \\
\hline Spiri & & 226 & 0.8643 & -2.43205 & 014 & 0.2 \\
\hline Xylan & 137.1303 & -0.91751 & 0.381865 & -2.4027 & 0.016275 & 0.224107 \\
\hline Acidin & 23.26258 & -1.91107 & 0.736444 & -2.595 & 0.009 & 0.224107 \\
\hline & 36.82908 & -1.56126 & 0.606102 & -2.5759 & 998 & 0.224107 \\
\hline Burkholderia metallica & 248.6665 & -1.41342 & 0.556534 & -2.53969 & 0.011095 & 0.224107 \\
\hline Halobacterium salinarum & 14.70663 & -2.20113 & 0.91074 & -2.41686 & 0.015655 & 0.224107 \\
\hline Bradymonas sediminis & 29.63995 & -1.7617 & 0.634467 & -2.77666 & 0.005492 & 0.224107 \\
\hline Xanthomonas oryzae & 41.13327 & -1.36206 & 0.540926 & -2.51802 & 0.011802 & 0.224107 \\
\hline Burkhold & 314.5218 & -1.53412 & 0.605614 & -2.53316 & 0.011304 & 0.224107 \\
\hline Azoarcus sp. DN11 & 140.899 & -1.44447 & 0.518906 & -2.78368 & 0.005375 & 0.224107 \\
\hline Candidatus Koribacter & 55.69674 & -1.38338 & 0.530398 & -2.60819 & 0.009102 & 0.224107 \\
\hline Halotalea alkalilenta & 38.02159 & -1.70741 & 0.61524 & -2.7752 & 0.005517 & 0.224107 \\
\hline Acaryochloris marina & 16.6701 & -2.28419 & 0.948355 & -2.40858 & 0.016015 & 0.224107 \\
\hline Komagataeibacter xylinus & 46.57891 & -2.51754 & 0.921638 & -2.73159 & 0.006303 & 0.224107 \\
\hline Gibbsiella quercinecans & 35.90542 & -1.40662 & 0.580321 & -2.42387 & 0.015356 & 0.224107 \\
\hline & 12.48747 & -2.55835 & 0.894399 & -2.86041 & 0.004231 & 0.224107 \\
\hline Hoeflea phototrophica & 23.54012 & -1.90729 & 0.741713 & -2.57147 & 0.010127 & 0.224107 \\
\hline & 31.0347 & 1.835378 & 0.707325 & 2.594817 & 0.009464 & 0.224107 \\
\hline Herbaspirillum seropedicae & 281.929 & -1.35456 & 0.485463 & -2.79024 & 0.005267 & 0.224107 \\
\hline Mycoplasma mycoides & 11.86656 & 3.589371 & 1.462071 & 2.454992 & 0.014089 & 0.224107 \\
\hline Collimonas arenae & 192.9469 & -1.42649 & 0.521306 & -2.73638 & 0.006212 & 0.224107 \\
\hline Bradyrhizobium sp. ORS 3257 & 294.1028 & -1.41598 & 0.539231 & -2.62592 & 0.008642 & 0.224107 \\
\hline Thiocystis violascens & 42.58135 & -1.73942 & 0.636023 & -2.73484 & 0.006241 & 0.224107 \\
\hline
\end{tabular}


Burkholderia oklahomensis

Halobacillus halophilus

Serratia sp. FGI94

Kitasatospora setae

Clostridium beijerinckii

Nitrospira defluvii

Dehalogenimonas formicexedens

Candidatus Promineofilum breve

Halomonas chromatireducens

Hyphomicrobium nitrativorans

Cystobacter fuscus

Rhizobium sp. CIAT894

Burkholderia seminalis

Komagataeibacter europaeus

Bradyrhizobiaceae bacterium SG-6C

Cloacibacillus porcorum

Halomicrobium mukohataei

Desulfococcus multivorans

Bordetella trematum

Agrobacterium vitis

Staphylococcus sp. M0911

Echinicola strongylocentroti

Caldisphaera lagunensis

Flavisolibacter tropicus

Methylomicrobium sp. wino1

Burkholderia territorii

Xanthomonas sacchari

Haloarcula sp. CBA1115

Paraburkholderia sprentiae

Cupriavidus pauculus

Caulobacter henricii

Limnohabitans sp. 103DPR2

Turneriella parva

Bacillus oceanisediminis

Pandoraea thiooxydans

Phaeobacter sp. LSS9

Caulobacter mirabilis

Lacunisphaera limnophila

Bordetella genomosp. 13

Pseudomonas sp. GR 6-02

Libanicoccus massiliensis

Desulfuromonas sp. DDH964

Anaeromyxobacter sp. Fw109-5

Alcanivorax xenomutans

Granulicella mallensis

Geobacter sp. M21

Cyanobium sp. NIES-981

Flammeovirgaceae bacterium 311

Collimonas fungivorans

Streptomyces venezuelae
183.6459

13.77836

14.49972

110.9923

110.8038

15.48937

11.2464

43.72492

15.85056

44.41045

157.3627

36.71597

223.2325

13.5014

122.1328

12.83235

10.71414

16.38143

95.39385

40.8151

428.3082

10.26174

3.236013

47.45105

9.102564

336.6107

96.88559

4.482871

852.0856

583.6967

62.36509

54.30918

11.18765

15.10755

225.6205

4.787443

124.2431

56.50022

167.2058

17.51244

33.71358

32.50297

110.3319

29.11469

58.99086

19.77467

61.175

10.91514

295.3755

169.6691

$-1.42531$

$-2.47951$

0.531557

$-2.68138$

$-2.64568$

0.0073320 .224107

$-2.59648$

0.937193

$-2.49891$

0.008153

0.224107

$-0.89525$

0.365408

$-2.44999$

0.012458

0.224107

1.515949

0.562076

2.697053

0.014286

0.224107

$-2.431220 .83008$

$-2.92889$

0.006996

0.224107

$-2.49264$

0.967946

$-2.57518$

0.003402

0.224107

$-1.41938$

0.547507

$-2.59243$

0.010019

0.224107

$-1.83618$

0.672856

$-2.72893$

0.00953

0.224107

$-1.982340 .66991$

$-2.95911$

0.006354

0.224107

$-0.944090 .394486$

$-2.39323$

0.003085

0.224107

$\begin{array}{ll}-1.98738 & 0.675231\end{array}$

$-2.94327$

0.016701

0.224107

$-1.3339$

0.530215

$-2.51577$

0.003248

0.224107

$-2.39926$

0.851444

$-2.81788$

0.011877

0.224107

$-1.52811$

0.524271

$-2.91474$

0.004834

0.224107

$-2.44859$

0.835742

$-2.92984$

0.00356

0.224107

$-2.84495$

0.991777

$-2.86854$

0.003391

0.224107

$-2.22387$

0.903366

$-2.46176$

0.004124

0.224107

$-1.30766$

0.536072

$-2.43934$

0.013826

0.224107

$-1.60629$

0.550412

$-2.91835$

0.014714

0.224107

1.661865

0.58572

2.837301

0.003519

0.224107

-2.73472 1.104525

$-2.47593$

0.00455

0.224107

$5.763062 \quad 2.402939$

2.398339

0.013289

0.224107

$-1.50273$

0.581036

$-2.58629$

0.01647

0.224107

$-3.011061 .041565$

$-2.8909$

0.009701

0.224107

$-1.501770 .578499$

$-2.59599$

0.003841

0.224107

$-1.23297$

0.488301

$-2.52502$

0.009432

0.224107

$-4.248941 .615442$

$-2.6302$

0.011569

0.224107

$-1.277760 .526304$

$-2.42779$

0.008533

0.224107

$-1.47123$

0.531997

$-2.76548$

0.015191

0.224107

$-1.29668$

0.533851

$-2.42892$

0.005684

0.224107

$-1.40602$

0.56621

$-2.48321$

0.015144

0.224107

$-2.01243$

0.839531

$-2.39709$

0.013021

0.224107

$-1.91048 \quad 0.78986$

$-2.41876$

0.016526

0.224107

$-1.341410 .504639$

$-2.65816$

0.015573

0.224107

$-4.340881 .632968$

$-2.65828$

0.007857

0.224107

$-1.33312$

0.531042

$-2.51038$

0.007854

0.224107

$-1.58933$

0.614751

$-2.58533$

0.01206

0.224107

-1.37202 0.50687

$-2.70684$

0.009729

0.224107

$-3.273 \quad 1.220748$

$-2.68114$

0.006793

0.224107

$-1.665010 .647436$

$-2.57169$

0.007337

0.224107

$-1.443610 .553884$

$-2.60633$

0.01012

0.224107

$-1.26684$

0.480295

$-2.63763$

0.009152

0.224107 
Sphingobium sp. RAC03

Thauera hydrothermalis

Herminiimonas arsenicoxydans

Rhizobium sp. NXC14

Clostridium perfringens

Acidianus brierleyi

Afipia sp. GAS231

Cupriavidus necator

Desulfomicrobium baculatum

Fluviicola taffensis

Enterobacteriaceae bacterium w6

Zoogloeaceae bacteirum Par-f-2

Synechococcus sp. KORDI-49

Aquabacterium olei

Lactobacillus paracollinoides

Betaproteobacteria bacterium GR16-43

Nitratireductor basaltis

Burkholderia glumae

Methylococcus capsulatus

Ectothiorhodospira sp. BSL-9

Verrucomicrobium sp. GAS474

Micavibrio aeruginosavorus

Sulfurimonas autotrophica

Limnohabitans sp. 63ED37-2

Ralstonia solanacearum

Acetobacter ascendens

Klebsiella quasivariicola

Rhodobacteraceae bacterium BAR1

Tolumonas auensis

Campylobacter curvus

Phreatobacter cathodiphilus

Desulfomicrobium orale

Sulfuriferula sp. AH1

Bradyrhizobium diazoefficiens

Yoonia vestfoldensis

Achromobacter denitrificans

Hyphomicrobium denitrificans

Bradyrhizobium lablabi

Pseudomonas alcaliphila

Burkholderia sp. MSMB617WGS

Paucibacter sp. KCTC 42545

Novosphingobium tardaugens

Ralstonia pseudosolanacearum

Paenibacillus mucilaginosus

Rhodopirellula baltica

Nitrosospira lacus

Pandoraea vervacti

Burkholderia sp. KJ006

Cupriavidus sp. USMAA1020

Hyphomonas sp. CACIAM 19H1
80.23206

$224.3951-1.46631$

$-1.51441$

78.81154

$-1.59131$

31.51846

93.67786

$-1.46653$

3.853375

1.164571

5.928903

261.6527

$-1.20287$

1629.097

$-1.24597$

18.01281

$-1.62133$

9.970238

$-2.29364$

13.24374

$-3.74665$

45.62121

$-2.08803$

9.668227

$-2.79142$

450.8692

$-1.44249$

10.3205

$-2.48505$

108.8443

$-1.41033$

16.9458

361.8653

$-2.47331$

63.60805

$-1.24674$

32.48223

$-1.63782$

$-1.32597$

40.57865

$-1.36045$

12.94135

$-2.38116$

2.545619

217.835

5.717671

$-1.3376$

27971.97

$-1.34916$

11.83898

$-2.45102$

9.252937

19.20399

$-2.58435$

10.08586

$-1.91635$

$-2.4149$

6.989436

$-2.89255$

130.9256

$-1.2503$

20.50755

$-1.69204$

21.2083

599.8436

$-1.86173$

$-1.39306$

27.89043

$-1.54594$

304.6644

71.18123

$-1.16281$

$-1.49688$

$-1.4308$

$-1.7324$

49.05745

13.2015

$-3.75676$

808.868

38.58774

1274.513

41.68867

7.411003

9.392277

189.6118

98.77178

187.9861

24.91596

$-1.46289$

0.611

$-2.47857$

0.0131910 .224107

$\begin{array}{llll}0.586054 & -2.50201 & 0.012349 & 0.224107\end{array}$

0.588896

$-2.70219$

0.006888

0.224107

0.561315

$-2.61267$

0.008984

0.224107

0.484188

2.405207

0.016163

0.224107

2.3512792 .521565

0.011683

0.224107

0.481276

$-2.49933$

0.012443

0.224107

0.517412

$-2.40807$

0.016037

0.224107

0.642867

0.941265

$-2.52203$

$-2.43677$

0.011668

0.224107

1.288795

$-2.9071$

0.014819

0.224107

0.709717

$-2.94206$

0.003648

0.224107

1.063184

$-2.62553$

0.00326

0.224107

0.557711

$-2.58644$

0.008651

0.224107

1.002636

$-2.47852$

0.009697

0.224107

0.502854

$-2.80465$

0.013193

0.224107

$\begin{array}{lll}0.84649 & -2.92184\end{array}$

0.005037

0.224107

0.516177

$-2.41533$

0.00348

0.224107

0.579487

$-2.82632$

0.015721

0.224107

0.537823

$-2.46545$

0.004709

0.224107

0.566545

$-2.40131$

0.013684

0.224107

0.980187

$-2.42929$

0.016336

0.224107

2.099057

2.723924

0.015128

0.224107

0.552492

$-2.42102$

0.006451

0.224107

0.514882

$-2.62033$

0.015477

0.224107

1.014238

$-2.41662$

0.008784

0.224107

1.026845

0.780461

$-2.51679$

0.015666

0.224107

$-2.45541$

0.011843

0.224107

0.869354

$-2.77781$

0.014073

0.224107

1.152646

$-2.50949$

0.005473

0.224107

$-2.95437$

0.012091

0.224107

0.423204

$-2.67847$

0.003133

0.224107

$-2.62889$

0.007396

0.224107

0.708181

$-2.66333$

0.008566

0.224107

0.523053

$-2.41008$

0.007737

0.224107

0.641449

$-2.39083$

0.015949

0.224107

0.01681

0.224107

0.54633

$-2.73989$

0.006146

0.224107

0.572384

$-2.49972$

0.012429

0.224107

$-2.50665$

0.012188

0.224107

$-2.93166$

0.003372

0.224107

$-2.5411$

0.011051

0.224107 


\begin{tabular}{|c|c|c|c|c|c|c|}
\hline Bradyrhizobium sp. WSM471 & 150.0065 & -1.89876 & 0.627978 & -3.0236 & 0.002498 & \\
\hline Streptococcus intermedius & 372.4424 & -1.68417 & 0.618498 & -2.723 & 0.006469 & 224107 \\
\hline Paraburkholderia terrae & 654.2759 & -1.44772 & 0.548045 & -2.64162 & 0.008251 & 0.224107 \\
\hline Sutterella megalosphaeroides & 45.37689 & -1.51882 & 0.589492 & -2.57649 & 0.009981 & 0.224107 \\
\hline Acidobacterium capsulatum & 43.02372 & -1.82708 & 0.65688 & -2.78145 & 0.005412 & 0.224107 \\
\hline Rhodoferax antarcticus & 142.5333 & -1.56055 & 0.517995 & -3.01268 & 0.00259 & 0.224107 \\
\hline Helicobacter pylori & 33.72427 & 1.696841 & 0.59081 & 2.872058 & 0.004078 & 0.224107 \\
\hline endosymbiont of unidentified scaly snail $i$ & 26.49894 & -1.44324 & 0.571011 & -2.52752 & 0.011487 & 0.224107 \\
\hline Orrella dioscoreae & 162.2748 & -1.32366 & 0.517369 & -2.55845 & 0.010514 & 0.224107 \\
\hline Nitrosomonas sp. AL212 & 6.404686 & -4.75388 & 1.867444 & -2.54566 & 0.010907 & 0.224107 \\
\hline Slackia heliotrinireducens & 51.92732 & -1.70029 & 0.688874 & -2.46822 & 0.013579 & 0.224107 \\
\hline Woeseia oceani & 12.90025 & -1.86035 & 0.724998 & -2.56601 & 0.010287 & 0.224107 \\
\hline Mariprofundus ferrinat & 5.946975 & -3.51469 & 1.305787 & -2.69163 & 0.00711 & 0.224107 \\
\hline Bacteroides vulgatus & 397.4096 & -1.87966 & 0.728031 & -2.58184 & 0.009828 & 0.224107 \\
\hline Melaminivora sp. SC2-9 & 516.0593 & -1.32113 & 0.55112 & -2.39717 & 0.016522 & 0.224107 \\
\hline Pseudomonas chlo & 338.696 & -1.00309 & 0.415335 & -2.41514 & 0.015729 & 0.224107 \\
\hline Brachy & 20.842 & 2.326842 & 0.970557 & 2.397431 & 0.016511 & 0.224107 \\
\hline Natrinema & 7.834716 & -3.32261 & 1.060789 & -3.13221 & 0.001735 & 0.224107 \\
\hline Aeromon & 123.4639 & -0.86863 & 0.358348 & -2.42399 & 0.015351 & 0.224107 \\
\hline Bordetella genor & 96.41652 & -1.22769 & 0.511632 & -2.39955 & 0.016415 & 0.224107 \\
\hline Borde & 104.5153 & -1.44403 & 0.600323 & -2.40542 & 0.016154 & 0.224107 \\
\hline Prevo & 149.1284 & -1.4598 & 0.551596 & -2.6465 & 0.008133 & 0.224107 \\
\hline Archa & 136.107 & -1.19376 & 0.472275 & -2.52768 & 0.011482 & 0.224107 \\
\hline Desulfovibrio desulfuricans & 45.15192 & -1.71739 & 0.567465 & -3.02643 & 0.002475 & 0.224107 \\
\hline Pelobacte & 15.69089 & -2.2998 & 0.821469 & -2.79962 & 0.005116 & 0.224107 \\
\hline Pseud & 847.2532 & -1.41218 & 0.565116 & -2.49893 & 0.012457 & 0.224107 \\
\hline Herminiimonas arsenitoxidans & 49.87558 & -1.84671 & 0.693857 & -2.66152 & 0.007779 & 0.224107 \\
\hline Bradyrhizobium sp. ORS 285 & 196.3878 & -1.24212 & 0.46359 & -2.67934 & 0.007377 & 0.224107 \\
\hline Niab & 48.947 & -1.67647 & 0.700269 & -2.39404 & 0.016664 & 0.224107 \\
\hline Ahnie & 23.62832 & -1.88525 & 0.67058 & -2.81138 & 0.004933 & 0.224107 \\
\hline Burkholderia insecticola & 502.5472 & -1.3348 & 0.539775 & -2.47288 & 0.013403 & 0.224107 \\
\hline Xanthomonas albilineans & 31.75394 & -1.96188 & 0.685388 & -2.86244 & 0.004204 & 0.224107 \\
\hline Achromobacter spanius & 226.4706 & -1.14245 & 0.471376 & -2.42365 & 0.015366 & 0.224107 \\
\hline Halomonas subglaciescola & 22.33265 & -1.95182 & 0.777587 & -2.5101 & 0.01207 & 0.224107 \\
\hline ium zucineum & 146.4734 & -1.37966 & 0.568804 & -2.42555 & 0.015285 & 0.224107 \\
\hline Micromonospora auratinigra & 84.22571 & -1.3853 & 0.529622 & -2.61564 & 0.008906 & 0.224107 \\
\hline Bordetella holmesii & 35.5238 & -1.30479 & 0.545471 & -2.39205 & 0.016755 & 0.224107 \\
\hline Paraburkholderia graminis & 532.5381 & -1.67755 & 0.659927 & -2.54202 & 0.011021 & 0.224107 \\
\hline Pandoraea pnomenusa & 446.7082 & -1.24602 & 0.521198 & -2.39068 & 0.016817 & 0.224107 \\
\hline Fusobacterium varium & 36.80244 & 1.481714 & 0.605508 & 2.447057 & 0.014403 & 0.224107 \\
\hline Dechloromonas sp. HYN0024 & 42.42003 & -1.53742 & 0.64164 & -2.39608 & 0.016572 & 0.224107 \\
\hline Rhodospirillum rubrum & 53.35708 & -1.1195 & 0.45301 & -2.47126 & 0.013464 & 0.224107 \\
\hline Xanthomonas translucens & 166.8761 & -1.29164 & 0.521885 & -2.47495 & 0.013325 & 0.224107 \\
\hline Sorangium cellulosum & 637.7292 & -1.32656 & 0.458445 & -2.8936 & 0.003808 & 0.224107 \\
\hline Desulfovibrio magneticus & 72.51278 & -1.19232 & 0.455145 & -2.61964 & 0.008802 & 0.224107 \\
\hline Azorhizobium caulinodans & 100.8095 & -1.05268 & 0.432306 & -2.43504 & 0.01489 & 0.224107 \\
\hline Porphyrobacter neustonensis & 37.32995 & -1.32995 & 0.50939 & -2.61087 & 0.009031 & 0.224107 \\
\hline Comamonas serinivorans & 396.7172 & -1.25979 & 0.472436 & -2.66658 & 0.007663 & 0.224107 \\
\hline Thioalkalivibrio sulfidiphilus & 55.95433 & -1.49395 & 0.586243 & -2.54835 & 0.010823 & 0.224107 \\
\hline Polaromonas sp. SP1 & 438.8968 & -1.31916 & 0.469877 & -2.80746 & 0.004993 & 0.224107 \\
\hline
\end{tabular}


Herbaspirillum rubrisubalbicans

Acidiphilium multivorum

Halogeometricum borinquense

Nitrobacter hamburgensis

Candidatus Desulforudis audaxviator

Delftia tsuruhatensis

Methylocystis rosea

Myxococcus xanthus

Arcobacter skirrowii

Syntrophobacter fumaroxidans

Azospirillum humicireducens

Oleiphilus messinensis

Pseudomonas kribbensis

Bradyrhizobium icense

Jeongeupia sp. USM3

Cupriavidus sp. USMAHM13

Gemmatirosa kalamazoonesis

Methylosinus trichosporium

Marinithermus hydrothermalis

Halomonas sp. GFAJ-1

Chitinophaga caeni

Micromonospora siamensis

Parvularcula bermudensis

Azoarcus sp. SY39

Staphylococcus cohnii

Bacterioplanes sanyensis

Chlorobaculum parvum

Janthinobacterium agaricidamnosum

Burkholderiales bacterium GJ-E10

Neisseria weaveri

Maricaulis maris

Acidovorax sp. RAC01

Marinobacter similis

Burkholderia sp. BDU6

Rhizobium jaguaris

Burkholderia pseudomultivorans

Thauera chlorobenzoica

Simiduia agarivorans

Streptomyces pristinaespiralis

Verrucomicrobium spinosum

Pelobacter sp. SFB93

Dickeya chrysanthemi

Rhizobium gallicum

Cupriavidus sp. USMAA2-4

Mesorhizobium loti

Nitrosomonas sp. Is79A3

Phyllobacterium zundukense

Indioceanicola profundi

Opitutaceae bacterium TAV5

Deinococcus sp. NW-56

\begin{tabular}{|c|c|c|c|c|c|}
\hline & & & & & \\
\hline & 157 & & & & \\
\hline & & & & & \\
\hline & -1.49163 & 591858 & -2.52025 & & \\
\hline 9944 & -2.61178 & 903583 & -2.89047 & 347 & 4107 \\
\hline 9.6257 & -1.37195 & 79 & -2.668 & & \\
\hline 35252 & -1.68523 & 699045 & -2.41076 & 919 & 107 \\
\hline 79959 & -1.69082 & 567727 & -2.978 & & \\
\hline 69524 & 4.4064 & 1.829601 & 2.408 & 16023 & 107 \\
\hline 13619 & -2.03226 & 807 & -2.51 & 97 & 07 \\
\hline .75493 & -1.51297 & 0.598628 & -2.527 & 91 & \\
\hline 08187 & -2.87083 & 123869 & -2.55441 & 637 & 107 \\
\hline .77939 & -1.78843 & 0.666787 & -2.68 & 315 & 107 \\
\hline 9.7313 & -1.22208 & 0.482163 & -2.53 & 0.01 & 107 \\
\hline 98897 & -1.36299 & & -2.7 & & \\
\hline 6.8894 & -1.97159 & 969 & -2.74 & 5102 & 4107 \\
\hline 3.7385 & -1.366 & & -2.8 & & \\
\hline 0.71884 & -1.56304 & 0.575 & $-2.71 \epsilon$ & 559 & 107 \\
\hline .42035 & -1.56 & 03 & -2.6 & & \\
\hline 564052 & -4.27952 & 1.41 & -3.0 & & \\
\hline 96834 & -1.59452 & 784 & -2.44 & & 107 \\
\hline 79.3109 & -0.865 & 1 & -2.4 & & 107 \\
\hline 484869 & -3.39807 & 593 & -3.0216 & 0.002514 & 0.224107 \\
\hline 83.27145 & -1.55247 & 0.595077 & -2.60885 & 085 & 4107 \\
\hline 249.362 & 1.284491 & 05 & 3.040897 & 0.0 & 107 \\
\hline 6.1952 & -2.74714 & 0.87 & -3.1 & 0.00176 & 4107 \\
\hline 17.8517 & -2.34173 & 0.846231 & $-2.7 €$ & 0.0 & 107 \\
\hline 438.6492 & -1.2803 & 82 & -2.4 & & \\
\hline 7.29262 & -1.48825 & 76 & -2.50725 & 0.01 & 4107 \\
\hline .74321 & -2.62728 & & & & 107 \\
\hline 23.46205 & -1.34985 & 412 & -2.58883 & 963 & 4107 \\
\hline 73 & -1.29 & & & & \\
\hline 14.32679 & -2.38013 & 9278 & -2.56 & 07 & 107 \\
\hline 126.7 & -1.327 & & & & \\
\hline 40.1633 & -1.483 & & & & \\
\hline 38.1 & -1.65616 & & -2.4 & & 107 \\
\hline 3.59065 & -1.71984 & 47 & -2.90001 & 32 & 107 \\
\hline 5.19922 & -2.42 & 17 & -2.51686 & 0.01 & 0.224107 \\
\hline 3 & -1.09304 & & -2.5 & & \\
\hline 31.869 & -1.46103 & 0.566955 & -2.57698 & 0.009967 & 0.224107 \\
\hline 0.12 & -2.842 & & -2.7 & & 107 \\
\hline 28.85487 & -1.43556 & 0.535499 & -2.68078 & 0.007345 & 224107 \\
\hline 62.63379 & -1.43613 & 0.550383 & -2.60933 & 0.00 & 107 \\
\hline 2029.833 & -1.39277 & 0.568388 & -2.45039 & 0.01427 & 0.224107 \\
\hline 33.6498 & -1.32935 & 0.518541 & -2.56363 & 0.010358 & 0.224107 \\
\hline 3.05872 & -2.39035 & 0.77261 & -3.09386 & 0.001976 & 0.224107 \\
\hline 23.16216 & -1.33657 & 0.532479 & -2.51009 & 0.01207 & 0.224107 \\
\hline 27 & -1.11814 & 458231 & -2.44013 & 0.014682 & 0.224107 \\
\hline .10312 & -1.36172 & 0.566204 & -2.40501 & 0.016 & 0.224107 \\
\hline 85.56665 & -1.38366 & 0.531384 & -2.60388 & 0.009218 & 0.224107 \\
\hline
\end{tabular}


Geobacter sulfurreducens

Methyloceanibacter caenitepidi

Staphylococcus haemolyticus

Burkholderia lata

Paenibacillus lentus

Thauera sp. K11

Magnetospirillum sp. ME-1

Cronobacter malonaticus

Methyloversatilis sp. RAC08

Methylocaldum marinum

Bradyrhizobium sp. 3 85S1MB

Amycolatopsis mediterranei

Variibacter gotjawalensis

Bacillus thuringiensis

Bosea sp. RAC05

Variovorax sp. PMC12

Herbaspirillum hiltneri

Vogesella sp. LIG4

Methanobrevibacter millerae

Bdellovibrio bacteriovorus

Mesorhizobium australicum

Xanthomonas citri

Ralstonia insidiosa

Clostridium kluyveri

Rubinisphaera brasiliensis

Stenotrophomonas sp. ZAC14D2_NAIMI4

Bradyrhizobium erythrophlei

Spiroplasma diminutum

Comamonas kerstersii

Candidatus Accumulibacter phosphatis

Granulicella tundricola

Rhizobacter gummiphilus

Laribacter hongkongensis

Haloplanus aerogenes

Chondromyces crocatus

Lysobacter sp. TY2-98

Methylobacterium sp. DM1

Variovorax sp. PAMC 28711

Halomonas sp. SF2003

Methylobacterium nodulans

Cupriavidus basilensis

Komagataeibacter saccharivorans

Pseudorhodoplanes sinuspersici

Streptomyces sp. CdTB01

Paucimonas lemoignei

Thermaerobacter marianensis

Granulibacter bethesdensis

Streptomyces sp. Sge12

Sphingobium yanoikuyae

Geobacter anodireducens
76.2734

22.46993

$4919.519 \quad 1.457589$

577.8981

7.812336

293.593

44.50726

4.97188

87.97234

59.82499

206.669

147.3114

64.95354

414.6642

195.4117

762.0929

188.5284

93.10032

4.696609

17.64614

106.4554

157.19

33222.26

50.31057

11.92803

23.89994

783.5728

5.275995

137.8465

96.31982

38.19342

621.0577

70.8138

23.00241

153.5447

96.12876

104.4969

462.6963

33.60731

172.8149

897.989

16.33488

78.44344

96.34725

35.49579

72.52865

54.16926

36.40445

435.8642

14.38141 $\begin{array}{lll}-1.82333 & 0.665596\end{array}$

0.7030

0.530547

0.527508

0.901335

$-2.21595$

0.433526

$-1.2813$

0.525219

$-4.39142$

1.758181

0.476416

0.517673

138265

0.527965

$-0.88874$

0.36888

$-1.38785$

1.775574

0.546641

$-1.13149$

0.619063

0.471723

$-1.24018$

0.514472

$-1.28189$

0.48406

$-1.30919$

0.484237

4.764426

1.981056

$-1.74586$

0.61167

$-1.26956$

0.447034

$-1.44687$

0.564516

$-1.23272$

0.508708

1.661903

0.695702

$-2.04237$

0.857874

$-2.76176$

1.15777

$-1.11107$

0.465908

5.890991

2.473138

$-1.06956$

0.449386

$-1.23112$

0.517229

$-1.38751$

0.585048

$-1.20258$

0.507042

0.459271

0.92295

$-1.18553$

0.499346

$-1.38807$

0.586589

$-0.84169$

0.355982

0.550961

0.665911

$-1.00222$

0.426611

$-1.24937$

0.531766

$-2.19588$

0.934468

$-1.16091$

0.493894

$-1.01833$

0.433362

$-1.3408$

0.571944

$-1.04581$

0.44956

$-0.99993$

0.431092

$-2.03698$

0.871207

$-1.29051$

0.556262

$-2.03191$

0.875356

$-2.73939$

$0.006155 \quad 0.224107$

$-2.68871$

0.007173

0.224107

2.747332

0.006008

0.224107

$-2.64636$

0.008136

0.224107

$-2.45852$

0.013951

0.224107

$-2.72463$

0.006437

0.224107

$-2.43955$

0.014705

0.224107

$-2.4977$

0.0125

0.224107

$-2.51728$

0.011827

0.224107

$-2.60514$

0.009184

0.224107

$-2.61883$

0.008823

0.224107

$-2.40929$

0.015984

0.224107

$-2.53887$

0.011121

0.224107

2.868164

0.004129

0.224107

$-2.39863$

0.016457

0.224107

$-2.41058$

0.015927

0.224107

$-2.6482$

0.008092

0.224107

$-2.70361$

0.006859

0.224107

2.404993

0.016173

0.224107

$-2.85425$

0.004314

0.224107

$-2.83997$

0.004512

0.224107

$-2.56303$

0.010376

0.224107

$-2.42323$

2.388815

0.015383

0.224107

$-2.38073$

0.016903

0.224425

$-2.38541$

0.017278

0.224912

$-2.38475$

0.01706

0.224912

2.381991

$-2.38005$

0.017091

0.224912

0.017219

0.224912

$-2.38023$

0.01731

0.224912

$-2.37161$

0.017302

0.224912

$-2.37175$

0.017711

0.226074

$-2.37323$

0.017704

0.226074

$-2.37562$

0.017633

0.226074

$-2.37417$ 
Deinococcus irradiatisoli

Bordetella sp. $\mathrm{H} 567$

Serpentinomonas raichei

Rubrivivax gelatinosus

Burkholderia stagnalis

Acidiferrobacter sp. SPIII_3

Bradyrhizobium sp. CCGE-LA001

Xylella fastidiosa

Melaminivora sp. SC2-7

Halomonas aestuarii

Methanoculleus marisnigri

Prosthecochloris sp. GSB1

Burkholderia sp. LA-2-3-30-S1-D2

Acidithiobacillus ferrooxidans

Myxococcus stipitatus

Herbaspirillum robiniae

Paraburkholderia caledonica

Clostridium butyricum

Burkholderia latens

Nitrosospira briensis

Sulfuricaulis limicola

Streptomyces sp. fd1-xmd

Symbiobacterium thermophilum

Hydrogenophaga crassostreae

Ensifer sojae

Klebsiella quasipneumoniae

Cupriavidus pinatubonensis

Roseateles depolymerans

Paenibacillus sp. RUD330

Acidovorax cattleyae

Burkholderia cepacia

Burkholderia sp. OLGA172

Terriglobus roseus

Staphylococcus kloosii

Halobiforma lacisalsi

Candidatus Solibacter usitatus

Frankia casuarinae

Desulfurivibrio alkaliphilus

Bradyrhizobium japonicum

Meiothermus ruber

Aeromonas sp. CA23

Streptomyces sp. CMB-StM0423

Paraburkholderia caffeinilytica

Massilia oculi

Stenotrophomonas sp. SAU14A_NAIMI4_

Marinobacterium aestuarii

Allochromatium vinosum

[Pseudomonas] mesoacidophila

Tsukamurella tyrosinosolvens

Paraburkholderia sp. SOS3
60.7301

108.8432

154.7881

871.2325

446.8427

34.02806

295.8029

18.7334

419.4541

33.73793

13.11792

10.10415

110.4239

11.40067

81.97943

305.8289

800.7273

46.93886

88.83024

5.201434

38.43135

85.87167

77.43769

263.6987

51.76777

25.43974

574.3504

710.6567

47.89124

299.9727

1564.457

2194.81

90.31632

108.9481

13.25768

71.19586

44.68926

16.59006

549.6361

41.33344

22.28956

73.59359

1377.635

533.3084

99.71287

31.25115

52.98542

357.2816

307.1934

874.5797
$-1.19167$

$-1.07939$

0.512503

$-2.32519$

$-2.34111$

$-2.34306$

$-2.33141$

$-2.32481$

$-2.34049$

$-2.32237$

$-2.33021$

$-2.32059$

$-2.33388$

$-2.34283$

$-2.32222$

$-2.32847$

$-2.3252$

$-2.32034$

$-2.33489$

$-1.14636$

$-1.23945$

1.584486

$-1.80098$

$-2.81934$

$-1.3036$

$-1.12869$

$-1.31646$

$-1.19724$

$-1.51158$

$-2.91458$

$-1.10597$

$-1.13229$

$-1.43781$

$-1.27568$

$-1.21456$

$-1.22233$

$-1.07805$

1.365803

$-1.57771$

$-1.14041$

$-1.15172$

$-1.86174$

$-1.17861$

$-1.28701$

$-1.62651$

$-1.33417$

$-1.24186$

$-1.16914$

$-1.34857$

$-1.38244$

$-1.14536$

$-1.26474$

$-0.91519$

$-1.2631$
0.547876

0.490969

0.528142

0.680148

0.777274

1.218312

0.563925

0.488508

0.573

0.520368

0.655449

1.267451

0.480747

0.491575

0.624474

0.554985

0.526572

0.531869

0.469736

0.595137

0.6892

0.497453

0.503531

0.813041

0.514959

0.562551

0.7116

0.584744

0.544128

0.514383

0.594392

0.608602

0.503644

0.557728

0.401594

0.555997
$-2.34682$

2.329619

$-2.31705$

$-2.31414$

$-2.31166$

$-2.31048$

$-2.29748$

$-2.30075$

$-2.30618$

$-2.29956$

$-2.30052$

$-2.3034$

$-2.30244$

$-2.29859$

$-2.30654$

$-2.29817$

$-2.29501$

2.294939

$-2.28919$

$-2.2925$

$-2.28728$

$-2.28985$

$-2.28876$

$-2.28782$

$-2.2857$

$-2.28163$

$-2.28229$

$-2.27291$

0.020062

0.019226

0.232992

0.232992

$\begin{array}{ll}0.019732 & 0.232992\end{array}$

0.0200820 .232992

$0.019258 \quad 0.232992$

$0.020213 \quad 0.232992$

0.0197950 .232992

0.0203090 .232992

0.0196020 .232992

$0.019138 \quad 0.232992$

$\begin{array}{ll}0.020221 & 0.232992\end{array}$

0.0198870 .232992

$\begin{array}{ll}0.020061 & 0.232992\end{array}$

0.0203220 .232992

0.019549

0.232992

0.018934

0.232992

0.019826

0.232992

0.020501

0.233801

0.02066

0.234881

0.020796

0.235692

0.020861

0.235696

0.021591

0.236593

0.021406

0.236593

0.0211

0.236593

0.021473

0.236593

0.021419

0.236593

0.021257

0.236593

0.02131

0.236593

0.021528

0.236593

0.021081

0.236593

0.021552

0.236593

$0.021733 \quad 0.23676$

0.0217370 .23676

0.022068

0.237317

0.021877

0.237317

0.022179

0.237317

0.02203

0.237317

0.022094

0.237317

0.022148

0.237317

0.022272

0.237608

0.022511

0.238765 
Pseudomonas xinjiangensis

Spiroplasma corruscae

Marinobacter sp. LQ44

Rhodoferax ferrireducens

Methylocystis sp. SC2

Pseudomonas sp. K2W31S-8

Hydrogenophaga sp. RAC07

Vulgatibacter incomptus

Pseudomonas sp. M30-35

Hyphomonas sp. Mor2

Enterococcus casseliflavus

Rhizobium favelukesii

Pandoraea oxalativorans

Klebsiella michiganensis

Myxococcus hansupus

Rhodospirillum centenum

Bradyrhizobium oligotrophicum

Pandoraea norimbergensis

Vibrio tritonius

Dyadobacter fermentans

Phycisphaera mikurensis

Bosea sp. PAMC 26642

Acidovorax avenae

Magnetospira sp. QH-2

Streptomyces niveus

Nitrospira japonica

Pseudomonas sp. MT-1

Halanaeroarchaeum sulfurireducens

Streptomyces albireticuli

Alicycliphilus denitrificans

Blastochloris viridis

Bordetella petrii

Synechococcus sp. KORDI-52

Microbulbifer thermotolerans

Devosia sp. 1507

Cupriavidus metallidurans

Gordonibacter urolithinfaciens

Massilia violaceinigra

Ketobacter alkanivorans

Nocardiopsis alba

Burkholderia sp. CCGE1002

Bradyrhizobium sp. 2 39S1MB

Dyella jiangningensis

Pseudomonas sp. S-6-2

Pseudomonas citronellolis

Gordonia terrae

Streptomyces bingchenggensis

Burkholderia pyrrocinia

Nonlabens spongiae

Acidisphaera sp. G45-3
21.13632

5.711168

14.75328

231.7414

26.37976

74.08487

526.5734

90.14097

15.47337

18.05355

12.14678

58.81716

155.5193

44.69368

80.66715

85.30798

206.9574

211.0258

5.19791

40.4326

54.93778

122.0971

1097.524

21.09579

140.423

24.43184

24.19147

7.095579

99.78524

1448.434

78.974

189.785

12.54499

9.885095

41.15662

1000.754

41.72742

210.1204

6.661748

91.43715

972.6326

208.015

93.41765

23.97187

182.1741

436.3728

116.095

481.0565

10.69074

67.0041
$-1.2856$

4.780918

$-1.60926$

$-1.20928$

$-1.49001$

$-1.12873$

$-1.10743$

$-1.30891$

$-1.44249$

$-1.94731$

$-3.17392$

$-1.43934$

$-1.30487$

$-1.67511$

$-1.22504$

$-1.17442$

$-1.10683$

$-1.19945$

$-3.40968$

$-1.28949$

$-1.27025$

$-1.08817$

$-1.21913$

$-1.3033$

$-1.0495$

$-1.45816$

$-1.73731$

$-2.48863$

$-0.9066$

$-1.12859$

$-1.26607$

$-1.19933$

$-2.54378$

$-1.70611$

$-1.49291$

$-1.22613$

$-1.97219$

$-1.04978$

$-2.75133$

$-0.83066$

$-1.19465$

$-1.21414$

$-1.12951$

$-1.40258$

$-0.93943$

$-1.11727$

$-1.00633$

$-1.20861$

$-2.02281$

$-1.17183$

$0.567747-2.26439$

$0.02355 \quad 0.239585$

$2.108112 \quad 2.267867$

0.023337

0.239585

0.707838

$-2.27348$

0.022997

0.239585

0.534223

$-2.26363$

0.023597

0.239585

0.65625

$-2.2705$

0.023178

0.239585

0.49879

$-2.26294$

0.02364

0.239585

0.488655

$-2.26629$

0.023434

0.239585

0.576346

$-2.27105$

0.023144

0.239585

0.637452

0.861536

$-2.26289$

0.023642

0.239585

$-2.26028$

0.023804

0.240552

1.404884

$-2.2592$

0.023871

0.240558

0.637576

$-2.25752$

0.023976

0.240583

0.578368

$-2.25612$

0.024063

0.240583

0.742516

$-2.25599$

$-2.25305$

0.024072

0.240583

0.543725

$-2.24595$

0.024256

0.24176

0.522906

$-2.23967$

0.024707

0.242329

0.494191

$-2.25035$

0.025112

0.242329

$-2.25002$

0.024427

0.242329

515403

$-2.24081$

0.024448

0.242329

0.575457

$-2.2398$

0.025038

0.242329

0.567127

$-2.24268$

0.025104

0.242329

0.543554

$-2.24289$

0.024917

0.242329

0.580594

$-2.24476$

0.024904

0.242329

0.467935

$-2.24284$

0.024783

0.242329

0.650293

$-2.24231$

0.024907

0.242329

0.772957

$-2.24761$

0.024941

0.242329

1.11694

$-2.22808$

0.024601

0.242329

0.407004

$-2.2275$

0.025875

0.242525

0.506088

$-2.23002$

0.025914

0.242525

0.568109

$-2.22857$

0.025746

0.242525

0.537923

$-2.22956$

0.025842

0.242525

1.138119

$-2.23508$

0.025776

0.242525

0.766369

$-2.22623$

0.025412

0.242525

0.667725

$-2.23582$

0.025999

0.242525

0.550386

$-2.22776$

0.025364

0.242525

0.885534

$-2.22712$ 
Thioflavicoccus mobilis

Streptomyces sp. TLI_053

Tistrella mobilis

Dokdonella koreensis

Gemmata obscuriglobus

Blastomonas fulva

Streptomyces dengpaensis

Sulfuricella denitrificans

Nitratireductor sp. OM-1

Croceicoccus marinus

Streptomyces griseus

Planococcus sp. PAMC 21323

Verminephrobacter eiseniae

Dyella japonica

Bordetella avium

Bernardetia litoralis

Burkholderia diffusa

Pseudomonas sp. UW4

Sandaracinus amylolyticus

Niastella koreensis

Paraburkholderia rhizoxinica

Streptomyces spongiicola

Cupriavidus oxalaticus

Paraburkholderia fungorum

Pandoraea apista

Streptomyces sp. Z022

Methylorubrum extorquens

Azospirillum sp. TSH58

gamma proteobacterium HdN1

Pseudomonas sp. CC6-YY-74

Erwinia amylovora

Pseudomonas cedrina

Leadbetterella byssophila

Variovorax paradoxus

Halomonas elongata

Thiomonas sp. X19

Cupriavidus taiwanensis

Ferriphaselus amnicola

Advenella mimigardefordensis

Rhizobium sp. N324

Sodalis glossinidius

Rubrobacter indicoceani

Xanthomonas vesicatoria

Streptomyces glaucescens

Burkholderiales bacterium JOSHI_001

Rhodoplanes sp. Z2-YC6860

Methylomonas methanica

Neisseria meningitidis

Mycolicibacterium vaccae

Azoarcus sp. CIB
37.54092

87.57887

134.711

219.7922

75.78514

39.21137

63.04067

41.01978

42.24016

66.26358

69.89716

12.03255

465.1445

135.6773

51.6194

21.95266

151.225

42.78683

185.9754

143.4488

117.8144

46.89278

424.9388

4549.335

178.8738

67.47411

343.5458

103.3218

17.6127

35.6421

14.34065

60.01945

8.055948

2106.268

35.71908

160.2465

2603.095

37.64612

23.06026

6.373504

9.284885

28.49722

40.09968

59.26876

600.4974

154.9823

9.014651

391.6516

233.6023

95.69597
$-1.10099$

$-0.88435$

0.498115

0.401858

$-1.14883$

0.52186

0.530101

0.551619

$-1.21359$

0.886226

$-1.2203$

0.554573

$-1.17549$

0.532942

$-1.31061$

0.594679

$-0.91335$

0.414325

$-1.22776$

0.558864

$-2.42712$

1.104045

$-1.21779$

0.554155

$-1.05422$

0.480465

$-1.24194$

0.5726

1.566264

0.715366

$-1.34592$

0.617978

$-1.27938$

$-0.98845$

0.590266

0.452377

$-1.16721$

0.537974

$-1.31423$

0.606006

$-1.04635$

0.482209

$-1.10101$

0.511215

$-1.24901$

0.576378

$-1.03159$

0.476535

$-1.12736$

0.517278

$-0.77228$

0.357477

$-1.22883$

0.56722

$-1.45699$

0.670084

$-1.22532$

0.560316

$-2.46362$

1.142334

$-1.21958$

0.562973

$-1.98305$

0.917215

$-1.04554$

0.482192

$-1.10958$

0.509105

$-1.23702$

0.566892

$-1.08899$

0.50141

$-1.2402$

0.576295

$-1.36586$

0.62503

$-4.74088$

2.193685

$-2.026170 .934405$

$-1.444$

0.67004

$-1.24481$

0.578518

$-1.015210 .465848$

$-1.12382$

0.514688

$-1.0834$

0.503667

$-2.58469$

1.19709

$-1.40355$

0.645769

$-0.92557 \quad 0.428444$

$-1.27717$

0.58459

$-2.21031$

$-2.20064$

0.027084

0.246333

$-2.20142$

0.027761

0.246701

$-2.20272$

0.027706

0.246701

$-2.20005$

0.027614

0.246701

$-2.20173$

0.027803

0.246701

$-2.20043$

0.027684

0.246701

$-2.20567$

0.027776

0.246701

$-2.2039$

0.027407

0.246701

$-2.20442$

0.027532

0.246701

$-2.19689$

0.027495

0.246701

$-2.19839$

0.028029

0.246895

$-2.19757$

0.027921

0.246895

$-2.19417$

0.02798

0.246895

$-2.16895$

2.189458

0.028223

0.248007

$-2.17793$

0.030086

0.248373

$-2.16746$

$-2.185$

$-2.16964$

$-2.16868$

$-2.16992$

$-2.15371$

$-2.16699$

$-2.16478$

$-2.17941$

$-2.16037$

$-2.16641$

0.028564

0.248373

0.029411

0.248373

0.0302

0.248373

0.028889

0.248373

0.030034

0.248373

0.030107

0.248373

0.030013

0.248373

0.031263

0.248373

0.030235

0.248373

0.030405

0.248373

0.029301

0.248373

$-2.17433$

0.030744

0.248373

$-2.18683$

03028

0.248373

$-2.15665$

0.02968

0.248373

$-2.16632$

0.028755

0.248373

$-2.16203$

0.031033

0.248373

$-2.16831$ 
Serratia sp. FDAARGOS_506

Burkholderia gladioli

Paraburkholderia aromaticivorans

Azospirillum brasilense

Fuerstia marisgermanicae

Herbaspirillum sp. meg3

Vitreoscilla filiformis

Moorella thermoacetica

Pandoraea sputorum

Roseomonas gilardii

Arachidicoccus sp. KIS59-12

Brachyspira intermedia

Acidovorax carolinensis

Chitinophaga sp. MD30

Pseudomonas brenneri

Paraburkholderia terricola

Gloeobacter violaceus

Paraburkholderia xenovorans

Variovorax sp. HW608

Pseudonocardia sp. HH130629-09

Paraburkholderia caribensis

Streptomyces roseochromogenus

Kushneria konosiri

Gluconacetobacter diazotrophicus

Burkholderia ambifaria

Geobacter sp. DSM 9736

Marichromatium purpuratum

Pseudomonas sp. LTJR-52

Pelodictyon luteolum

Streptomyces sp. S8

Porphyrobacter sp. CACIAM 03H1

Burkholderia sp. CCGE1003

Oxalobacter formigenes

Paraburkholderia phymatum

Methanosaeta harundinacea

Bradyrhizobium sp.

Mycobacterium shigaense

Pseudomonas furukawaii

Halomonas beimenensis

Agrobacterium rhizogenes

Actinoplanes friuliensis

Epibacterium mobile

Serratia rubidaea

Elizabethkingia miricola

Tsukamurella paurometabola

Methylobacillus flagellatus

Sinorhizobium medicae

Desulfovibrio sp. FW1012B

Frankineae bacterium MT45

Serratia proteamaculans

\begin{tabular}{|c|c|c|c|c|c|}
\hline & & & & & \\
\hline & & & -2.16268 & & \\
\hline & & 570974 & & & \\
\hline & -1.19477 & .546621 & -2.18574 & & \\
\hline 63039 & -1.71304 & 787774 & -2.17453 & & \\
\hline & -1.2827 & 585587 & -2.19046 & & \\
\hline 31 & -1.02594 & 0.471858 & -2.17 & & 3373 \\
\hline 17 & -1.38047 & 582 & -2.1 & & \\
\hline 146.668 & -1.30517 & 0.605452 & -2.1557 & 0.031107 & \\
\hline 38.8228 & -1.07275 & 691 & -2.15884 & 62 & \\
\hline 2.25 & -1.29089 & & -2.1 & & \\
\hline .038 & 2.796356 & 1.30 & 2.149702 & 0.03 & \\
\hline 53.8 & -1.14607 & 0.533 & -2.14871 & 0.03 & \\
\hline .20064 & -1.26649 & 186 & -2.1 & 0.03 & \\
\hline .00 & -1.2776 & & & & \\
\hline 30.0 & -1.1584 & 0.54 & -2.1 & 0.032 & 137 \\
\hline 3.23 & -1.09 & 0.5 & -2.13 & 0.0 & \\
\hline 544 & -1.10216 & 0.51 & -2.13622 & 0.03 & \\
\hline 97.1 & -1.08644 & 85 & & & \\
\hline 14.1 & -0.9408 & 0.4 & -2.1 & & \\
\hline 01. & -1.08925 & 86 & -2.1 & 74 & \\
\hline .0 & -0.96029 & & -2.1 & & \\
\hline .63598 & -1.20988 & 0.568267 & -2.12907 & 0.033248 & 255924 \\
\hline 77.53 & -1.08639 & 0.510622 & -2.12758 & 0.033372 & 131 \\
\hline 48.3 & -1.19163 & 0.56 & -2.1 & 0.03 & 564 \\
\hline 15.80 & -1.7519 & 57 & -2.09093 & 0.03 & 64 \\
\hline 60.2 & -0.95185 & 0.45 & -2.0 & & \\
\hline 38.39712 & -1.31777 & & -2.1 & & \\
\hline 1.02 & -1.79246 & 0.85 & -2.09967 & 0.03 & \\
\hline 36.3 & -1.62 & & & & \\
\hline 51.50 & -1.09245 & 0.5 & -2.0 & & 664 \\
\hline 72.0 & -1.199 & & & & \\
\hline 10.61 & -1.84959 & 0.87 & -2.10278 & 485 & \\
\hline 1. & -1.15791 & & & & \\
\hline 132 & & & & & \\
\hline 77.4 & -1.108 & & -2.0 & & \\
\hline 104.7886 & -0.87249 & 145 & -2.09158 & 0.03 & \\
\hline 2.36 & -1.04603 & & -2.1 & 0.03 & \\
\hline 93.39 & -1.18472 & & -2.1 & & \\
\hline 47.52579 & -1.29887 & 0.620136 & -2.09449 & 0.036216 & 0.256664 \\
\hline 158.9 & -0.93911 & & -2.1 & & \\
\hline 19.94595 & -1.33276 & 0.6330 & -2.10521 & 73 & \\
\hline 22.38 & -2.09034 & 0.98 & -2.1 & & \\
\hline 5.940043 & -3.6775 & 1.759157 & -2.09049 & 0.03 & 0.25 \\
\hline 155.3346 & -0.84925 & 0.4022 & -2.11149 & 173 & 664 \\
\hline 15.0711 & -1.48653 & 707903 & -2.09991 & 0.035737 & 0.256664 \\
\hline 35.97563 & -1.36194 & 0.642506 & -2.11974 & 0.034028 & 0.256664 \\
\hline & -1.18093 & 0.558549 & -2.11429 & 0.034491 & 0.256664 \\
\hline 70.63188 & -0.89217 & 0.421603 & -2.116 & 0.034334 & 0.256664 \\
\hline 112.381 & 1.755514 & 0.837136 & 2.097048 & 0.035989 & 0.256664 \\
\hline
\end{tabular}


Pseudonocardia sp. AL041005-10

Pseudomonas sp. DY-1

Rhodopseudomonas palustris

Staphylococcus virus Sextaec

[Polyangium] brachysporum

Methanosarcina barkeri

Dyella thiooxydans

Microbulbifer sp. A4B17

Cyanobium gracile

Mycobacterium paragordonae

Polaromonas sp. JS666

Ottowia sp. oral taxon 894

Conexibacter woesei

Streptomyces albus

Azoarcus sp. BH72

Nocardiopsis dassonvillei

Burkholderia sp. YI23

Prochlorococcus marinus

Streptomyces pratensis

Mitsuaria sp. 7

Mycobacterium sp. EPa45

Simplicispira suum

Breoghania sp. L-A4

Bacillus glycinifermentans

Pseudogulbenkiania sp. NH8B

Burkholderia sp. PAMC 26561

Yangia pacifica

Curvibacter sp. AEP1-3

Filimonas lacunae

Citrobacter amalonaticus

Massilia sp. NR 4-1

Rhodoferax koreense

Cedecea neteri

Martelella mediterranea

Burkholderia sp. BDU8

Sinorhizobium americanum

Xanthomonas arboricola

Sulfolobus islandicus

Methylomonas koyamae

Rhodococcus sp. PBTS 2

Bradyrhizobium ottawaense

Pseudomonas savastanoi

Pseudomonas knackmussii

Bosea vaviloviae

Burkholderia sp. Bp7605

Desulfobulbus sp. ORNL

Halomonas sp. K0116

Stappia sp. ES.058

Serpentinomonas mccroryi

Propionibacterium freudenreichii

262.2201

58.18207

$-0.78314$

0.37061

$-2.11312$

0.03459

0.256664

1054.245

$-1.37001$

0.645705

$-2.12172$

0.033861

0.256664

359.2255

0.441538

$-2.09548$

0.036128

0.256664

409.9923

76312

1.778589

2.11579

0.034363

0.256664

19.82844

0.545818

$-2.0991$

0.035808

0.256664

165.4473

2.456867

1.167205

2.104915

0.035299

0.256664

9.624404

53.7494

$-1.06406$

0.50868

$-2.0918$

0.036456

0.256664

197.0944

$-1.03436$

0.771777

$-2.1044$

0.035343

0.256664

492.9448

$-0.80964$

0.493412

115.2138

$-1.15581$

0.386046

0.036052

0.256664

198.5958

$-1.10955$

0.544734

0.035969

0.256664

92.07557

$-0.98208$

0.524854

$-2.12178$

0.033856

0.256664

58.29702

$-0.86344$

0.467733

$-2.11403$

0.034513

0.256664

173.9457

$-2.05565$

0.035758

0.256664

227.8715

$-0.8539$

0.971184

$-2.09739$

0.035959

0.256664

67.67244

$-1.27262$

0.405848

$-2.11665$

0.03429

0.256664

$-2.104$

0.035379

0.256664

20.74386

0.954356

0.60769

$-2.09419$

0.036243

0.256664

1151.459

$-2.97862$

2.112649

0.034631

0.256664

127.5897

$-1.03448$

$-2.10045$

0.035689

0.256664

391.6697

$-1.01604$

0.496048

$-2.08545$

0.037028

0.257176

58.51215

$-1.02375$

0.487025

$-2.08621$

0.036959

0.257176

13.21588

$-1.3225$

$-2.08821$

0.036778

0.257176

$-2.0853$

0.037042

0.257176

104.4173

$-1.95583$

0.937893

$-2.08535$

0.037038

0.257176

351.3319

$-1.01154$

0.485482

$-2.08358$

0.037198

0.257766

75.54985

$-1.21689$

0.58444

$-2.08214$

0.037329

0.258182

192.9235

$-1.00536$

0.483407

$-2.07974$

0.037549

0.25921

97.39296

$-1.08324$

0.521353

$-1.107$

0.532757

$-2.07775$

0.037733

0.259493

28.90698

$-2.33878$

1.126606

$-2.07788$

0.037721

0.259493

219.5538

$-0.96379$

0.464184

$-2.07595$

0.037898

0.25965

928.1802

$-1.12224$

0.541048

$-2.0763$

0.037866

0.25965

47.05562

$-1.07903$

0.521237

$-2.0742$

0.038061

0.260275

67.62622

$-1.18819$

0.57387

$-2.07013$

0.03844

0.260705

133.055

$-1.3215$

0.638506

$-2.07048$

0.038408

0.260705

34.15315

$-1.25356$

0.605062

$-2.06968$

0.038482

0.260705

31.93614

$-1.37089$

0.662014

$-2.07179$

0.038285

0.260705

5.833522

4.413886

2.133882

$-2.07078$

0.038379

0.260705

4.602243

$-3.19111$

1.543635

2.068477

0.038595

0.260784

383.4314

1.321351

0.639138

$-2.06727$

0.038709

0.260784

689.9988

$-1.0832$

0.52462

2.067396

0.038697

0.260784

13.81628

$-2.87858$

1.393951

$-2.06473$ 
Brenneria goodwinii

Streptomyces reticuli

Synechococcus sp. PCC 7336

Brevibacterium siliguriense

Pseudomonas resinovorans

Geobacter bemidjiensis

Clostridium novyi

Methylibium petroleiphilum

Bosea sp. AS-1

Mesorhizobium oceanicum

Rhodobaca barguzinensis

Mycolicibacterium rhodesiae

Aureimonas sp. AU20

Bifidobacterium scardovii

Atopobium parvulum

Paraburkholderia phytofirmans

Burkholderia sp. JP2-270

Natronococcus occultus

Halioglobus pacificus

Olsenella sp. GAM18

Azotobacter chroococcum

Clostridium sp. DL-VIII

Luteibacter rhizovicinus

Arcobacter sp. L

Burkholderia pseudomallei

Frankia inefficax

Chlorobium chlorochromatii

Paenibacillus ihbetae

Staphylococcus sp. AntiMn-1

Thauera humireducens

Chelatococcus sp. CO-6

Synechococcus sp. KORDI-100

Delftia acidovorans

Lysobacter maris

Rhodovulum sp. P5

Nitrosomonas ureae

Rhizobium sp. ACO-34A

Shewanella loihica

Streptomyces sp. Tu6071

Aromatoleum aromaticum

Marinobacter salarius

Mesorhizobium opportunistum

Desulfotalea psychrophila

Arcobacter mytili

Lysobacter enzymogenes

Polaribacter sp. SA4-10

Salipiger profundus

Burkholderia sp. NRF60-BP8

Pontimonas salivibrio

Anaeromyxobacter dehalogenans

13.03815

64.84636

$-1.46805$

0.712599

$-2.06013$

0.0393860 .261825

14.89526

$-1.11503$

0.541115

$-2.06062$

0.039339

0.261825

245.1817

$-1.53209$

0.745303

$-2.05566$

0.039815

0.261839

130.0303

0.456697

$-2.05451$

0.039926

0.261839

25.51809

.543288

$-2.05357$

0.040017

0.261839

36.54554

704.7983

$-1.55488$

0.757114

$-2.0537$

0.040005

0.261839

161.1735

77.50083

$-1.10585$

0.590828

2.050819

0.040285

0.262155

15.88995

$-1.00911$

0.539562

$-2.04954$

0.04041

0.262155

137.8251

$-0.90257$

0.491839

$-2.05171$

0.040198

0.262155

90.24364

$-1.63082$

$-2.04875$

0.040487

0.262155

44.07463

$-0.8329$

0.79583

$-2.04921$

0.040441

0.262155

149.9146

$-1.17728$

0.406848

$-2.0472$

0.040638

0.262155

3733.867

$-0.85311$

$-2.04716$

0.040642

0.262155

$-1.27472$

6612

$-2.04773$

0.040587

0.262155

$-1.11252$

626288

$-2.03536$

0.041815

0.263101

209.7013

$-1.10253$

0.546789

$-2.03464$

13.91119

$-1.69151$

0.541054

$-2.03775$

0.041887

0.263101

12.6363

$-1.96367$

0.832501

$-2.0318$

0.041575

0.263101

21.72054

$-1.31784$

0.968178

$-2.02821$

0.04217

0.263101

120.8283

$-1.06854$

0.648255

$-2.0329$

0.042538

0.263101

31.66012

1.08755

0.524982

$-2.03539$

0.042062

0.263101

82.61218

$-0.89106$

0.536086

2.028686

0.041812

0.263101

11.34484

3.563391

0.438666

$-2.03129$

0.04249

0.263101

2104.637

$-1.19598$

1.757404

2.027644

0.042226

0.263101

117.5753

$-0.98081$

0.589101

$-2.03017$

0.042597

0.263101

4.539451

$-2.49079$

0.480428

$-2.04153$

0.042339

0.263101

7.766523

$-2.00743$

1.222761

$-2.03702$

0.041198

0.263101

27.19047

$-2.30598$

0.984119

$-2.03982$

0.041648

0.263101

98.98766

$-1.17122$

136645

$-2.02876$

0.041368

0.263101

126.0558

$-1.07671$

0.57742

$-2.02837$

0.042483

0.263101

5.215298

$-2.5144$

0.530865

$-2.02822$

0.042522

0.263101

626.2661

$-1.24454$

1.235967

$-2.03436$

0.042538

0.263101

105.8091

$-1.01145$

0.609869

$-2.04067$

0.041916

0.263101

49.5127

$-1.10921$

$-2.04281$

0.041284

0.263101

34.72659

$-1.43492$

0.543683

$-2.04017$

0.041071

0.263101

69.26793

$-0.9807$

0.704023

$-2.03818$

0.041333

0.263101

21.20789

$-1.19566$

0.483554

$-2.02811$

0.041532

0.263101

14.60313

$-2.96318$

0.585677

$-2.04149$

0.042549

0.263101

89.44839

$-1.077$

$-2.02981$

0.041202

0.263101

20.38511

$-1.41653$

0.531857

$-2.02499$

0.042376

0.263101

105.7083

$-1.10053$

0.700312

$-2.02272$

0.042869

0.264333

4.461436 
Frateuria aurantia

Streptococcus pluranimalium

Leuconostoc garlicum

Actinoplanes derwentensis

Pseudarthrobacter chlorophenolicus

Cronobacter muytjensii

Mesorhizobium japonicum

Streptomyces cyaneogriseus

Erythrobacter sp. Alg231-14

Methanococcus maripaludis

Myxococcus macrosporus

Streptomyces sp. Mg1

Clostridium saccharobutylicum

Ethanoligenens harbinense

Paraburkholderia phenoliruptrix

Burkholderia plantarii

Kurthia sp. $11 \mathrm{kri321}$

Massilia sp. WG5

Fimbriimonas ginsengisoli

Cupriavidus nantongensis

Xylella taiwanensis

Mesorhizobium ciceri

Kitasatospora albolonga

Hydrogenophaga sp. PBC

Salinivirga cyanobacteriivorans

Dickeya dianthicola

Pantoea stewartii

Leptolyngbya sp. 0-77

Mycobacterium marseillense

Acidovorax citrulli

Hyphomonas neptunium

Streptomyces albidoflavus

Methyloceanibacter sp. wino2

Xenorhabdus nematophila

Pseudomonas balearica

Chromobacterium rhizoryzae

Methanosarcina horonobensis

Janthinobacterium sp. 1_2014MBL_MicD

Opitutus sp. GAS368

Streptosporangium roseum

Enterococcus thailandicus

Ramlibacter tataouinensis

Streptomyces globisporus

Sphingomonas wittichii

Roseobacter litoralis

Paraburkholderia hospita

Natronomonas moolapensis

Acidaminococcus fermentans

Natronobacterium gregoryi

Ottowia oryzae

\begin{tabular}{|c|c|c|c|c|c|}
\hline & & & & & \\
\hline 91556 & -1.70322 & 0.845563 & -2.0143 & 0.043978 & 0.266014 \\
\hline & -3.00721 & 1.494028 & -2.01282 & 0.044133 & 0.266014 \\
\hline .4208 & -0.78017 & 0.388448 & -2.00843 & 0.044598 & 0.266014 \\
\hline .5971 & -0.739 & 0.368554 & -2.00515 & 0.044947 & 0.266014 \\
\hline .0115 & -2.14676 & 1.06678 & -2.01238 & 0.04418 & 0.266014 \\
\hline .81095 & -1.25336 & 0.623915 & -2.00886 & 0.044552 & 0.266014 \\
\hline 2.08195 & -1.48786 & 0.738573 & -2.01451 & 0.043956 & 0.266014 \\
\hline 2.05674 & -1.87724 & 0.935382 & -2.00693 & 0.044757 & 0.266014 \\
\hline 4.05558 & 1.98705 & 0.991545 & 2.003994 & 0.045071 & 0.266014 \\
\hline 51.0961 & -0.99519 & 0.494156 & -2.01393 & 0.044017 & 0.266014 \\
\hline .54824 & -1.22074 & 0.609279 & -2.00357 & 0.045116 & 0.266014 \\
\hline .45142 & 1.499813 & 0.745604 & 2.01154 & 0.044268 & 0.266014 \\
\hline .12761 & -1.53298 & 0.764733 & -2.00459 & 0.045007 & 0.266014 \\
\hline 26.9914 & -1.24695 & 0.621952 & -2.0049 & 0.044974 & 0.266014 \\
\hline 563.499 & -1.09217 & 0.542665 & -2.0126 & 0.044156 & 0.266014 \\
\hline 17.23796 & -2.05974 & 1.026485 & -2.0 & 0.044793 & 6014 \\
\hline 64.2959 & -1.12449 & 0.562029 & -2.00076 & 0.045418 & 0.266873 \\
\hline 1.57985 & -1.23512 & 0.617323 & -2.0 & 5417 & 266873 \\
\hline 42.6339 & -1.26787 & 0.633878 & -2.00017 & 0.045481 & 0.266873 \\
\hline 8.72016 & -1.95007 & 0.976018 & -1.99799 & 0.045718 & 0.267828 \\
\hline 359.9747 & -1.03541 & 0.518578 & -1.99663 & 0.045865 & 0.267831 \\
\hline 63.8445 & -1.10399 & 0.552929 & -1.99663 & 0.045865 & 0.267831 \\
\hline 693.7178 & -1.06431 & 0.533235 & -1.99594 & 0.04594 & 0.267836 \\
\hline 5.547855 & -2.78123 & 1.396127 & -1.99211 & 0.04636 & 0.269849 \\
\hline 24.96843 & -1.91751 & 0.963438 & -1.99028 & 0.04656 & 0.270153 \\
\hline 16.47738 & -1.48857 & 0.747668 & -1.99095 & 0.046487 & 0.270153 \\
\hline 24.04206 & -1.38033 & 0.695053 & -1.98593 & 0.047041 & 0.27251 \\
\hline 75.22022 & -0.79518 & 0.400855 & -1.98371 & 0.047288 & 0.273504 \\
\hline 425.9206 & -1.05537 & 0.532935 & -1.98031 & 0.047669 & 0.275204 \\
\hline 16.44598 & -1.70906 & 0.864743 & -1.97638 & 0.048112 & 0.275204 \\
\hline 12.53208 & -3.5195 & 1.780346 & -1.97686 & 0.048057 & 0.275204 \\
\hline 43.18165 & -1.05037 & 0.53063 & -1.97947 & 0.047763 & 0.275204 \\
\hline 5.084416 & -3.28662 & 1.661574 & -1.97801 & 0.047927 & 0.275204 \\
\hline 69.23482 & -0.9724 & 0.491891 & -1.97686 & 0.048058 & 0.275204 \\
\hline 67.74598 & -0.94548 & 0.478121 & -1.97748 & 0.047987 & 0.275204 \\
\hline 4.350607 & -3.56039 & 1.803624 & -1.97402 & 0.048379 & 0.275388 \\
\hline 202.557 & -1.04152 & 0.52913 & -1.96837 & 0.049026 & 0.275388 \\
\hline 65.958 & -1.06649 & 0.541694 & -1.96881 & 0.048975 & 0.275388 \\
\hline 119.7382 & -0.74123 & 0.375735 & -1.97275 & 0.048524 & 0.275388 \\
\hline 7.18122 & -2.1847 & 1.107472 & -1.97269 & 0.04853 & 0.275388 \\
\hline 1399.853 & -1.0277 & 0.52088 & -1.97301 & 0.048495 & 0.275388 \\
\hline 81.51059 & -1.07554 & 0.546563 & -1.96782 & 0.049089 & 0.275388 \\
\hline 220.3546 & -0.95237 & 0.483367 & -1.97029 & 0.048805 & 0.275388 \\
\hline 17.0225 & -1.62649 & 0.824908 & -1.97172 & 0.048641 & 0.275388 \\
\hline 1034.551 & -1.11061 & 0.563491 & -1.97094 & 0.048731 & 0.275388 \\
\hline 9.207816 & -2.12172 & 1.078393 & -1.96748 & 0.049128 & 0.275388 \\
\hline 13.4747 & -1.59156 & 0.808353 & -1.9689 & 0.048965 & 0.275388 \\
\hline 5.544264 & -3.07348 & 1.559602 & -1.97068 & 0.048761 & 0.275388 \\
\hline 28.7981 & -1.13327 & 0.576247 & -1.96665 & 0.049224 & 0.275502 \\
\hline
\end{tabular}


Thermomicrobium roseum

Pseudoalteromonas arctica

Sinorhizobium fredii

Candidatus Thiodictyon syntrophicum

Myxococcus fulvus

Burkholderia cenocepacia

Xanthomonas phaseoli

Stenotrophomonas sp. YAU14D1_LEIMI4

Polaromonas naphthalenivorans

Paenibacillus swuensis

Pseudomonas alcaligenes

Comamonas aquatica

Bradyrhizobium sp. SK17

Arcobacter ellisii

Leptothrix cholodnii

Aminomonas paucivorans

Burkholderia stabilis

Robiginitalea biformata

Chelatococcus daeguensis

Thermogutta terrifontis

Halobacterium sp. DL1

Pseudohongiella spirulinae

Streptomyces albulus

Streptomyces sp. CB09001

Bradyrhizobium sp. ORS 278

Zobellella denitrificans

Paenibacillus glucanolyticus

Cryobacterium sp. LW097

Pseudomonas xanthomarina

Sphingomonas sp. MM-1

Lactobacillus jensenii

Campylobacter gracilis

Serratia marcescens

Streptococcus agalactiae

Diaphorobacter polyhydroxybutyrativora

Salinarchaeum sp. Harcht-Bsk1

Endozoicomonas montiporae

Pseudomonas saudiphocaensis

Thiolapillus brandeum

Streptomyces alboflavus

Thiomonas intermedia

Pirellula staleyi

Halothiobacillus neapolitanus

Rhodococcus sp. WB1

Pseudomonas asplenii

Streptomyces sp. NEAU-S7GS2

Candidatus Babela massiliensis

Alkalilimnicola ehrlichii

Mycoplasma hominis

Staphylococcus stepanovicii
12.64715

7.305411

283.3686

82.00162

134.9048

1950.213

150.2232

31.45561

498.5703

9.254763

82.05727

410.442

551.2775

10.05856

683.3294

10.99231

606.8436

14.77055

82.9833

6.162704

23.36695

3.3028

86.21858

32.14666

193.1088

61.95516

18.70821

102.6738

27.51978

155.0713

44.79921

125.5727

242.9782

674.1185

1039.434

12.57477

4.415775

23.27971

10.8845

67.81868

234.8241

13.2394

19.94744

28.08025

13.40002

107.4546

3.498963

41.17225

6.03001

88.28388
$-1.74865$

$-3.95734$

0.89027

$-0.87538$

2.017755

$-1.00315$

0.446333

0.51131

0.450161

1.07193

0.548526

$-1.41585$

0.723196

$-2.16086$

$-0.9907$

1.106212

$-1.7319$

0.50752

0.886943

$-0.96993$

0.495196

$-0.94836$

0.484483

$-0.97552$

0.49865

2.957699

1.514918

$-1.06929$

0.547883

$-1.38624$

0.709193

$-1.01307$

0.517895

$-1.59696$

0.819002

$-1.26805$

0.650195

$-1.79232$

0.920878

$-1.54771$

0.795396

$-3.09452$

1.589308

$-0.76582$

0.393526

$-1.28008$

0.658819

$-0.91796$

0.473016

$-1.08989$

0.563237

$-1.64842$

0.852577

$-0.98546$

0.510291

$-1.15329$

0.59714

$-1.04682$

0.542441

1.463548

0.758449

$-1.34745$

$-0.79985$

0.699903

0.415473

0.533054

026693

0.59387

$-1.14242$

0.861671

$-2.66958$

1.384247

$-1.31679$

0.684534

$-1.63316$

0.848701

$-0.92165$

0.478328

$-0.93972$

0.492893

$-1.41308$

0.738191

$-1.35202$

0.70621

$-2.13676$

1.121659

$-2.27351$

1.190504

$-0.86132$

0.449975

4.574197

2.396281

$-0.99158$

0.519255

2.800669

1.468767

1.390734

0.729227

$-1.96418$

$-1.96126$

$-1.96127$

0.0495

0.276677

$-1.96192$

0.049849

0.277293

$-1.95526$

0.277293

$-1.9542$

0.277293

$-1.95777$

0.050552

0.278046

$-1.95339$

0.050677

0.278046

$-1.95204$

0.050257

0.278046

$-1.95266$

0.278046

$-1.95868$

0.278046

$-1.95747$

0.05086

0.278046

$-1.95632$

0.05015

0.278046

1.952382

0.050292

0.278046

$-1.95167$

0.278046

$-1.95467$

0.050893

0.278046

$-1.95613$

0.050978

0.278046

$-1.94988$

0.050622

0.278046

$-1.95026$

0.05045

0.278046

0.05119

0.278369

$-1.94632$

0.051145

0.278369

$-1.94583$

0.051616

0.279336

$-1.94709$

0.051675

0.279336

$-1.94606$

$-1.94299$

0.051524

0.279336

0.051648

0.279336

$-1.94066$

0.052017

0.280769

$-1.93504$

0.052299

0.281875

$-1.93346$

0.052985

0.285146

$-1.93117$

0.053179

0.285771

$-1.93136$

0.053461

0.28644

$-1.92983$

0.053438

0.28644

1.92966

0.053627

0.286601

$-1.92519$

$-1.92516$

0.053649 
Frankia symbiont of Datisca glomerata

Burkholderia vietnamiensis

Nocardia terpenica

Desulfovibrio fairfieldensis

Kiritimatiella glycovorans

Castellaniella defragrans

Paludisphaera borealis

Methylorubrum populi

Megasphaera hexanoica

Rhodanobacter denitrificans

Mycobacterium colombiense

Chromobacterium sp. IIBBL 112-1

Paenibacillus sp. FSL R5-0345

Leuconostoc lactis

Actinomyces sp. oral taxon 414

Lysobacter antibioticus

Melittangium boletus

Sphingomonas sp. YZ-8

Paenibacillus sp. FSL R7-0331

Sphingomonas koreensis

Nocardiopsis gilva

Cellulomonas fimi

Bacillus weihaiensis

Pseudomonas sabulinigri

Mycolicibacterium litorale

Burkholderia ubonensis

Novosphingobium sp. PP1Y

Aeromonas sp. ASNIH3

Rhizobium tropici

Burkholderia multivorans

Halomonas sp. JS92-SW72

Luteimonas sp. JM171

Desulfococcus oleovorans

Mycolicibacterium vanbaalenii

Geobacter uraniireducens

Cellvibrio sp. PSBB006

Kosakonia cowanii

Bifidobacterium actinocoloniiforme

Stenotrophomonas sp. ZAC14D2_NAIMI4

Chlorobaculum limnaeum

Alicyclobacillus acidocaldarius

Caulobacter vibrioides

Sphingomonas sanxanigenens

Sulfurifustis variabilis

Achromobacter xylosoxidans

Polynucleobacter asymbioticus

Crenobacter sp. K1W11S-77

Bacillus sp. FJAT-45348

Buchnera aphidicola

Rhizobium phaseoli
78.71488

812.7878

154.7023

20.84675

27.26604

191.56

95.08039

285.1172

12.34926

175.5372

101.3558

33.47682

5.208972

5.941719

935.0356

106.6581

88.39072

31.72271

10.36784

174.8556

82.53712

236.6279

6.183021

36.37352

118.8565

1162.147

52.39447

288.7873

49.40322

1142.131

151.737

75.19359

9.186825

207.6783

18.8806

10.75844

43.18606

18.24168

38.88718

20.71421

118.594

203.1776

158.6225

63.67082

946.3929

14.35176

94.38934

20.22851

79.10302

132.3072
$-0.74343$

$-1.211210 .634738$

$-0.90751$

0.47582

0.757574

0.67829

$-1.29883$

$-1.04101$

$-0.84548$

$-0.76563$

$-1.71414$

$-0.9775$

$-0.74772$

$-2.93681$

$-2.36001$

$-3.67134$

$-1.03656$

$-0.93921$

$-0.90393$

$-1.26917$

$-1.71918$

$-0.9039$

$-0.80372$

$-0.85757$

$-2.23057$

$-1.00221$

$-0.70119$

$-1.00326$

$-1.07296$

$-1.23714$

$-1.11723$

$-1.01234$

$-1.11986$

$-1.03208$

$-1.43274$

$-0.77529$

$-1.30771$

$-1.37913$

$-1.42138$

$-1.13187$

$-1.70658$

$-1.66364$

$-0.98751$

$-0.8825$

$-0.7777$

$-0.91689$

$-0.88429$

$-2.27547$

$-1.10935$

2.427333

0.99804

$-1.17684$
0.543073

0.439851

0.400737

0.893443

0.511719

0.392404

1.538028

1.227408

1.919987

0.540281

0.490977

0.470574

0.665829

0.898991

0.473785

0.422132

0.450484

1.172859

0.5271

0.36908

0.529369

0.566625

0.653089

0.588847

0.533916

0.591243

0.544768

0.755862

0.409413

0.688799

0.728484

0.751609

0.598449

0.903168

0.88072

0.523306

0.467578

0.41213

0.4862

0.469097

1.207767

0.588904

1.289022

0.530162

0.626093
$-1.90992$

$-1.9082$

0.056143

0.286897

$-1.90725$

$-1.908$

$-1.91486$

$-1.91689$

$-1.9222$

$-1.91055$

$-1.91858$

$-1.91023$

$-1.90549$

$-1.90947$

$-1.92276$

$-1.91217$

$-1.91856$

$-1.91294$

$-1.92091$

$-1.90616$

$-1.91234$

$-1.90784$

$-1.90396$

$-1.90366$

$-1.90183$

$-1.90137$

$-1.89984$

$-1.8952$

$-1.89359$

$-1.89428$

$-1.89733$

$-1.89607$

$-1.89408$

$-1.89454$

$-1.89551$

$-1.89367$

$-1.89854$

$-1.89315$

$-1.89112$

$-1.89134$

$-1.88955$

$-1.88895$

$-1.88706$

$-1.88738$

$-1.88702$

$-1.88583$

$-1.8851$

$-1.88403$

$-1.88376$

1.88308

1.882518

$-1.87966$

0.056365

0.286897

0.056488

0.286897

$\begin{array}{ll}0.056391 & 0.286897\end{array}$

$\begin{array}{ll}0.05551 & 0.286897\end{array}$

$\begin{array}{ll}0.055252 & 0.286897\end{array}$

$0.05458 \quad 0.286897$

$\begin{array}{ll}0.056062 & 0.286897\end{array}$

$\begin{array}{ll}0.055038 & 0.286897\end{array}$

$\begin{array}{ll}0.056103 & 0.286897\end{array}$

$\begin{array}{ll}0.056716 & 0.286897\end{array}$

$\begin{array}{ll}0.056202 & 0.286897\end{array}$

$0.05451 \quad 0.286897$

0.055854

0.286897

0.05504

0.286897

0.055755

0.286897

0.054743

0.286897

0.05663

0.286897

0.055832

0.286897

0.056412

0.286897

0.056915

0.286981

0.056955

0.286981

0.057194

0.28769

0.057253

0.28769

0.057454 
Neorhizobium sp. NCHU2750

Ochrobactrum pituitosum

Streptomyces rubrolavendulae

Staphylococcus lutrae

Ferrimonas balearica

Sphingobium cloacae

Staphylococcus succinus

Burkholderia sp. MSMB0266

Sphingopyxis sp. FD7

Azoarcus olearius

Luteitalea pratensis

Mycolicibacterium smegmatis

Sulfitobacter sp. AM1-D1

Chromobacterium vaccinii

Rivularia sp. PCC 7116

Pseudomonas sp. CMR12a

Pediococcus damnosus

Bordetella sp. $\mathrm{N}$

Gammapapillomavirus 9

Mycolicibacterium thermoresistibile

Collimonas pratensis

Plantibacter flavus

Jeotgalibaca sp. PTS2502

Alteromonas australica

Sagittula sp. P11

Chelativorans sp. BNC1

Sphingomonas indica

Mycobacterium dioxanotrophicus

Bordetella pseudohinzii

Novosphingobium aromaticivorans

Enterococcus sp. FDAARGOS_553

Niabella ginsenosidivorans

Marinobacter hydrocarbonoclasticus

Bifidobacterium choerinum

Kozakia baliensis

Vagococcus penaei

Pseudomonas prosekii

Nostoc sp. 'Peltigera membranacea cyanc

Thauera sp. MZ1T

Streptomyces actuosus

Sulfuritalea hydrogenivorans

Oceanimonas sp. GK1

Thermodesulfovibrio yellowstonii

Rubrobacter xylanophilus

Pseudomonas palleroniana

Caulobacter flavus

Nocardia nova

Paenibacillus crassostreae

Brenneria sp. EniD312

Nitrospira moscoviensis
50.45074

25.98573

$-1.04627$

$-1.89168$

69.18274

$-0.87941$

36.78856

1.804659

16.0061

$-1.09644$

127.1983

$-0.90787$

376.76

$-1.74933$

138.5799

51.45149

141.3675

122.1369

193.3618

46.54172

108.4141

15.19092

54.00917

5.075937

119.5857

5.454305

131.5326

193.4353

132.1549

12.00188

7.102789

73.9865

57.06935

66.31145

153.4899

90.18769

52.81291

8.847082

45.12254

28.48731

30.18504

11.11121

8.774255

43.89066

8.43543

144.4025

78.76107

193.4989

30.99249

6.032266

68.08941

34.3598

167.8679

135.9707

4.403988

17.86562

26.88746

$-1.38062$

$-1.25521$

$-1.20589$

$-0.86405$

$-0.6166$

$-1.04465$

.88435

$-1.28003$

$-2.74679$

$-0.9828$

0.66712

$-0.87585$

$-1.63203$

$-2.58168$

$-0.86485$

$-0.78379$

$-0.86173$

$-0.54301$

$-1.18878$

$-0.89339$

$-2.439$

$-1.09246$

$-0.96697$

$-1.78519$

$-1.28672$

3.099543

$-0.92601$

$-0.86586$

$-1.09661$

$-0.83875$

$-0.91225$

$-0.79788$

$-0.71521$

$-2.24735$

$-1.24671$

$-1.08832$
0.556843

1.006811

0.467781

0.961593

0.585234

0.484535

0.934727

0.737976

0.67171

0.645689

0.462351

0.330104

0.559805

0.47415

1.058873

0.687149

1.473279

0.527665

2.833752

358437

0.515454

0.471187

0.878255

1.38925

0.465849

0.422127

0.464523

0.292695

0.64094

0.481922

1.316217

0.590392

0.522532

0.540886

0.763

0.9644

0.69576

1.676444

0.50102

0.468794

0.594621

0.454694

1.305382

0.557953

0.495975

0.433665

0.38877

1.223749

0.678803

0.592655
$-1.87894$

$-1.87888$

$-1.87996$

1.87674

$-1.87351$

$-1.87368$

$-1.87149$

$-1.87082$

$-1.86868$

$-1.8676$

$-1.86881$

$-1.86789$

$-1.86609$

$-1.86512$

1.862285

$-1.86281$

$-1.86441$

$-1.86255$

$-1.86217$

$-1.86119$

$-1.85827$

$-1.85883$

$-1.85827$

$-1.85833$

$-1.8565$

$-1.85677$

$-1.85508$

$-1.85522$

$-1.85474$

$-1.8538$

$-1.85303$

$-1.85039$

$-1.85055$

$-1.8501$

$-1.85027$

$-1.85109$

$-1.84937$

1.848879

$-1.84826$

$-1.847$

$-1.84422$

$-1.84465$

$-1.84366$

$-1.84253$

$-1.8393$

$-1.83984$

$-1.83967$

$-1.83645$

$-1.83664$

$-1.83635$

$0.060253 \quad 0.291139$

$0.060261 \quad 0.291139$

$0.060114 \quad 0.291139$

$0.060554 \quad 0.292168$

$0.060998 \quad 0.293534$

$0.060974 \quad 0.293534$

$0.061278 \quad 0.294488$

$\begin{array}{ll}0.061371 & 0.294546\end{array}$

$\begin{array}{lll}0.061667 & 0.295135\end{array}$

$0.061818 \quad 0.295135$

$0.061649 \quad 0.295135$

$\begin{array}{lll}0.061777 & 0.295135\end{array}$

$0.062029 \quad 0.295755$

$\begin{array}{ll}0.062164 & 0.296012\end{array}$

$\begin{array}{lll}0.062563 & 0.296049\end{array}$

$0.062489 \quad 0.296049$

$0.062265 \quad 0.296049$

$0.062526 \quad 0.296049$

$\begin{array}{lll}0.062579 & 0.296049\end{array}$

$\begin{array}{ll}0.062717 & 0.29632\end{array}$

$\begin{array}{ll}0.063131 & 0.296732\end{array}$

$0.063052 \quad 0.296732$

$\begin{array}{ll}0.063131 & 0.296732\end{array}$

$0.063123 \quad 0.296732$

$\begin{array}{lll}0.063382 & 0.297144\end{array}$

$\begin{array}{lll}0.063345 & 0.297144\end{array}$

$0.063585 \quad 0.297172$

$\begin{array}{ll}0.063565 & 0.297172\end{array}$

$0.063633 \quad 0.297172$

$\begin{array}{ll}0.063768 & 0.297421\end{array}$

$\begin{array}{ll}0.063877 & 0.29755\end{array}$

$\begin{array}{ll}0.064257 & 0.297612\end{array}$

$0.064234 \quad 0.297612$

$0.0643 \quad 0.297612$

$0.064275 \quad 0.297612$

$0.064157 \quad 0.297612$

$0.064404 \quad 0.297666$

0.0644750 .297666

$\begin{array}{ll}0.064565 & 0.297704\end{array}$

$\begin{array}{lll}0.064747 & 0.298166\end{array}$

$\begin{array}{lll}0.06515 & 0.299265\end{array}$

$0.065088 \quad 0.299265$

$0.065233 \quad 0.299265$ 
Aminobacter sp. MSH1

Mannheimia varigena

Janthinobacterium sp. Marseille

Mycobacterium tuberculosis

Lactobacillus sp. HBUAS52074

Delftia sp. Cs1-4

Planctomyces sp. SH-PL14

Desulfovibrio africanus

Shewanella algae

Aeromicrobium sp. A1-2

Polynucleobacter acidiphobus

Pseudomonas simiae

Staphylococcus capitis

Sphingopyxis macrogoltabida

Azospirillum sp. CFH 70021

Olleya sp. Bg11-27

Limnochorda pilosa

Planococcus rifietoensis

Candidatus Nitrospira inopinata

Streptomyces olivoreticuli

Burkholderia sp. CCGE1001

Mycobacterium kansasii

Sphingobium hydrophobicum

Micromonospora echinaurantiaca

Caulobacter sp. K31

Geobacter lovleyi

Burkholderia sp. MSMB1588

Achromobacter sp. AONIH1

Steroidobacter denitrificans

Burkholderia sp. KK1

Granulosicoccus antarcticus

Streptomyces atratus

Gallionella capsiferriformans

Beutenbergia cavernae

Altererythrobacter mangrovi

Pedobacter cryoconitis

Brachyspira murdochii

Neisseria sp. 10022

Pannonibacter phragmitetus

Cupriavidus gilardii

Zymobacter palmae

Tateyamaria omphalii

Microbacterium paludicola

Burkholderia sp. MSMB0856

Bordetella flabilis

Pseudomonas yamanorum

Ereboglobus luteus

Acidobacteriaceae bacterium SBC82

Microbacterium sp. BH-3-3-3

Actinomyces sp. 2129
102.1976

7.800193

62.87962

256.816

4.362128

525.8989

61.98355

39.39503

17.42248

117.9728

5.131239

4.418808

44447.03

148.8174

181.6987

6.753192

91.48088

10.76032

16.97039

86.18801

832.2561

100.0878

74.98268

91.5616

179.1832

11.03848

115.975

273.0072

35.60071

299.2295

8.714046

84.07775

31.8253

117.0721

24.30338

15.38631

12.51356

25.00185

170.9858

581.5004

15.96485

24.56121

173.7345

160.8559

108.8996

94.0841

30.62462

24.90365

145.9816

134.9698
$-0.9132$

0.496865

$-2.07046$

1.128012

$-0.97644$

0.533382

$-2.89717$

1.579726

$-2.38023$

301014

$-1.03116$

0.563435

$-0.80975$

0.442272

$-1.1135$

0.607403

$-1.35429$

0.739879

$-0.94974$

0.518496

$-1.84209$

1.006415

$-4.20827$

2.300402

1.142181

0.623579

0.561237

0.

2.347426

494924

$-0.94504$

1.285038

$-2.08933$

0.517635

$-1.7917$

0.98074

$-0.9112$

0.499994

$-1.19102$

0.65355

$-0.64445$

0.354266

$-0.98467$

0.540799

$-0.98017$

0.540456

$-0.94647$

0.522061

$-1.24758$

0.68762

$-1.7819$

0.982518

$-0.969$

0.534314

$-0.939730 .518256$

$-1.043620 .57493$

$-1.34802$

0.741938

$-0.8293$

0.457075

$-1.14106$

0.627364

$-0.85304$

0.469061

$-1.05088$

0.579403

$-1.61521$

0.887773

2.263997

1.247061

$-1.46275$

0.80737

$-0.84731$

0.468424

$-0.92789$

0.513099

$-1.19608$

0.66181

$-1.33513$

0.739984

$-0.84249$

0.467058

$-1.092$

0.605851

$-0.86629$

0.480751

$-1.00161$

0.555685

$-1.27065$

0.705518

$-1.10988$

0.616472

$-0.84196$

0.468193

$-0.63959$

0.355552
$-1.83793$

$-1.83549$

$-1.83066$

$-1.83397$

$-1.82952$

$-1.83013$

$-1.83088$

$-1.83321$

$-1.83042$

$-1.83172$

$-1.83035$

$-1.82936$

1.831653

$-1.82628$

$-1.82801$

1.826737

$-1.82569$

$-1.82584$

$-1.82689$

$-1.82243$

$-1.82238$

$-1.81912$

$-1.82077$

$-1.81359$

$-1.81295$

$-1.81435$

$-1.81361$

$-1.81354$

$-1.81325$

$-1.81522$

$-1.8169$

$-1.81437$

$-1.81881$

$-1.81861$

$-1.81374$

$-1.8194$

1.815467

$-1.81174$

$-1.80886$

$-1.8084$

$-1.80729$

$-1.80427$

$-1.80382$

$-1.80243$

$-1.80195$

$-1.80247$

$-1.80101$

$-1.80037$

$-1.79832$

$-1.79886$

$\begin{array}{ll}0.066073 & 0.301147\end{array}$

0.0664330 .301349

0.0671510 .301356

0.0666590 .301356

0.0673220 .301356

$0.06723 \quad 0.301356$

0.0671180 .301356

0.0667710 .301356

0.0671870 .301356

0.0669940 .301356

0.0671980 .301356

0.0673450 .301356

0.0670030 .301356

$0.067808 \quad 0.301599$

0.0675480 .301599

0.0677390 .301599

0.0678970 .301599

0.0678750 .301599

0.0677160 .301599

0.068390 .303079

$\begin{array}{ll}0.068397 & 0.303079\end{array}$

0.0688940 .303558

0.0686410 .303558

0.0697410 .303558

0.069840 .303558

0.0696240 .303558

0.0697380 .303558

0.0697480 .303558

0.0697940 .303558

0.069490 .303558

0.0692330 .303558

0.0696210 .303558

0.068940 .303558

0.0689710 .303558

0.0697180 .303558

0.0688510 .303558

0.0694520 .303558

0.0700260 .304003

0.0704730 .305522

0.0705440 .305522

0.0707170 .305906 
Azospirillum thiophilum

Staphylococcus pseudintermedius

Kitasatospora aureofaciens

Coriobacteriaceae bacterium 68-1-3

Polynucleobacter difficilis

Deinococcus maricopensis

Pseudomonas sp. 09C 129

Streptomyces sp. P3

Orientia tsutsugamushi

Oligella urethralis

Raoultella planticola

Pigmentiphaga sp. $\mathrm{H} 8$

Thermus oshimai

Pseudomonas sp. 7SR1

Streptomyces fulvissimus

Rhodobiaceae bacterium

Acidovorax sp. T1

Neisseria elongata

Serratia sp. YD25

Halorubrum lacusprofundi

Massilia armeniaca

Nitrosospira multiformis

Frankia sp. EAN1pec

Auraticoccus monumenti

Gammaproteobacteria bacterium ESL007

Burkholderia anthina

Paenibacillus borealis

Celeribacter ethanolicus

blood disease bacterium A2-HR MARDI

Paenibacillus naphthalenovorans

Frankia sp. QA3

Saccharothrix espanaensis

Isoptericola variabilis

Microchaete diplosiphon

Aliivibrio salmonicida

Candidatus Filomicrobium marinum

Bordetella bronchialis

Xanthomonas campestris

Pseudomonas fulva

Methylovorus sp. MP688

Streptomyces cattleya

Xanthomonas gardneri

Gloeobacter kilaueensis

Brevundimonas subvibrioides

Devosia sp. A16

Parvimonas micra

Stenotrophomonas sp. SAU14A_NAIMI4_

Pandoraea pulmonicola

Bacillus sp. OxB-1

Mesorhizobium amorphae
119.0085

298.2127

91.31495

43.63453

9.480608

52.50943

12.69457

88.49443

5.182215

8.309732

23.01161

198.5496

18.03601

49.22139

55.12739

5.824754

967.1438

438.1565

15.53451

19.75794

204.2477

10.43868

126.5244

130.2706

6.135442

161.5903

9.926872

28.09292

288.3029

8.722689

78.48505

162.8732

151.0538

9.294751

3.789904

16.78256

148.177

805.2264

277.0791

13.07444

119.6235

25.97548

74.69403

115.7241

84.6221

249.088

4.897416

240.1463

3.749227

155.0149
$-0.80687$

0.448664

$-1.79838$

0.072117

0.072232

1.797653

0.072346

0.447286

$-1.79693$

0.072576

$-1.79549$

$-1.79418$

0.072784

$-1.79248$

0.073056

$-1.79293$

0.072984

$-1.79169$

0.073182

$-0.87583$

0.488826

2.036495

2.04405

1.141902

$-1.62882$

0.910462

$-0.8648$

0.483968

$-0.86791$

$-0.90485$

0.486321

0.5069

0.964245

$-0.94288$

0.528813

0.563736

$-1.78215$

$-1.47049$

0.825519

$-1.54519$

0.867592

$-0.88559$

0.497488

$-1.59409$

0.895808

$-0.68225$

0.383715

$-0.75784$

0.426241

$-2.62128$

1.476256

$-1.22124$

0.687308

$-1.64616$

0.926994

0.736476

0.780772

0.895575

0.540521

$-0.95831$

0.425379

$-0.73213$

0.413653

3.5359

1.998674

1.550684

$-1.76841$

$-1.34045$

0.758726

$-0.85938$

0.486914

0.870748

0.493774

0.549368

$-1.75981$

1.432756

$-0.80795$

0.459113

$-2.05811 .168666$

$-1.12479$

0.638645

0.478784

$-0.91803 \quad 0.521604$

$\begin{array}{ll}-0.91347 & 0.519292\end{array}$

$-4.356542 .477702$

$\begin{array}{ll}-0.93773 & 0.533989\end{array}$

$-2.280151 .302132$

$-0.87512$
1.789484

$-1.79004$

$-1.789$

$-1.78689$

$-1.78583$

$-1.78465$

$-1.78506$

$-1.78271$

$-1.78301$

$-1.7813$

$-1.78101$

$-1.78012$

$-1.7795$

$-1.77802$

$-1.77796$

$-1.77563$

$-1.77685$

$-1.7758$

$-1.77647$

$-1.77467$

$-1.77315$

$-1.77294$

$-1.76955$

$-1.76991$

1.769122

$-1.76671$

$-1.76495$

1.763454

$-1.76339$

$-1.76362$

$-1.76107$

$-1.76122$

$-1.75975$

$-1.76001$

$-1.75906$

$-1.7583$

$-1.75608$

$-1.75109$

0.073537

0.073448

0.073615

0.073955

0.074126

0.074319

0.074251

0.074633

0.074585

0.074724

0.074864

0.07491

0.075057

0.075158

0.0754

0.07541

0.075795

0.075593

0.075765

0.075655

0.075952

0.076204

0.076239

0.076803

0.076743

0.076873

0.076992

0.077277

0.077572

0.077824

0.077835

0.077796

0.078439

0.078227

0.078202

0.07845

0.078405

0.078567

0.078697

0.079074

0.07993

$\begin{array}{ll}-1.75087 & 0.079967\end{array}$

0.30834

0.308427

0.308553

0.309168

0.309693

0.310126

0.310126

0.310299

0.311045

0.311045

0.311045

0.31212

0.312481

0.312569

0.312569

0.313166

0.313166

0.313187

0.313245

0.313245 


\begin{tabular}{|c|c|c|c|c|c|c|}
\hline Streptacidiphilus sp. DSM 106435 & 94.36827 & -0.67862 & 0.387458 & -1.75147 & 0.079866 & 0.3204 \\
\hline Micromonospora chokoriensis & 60.12547 & -0.90422 & 0.516075 & -1.75211 & 0.079755 & 0.3204 \\
\hline Arcobacter nitrofigilis & 11.55169 & 2.552291 & 1.456601 & 1.752224 & 0.079735 & 0.3204 \\
\hline Bifidobacterium asteroides & 20.23445 & -1.27544 & 0.72846 & -1.75087 & 0.079968 & 0.3204 \\
\hline Erythrobacter sp. HL-111 & 58.30601 & -0.89687 & 0.512155 & -1.75117 & 0.079916 & 0.3204 \\
\hline Rhizobium sp. IE4771 & 27.87624 & -2.23235 & 1.272815 & -1.75387 & 0.079453 & 0.3204 \\
\hline Kushneria marisflavi & 11.90917 & -1.59704 & 0.910165 & -1.75467 & 0.079315 & 0.3204 \\
\hline Streptomyces sp. CFMR 7 & 41.6268 & -1.63326 & 0.931539 & -1.7533 & 0.079551 & 0.3204 \\
\hline Sphingopyxis sp. QXT-31 & 71.33149 & -0.91976 & 0.525715 & -1.74955 & 0.080196 & 0.320609 \\
\hline Arcobacter suis & 6.547352 & 2.488386 & 1.422176 & 1.749704 & 0.080169 & 0.320609 \\
\hline Ralstonia mannitolilytica & 11494 & -0.8824 & 0.504784 & -1.74807 & 0.080452 & 0.321276 \\
\hline Halorientalis sp. IM1011 & 23.96594 & -1.27259 & 0.729951 & -1.74339 & 0.081266 & 0.321496 \\
\hline Candidatus Phaeomarinobacter ectocarpi & 14.37801 & -1.48635 & 0.851399 & -1.74577 & 0.080851 & 0.321496 \\
\hline Leptospira santarosai & 38.59238 & 1.373825 & 0.787114 & 1.745396 & 0.080916 & 0.321496 \\
\hline Geobac & 50.87098 & -1.0812 & 0.620046 & -1.74374 & 0.081204 & 0.321496 \\
\hline Mycobacterium sp. djl-10 & 192.1661 & -0.54337 & 0.31152 & -1.74427 & 0.081112 & 0.321496 \\
\hline Comamonas testosteroni & 552.2892 & -0.91908 & 0.526605 & -1.74529 & 0.080935 & 0.321496 \\
\hline tivorans & 52.9257 & -0.9013 & 0.516168 & -1.74614 & 0.080786 & 0.321496 \\
\hline ndongense & 98.90718 & -1.00499 & 0.576039 & -1.74466 & 0.081045 & 0.321496 \\
\hline Agrobacterium tumefaciens & 396.8989 & -0.70766 & 0.405959 & -1.74318 & 0.081302 & 0.321496 \\
\hline Geminocystis herdm & 9.551816 & 1.95236 & 1.120475 & 1.742439 & 0.081432 & 0.321659 \\
\hline Devriesea agamarum & 48.65011 & -0.71478 & 0.410596 & -1.74084 & 0.081712 & 0.322096 \\
\hline Avibacterium paragallinarum & 3.706382 & -2.64051 & 1.517649 & -1.73987 & 0.081882 & 0.322096 \\
\hline Muricauda ruestringensis & 6.519888 & -2.06242 & 1.185688 & -1.73943 & 0.081959 & 0.322096 \\
\hline Thioal & 36.31577 & -0.85038 & 0.488926 & -1.73928 & 0.081985 & 0.322096 \\
\hline Pseud & 52.86212 & -0.99213 & 0.569993 & -1.7406 & 0.081753 & 0.322096 \\
\hline Escherichia coli & 616.2566 & 0.729752 & 0.419959 & 1.737676 & 0.082268 & 0.322859 \\
\hline Lelliottia nimipressu & 5.317246 & -2.12101 & 1.222106 & -1.73554 & 0.082646 & 0.323993 \\
\hline Shewa & 5.01105 & -1.5999 & 0.923127 & -1.73312 & 0.083073 & 0.325319 \\
\hline Mycoli & 298.8054 & -0.77313 & 0.446689 & -1.73081 & 0.083486 & 0.326582 \\
\hline Oligotropha carboxidovorans & 76.80737 & -0.84394 & 0.488402 & -1.72796 & 0.083995 & 0.328221 \\
\hline Providencia sp. WCHPr000369 & 5.586186 & -2.40331 & 1.392262 & -1.72619 & 0.084313 & 0.32911 \\
\hline Variovorax boronicumulans & 869.2076 & -0.88162 & 0.511194 & -1.72463 & 0.084595 & 0.329857 \\
\hline Ochrobactrum sp. A44 & 41.22513 & -1.07346 & 0.623051 & -1.72291 & 0.084905 & 0.330072 \\
\hline Lactob & 230.8906 & -1.13899 & 0.661532 & -1.72175 & 0.085115 & 0.330072 \\
\hline Pseudanabaena sp. PCC 7367 & 6.79438 & -1.82623 & 1.060386 & -1.72223 & 0.085028 & 0.330072 \\
\hline Nocardia farcinica & 242.1051 & -0.58825 & 0.341593 & -1.72207 & 0.085056 & 0.330072 \\
\hline Advel & 30.4198 & -1.359 & 0.789514 & -1.72131 & 0.085194 & 0.330072 \\
\hline Martelella endophytica & 42.64492 & -0.96935 & 0.562629 & -1.72289 & 0.084908 & 0.330072 \\
\hline Plesiomonas shigelloides & 16.87903 & -1.30556 & 0.758963 & -1.72019 & 0.085397 & 0.330506 \\
\hline Streptomyces sp. SirexAA-E & 59.06379 & -0.81697 & 0.475657 & -1.71755 & 0.085878 & 0.330608 \\
\hline Aquitalea magnusonii & 57.3426 & -0.85124 & 0.495508 & -1.71792 & 0.085811 & 0.330608 \\
\hline Brachyspira hampsonii & 5.189282 & 2.877292 & 1.674272 & 1.718533 & 0.085699 & 0.330608 \\
\hline Neisseria sp. 10023 & 44.81292 & -1.14256 & 0.664789 & -1.71869 & 0.085671 & 0.330608 \\
\hline Thermosediminibacter oceani & 4.045599 & -2.04143 & 1.187201 & -1.71953 & 0.085518 & 0.330608 \\
\hline Burkholderia sp. HB1 & 608.3306 & -1.08623 & 0.632858 & -1.71639 & 0.08609 & 0.331075 \\
\hline Rhodothermus marinus & 64.47594 & -0.9479 & 0.552607 & -1.71533 & 0.086285 & 0.331466 \\
\hline Arcobacter halophilus & 10.5169 & 2.642819 & 1.541592 & 1.714344 & 0.086465 & 0.331466 \\
\hline Halomonas sp. 1513 & 50.38649 & -0.93112 & 0.543065 & -1.71457 & 0.086423 & 0.331466 \\
\hline Acidovorax sp. 1608163 & 598.3842 & -0.98229 & 0.573653 & -1.71234 & 0.086835 & 0.331915 \\
\hline
\end{tabular}


Pedobacter steynii

Burkholderiales bacterium YL45

Thioalkalivibrio versutus

Francisella noatunensis

Streptomyces sp. Go-475

Pseudomonas soli

Vibrio gazogenes

Pseudoalteromonas spongiae

Sphingopyxis fribergensis

Idiomarina sp. OT37-5b

Asticcacaulis excentricus

Rhizobium leguminosarum

Altererythrobacter atlanticus

Acinetobacter sp. TTH0-4

Burkholderia sp. IDO3

Chryseobacterium sp. 6424

Spirosoma aerolatum

Altererythrobacter ishigakiensis

Nitrospirillum amazonense

Streptococcus himalayensis

Hymenobacter sedentarius

Mycolicibacterium hassiacum

Oblitimonas alkaliphila

Verrucomicrobia bacterium IMCC26134

Streptococcus uberis

Rummeliibacillus stabekisii

Thauera aromatica

Euzebyella marina

Streptococcus macedonicus

Pseudomonas syringae

Synechococcus sp. SynAce01

Sedimenticola thiotaurini

Micromonospora coriariae

Sanguibacter keddieii

Neoasaia chiangmaiensis

Burkholderia contaminans

Pseudomonas oryzae

Sphingobium sp. TKS

Lysobacter capsici

Streptomyces globosus

Vibrio coralliilyticus

Bacillus circulans

Agrobacterium sp. RAC06

Desulfomonile tiedjei

Saccharomonospora marina

Halothiobacillus sp. LS2

Micromonospora echinofusca

Mesorhizobium sp. WSM1497

Neorhizobium sp. SOG26

Burkholderia sp. MSMB0852

21.35733

5.363077

31.28903

$-1.49969$

0.875878

$-1.71222$

$0.086856 \quad 0.331915$

1.769746

$-2.68214$

1.566183

$-1.71253$

0.086798

0.331915

77.40561

0.629129

$-1.71149$

0.086991

0.33208

3.148022

2.890389

1.709516

0.47693

$-1.70975$

0.087355

0.332774

6.958964

$-3.72023$

2.189719

$-1.69895$

0.087313

0.332774

14.81151

$-2.13919$

1.262473

$-1.69444$

0.089328

0.333467

62.17913

2.175774

1.278147

1.702288

0.090182

0.333467

5.270016

$-0.93851$

0.553226

$-1.69643$

0.088701

0.333467

33.76747

$-2.41382$

1.416987

$-1.70349$

0.089804

0.333467

381.3384

$-1.21921$

0.714306

$-1.70685$

0.088476

0.333467

27.1061

$-0.67838$

0.397191

$-1.70793$

0.08785

0.333467

34.13915

$-1.03671$

0.611322

$-1.69585$

0.087649

0.333467

319.6481

1.187238

0.700296

1.695336

0.089915

0.333467

18.15334

$-0.98781$

0.581014

$-1.70016$

0.090012

0.333467

20.28975

$-1.4977$

0.878423

$-1.70499$

0.089101

0.333467

7.097296

$-1.25531$

0.739071

$-1.6985$

171.3197

$-1.7836$

1.04706

$-1.70344$

0.088196

0.333467

14.17712

$-0.88595$

0.522006

$-1.69721$

$-1.69404$

$-1.7031$

78.85991

$-0.83707$

162.0379

$-0.60957$

0.491497

6.548464

$-2.15778$

0.358222

$-1.70166$

32.95982

$-0.91876$

1.268081

$-1.70161$

0.542706

$-1.69292$

0.804223

$-1.69398$

1.331369

$-1.69376$

6.376656

$-2.25501$

0.56416

$-1.6934$

4.275071

$-0.95535$

1.373013

3.824052

$-3.99985$

2.353802

$-1.70209$

321.8453

$-0.77556$

0.455668

15.0754

$-1.22256$

0.718839

17.09337

$-1.33826$

0.785344

60.71929

$-0.87931$

0.517221

151.1403

$-0.68433$

0.402232

37.27862

$-1.08412$

0.640305

425.4124

$-0.92636$

0.543127

69931

$-1.70202$

$-1.70074$

$-1.70404$

$-1.70007$

$-1.70134$

$-1.69313$

0.089414

0.333467

0.088486

0.333467

113.6496

$-1.00423$

0.593

$-1.7056$

66.15204

$-0.91007$

0.538116

157.9632

$-1.08637$

0.642876

$-1.69347$

0.089657

0.333467

74.53419

$-0.70564$

0.417582

$-1.69121$

$-1.68986$

$-1.68983$

0.766013

19.36378

$-1.29465$

26.78644

1.223305

0.723289

$-1.69011$

0.090258

0.333467

0.08855

0.333467

0.08882

0.333467

0.088828

0.333467

0.090471

0.333467

0.090269

0.333467

69.1265

$-0.93883$

0.556662

1.691307

0.090312

0.333467

0.090379

0.333467

4.408938

$-2.36218$

1.400193

$-1.6865$

$-1.68704$

0.088739

0.333467

0.08926

0.333467

110.7646

$-0.77023$

0.456642

$-1.68673$

$-1.68585$

0.088751

0.333467

0.088992

0.333467

0.088373

0.333467

0.089118

0.333467

0.088879

0.333467

23.40389

$-1.08099$

0.641213 
Spiroplasma helicoides

Desulfovibrio vulgaris

Arcobacter marinus

Micromonospora purpureochromogenes

Gemmobacter sp. HYN0069

Streptomyces anulatus

Brevibacillus brevis

Actinomadura amylolytica

Halomonas sp. N3-2A

Microbulbifer agarilyticus

Pelobacter acetylenicus

Clostridium taeniosporum

Bradyrhizobium sp. S23321

Pseudomonas cichorii

Achromobacter sp. MFA1 R4

Clostridium argentinense

Desulfatibacillum alkenivorans

Magnetospirillum gryphiswaldense

Porphyrobacter sp. LM 6

Tannerella forsythia

Campylobacter lari

Pseudomonas sp. R2A2

Streptomyces lunaelactis

Lysobacter gummosus

Methylotenera mobilis

Chitinophaga pinensis

Saccharomonospora cyanea

Streptomyces sp. SM18

Runella sp. HYN0085

Singulisphaera acidiphila

Nocardia sp. CFHSO054

Sinorhizobium sp. CCBAU 05631

Streptomyces sp. Tue 6075

Achromobacter insolitus

Agrobacterium fabrum

Desulfuromonas soudanensis

Thermotoga caldifontis

Dinoroseobacter shibae

Acidovorax sp. KKS102

Pandoraea faecigallinarum

Corynebacterium resistens

Rhodobacter sp. CZR27

Pseudomonas guangdongensis

Anaerolinea thermophila

Microbulbifer aggregans

Proteiniphilum saccharofermentans

Prauserella marina

Aggregatibacter aphrophilus

Desulfovibrio gigas

Akkermansia muciniphila

\begin{tabular}{|c|c|c|c|c|c|}
\hline & & & & & \\
\hline .49673 & -0.79337 & 0.473837 & -1.67436 & 0.094061 & 0.34151 \\
\hline 31441 & 1.919232 & 1.146693 & 1.67371 & 0.094188 & 0.341629 \\
\hline 82.9032 & -0.69616 & 0.41639 & -1.67188 & 0.094547 & 0.342593 \\
\hline .78205 & -0.92206 & 0.551687 & -1.67135 & 0.094653 & 0.342635 \\
\hline .88789 & -0.71011 & 0.425092 & -1.67049 & 0.094823 & 0.342907 \\
\hline 14961 & -1.00465 & 0.602229 & -1.66822 & 0.095272 & 0.344192 \\
\hline 11.5583 & -0.73764 & 0.44233 & -1.66763 & 0.095389 & 0.344272 \\
\hline 027319 & -3.66377 & 2.199166 & -1.66598 & 0.095717 & 0.345114 \\
\hline .40405 & -0.95346 & 0.573224 & -1.66332 & 0.096248 & 0.346682 \\
\hline 2.74956 & -1.1572 & 0.696817 & -1.66069 & 0.096776 & 0.34824 \\
\hline 1.15987 & 1.476696 & 0.89132 & 1.656752 & 0.09757 & 0.350403 \\
\hline 90.8588 & -0.83183 & 0.501944 & -1.65722 & 0.097475 & 0.350403 \\
\hline 1.96608 & -1.16441 & 0.703227 & -1.65581 & 0.097761 & 0.350745 \\
\hline 141.032 & -0.8812 & 0.532593 & -1.6 & $0.0 s$ & 309 \\
\hline 30.78684 & 1.102099 & 0.666612 & 1.653285 & 0.098273 & 0.351541 \\
\hline 28.40075 & -1.03919 & 0.628457 & -1.6 & 0.098217 & 351541 \\
\hline 407.169 & -0.99977 & 0.605016 & -1.65247 & 0.098439 & 0.351714 \\
\hline 1.68737 & -1.07939 & 0.653531 & -1.65162 & 0.098612 & 0.351714 \\
\hline 159.9991 & -1.02113 & 0.61825 & -1.65164 & 0.098608 & 0.351714 \\
\hline 19.62401 & 1.658072 & 1.00427 & 1.651023 & 0.098734 & 0.351736 \\
\hline 21.81279 & -1.26962 & 0.769164 & -1.6 & 0.098811 & 0.351736 \\
\hline 57.73603 & -0.81937 & 0.496539 & -1.65017 & 0.098909 & 0.351741 \\
\hline 88.95343 & -1.02089 & 0.619677 & -1.64746 & 0.099463 & 0.353367 \\
\hline 8.340136 & -1.37126 & 0.83286 & -1.64645 & 0.099671 & 0.35376 \\
\hline 51.19293 & -0.92734 & 0.564357 & -1.64317 & 0.10 & 0.354445 \\
\hline 110.1474 & -0.86234 & 0.524654 & -1.64363 & 0.100252 & 0.354445 \\
\hline 65.62044 & -0.76987 & 0.468298 & -1.64397 & 0.100183 & 0.354445 \\
\hline 10.24613 & -1.60106 & 0.974846 & -1.64237 & 0.100512 & 0.354445 \\
\hline 62.67654 & -0.82351 & 0.501263 & -1.64286 & 0.100412 & 0.354445 \\
\hline 168.8619 & -0.50176 & 0.305539 & -1.64221 & 0.100546 & 0.354445 \\
\hline 6.244487 & -2.40599 & 1.462711 & -1.64488 & 0.099994 & 0.354445 \\
\hline 40.86685 & -1.21335 & 0.740272 & -1.6 & 0.1012 & 0.356061 \\
\hline 119.0166 & -0.74118 & 0.452188 & -1.6 & 0.101194 & 0.356061 \\
\hline 55.90565 & -0.84975 & 0.519345 & -1.63619 & 0.101799 & 0.357821 \\
\hline 49.56787 & -0.91674 & 0.560611 & -1.63526 & 0.101996 & 0.358166 \\
\hline 4.149385 & -2.92396 & 1.789882 & -1.6336 & 0.102342 & 0.359036 \\
\hline 60.84419 & -0.89443 & 0.54821 & -1.63154 & 0.102776 & 0.359894 \\
\hline 1336.223 & -0.78816 & 0.483087 & -1.6315 & 0.102785 & 0.359894 \\
\hline 171.7452 & -0.83797 & 0.513932 & -1.63051 & 0.102994 & 0.36028 \\
\hline 1930.986 & -0.71319 & 0.437709 & -1.62937 & 0.103234 & 0.360428 \\
\hline 108.0556 & -0.78106 & 0.479268 & -1.62969 & 0.103166 & 0.360428 \\
\hline 41.95308 & -0.92456 & 0.567782 & -1.62837 & 0.103446 & 0.36082 \\
\hline 5.107616 & -1.6331 & 1.003773 & -1.62696 & 0.103745 & 0.360955 \\
\hline 13.39669 & -1.45815 & 0.895928 & -1.62753 & 0.103625 & 0.360955 \\
\hline 17.44108 & -1.46191 & 0.898719 & -1.62665 & 0.103811 & 0.360955 \\
\hline 79.87909 & -0.52504 & 0.322842 & -1.62631 & 0.103884 & 0.360955 \\
\hline 148.299 & -1.04548 & 0.643037 & -1.62586 & 0.10398 & 0.360955 \\
\hline 25.5026 & -0.95413 & 0.587291 & -1.62462 & 0.104244 & 0.361523 \\
\hline 78.52703 & -1.51398 & 0.932543 & -1.62349 & 0.104484 & 0.361668 \\
\hline
\end{tabular}


Vibrio breoganii

Starkeya novella

Bacillus lehensis

Agrococcus carbonis

Brevirhabdus pacifica

Sphingobacterium sp. 21

Immundisolibacter cernigliae

Amycolatopsis albispora

Macrococcus canis

Massilia sp. YMA4

Amycolatopsis orientalis

Nostocales cyanobacterium HT-58-2

Fluoribacter dumoffii

Actinomyces sp. Z16

Geobacter daltonii

Algoriphagus sp. M8-2

Phaeobacter piscinae

Kosakonia oryzae

Microbacterium sp. PAMC 28756

Microbacterium sp. TPU 3598

Sulfitobacter pseudonitzschiae

Eggerthella lenta

Pectobacterium carotovorum

Methylophilus sp. TWE2

Acidovorax sp. JS42

Neorhizobium galegae

Synechococcus sp. NIES-970

Pseudoalteromonas piscicida

Reinekea forsetii

Beggiatoa leptomitoformis

Massilia putida

Pelagibacterium halotolerans

Mycobacteroides saopaulense

Pseudomonas monteilii

Parageobacillus genomosp. 1

Eikenella corrodens

Pseudodesulfovibrio aespoeensis

Lachnospiraceae bacterium GAM79

Lactobacillus brevis

Brachyspira hyodysenteriae

Rhodoferax saidenbachensis

Haloferax mediterranei

Hyphomicrobium sp. MC1

Pseudomonas sp. TCU-HL1

Serratia ficaria

Rhodobacter sphaeroides

Mycobacterium sp. MS1601

Cellulophaga Iytica

Pelagibaca abyssi

Erwinia persicina

\begin{tabular}{|c|c|c|c|c|c|}
\hline & & & & & \\
\hline 108.578 & -0.75465 & 0.464983 & -1.62295 & 0.1046 & 0.361725 \\
\hline 2.81958 & -1.37484 & 0.847945 & -1.62137 & 0.104937 & 0.362547 \\
\hline 135.5621 & -0.69688 & 0.430045 & -1.62047 & 0.10513 & 0.36287 \\
\hline .19418 & -0.86573 & 0.534484 & -1.61975 & 0.105286 & 0.363062 \\
\hline .24445 & -0.98819 & 0.610958 & -1.61744 & 0.105784 & 0.36333 \\
\hline .92124 & -0.74176 & 0.458369 & -1.61825 & 0.105608 & 0.36333 \\
\hline 168.1831 & -0.58643 & 0.362649 & -1.61707 & 0.105863 & 0.36333 \\
\hline 20.41141 & 1.7139 & 1.059468 & 1.617699 & 0.105727 & 0.36333 \\
\hline 256.2995 & -0.78763 & 0.486974 & -1.6174 & 0.105792 & 0.36333 \\
\hline 74.90351 & -0.63974 & 0.396053 & -1.61529 & 0.106247 & 0.364304 \\
\hline 16.6328 & 1.686077 & 1.044392 & 1.614411 & 0.106438 & 0.364399 \\
\hline 3.142758 & -3.21958 & 1.994483 & -1.61424 & 0.106475 & 0.364399 \\
\hline 199.2993 & -0.66098 & 0.409675 & -1.61342 & 0.106654 & 0.36467 \\
\hline 9.168766 & -1.42662 & 0.885225 & -1.6116 & 0.10705 & 0.365679 \\
\hline 4.222434 & -2.2265 & 1.384474 & -1.6082 & 0.107792 & 0.367203 \\
\hline 23.32903 & -1.27198 & 0.790951 & -1.60817 & 0.107799 & 0.367203 \\
\hline 136.0034 & -0.82895 & 0.51529 & -1.6087 & 0.107682 & 0.367203 \\
\hline 174.9743 & -0.77389 & 0.482079 & -1.60531 & 0.108425 & 0.36725 \\
\hline 160.516 & -0.68776 & 0.42823 & -1.60605 & 0.108263 & 0.36725 \\
\hline 25.04862 & -1.16383 & 0.724092 & -1.6073 & 0.10799 & 0.36725 \\
\hline 49.42604 & -0.67458 & 0.41977 & -1.60701 & 0.108051 & 0.36725 \\
\hline 91.11551 & -0.92166 & 0.574286 & -1.60488 & 0.108519 & 0.36725 \\
\hline 17.27466 & -1.62969 & 1.014455 & -1.60646 & 0.108172 & 0.36725 \\
\hline 1362.053 & -0.94357 & 0.587788 & -1.60528 & 0.108431 & 0.36725 \\
\hline 93.96859 & -0.78399 & 0.488748 & -1.60408 & 0.108697 & 0.367508 \\
\hline 6.400466 & -1.88724 & 1.17718 & -1.60319 & 0.108892 & 0.367828 \\
\hline 5.38558 & 2.40795 & 1.503241 & 1.601838 & 0.109191 & 0.368348 \\
\hline 5.135402 & -2.1062 & 1.315076 & -1.60158 & 0.109249 & 0.368348 \\
\hline 10.36463 & -1.45861 & 0.911355 & -1.60049 & 0.109491 & 0.368822 \\
\hline 422.0878 & -0.78573 & 0.491425 & -1.59888 & 0.109847 & 0.369679 \\
\hline 38.05828 & -0.78326 & 0.490129 & -1.59807 & 0.110027 & 0.369942 \\
\hline 72.65984 & -0.87854 & 0.550078 & -1.59711 & 0.11024 & 0.370318 \\
\hline 217.6945 & -0.88309 & 0.554493 & -1.5926 & 0.111249 & 0.371876 \\
\hline 4.104493 & -2.31793 & 1.455102 & -1.59296 & 0.111168 & 0.371876 \\
\hline 90.71553 & -0.83443 & 0.524039 & -1.5923 & 0.111317 & 0.371876 \\
\hline 33.72004 & -1.04847 & 0.658343 & -1.59259 & 0.111252 & 0.371876 \\
\hline 143.35 & -1.14362 & 0.717637 & -1.5936 & 0.111027 & 0.371876 \\
\hline 13.33234 & -1.6977 & 1.065976 & -1.59263 & 0.111244 & 0.371876 \\
\hline 15.55796 & 1.93982 & 1.219651 & 1.590472 & 0.111729 & 0.372907 \\
\hline 275.4861 & -0.89161 & 0.560837 & -1.58978 & 0.111885 & 0.373016 \\
\hline 2.729456 & -2.73528 & 1.720936 & -1.58942 & 0.111966 & 0.373016 \\
\hline 32.35748 & -1.31598 & 0.828423 & -1.58853 & 0.112166 & 0.373341 \\
\hline 54.11325 & -0.83792 & 0.527729 & -1.58778 & 0.112336 & 0.373564 \\
\hline 122.1843 & -1.03652 & 0.654015 & -1.58485 & 0.113 & 0.373723 \\
\hline 312.4519 & -0.78144 & 0.492751 & -1.58587 & 0.112768 & 0.373723 \\
\hline 159.6257 & -0.5218 & 0.329076 & -1.58566 & 0.112817 & 0.373723 \\
\hline 12.65222 & 1.727769 & 1.089992 & 1.585122 & 0.112939 & 0.373723 \\
\hline 60.13212 & -0.80957 & 0.510577 & -1.58559 & 0.112832 & 0.373723 \\
\hline 54.58028 & -1.20794 & 0.761764 & -1.58571 & 0.112805 & 0.373723 \\
\hline
\end{tabular}


Erythrobacter gangjinensis

Cronobacter condimenti

Azospirillum lipoferum

Coxiella burnetii

Thiobacillus denitrificans

Pseudomonas sp. HLS-6

Nonomuraea sp. ATCC 55076

Natronolimnobius aegyptiacus

Gardnerella vaginalis

Deferribacter desulfuricans

Actinomyces hongkongensis

Actinoalloteichus sp. GBA129-24

Cytophagales bacterium TFI 002

Arcobacter butzleri

Deinococcus sp. S14-83

Mycolicibacterium phlei

Chthonomonas calidirosea

Maritalea myrionectae

Cellvibrio japonicus

Bradyrhizobium sp. BTAi1

Thermobacillus composti

Flavobacterium pallidum

Staphylococcus pasteuri

Delftia sp. HK171

Altererythrobacter namhicola

Chromatiaceae bacterium 2141T.STBD.0c

Streptococcus mitis

Sphingobium sp. EP60837

Aggregatibacter segnis

Novosphingobium resinovorum

Sphingobium sp. SCG-1

Pedobacter sp. eg

Leptospira interrogans

Thermobaculum terrenum

Streptomyces autolyticus

Thermoanaerobacter kivui

Akkermansia glycaniphila

Streptomyces vietnamensis

Rhizobium sp. NXC24

Paenibacillus graminis

Candidatus Methylopumilus turicensis

Alcanivorax pacificus

Lacinutrix sp. Bg11-31

Victivallales bacterium CCUG 44730

Mycolicibacterium chubuense

Aquimarina sp. BL5

Brevundimonas naejangsanensis

Aeromicrobium erythreum

Kyrpidia tusciae

Trichodesmium erythraeum
20.94506

16.49018

180.9168

8.73263

62.67963

28.46201

240.9809

20.19118

384.4255

8.206502

467.4825

26.58332

4.304183

15.8714

18.20075

1447.373

10.95512

11.17543

14.20651

286.921

47.73105

3.818577

6970.929

187.8111

41.34189

40.40899

3692.679

26.00183

148.3305

153.0016

47.84156

34.4689

13.26394

5.468874

36.88389

6.362067

15.62095

79.25955

40.60618

4.333952

2.858122

45.48716

9.869357

22.55591

196.642

16.0016

328.4524

175.2971

15.52271

17.36313
$-1.031210 .651388$

$-1.78419$

1.12688

$-0.72178$

0.455727

$-2.02282$

1.278155

$-0.87347$

0.526333

0.552673

$-0.60928$

0.385657

$-1.46901$

0.930422

0.998573

0.633347

2.402321

1.524703

$-0.77562$

0.492291

$-3.3897$

2.153126

$-1.70654$

1.085419

1.934537

1.230432

$-1.0873$

0.691772

$-0.86308$

0.549437

$-1.64392$

1.046471

$-1.42282$

0.90621

$-1.10963$

0.707056

$-0.82023$

0.52288

$-1.03735$

0.661165

$-2.69548$

1.720971

$-0.76426$

0.488305

$-0.93384$

0.596699

$-0.80035$

0.511753

$-0.6863$

0.439021

$-0.85252$

0.546252

$-1.14948$

0.736603

$-0.93743$

0.601092

$-0.73384$

0.471184

$-0.78817$

0.506089

$-1.09622$

0.703788

2.091515

1.342244

$-1.87052$

1.200982

$-1.76864$

1.134111

$-2.75357$

1.769408

$-1.51634$

0.974714

$-0.70702$

0.455291

$-0.90604$

0.58376

$-2.30153$

1.484685

$-2.50039$

1.61438

$-1.10068$

0.711463

1.832459

1.184956

$-0.93706$

0.606975

$-0.82615$

0.535149

1.423632

0.921615

$-0.74862$

0.48468

0.437722

0.67526

1.117688

$-1.72442$

0.950454

$-1.58309$

0.113401

0.113353

0.374029

$-1.5833$

0.113242

0.374029

$-1.58379$

0.113511

0.374029

$-1.58261$

$-1.58176$

0.113705

0.374052

$-1.58045$

0.114004

0.374353

$-1.57984$

0.114143

0.374998

$-1.57886$

0.114368

0.375114

1.576661

0.114874

0.375514

1.575599

0.115118

0.376835

$-1.57553$

0.115135

0.377012

$-1.57432$

0.115415

0.377012

$-1.57224$

0.115894

0.377588

1.572242

0.115894

0.378477

$-1.57176$

0.116006

0.378477

$-1.57084$

0.116219

0.378503

$-1.57092$

0.116202

0.378519

$-1.57007$

0.116398

0.378519

$-1.56936$

0.116563

0.378762

$-1.56868$

0.116722

0.378799

$-1.56897$

0.116655

0.378799

$-1.56625$

0.117289

0.378799

$-1.56513$

0.117553

0.380302

$-1.56502$

0.117579

0.380561

$-1.56395$

0.11783

0.380561

$-1.56325$

0.117994

0.381037

$-1.56067$

0.118602

0.381226

$-1.56052$

0.118638

0.382627

$-1.55955$

0.118866

0.382627

$-1.55744$

0.119367

0.38266

$-1.55736$

0.119384

0.38266

$-1.5576$ 
Sphingomonas hengshuiensis

Acidithiobacillus caldus

Arachidicoccus sp. BS20

Salinicola tamaricis

Wenzhouxiangella marina

Streptomyces lydicus

Erythrobacter flavus

Kitasatospora sp. MMS16-BH015

Pectobacterium atrosepticum

Terriglobus saanensis

Mycobacterium sp. JS623

Lachnospiraceae bacterium Choco86

Mycolicibacterium fortuitum

Actinoalloteichus sp. AHMU CJ021

Corynebacterium humireducens

Muribaculum intestinale

Microbacterium sp. LKL04

Desulfitobacterium hafniense

Paracoccus mutanolyticus

Bacillus cereus

Bifidobacterium animalis

Pseudodesulfovibrio indicus

Pseudomonas sp. PONIH3

Mesoplasma florum

Synechococcus sp. RCC307

Bifidobacterium angulatum

Erythrobacter litoralis

Actinobacillus porcitonsillarum

Vagococcus teuberi

Beijerinckia indica

Weissella jogaejeotgali

Nonlabens marinus

Anderseniella sp. Alg231-50

Corynebacterium maris

Sulfitobacter sp. JL08

Pandoravirus dulcis

Pseudomonas sp. LH1G9

Croceicoccus naphthovorans

Komagataeibacter medellinensis

Wigglesworthia glossinidia

Komagataeibacter nataicola

Klebsiella sp. FDAARGOS_511

Bacteroidales bacterium CF

Plautia stali symbiont

Thermoanaerobacterium thermosaccharc

Streptomyces sp. SCSIO 03032

Neisseria polysaccharea

Ensifer adhaerens

Staphylococcus sciuri

Geobacillus thermodenitrificans
145.6016

21.81237

38.70675

76.61274

40.12075

175.4628

39.08294

115.604

14.84305

37.12489

168.52

60.27843

22.81722

275.6765

933.676

21.7964

114.331

5.89717

79.83753

723.8069

68.67708

49.63254

27.58468

14.75998

16.17561

41.0549

102.894

9.958205

9.70085

19.71834

6.217357

3.667307

15.96214

898.6322

11.1737

2.151771

21.03186

90.32162

16.36747

5.241512

30.73407

6.736426

4.117773

45.78714

27.05684

101.43

37.68036

150.9668

92.07115

3.50674

$-0.77324$

$-1.02088$

0.502304

$-1.53938$

0.123711

0.391016

$-0.92313$

0.663708

$-1.53814$

0.124014

0.391634

$-0.79713$

0.601178

$-1.53554$

0.124651

0.393304

$-0.72644$

0.520082

$-1.53271$

0.125348

0.394839

$-0.47984$

0.473968

$-1.53268$

0.125354

0.394839

$-0.95721$

0.6254

$-1.5317$

0.125596

0.395257

$-0.67053$

0.438167

$-1.53056$

0.125878

0.395664

$-1.56766$

1.024711

$-1.5303$

0.125942

0.395664

$-1.00132$

0.654916

$-1.52985$

0.126053

0.395668

$-0.55562$

0.363507

$-1.52894$

0.12628

0.395695

$-1.5285$

0.126388

0.395695

-1.11406

0.728761

$-1.5287$

0.126339

0.395695

$-1.31797$

0.862736

$-1.52767$

0.126595

0.396005

0.745431

0.488394

1.526291

0.126938

0.396734

$-0.44286$

0.29026

$-1.52572$

0.127079

0.396837

$-1.51672$

0.994839

$-1.52459$

0.127361

0.397375

$-0.72522$

0.476687

$-1.52138$

0.128166

0.398178

$-1.85722$

1.220147

$-1.52213$

0.127977

0.398178

$-0.73598$

0.483607

$-1.52186$

0.128044

0.398178

0.664877

0.436879

1.521877

0.12804

0.398178

$\begin{array}{ll}-0.68448 & 0.449788\end{array}$

$-1.52177$

0.128066

0.398178

$-0.78608$

0.516921

$-1.52069$

0.128337

0.398371

$-2.05264$

1.350616

$-1.51978$

0.128565

0.398739

2.357706

1.558028

1.513264

0.130213

0.398769

$-1.43386$

0.946407

$-1.51505$

0.129759

0.398769

$-1.0886$

0.719562

$-1.51286$

0.130315

0.398769

$-0.7789$

0.514414

$-1.51414$

0.12999

0.398769

$-1.73302$

1.144046

$-1.51482$

0.129819

0.398769

$-1.368980 .90222$

$-1.51735$

0.129179

0.398769

$-1.054170 .694123$

$-1.51871$

0.128837

0.398769

$-1.913571 .263625$

$-1.51435$

0.129937

0.398769

$-2.394581 .582114$

$-1.51353$

0.130144

0.398769

$-1.51671$

0.12934

0.398769

$-0.44341$

0.292242

$-1.51728$

0.129195

0.398769

$-1.661061 .097999$

$-1.51281$

0.130329

0.398769

$-3.175692 .095585$

$-1.51542$

0.129666

0.398769

$-1.5172$

0.129216

0.398769

$-1.51553$

0.129637

0.398769

$-1.51599$

0.129522

0.398769

$-1.366490 .901385$

1.512243

0.130472

0.398871

$-1.511$

0.130788

0.399503

$-1.193730 .790027$

$-1.51041$

0.130939

0.399628

$-2.241531 .485939$

$-1.50849$

0.131429

0.400451 
Sphingomonas sp. NIC1

Microcystis aeruginosa

Streptomyces katrae

Pseudomonas aeruginosa

Actinoplanes missouriensis

Pseudomonas poae

Salinimonas sp. N102

Legionella hackeliae

Blastomonas sp. RAC04

Pantoea sp. PSNIH2

Streptococcus sp. NPS 308

Streptomyces clavuligerus

Halopiger xanaduensis

Streptomyces lincolnensis

Chloracidobacterium thermophilum

Mannheimia haemolytica

Bordetella hinzii

Cronobacter dublinensis

Pseudovibrio sp. FO-BEG1

Denitrobacterium detoxificans

Amycolatopsis sp. AA4

Chloroflexus aurantiacus

Celeribacter indicus

Bordetella sp. HZ2O

Desulfurobacterium thermolithotrophum

Phaeobacter gallaeciensis

Phaeobacter porticola

Rhodococcus fascians

Streptomyces malaysiensis

Streptococcus oralis

Streptomyces hygroscopicus

Blattabacterium punctulatus

Streptomyces sp. 3214.6

Pelodictyon phaeoclathratiforme

Runella slithyformis

Aggregatibacter actinomycetemcomitans

Pasteurella multocida

Saccharomonospora viridis

Pseudomonas syringae group genomosp.

Sphaerochaeta pleomorpha

Thermobifida fusca

Rhodothermaceae bacterium RA

Pseudomonas trivialis

Sphingopyxis sp. MG

Mariniflexile sp. TRM1-10

Shewanella sp. ANA-3

Micromonospora inositola

Truepera radiovictrix

Bacillus amyloliquefaciens

Clostridium cellulovorans
160.1681

5.264293

62.94547

3462.355

191.094

64.963

3.13673

4.074964

33.9244

4.2871

707.6592

79.32055

21.74257

71.87436

13.06259

5.611204

134.713

14.49568

8.058859

14.02252

129.3631

7.831384

66.02351

18.09669

3.639186

66.38695

8.666329

428.6191

34.10801

4626.866

297.5371

3.457677

71.30662

2.565901

12.57428

15.1415

491.5084

72.03617

68.52804

3.507212

54.52161

40.58339

86.96475

13.75232

13.74779

4.650979

83.63449

41.16298

5.961546

24.28007
$-0.84872$

$-2.53568$

$-0.7145$

$-0.7492$

$-0.64036$

$-1.09784$

0.564778

1.686258

0.47496

0.498246

0.426278

0.731034

$-2.57639$

1.716443

$-2.23985$

1.493073

0.740239

$-4.1604$

2.777066

$-0.83443$

0.557181

$-0.72165$

0.48204

$-1.2732$

$-0.83706$

0.851137

0.56023

0.81

$-1.21038$

$-1.71445$

1.147526

$-0.81384$

0.544572

0.814543

$-1.46856$

0.846947

0.985195

$-0.59814$

0.401682

$-1.62727$

1.093998

$-0.69839$

$-0.89215$

0.469912

0.601413

$-2.34415$

1.58102

$-0.72288$

0.487524

$-1.65456$

1.117885

1.017459

0.68742

$-1.38944$

0.938301

$-0.79895$

0.539858

$-0.69216$

0.468155

$-3.85484$

2.60799

0.495836

1.735449

$-1.38751$

0.940634

$-1.59073$

1.079068

$-0.73913$

0.502195

$-0.64217$

0.436821

$-1.46683$

0.99821

$-1.91779$

1.30534

$-0.6077$

0.413876

$-1.39098$

0.948161

$-0.73636$

0.50277

$-2.6408$

1.802789

$1.53343 \quad 1.046554$

$-2.21176$

1.511465

$-0.67862$

0.463655

$-0.79983$

0.546825

$-2.30692$

1.578767

0.958575

0.656206

$-1.50275$

0.132904

0.401582

$-1.50373$

0.13265

0.401582

$-1.50433$

0.132496

0.401582

$-1.50368$

0.132663

0.401582

$-1.50222$

0.133041

0.401663

$-1.50177$

0.133157

0.401678

$-1.50101$

0.133354

0.40194

$-1.50016$

0.133573

0.402267

$-1.4985$

$-1.49813$

0.134003

0.403187

$-1.49759$

0.1341

0.403187

$-1.49708$

0.13424

0.403274

$-1.49588$

0.134373

0.403342

$-1.49413$

0.134685

0.403943

$-1.4943$

0.135141

0.404052

$-1.49404$

0.135097

0.404052

$-1.49446$

0.135165

0.404052

$-1.49025$

$-1.49007$

0.135056

0.404052

0.136158

0.406165

$-1.49063$

0.136207

0.406165

$-1.48909$

0.136059

0.406165

$-1.48745$

0.136464

0.406598

$-1.48622$

0.136896

0.407551

$-1.48342$

0.137222

0.408188

$-1.48268$

0.137962

0.409972

0.13816

0.409972

$-1.48276$

$-1.48008$

0.138138

0.409972

1.480113

0.138852

0.410808

$-1.48081$

0.138843

0.410808

$-1.47992$

0.138658

0.410808

$-1.47848$

0.138893

0.410808

$-1.47809$

0.139279

0.411591

$-1.47749$ 
Caulobacter segnis

Neisseria zoodegmatis

Magnetospirillum magneticum

Caldithrix abyssi

Weeksella virosa

Peptoclostridium acidaminophilum

Actinobacteria bacterium IMCC26256

Yersinia rohdei

Hirschia baltica

Aeromicrobium marinum

Streptomyces sp. 3211

Bacillus infantis

Nocardioides sp. JS614

Micromonospora krabiensis

Micromonospora narathiwatensis

Roseibacterium elongatum

Mycolicibacter terrae

Jonquetella anthropi

Pseudomonas antarctica

Yersinia similis

Gilliamella apicola

Stackebrandtia nassauensis

Jeotgalibaca dankookensis

Streptomyces scabiei

Gemmatimonas aurantiaca

Defluviimonas alba

Plantibacter sp. PA-3-X8

Serratia sp. 3ACOL1

Streptomyces cavourensis

Staphylococcus condimenti

Halomonas hydrothermalis

Pseudomonas azotoformans

Nitrosomonas eutropha

Rhodococcus sp. YL-1

Thioalkalivibrio sp. K90mix

Gordonia polyisoprenivorans

Actinoplanes sp. N902-109

Micropruina glycogenica

Mycobacterium avium

Kangiella koreensis

Pseudoalteromonas atlantica

Ezakiella massiliensis

Acinetobacter sp. SWBY1

Olsenella uli

Streptomyces sp. HNM0039

Catenulispora acidiphila

Nocardioides sp. CF8

Arcanobacterium haemolyticum

Desulfarculus baarsii

Streptococcus pseudopneumoniae
159.911

34.15242

77.23386

3.47438

18.92034

5.975631

4.264456

4.155273

1.725429

124.3441

37.14771

9.335975

270.9308

116.7286

57.31809

39.11939

75.49085

48.97074

115.7211

2.968483

17.34115

108.2195

6.542669

75.52939

53.20161

110.8579

174.0809

2.421462

15.79052

61.98481

39.5451

140.881

6.126468

129.2982

25.3627

342.2178

145.0974

170.0668

255.0214

3.122941

12.68825

571.3083

166.8579

62.07051

48.35967

121.0724

282.1017

64.38764

45.14044

546.3913

$-0.80967$

$-0.97855$

$-0.78708$

0.55441

$-1.46043$

$0.144173 \quad 0.418931$

$-2.01198$

0.669622

$-1.46134$

0.143922

0.418931

$-1.85796$

1.379176

$-1.45979$

0.144348

0.419106

$-1.85002$

1.27626

0.144612

0.419537

2.01364

1.274073

0.145454

0.421642

$-2.1635$

1.386444

$-1.45205$

0.146487

0.423287

$-2.85972$

$-1.45238$

0.146397

0.423287

$-0.71484$

0.493763

$-1.45229$

0.14642

0.423287

$-0.74009$

0.510791

$-1.4522$

0.146445

0.423287

$-1.35895$

0.938654

$-1.44775$

0.147688

0.423911

$-0.70799$

0.489247

$-1.44891$

0.147364

0.423911

$-0.71142$

0.491326

$-1.44777$

0.147683

0.423911

$-1.4471$

$-1.44796$

0.147868

0.423911

$-0.76368$

0.527221

$-1.4485$

0.147629

0.423911

$-0.84219$

0.581909

$-1.4473$

0.147477

0.423911

$-0.54159$

0.373634

$-1.44951$

0.147814

0.423911

$-1.21424$

0.837998

$-1.44898$

0.147194

0.423911

$-0.87771$

0.606225

$-1.44782$

0.147344

0.423911

$-2.49285$

1.724079

$-1.4459$

0.147666

0.423911

1.355503

0.938287

1.444657

0.148205

0.424542

$-0.46143$

0.319966

$-1.44214$

0.148554

0.425209

$-1.60255$

1.111139

$-1.44226$

0.149263

0.426314

$-0.59167$

0.4103

$-1.44204$

0.14923

0.426314

$-0.71107$

0.493265

$-1.44156$

0.149292

0.426314

$-0.82093$

0.570139

$-1.43988$

0.149428

0.426367

$-0.74229$

0.515448

$-1.44008$

0.149901

0.426379

3.616059

2.509194

1.441123

0.149845

0.426379

$-1.5531$

1.078401

$-1.44019$

0.14955

0.426379

0.8270580 .57456

1.439464

0.149813

0.426379

$-1.4378$

0.150019

0.426382

$-0.89187$

0.620301

0.150491

0.427239

$-0.88899$

0.618542

$-1.43724$

0.15065

0.427239

$-1.43675$

0.15079

0.427239

$\begin{array}{lll}-1.05019 & 0.730897 & -1.43685\end{array}$

0.150761

0.427239

$-1.4354$

0.151174

0.427356

$-0.62465$

$0.435186-1.43536$

$-0.6411$

0.446406

$-1.43613$

0.151184

0.427356

$-0.54671$

0.38127

$-1.43391$

0.150966

0.427356

$-0.63352$

0.441616

$-1.43454$

0.151597

0.427632

$-2.639451 .840898$

$-1.43378$

0.151417

0.427632

$-2.26272 \quad 1.579115$

$-1.43291$

0.151634

0.427632

0.710018

0.495538

1.432824 
Pimelobacter simplex

Methylobacterium sp. 4-46

Campylobacter hyointestinalis

Sphingorhabdus flavimaris

Leifsonia sp. 21MFCrub1.1

Lactobacillus sakei

Candidatus Puniceispirillum marinum

Fibrella aestuarina

Mycolicibacterium rutilum

Erythrobacter sp. YH-07

Caldilinea aerophila

Microbacterium chocolatum

Pseudopedobacter saltans

Pajaroellobacter abortibovis

Azoarcus communis

Coriobacterium glomerans

Shewanella sp. M2

Pseudomonas sp. NCO2

Methylocella silvestris

Pseudomonas sp. MYb193

Tatumella citrea

Altererythrobacter sp. B11

Clostridium sp. JN-1

Streptomyces sp. 2323.1

Planococcus sp. Y42

Methylomonas denitrificans

Rhodocyclaceae bacterium

Cylindrospermum stagnale

Deinococcus puniceus

Rhodobacteraceae bacterium

Corynebacterium glyciniphilum

Bacillus paralicheniformis

Austwickia chelonae

Acidovorax ebreus

Leisingera methylohalidivorans

Nitrobacter winogradskyi

Spiroplasma litorale

Aerococcus christensenii

Mycoplasma capricolum

Stenotrophomonas sp. Pemsol

Arcobacter cryaerophilus

Marinovum algicola

Fusobacterium ulcerans

Amycolatopsis methanolica

Propionimicrobium sp. Marseille-P3275

Pseudomonas salegens

Bacillus sonorensis

Deinococcus proteolyticus

Celeribacter manganoxidans

Streptococcus sp. Z15
235.7532

173.9763

4.745509

16.43679

216.7935

35.9199

3.616058

43.89011

165.4134

23.62689

17.79206

158.5408

26.90762

2.286728

157.6845

20.91048

7.943657

95.49023

51.33165

86.826

12.06048

52.40961

17.09185

74.05311

25.92695

18.7122

52.53297

3.086043

39.48774

7.452796

472.4967

15.47695

63.93355

914.8676

33.73402

43.35834

2.57646

54.18105

2.471411

36.89535

11.09761

58.81083

9.89486

140.6791

163.968

20.76501

9.365089

167.0675

42.0459

5.687028

$\begin{array}{ll}-0.60583 & 0.425729\end{array}$

$-0.67873$

0.47683

$-1.42305$

0.154721

0.154612

0.432314

2.433028

1.711941

$-1.42343$

0.155256

0.432314

$-1.07654$

0.757275

1.42121

$-1.42159$

$-0.7569$

0.533106

$-1.41979$

0.155145

0.433144

$-1.07254$

0.756507

$-1.41775$

0.155669

0.433144

$-1.86348$

1.31833

$-1.41352$

0.156263

0.433964

$-1.02256$

0.724684

$-1.41104$

0.157504

0.435288

$-0.47219$

0.334406

$-1.41202$

0.158233

0.438407

$-1.07117$

0.759473

$-1.41042$

0.157945

0.439225

$-0.94503$

0.670203

$-1.41006$

0.158417

0.439225

$-0.75945$

0.538321

$-1.41077$

0.158522

0.439225

$-1.08227$

0.767502

$-1.41012$

0.158312

0.439225

$-3.24481$

2.302157

$-1.40946$

0.158504

0.439225

$-0.72611$

0.515425

$-1.40875$

0.158698

0.439225

$-1.06672$

0.757438

$-1.40833$

0.158908

0.439378

$-2.01381$

1.43192

$-1.40637$

0.159034

0.439626

$-1.04451$

0.7431

$-1.40561$

0.159615

0.43964

$-0.88128$

0.626712

$-1.4062$

0.159839

0.439936

$-0.87192$

0.620716

$-1.40471$

0.159663

0.439936

$-1.13769$

0.809532

$-1.40536$

0.160109

0.439936

$-0.75072$

0.534412

$-1.40476$

0.159914

0.439936

1.272592

0.905407

1.405547

0.160093

0.439936

$-0.66375$

0.471916

$-1.4065$

0.159859

0.439936

1.359727

0.968431

1.404052

0.159575

0.439936

$-1.12217$

0.799609

$-1.4034$

$-0.96371$

0.687549

$-1.40166$

0.160303

0.439936

$-2.23788$

1.597951

$-1.40047$

0.160497

0.440139

$-0.81146$

0.579451

$-1.4004$

0.161016

0.440338

$-1.41588$

1.012171

$-1.39885$

0.161373

0.441428

$\begin{array}{lll}-0.66374 & 0.474384 & -1.39916\end{array}$

0.161394

0.4418

$-1.418551 .01352$

$-1.39963$

0.161857

0.4418

$-0.620240 .443618$

$-1.39813$

0.161765

0.44207

-0.89807 0.642741

$-1.39725$

0.161624

0.44207

$-0.80820 .578403$

$-1.3973$

0.162073

0.44207

0.162339

0.442327

$-0.873440 .626191$

$-1.39485$

0.162323

0.44239

0.16306

0.44239

3.240006

2.323285

1.394579

0.163143

0.443914

$-0.945210 .678419$

$-1.39326$

0.163541

0.443914

$3.516212 \quad 2.526954$

1.391483

0.164079

0.444666

$-0.874250 .627854$

$-1.39244$

0.163788

0.444799

$\begin{array}{lll}1.840272 & 1.321984 & 1.392053\end{array}$

0.163906

0.444799

$-0.790710 .56809$

$-1.39187$

0.163962 
Luteimonas sp. 100111

Lactobacillus apis

Grimontia hollisae

Sphingobium japonicum

Tumebacillus algifaecis

Streptococcus sp. ChDC B345

Paenibacillus sabinae

Lactobacillus acetotolerans

Cryobacterium sp. GCJ02

Aeromicrobium sp. 592

Sebaldella termitidis

Nostoc sp. PCC 7107

Thermomonospora curvata

Roseburia hominis

Cobetia marina

Methanosarcina mazei

Polaribacter sp. BM10

Tumebacillus avium

Streptococcus gallolyticus

Oceanithermus profundus

halophilic archaeon DL31

Hungateiclostridium clariflavum

Salinibacter ruber

Magnetospirillum sp. XM-1

Aeromonas schubertii

Sphingomonas panacis

Streptococcus parasanguinis

Brochothrix thermosphacta

Janthinobacterium svalbardensis

Nitrosococcus halophilus

Mycolicibacter sinensis

Thermus parvatiensis

Rhodobacter capsulatus

Arsenicicoccus sp. oral taxon 190

Salinispira pacifica

Streptococcus halotolerans

Pseudomonas agarici

Desulfotomaculum nigrificans

Shimwellia blattae

Corynebacterium xerosis

Kutzneria albida

Enterococcus durans

Vibrio scophthalmi

Actinobacteria bacterium IMCC25003

Gammaproteobacteria bacterium DM2

Deinococcus wulumuqiensis

Bifidobacterium pseudolongum

Gammapapillomavirus 15

Xanthomonas cassavae

Pseudomonas sp. ATCC 13867
86.01518

11.80287

3.370998

38.14332

7.449483

1280.076

17.6766

4.04866

90.3907

216.8297

13.15585

7.108959

102.9473

90.34015

34.65311

10.33459

3.668209

27.27793

9.671654

66.81201

3.835051

14.53361

29.62257

68.74627

21.70778

165.7168

1655.378

311.7704

118.4549

16.11078

99.71662

2.456795

68.53574

217.9951

3.104442

10.70163

43.29298

6.462946

860.1553

779.4126

169.0952

10.71591

17.91297

5.737364

3.725334

585.4156

44.29696

15.35194

67.66888

92.00048
$-0.78769$

0.56856

1.83152

1.481144

0.6919

0.949413

0.649694

$-0.89768$

$-1.22337$

0.885483

$-2.72171$

1.969653

$-0.7595$

0.549609

$-0.69408$

0.502527

1.42838

1.034516

$-2.19559$

1.591391

0.46925

0.814193

$-1.12233$

$-0.94937$

0.68928

2.125815

1.545099

3.341081

2.43062

$-0.89298$

$-1.55657$

0.649922

1.13332

$-0.66744$

0.486323

$-2.01028$

1.465424

$-1.08574$

0.791834

$-0.83975$

0.613221

$-0.72622$

0.53005

$-0.85147$

0.621959

$-0.78685$

0.574485

$-0.81556$

1.247567

0.595504

0.911816

$-0.68316$

0.499685

$-0.8838$

0.646982

$-0.59852$

0.438066

$-3.36447$

2.464498

$-0.8028$

0.588546

$-0.56496$

0.414506

$-2.33289$

1.713246

$-1.40837$

1.035163

$-0.78703$

0.578374

$-1.5066$

1.10932

$-1.61168$

1.18673

$-0.54221$

0.399141

$-0.44567$

0.328016

$-1.49541$

1.103957

$-1.65849$

1.22627

$-1.68594$

1.24731

$-2.15862$

1.599474

$-0.79583$

0.590759

$-0.65245$

0.484119

$-2.15047$

1.594688

$-0.81134$

0.602107

$-0.6104$

0.452967
$-1.38541$

1.385212

$-1.38451$

$-1.38417$

$-1.38576$

$-1.3817$

$-1.38158$

$-1.38182$

$-1.38189$

$-1.38117$

1.380723

$-1.37967$

$-1.37894$

$-1.37845$

$-1.37733$

1.375843

1.37458

$-1.37398$

$-1.37346$

$-1.37243$

$-1.37181$

$-1.37117$

$-1.36941$

$-1.3701$

$-1.36902$

$-1.36967$

$-1.36953$

1.368222

$-1.36717$

$-1.36603$

$-1.36628$

$-1.36517$

$-1.36404$

$-1.36298$

$-1.36168$

$-1.36053$

$-1.36077$

$-1.35813$

$-1.35808$

$-1.35845$

$-1.35867$

$-1.35459$

$-1.35247$

$-1.35166$

$-1.34958$

$-1.34713$

$-1.34771$

$-1.34852$

$-1.34751$

0.1659290 .446513

0.1659880 .446513

0.1662040 .446513

0.1663070 .446513

0.1658190 .446513

0.1670640 .447322

$0.1671 \quad 0.447322$

0.1670260 .447322

$0.167007 \quad 0.447322$

0.1672260 .44733

$\begin{array}{ll}0.167364 & 0.44737\end{array}$

$0.167689 \quad 0.44791$

$0.167914 \quad 0.44818$

0.1680630 .448251

0.168410 .448846

0.168870 .449743

0.1692620 .450457

$0.169448 \quad 0.450624$

$0.169609 \quad 0.45072$

0.169930 .451245

0.1701230 .451428

$0.170322 \quad 0.451627$

$0.17087 \quad 0.451758$

0.1706550 .451758

0.1709930 .451758

0.170790 .451758

0.1708330 .451758

0.1712430 .45209

0.1715710 .452629

0.1719290 .452916

0.1718510 .452916

0.1721980 .453297

0.1725540 .453906

0.1728890 .454458

$0.1733 \quad 0.455211$

0.1736630 .455504

$0.173587 \quad 0.455504$

$0.174424 \quad 0.456221$

0.1744370 .456221

0.1743220 .456221

0.1742510 .456221

0.1755480 .458797

0.1762260 .460237

0.1764840 .460579

0.177150 .461986

0.1779370 .462383 
Martelella sp. AD-3

Morganella morganii

Pseudopropionibacterium propionicum

Petrimonas mucosa

Rhodococcus qingshengii

Roseobacter denitrificans

Faecalitalea cylindroides

Yangia sp. CCB-MM3

Streptomyces avermitilis

Staphylococcus aureus

Methylophaga nitratireducenticrescens

Archaeoglobus veneficus

Marinobacter sp. es.042

Stanieria cyanosphaera

Streptomyces puniciscabiei

Cellulomonas sp. PSBB021

Enterobacter hormaechei

Pseudomonas sp. MRSN12121

Pedobacter sp. PACM 27299

Methylovorus glucosotrophus

Desulfallas gibsoniae

Prevotella enoeca

Paracoccus yeei

Psychrobacter sp. DAB_AL43B

Microvirga sp. 17 mud 1-3

Streptomyces sp. 4F

Oceanisphaera profunda

Acinetobacter johnsonii

Bacillus smithii

Pseudomonas psychrophila

Sideroxydans lithotrophicus

Actinoalloteichus sp. ADI127-7

Cellulomonas flavigena

Streptomyces sp. 2114.2

Propionibacterium phage PAC1

Limnobaculum parvum

Staphylococcus virus SEP9

Rhizobium sp. 11515TR

Octadecabacter arcticus

Zhihengliuella sp. ISTPL4

Corallococcus coralloides

Luteimonas sp. 83-4

Stenotrophomonas acidaminiphila

Corynebacterium ureicelerivorans

Neisseria gonorrhoeae

Streptomyces coelicolor

Lactococcus sp. 1JSPR-7

Streptococcus sp. oral taxon 431

Bacillus litoralis

Blastococcus saxobsidens

\begin{tabular}{|c|c|c|c|c|c|}
\hline & & & & & \\
\hline .87002 & -1.1767 & 0.876421 & -1.34262 & 0.179395 & 0.465038 \\
\hline & -0.74622 & 0.556054 & -1.34199 & 0.179598 & 0.465038 \\
\hline 39504 & -1.18045 & 0.879573 & -1.34208 & 0.179572 & 0.465038 \\
\hline .4869 & -1.60943 & 1.198709 & -1.34264 & 0.179389 & 0.465038 \\
\hline 906736 & -1.22444 & 0.913767 & -1.33999 & 0.180249 & 0.465439 \\
\hline .66969 & -1.09909 & 0.819783 & -1.34071 & 0.180015 & 0.465439 \\
\hline 78.8124 & -0.59738 & 0.445761 & -1.34014 & 0.180199 & 0.465439 \\
\hline 49.83058 & -0.61485 & 0.458868 & -1.33994 & 0.180265 & 0.465439 \\
\hline 1773.23 & 0.951074 & 0.710572 & 1.338462 & 0.180746 & 0.46635 \\
\hline .884178 & -2.14722 & 1.608442 & -1.33497 & 0.181886 & 0.467056 \\
\hline 3.84057 & -1.94465 & 1.454703 & -1.3368 & 0.181287 & 0.467056 \\
\hline 14.21935 & -1.14075 & 0.853084 & -1.33721 & 0.181155 & 0.467056 \\
\hline 295935 & 1.965052 & 1.47209 & 1.334872 & 0.181918 & 0.467056 \\
\hline 65.24967 & -0.50514 & 0.378296 & -1.3353 & 0.181777 & 0.467056 \\
\hline 131.526 & -0.5739 & 0.42956 & -1.33602 & 0.181544 & 0.467056 \\
\hline 94.64144 & -1.21767 & 0.911583 & -1.33578 & 1622 & 7056 \\
\hline 25.08864 & -1.43075 & 1.072411 & -1.33414 & 0.182157 & 0.467339 \\
\hline 14.67225 & -1.26943 & 0.95209 & 333 & 2432 & 7716 \\
\hline 10.31124 & -1.51218 & 1.13543 & -1.33181 & 0.182922 & 0.468163 \\
\hline 7.542768 & -1.357 & 1.018856 & -1.33188 & 0.182898 & 0.468163 \\
\hline 123.9329 & -0.77071 & 0.578788 & -1.3316 & 0.182993 & 0.468163 \\
\hline 1032.3 & -0.80908 & 0.608247 & -1.33018 & 0.183458 & 0.469025 \\
\hline 19.60735 & -1.31882 & 0.992914 & -1.32823 & 0.184102 & 0.469705 \\
\hline 58.90435 & -0.65068 & 0.489761 & -1.32856 & 0.183994 & 0.469705 \\
\hline 56.52752 & -0.73939 & 0.556688 & -1.3282 & 0.184112 & 0.469705 \\
\hline 4.597922 & -1.75706 & 1.323561 & -1.32752 & 0.184336 & 0.469948 \\
\hline 424.2008 & -0.74319 & 0.560227 & -1.32659 & 0.184646 & 0.470407 \\
\hline 6.130459 & -1.59233 & 1.201459 & -1.32533 & 0.185062 & 0.471138 \\
\hline 82.8616 & -0.76861 & 0.581224 & -1.3224 & 0.186036 & 0.472954 \\
\hline 51.19056 & -0.78417 & 0.592868 & -1.32267 & 0.185944 & 0.472954 \\
\hline 3.315727 & -3.79327 & 2.87073 & -1.32136 & 0.186381 & 0.473501 \\
\hline 120.049 & -0.6618 & 0.501185 & -1.32046 & 0.186681 & 0.473933 \\
\hline 2.754571 & -3.52682 & 2.672227 & -1.31981 & 0.1869 & 0.474157 \\
\hline 18.72886 & -1.99744 & 1.516155 & -1.31744 & 0.187692 & 0.475835 \\
\hline 8.652402 & -1.27842 & 0.97072 & -1.31698 & 0.187844 & 0.475888 \\
\hline 107.0409 & 2.299168 & 1.747757 & 1.315496 & 0.188343 & 0.476821 \\
\hline 69.59405 & -0.73804 & 0.561531 & -1.31434 & 0.188732 & 0.477474 \\
\hline 7.855219 & -1.25181 & 0.953135 & -1.31336 & 0.189063 & 0.477979 \\
\hline 114.407 & -0.55906 & 0.426567 & -1.31059 & 0.189995 & 0.478245 \\
\hline 121.8942 & -0.73705 & 0.561787 & -1.31197 & 0.189529 & 0.478245 \\
\hline 96.95985 & -0.67703 & 0.516374 & -1.31113 & 0.189813 & 0.478245 \\
\hline 196.9742 & -0.62999 & 0.480477 & -1.31117 & 0.1898 & 0.478245 \\
\hline 11336.12 & -0.71276 & 0.544217 & -1.3097 & 0.190298 & 0.478245 \\
\hline 33.95886 & -1.24742 & 0.95284 & -1.30916 & 0.190481 & 0.478245 \\
\hline 21.69228 & -2.12492 & 1.62191 & -1.31013 & 0.190151 & 0.478245 \\
\hline 3.849008 & -1.75652 & 1.341586 & -1.30928 & 0.190438 & 0.478245 \\
\hline 898.0551 & -0.85579 & 0.652593 & -1.31137 & 0.189732 & 0.478245 \\
\hline 23.22759 & -1.00858 & 0.770404 & -1.30915 & 0.190483 & 0.478245 \\
\hline 229.3941 & -0.6274 & 0.480034 & -1.307 & 0.191213 & 0.478434 \\
\hline
\end{tabular}


Altererythrobacter dongtanensis

Arcobacter bivalviorum

Parasaccharibacter apium

Aeromonas veronii

Propionibacterium virus SKKY

Haliangium ochraceum

Paenibacillus polymyxa

Algibacter alginicilyticus

Sulfitobacter sp. SK011

Ketogulonicigenium vulgare

Streptomyces chartreusis

Clostridium carboxidivorans

Gynuella sunshinyii

Corynebacterium mycetoides

Pseudomonas thivervalensis

Zymomonas mobilis

Solimonas sp. K1W22B-7

Acinetobacter sp. WCHA45

[Mannheimia] succiniciproducens

Natrinema sp. J7-2

Halomonas sp. HL-93

Vibrio mimicus

Devosia sp. $\mathrm{H} 5989$

Tamlana sp. UJ94

Planctopirus limnophila

Gramella flava

Microvirga ossetica

Corynebacterium halotolerans

Rufibacter tibetensis

Spiroplasma cantharicola

Hahella chejuensis

Microvirgula aerodenitrificans

Corynebacterium sp. 2183

Aquiflexum balticum

Shewanella woodyi

Bacteroides thetaiotaomicron

Haematospirillum jordaniae

Acetoanaerobium sticklandii

Halorubrum trapanicum

Mycoplasma fermentans

Stenotrophomonas sp. ESTM1D_MKCIP4

Shewanella benthica

Enterobacter sp. 638

Agromyces sp. 30A

Streptomyces davaonensis

Tessaracoccus sp. T2.5-30

Geodermatophilus obscurus

Shewanella sp. FDAARGOS_354

Micromonospora zamorensis

Frankia alni

$\begin{array}{rrrrrr}46.95291 & -0.68029 & 0.520037 & -1.30816 & 0.190818 & 0.478434 \\ 9.190749 & 1.638944 & 1.253396 & 1.307603 & 0.191008 & 0.478434 \\ 12.39909 & -1.33052 & 1.017507 & -1.30762 & 0.191001 & 0.478434 \\ 62.28356 & -0.63427 & 0.485291 & -1.30699 & 0.191216 & 0.478434 \\ 15.43086 & -1.78346 & 1.365374 & -1.3062 & 0.191483 & 0.478774 \\ 181.017 & -0.59119 & 0.452794 & -1.30565 & 0.191673 & 0.478919 \\ 112.5299 & 1.058582 & 0.811203 & 1.304952 & 0.191909 & 0.47918 \\ 8.947162 & 1.320333 & 1.012794 & 1.303654 & 0.192352 & 0.479955 \\ 13.40609 & -1.23064 & 0.945568 & -1.30149 & 0.193092 & 0.480485 \\ 30.68701 & -0.86819 & 0.666917 & -1.3018 & 0.192986 & 0.480485 \\ 68.15836 & -0.69204 & 0.531658 & -1.30166 & 0.193032 & 0.480485 \\ 19.22412 & 1.048755 & 0.80569 & 1.301686 & 0.193024 & 0.480485 \\ 4.16487 & -1.68763 & 1.297228 & -1.30095 & 0.193275 & 0.480612 \\ 2086.245 & -0.48685 & 0.3744 & -1.30034 & 0.193486 & 0.480806 \\ 31.17718 & -1.09068 & 0.839336 & -1.29946 & 0.193786 & 0.480857 \\ 16.20191 & -0.99203 & 0.763452 & -1.29941 & 0.193805 & 0.480857 \\ 122.3893 & -0.71591 & 0.551071 & -1.29912 & 0.193903 & 0.480857 \\ 87.97641 & 0.830122 & 0.639605 & 1.297868 & 0.194333 & 0.481596 \\ 4.549968 & -2.21618 & 1.708795 & -1.29692 & 0.194657 & 0.482071 \\ 7.477352 & -1.6266 & 1.255164 & -1.29593 & 0.195001 & 0.482593 \\ 6.507057 & -2.81638 & 2.174486 & -1.2952 & 0.195253 & 0.482889 \\ 3.127178 & -2.55273 & 1.97192 & -1.29454 & 0.195479 & 0.48312 \\ 63.23899 & -0.55595 & 0.429801 & -1.29351 & 0.195836 & 0.483345 \\ 5.383932 & 2.682052 & 2.073233 & 1.293657 & 0.195784 & 0.483345 \\ 4.148962 & -1.56398 & 1.214565 & -1.28769 & 0.197855 & 0.48339 \\ 4.68196 & -1.70007 & 1.318856 & -1.28905 & 0.19738 & 0.48339 \\ 133.309 & -0.6681 & 0.516853 & -1.29263 & 0.196138 & 0.48339 \\ 1266.555 & -0.33265 & 0.257799 & -1.29035 & 0.196929 & 0.48339 \\ 15.91245 & -0.94671 & 0.735404 & -1.28733 & 0.19798 & 0.48339 \\ 3.563623 & 2.937292 & 2.279197 & 1.28874 & 0.197489 & 0.48339 \\ 16.33761 & -0.82148 & 0.638047 & -1.2875 & 0.197921 & 0.48339 \\ 84.38277 & -0.55222 & 0.430655 & -1.28227 & 0.199746 & 0.485789 \\ 127.9709 & -0.55018 & 0.42908 & -1.28223 & 0.199764 & 0.485789 \\ 292.6087 & -0.67986 & 0.531314 & -1.27957 & 0.200695 & 0.487729 \\ 13.30108 & 1.383904 & 1.083355 & 1.277425 & 0.201452 & 0.489118 \\ 64.38884 & -0.54411 & 0.42615 & -1.27681 & 0.201671 & 0.489118 \\ 98.2108 & -0.6138 & 0.480696 & -1.2769 & 0.201636 & 0.489118\end{array}$


Polaribacter vadi

Deinococcus ficus

Mannheimia sp. USDA-ARS-USMARC-126

Parabacteroides distasonis

Sphingobium sp. SYK-6

Acinetobacter junii

Pragia fontium

Bacillus coagulans

Rhodovulum sulfidophilum

Aeromonas dhakensis

Paracoccus sp. SC2-6

Vibrio furnissii

Paenibacillus beijingensis

Rhodococcus biphenylivorans

Lentibacillus amyloliquefaciens

Microbacterium sp. Y-01

Shewanella sp. WE21

Calothrix parietina

Haemophilus sp. oral taxon 036

Nocardia brasiliensis

Nocardioides sp. 603

Treponema brennaborense

Streptomyces sp. CCM_MD2014

Corynebacterium sp. 2184

Vibrio natriegens

Cnuibacter physcomitrellae

Streptomyces pluripotens

Streptococcus marmotae

Sphingobium chlorophenolicum

Shewanella oneidensis

Altererythrobacter sp. NS1

Anoxybacillus flavithermus

Lactobacillus helveticus

Leclercia adecarboxylata

Hoyosella subflava

Streptomyces nodosus

Burkholderia thailandensis

Marmoricola scoriae

Oceanobacillus sp. 160

Halopenitus persicus

Psychrobacter sp. AntiMn-1

Methylobacterium currus

Ehrlichia ruminantium

Nocardioides sp. 78

Pseudarcicella sp. HME7025

Winogradskyella sp. RHA_55

Corynebacterium sphenisci

Rhodobacter sp. LPB0142

Carnobacterium inhibens

Klebsiella pneumoniae
69.41816

3.404922

70.49027

84.76941

225.9786

2.579386

13.90044

72.90301

4.995763

141.9544

9.492429

12.07497

112.1182

7.539801

204.3947

12.96449

12.08213

63.7453

274.8674

222.7609

4.9014

46.98279

1473.024

6.235702

207.6842

58.70019

8.828842

44.90604

2.849486

82.63247

51.51324

80.28939

43.36665

83.47393

63.30756

558.6745

325.8691

5.885886

9.400056

26.15277

135.3729

3.579978

167.9322

14.13247

5.795058

386.3147

92.09139

14.04082

523.6375

\subsection{1}

$-0.6726$

$-2.338$

$-1.39564$

$-0.4974$

$-0.69076$

$-1.90838$

$-0.78393$

$-0.72859$

$-1.88616$

$-0.67737$

$-1.22843$

$-0.90879$

$-1.41657$

$-1.18501$

$-0.62936$

$-1.2835$

$-1.50008$

$-1.011$

$-0.39925$

$-0.52216$

$-1.55408$

$-0.67336$

$-0.37733$

$-2.5736$

$-0.74069$

$-0.60881$

$-1.2821$

$-0.83482$

$-2.96766$

$-0.67798$

$-1.09784$

$-1.0764$

$-1.79662$

$-0.58745$

$-0.54873$

$-0.69897$

$-0.52314$

$-1.68565$

$-1.19609$

$-1.19268$

$-0.57555$

3.094148

$-0.65984$

$-1.11563$

$-1.78537$

$-0.41488$

$-0.65676$

$-0.98727$

0.52726

0.794161

0.424344

527415

1.834532

415

390575

0.616841

573695

1.484321

0.969236

0.718849

1.121662

0.938129

0.801761

0.316871

0.534905

1.021655

2.362696

0.876556

1.434466

1.276292

0.201852

0.489233

$-1.27527$

0.202213

0.489781

$-1.27444$

0.202507

0.490168

$-1.27291$

0.20305

0.490596

$-1.27351$

0.202836

0.490596

$-1.2728$

0.203089

0.490596

$1.502371-1.27025$

0.203996

0.491705

$-1.27088$

0.203771

0.491705

$-1.26999$

0.204088

0.491705

$-1.27072$

0.203827

0.491705

$-1.269$

0.204442

0.492229

$-1.26742$

0.205006

0.493262

$-1.26423$

0.206146

0.495678

$-1.26292$

0.206619

0.496158

$-1.26316$

0.206532

0.496158

$0.49854-1.2624$

0.206805

0.496277

$1.01756-1.26135$

0.207183

0.49653

$1.189113-1.26151$

0.207124

0.49653

$-1.26097$

0.20732

0.49653

$-1.25997$

0.20768

0.497067

$0.414559-1.25954$

0.207834

0.497107

$-1.25858$

0.208183

0.497288

$-1.25884$

0.208087

0.497288

$-1.25534$

0.209357

0.498758

$-1.25461$

0.20962

0.498758

$-1.25423$

0.209758

0.498758

$-1.25427$

0.209744

0.498758

$-1.25492$

0.209507

0.498758

$-1.2555$

0.209297

0.498758

$-1.25605$

0.209099

0.498758

$-1.25237$

0.210436

0.499347

$-1.25244$

0.210409

0.499347

$-1.2519$

0.210608

0.499347

$-1.25247$

0.2104

0.499347

$-1.25166$

0.210692

0.499347

$-1.25085$

0.210989

0.499401

$-1.25096$

0.210948

0.499401

$-1.24882$

0.211732

0.499824

$-1.24836$

0.211898

0.499824

$-1.24811$

0.211993 
Pantoea alhagi

Rathayibacter tritici

Streptomyces griseochromogenes

Microbacterium testaceum

Gordonia sp. 1D

Lactobacillus amylolyticus

Corynebacterium testudinoris

Arthrobacter crystallopoietes

Streptomyces gilvosporeus

Thiomicrospira aerophila

Microbacterium sp. No. 7

Sphingobium sp. YG1

Dietzia lutea

Gammapapillomavirus 11

Propionibacterium virus P144

Vibrio tapetis

Sphingomonas sp. Cra20

Synechococcus sp. CC9605

Brevundimonas sp. GW460-12-10-14-LB2

Yersinia aldovae

Pseudonocardia dioxanivorans

Sphingobium sp. YBL2

Pseudomonas mendocina

Salinispora arenicola

Pseudomonas sihuiensis

Nakamurella sp. s14-144

Flavobacterium sp. CJ74

Halorhodospira halochloris

Idiomarina loihiensis

Bibersteinia trehalosi

Streptococcus sp. oral taxon 064

Fibrobacter succinogenes

Candidatus Bipolaricaulis anaerobius

Jonesia denitrificans

Halioglobus japonicus

Microbacterium sp. 10M-3C3

Agromyces aureus

Trueperella pyogenes

Fusobacterium nucleatum

Ruegeria sp. AD91A

Staphylococcus phage StB12

Campylobacter hominis

Microcella alkaliphila

[Enterobacter] lignolyticus

Amycolatopsis keratiniphila

Yersinia ruckeri

Neisseria mucosa

Geminocystis sp. NIES-3708

Haemophilus haemolyticus

Alistipes shahii
20.7442

82.04586

71.83927

118.6332

258.7029

3.804422

983.0053

151.1168

87.64134

5.935203

264.9153

114.6589

1454.156

33.06983

22.33976

3.381637

119.8524

17.2385

325.8829

2.97714

192.6575

67.59588

424.2076

39.80362

58.21296

38.2789

18.41253

6.441646

6.541659

8.049973

780.8899

7.725801

4.872993

40.28468

13.3845

170.6426

119.8327

124.9683

496.5736

12.44978

75.11767

16.30385

84.2859

21.44221

112.3321

8.433607

369.5458

14.18361

198.4342

95.15295
$-0.94665$

$-0.62518$

$-0.69789$

0.762464

0.503533

$-0.53082$

0.56379

0.428862

0.465778

1.851806

0.40944

0.330952

$-0.46154$

0.372814

$-0.472$

0.381284

$-1.86684$

1.515159

$-0.56614$

0.459394

$-0.68474$

0.555331

$-0.80889$

1.895031

0.655973

1.537069

1.742162

1.412694

$-2.44735$

1.984448

$-0.69959$

$-1.30589$

0.567031

0.65168

1.056993

0.52893

$-1.88951$

1.53093

$-0.48346$

0.392714

$-0.74379$

0.604121

$-0.72413$

0.589952

$-0.65615$

0.534486

$-0.55994$

0.456088

$-0.68317$

0.556212

$-1.07805$

0.879283

$-1.38979$

1.131452

$-1.49062$

1.212777

$-1.31783$

1.071726

$-0.72616$

0.591016

$-1.1169$

0.911027

$-1.62609$

1.326324

$-0.58735$

0.477687

$-0.8786$

0.716023

$-0.48805$

0.398722

$-0.55903$

0.456647

$-0.32903$

0.268913

$-0.55551$

0.454066

$-1.18526$

0.969249

0.957381

0.78334

$-1.1684$

0.956386

$-0.53085$

0.434517

$-0.78284$

0.641055

$-0.50183$

0.411232

$-1.30653$

1.070588

$-0.93192$

1.193666

0.764675

0.978738

0.617361

0.75255

0.555236
$-1.24156$

$-1.24158$

$-1.23786$

$-1.23773$

$-1.23902$

$-1.23744$

$-1.23716$

$-1.23799$

$-1.23793$

$-1.23211$

$-1.23236$

$-1.23304$

$-1.23311$

1.232886

1.23322

$-1.23326$

$-1.23378$

$-1.23547$

$-1.23207$

$-1.23422$

$-1.23107$

$-1.23119$

$-1.22744$

$-1.22763$

$-1.22771$

$-1.22826$

$-1.22606$

$-1.22833$

$-1.22909$

$-1.22964$

$-1.22866$

$-1.22598$

$-1.22602$

$-1.22957$

$-1.22706$

$-1.22403$

$-1.2242$

$-1.22355$

$-1.22341$

$-1.22286$

1.222178

$-1.22168$

$-1.2217$

$-1.22118$

$-1.2203$

$-1.22039$

$-1.21871$

1.219597

$-1.21898$

$-1.21907$

0.2143990 .502566

0.214390 .502566

$\begin{array}{lll}0.215767 & 0.504107\end{array}$

0.2158150 .504107

0.2153370 .504107

0.2159250 .504107

0.2160260 .504107

0.215720 .504107

$0.215744 \quad 0.504107$

0.2179090 .504974

0.2178150 .504974

$0.217562 \quad 0.504974$

0.2175350 .504974

0.2176180 .504974

$0.217494 \quad 0.504974$

$0.217478 \quad 0.504974$

0.2172850 .504974

0.2166550 .504974

0.2179250 .504974

$0.217122 \quad 0.504974$

0.2182980 .505195

0.2182520 .505195

0.2196570 .505432

0.2195850 .505432

0.2195560 .505432

0.219350 .505432

0.2201770 .505432

0.2193250 .505432

0.2190360 .505432

0.2188330 .505432

0.2191990 .505432

0.2202060 .505432

0.2201930 .505432

0.2188580 .505432

0.2198010 .505432

0.2209430 .506382

0.2208760 .506382

$\begin{array}{lll}0.22112 & 0.506382\end{array}$

0.2211770 .506382

0.2213810 .50653

0.2216410 .506598 
Thalassospira xiamenensis

Oscillatoria acuminata

Streptomyces qaidamensis

Enterobacteriaceae bacterium w17

Micromonospora viridifaciens

Caldicellulosiruptor saccharolyticus

Vibrio anguillarum

Neisseria sp. KEM232

Pseudoalteromonas agarivorans

Dietzia sp. oral taxon 368

Intrasporangium calvum

Altererythrobacter epoxidivorans

Candidatus Nucleicultrix amoebiphila

Shewanella japonica

Marinobacter sp. Arc7-DN-1

Streptomyces sp. ETH9427

Humibacter sp. BT305

Leifsonia xyli

Aeromonas caviae

Tabrizicola sp. K13M18

Streptomyces sp. RTd22

Pantoea ananatis

Streptococcus equi

Cellulosimicrobium cellulans

Micromonospora tulbaghiae

Rhodococcus sp. P1Y

Lutibacter profundi

Leuconostoc gelidum

Deinococcus soli Cha et al. 2016

Leclercia sp. LSNIH1

Mucilaginibacter mallensis

Tessaracoccus aquimaris

Thermococcus pacificus

Niveispirillum cyanobacteriorum

Corynebacterium uterequi

Hungateiclostridium saccincola

Owenweeksia hongkongensis

Hymenobacter nivis

Aerococcus urinaehominis

Ketogulonicigenium robustum

Planctomyces sp. SH-PL62

Candidatus Saccharibacteria oral taxon Tr

Hymenobacter sp. DG25A

Photobacterium gaetbulicola

[Eubacterium] hallii

Kineococcus radiotolerans

Serratia sp. ATCC 39006

Sphingopyxis sp. EG6

Arthrobacter sp. DCT-5

Bordetella genomosp. 6
16.05365

2.70113

64.58409

2.059422

77.83662

6.615658

17.01305

80.15289

2.400646

5915.23

166.6742

20.96323

1.510949

3.088212

20.51358

42.48779

231.4551

278.5884

24.92744

51.14698

122.8612

38.70233

59.19181

123.0118

66.52734

146.2682

11.97098

92.55287

37.7251

11.30758

40.03396

163.6911

3.676169

82.23331

804.5438

11.11172

3.684997

65.32261

3.765306

25.36697

117.5707

52.05183

28.72505

6.320813

92.18346

129.6845

9.039923

67.09231

149.2683

18.35488 $\begin{array}{lll}-0.78073 & 0.641161\end{array}$

$-2.08892$

$-0.61379$

1.716549

$-3.10697$

0.504513

2.554035

$-0.53316$

0.43845

$-1.91305$

1.573577

1.147797

0.945187

$-0.62882$

0.518082

$-3.32894$

2.74244

$-0.7661$

0.631519

$-0.51241$

0.422541

$-0.93082$

0.769039

$-2.66091$

2.199554

$-2.42064$

2.000046

$-0.83907$

0.693562

$-0.65178$

0.540121

$-0.64342$

0.533774

$-0.47943$

0.396685

$-1.40873$

1.166778

$-0.65571$

0.543643

$-0.46168$

0.382093

$-0.62821$

0.520403

$-0.72041$

0.597403

$-0.59679$

0.494956

$-0.72707$

0.602621

0.536337

0.445574

1.142527

0.948867

$-0.77222$

0.641737

$-0.827$

0.686579

$-1.5604$

1.296594

$-0.8102$

0.673584

$-0.4179$

0.347789

$-2.2138$

1.842514

$-0.66496$

0.553611

$-0.3548$

0.295421

1.157182

0.964342

$-1.66741$

1.39337

$-0.66628$

0.555972

$-1.75539$

1.468036

$-0.68813$

0.575459

$-0.67612$

0.5649

$-0.90135$

0.818108

0.753961

$-1.51461$

0.683711

1.267006

$-0.74384$

0.620899

$-0.54654$

0.456185

$-1.1409$

0.952891

$-0.67156$

0.562071

$-0.52556$

0.440359

$-1.352411 .134476$
$-1.21768$

$-1.21693$

$-1.2166$

$-1.2165$

$-1.21602$

$-1.21573$

1.21436

$-1.21375$

$-1.21386$

$-1.21311$

$-1.2127$

$-1.21037$

$-1.20975$

$-1.21029$

$-1.2098$

$-1.20674$

$-1.20542$

$-1.20858$

$-1.20737$

$-1.20615$

$-1.20831$

$-1.20717$

$-1.2059$

$-1.20574$

$-1.20651$

1.203699

1.204097

$-1.20333$

$-1.20452$

$-1.20346$

$-1.20282$

$-1.2016$

$-1.20151$

$-1.20114$

$-1.20098$

1.19997

$-1.19667$

$-1.19841$

$-1.19574$

$-1.1958$

$-1.19688$

$-1.19549$

1.19657

$-1.19543$

$-1.19801$

$-1.19807$

$-1.1973$

$-1.1948$

$-1.19347$

$-1.1921$

$0.223345 \quad 0.507513$

0.2236310 .507588

0.2237570 .507588

0.2237960 .507588

0.2239770 .507613

0.2240860 .507613

0.224610 .508373

0.2248410 .508373

$0.2248 \quad 0.508373$

0.225090 .508619

0.2252460 .508655

$0.226136 \quad 0.50994$

$0.226375 \quad 0.50994$

$0.226167 \quad 0.50994$

$0.226354 \quad 0.50994$

$0.227534 \quad 0.510534$

$0.228042 \quad 0.510534$

$0.226824 \quad 0.510534$

0.2272890 .510534

0.2277610 .510534

$0.22693 \quad 0.510534$

$0.227367 \quad 0.510534$

0.2278550 .510534

$0.22792 \quad 0.510534$

0.227620 .510534

0.2287060 .510768

0.2285520 .510768

0.2288490 .510768

0.228390 .510768

0.2287970 .510768

0.2290450 .510892

0.2295170 .511227

$0.229553 \quad 0.511227$

0.2296980 .511227

0.2297570 .511227

0.2301510 .51179

0.2314330 .512281

0.2307580 .512281

0.2317970 .512281

0.2317740 .512281

0.2313520 .512281

0.2318970 .512281 
Thermanaerovibrio acidaminovorans

Janthinobacterium sp. LM6

Actinomyces oris

Sinorhizobium meliloti

Acetobacter aceti

Klebsiella oxytoca

Halorhodospira halophila

Methanobrevibacter ruminantium

Streptomyces sp. FR-008

Olsenella umbonata

Rhizobium etli

Pontibacter akesuensis

Corynebacterium marinum

Alteromonas mediterranea

Phenylobacterium sp. HYN0004

Lactobacillus gasseri

Maribacter cobaltidurans

Colwellia sp. Arc7-D

Planococcus antarcticus

Clostridium chauvoei

Actinosynnema pretiosum

Agrobacterium sp. H13-3

Francisella tularensis

Methylomonas sp. DH-1

Corynebacterium aquilae

Erythrobacter atlanticus

Carnobacterium sp. CP1

Treponema succinifaciens

Mucinivorans hirudinis

Hoeflea sp. IMCC20628

Anaeromyxobacter sp. K

Flavobacterium indicum

Lysinibacillus sp. Marseille-P5727

Saccharopolyspora erythraea

Propionibacterium phage PA1-14

Streptomyces olivaceus

Veillonella rodentium

Arthrobacter sp. FB24

Lactobacillus zymae

Streptococcus pneumoniae

Micromonospora aurantiaca

Sinomonas atrocyanea

Corynebacterium geronticis

Tenacibaculum maritimum

[Mycobacterium] stephanolepidis

Methanosphaera stadtmanae

Lactobacillus acidipiscis

Salinisphaera sp. LB1

Olsenella sp. Marseille-P2300

Neomicrococcus aestuarii

\begin{tabular}{|c|c|c|c|c|c|}
\hline & & & & & \\
\hline & -0.72492 & 0.609687 & -1.189 & 0.23444 & \\
\hline & -0.59796 & 0.503318 & -1.18803 & 0.234821 & ז. \\
\hline 478 & -0.53097 & .447275 & -1.18713 & 5177 & \\
\hline & -0.7 & & & & \\
\hline & -0.67227 & 0.567144 & -1.18537 & 0.235873 & \\
\hline 44 & -0.79 & 0.674388 & -1.18551 & 15 & 67 \\
\hline & 3.20 & 753 & 1.181702 & & \\
\hline & & & -1.1 & & \\
\hline & -0.81 & 235 & -1.18009 & 964 & \\
\hline & -0.5 & 582 & -1.17887 & 45 & \\
\hline & & 999 & & & \\
\hline & -0.3 & 759 & -1.17 & 76 & \\
\hline & -1.696 & 1.441017 & -1.17726 & 91 & \\
\hline & -0.68 & 0.578162 & -1.17678 & & \\
\hline & & 818 & 1.17 & 32 & \\
\hline 087 & -1.99 & 033 & -1.17524 & 99 & \\
\hline & -1.7 & 1.508041 & -1.17525 & & \\
\hline & -1.5 & & -1.17441 & & \\
\hline & 0.98 & & 1.1743 & & \\
\hline & -0.7 & 1855 & -1.17265 & 0.24 & \\
\hline & -3.4 & & -1.17298 & & \\
\hline & 1.507 & & 1.171759 & 94 & \\
\hline & -1.3 & 1.1 & -1.17199 & & \\
\hline & -0.4 & & -1.17021 & & \\
\hline & -1.1 & 0.9 & & & \\
\hline & -1.2 & 1.0 & -1.170 & & \\
\hline & & 1.605153 & 1.168804 & & \\
\hline & -1.52 & & -1.16778 & & \\
\hline & -0.7 & & -1.1 & & \\
\hline & -0.94 & 0.80 & -1.16606 & & \\
\hline & 1.533707 & & 1.164295 & & \\
\hline & 0.89 & & 1.165 & & \\
\hline & -0.41 & 0.3 & -1.16392 & 0.24 & \\
\hline & & & & & \\
\hline & -0.63 & & -1.16408 & 0.24 & \\
\hline & -0.6 & & -1.1 & & \\
\hline & $-0.4 \subseteq$ & 0.4 & & & \\
\hline & & 1.784327 & -1.16134 & 0.245 & \\
\hline & -0.62 & & -1.161 & & \\
\hline & -1.05 & 0.90 & -1.159 & & \\
\hline & & & & & \\
\hline & -0.42412 & 0.366366 & -1.15764 & 0.247013 & \\
\hline & & & 1.157083 & & \\
\hline & -0.69907 & 0.603989 & -1.15742 & & \\
\hline & 2.435157 & 2.106776 & 1.155869 & 0.247735 & \\
\hline & -2.16749 & 1.874643 & -1.15622 & 0.247593 & \\
\hline & & 0.529809 & -1.15402 & & \\
\hline & & 0.509156 & -1.15414 & 0.248 & \\
\hline & -0 & 0.493401 & -1.15388 & 0.248 & 0.531 \\
\hline
\end{tabular}


Streptococcus phage SpSL1

Barnesiella viscericola

Streptomyces noursei

Hymenobacter sp. sh-6

Gordonia sp. MMS17-SY073

Kibdelosporangium phytohabitans

Methanobacterium lacus

Staphylococcus hominis

Propionibacterium phage PHL010M04

Corynebacterium sp. NML98-0116

Pseudomonas psychrotolerans

Streptomyces peucetius

Erysipelotrichaceae bacterium GAM147

Calditerrivibrio nitroreducens

Legionella spiritensis

Prevotella scopos

Comamonas thiooxydans

Sphaerochaeta globosa

Clavibacter michiganensis

Candidatus Symbiobacter mobilis

Pantoea rwandensis

Spongiibacter sp. IMCC21906

Staphylococcus phage StB20

Lentzea guizhouensis

Actinobacillus equuli

Alteromonas sp. BL110

Xenorhabdus hominickii

Enterobacter kobei

Methylophaga frappieri

Alteromonas macleodii

Gammapapillomavirus 3

Laceyella sacchari

Polaribacter sp. KT25b

Serratia plymuthica

Glaesserella parasuis

Flammeovirga sp. MY04

Streptomyces sp. GSSD-12

Actinobacteria bacterium YIM 96077

Spirosoma rigui

Nakamurella multipartita

Sulfitobacter sp. D7

Roseiflexus castenholzii

Bacteriovorax stolpii

Paenibacillus stellifer

Brevundimonas sp. LM2

Streptomyces sp. 11-1-2

Sphingomonas taxi

Streptomyces griseorubiginosus

Haloferax gibbonsii

Corynebacterium provencense
1.61991

57.19991

50.1943

31.57819

241.4006

158.5203

1.623347

7344.603

8.588527

2571.503

166.3706

63.65449

52.18914

2.598411

2.249404

226.2053

253.4627

4.682374

448.1375

49.95641

15.69162

2.469078

96.09325

137.9165

34.94616

6.183973

2.589227

2.863937

3.314837

25.59323

2.35505

2.846878

11.59192

56.44757

7.831356

15.12471

82.9814

70.28256

34.53393

174.8161

21.95574

9.793945

5.021032

9.892957

144.589

27.89076

274.554

69.01961

21.4087

302.1797
$-2.76305 \quad 2.394117$

$-0.86851$

0.752623

$-0.66563$

0.577038

$-0.56634$

0.491871

-0.55347 0.480904

$-0.45592$

0.396788

3.354075

2.924996

$-0.56153$

0.489802

$-1.87139$

0.493665

1.631426

$-0.53299$

$-0.43586$

0.430865

0.465241

0.380783

1.093446

0.95563

2.471934

2.16305

$-1.88766$

1.651326

$-0.59743$

$-0.78507$

0.522456

0.687647

1.550156

1.357382

$-0.49431$

0.433544

$-0.77559$

0.680573

$-0.83565$

0.734267

$-1.8338$

1.611877

1.015614

$-0.32882$

0.893915

0.289603

$-2.23655$

1.973166

$-1.43383$

1.265808

$-1.96897$

1.739642

$-2.26746$

2.004885

$-1.59776$

1.413546

$-1.07802 \quad 0.95434$

-3.29737 2.920547

$-1.68612$

1.597293

1.493971

1.415902

0.611977

0.543302

$-1.309151 .163015$

1.1310421 .005009

$-0.57902$

0.514077

$-0.53845$

$-0.76874$

0.478209

$-0.57934$

0.682254

0.514132

$-0.80647$

0.717353

$-0.96414$

0.857422

$-1.68229$

1.497342

$-0.91243$

0.812292

$-0.5061$

0.450757

$-1.14396$

1.020492

$-0.63625$

0.567675

$-0.60332$

0.537918

$\begin{array}{ll}-0.65228 & 0.581586\end{array}$

$-0.46820 .417728$
$-1.1541$

0.248459

$-1.15398$

0.248508

$-1.15352$

0.248696

$-1.1514$

0.249567

$-1.15089$

0.249776

0.250548

$-1.14902$

0.251508

1.146694

$-1.14645$

0.251609

$-1.14709$

0.251346

1.145754

0.251897

$-1.14562$

0.251953

$-1.14465$

0.252354

1.144215

0.252535

1.1428

$-1.14312$

0.253122

0.252989

$-1.1435$

0.25283

0.25359

$-1.14167$

0.253446

1.14202

$-1.14015$

0.254223

$-1.13961$

0.254447

0.255088

$-1.13808$

0.255255

1.136142

$-1.13543$

0.255897

0.256194

0.257012

$-1.13348$

$-1.13274$

0.257324

$-1.13183$

0.257707

$-1.13097$

$-1.13032$

0.258068

0.258342

$-1.1296$

0.258647

$-1.12903$

0.258887

$-1.12862$

0.259059

1.12811

1.126402

0.259274

0.259995

$-1.12566$

0.260311

1.125405

0.260418

$-1.12633$

0.260028

$-1.12597$

0.260178

$-1.12677$

0.25984

$-1.12683$

0.259814

$-1.12423$

0.260916

$-1.12447$

0.260815

$-1.12352$

0.261219

0.261318

0.261534

$-1.12277$

$-1.12099$

0.262294

$-1.1208$

0.262371

$-1.12158$

0.262043

$-1.12156$

0.262049

$-1.12082$ 


\begin{tabular}{|c|c|c|c|c|c|c|}
\hline Corynebacterium vitaeruminis & 1206.081 & -0.33208 & 0.29648 & -1.12007 & 0.262686 & \\
\hline Acetobacter senegalensis & 3.006669 & -2.40172 & 2.145836 & -1.11925 & 0.263034 & \\
\hline lostridium acetobutylicum & 25.23215 & 0.699535 & 0.625028 & 1.119207 & 0.263052 & 0.5456 \\
\hline Acidothermus cellulolyticus & 29.18847 & -0.75224 & 0.672072 & -1.11929 & 0.263017 & 0.5456 \\
\hline Pseudonocardia sp. HH130630-07 & 217.0286 & -0.47353 & 0.423363 & -1.11849 & 0.263358 & .545614 \\
\hline Weissella cibaria & 16.79052 & -1.1696 & 1.045514 & -1.11868 & 0.263276 & 0.545614 \\
\hline Halomonas sp. A3H3 & 35.57312 & -0.76563 & 0.685096 & -1.11754 & 0.263762 & 0.545664 \\
\hline Fusobacterium gonidiaformans & 3.313697 & -1.47276 & 1.317774 & -1.11761 & 0.263732 & 0.545664 \\
\hline Propionibacterium virus Pacnes 201215 & 16.36772 & 1.679375 & 1.502961 & 1.117378 & 0.263833 & 0.545664 \\
\hline Pseudolabrys taiwanensis & 115.7712 & -0.52829 & 0.47321 & -1.11639 & 0.264254 & 0.545916 \\
\hline Escheric & 14.29861 & -1.05909 & 0.94849 & -1.11661 & 0.264162 & 0.545916 \\
\hline Cutibacterium granulosum & 14835.04 & -0.69198 & 0.620137 & -1.11585 & 0.264485 & 0.545944 \\
\hline Synec & 1.326539 & -2.47958 & 2.222525 & -1.11566 & 0.264568 & 5944 \\
\hline Coryr & 476.9018 & -0.43116 & 0.386615 & -1.11522 & 0.264758 & 0.545981 \\
\hline Halomona & 20.19256 & -0.8586 & 0.770245 & -1.11471 & 0.264976 & 0.545981 \\
\hline Dicke & 47.376 & -0.66636 & 0.597868 & -1.11457 & 0.265036 & 0.545981 \\
\hline Acinetobacter inc & 41.11398 & -0.70611 & 0.633748 & -1.11418 & 0.2652 & 0.546009 \\
\hline Meth & 157.4638 & -0.49453 & 0.44438 & -1.11285 & 0.265774 & 0.54605 \\
\hline Streptomyce & 53.39694 & -0.52216 & 0.468976 & -1.1134 & 0.265537 & 0.54605 \\
\hline Rueg & 61.471 & -0.5 & 0.521 & -1.11217 & 5065 & \\
\hline Shew & 3.16801 & -1.91594 & 1.723083 & -1.11193 & 0.266169 & 0.54605 \\
\hline Rhizo & 33.83279 & -2.74184 & 2.461867 & -1.11372 & 0.265398 & 0.54605 \\
\hline Chro & 52.27823 & -1.38261 & 1.243701 & -1.11169 & 0.266271 & 0.54605 \\
\hline Stenot & 20.67502 & -1.20864 & 1.087145 & -1.11176 & 0.266243 & 0.54605 \\
\hline Candic & 1.353466 & -2.50109 & 2.251317 & -1.11095 & 0.266592 & 0.546401 \\
\hline Coryr & 1570.909 & -0.2629 & 0.237444 & -1.10721 & 0.268205 & 0.549396 \\
\hline Polar & 13.31793 & 1.162107 & 1.050467 & 1.106276 & 0.268607 & 0.549911 \\
\hline Lachr & 37.9607 & -0.68491 & 0.61955 & -1.1055 & 0.268943 & 0.550289 \\
\hline Vibrio harveyi & 3.38187 & -1.59402 & 1.442568 & -1.10499 & 0.269164 & 0.550432 \\
\hline Calo & 20.76849 & 1.205633 & 1.091453 & 1.104613 & 0.269327 & 0.550457 \\
\hline Acidipropionibacterium virtanenii & 100.7619 & -0.46196 & 0.418678 & -1.10338 & 0.269862 & 0.55124 \\
\hline Pantoea sp. PSNIH1 & 94.39948 & 0.97111 & 0.880528 & 1.102873 & 0.270083 & 0.551381 \\
\hline Lactobacillus ruminis & 12.45566 & -1.44921 & 1.314853 & -1.10219 & 0.27038 & 0.551381 \\
\hline Pediococcus & 9.942272 & -0.90627 & 0.823301 & -1.10078 & 0.270992 & 0.551381 \\
\hline Strep & 70.75945 & -0.52044 & 0.47239 & -1.10172 & 0.270584 & 0.551381 \\
\hline Zhongshania & 4.95992 & -1.27583 & 1.158294 & -1.10147 & 0.27069 & 0.551381 \\
\hline Sphir & 14.64208 & -3.19425 & 2.901564 & -1.10087 & 0.270952 & 0.55 \\
\hline Alter & 29.68262 & -0.60705 & 0.551345 & -1.10104 & 0.27088 & 0.551381 \\
\hline Fried & 196.8453 & -0.40086 & 0.364356 & -1.10018 & 0.271254 & 0.551605 \\
\hline Rhodococcus py & 130.5745 & -0.90816 & 0.82678 & -1.09843 & 0.272017 & 0.552539 \\
\hline Gilvibacter sp. SZ-19 & 1.786487 & -1.96105 & 1.785046 & -1.0986 & 0.271943 & 0.552539 \\
\hline Dicke & 26.9344 & -0.71731 & 0.65334 & -1.09792 & 0.27224 & 0.552684 \\
\hline Lacimicrobium all & 16.2762 & -0.79129 & 0.721375 & -1.09692 & 0.272676 & 0.55326 \\
\hline Xanth & 174.2888 & -0.55653 & 0.507957 & -1.09562 & 0.273247 & 0.553794 \\
\hline Stigmatella aurantiaca & 84.20451 & -0.54947 & 0.501542 & -1.09556 & 0.273273 & 0.553794 \\
\hline Mucilaginibacter gotjawali & 38.73235 & -0.52797 & 0.482196 & -1.09493 & 0.273548 & 0.553794 \\
\hline Nostoc flagelliforme & 10.02878 & 1.292311 & 1.179975 & 1.095202 & 0.273428 & 0.553794 \\
\hline Pseudoalteromonas tunicata & 12.14136 & -1.12994 & 1.034093 & -1.09268 & 0.274533 & 0.55517 \\
\hline & 30.91099 & -0.86246 & 0.789162 & -1.09288 & 0.274448 & 0.55517 \\
\hline Acidihalobacter prosperus & 65.95996 & -0.56019 & 0.512947 & -1.0921 & 0.27479 & 0.555381 \\
\hline
\end{tabular}


Mycobacterium sp. VKM Ac-1817D

Pseudomonas vancouverensis

Agrococcus jejuensis

Propionibacterium virus PHL152M00

Pseudomonas sp. phDV1

Paenibacillus xylanexedens

Arthrobacter sp. PAMC 25486

Porcine type-C oncovirus

Pusillimonas sp. T7-7

Bifidobacterium thermophilum

Prevotella fusca

Jatrophihabitans sp. GAS493

Chromobacterium sp. ATCC 53434

Pseudomonas sp. LAB-08

Parabacteroides sp. CT06

Ferroplasma acidarmanus

Yersinia entomophaga

Porphyromonas asaccharolytica

Lacinutrix venerupis

Verrucomicrobia bacterium

Candidatus Sulcia muelleri

Achromobacter sp. B7

Fermentimonas caenicola

Pseudomonas sp. R5-89-07

Yersinia frederiksenii

Porphyromonas gingivalis

Anaerostipes hadrus

Alcaligenes faecalis

Clostridiaceae bacterium 14 S0207

Mesorhizobium sp. DCY119

cyanobacterium endosymbiont of Epither

Ralstonia pickettii

Vibrio nigripulchritudo

Methylobacterium sp. 17Sr1-28

Acetobacterium sp. KB-1

Corynebacterium glutamicum

Candidatus Ruthia magnifica

Heliobacterium modesticaldum

Gordonibacter pamelaeae

Halothermothrix orenii

Acinetobacter schindleri

Pseudomonas lini

Rhodococcus sp. PBTS 1

Sphingopyxis alaskensis

Paenibacillus lautus

Caulobacteraceae bacterium OTSz_A_27:

Thalassococcus sp. SH-1

beta proteobacterium $\mathrm{CB}$

Bacillus altitudinis

Corynebacterium cystitidis
26.97013

51.95473

150.4997

18.56369

108.913

17.74542

108.0861

4.869026

21.30003

27.82

121.9641

65.54295

119.8333

54.56264

155.7966

1.901149

5.225072

1397.51

8.651035

60.17006

6.93159

117.9905

8.979876

47.58309

15.77379

225.6087

222.5064

97.85511

18.96326

61.59315

3.424317

75648.99

8.637554

106.7454

4.739426

678.6779

1.470308

27.92937

40.16985

4.373387

159.2481

17.65968

518.7578

37.68984

3.008564

53.31244

55.71235

4.523155

14.94425

623.0924
$-0.56858$

$-0.7334$

0.521376

0.672156

$-0.50808$

0.465766

$-1.60387$

1.471386

$-0.69382$

0.637298

$-1.158$

1.063478

$-0.37423$

0.343622

1.9121

2.080495

0.680355

0.5

0.503015

$-0.49547$

0.455968

$-0.45663$

0.420895

0.51439

$-0.7107$

0.474342

0.655357

$-1.23052$

1.1341

2.63617

2.860911

2.6361

$-1.33774$

0.872656

0.806451

1.516346

1.402945

$-0.62254$

0.576226

1.402993

1.300226

$-0.57261$

0.531415

$-1.28948$

1.197017

$-0.67139$

0.623579

$-1.26559$

1.176164

$-0.78934$

0.734322

$-0.78662$

0.731809

$-0.52393$

0.48799

0.976631

0.909788

$-0.52849$

0.492542

2.667009

2.493311

$-0.52626$

0.491957

$-1.33213$

1.247129

$-0.54163$

0.506942

$-1.23584$

1.156365

$-0.41472$

0.388988

$-2.61633$

2.45276

$-0.74319$

0.697601

$-0.81611$

0.765309

1.613875

1.513009

$-0.77699$

0.729021

$-0.75504$

0.708728

0.544245

0.509927

$-0.54048$

0.507611

$-1.97146$

1.852172

$-0.653810 .614678$

$-0.51864$

0.487624

$-1.86544$

1.755042

2.803512

2.642671

$-0.34749$

$-1.09053$

0.27548

0.555849

$-1.09112$

0.275219

0.555849

$-1.09085$

0.275337

0.555849

$-1.09004$

0.275696

0.555976

$-1.08869$

0.276292

0.556253

$-1.08888$

0.276205

0.556253

$-1.08908$

0.276119

1.088068

0.276565

0.556253

$-1.08746$

0.276834

0.556496

1.086712

0.277164

0.556729

$-1.08663$

0.277202

0.556854

$-1.08491$

0.277962

0.556854

$-1.08443$

0.278176

0.557253

$-1.08445$

0.278165

0.557253

$-1.08501$

0.277919

0.557253

1.085253

0.27781

0.557253

$-1.0841$

0.27832

0.557253

1.082095

0.27921

0.557253

1.08083

$-1.08038$

0.279773

0.558728

1.079038

0.279974

0.559545

$-1.07752$

0.280571

0.55964

$-1.07724$

$-1.07668$

0.281249

0.560526

$-1.07603$

0.281372

0.56151

0.281625

0.56151

$-1.07493$

0.281913

0.561705

$-1.07493$

0.282407

0.561972

$-1.0749$

0.282419

0.562365

$-1.07366$

0.282977

0.562365

1.073471

0.28306

0.563024

$-1.07299$

0.283274

0.563024

1.069665

0.28477

0.563143

$-1.06972$ 
Bordetella bronchiseptica

Vibrio rumoiensis

Porphyrobacter HT-58-2

Streptomyces xiamenensis

Sphingomonas sp. KC8

Pseudomonas silesiensis

Campylobacter sputorum

Corynebacterium efficiens

Fusobacterium sp. oral taxon 203

Nostoc piscinale

Burkholderia sp. PAMC 28687

Mycobacteroides immunogenum

Aquimarina sp. AD1

Rhodococcus erythropolis

Calothrix sp. NIES-2100

Phytobacter ursingii

Cytophaga hutchinsonii

Alistipes sp. Marseille-P5997

Anabaenopsis circularis

Lactobacillus acidophilus

Burkholderia dolosa

Lactococcus virus Bibb29

Thermus sp. CCB_US3_UF1

Actinoplanes teichomyceticus

Arcobacter trophiarum

Flavonifractor plautii

Micromonospora echinospora

Thiomonas arsenitoxydans

Taylorella asinigenitalis

Corynebacterium choanis

Pseudomonas brassicacearum

Francisella philomiragia

Rubrobacter radiotolerans

Geobacter sp. M18

Deinococcus deserti

Paracoccus denitrificans

alpha proteobacterium HIMB5

Eubacterium limosum

Sneathia amnii

Megasphaera elsdenii

Alteromonas sp. RKMC-009

Corynebacterium falsenii

Streptomyces sp. TN58

Mycolicibacterium aurum

Marinobacter sp. LV10R510-11A

Pseudomonas parafulva

Celeribacter marinus

Bacillus methanolicus

Rhodococcus sp. 008

Thermoactinomycetaceae bacterium SCS
257.8106

12.61903

37.87962

83.553

50.38939

64.19303

14.30488

803.9635

6.654569

5.472668

236.2181

80.29481

9.026593

689.5825

18.6123

12.294

29.5245

43.2344

46.333

13.06926

416.9619

3.08782

17.42723

176.14

7.09531

160.9892

87.47192

98.80836

7.760442

206.3401

38.20576

13.27601

45.95838

88.815

45.88882

1890.155

1.771171

42.19352

9.595161

25.1322

5.449854

732.6541

57.41133

144.1818

6.719756

101.6635

9.197752

8.141406

105.2265

16.74398
$-0.622260 .586191$

$-1.017940 .96023$

$-0.68537$

0.646746

$-0.474570 .447797$

$-0.5597$

0.52927

$-0.59219$

0.559911

1.193435

1.127974

$-0.32494$

0.307062

$-1.16767$

1.106746

$-1.91854$

1.819159

$-0.68625$

0.651109

$-0.51966$

0.492911

1.395934

1.324478

$-0.57886$

1.3097

0.548473

$-1.3057$

1.242107

1.247301

0.885327

0.843782

$-0.87328$

1.283677

0.825932

$-0.49148$

1.220718

0.468504

2.263123

2.149197

$-0.8779$

0.833875

0.

544117

(28303

$-0.72632$

1.473275

0.691802

$-0.52693$

0.502831

$-0.68112$

0.650657

$-1.46111$

1.392102

$-0.31678$

0.301997

$-0.82539$

0.785167

1.650957

1.5764

$-0.66487$

$-0.62109$

0.634231

0.592781

$-0.57351$

0.546283

$-0.63858$

0.610562

$-2.20167$

2.104126

$-0.80389$

0.768948

$-1.06488$

1.018296

$-0.66453$

0.635871

$-1.1685$

1.119975

$-0.40074$

0.384183

$-0.56649$

0.5448

$-0.45886$

0.4422

$-1.1798$

1.136904

$-0.52826$

0.509167

$-1.15654$

1.114195

$-0.97742$

0.940264

$-1.1409$

1.098771

$-0.78676$
$-1.06153$

$-1.0601$

$-1.05972$

$-1.0598$

$-1.05749$

$-1.05765$

1.058034

$-1.05823$

$-1.05505$

$-1.05463$

$-1.05398$

$-1.05427$

1.05395

$-1.0554$

1.054418

$-1.04682$

1.049236

$-1.04936$

$-1.05152$

1.051575

$-1.04904$

1.053009

$-1.05279$

$-1.05173$

1.048085

$-1.04989$

$-1.04792$

$-1.04681$

$-1.04957$

$-1.04896$

$-1.05123$

1.047296

$-1.0483$

$-1.04776$

$-1.04983$

$-1.04588$

$-1.04636$

$-1.04544$

$-1.04574$

$-1.04507$

$-1.04333$

$-1.04311$

$-1.0398$

$-1.0376$

$-1.03773$

$-1.03751$

$-1.03801$

$-1.03952$

$-1.03834$

$-1.03936$

0.288448

0.2891

0.567534

0.289274

0.567625

0.289236

0.567625

0.290286

0.567625

0.290217

0.568385

0.29004

0.568385

0.289951

0.291405

0.291595

0.568385

0.291894

0.291758

0.291906

0.29124

0.291692

0.295183

0.294069

0.294012

0.29302

0.292994

0.294161

0.292337

0.292435

0.292921

0.2946

0.293767

0.294676

0.295185

0.293915

0.294197

0.293155

0.294963

0.294499

0.294747

0.293795 
Azoarcus sp. KH32C

Ruminococcus albus

Hartmannibacter diazotrophicus

Marinifilaceae bacterium SPP2

Prosthecochloris sp. CIB 2401

Polynucleobacter necessarius

Mycobacterium sp. QIA-37

Halorhabdus tiamatea

Cloacibacterium normanense

Hafnia paralvei

Pasteurella dagmatis

Jeotgalicoccus saudimassiliensis

Streptococcus constellatus

Defluviitoga tunisiensis

Melissococcus plutonius

Methylobacterium sp. XJLW

Candidatus Nitrosotenuis cloacae

Sphingorhabdus sp. YGSMI21

Flavobacteriaceae bacterium 3519-10

Aeromonas media

Streptococcus equinus

Sphingorhabdus sp. M41

Pseudomonas viridiflava

Olleya aquimaris

Candidatus Gracilibacteria bacterium $\mathrm{HO}^{-}$

Capnocytophaga cynodegmi

Human alphaherpesvirus 1

Ruminococcus champanellensis

Gemmata sp. SH-PL17

Streptococcus porcinus

Haloarcula taiwanensis

Actinomyces sp. Chiba101

Rhizobium sp. NT-26

Acidihalobacter ferrooxidans

Desulfobacter postgatei

Legionella pneumophila

Edwardsiella hoshinae

Rhodococcus aetherivorans

Nocardia seriolae

Pseudomonas sp. R3-52-08

Rhodococcus sp. WMMA185

Corynebacterium stationis

Vibrio alginolyticus

Rhodococcus ruber

Rhizobium sp. Y9

Oceanicoccus sagamiensis

Paracoccus sp. BM15

Eggerthella sp. YY7918

Pseudomonas fuscovaginae

Paenarthrobacter aurescens
158.7951

15.42802

64.51413

11.02632

4.885376

54.76298

27.85269

12.04385

82.70067

4.148553

4.785274

14.1717

51.49121

1.980068

12.57627

59.10773

39.75062

27.03277

9.998436

31.14365

197.246

15.83717

49.48231

8.402021

2.064386

5.24301

11.04541

20.78577

59.26931

9.453476

1.883493

165.6655

60.66799

34.92117

2.229722

44.13081

18.15147

108.8986

180.0803

1.803279

84.54377

1298.976

5.23971

226.0934

65.73088

4.667481

82.5221

10.58287

28.63068

96.26855 $\begin{array}{lll}-0.57915 & 0.557396\end{array}$

1.044743

1.084175

0.535035

0.983728

1.516662

0.480275

1.0562

$-1.09069$

$-1.0673$

1.034046

$-0.50831$

0.492675

$-1.52775$

1.484426

$-1.31323$

1.276106

$-0.99305$

0.964319

$-0.65718$

0.638254

2.477672

2.40991

1.22269

1.145747

$-0.71348$

0.694788

$-0.643$

0.626639

$-1.0768$

1.050286

$-0.65012$

0.634523

$-0.68116$

0.664271

$-0.71887$

0.701599

0.637144

0.623483

1.512192

1.480008

2.456694

2.413297

1.384997

1.361899

$-2.63325$

2.588501

$-1.15595$

1.135868

$-0.54131$

0.53212

$-0.93923$

0.925378

$-2.09432$

2.067555

$-0.44188$

0.437053

$-0.62175$

0.614581

$-0.60329$

0.596158

$-1.42035$

1.403805

0.520555

0.515118

$-0.92999$

0.920349

$-0.69777$

0.690077

$-0.35868$

0.35571

$-2.91154$

2.887993

$-0.51959$

0.516085

$-0.31253$

0.31071

$-1.19589$

1.189411

$-0.29991$

0.29840

$-1.305741 .298983$

$-1.2483$

1.243382

$-0.49935$

0.498024

$-1.14544$

1.142356

$-0.75788$

0.755401

$-1.01078$
$-1.03903$

1.037743

$-1.03687$

1.036007

$-1.03446$

$-1.03479$

$-1.03265$

$-1.03216$

$-1.03173$

$-1.02919$

$-1.02909$

$-1.02979$

$-1.02965$

1.028118

1.028274

$-1.02747$

$-1.02691$

$-1.02611$

$-1.02525$

$-1.02459$

$-1.02542$

$-1.02462$

1.021911

1.021746

1.017983

1.01696

$-1.01729$

$-1.01768$

$-1.01726$

$-1.01497$

$-1.01294$

$-1.01104$

$-1.01166$

$-1.01197$

$-1.01179$

1.010555

$-1.01047$

$-1.01115$

$-1.00834$

$-1.00815$

$-1.00678$

$-1.00587$

$-1.00545$

$-1.00503$

$-1.0052$

$-1.00395$

$-1.00267$

$-1.0027$

$-1.00329$

$1.009728-1.00104$

0.298793

0.29939

0.57286

0.3001990 .573594

0.3009210 .574371

0.3007680 .574371

0.3017650 .57568

0.3019990 .575824

0.3021980 .575902

0.3033910 .577057

0.3034380 .577057

$0.303108 \quad 0.577057$

0.303173

0.303894

0.577057

0.303821

0.57732

0.304198

0.57732

0.304464

0.577595

0.304841

0.577799

0.305247

0.578212

0.305558

0.578366

0.305165

0.578366

0.305544

0.578366

0.306823

0.578366

0.306901

0.580305

0.308686

0.580305

0.309173

0.583084

0.309017

0.583084

0.308829

0.583084

0.309028

0.583084

0.310119 
Microlunatus phosphovorus

Lysinibacillus fusiformis

Citromicrobium sp. JL477

archaeon AArc-SI

Pseudomonas versuta

Congregibacter litoralis

Cyanothece sp. ATCC 51142

Shewanella sp. Pdp11

Amycolatopsis japonica

Spiroplasma alleghenense

Methylobacterium sp. 17Sr1-1

Novosphingobium pentaromativorans

Brevundimonas sp. DS20

Pseudomonas granadensis

Streptomyces parvulus

Lactobacillus paragasseri

Cycloclasticus sp. PY97N

Sphaerospermopsis kisseleviana

Vibrio vulnificus

Dactylococcopsis salina

Hymenobacter swuensis

Bacillus krulwichiae

Paludibacter propionicigenes

Bartonella apis

Arthrobacter sp. QXT-31

Pluralibacter gergoviae

Methanocella conradii

Nakamurella panacisegetis

Sphingopyxis sp. 113P3

Lawsonia intracellularis

Microcystis sp. MC19

Stenotrophomonas sp. MYb57

Nostoc punctiforme

Auritidibacter sp. NML130574

Citrobacter koseri

Clostridium scatologenes

Streptococcus sanguinis

Streptococcus acidominimus

Campylobacter pinnipediorum

Neisseria sicca

Chryseobacterium balustinum

Staphylococcus felis

Chromohalobacter salexigens

[Eubacterium] minutum

Saccharomonospora azurea

Aeromonas salmonicida

Haemophilus influenzae

Acinetobacter equi

Azotobacter vinelandii

Sphingobium herbicidovorans
140.8684

8.031135

50.24868

6.077287

46.92162

4.11486

5.542014

1.522225

99.01974

2.441044

104.8628

63.65411

354.8618

21.82598

78.59113

4.078323

1.774646

7.102881

36.00525

2.427895

59.20125

9.672137

13.48583

15.95532

140.5169

52.15981

1.312864

100.0874

64.56392

5.536564

2.324548

35.67567

23.54981

144.7557

27.56241

14.4951

2202.882

17.25502

11.0795

401.3327

64.71023

45.27502

30.90597

11.98866

71.01589

52.80397

188.106

17.93035

138.4639

33.75025
$-0.40209$

$-1.21471$

$-0.56002$

$-1.07645$

$-0.65047$

$-1.5991$

1.626828

$-2.66911$

$-0.47541$

2.361922

$-0.48576$

$-0.58535$

$-0.44279$

0.97651

$-0.39136$

$-2.10502$

$-2.88565$

1.55157

$-0.62891$

$-2.18793$

0.527931

$-1.05584$

$-1.00587$

$-0.80648$

$-0.50574$

$-0.46575$

$-2.45864$

$-0.4274$

$-0.63083$

$-1.3555$

$-2.19168$

$-0.83886$

1.311879

$-0.31193$

$-0.6111$

1.137185

$-0.61123$

$-1.013$

1.138161

$-0.76704$

$-0.69562$

0.579106

$-0.48578$

$-1.10212$

$-0.33992$

$-0.47065$

$-0.6634$

0.962708

$-0.51013$

$-0.62925$
0.401659

1.216195

0.560579

1.077078

0.651898

1.602405

1.63612

2.688828

0.479354

2.379934

0.488972

0.588433

0.444265

0.983696

0.394196

2.117978

2.905345

1.562346

0.633899

2.206454

0.530405

1.063271

1.014447

0.814213

0.511183

0.470741

2.487131

0.432357

0.639204

1.376312

2.225762

0.851724

1.331133

0.316354

0.620891

1.155388

0.622067

1.031369

1.159605

0.78242

0.70986

0.590879

0.495855

1.124211

0.347211

0.481006

0.678106

0.986246

0.522777

0.644022

$-1.00106$

$-0.99878$

$-0.999$

$-0.99942$

$-0.99781$

$-0.99794$

0.99432

$-0.99267$

$-0.99178$

0.992432

$-0.99343$

$-0.99476$

$-0.99669$

0.992695

$-0.99282$

$-0.99388$

$-0.99322$

0.993103

$-0.99213$

$-0.99161$

0.995336

$-0.99301$

$-0.99155$

$-0.9905$

$-0.98936$

$-0.9894$

$-0.98854$

$-0.98852$

$-0.9869$

$-0.98488$

$-0.98469$

$-0.9849$

0.985536

$-0.98601$

$-0.98423$

0.984245

$-0.98258$

$-0.98219$

0.981507

$-0.98035$

$-0.97993$

0.980075

$-0.97968$

$-0.98035$

$-0.97899$

$-0.97847$

$-0.97831$

0.976134

$-0.97581$

0.316796

0.317903

0.590747

0.317795

0.591878

0.317591

0.591878

0.31837

.

0.31831

0.320067

0.320873

0.592142

0.321306

0.592663

0.320987

0.320502

0.592663

0.320502

0.319851

0.318917

0.320858

0.320799

0.32028

0.320602

0.32066

0.321136

0.32139

0.319573

0.320705

0.32142

0.321931

0.322489

0.322468

0.322886

0.322896

0.323691

.592663 
Glutamicibacter halophytocola

Corynebacterium crudilactis

Aeromonas sp. ASNIH1

Asaia bogorensis

Veillonella parvula

Borrelia hermsii

Methanosarcina sp. MTP4

Clostridium ljungdahlii

Dietzia timorensis

Labrenzia sp. VG12

Magnetococcus marinus

Persicobacter sp. JZB09

Streptomyces sp. CNQ-509

Pseudomonas plecoglossicida

Isosphaera pallida

Gramella sp. SH35

Arthrobacter sp. U41

Lactobacillus casei

Confluentimicrobium sp. EMB200-NS6

Amphibacillus xylanus

Staphylococcus virus IPLAC1C

Kordia sp. SMS9

Streptomyces fungicidicus

Pediococcus claussenii

Aquaspirillum sp. LM1

Clostridium drakei

Thermodesulfobium narugense

Corynebacterium pseudopelargi

Corynebacterium renale

Aminobacter aminovorans

Tenericutes bacterium $\mathrm{MO}-\mathrm{XQ}$

Propionibacterium phage PHL150M00

Sphingomonas sp. LK11

Isoptericola dokdonensis

Brucella sp. 09RB8910

Propionibacterium virus P100D

Corynebacterium glaucum

Prevotella jejuni

Candidatus Aquiluna sp. UB-MaderosW2r

Pseudomonas corrugata

Clostridioides difficile

Tenacibaculum jejuense

Rufibacter sp. DG15C

Plantactinospora sp. BC1

Citrobacter werkmanii

Lactobacillus delbrueckii

Propionibacterium phage Moyashi

Corynebacterium variabile

Spirochaeta thermophila

Pseudomonas veronii
135.1405

321.348

1.70023

10.27059

3573.936

4.704541

3.41476

1.694089

294.1672

31.88723

2.057418

11.54127

74.5662

97.61887

11.97447

6.494858

189.8548

11.94775

43.69456

4.242486

206.3285

7.791072

62.17828

3.775746

40.46599

10.88906

1.399142

652.291

727.0634

109.3758

7.353228

8.950594

173.1344

149.6371

5.348766

22.79551

1591.651

431.9965

7.14929

35.05887

423.0596

11.72815

7.816894

66.09897

22.0593

45.91635

10.16838

463.4011

18.43473

34.51016
$-0.39108$

$-0.35096$

0.400335

$-0.97689$

0.328622

$-0.9761$

0.329017

$-2.83081$

0.72174

2.902606

$-0.97526$

0.329429

0.740053

0.552121

$-0.97526$

0.329432

$-0.97151$

0.331296

2.131566

0.973113

0.330497

0.972221

0.33094

$-0.97169$

0.331204

$-2.82616$

2.908499

$-0.40738$

0.418965

$-0.5731$

0.589919

$-1.84341 .896444$

$-0.8295 \quad 0.85444$

$-0.5177$

0.533269

$-0.45595$

0.470566

$-0.71263$

0.737164

$-1.1648$

1.206252

$-0.3582$

0.370731

$-1.05421$

1.092414

$-0.56159$

0.582113

$-1.36594$

1.415165

$-1.811571 .878472$

1.3412881 .386756

$-0.400150 .414042$

$-1.67378 \quad 1.73259$

$-0.50433$

0.522876

1.422705

1.472379

2.667064

2.767231

$-0.34241$

0.357072

$-0.2748$

0.286476

$-0.528150 .551828$

$1.321591 \quad 1.38095$

$-1.533791 .604578$

$-0.49005$

0.513354

$-0.41566$

0.435918

$-1.724841 .811305$

$1.40193 \quad 1.473107$

$-0.343890 .361265$

$-0.50217$

0.528061

$-0.78366$

0.825734

$-0.774550 .816086$

0.4418680 .465607

$1.238367 \quad 1.305835$

$-1.09721 .158145$

$-0.641180 .677487$

$-1.040181 .099667$

$-0.526260 .556921$

$-1.7191 \quad 1.818658$

$-0.396220 .419316$

$-0.65710 .696028$

$-0.97999 \quad 1.04028$

$-0.97234$

0.330881

$-0.97149$

0.331305

$-0.97203$

0.331036

$-0.97081$

0.331641

$-0.97081$

0.331641

$-0.96894$

0.332577

$-0.96672$

0.333685

$-0.96563$

0.334227

$-0.9662$

0.333943

$-0.96503$

0.334529

$-0.96474$

0.334675

$-0.96522$

0.334436

$-0.96438$

0.334853

0.967212

0.333438

$-0.96644$

0.333826

$-0.96606$

0.334015

$-0.96453$

0.334781

0.966263

0.333912

0.963802

0.335145

$-0.95894$

0.337588

$-0.95926$

337429

$-0.95709$

0.33852

0.957016

0.338559

$-0.95588$

0.339132

$-0.95461$

0.339775

$-0.95353$

0.340323

$-0.95227$

0.340962

0.951683

0.341258

$-0.95189$

0.341151

$-0.95096$

0.341623

$-0.94904$

0.3426

$-0.94911$

0.342567

0.949015

0.342613

0.948333

0.34296

$-0.94738$

0.343445

$-0.94641$

0.59804

0.59804

0.59804

0.59804

0.599347

0.599347

0.599347

0.599347

0.599347

0.599347

0.599347

0.59936

0.59936

0.600751

0.601282

0.601282

0.601282

0.601282

0.601282

0.601282

0.601282

0.601282

0.601282

0.601282

0.601282

0.601282

0.601508

0.605295

0.605295

0.60644

0.60644

0.607166 
Ruminococcaceae bacterium CPB6

Mycoplasma bovirhinis

Streptomyces sp. SAT1

Christensenella sp. Marseille-P3954

Haemophilus pittmaniae

Microbacterium foliorum

Synechococcus sp. JA-3-3Ab

Mycobacteroides chelonae

Enterococcus sp. FDAARGOS_375

Mycolicibacterium goodii

Streptococcus sp. FDAARGOS_192

Lactobacillus rhamnosus

Saprospira grandis

Parascardovia denticolens

Shewanella putrefaciens

Streptococcus respiraculi

Pseudoalteromonas translucida

Cellulomonas gilvus

Enterobacter sp. FY-07

Geobacillus subterraneus

Corynebacterium ulcerans

Halodesulfurarchaeum formicicum

Sphingobium sp. MI1205

Rhodobacter blasticus

Jeotgalibacillus malaysiensis

Bacillus butanolivorans

Corynebacterium striatum

Aurantimicrobium sp. MWH-Mo1

Pseudomonas sp. URMO17WK12:I11

Exiguobacterium sibiricum

Georgenia sp. ZLJ0423

Bacillus halodurans

Rufibacter sp. DG31D

Kingella kingae

Exiguobacterium sp. AT1b

Rathayibacter toxicus

Paenibacillus odorifer

Propionibacterium virus Attacne

Maribacter sp. 1_2014MBL_MicDiv

Carnobacterium maltaromaticum

Xenorhabdus poinarii

Teredinibacter turnerae

Kosakonia sacchari

Capnocytophaga ochracea

Sulfurihydrogenibium sp. YO3AOP1

Corynebacterium callunae

Pseudomonas cremoricolorata

Lactobacillus koreensis

Staphylococcus saprophyticus

Candidatus Planktophila limnetica
20.79785

3.038844

80.4446

$-0.74822$

0.795041

2.772301

20.60831

49.82168

0.476183

$-0.73661$

0.787328

292.4023

$-0.69543$

0.743475

$-0.50530 .54002$

48.95583

$-0.70711$

0.755995

600.479

$-0.6227$

0.667109

2.495215

154.1995

135.7303

$-2.43632$

2.618066

$-0.30264$

0.325362

34.95895

0.703524

0.754436

1.876089

100.5403

2.726982

$-0.59787$

0.64256

14.07685

6.257735

124.8243

15.2948

2.257007

548.1974

2.686535

28.17291

73.70487

5.54098

12.98859

9726.228

34.71419

53.85435

14.12395

134.0624

2.076487

12.13726

16.48228

50.95955

26.32474

4.856476

63.4245

1.532582

78.63776

2.018279

9.745359

13.88311

54.06362

1.787633

478.9347

44.48859

1.373649

566.6933

3.621198

0.62359

$-0.58055$

2.586827

0.861526

$-0.80381$

2.416269

2.249509

0.464441

$-0.72764$

0.781173

$-2.253412 .415509$

$-0.342180 .36805$

$-1.32491 \quad 1.427475$

$-0.69785$

0.752181

$-0.4276$

0.461123

$-1.10721$

1.195607

$-0.82309$

0.889599

$-0.28128 \quad 0.30437$

$-0.55990 .606232$

$-0.621130 .672888$

$1.02015 \quad 1.10864$

$-0.3399$

0.369411

$-1.75031 \quad 1.901831$

-0.92902 1.009471

$-0.844020 .917646$

$-0.782790 .851419$

$-0.438250 .47756$

$-1.085111 .182074$

1.3069681 .426552

$-2.675792 .922104$

$-0.589380 .644594$

$-2.04424 \quad 2.234064$

$-0.822540 .899139$

$-0.82822$

0.905601

$-0.76125$

0.833902

2.046095

2.242059

$\begin{array}{ll}-0.33603 & 0.368575\end{array}$

$-0.48046$

0.527848

$-2.49786$

2.745932

0.462256

0.507934

1.686763

1.854544
$-0.94111$

0.939661

$-0.93745$

$-0.93558$

$-0.93538$

$-0.9357$

$-0.93533$

$-0.93344$

$-0.93058$

$-0.93015$

0.932516

$-0.93044$

$-0.93349$

$-0.93098$

$-0.93138$

$-0.93301$

0.930984
$-0.93063$

$-0.93148$

$-0.93289$

$-0.92815$

$-0.92776$

$-0.92731$

$-0.92606$

$-0.92523$

$-0.92415$

$-0.92357$

$-0.92308$

0.920181

$-0.92013$

$-0.92033$

$-0.9203$

$-0.91977$

$-0.9194$

$-0.91769$

$-0.91797$

0.916173

$-0.91571$

$-0.91435$

$-0.91503$

$-0.91481$

$-0.91455$

$-0.91288$

0.912596

$-0.91169$

$-0.91022$

$-0.90966$

0.910071

0.90953
$-0.9297$
0.346646

0.347392

0.348528

0.614871

0.615892

0.349491

0.617605

0.34959

0.61833

0.349428

0.61833

0.349616

0.61833

$0.35207 \quad 0.619149$

0.3522920 .619149

$0.35107 \quad 0.619149$

0.3521410 .619149

0.3505660 .619149

0.351862

0.619149

0.3516580 .619149

0.3508150 .619149

0.3518620 .619149

0.3520440 .619149

0.3516070 .619149

0.3508760 .619149

0.3525260 .619261

0.353330 .620373

0.3535310 .620427

$0.353767 \quad 0.620542$

0.3544130 .621376

0.3548440 .621832

0.3554090 .622522

$\begin{array}{ll}0.35571 & 0.622749\end{array}$

0.3559650 .622896

0.3574780 .624392

0.3575070 .624392

0.35740 .624392

0.357415

0.624392

0.3576950 .624422

0.3578880 .624459

$\begin{array}{lll}0.35878 & 0.625415\end{array}$

0.358634

0.625415

0.3595760 .626503

0.3598210 .62663

0.3605340 .626672

0.3601760 .626672

0.3602920 .626672

0.3604290 .626672

$0.361306 \quad 0.627672$

0.3614550 .627672

0.3619320 .6282

0.3627090 .628976

0.3630020 .628976

0.3627850 .628976

$0.36307 \quad 0.628976$ 
Corynebacterium ammoniagenes

Staphylococcus virus $\mathrm{PH} 15$

Pseudarthrobacter phenanthrenivorans

Actinomyces meyeri

Proteus mirabilis

Lactococcus virus 712

Gemmatimonas phototrophica

Lactobacillus crustorum

Pseudomonas sp. SWI36

Streptomyces lavendulae

Clostridiales bacterium CCNA10

Natrialba magadii

Propionibacterium virus PHL025M00

Bacillus gobiensis

Alcanivorax dieselolei

Halorhabdus utahensis

Oceanobacillus kimchii

Formosa agariphila

Flavobacterium arcticum

Aeromonas sp. ASNIH4

Staphylococcus virus IPLA5

Citrobacter freundii complex sp. CFNIH3

Synechocystis sp. PCC 6714

Haloterrigena daqingensis

Actinomyces gaoshouyii

Escherichia marmotae

Flavobacterium anhuiense

Actinopolymorpha singaporensis

Pseudomonas sp. LG1E9

Chondrocystis sp. NIES-4102

Gordonia sp. YC-JH1

Streptomyces sp. MOE7

Haloterrigena turkmenica

Prevotella ruminicola

Betapapillomavirus 2

Pseudomonas pohangensis

Sporosarcina sp. PTS2304

Piscirickettsia salmonis

Paenibacillus sp. CAA11

Staphylococcus lugdunensis

Enterococcus gilvus

Spiroplasma apis

Nitrososphaera viennensis

Chryseobacterium gallinarum

Bartonella ancashensis

Thermus thermophilus

Methanocella arvoryzae

Nostoc sphaeroides

Virgibacillus sp. Bac332

Shewanella psychrophila
1374.012

163.8628

171.5946

364.005

32.42791

1.497609

31.58785

4.954446

1.485692

63.4591

87.58148

9.523419

16.04999

3.247681

73.7138

3.086371

8.206804

12.59765

9.865663

4.763205

109.7757

3.397792

1.469763

13.77563

103.1988

5.012953

49.89098

85.42234

67.58125

20.90081

240.8738

22.78762

28.71161

40.28198

22.63241

35.18932

2.681659

9.608859

4.567308

2286.443

7.887441

5.245249

1.420643

30.28538

1.706309

64.2009

3.132203

7.014432

10.18956

2.150224
$-0.2657$

1.111656

$-0.37845$

$-0.42682$

0.706118

$-2.64683$

$-0.51402$

$-1.21008$

$-2.63561$

$-0.39002$

$-0.60244$

$-0.89939$

$-1.22227$

$-1.16938$

$-0.52901$

$-1.36055$

1.060405

0.959546

0.98513

$-1.43542$

0.971268

1.905756

$-2.61657$

$-0.86789$

$-0.32372$

$-1.63365$

$-0.62799$

$-0.35257$

$-0.52534$

$-0.70631$

$-0.38181$

$-0.89649$

$-0.48095$

$-0.46611$

$-1.19625$

$-0.60475$

$-1.93731$

0.946761

$-1.14613$

0.461323

$-1.02198$

1.628528

$-2.56819$

$-0.6883$

$-2.20936$

$-0.51797$

$-1.65043$

1.623797

$-0.99688$

$-1.663$

$-0.90829$

0.363726

0.629812

225306

0.907248

0.364276

0.630464

0.417851

$-0.90571$

0.36509

0.630679

0.471162

0.779635

$-0.90588$

0.365001

0.630679

2.922194

0.905703

0.365093

0.630679

0.568866

$-0.90577$

0.365059

0.630679

1.339522

$-0.90359$

0.36621

0.632217

2.92223

$-0.90337$

0.366331

0.632217

0.432358

$-0.90192$

0.367101

0.632645

$-0.90207$

0.367018

0.632645

0.667743

0.998928

$-0.9022$

0.36695

0.632645

$-0.90035$

0.367932

0.633776

1.358996

$-0.89939$

0.368445

0.634356

1.300659

$-0.89907$

0.368617

0.634356

0.590946

$-0.8952$

0.370681

0.634905

1.516392

$-0.89723$

0.369598

0.634905

1.183604

0.895912

0.3703

0.634905

0.89675

0.369852

0.634905

0.369612

0.634905

1.59938

$-0.89748$

0.369461

0.634905

1.083515

0.896405

0.370037

0.634905

2.127746

0.895669

0.37043

0.634905

2.92229

$-0.89538$

0.370581

0.634905

0.967927

$-0.89664$

0.369909

0.634905

0.362329

$-0.89343$

0.371627

0.636225

$-0.89187$

0.372464

0.637359

0.704758

$-0.89107$

0.37289

0.637787

0.395845

$-0.89068$

0.373101

0.637848

$-0.88914$

0.373928

0.638662

0.794365

$-0.88915$

0.373924

0.638662

0.429747

$-0.88846$

0.374292

0.638984

1.010677

$-0.88702$

0.375069

0.64001

0.542984

$-0.88575$

0.375755

0.64088

0.526849

$-0.88471$

0.376311

0.641398

$-0.8836$

0.376914

0.641398

0.684204

$-0.88387$

0.376768

0.641398

$-0.88355$ 
Fischerella sp. NIES-3754

Erysipelothrix sp. 15TAL0474

Rhodothermaceae bacterium

Thermus aquaticus

Gluconobacter oxydans

Prosthecochloris sp. HL-130-GSB

Betapapillomavirus 3

Methylobacterium phyllosphaerae

Staphylococcus phage BP39

Bacillus marisflavi

Tessaracoccus flavus

Bifidobacterium longum

Verrucosispora maris

Modestobacter marinus

Staphylococcus virus IPLA7

Pseudomonas sp. Leaf58

Geminocystis sp. NIES-3709

Pelosinus fermentans

Acinetobacter sp. TGL-Y2

Oscillibacter valericigenes

Ilyobacter polytropus

Rhodococcus sp. p52

Clostridium sp. JN500901

Bacillus cellulosilyticus

Micromonospora sp. WMMA2032

Enterobacter sp. SA187

Streptococcus australis

Francisella halioticida

Microbacterium sp. CGR1

Opitutus terrae

Syntrophus aciditrophicus

Clostridium estertheticum

Arthrobacter sp. ATCC 21022

Lactococcus virus $\mathrm{P008}$

[Clostridium] cellulosi

Micromonospora rifamycinica

Liberibacter crescens

Bacillus atrophaeus

Methanofollis liminatans

Pseudomonas sp. TMW 2.1634

Nostoc sp. CENA543

Streptomyces nigra

Pseudoalteromonas carrageenovora

Candidatus Endolissoclinum faulkneri

Streptococcus ruminantium

Treponema azotonutricium

Psychrobacter sp. PRwf-1

Microlunatus soli

Pseudomonas libanensis

Collinsella aerofaciens
$15.15623 \quad 1.020758 \quad 1.159678$

$\begin{array}{rrrrrr}1.943653 & -1.68054 & 1.910935 & -0.87944 & 0.379165 & 0.642415 \\ 55.85625 & -0.58218 & 0.662396 & -0.8789 & 0.379454 & 0.642415 \\ 5.321468 & -0.97605 & 1.113142 & -0.87684 & 0.380571 & 0.642766 \\ 38.3231 & -0.64965 & 0.741423 & -0.87623 & 0.380907 & 0.643035 \\ 2.566201 & -1.52275 & 1.738587 & -0.87586 & 0.381108 & 0.643075 \\ 57.24069 & -2.05655 & 2.351455 & -0.87459 & 0.381799 & 0.643943 \\ 67.7827 & -0.7845 & 0.897471 & -0.87412 & 0.382055 & 0.644075 \\ 1.401003 & -2.55076 & 2.922506 & -0.8728 & 0.382773 & 0.644665 \\ 9.676364 & -0.85671 & 0.982071 & -0.87235 & 0.383015 & 0.644665 \\ 89.584 & -0.46753 & 0.535829 & -0.87253 & 0.382921 & 0.644665 \\ 677.1168 & -0.47464 & 0.544401 & -0.87185 & 0.38329 & 0.644665 \\ 91.70869 & -0.41492 & 0.475813 & -0.87203 & 0.383194 & 0.644665 \\ 252.9546 & -0.41882 & 0.481462 & -0.86989 & 0.38436 & 0.646165\end{array}$

$\begin{array}{lllllll}149.5495 & 0.940625 & 1.082184 & 0.869192 & 0.384742 & 0.646211\end{array}$

$\begin{array}{lllllll}44.16587 & -0.45446 & 0.522692 & -0.86947 & 0.384591 & 0.646211\end{array}$

$\begin{array}{lllllll}14.83376 & 1.044114 & 1.202242 & 0.868472 & 0.385136 & 0.646573\end{array}$

$\begin{array}{lllllll}9.432242 & -0.86951 & 1.001668 & -0.86806 & 0.385359 & 0.646649\end{array}$

$\begin{array}{lllllll}15.90938 & 0.73973 & 0.853737 & 0.866461 & 0.386237 & 0.647526\end{array}$

$\begin{array}{lllllll}19.7868 & -0.95455 & 1.10143 & -0.86664 & 0.386137 & 0.647526\end{array}$

$\begin{array}{lllllll}6.593252 & 1.11851 & 1.291805 & 0.865851 & 0.386572 & 0.647788\end{array}$

$\begin{array}{lllllll}144.5675 & -0.77432 & 0.895045 & -0.86512 & 0.386971 & 0.648073\end{array}$

$\begin{array}{lllllll}9.47773 & 0.968061 & 1.119285 & 0.864892 & 0.387098 & 0.648073\end{array}$

$\begin{array}{lllllll}6.909265 & -0.93161 & 1.07806 & -0.86415 & 0.387505 & 0.648456\end{array}$

$\begin{array}{lllllll}75.39761 & -0.44274 & 0.513157 & -0.86278 & 0.388258 & 0.649417\end{array}$

$\begin{array}{lllllll}20.49291 & -0.6711 & 0.778618 & -0.86191 & 0.388737 & 0.64992\end{array}$

$\begin{array}{lllllll}156.2223 & -0.4809 & 0.558961 & -0.86035 & 0.389598 & 0.649943\end{array}$

$\begin{array}{lllllll}3.749897 & 1.606188 & 1.86709 & 0.860263 & 0.389644 & 0.649943\end{array}$

$\begin{array}{lllllll}53.58788 & -0.80879 & 0.93995 & -0.86046 & 0.389535 & 0.649943\end{array}$

$\begin{array}{lllllll}40.48558 & -0.43589 & 0.506688 & -0.86027 & 0.389638 & 0.649943\end{array}$

$\begin{array}{lllllll}3.527854 & -1.05973 & 1.23106 & -0.86082 & 0.389335 & 0.649943\end{array}$

$\begin{array}{lllllll}39.24749 & 0.704549 & 0.819396 & 0.85984 & 0.389877 & 0.650034\end{array}$

$\begin{array}{lllllll}109.2654 & -0.67947 & 0.792174 & -0.85773 & 0.391042 & 0.650357\end{array}$

$\begin{array}{lllllll}5.22076 & -1.58228 & 1.84396 & -0.85809 & 0.390844 & 0.650357\end{array}$

$\begin{array}{lllllll}6.416357 & -1.20786 & 1.407275 & -0.8583 & 0.390728 & 0.650357\end{array}$

$\begin{array}{lllllll}53.36123 & -0.41724 & 0.486558 & -0.85754 & 0.391144 & 0.650357\end{array}$

$\begin{array}{lllllll}1.89774 & -1.79911 & 2.096237 & -0.85826 & 0.39075 & 0.650357\end{array}$

$\begin{array}{lllllll}2.122646 & -2.1045 & 2.450014 & -0.85897 & 0.390355 & 0.650357\end{array}$

$\begin{array}{lllllll}16.21671 & -0.66593 & 0.777695 & -0.85628 & 0.391842 & 0.651219\end{array}$

$\begin{array}{lllllll}22.51887 & -1.11163 & 1.299126 & -0.85567 & 0.392178 & 0.651482\end{array}$

$\begin{array}{lllllll}11.85924 & -1.15268 & 1.351345 & -0.85299 & 0.393667 & 0.652655\end{array}$

$\begin{array}{lllllll}62.94156 & -0.36121 & 0.423038 & -0.85386 & 0.393184 & 0.652655\end{array}$

$\begin{array}{lllllll}1.677837 & -2.14024 & 2.509728 & -0.85278 & 0.393782 & 0.652655\end{array}$

$\begin{array}{lllllll}3.214359 & 1.723529 & 2.020453 & 0.853041 & 0.393637 & 0.652655\end{array}$

$\begin{array}{lllllll}11.09519 & -0.85545 & 1.001998 & -0.85374 & 0.393248 & 0.652655\end{array}$

$\begin{array}{lllllll}2.56176 & -1.19726 & 1.40722 & -0.8508 & 0.394881 & 0.653422\end{array}$

$\begin{array}{lllllll}33.21771 & 1.033922 & 1.215481 & 0.850628 & 0.394976 & 0.653422\end{array}$

$\begin{array}{lllllll}108.4185 & -0.35637 & 0.418811 & -0.85091 & 0.394821 & 0.653422\end{array}$

$\begin{array}{lllllll}24.47984 & 0.80462 & 0.945744 & 0.85078 & 0.394892 & 0.653422\end{array}$

$\begin{array}{llllll}320.083 & -0.55024 & 0.647096 & -0.85033 & 0.395143 & 0.653422\end{array}$ 
Neisseriaceae bacterium DSM 100970

Pseudomonas sp. XWY-1

Bifidobacterium catenulatum

Cronobacter universalis

Deinococcus geothermalis

Arthrobacter sp. ZXY-2

Alloactinosynnema sp. L-07

Tetragenococcus koreensis

Pseudomonas sp. LBUM920

Arcobacter molluscorum

Neisseria lactamica

Rhodococcus phage RRH1

Segniliparus rotundus

Jiangella sp. DSM 45060

Aeromonas rivipollensis

Pseudomonas sp. 31-12

Bacteroides fragilis

Tessaracoccus sp. Marseille-P5995

Dickeya sp. NCPPB 3274

Paenibacillaceae bacterium GAS479

Cellulosimicrobium sp. TH-20

Candidatus Protochlamydia amoebophila

Rhodococcus hoagii

Actinoalloteichus hoggarensis

Streptococcus parauberis

Methanococcoides methylutens

Exiguobacterium sp. ZWU0009

Thermacetogenium phaeum

Campylobacter iguaniorum

Campylobacter hepaticus

Agarivorans gilvus

Corynebacterium terpenotabidum

Arcanobacterium phocae

Vibrio sp. HBUAS61001

Mariprofundus aestuarium

Aeromonas sp. ASNIH2

Methanobrevibacter sp. AbM4

Serratia sp. FS14

Desulfobacca acetoxidans

Thioclava nitratireducens

Lactobacillus parabuchneri

Paenibacillus sp. JDR-2

Lelliottia sp. WB101

Dichelobacter nodosus

Sulfitobacter sp. SK012

Pseudomonas sp. FDAARGOS_380

Thermobispora bispora

Wenyingzhuangia fucanilytica

Bacteroides caccae

Rahnella sp. Y9602
5.078335

23.4939

$-1.08873$

1.281083

$-0.94309$

1.111352

40.18085

0.633443

0.747173

4.617655

$-1.43944$

1.700995

29.01936

$-0.4335$

0.512939

110.3972

$-0.65224$

0.771419

122.1827

$-0.35616$

0.421197

6.038261

$-1.08303$

1.281816

69.8274

0.429018

0.508415

12.99269

1.112003

1.319579

74.87122

$-0.67134$

0.799264

1.225253

$-2.35189$

2.798719

80.29743

158.8359

$-0.40305$

0.479666

15.93247

$-0.32959$

0.392042

$-0.8271$

0.985652

33.88698

0.562531

0.671738

308.6103

$-0.56162$

0.670476

346.9454

5.113858

$-0.46927$

0.560908

4.480595

$-1.42228$

1.703888

170.1382

$-0.97627$

1.169163

$-0.40753$

0.488288

1.726901

211.1569

$-1.9014$

2.284429

110.6381

$-0.28386$

0.34104

$-0.34844$

0.418638

14.28806

$-0.67516 \quad 0.81178$

1.645477

$-1.9041$

2.293258

9.645781

5.132374

3.672311

$-2.38304$

2.869938

$-1.11177$

1.340955

2.76979

1.668606

2.013977

2.625827

1.687249

2.037407

392.7399

31.15839

$-1.35341$

1.636257

$-0.35338$

0.427245

$-0.44408$

0.536854

5.913425

1.07056

1.297492

2.343609

$-1.45485$

1.762715

1.270206

$-2.41205$

2.922994

4.152885

1.54931

1.876589

1.270197

$-2.41028$

2.923

9.593961

$-0.78002$

0.946136

58.29287

$-0.46573$

0.565817

4.713923

3.87794

13.34152

3.522979

1.445784

1.756362

$-0.93538$

1.136181

$-0.66969$

0.813994

$-1.0589$

1.288553

8.198431

$-0.73222$

0.890901

153.248

0.609687

0.742298

80.31658

9.282481

$-0.36508$

0.444408

88.32285

0.930624

1.134029

$-0.78111$

0.953834

8.656528

$-0.84985$

0.395408

0.396107

$-0.84859$

0.396557

0.847786

0.397423

0.398041

$-0.84551$

0.397827

$-0.84559$

0.397784

$-0.84492$

0.398156

0.843833

0.398762

0.842695

0.399399

$-0.83995$

0.400934

$-0.84035$

0.400714

$-0.84027$

0.400759

$-0.84069$

0.400519

$-0.83914$

0.401389

0.837426

0.402353

$-0.83764$

0.402232

$-0.83662$

0.402806

$-0.83472$

0.403873

$-0.83501$

0.40371

$-0.8346$

0.403941

$-0.83233$

0.405224

$-0.83234$

0.405217

$-0.83231$

0.405235

$-0.8317$

0.405576

$-0.8303$

0.406368

$-0.83035$

0.406344

$-0.82909$

0.407055

0.828513

0.40738

0.828136

0.407594

$-0.82714$

0.408158

$-0.82712$

0.408168

$-0.82718$

0.408134

0.8251

0.409315

$-0.82535$

0.409175

$-0.8252$

0.40926

0.825599

0.409032

$-0.82459$

0.409604

$-0.82442$

0.409699

0.653564

0.654422

0.654868

0.656001

0.65602

0.65602

0.65602

0.65602

0.656722

0.657472

0.658807

0.658807

0.658807

0.658807

0.659257

0.660244

0.660244

0.660689

0.661655 
Paenibacillus sp. 320-W

Deinococcus gobiensis

Rahnella sp. ERMR1:05

Actinoalloteichus hymeniacidonis

Haloferax volcanii

Cryobacterium arcticum

Methylobacterium sp. C1

Paenibacillus sp. FSL H7-0357

Rhodococcus sp. MTM3W5.2

Acinetobacter sp. WCHA55

Tenacibaculum mesophilum

Enterobacter asburiae

Propionibacterium virus PHL112N00

Paenibacillus physcomitrellae

Arthrobacter sp. YC-RL1

Desulfitobacterium metallireducens

Dermabacter vaginalis

Streptococcus infantarius

Solibacillus sp. R5-41

Streptomyces violaceusniger

Micromonospora sp. L5

Alistipes finegoldii

Rhodovulum sp. MB263

Bacillus anthracis

Sphingobacterium sp. B29

Pseudomonas taetrolens

Spirosoma radiotolerans

Methylocystis bryophila

Octadecabacter antarcticus

Halobacillus mangrovi

Pseudomonas extremaustralis

Corynebacterium minutissimum

Providencia alcalifaciens

Halalkalicoccus jeotgali

Thioalkalivibrio paradoxus

Lactobacillus kunkeei

Haloarcula marismortui

Propionibacterium phage QueenBey

Miniimonas sp. S16

Arthrobacter alpinus

Corynebacterium urealyticum

Sphingobacterium mizutaii

Pseudomonas sp. CCOS 191

Lactobacillus murinus

Bacteroides zoogleoformans

Candidatus Atelocyanobacterium thalass:

Clostridium septicum

Chryseobacterium sp. IHB B 17019

Mesorhizobium huakuii

Halobacterium hubeiense
15.7617

71.40189

34.94053

75.04724

11.54116

103.5468

156.3839

14.00009

158.7464

137.051

2.176568

35.82203

24.91381

7.296847

188.6641

3.849914

1220.56

31.25742

11.84174

44.26954

1.216231

113.7298

67.18723

65.60498

45.67979

34.84849

21.75933

60.79909

13.19424

5.756139

35.57829

48137.29

7.681949

19.69209

38.00992

2.412004

1.206285

5.686926

152.8033

289.5179

1689.228

19.7014

37.19322

2.320139

63.27881

3.88411

18.89242

26.64396

52.03623

14.02264
$-0.67294$

$-0.40322$

0.822647

$-0.50898$

0.493154

$-0.43562$

0.624155

0.534735

$-0.67155$

0.825891

$-0.38168$

0.468985

$-0.57874$

0.712515

$-0.69184$

0.849806

$-0.3182$

0.391694

0.518751

0.63837

$-1.98397$

2.440165

$-0.62563$

0.771519

1.089862

1.344793

$-0.8649$

1.06787

$-0.32635$

0.402989

$-1.20754$

1.493862

$-0.31768$

0.393681

$-0.92476$

1.145842

$-0.72623$

0.901191

$-0.47604$

0.590965

$-2.35152$

2.923222

$-0.51831$

0.645369

$-0.45857$

0.571057

1.501531

1.86244

0.85746

1.066571

$-0.5385$

0.670371

$-0.558690 .695384$

$-0.525470 .65318$

$-0.848041 .053628$

$-0.944061 .173268$

$-0.4503$

0.561102

$-0.21537$

0.269042

$-1.04167$

1.302235

$-0.525350 .658305$

$-0.517210 .645407$

$-1.42174 \quad 1.77684$

$-2.334352 .923288$

$-1.686052 .108406$

$-0.369090 .461903$

$-0.252080 .314911$

$-0.30492$

0.381516

$-0.64343$

0.803338

$-0.62715$

0.785306

$-1.51664$

1.892252

$-0.71792$

0.899661

1.482546

1.858711

0.692002

0.868402

0.623056

0.781989

$-0.76321$

0.959092

$-0.493240 .619442$

$-0.81801$

$-0.81763$

0.41335

0.668044

$-0.81546$

0.413566

0.668096

$-0.81465$

0.414807

0.669804

$-0.81312$

0.415275

0.670262

$-0.81385$

0.41615

0.6704

$-0.81225$

0.415731

0.6704

$-0.81412$

0.416651

0.6704

$-0.81237$

0.415577

0.6704

0.812618

0.416581

0.6704

$-0.81305$

0.416437

0.6704

$-0.8109$

0.416192

0.6704

0.810431

0.417423

0.671346

$-0.80993$

0.417693

0.671461

$-0.80981$

0.417978

0.671461

$-0.80833$

0.418048

0.671461

$-0.80695$

0.418898

0.672529

$-0.80706$

0.419693

0.673211

$-0.80585$

0.419632

0.420328

0.673211

$-0.80553$

0.420514

0.673295

$-0.80443$

0.421149

0.673295

$-0.80313$

0.421901

0.673295

$-0.80302$

0.421966

0.673295

0.806217

0.420118

0.673295

0.803941

0.421431

0.673295

$-0.80329$

0.421809

0.673295

$-0.80343$

0.421725

0.673295

$-0.80448$

0.421121

0.673295

$-0.80487$

0.420893

0.673295

$-0.80464$

0.421027

0.673295

$-0.80252$

0.422252

0.673295

$-0.80052$

0.42341

0.673456

$-0.79991$

0.423762

0.67351

$-0.79804$

0.424847

0.67351

$-0.80138$

0.422914

0.67351 
Neisseria cinerea

Geobacillus sp. GHH01

Lacinutrix sp. 5H-3-7-4

Novosphingobium sp. P6W

Leptolyngbya sp. NIES-3755

Lactobacillus mucosae

Paracoccus zhejiangensis

Aerococcaceae bacterium ZY16052

Paraoerskovia marina

Sphingopyxis terrae

Mucilaginibacter sp. HYN0043

Lactobacillus pentosus

Azospira oryzae

Vibrio rotiferianus

Paraglaciecola psychrophila

Halostagnicola larsenii

Cyanobacterium aponinum

Psychrobacter sp. P11G3

Desulfohalobium retbaense

Methylobacterium sp. 17SD2-17

Bacillus clausii

Gryllotalpicola sp. 2DFW10M-5

Sphingopyxis sp. WS5A3p

Corynebacterium doosanense

Gramella sp. MAR_2010_147

Streptococcus sp. JS71

Roseiflexus sp. RS-1

Streptococcus suis

Sphingobium baderi

Candidatus Coxiella mudrowiae

Paeniclostridium sordellii

Staphylococcus argenteus

Altererythrobacter sp. ZODW24

Vibrio azureus

Xenorhabdus bovienii

Aeromicrobium choanae

Mycobacterium marinum

Haliscomenobacter hydrossis

Amycolatopsis sp. BJA-103

Myroides profundi

Bacillus sp. FJAT-42376

Spirosoma montaniterrae

Paracoccus contaminans

Corynebacterium epidermidicanis

Actinobacillus pleuropneumoniae

Glaciecola nitratireducens

Acetohalobium arabaticum

Hymenobacter sp. PAMC 26554

Pediococcus inopinatus

Aurantimicrobium minutum

69.54491

0.378355

13.48961

$-0.66083$

0.830242

$-0.79594$

0.426065

0.673793

82.04778

2.336925

2.940915

0.794625

0.426832

0.67455

1.371803

$-0.36238$

1.079387

0.794016

0.427186

0.674644

2.59413

$-1.87646$

0.456461

$-0.79389$

0.427262

0.674644

224.9006

$-1.28956$

.365974

$-0.7931$

0.427718

0.675071

24.06427

$-0.42848$

1.62858

$-0.79183$

0.42846

0.6755

71.01054

$-0.69347$

0.541151

$-0.7918$

0.428476

0.6755

52.26026

$-0.32135$

0.876282

$-0.79137$

0.428725

0.6755

36.53041

$-0.50113$

.406077

$-0.79136$

0.428732

0.6755

13.43328

0.526297

0.633525

$-0.79102$

0.428933

0.675523

202.8756

$-0.64837$

0.665898

0.790356

0.42932

0.675736

0.886837

$-0.49338$

0.820569

$-0.79015$

0.42944

0.675736

6.874587

2.312352

$-0.78943$

0.429861

0.676054

$10.95685-0.87159 \quad 1.107799$

$-1.28726$

0.789169

0.430013

0.676054

$-0.78744$

0.431023

0.677349

$\begin{array}{lll}4.813233 & 1.074789 & 1.367455\end{array}$

$-0.78678$

0.431413

0.677668

34.15872

$-0.62442$

0.794196

0.785978

0.431881

0.677818

3.306457

$-1.11417$

1.418422

$-0.78622$

0.431736

0.677818

143.0721

$-0.39547$

0.503807

$-0.7855$

0.432163

0.677968

$\begin{array}{lll}9.090024 & -0.79946 & 1.020225\end{array}$

$-0.78497$

0.432469

0.678157

104.3649

$-0.351930 .448966$

54.96362

$-0.406540 .52007$

$-0.78361$

0.433268

0.678824

1295.68

$-0.25925$

0.332522

$-0.78388$

0.433113

0.678824

5.321381

$-1.121811 .440563$

$-0.7817$

0.434389

0.680287

$-0.77965$

0.435598

0.681887

107.8773

$-0.631330 .812082$

$-0.77873$

0.436137

0.682438

16.39999

$-0.58579$

0.75374

$-0.77743$

0.436907

0.683085

164.1955

0.373389

0.480505

$-0.77717$

0.437057

0.683085

85.23127

$-0.43561$

0.560822

0.777075

0.437115

$-0.77674$

0.437312

0.683085

$2.547471 \quad-1.27571 \quad 1.644033$

$-0.77596$

0.437771

0.6831

$\begin{array}{lll}27.64965 & 0.525217 & 0.676946\end{array}$

0.775863

0.43783

0.683322

87.14592

0.4106610 .52976

0.775183

0.438232

0.683322

8.419664

$-0.65879$

0.850058

$-0.77499$

0.438345

0.683432

2.34114

1.8592772 .401498

0.774216

0.438803

0.683432

12.77251

$-0.712890 .920899$

$-0.77413$

0.438855

0.683432

211.0762

$-0.40427$

0.522332

$-0.77398$

0.438943

0.683432

75.78012

0.449686

0.581112

25.23915

0.393331

0.509487

0.773837

0.439027

0.683432

78.99791

$-0.361750 .46797$

0.772014

0.440106

0.683432

0.727532

2.26332 .933126

$-0.77302$

0.439509

0.683733

6.228378

$-0.883581 .145596$

10.8761

$-0.60623$

0.785876

0.771634

0.440331

0.683733

$-0.77129$

0.440536

0.683733

538.9385

$-0.3897$

0.504314

$-0.77141$

0.440462

0.683733

724.5554

$-0.239210 .310002$

$-0.77274$

0.439676

0.683733

$-0.77164$

0.440328

0.683733

$\begin{array}{lll}7.759103 & -1.08212 & 1.404904\end{array}$

$-0.77024$

0.441155

0.683733

$\begin{array}{lll}1.624574 & -1.77653 & 2.312088\end{array}$ 
Flexistipes sinusarabici

Lactobacillus paraplantarum

Rhodococcus coprophilus

Bacillus safensis

Polynucleobacter duraquae

Pseudomonas sp. DR 5-09

Fusobacterium periodonticum

Kosakonia sp. CCTCC M2018092

Nitrosococcus oceani

Chryseobacterium sp. H3001

Gammapapillomavirus 19

Acidithiobacillus ferrivorans

Paraphotobacterium marinum

Euzebya sp. DY32-46

Finegoldia magna

Methylovulum psychrotolerans

Hymenobacter sp. APR13

Weissella koreensis

Corynebacterium jeikeium

Anabaena sp. 90

Porphyromonas crevioricanis

Melioribacter roseus

Klebsiella variicola

Dechloromonas aromatica

Corynebacterium casei

Bacteroides heparinolyticus

Flavobacterium crassostreae

Aquimarina sp. AD10

Mycobacterium sp. WY10

Mixta gaviniae

Cellvibrio sp. PSBB023

Shewanella violacea

Rhizobium sp. TAL182

Pseudomonas arsenicoxydans

Sporosarcina psychrophila

Enterobacter roggenkampii

Polaribacter sp. SA4-12

Calyptogena okutanii thioautotrophic gill

Lactobacillus backii

Pseudomonas sp. B10

Desulfurispirillum indicum

Deinococcus actinosclerus

Friedmanniella sagamiharensis

Staphylococcus schleiferi

Thalassolituus oleivorans

Mycobacterium haemophilum

Candidatus Planktophila dulcis

endosymbiont of Acanthamoeba sp. UWC

Oscillibacter sp. PEA192

Mycetocola sp. 449
2.172556

1.976891

122.6033

6.926376

1.840121

24.29218

162.0089

9.996676

6.920467

14.09964

1.842238

13.70632

8.774556

80.05848

7219.456

10.23976

55.74775

4.935955

11923.79

2.648027

20.64839

2.229902

34.23938

58.51576

1538.904

125.2898

3.818162

13.87867

27.9315

38.13691

16.081

1.739571

1.0788

48.37461

11.68207

19.85177

12.02054

2.778131

15.14302

32.38097

6.834191

86.32679

307.3162

59.25783

8.042833

67.90021

19.92122

1.382694

95.22366

70.66591

$$
-1.57826
$$

$-1.7222$

2.0683

$-0.46745$

2.257672

0.99795

0.613388

$-1.25158$

1.31216

$-0.6523$

1.645863

$-0.37376$

0.857524

$-0.8822$

0.490987

$-0.82772$

1.09047

$-0.87567$

1.154963

2.052581

2.709176

$-0.795161 .048868$

0.813901

1.07441

$-0.29791$

0.393459

$-0.29464$

0.389638

$-0.808491 .069549$

$-0.34586$

0.457931

$-1.15887$

1.537034

$-0.2998$

0.39759

$-1.31566$

1.745757

$-0.663130 .881315$

$-1.331831 .770506$

$-0.70969$

0.943076

$-0.31845$

0.424152

$\begin{array}{ll}-0.21577 & 0.287608\end{array}$

$-0.56484 \quad 0.75384$

$-1.148431 .534193$

$0.738526 \quad 0.98645$

$-0.614720 .820376$

$-0.414530 .553437$

$-0.477510 .640232$

$-2.05904 \quad 2.764247$

$-2.178762 .923926$

0.5053950 .67827

$-0.826151 .109835$

$0.88221 \quad 1.185825$

0.9598651 .291323

$-1.230311 .656007$

$-0.783891 .055675$

$-0.725420 .978112$

$-0.752341 .016726$

$-0.333060 .450036$

$-0.39635$

0.536801

$0.38284 \quad 0.518585$

$-0.794551 .075395$

$\begin{array}{ll}-0.31589 & 0.427677\end{array}$

$-0.631710 .85712$

$-1.742242 .365932$

-0.5189 0.704834

$-0.260310 .353653$

$-0.76307$

0.44542

0.689195

$-0.76282$

0.445571

0.689195

$-0.76208$

0.446013

0.689586

$-0.76054$

0.446931

0.689926

$-0.76044$

0.446991

0.689926

$-0.76068$

0.446847

0.689926

$-0.76123$

0.446518

0.689926

$-0.75978$

0.447387

0.690244

$-0.75905$

0.447821

0.69062

$-0.75818$

0.448342

0.690851

0.75764

0.448666

0.690851

$-0.75811$

0.448382

0.690851

0.757533

0.44873

0.690851

$-0.75716$

0.448952

0.6909

$-0.75619$

0.449533

0.691463

$-0.75592$

0.449698

0.691463

$-0.75527$

0.450087

0.691769

$-0.75397$

0.45087

0.692387

$-0.75404$

0.450823

0.692387

$-0.75363$

0.45107

0.692402

$-0.75243$

0.451791

0.692819

$-0.75223$

0.451913

0.692819

$-0.75253$

0.451732

0.692819

$-0.75079$

0.45278

0.693857

$-0.75021$

0.453131

0.694101

$-0.74929$

0.453684

0.69416

$-0.74856$

0.454123

0.69416

0.748671

0.454056

0.69416

$-0.74931$

0.453668

0.69416

$-0.749$

0.453854

0.69416

$-0.74584$

0.455766

0.696378

$-0.74488$

0.456343

0.696383

$-0.74515$

0.456181

0.696383

0.745124

0.456197

0.696383

$-0.74439$

0.456641

0.696545

0.743963

0.456899

0.696646

0.743319

0.457289

0.696948

$-0.74294$

0.457518

0.697007

$-0.74255$

0.457757

0.697078

$-0.74165$

0.4583

0.697613

$-0.73997$ 
Pseudomonas oryzihabitans

Corynebacterium singulare

Desulfobacula toluolica

Flavobacterium commune

Arthrobacter sp. ERGS1:01

Streptosporangium sp. 'caverna'

Capnocytophaga haemolytica

Tenacibaculum todarodis

Methanobrevibacter olleyae

Campylobacter jejuni

Dietzia psychralcaliphila

Filifactor alocis

Halothece sp. PCC 7418

Gammapapillomavirus 22

Sinorhizobium sp. RACO2

Pelosinus sp. UFO1

Thiohalobacter thiocyanaticus

Pseudomonas koreensis

Propionibacterium virus $\mathrm{P} 100 \mathrm{~A}$

Phycicoccus dokdonensis

Frondihabitans sp. PAMC 28766

Cellulophaga baltica

Rickettsiales endosymbiont of Stachyamc

Microbacterium hominis

Pararhodospirillum photometricum

Edwardsiella tarda

Paracoccus aminovorans

Corynebacterium kroppenstedtii

Streptococcus gordonii

Betapapillomavirus 1

Xanthomonas euvesicatoria

Edwardsiella ictaluri

Acidaminococcus intestini

Lactobacillus terrae

Lysinibacillus sp. YS11

Leuconostoc carnosum

Alteromonas naphthalenivorans

Tatumella ptyseos

Gordonia iterans

Curtobacterium sp. MR_MD2014

Capnocytophaga sp. ChDC OS43

Propionibacterium virus PHL113M01

Mycobacterium sp. PYR15

Oscillatoria nigro-viridis

Streptomyces sp. 769

Streptomyces pactum

Chloroflexus aggregans

Bacillus thermoamylovorans

Streptococcus iniae

Corynebacterium deserti

\subsection{3}

6418.27

2.117441

23.96487

154.3344

96.51279

10.82364

7.12372

4.804631

62.08163

434.2733

79.96276

3.24107

1.050335

91.39436

9.615714

39.55254

194.4013

7.791832

232.2839

165.7333

7.573067

2.756008

293.3354

49.39742

21.09068

212.1909

33651.21

6524.031

405.6211

41.62786

4.171994

6.858534

3.478834

15.23395

68.28045

1.974433

5.041452

288.0625

159.546

90.63845

21.93323

32.80697

18.91605

54.04159

55.07722

11.04949

5.984187

11.76345

561.2182

\begin{tabular}{rrr}
-0.37074 & 0.504075 & -0.7 \\
-0.24 & 0.326583 & -0.7 \\
-1.05824 & 1.442167 & -0.7 \\
\hline
\end{tabular}

$-0.73548$

$-0.73489$

$-0.73379$

$-0.7337$

$-0.73314$

$-0.73227$

$-0.73182$

0.730594

0.730479

0.732135

$-0.73162$

$-0.7308$

$-0.73108$

$-0.72934$

$-0.72894$

0.728072

$-0.72656$

$-0.72519$

$-0.72414$

$-0.72264$

$-0.72154$

$-0.3037$

0.498861

$0.902302 \quad 1.249718$

$1.590282 \quad 2.20491$

$-0.42456$

0.58876

$-0.42507$

0.590366

$\begin{array}{ll}-0.82127 & 1.140987\end{array}$

$-0.420650 .583968$

$-0.55006$

0.764483

$-0.40413$

0.561596

$-0.85632$

1.192711

$-0.57472$

0.800019

$1.335771 \quad 1.860239$

0.9966411 .387527

$-1.09083$

1.522821

$-0.845641 .181907$

$-0.778971 .088856$

$-1.478752 .070791$

$-1.151921 .612584$

$-0.360810 .50598$

$-0.357120 .501932$

$-0.471020 .663215$

$-1.025531 .444118$

$-0.7305$

1.029234

0.662487

0.933104

$-0.397270 .560299$

$-0.357870 .505109$

$-0.82443$

1.165312

$-0.76605$

1.085282

$-0.642490 .911448$

$-0.227190 .322603$
0.722005

0.721246

$-0.7211$

$-0.72001$

$-0.71979$

$-0.72033$

$-0.71952$

$-0.71961$

$-0.71796$

$-0.71838$

0.718065

0.718286

$-0.71632$

$-0.71549$

$-0.7154$

$-0.7141$

$-0.71433$

$-0.71309$

$-0.71149$

$-0.71021$

$-0.71014$

$-0.70975$

0.709982

$-0.70903$

$-0.70849$

$-0.70748$

$-0.70586$

$-0.70491$

$-0.70425$
0.462047

0.462404

0.463078

0.700094

0.463134

0.463474

0.464006

0.701089

0.464277

0.701212

0.465027

0.701212

0.465097

0.701212

0.464086

0.701212

0.464402

0.701212

0.464901

0.701212

0.464731

0.701212

0.465793

0.701212

0.466035

0.701969

0.466569

0.702044

0.467493

0.702558

0.468334

0.703657

0.468981

0.704632

0.469898

0.705313

0.470577

0.7064

0.470292

0.70666

0.470758

0.70666

0.470848

0.70666

0.471519

0.70666

0.471656

0.706666

0.471325

0.706666

0.471823

0.706666

0.471766

0.706666

0.472779

0.706666

0.472523

0.706934

0.472717

0.706934

0.472581

0.706934

0.473793

0.706934

0.474305

0.708159

0.474363

0.708429

0.475166

0.708429

0.475022

0.709045

$\begin{array}{ll}475788 & 0.709682\end{array}$

0.4767790 .71087

0.4775740 .711311

$0.477617 \quad 0.711311$

0.4778570 .711311

$0.477716 \quad 0.711311$

$0.478303 \quad 0.711683$

$0.47864 \quad 0.711894$

$0.47927 \quad 0.71254$

0.4802770 .713746

$0.480867 \quad 0.71433$

$0.481278 \quad 0.714648$ 
Saccharomonospora glauca

Staphylococcus hyicus

Corynebacterium timonense

Nitrosomonas europaea

Allokutzneria albata

Tatlockia micdadei

Bacteroides salanitronis

Klebsiella aerogenes

Frondihabitans sp. 762 G35

Leuconostoc citreum

Sediminicola sp. YIK13

Mycoplasma cynos

Virgibacillus sp. Bac330

Odoribacter splanchnicus

Streptomyces sp. CLI2509

Corynebacterium atypicum

Propionibacterium virus PHL095N00

Mycobacterium canettii

Bacteroides ovatus

Labrenzia aggregata

Enterococcus faecium

Streptomyces ambofaciens

Rhodococcus rhodochrous

Brevundimonas vesicularis

Halanaerobium praevalens

Haemophilus parainfluenzae

Paenibacillus riograndensis

Rothia dentocariosa

Corynebacterium imitans

Acetobacter tropicalis

Nostoc sp. PCC 7524

Gordonia phthalatica

Aerococcus viridans

Methylotenera versatilis

Streptococcus phage $\mathrm{PH} 10$

[Eubacterium] rectale

Cyanothece sp. PCC 7424

Acinetobacter haemolyticus

Marinilactibacillus sp. 15R

Enterococcus mundtii

Chryseobacterium indoltheticum

Corynebacterium kutscheri

Shewanella frigidimarina

Parachlamydia acanthamoebae

Gemella sp. oral taxon 928

Microbacterium sp. XT11

Exiguobacterium sp. U13-1

Candidatus Sodalis pierantonius

Bacillus subtilis

Mahella australiensis
94.88189

20.86081

2042.604

5.238726

149.9878

0.878065

38.61845

42.70814

128.3171

30.20358

4.6465

1.515492

1.957838

85.25252

9.286774

925.9296

21.79268

79.37398

157.7936

14.05478

159.0046

75.77955

148.8623

311.6989

8.6416

1337.631

6.854662

6855.35

2644.953

0.966196

1.812122

195.9138

42.82722

8.472857

9.398949

392.8152

9.154175

84.45078

13.37798

13.85768

79.47071

188.6637

4.428938

2.038783

3.313357

123.8944

11.1064

2.412472

249.3023

2.843817 $\begin{array}{ll}-0.3551 & 0.504912\end{array}$

0.615640 .876836

$-0.297110 .423205$

$-0.91928$

1.311259

$-0.25525$

0.364215

$-1.87645$

2.679032

$-0.60826$

0.868149

$-0.4054$

0.579948

$-0.37255$

0.533266

$-0.79584$

1.139177

$-0.94432$

1.350666

$1.752801 \quad 2.517831$

$-1.45109$

2.085221

0.599905

0.86339

$-1.26491 .822164$

-0.248 0.35665

$-1.037191 .494545$

0.354489

0.510355

$-0.51999$

0.747975

$-0.81667 \quad 1.178219$

$-0.418820 .606283$

$-0.301640 .43748$

$-0.319260 .461935$

$-0.37579$

0.544683

0.652123

$-0.46225$

0.945791

$-0.88885$

1.287512

$-0.48722$

0.705419

$-0.23682$

0.343158

$-2.01789$

2.924662

$-1.46343$

2.120102

$-0.27307$

0.397666

0.532843

0.77628

$-0.76265$

1.110224

$-1.13003$

1.645613

0.511325

0.74582

0.900999

1.315199

0.415736

0.607325

$-0.65962 \quad 0.96467$

0.6077370 .890254

$\begin{array}{ll}-0.4323 & 0.633512\end{array}$

$-0.25782$

0.377693

1.016898

1.491095

$-1.54736$

2.272191

$-1.291151 .896981$

$-0.367670 .540915$

$-1.098571 .617191$

$-1.632672 .403678$

0.4563310 .672262

$-1.0928 \quad 1.61144$

$-0.70328$

0.481879

0.71525

$\begin{array}{ll}0.702116 & 0.482607\end{array}$

0.715817

$-0.702040 .482655$

0.715817

$-0.70106$

0.483263

0.716148

$-0.70083$

0.483408

0.716148

$-0.70042$

0.483665

0.716148

$-0.70064$

0.483528

0.716148

$-0.69902$

0.484538

0.716655

$-0.69861$

0.484794

0.716655

$-0.69861$

0.484796

0.716655

$-0.69915$

0.484458

0.716655

0.696155

0.486332

0.718583

$-0.69589$

0.486495

0.718583

0.694825

0.487165

0.718603

$-0.69418$

0.487571

0.718603

$-0.69535$

0.486837

0.718603

$-0.69398$

0.487694

0.718603

0.694593

0.48731

0.718603

$-0.6952$

0.486929

0.718603

$-0.69314$

0.488223

0.719092

$-0.69081$

0.489688

0.719255

$-0.6895$

0.490509

0.719255

$-0.69113$

0.489481

0.719255

$-0.68992$

0.490243

0.719255

0.6895

0.490509

0.719255

$-0.69015$

0.490101

0.719255

$-0.69036$

0.489966

0.719255

$-0.69068$

0.489769

0.719255

$-0.69013$

0.490111

0.719255

$-0.68996$

0.490222

0.719255

$-0.69026$

0.490029

0.719255

$-0.68668$

0.492287

0.720949

0.686406

0.492457

0.720949

$-0.68693$

0.492127

0.720949

$-0.68669$

0.492275

0.720949

0.685588

0.492973

0.721414

0.685067

0.493302

0.721605

0.684536

0.493637

0.721805 
Streptomyces collinus

Mycobacterium leprae

Capnocytophaga sp. oral taxon 323

Flavobacterium sp. HYN0086

Rhodobacteraceae bacterium QY30

Simonsiella muelleri

Vibrio campbellii

Alkaliphilus oremlandii

Salmonella enterica

Methylomicrobium alcaliphilum

Pseudomonas amygdali

Pseudomonas reinekei

Methylobacterium sp. 17Sr1-43

Luteipulveratus mongoliensis

Lachnoanaerobaculum umeaense

Pseudomonas sp. CMR5c

Desulfurella acetivorans

Enterococcus sp. CR-Ec1

Rhodococcus sp. H-CA8f

Idiomarinaceae bacterium HL-53

Pseudomonas alkylphenolica

Pontibacter korlensis

Mycobacterium sp. DL90

Citrobacter freundii complex sp. CFNIH2

Selenomonas ruminantium

Rhodococcus sp. B7740

Microterricola viridarii

Streptococcus merionis

Legionella longbeachae

Frischella perrara

Fastidiosipila sanguinis

Lactobacillus alimentarius

Faecalibaculum rodentium

Cyanothece sp. PCC 7822

Winogradskyella sp. PC-19

Salinicoccus halodurans

Pseudomonas sp. Lz4W

Wolinella succinogenes

Tessaracoccus flavescens

Planktothrix agardhii

Bacillus sp. 1NLA3E

Oscillatoriales cyanobacterium JSC-12

Lactobacillus amylovorus

Candidatus Arthromitus sp. SFB-rat-Yit

Streptomyces sp. ZFG47

Pseudodesulfovibrio piezophilus

Cronobacter sakazakii

Commensalibacter sp. AMU001

Citrobacter rodentium

Hydrogenophilus thermoluteolus
59.8061

29.20433

$\begin{array}{lll}0.32771 & 0.483404\end{array}$

56.13199

$-0.40697$

0.601081

40.68405

$-0.5428$

0.804676

71.04231

$-0.68134$

1.009678

27.33765

$-0.37442$

0.553914

11.64539

$-0.75456$

1.117859

9.52491

0.867603

1.284971

299.4343

0.74922

1.109791

4.070766

0.253099

0.375646

0.938754

$-0.90328$

1.342132

25.97337

$-1.9683$

2.924905

151.4326

156.1569

0.483238

0.72013

$-0.32976$

0.491879

53.26615

$-0.27144$

0.405472

35.51343

$-0.44897$

0.671601

4.016656

0.925602

142.9231

2.156615

80.46018

10.1944

149.7812

0.912877

11.10188

227.5711

209.3957

13.12323

1.111643

5.405845

174.3362

2.534988

5.919383

10.16455

3.470829

7.0061

19.36927

1.930222

217.9197

2.596685

8.117397

11.15563

14.82688

6.211624

73.36317

1.471804

47.69821

1.697364

10.87553

33.33176
0.4263680 .638245

1.171238

$-1.95182$

1.754308

2.924988

0.684857

1.029973

$-1.19418$

1.797282

$-0.3465$

0.522973

$-0.61122$

0.924694

$-0.28358$

0.429547

$-1.93149$

2.925091

$-0.556470 .843326$

$-0.277650 .422084$

$-0.26847$

0.408237

$-0.49488$

0.753646

$-1.60084$

2.440451

$-0.777431 .186262$

0.3882520 .596023

$-1.064131 .631292$

$-0.91797 \quad 1.408523$

$0.924228 \quad 1.418212$

$1.075836 \quad 1.648784$

$-0.910581 .394525$

$-0.790471 .211592$

$-1.48272 \quad 2.269129$

$-0.282460 .433724$

$-1.04921 .611897$

$-0.690131 .060808$

$-0.593140 .914103$

$-0.61005$

0.939818

$1.051468 \quad 1.625813$

$-0.27159$

0.420077

$-1.38696$

2.149203

$-0.47959$

0.744884

1.345969

2.091668

$-0.73306$

1.140193

0.427328

$-0.67793$

$-0.67706$

$-0.67456$

0.497817

0.724134

$-0.67481$

0.499957

0.724642

$-0.67596$

0.499795

0.725216

$-0.67501$

0.499067

0.725216

0.675193

0.499671

0.725216

0.675101

0.499553

0.725216

0.67377

0.499612

0.725216

$-0.67302$

0.500458

0.725216

$-0.67295$

0.500935

0.725654

0.671042

0.500978

0.725829

$-0.67041$

0.502193

0.725829

$-0.66944$

0.502598

0.727301

$-0.66851$

0.503214

0.727597

0.668032

0.503811

0.728198

0.667635

0.504113

0.728773

$-0.66729$

0.504366

0.728921

0.664928

0.504586

0.728997

$-0.66444$

0.506097

0.729025

$-0.66255$

0.506411

0.730917

$-0.661$

0.507616

0.73253

$-0.66019$

0.508612

0.733675

$-0.66032$

0.509135

0.733848

$-0.65985$

0.509049

0.733848

$-0.65781$

0.509351

0.73387

$-0.65763$

0.510657

0.735339

$-0.65665$

0.510775

0.735339

$-0.65596$

0.511407

0.735957

$-0.65536$

0.511849

0.736301

0.651406

0.512235

0.736565

$-0.65232$

0.514785

0.737755

$-0.65172$

0.514193

0.737755

0.651685

0.514581

0.737755

0.652502

0.514604

0.737755

$-0.65296$

0.514077

0.737755

$-0.65242$

0.513779

0.737755

$-0.65343$

0.514127

0.737755

$-0.65125$ 
Moraxella bovoculi

Clostridium baratii

Flavobacteriaceae bacterium UJ101

[Bacillus] selenitireducens

Streptomyces violaceoruber

Weissella viridescens

Selenomonas sp. oral taxon 136

Acinetobacter sp. ACNIH1

Ruminococcus bicirculans

Candidatus Planktophila versatilis

Nitrosomonas communis

Paenalcaligenes hominis

Aerococcus urinae

Planococcus sp. MB-3u-03

Tenacibaculum sp. DSM 106434

Polymorphum gilvum

Treponema pedis

Chryseobacterium camelliae

Actinomyces radicidentis

Gordonibacter massiliensis

Lachnoclostridium phocaeense

Propionibacterium phage PHL055N00

Brachybacterium sp. VR2415

Enterobacter cloacae

Streptomyces xinghaiensis

Pseudomonas sp. Z003-0.4C(8344-21)

Erwinia gerundensis

Chryseobacterium sp. T16E-39

Kytococcus sedentarius

Methylobacterium brachiatum

Pseudodesulfovibrio profundus

Exiguobacterium sp. N4-1P

Mycobacterium sp. YC-RL4

Desulfosporosinus meridiei

Actinobacteria bacterium IMCC26077

Vibrio cholerae

Pseudarthrobacter sulfonivorans

Actinopolyspora erythraea

Denitrovibrio acetiphilus

Chlorobaculum tepidum

Synechococcus lividus

Dehalogenimonas lykanthroporepellens

Pseudonocardia sp. EC080625-04

Rhizobium sp. Kim5

Staphylococcus sp. SDB 2975

Syntrophothermus lipocalidus

Treponema sp. OMZ 838

Ndongobacter massiliensis

Cryptobacterium curtum

Acetobacter ghanensis

14.70808

48.71602

0.777729

1.216078

0.639539

0.522473

0.74482

8.907819

0.377488

0.590558

0.639205

0.52269

0.74482

1.596726

0.7309

1.143602

0.639121

0.522744

0.74482

49.02672

$-1.35369$

2.119831

$-0.63858$

0.523094

0.74482

4.148232

0.560258

$-0.63871$

0.523015

0.74482

64.26915

$-1.11901$

1.753514

$-0.63815$

0.523373

0.744926

91.03416

$-0.41895$

0.658761

101.7301

17.93337

$-0.46514$

0.731441

$-0.63596$

0.5248

0.745539

2.415211

0.881

$-0.63592$

0.524829

0.745539

$-0.46320 .727842$

$-0.63673$

0.524301

0.745539

2.415211

$-0.97389$

1.529049

$-0.6364$

0.524513

0.745539

3.948231

$-0.85582$

1.347746

$-0.63692$

0.524175

0.745539

$-0.635$

0.525427

0.746098

55.63541

$-0.37592$

0.593104

15.58574

9.375573

$-0.72993$

1.152549

$-0.63382$

0.526198

0.746902

97.2614

0.756506

1.196257

0.526523

0.747071

97.2614

$-0.31573$

0.499555

0.632395

0.527129

0.747112

2.530392

$-0.95698$

1.512572

$-0.63202$

0.527373

0.747112

17.39442

227.1635

$-0.49555$

0.783923

$-0.63268$

0.526942

0.747112

32.49234

$-0.25534$

0.404573

$-0.63214$

0.527294

0.747112

34.43915

$-0.41453$

0.659309

$-0.63113$

0.527955

0.747646

21.65799

$-0.656891 .045288$

$-0.62873$

0.529528

0.749057

$-0.62843$

0.529724

0.749057

301.1027

357.7308

0.921893

1.466025

0.628838

0.529455

0.749057

$-0.32026$

0.509689

$-0.62835$

0.529775

0.749057

72.01713

0.30176

0.481069

$-0.62726$

0.530489

0.749774

34.58269

$-0.23892$

0.381157

$-0.62682$

0.530779

0.749893

117.8698

16.9486

$-0.48976$

0.783258

$-0.62529$

0.53178

0.751015

510.578

0.485394

0.777079

0.624639

0.532208

0.751328

0.757

0.623566

0.532913

0.752031

$-0.2832$

0.454453

$-0.62317$

0.533171

0.752104

276.3921

0.378787

0.609078

0.621903

0.534006

0.752698

16.83358

$-0.50988$

0.819564

9.244863

$-0.67559$

1.098531

$-0.62213$

0.533855

0.752698

$-0.615$

0.538556

0.753265

196.8681

$-0.3208$

0.517221

2.400234

$-1.08393$

1.751192

$-0.62023$

0.535104

0.753265

1.110209

$-1.49632$

2.433413

$-0.61897$

0.535939

0.753265

25.43088

$-0.54097$

0.874974

119.9811

$-0.2221$

0.359031

$-0.61491$

0.538616

0.753265

$-0.61827$

0.536397

0.753265

76.71077

$-0.21257$

0.345238

$-0.61861$

0.536176

0.753265

1.329056

$-1.22931$

1.990542

$-0.61573$

0.538075

0.753265

23.16387

$-0.4266$

0.687036

$-0.61758$

0.536855

0.753265

6.817319

$-0.90144$

1.461971

$-0.62093$

0.534649

0.753265

1.422571

$-1.22501$

1.97722

$-0.6166$

0.537502 
Clostridium formicaceticum

Bacteroides coprosuis

Rhodococcus sp. BH4

Pediococcus acidilactici

Bartonella grahamii

Bifidobacterium dentium

Thalassotalea crassostreae

Aurantimicrobium sp. MWH-Uga1

Psychrobacter alimentarius

Sulfurimonas gotlandica

Clostridium bornimense

Pseudomonas pseudoalcaligenes

Pseudomonas entomophila

Mycoplasma canis

Propionibacterium virus P101A

Thiomicrospira cyclica

Lactococcus garvieae

Terribacillus goriensis

Bathymodiolus septemdierum thioautotr

Olsenella sp. oral taxon 807

Paenibacillus bovis

Bacteroides caecimuris

Sphingomonas melonis

Capnocytophaga sp. oral taxon 864

Dietzia sp. JS16-p6b

Salmonella bongori

Actinomyces pacaensis

Actinomyces sp. VUL4_3

Pseudomonas sp. S09G 359

Methanosarcina lacustris

Dermacoccus nishinomiyaensis

Metakosakonia sp. MRY16-398

Leptotrichia sp. oral taxon 212

Xanthomonas hortorum

Lachnoclostridium sp. YL32

Treponema denticola

Jiangella alkaliphila

Brevibacterium linens

Pseudomonas sp. LPH1

Mogibacterium diversum

Streptomyces sp. PAMC 26508

Thioploca ingrica

Campylobacter concisus

Janibacter indicus

Ichthyobacterium seriolicida

Methanohalophilus mahii

Sulfurovum lithotrophicum

Leptospira biflexa

Staphylococcus muscae

Dialister pneumosintes

\begin{tabular}{|c|c|c|c|c|c|}
\hline & & & & & \\
\hline & -0.62326 & 1.010979 & -0.61649 & 0.537572 & \\
\hline .98754 & 0.632618 & 1.030629 & 0.613817 & 539336 & \\
\hline & 1.000825 & 1.63344 & 0.61271 & 068 & \\
\hline & -1.71836 & 5068 & -0.61259 & & \\
\hline & 0.416658 & 0.680403 & 0.61237 & & \\
\hline & -1.30384 & 2.129416 & -0.6123 & 0.54034 & \\
\hline & & & -0.61332 & & \\
\hline & -0.60056 & 0.980215 & -0.61268 & 0.54009 & \\
\hline & 1.254849 & 2.052046 & 0.611511 & & \\
\hline & 0.45 & & 0.6 & & \\
\hline & & & & & \\
\hline & -0.33 & 0.5 & -0.6102 & & \\
\hline & 1.348503 & 2.213 & 0.609268 & & \\
\hline & 1.05 & 1.7 & 0.610054 & & \\
\hline & & & & & \\
\hline & -0.719 & 1.1796 & -0.61024 & & \\
\hline & & & & & \\
\hline & & & -0.60755 & & \\
\hline & 0.426397 & 0.702 & 0.606617 & 0.544105 & \\
\hline & -0.72588 & & -0.60547 & & \\
\hline & 0.581239 & 0.964 & 0.60 & & \\
\hline & & 0.594105 & -0.60202 & & \\
\hline & -0.40994 & 0.681239 & -0.60176 & 0.547335 & \\
\hline & & & & & \\
\hline & -0.9 & & -0.6 & & \\
\hline & -0.2 & 0.4 & -0. & & \\
\hline & & & & & \\
\hline & & & & & \\
\hline & & & & & \\
\hline & 0.3905 & & $0.5 s$ & & \\
\hline & & & & & \\
\hline & & 0.6 & & & \\
\hline & & & & & \\
\hline & 0.5514 & 0.924485 & 0.596481 & 0.550854 & \\
\hline & & & & & \\
\hline & -0.28222 & 0.475 & -0.59375 & & \\
\hline & & & & & \\
\hline & -0.393 & 0.6613 & -0.59499 & & \\
\hline & & & -0.5 & & \\
\hline & & & & & \\
\hline & 1.1164 & & 0.592 & & \\
\hline & & & $-0.5 s$ & 42 & \\
\hline & -0.27117 & 0.459438 & -0.59022 & 5504 & \\
\hline & 1.330141 & 2.255147 & 0.589825 & .555308 & \\
\hline & -1.72437 & & -0.58928 & & \\
\hline & & & 0.586761 & 0.557364 & \\
\hline & 1.319506 & 2.248723 & & & \\
\hline & -0.43351 & & -0.58696 & 0.557229 & \\
\hline & -0.5487 & 0.936934 & -0.58563 & 0.558125 & 0.7661 \\
\hline
\end{tabular}


Klebsiella sp. M5al

Lactococcus phage jm2

Bacteroides dorei

Hymenobacter sp. DG25B

Pleurocapsa minor

Methanoregula formicica

Lactococcus lactis

Brevibacillus laterosporus

Carboxydothermus hydrogenoformans

Intestinimonas butyriciproducens

Legionella anisa

Polaribacter sp. Hel1_33_78

Christensenella minuta

Sphingomonas sp. LM7

Mobiluncus curtisii

Gallaecimonas sp. HK-28

Calothrix brevissima

Arthrobacter sp. YN

Corynebacterium lactis

Bacillus sp. FJAT-22090

Corynebacterium frankenforstense

Gramella salexigens

Spiroplasma taiwanense

Sulfurihydrogenibium azorense

Paenibacillus terrae

Gordonia bronchialis

Fictibacillus arsenicus

endosymbiont 'TC1' of Trimyema compre

Flavivirga eckloniae

Staphylococcus simulans

Saccharophagus degradans

Curtobacterium pusillum

Paracoccus aminophilus

Pseudomonas fluorescens

Mycobacterium paraintracellulare

Erwinia pyrifoliae

Stanieria sp. NIES-3757

Arthrobacter sp. Rue61a

Cohaesibacter sp. ES.047

Halanaerobium hydrogeniformans

Saccharospirillum mangrovi

Synechococcus sp. WH 7803

Pseudomonas sp. NS1(2017)

Shewanella baltica

Cutibacterium avidum

Staphylococcus xylosus

Acinetobacter sp. NCu2D-2

Pedobacter heparinus

Flavobacterium columnare

Enterobacteriaceae bacterium strain FGI
1.466851

11.3286

436.5424

46.37614

1.538336

1.448134

2076.397

17.96382

1.837831

58.93604

6.909773

6.31268

43.63595

102.6458

672.5198

9.425586

14.47362

112.1181

727.7602

7.651476

1514.183

2.119074

1.220876

1.729084

5.568481

740.5477

6.218766

5.985205

9.305161

559.9691

4.859632

296.0052

82.50917

1367.327

3.956822

2.138272

9.99332

95.97681

14.22755

8.272828

22.86648

9.917436

36.25893

114.4868

4169.113

163.7821

20.5502

29.12668

28.29052

6.832064
$-1.65862$

2.833477

0.89031

1.524148

$-0.54497$

0.935189

$-0.2959$

0.507569

$-1.243$

2.132658

$-1.153131 .979976$

$-0.37432$

0.643297

0.416963

0.716697

$-1.12141$

1.928709

$-0.54095$

0.932266

$-0.676211 .166218$

$0.800961 \quad 1.384884$

$-0.498850 .865985$

$-0.29047$

0.504279

$-0.38685$

0.672522

$-0.4835$

0.840655

0.921718

1.605092

$-0.20631$

0.360296

$-0.22573$

0.394131

0.575602

1.00511

$-0.20706$

0.361255

$-0.952981 .668552$

$1.635335 \quad 2.870328$

$-1.31709$

2.3123

$-0.62501 \quad 1.105164$

$-0.32376$

0.572538

$0.729893 \quad 1.287773$

-0.68189 1.204586

$0.689602 \quad 1.216091$

$-0.290140 .514333$

$-0.805821 .429325$

$-0.29090 .514139$

$\begin{array}{ll}-0.28974 & 0.513887\end{array}$

$-0.26440 .467714$

$-1.293492 .291344$

$-1.375412 .429462$

$0.730377 \quad 1.291998$

$-0.5154 \quad 0.909383$

$-0.354860 .628249$

$0.679267 \quad 1.196986$

$-0.39832$

0.705772

$-0.602131 .063152$

$-0.3867$

0.686402

0.4461210 .792379

0.3039190 .541848

0.261952

0.467621

$-0.44347$

0.791204

$-0.36373$

0.64789

0.4111870 .734118

$-0.681991 .216144$

$-0.58536$

$-0.58413$

$0.558303 \quad 0.766166$

$-0.58274$

0.55913

0.767012

$-0.58297$

0.560069

0.767431

$-0.58284$

0.559913

0.767431

$-0.58239$

0.560002

0.767431

$-0.58188$

0.560302

0.767445

0.581785

$-0.58143$

$-0.58025$

$-0.57984$

0.57836

$-0.57604$

$-0.576$

$-0.57522$

$-0.57515$

0.574246

$-0.57261$

$-0.57272$

0.572676

$-0.57318$

$-0.57114$

0.569738

$-0.5696$

$-0.56554$

0.560648

0.767445

0.560712

0.767445

0.560949

0.767482

0.561744

0.76828

0.562026

0.768376

0.563021

0.769448

0.564585

0.771045

0.564614

0.771045

0.5651440 .771256

0.5651920 .771256

0.5658010 .771798

0.566908

0.772149

0.566834

0.772149

0.566864

0.772149

0.566524

0.772149

0.567905

0.773218

0.568855

0.774057

0.5689470 .774057

$-0.56548$

0.57171

0.774232

0.566787

0.571749

0.774232

$-0.56608$

0.570859

0.774232

0.567065

0.774232

$-0.56411$

0.774232

$-0.56378$

0.572682

0.774232

$-0.5658$

0.572906

0.774232

$-0.56381$

0.571529

0.774232

$-0.5653$

0.57288

0.774232

$-0.56451$

0.571867

0.774232

$-0.56614$

0.572406

0.774232

0.565308

0.571301

0.774232

$-0.56675$

0.571864

0.774232

$-0.56483$

0.570881

0.774232

0.567481

0.774232

$-0.56438$

0.570387

0.774232

$-0.56636$

0.572499 
Kyrpidia spormannii

Streptococcus pantholopis

Mycobacteroides abscessus

Staphylococcus pettenkoferi

Legionella oakridgensis

Virgibacillus necropolis

Thermoanaerobacter wiegelii

Glutamicibacter arilaitensis

Glaesserella sp. 15-184

Haloarculaceae archaeon HArcel1

Spiribacter salinus

Janthinobacterium sp. B9-8

Acidiphilium cryptum

Corynebacterium diphtheriae

Candidatus Hoaglandella endobia

Agromyces flavus

Kocuria turfanensis

secondary endosymbiont of Ctenarytaina

Bifidobacterium indicum

Propionibacterium phage PHL067M10

Ruegeria sp. TM1040

Planococcus halocryophilus

Prosthecochloris aestuarii

Synechococcus sp. JA-2-3B'a(2-13)

Weissella ceti

Candidatus Thioglobus autotrophicus

Vibrio fluvialis

Sphingobium indicum

Aerococcus urinaeequi

Streptococcus pasteurianus

Chryseobacterium sp. G0162

Cellulosilyticum lentocellum

Betapapillomavirus 5

Aeromonas sp. ASNIH7

Nostoc sp. 'Lobaria pulmonaria (5183) cy:

Selenomonas sputigena

Butyrivibrio proteoclasticus

Selenomonas sp. oral taxon 478

Pseudoalteromonas luteoviolacea

Virgibacillus phasianinus

Acholeplasma laidlawii

Virgibacillus halodenitrificans

Chryseobacterium sp. G0186

Citrobacter farmeri

Ammonifex degensii

Helicobacter cetorum

Zunongwangia profunda

Lactococcus virus bIL170

Histophilus somni

Vibrio casei
1.822326

6.242739

226.0084

2475.621

9.13599

9.03172

3.612508

134.0269

8.676938

2.995442

12.45975

21.78864

46.18025

2730.728

0.657266

110.1615

736.9608

0.710921

1.225432

17.95491

18.4426

2.931909

1.31953

10.37328

0.977644

5.574127

16.95097

29.05985

54.69368

9.484286

7.952161

14.96758

12.51404

2.804884

11.26939

81.03896

12.85637

48.21641

10.69614

3.834113

1.492889

10.28294

12.92949

8.429243

5.935526

1.112524

11.89488

5.017944

10.25159

31.50329
$-1.38928 \quad 2.478887$

$-0.68404$

1.219398

0.381437

0.684227

208533

1.267

0.70586

523892

404836

$-0.21724$

0.392795

$-0.54694$

0.989417

$-0.907311 .652553$

$-0.357740 .65206$

$-0.46211$

0.842985

$-0.53902$

0.987702

$-0.15901$

0.292452

1.593223

2.931214

$-0.22969$

0.42225

$-0.24204$

0.445602

$-1.57797$

2.926992

$-1.29012$

2.394907

$-0.869961 .615745$

$-0.364690 .678914$

$-1.095392 .038794$

$-1.28128$

2.384542

$-0.74125$

1.382024

$-1.4742 \quad 2.752048$

$-0.73677$

1.37923

$-0.42291$

0.791291

$-0.60992$

1.141177

0.426647

0.800227

$-0.73396$

1.376845

$-0.67707$

1.268665

0.495562

0.938543

$-0.9048$

1.710915

$-1.36087$

2.572516

$-0.576 \quad 1.08801$

0.2878310 .544384

$0.547047 \quad 1.029625$

$-0.45476$

0.856049

0.607614

1.147058

$-0.65896$

1.247983

$-1.03676$

1.962752

0.666468

1.262909

0.498958

0.941311

$-0.64803$

1.21791

$-0.64699$

1.22067

$-1.542412 .922411$

0.619339

1.170236

1.128676

2.140688

$-0.43674$

0.829713

$-0.52472$

0.996901
$-0.56045$

$-0.56097$

$-0.5618$

0.559773

0.557626

$-0.55711$

0.556615

$-0.55305$

$-0.55279$

$-0.54904$

$-0.54864$

$-0.54819$

$-0.54573$

$-0.54372$

0.543537

$-0.54396$

$-0.54318$

$-0.53911$

$-0.5387$

$-0.53843$

$-0.53717$

$-0.53727$

$-0.53733$

$-0.53635$

$-0.53567$

$-0.53419$

$-0.53446$

$-0.53447$

0.533157

$-0.53308$

$-0.53369$

0.528013

$-0.52884$

$-0.529$

$-0.52941$

0.528727

0.531307

$-0.53123$

0.529715

$-0.52802$

$-0.52822$

0.527724

0.530067

$-0.53209$

$-0.53003$

$-0.52779$

0.529242

0.527249

$-0.52637$

$-0.52635$
$0.575175 \quad 0.774443$

0.574820 .774443

0.5742560 .774443

$0.575634 \quad 0.774466$

0.57710 .776152

$\begin{array}{ll}0.577451 & 0.776337\end{array}$

0.577790 .776506

0.5802290 .779451

0.580410 .779451

$0.58298 \quad 0.782613$

0.5832550 .782693

0.5835640 .78282

0.5852520 .784795

0.5866330 .785948

0.586760 .785948

$\begin{array}{ll}0.586471 & 0.785948\end{array}$

0.5870060 .785988

0.5898110 .789453

0.5900970 .789503

0.5902830 .789503

0.5911490 .789791

0.5910790 .789791

0.5910410 .789791

0.5917170 .790259

0.5921840 .790593

0.5932130 .791095

0.5930240 .791095

$0.59302 \quad 0.791095$

$0.593925 \quad 0.79125$

0.5939810 .79125

$0.593559 \quad 0.79125$

0.5974910 .791554

0.5969150 .791554

$0.596803 \quad 0.791554$

$0.596524 \quad 0.791554$

0.5969950 .791554

$0.595206 \quad 0.791554$

0.5952580 .791554

$0.59631 \quad 0.791554$

$\begin{array}{lll}0.597487 & 0.791554\end{array}$

$0.597347 \quad 0.791554$

0.5976910 .791554

0.5960660 .791554

0.5946660 .791554

0.5960910 .791554

$0.597647 \quad 0.791554$

$\begin{array}{ll}0.596637 & 0.791554\end{array}$

$0.59802 \quad 0.791702$

0.5986320 .791955

0.5986460 .791955 
Deinococcus swuensis

Streptomyces hundungensis

Calothrix sp. NIES-4101

Erwinia tasmaniensis

Lonsdalea britannica

Sphingomonas sp. FARSPH

Acholeplasma oculi

Serratia sp. MYb239

Gallibacterium anatis

Hymenobacter sp. PAMC 26628

Lactococcus raffinolactis

Streptomyces sp. WAC00288

Maribacter sp. T28

Campylobacter insulaenigrae

Chloroherpeton thalassium

Chryseobacterium sp. $\mathrm{H} 6466$

[Clostridium] bolteae

Acinetobacter sp. ACNIH2

Psychrobacter cryohalolentis

Rhizobium sp. IRBG74

Chryseobacterium lactis

Chryseobacterium taklimakanense

Halobacteroides halobius

Trichormus variabilis

Chlorobium limicola

Chlorobium phaeovibrioides

Streptococcus salivarius

Kangiella sediminilitoris

Clostridiales bacterium 70B-A

Streptomyces formicae

Nocardioides dokdonensis

Bacillus licheniformis

Chryseobacterium sp. F5649

Ornithobacterium rhinotracheale

Nocardia cyriacigeorgica

Stenotrophomonas rhizophila

Desulfovibrio salexigens

[Clostridium] sphenoides

Propionibacterium virus PHL171M01

Thermoplasmatales archaeon BRNA1

Providencia stuartii

Bacteroides cellulosilyticus

Vibrio sp. 2521-89

[Clostridium] saccharolyticum

Mageeibacillus indolicus

Psychrobacter sp. P11F6

Oleispira antarctica

Bacillus lentus

Carnobacterium divergens

Corynebacterium pseudotuberculosis
62.11113

90.85365

$\begin{array}{ll}-0.23141 & 0.441927\end{array}$

$-0.20997$

0.403336

8.190178

0.630093

1.209921

14.47682

0.582587

1.119436

5.597545

$-0.70968$

1.3606

230.0515

$-0.29732$

0.569086

1.126446

$-1.2691$

2.435512

0.596184

6.904158

89.02317

1.530435

2.932022

0.649608

1.24516

74.01535

$-0.27611$

0.529785

63.6499

$-0.51267$

0.98741

$-0.32363$

0.624554

10.44759

0.655404

1.266002

3.515547

1.057015

2.044525

2.819436

0.790168

1.528307

273.1566

0.357759

0.697924

47.74175

$-0.48027$

0.933788

48.783

54.97104

$-0.43082$

0.838276

82.00514

$-0.57595$

1.123515

21.22421

0.546799

1.064834

0.410628

0.799275

82.40274

$-0.33734$

0.655394

7.687104

2.053944

8.259423

0.726339

1.416517

$-1.38318$

2.681112

$-0.544541 .059762$

2.077012

1.0547442 .056659

1509.663

0.333457

1.208462

$-1.13724$

0.648999

7.667574

$-0.65843$

2.208338

94.23941

$-0.17829$

1.287569

246.6735

$-0.21135$

0.348393

1.420892

$-1.31326$

0.413175

251.9203

0.358947

2.573281

46.77361

$-0.56316$

0.704808

309.8859

$-0.17121$

1.106318

252.5241

$-0.25544$

0.336504

4.534295

$-0.63525$

0.502463

19.13242

11.88012

$-0.50646$

1.250068

0.997499

0.662619

1.77373

9.198884

2.927519

181.5827

2.017044

31.30099

34.28434

98.2579

7.505624

10.86574

81.83107

$-1.48105$

1.133368

$-0.57157$

0.783105

2.691102

$-0.526351 .050072$

$-0.4958$

0.988453

0.409028

0.816164

$-0.68794$

1.373018

$-0.554221 .107239$

$-0.333850 .667413$

315.54

$-0.52364$

$-0.52059$

0.600526

0.794153

0.520772

0.602652

0.794513

0.520429

0.602526

0.794513

$-0.5216$

0.602764

0.794513

$-0.52245$

0.601951

0.794513

$-0.52108$

0.60136

0.794513

0.521973

0.602309

0.794513

0.521706

$-0.52118$

0.601689

0.794513

$-0.51921$

0.601875

0.794513

$-0.51818$

0.602242

0.794513

0.517696

0.516998

0.517022

0.512605

$-0.51433$

$-0.51394$

$-0.51263$

0.513506

0.513751

0.603616

0.795348

0.604334

0.796005

0.604671

0.796161

0.605158

0.796226

0.605141

0.796226

$-0.51472$

0.608228

0.79652

0.607023

0.79652

0.607297

0.79652

0.608209

0.79652

0.607598

0.79652

0.607426

0.79652

0.512764

0.60675

0.79652

$-0.5159$

0.608116

0.79652

$-0.51383$

0.605926

0.79652

0.512843

0.607373

0.79652

0.513801

0.608061

0.79652

$-0.51498$

0.607391

0.79652

$-0.51137$

0.606569

0.79652

$-0.51175$

0.609089

0.796787

$-0.51152$

0.608827

0.796787

$-0.51035$

0.60899

0.796787

0.509283

0.60981

0.797443

$-0.50904$

0.610554

0.798001

$-0.50879$

0.610722 
Flavobacterium sp. MEBiC07310

Halocynthiibacter arcticus

Spirosoma pollinicola

Thermosipho melanesiensis

Stenotrophomonas sp. WZN-1

Nocardia sp. Y48

Thermodesulfobium acidiphilum

Psychromicrobium lacuslunae

Pseudomonas mandelii

[Haemophilus] ducreyi

Candidatus Cyclonatronum proteinivorun

Aerococcus sanguinicola

Pseudoalteromonas donghaensis

Natronomonas pharaonis

Thermosulfidibacter takaii

Novosphingobium sp. THN1

Planococcus plakortidis

Plantactinospora sp. KBS50

Bacillus kochii

Dokdonia sp. PRO95

Erythrobacter sp. KY5

Leptospirillum ferriphilum

cyanobacterium endosymbiont of Rhopal

Sulfurospirillum cavolei

Corynebacterium sp. ATCC 6931

Paenibacillus yonginensis

Shinella sp. HZN7

Raphidiopsis curvata

Lactococcus virus CB13

Antarctobacter heliothermus

Anaerotignum propionicum

Pontibacter actiniarum

Pseudoxanthomonas suwonensis

Desulfitobacterium dehalogenans

Dehalococcoides mccartyi

Psychrobacter sp. P11G5

Syntrophomonas wolfei

Flavobacterium album

Mycoplasma californicum

Methylobacterium radiotolerans

Micrococcus luteus

Rothia mucilaginosa

Roseovarius mucosus

Moraxella ovis

Gloeocapsa sp. PCC 7428

Methanobrevibacter smithii

Streptomyces sp. M56

Paenibacillus kribbensis

Capnocytophaga sp. oral taxon 878

Moraxella catarrhalis
6.653669

7.094612

38.27018

1.369305

29.8104

158.6193

1.346752

55.0459

79.13098

22.73873

4.7669

6.280024

2.725299

5.895068

1.795986

73.73779

7.587014

87.59407

7.502631

0.638415

16.03805

2.147631

3.970923

1.298506

8568.576

4.750685

273.6014

1.089146

10.57659

25.48678

7.553672

22.72943

207.9272

1.942708

19.26051

35.75393

8.446063

16.85387

1.019237

141.4352

51832.1

3759.909

6.695105

3.157621

4.531762

22.83093

28.46748

7.966506

23.58313

31.67149
$-0.720551 .442635$

0.509057

1.020023

0.54533

2.85682

1.106193

0.405688

2.874727

$-1.42779$

$-0.2989$

0.60277

0.355142

0.716905

$-0.3222$

0.651967

$-0.67094$

1.357642

$-0.515121 .044227$

$-0.94019$

1.909757

$-0.63375$

1.288006

$-0.952521 .933986$

$-0.233750 .475919$

$-0.763241 .554183$

$-0.24007$

0.489691

0.539123

1.099875

$-1.43407$

2.927788

$-0.394940 .807168$

$1.301139 \quad 2.66062$

0.9916132 .028768

$-1.270012 .601514$

$-0.304940 .626011$

0.7440631 .52902

$-0.26706$

1.52902

$-1.3105$

2.698131

$-0.803881 .660663$

$-0.3153 \quad 0.65237$

$-0.559311 .157748$

$-0.327310 .679196$

$-0.20793$

0.432243

$-0.91255$

1.900729

$-0.493381 .030464$

$-0.44504$

0.619959

1.301371

$-0.28347$

0.595088

$-1.30062$

2.731554

$-0.32113$

0.676173

$-0.27679$

0.582967

$-0.27916$

0.588643

$-0.67004$

1.41943

1.329568

2.820618

$-0.68236$

1.44235

0.551183

1.166107

$-0.58021$

1.230553

0.525507

1.11127

$-0.35568$

0.753713

$-0.47063$

0.998947
0.929533

$-0.49947$

0.499064

$-0.49827$

$-0.49602$

$-0.4959$

$-0.49676$

$-0.49667$

$-0.49588$

0.495382

$-0.49419$

$-0.4942$

$-0.49331$

$-0.49231$

$-0.49204$

$-0.49251$

$-0.49115$

$-0.49109$

$-0.49024$

0.490167

$-0.48981$

$-0.4893$

0.489036

0.488776

$-0.48818$

$-0.48711$

0.486627

$-0.48558$

$-0.48571$

$-0.48407$

$-0.48331$

$-0.4831$

$-0.4819$

$-0.48105$

$-0.48011$

$-0.47879$

$-0.47878$

0.476389

$-0.47635$

$-0.47615$

$-0.47492$

$-0.47479$

$-0.47424$

$-0.47205$

0.471375

$-0.47309$

0.472669

$-0.4715$

0.472888

$-0.47191$
$0.617448 \quad 0.801955$

$0.617734 \quad 0.80204$

0.6182960 .802483

0.6198780 .803239

0.6199650 .803239

0.6193560 .803239

0.6194210 .803239

0.6199820 .803239

0.620330 .803404

0.6211710 .80392

0.6211680 .80392

0.6217960 .804443

0.6225010 .804746

0.6226930 .804746

0.6223550 .804746

0.623320 .805042

0.6233650 .805042

$0.623964 \quad 0.80531$

$0.624015 \quad 0.80531$

0.6242660 .805347

0.6246320 .805438

0.6248160 .805438

0.6250 .805438

$0.62542 \quad 0.805694$

0.626180 .806386

0.6265230 .806543

0.6272680 .806931

0.6271740 .806931

0.6283370 .808021

0.6288760 .80833

$0.629022 \quad 0.80833$

0.6298760 .809141

0.6304820 .809634

0.6311510 .810207

$0.632087 \quad 0.81085$

0.6320970 .81085

0.6337970 .812393

0.6338260 .812393

0.633970 .812393

0.6348420 .813057

0.6349350 .813057

0.6353290 .813275

0.6368940 .813291

0.6373730 .813291

0.636150 .813291

0.6364490 .813291

0.6372830 .813291

0.6362930 .813291

$0.636993 \quad 0.813291$

$\begin{array}{lll}-0.47113 & 0.637547 & 0.813291\end{array}$ 
Oceanobacillus iheyensis

23.34056

Candidatus Methanomassiliicoccus intest 1.200894

Acinetobacter sp. WCHAc010005

Aliivibrio fischeri

Lactobacillus bombi

Thermanaeromonas toyohensis

Ornithinimicrobium sp. AMA3305

Pseudoalteromonas rubra

Lactobacillus oligofermentans

Kocuria sp. BT304

Polynucleobacter wuianus

Enterobacteriaceae bacterium ENNIH1

Aneurinibacillus soli

Prevotella intermedia

Paenibacillus sp. FSL P4-0081

Propionibacterium virus PHL199M00

Peptostreptococcaceae bacterium oral ta

Pseudomonas lurida

Mogibacterium pumilum

Dermatophilus congolensis

Thermoproteus uzoniensis

Listeria ivanovii

Desulfotomaculum ferrireducens

Actinosynnema mirum

Azospirillum sp. M2T2B2

Myroides odoratus

Geitlerinema sp. PCC 7407

Campylobacter fetus

Sphingorhabdus sp. Alg231-15

Lactobacillus coryniformis

Glaciecola sp. THG-3.7

Myroides odoratimimus

Ochrobactrum anthropi

Enterobacter cancerogenus

Propionibacterium virus PHL132N00

Flavobacterium psychrophilum

Virgibacillus sp. 6R

Lactobacillus fuchuensis

Acidipropionibacterium acidipropionici

Aureitalea sp. RR4-38

Moorea producens

Stenotrophomonas maltophilia

Clostridium cochlearium

Marinomonas primoryensis

Methanoculleus bourgensis

Acholeplasma palmae

Xanthomonas vasicola

Staphylococcus warneri

Paenisporosarcina sp. K2R23-3

Nonlabens dokdonensis
10.05454

12.41464

3.798509

1.499828

201.8128

0.990567

3.522882

1491.664

1.184696

2.454953

2.472364

861.5035

16.52379

4.288633

895.5748

28.83756

65.40483

63.49781

5.390044

14.3437

1.696962

32.76583

81.23121

13.35885

31.299

13.79167

5.091144

3.876434

5.117115

3.013234

133.2418

31.26337

42.83777

17.37316

11.6115

4.460496

202.3756

2.907435

8.931183

1932.217

13.63619

4.04344

1.341292

9.308053

86.14042

2393.172

4.603728

1.221663 $\begin{array}{lll}-0.41718 & 0.883042\end{array}$

1.018595

2.162211

$-0.54591$

1.159841

0.493392

1.051542

$-0.6739$

1.436267

$-1.09297$

2.328059

$-0.177$

0.378665

$-1.365452 .923281$

$-0.706271 .514038$

$-0.27380 .590519$

$1.213944 \quad 2.615294$

$-1.02976$

2.229316

$-0.87736$

1.891001

$-0.21086$

0.456099

$-0.5006$

1.080822

$-0.92086$

1.991579

0.258033

0.558671

$-0.57111$

1.235252

0.330515

0.713713

$-0.23767$

0.514581

0.758625

1.655034

$-0.407$

0.888144

$\begin{array}{ll}-1.05127 & 2.300077\end{array}$

$-0.97924$

2.145642

$-0.295230 .647418$

0.4856481 .065212

$-0.320570 .703962$

0.53518

1.176672

$-0.57669$

1.269546

$-0.65431 \quad 1.4458$

$-0.611261 .351786$

$1.273259 \quad 2.814087$

$-0.31016$

0.685458

$-0.32457$

0.718514

0.663517

1.469607

0.339216

0.752053

$0.469778 \quad 1.043746$

$-0.851091 .892405$

$-0.18083$

0.402011

$-0.73651 .638824$

0.4406760 .983403

$-0.24103$

0.539477

0.468813

1.049904

0.61365

1.379738

1.2606752 .835163

$0.510562 \quad 1.147019$

$-0.36072$

0.812668

$-0.192170 .434651$

$-0.597551 .357931$

$-1.273252 .916629$

$-0.47244$

0.636615

0.813291

0.471089

0.637577

0.813291

$-0.47067$

0.637874

0.813384

0.469208

0.638921

0.813867

$-0.4692$

0.638924

0.813867

$-0.46948$

0.638727

0.813867

$-0.46742$

0.640196

0.815203

$-0.4671$

0.640431

0.815217

$-0.46648$

0.640869

0.815489

$-0.46366$

0.642892

0.81656

0.464171

0.642525

0.81656

$-0.46192$

0.644142

0.81656

$-0.46397$

0.642671

0.81656

$-0.46231$

0.643862

0.81656

$-0.46317$

0.643244

0.81656

$-0.46238$

0.64381

0.81656

0.461869

0.644175

0.81656

$-0.46234$

0.643837

0.81656

0.463092

0.643298

0.81656

$-0.46186$

0.64418

0.81656

0.458374

$-0.45826$

0.646684

0.819266

$-0.45706$

0.646765

0.819266

$-0.45639$

0.64763

0.820076

$-0.45601$

0.648113

0.820258

0.455917

0.648386

0.820258

$-0.45537$

0.64845

0.820258

0.454825

0.64884

0.820466

$-0.45425$

0.649235

0.82068

$-0.45256$

0.649647

0.820916

$-0.45219$

0.650865

0.821653

0.452459

0.651134

0.821653

$-0.45248$

0.650938

0.821653

$-0.45173$

0.650922

0.821653

0.451493

0.651463

0.821714

0.451054

0.651634

0.821714

0.450089

0.651951

0.821829

$-0.44974$

0.652646

0.822166

$-0.44982$

0.652897 
Bacillus horikoshii

Pseudomonas putida

Alcaligenes aquatilis

Anabaena cylindrica

Pseudomonas sp. LG1D9

Streptomyces bacillaris

Raoultella ornithinolytica

Streptococcus phage EJ-1

Caldicellulosiruptor owensensis

Vitreoscilla sp. C1

Listeria seeligeri

Acinetobacter venetianus

Staphylococcus nepalensis

secondary endosymbiont of Trabutina me

Marinomonas posidonica

Pseudomonas sp. VLB120

Acholeplasma brassicae

Bacillus beveridgei

Methylobacterium sp. AMS5

Clostridium sp. SY8519

Buttiauxella sp. 3AFRM03

Pectobacterium wasabiae

Rhodococcus jostii

Candidatus Pantoea carbekii

Anaerococcus mediterraneensis

Legionella sainthelensi

Siansivirga zeaxanthinifaciens

Oceanisphaera avium

Nodularia spumigena

Nonlabens sp. MB-3u-79

Croceibacter atlanticus

Pelobacter propionicus

Salegentibacter sp. T436

Pseudomonas sp. 02C 26

Geobacillus sp. WCH70

Pseudoalteromonas espejiana

Tepidanaerobacter acetatoxydans

Sporosarcina ureae

Candidatus Thioglobus singularis

Brevefilum fermentans

Staphylococcus phage 23MRA

Rhizorhabdus dicambivorans

Chryseobacterium nakagawai

Acinetobacter sp. WCHAc010034

Marivirga tractuosa

Leptolyngbya sp. PCC 7376

Acinetobacter radioresistens

Staphylococcus phage StB20-like

Campylobacter coli

Corynebacterium aurimucosum

7.035813

1796.852

0.507452

1.163547

0.436125

0.662746

0.828576

30.29324

$-0.23966$

0.546346

$-0.43866$

0.660908

0.828576

8.771001

$-0.35556$

0.813517

$-0.43706$

0.662065

0.828576

37.57962

.649584

1.486121

0.437101

0.662038

0.828576

52.03756

.547475

$-0.4368$

0.662259

0.828576

119.053

$-0.41366$

0.94796

$-0.43637$

0.66257

0.828576

1.202745

$\begin{array}{ll}-0.26741 & 0.613638\end{array}$

$-0.43578$

0.662997

0.828576

2.307464

2.64796

$-0.43604$

0.662809

0.828576

19.86299

$-0.97177 \quad 2.214634$

$-0.43879$

0.660811

0.828576

1.583479

$-0.43513$

0.994148

$-0.43769$

0.661609

0.828576

264.8922

$-0.92199$

2.11535

$-0.43586$

0.662942

0.828576

200.5516

$-0.25255$

0.580349

$-0.43518$

0.663435

0.828838

1.985765

0.587768

$-0.43477$

0.66373

0.828923

5.400253

1.179573

2.716892

0.434163

0.66417

0.829187

$\begin{array}{lll}0.562255 & -1.26462 & 2.928832\end{array}$

0.432378

0.665467

0.830521

8.45122

1.154085

$-0.43178$

0.665899

0.830683

2.942051

0.498069

0.431571

0.666053

0.830683

55.09626

$-0.60999$

1.414815

$-0.43115$

0.666362

0.830783

13.04648

.2513

$-0.43044$

0.666879

0.831142

12.09545

$-0.43241 .013602$

$-0.4266$

0.669672

0.832627

4.836649

$-0.51278 \quad 1.200016$

$-0.42731$

0.669153

0.832627

92.09618

$-0.623041 .459866$

$-0.42678$

0.669542

0.832627

0.915653

$-0.19273$

0.451515

$-0.42686$

0.669482

0.832627

907.6782

$-0.42745$

0.669052

0.832627

3.934557

0.187063

0.438484

0.426614

0.669661

0.832627

4.632705

$0.868652 \quad 2.029334$

0.428048

0.668616

0.832627

2.301972

$-0.613161 .446065$

$-0.42402$

0.671551

0.834393

8.221517

$-0.812281 .915548$

$-0.42405$

0.671533

0.834393

3.632723

$-0.586081 .386823$

$-0.42261$

0.672581

0.835102

2.658903

$\begin{array}{ll}-0.738 & 1.746024\end{array}$

$-0.42267$

0.672533

0.835102

36.60889

$-0.652031 .544036$

$-0.42229$

0.672815

0.835108

8.551377

$-0.22672$

0.537585

$-0.42174$

0.673218

0.835323

105.8297

1.31738

$-0.4208$

0.673901

0.835675

6.4422

0.607403

$-0.42072$

0.673961

0.835675

0.554294

$-0.634351 .511351$

$-0.41973$

0.674685

0.836003

5.625683

$-1.229852 .929062$

$-0.41988$

0.674574

0.836003

22.14516

$-0.50317$

1.202737

$-0.41835$

0.675689

0.836393

19.61974

0.33139

0.791843

$-0.4185$

0.67558

0.836393

1.603207

0.947605

0.41863

0.675486

0.836393

40.2809

0.9617092 .300675

0.418012

0.675939

0.836417

123.3177

1.118856

2.679201

0.417608

0.676234

0.836419

18.03935

$-0.24077$

0.576863

$-0.41738$

0.6764

0.836419

30.67336

1.066556

$-0.41562$

0.677691

0.837445

9.940672

$-0.2925$

0.703653

$-0.41569$

0.677638

0.837445

3.073436

0.403531

0.973227

0.414632 
Candidatus Desulfofervidus auxilii

Flavobacterium branchiophilum

Lactococcus phage P680

Echinicola vietnamensis

Streptococcus cristatus

Brachybacterium sp. VM2412

Tenericutes bacterium MZ-XQ

Micromonospora sp. B006

Acetobacter pasteurianus

Listeria monocytogenes

Gramella forsetii

Nitrosococcus watsonii

Rhodococcus opacus

Proteus vulgaris

Staphylococcus epidermidis

Psychrobacter sp. YP14

Betapapillomavirus 4

Aequorivita sublithincola

Streptomyces koyangensis

Pedobacter ginsengisoli

Prevotella melaninogenica

Sphingopyxis granuli

Taylorella equigenitalis

Bordetella parapertussis

Enterococcus cecorum

Mordavella sp. Marseille-P3756

Nostoc sp. NIES-4103

Draconibacterium orientale

Psychromonas ingrahamii

Thermoanaerobacterium sp. RBIITD

Methanobacterium paludis

Acetomicrobium mobile

Candidatus Saccharibacteria bacterium YI

Marivivens sp. JLT3646

Trichormus azollae

Microbacterium sp. 1.5R

Lelliottia amnigena

Vibrio owensii

Paenibacillus sp. FSL H7-0737

Acinetobacter baumannii

Staphylococcus phage IME-SA4

Desulfovibrio alaskensis

Shewanella sediminis

Auricoccus indicus

Fusobacterium mortiferum

Leclercia sp. LSNIH3

Dehalobacterium formicoaceticum

Colwellia beringensis

Prevotella sp. oral taxon 299

Propionibacterium acidifaciens
2387595

14.38768

12.48882

8.576001

800.5137

277.2307

1.465948

56.93498

6.263376

72.50805

3.130071

0.533024

297.2736

19.5825

69970.97

10.40953

5.435792

4.040952

53.5773

26.07781

951.2611

119.0801

2.323634

3.81815

12.45314

35.76909

25.5645

10.3725

9.734738

7.850871

0.662725

1.229395

0.993982

4.715202

5.588451

41.06352

63.09362

1.784425

0.665872

747.447

27.51637

13.36393

5.59329

42.39638

12.80514

82.56483

8.429786

2.032971

113.1274

267.7228
$-1.00327$

2.427122

0.366452

0.887012

0.73851

1.800732

$-0.4268$

1.039821

$-0.26853$

0.654927

$-0.19295$

0.471825

$-0.95193$

2.327272

$-0.21776$

0.532471

$-0.61766$

1.516003

0.193545

0.475033

$-0.62826$

1.544367

$-1.19007$

2.929332

$-0.15503$

0.382505

0.388644

0.960321

0.208981

0.517213

0.636004

1.583414

$-0.68381$

1.706102

0.701405

1.754581

$-0.22596$

0.566234

$-0.25791$

0.647733

$-0.18361$

0.462534

$-0.31185$

0.789682

0.889749

2.249631

$-0.98665$

2.50398

$-0.37797$

0.955352

$-0.46562$

1.180176

0.3489540 .885276

0.3965921 .004192

$-0.410141 .039845$

0.4268371 .086098

$\begin{array}{ll}1.14661 & 2.928878\end{array}$

$\begin{array}{ll}-0.94392 & 2.406708\end{array}$

$-1.07002$

2.724467

$-0.56469$

1.443424

0.6492121 .659807

$-0.351920 .894955$

$-0.257440 .657942$

$-1.07715 \quad 2.747139$

1.1385812 .928688

0.1957270 .501684

$-0.355320 .915521$

$-0.462491 .193693$

$-0.602591 .545995$

0.3117310 .803976

0.3201270 .825205

$-0.47124 \quad 1.209887$

$\begin{array}{ll}-0.47457 & 1.224407\end{array}$

$-0.810782 .090026$

$-0.219820 .566852$

$-0.204870 .530063$

$-0.41336$

0.413131

0.679343

0.67951

0.837635

$-0.41012$

0.681719

0.837635

$-0.41046$

0.681469

0.839382

$-0.41001$

0.681796

0.839382

$-0.40895$

0.682579

0.839382

$-0.40903$

0.682517

0.839494

$-0.40895$

0.682573

0.839494

$-0.40743$

0.683696

0.839494

0.407436

0.683688

0.840299

$-0.40681$

0.684149

0.840299

$-0.40626$

0.684551

0.840573

$-0.40531$

0.404703

0.685251

0.840783

0.404052

0.685696

0.841358

0.401666

0.686175

0.84162

0.68793

0.841924

$-0.4008$

0.688567

0.843792

0.399756

$-0.39905$

0.689336

0.844289

$-0.39818$

0.689855

0.844947

$-0.39696$

0.6905

0.845299

$-0.3949$

0.691399

0.845804

0.395509

0.692914

0.84662

$-0.39403$

0.692467

0.846981

$-0.39563$

0.693556

0.846981

$-0.39453$

0.692377

0.846981

0.693188

0.846981

0.394175

0.693452

0.846981

0.394936

0.69289

0.846981

$-0.39442$

0.69327

0.846981

0.393001

0.694319

0.846981

0.391485

0.695439

0.847036

$-0.3922$

0.694909

0.847036

$-0.39274$

0.694508

0.847036

$-0.39122$

0.695635

0.847036

0.391137

0.695696

0.847036

$-0.39323$

0.694151

0.847036

$-0.39128$

0.695588

0.847036

$-0.3921$

0.694984

0.847036 
Helicobacter apodemus

Desulfovibrio piger

Staphylococcus virus CNPH82

Microbacterium aurum

Flavobacterium magnum

Erythrobacter seohaensis

Bifidobacterium bifidum

Natranaerobius thermophilus

Corynebacterium phocae

Octadecabacter temperatus

Bifidobacterium breve

Listeria welshimeri

Propionibacterium virus Stormborn

Bacillus vallismortis

Peptoniphilus sp. ING2-D1G

Paenibacillus sp. FSL R7-0273

Staphylococcus equorum

Staphylococcus phage CNPx

Anoxybacillus amylolyticus

Arthrobacter sp. PGP41

Betapapillomavirus 6

Pseudomonas sp. StFLB209

Planococcus maritimus

Capnocytophaga canimorsus

Actinobacillus succinogenes

Vibrio crassostreae

Candidatus Nitrosoglobus terrae

Polaribacter sp. MED152

Cohnella sp. 18JY8-7

Cyanothece sp. PCC 7425

Desulfotomaculum ruminis

Flavobacteriaceae bacterium MAR_2010_

Syntrophobotulus glycolicus

Mycobacterium intracellulare

Winogradskyella sp. PG-2

Mycoplasma hyosynoviae

Streptococcus thermophilus

Serratia liquefaciens

Bifidobacterium coryneforme

Microbacterium sp. ABRD_28

Mucilaginibacter sp. BJC16-A31

Brevibacillus formosus

Sphingobacterium sp. ML3W

Gordonia sp. KTR9

Yersinia enterocolitica

Pseudoclostridium thermosuccinogenes

Blautia sp. N6H1-15

Nonlabens sp. Hel1_33_55

Lactobacillus amylophilus

Tenacibaculum sp. SZ-18
2.799473

20.92251

43.03032

709.44

4.517698

20.61385

127.2458

6.097417

1445.803

1.881912

133.9996

2.14042

11.89137

1.46372

109.1973

8.103476

128.1794

56.69054

5.134641

98.4063

4.070401

71.68849

1.866382

16.86138

4.567807

3.510364

3.958829

12.43537

22.79346

5.52233

2.557323

3.078502

1.533232

145.1481

7.551654

0.258975

1137.745

53.95808

3.227887

143.1606

27.83651

5.708581

37.3723

461.5452

53.37497

6.805193

56.16492

3.480455

1.80942

13.45799
$-0.68606$

$-0.23294$

1.775312

0.603677

0.450351

1.16988

0.581882

1.335773

0.51298

0.580496

$-0.21289$

0.55579

0.560182

1.461716

0.09162

0.238768

$-0.73633$

1.925837

0.169788

0.444471

0.78537

2.06289

$-0.63485$

1.666206

$-0.97391$

2.563381

0.21751

0.573121

$-0.47198$

1.243117

$-0.21706$

0.573986

0.939611

2.483417

$-0.501$

1.326909

$-0.13028$

0.345333

$-0.7675$

2.033819

$\begin{array}{lll}-0.22247 & 0.589034\end{array}$

0.7872592 .091779

0.310874

0.587954

0.819453

0.573307

$-0.44149$

$-0.23208$

$-0.51213$

$-0.61077$

$0.65001 \quad 1.753237$

$-0.938532 .533252$

$-0.232470 .630884$

0.4362281 .183245

1.0846882 .940784

$-0.221350 .60221$

0.2573540 .697834

$-0.80622 .185865$

$\begin{array}{ll}-0.1761 & 0.478608\end{array}$

$-0.25988$

0.706744

$-0.49708$

1.351854

$-0.28845$

0.785977

$-0.20427$

0.557433

0.192746

0.526347

0.503231

1.37801

0.315815

0.8666

$-0.5147 \quad 1.416205$

$-0.766782 .114866$

0.3140110 .86895

$-0.38644$

0.699167

0.847574

$-0.38587$

0.699595

0.84781

0.384953

0.700272

0.848348

0.38441

0.700678

0.84838

$-0.38403$

0.700956

0.84838

0.383045

0.701686

0.84838

$-0.38303$

0.701697

0.84838

0.383236

0.701545

0.84838

0.383721

0.701185

0.84838

$-0.38234$

0.702208

0.848715

0.382001

0.702461

0.848739

0.380714

0.703416

0.849328

$-0.38102$

0.703192

0.849328

$-0.37993$

0.703997

0.849554

0.379518

0.704303

0.849554

$-0.37967$

0.704188

0.849554

$-0.37817$

0.705307

0.849881

0.378354

0.705168

0.849881

$-0.37757$

0.705753

0.849881

$-0.37727$

0.705976

0.849881

$-0.37737$

0.7059

0.849881

$-0.37768$

0.705665

0.849881

0.376359

0.70665

0.850085

0.37578

0.70708

0.850085

0.376591

0.706478

0.850085

0.375945

0.706958

0.850085

0.375158

0.707543

0.85036

$-0.3726$

0.709442

0.851799

$-0.37266$

0.709402

0.851799

$-0.37284$

0.709268

0.851799

$-0.37116$

0.710517

0.370748

0.710825

0.852808

$-0.37048$

0.711022

0.852851

$-0.36848$

0.712517

0.852851

0.368671

0.712373

0.85293

0.368843

0.712245

0.85293

$-0.36757$

0.713197

0.85293

0.36879

0.712284

0.85293 
Actinomyces sp. oral taxon 897

Human papillomavirus

Paenibacillus sp. IHBB 10380

Thermoclostridium stercorarium

Ralstonia phage 1 NP-2014

Brachybacterium sp. P6-10-X1

Ochrobactrum pseudogrignonense

Staphylococcus phage StB27

Synechococcus sp. PCC 7502

Propionibacterium virus PAD20

Bdellovibrio exovorus

Streptococcus pyogenes

Geosporobacter ferrireducens

Paracoccus sp. CBA4604

Desulfosporosinus youngiae

Kocuria flava

Mycobacterium sp. KMS

Lactococcus virus SI4

Scytonema sp. HK-05

Leminorella richardii

Acinetobacter wuhouensis

Flavobacterium kingsejongi

Lactococcus phage 340

Propionibacterium virus P91

Hafnia alvei

Celeribacter baekdonensis

Komagataeibacter hansenii

Leptospira alstonii

Adlercreutzia equolifaciens

Enterobacter bugandensis

Riemerella anatipestifer

Pseudarthrobacter equi

[Eubacterium] cellulosolvens

Escherichia fergusonii

Photobacterium damselae

Pseudoxanthomonas spadix

Lactococcus piscium

Lactobacillus salivarius

Propionibacterium virus PAS50

Geobacillus genomosp. 3

Corynebacterium camporealensis

Acinetobacter oleivorans

Sphingomonas sp. JJ-A5

Shewanella piezotolerans

Thermosynechococcus elongatus

Meiothermus silvanus

Corynebacterium mustelae

Psychrobacter urativorans

Lactobacillus agilis

Kocuria rhizophila
225.2534

2.12499

2.272524

4.151249

2.31767

325.3151

29.83758

26.31108

0.936659

16.67764

1.356188

323.1394

10.44396

133.4598

6.661576

634.8743

5.502408

14.29614

19.00205

10.91454

58.84893

8.16623

4.770429

26.18515

15.54678

15.9752

22.79728

0.517818

117.8724

1.799966

18.85493

109.3067

10.2004

1.336828

25.30255

116.5554

87.43901

58.43129

16.76842

6.85436

3742.403

9.393146

53.70034

2.161896

1.829159

34.64527

343.1072

14.30522

1.849668

1687.751 $\begin{array}{lll}-0.14814 & 0.409929\end{array}$

$-0.97259$

2.702413

$-0.63049$

1.756435

$-0.545191 .518001$

0.954655

2.660107

$-0.16604$

0.465204

$-0.32724$

0.920558

0.28699

0.810572

$-0.80057$

1.3999

0.91383

2.594049

0.162686

0.461832

0.316526

0.896382

$-0.198$

0.565386

$-0.34973$

0.998362

$-0.1648$

0.471692

$-0.92704$

2.651648

$-0.50072$

1.434889

$-0.34185$

0.9814

$-0.3085$

0.889819

0.250636

0.733856

$-0.37042$

1.079408

0.745437

2.166274

$-0.50455$

1.479418

$-0.46558 \quad 1.35444$

$\begin{array}{ll}-0.23219 & 0.68019\end{array}$

$-0.25366$

0.68019

1.003826

2.931288

$-0.23051$

0.672837

$-0.94077$

2.746922

$-0.26435$

0.77252

$-0.16306$

0.475409

$-0.38249$

1.121647

$-0.89196$

2.599188

$-0.33213$

0.975807

$-0.19122$

0.562033

0.260574

0.767942

$-0.16716$

0.492554

$-0.52509$

1.548492

$-0.488421 .441133$

0.084052

0.248643

0.382081

1.129561

$-0.19626$

0.581233

$-0.551151 .644837$

$-0.9194$

2.742904

$-0.23742$

0.709104

0.133657

0.398874

$-0.32499$

0.973502

$-0.647671 .938666$

$-0.19642$

$-0.36137$

$-0.3599$

$-0.35896$

0.71782

0.855931

$-0.35915$

0.718924

0.856961

0.358878

0.857027

$-0.35693$

0.719482

0.857027

19686

0.857027

$-0.35547$

0.721145

0.858484

0.354058

0.722234

0.859498

$-0.35368$

0.723295

0.86048

0.352803

0.72358

0.860537

$-0.35228$

0.724236

0.860674

0.352263

0.724628

0.860674

0.353115

0.724641

0.860674

$-0.3502$

0.724002

0.860674

$-0.3503$

0.726187

0.861948

$-0.34938$

0.726114

0.861948

$-0.34961$

0.726807

0.862121

$-0.34896$

0.726631

0.862121

$-0.34833$

0.727119

0.86221

$-0.3467$

0.727596

0.862494

0.341533

0.728813

0.863655

$-0.34317$

0.732703

0.864905

0.34411

0.731472

0.864905

$-0.34104$

0.730763

0.864905

$-0.34374$

.733071

0.864905

$-0.34137$

0.73104

0.864905

$-0.34088$

0.732827

0.864905

0.342452

0.733196

0.864905

$-0.3426$

0.732011

0.864905

$-0.34248$

0.731902

0.864905

$-0.3422$

0.731988

0.864905

$-0.34299$

0.732202

0.864905

$-0.34101$

0.731607

0.864905

$-0.34317$

0.7331

0.864905 
Moraxellaceae bacterium HYN0046

Curtobacterium sp. BH-2-1-1

Molluscum contagiosum virus

Pantoea agglomerans

[Eubacterium] eligens

Formosa sp. Hel3_A1_48

Calothrix sp. 336/3

Vibrio tubiashii

Aeribacillus pallidus

Pantoea sp. At-9b

Brachybacterium ginsengisoli

Sulfurospirillum sp. JPD-1

Polaribacter sp. ALD11

Pseudoalteromonas piratica

Eubacterium callanderi

Bacillus cytotoxicus

Microbacterium sp. str. 'China'

Microbacterium pygmaeum

Tenacibaculum dicentrarchi

Propionibacterium virus PHL070N00

Roseomonas sp. FDAARGOS_362

Propionibacterium virus P105

Psychroflexus torquis

Bacteroides helcogenes

Spiroplasma turonicum

Cyclobacterium marinum

Maribacter sp. HTCC2170

Dictyoglomus turgidum

Mesoplasma entomophilum

Moraxella bovis

Jannaschia sp. CCS1

Kosmotoga olearia

Sulfurospirillum sp. UCHOO1

Pseudoalteromonas phenolica

Planococcus donghaensis

Propionibacterium phage PHL082M00

Agarilytica rhodophyticola

Halomicronema hongdechloris

Lelliottia jeotgali

Basilea psittacipulmonis

Treponema putidum

Corynebacterium genitalium

Pseudomonas fragi

Streptococcus anginosus

Lactobacillus ginsenosidimutans

Propionibacterium virus Kubed

Providencia sneebia

Propionibacterium virus PHL301M00

Labrenzia sp. CP4

Candidatus Kinetoplastibacterium galatii

\begin{tabular}{|c|c|c|c|c|c|}
\hline & & & & & \\
\hline 9624 & -0.1701 & 0.513756 & -0.33109 & 0.740574 & \\
\hline 960.54 & -0.95571 & 2.88545 & -0.33122 & 0.740479 & 0 \\
\hline & -0.16228 & 0.490424 & -0.3309 & .740724 & \\
\hline & -0.26 & 0.807636 & 2942 & .741836 & \\
\hline 4051 & -0.95999 & 2.925005 & -0.3282 & 0.74276 & \\
\hline 0527 & -0.72868 & 2.225064 & -0.32749 & .743298 & \\
\hline & -0.3862 & & -0.3 & .744965 & \\
\hline & 0.455592 & 1.400244 & 0.325366 & 0.744904 & \\
\hline 2989 & -0.25322 & 0.779403 & -0.32489 & .745264 & \\
\hline & -0.16 & 0.524641 & & & \\
\hline & -0.7 & & -0.3 & 86 & \\
\hline & 0.506428 & 1.571994 & 0.32 & 34 & \\
\hline & -0.77569 & 2.413006 & -0.3 & 59 & \\
\hline & -0.4 & 1.526533 & & 97 & \\
\hline & -0.3 & 1.10 & -0 . & & \\
\hline & -0.28348 & 0.8885 & -0.31904 & 0.749 & \\
\hline & -0.16 & & & & \\
\hline & 0.439684 & 1.384 & 0.317609 & & \\
\hline & -0.40918 & 1.2917 & -0.31675 & 0.75 & \\
\hline & -0.1723 & & -0.31592 & & \\
\hline & 0.555653 & 1.759583 & 0.31 & & \\
\hline & 0.491661 & 1.560405 & 0.315085 & & \\
\hline 7245 & -0.32283 & 1.025432 & -0.31483 & 0.752894 & 0 87677 \\
\hline & 0.59 & & & & \\
\hline 4 & -0.2 & 0.9 & & & \\
\hline 63 & -0.38133 & 1.22 & & & \\
\hline & 0.702044 & & & & \\
\hline & & & & & \\
\hline & -0.3 & 1.00 & & & \\
\hline 9472 & -0.22114 & 0.723993 & & 0.760 & \\
\hline & -0.892 & 2.927 & & & \\
\hline & & & & & \\
\hline 531041 & -0.58113 & 1.906857 & & 0.760 & \\
\hline & -0.62245 & 2.051209 & -0.30345 & 0.761545 & \\
\hline & & & & & \\
\hline & & 1.764929 & 0.301967 & 577 & \\
\hline & & & & & \\
\hline & -0.27125 & 0.901638 & -0.30084 & 0.7635 & \\
\hline & -0.440 & & & 34 & \\
\hline & 0.526013 & 1.756972 & 0.299386 & 0.764646 & \\
\hline & & & -0.298 & & \\
\hline & -0.20526 & 0.689686 & -0.297 & 96 & \\
\hline 1063 & -0.17088 & 0.574259 & -0.29757 & 0.766032 & \\
\hline & -0.40806 & 1.375543 & -0.29665 & & \\
\hline 9.444075 & 0.769979 & 2.602923 & 0.295813 & & \\
\hline & & 1.451934 & -0.29511 & & \\
\hline & 0.777817 & 2.648215 & 0.293714 & 0.768977 & 088067 \\
\hline & -0.5809 & & -0.29213 & 0.770191 & \\
\hline & -0.71603 & 2.452561 & -0.29195 & 0.770324 & 0.889 \\
\hline
\end{tabular}


Cyclobacterium amurskyense

Caldicellulosiruptor obsidiansis

Brevibacterium sandarakinum

Candidatus Fukatsuia symbiotica

[Clostridium] ultunense

Lactobacillus reuteri

Bacillus pseudomycoides

Gammapapillomavirus 13

Actinomyces radingae

Lactococcus virus ASCC191

Candidatus Kuenenia stuttgartiensis

Brachybacterium faecium

Halohasta litchfieldiae

Flagellimonas sp. HME9304

Staphylococcus carnosus

Desulfosporosinus acidiphilus

Legionella fallonii

Ruminiclostridium cellulolyticum

Deinococcus peraridilitoris

Photobacterium profundum

Propionibacterium phage BruceLethal

Lactobacillus kullabergensis

Calothrix sp. PCC 7507

Propionibacterium virus Ouroboros

Lactobacillus heilongjiangensis

Renibacterium salmoninarum

Cellulophaga algicola

Micromonospora coxensis

Arenibacter algicola

Paenibacillus durus

Alkalitalea saponilacus

Helicobacter cinaedi

Weissella paramesenteroides

Bacillus mycoides

Candidatus Planktophila lacus

Chryseobacterium sp. G0201

Arcticibacterium luteifluviistationis

Mycobacterium chimaera

Gordonia virus Wizard

Pseudomonas sp. s211(2017)

Scytonema sp. NIES-4073

Rickettsiales bacterium Ac37b

Marinomonas mediterranea

Vibrio splendidus

Chryseobacterium carnis

Sulfurovum sp. NBC37-1

Hydrogenovibrio crunogenus

Staphylococcus phage SAP-2

Novibacillus thermophilus

Microcoleus sp. PCC 7113

11.1948

0.833397

274.8613

0.307203

1.050462

$-0.849072 .923986$

0.292446

0.769946

0.88938

9.078491

$-0.15512$

0.535353

$-0.29038$

0.771524

$-0.28975$

0.772008

0.889631

19.49334

$-0.28873$

0.994506

$-0.29033$

0.771565

0.889631

28.73196

0.249002

0.858622

0.290002

0.771815

0.889631

33.90194

$-0.22581$

0.777128

$-0.29058$

0.771376

0.889631

1.425669

0.147313

0.506447

0.290875

0.771147

368.8671

$-0.84104$

2.916804

$-0.28834$

0.773084

0.889631

9.01762

0.16908

0.585922

$-0.28857$

0.772909

0.889631

1.083298

$-0.40452$

1.403457

$-0.28823$

0.773168

0.889841

416.5101

$-0.63898$

2.212264

$-0.28884$

0.772707

0.889841

4.381328

$-0.14509$

0.505146

$-0.28722$

0.773947

0.889841

1.009486

44.05527

1.455782

$-0.28613$

0.77478

0.889841

1.339012

$-0.28524$

0.775459

0.890455

4.214236

$-0.1794$

0.628265

$-0.28554$

0.775228

0.891132

6.810306

$-0.66733$

2.352718

$-0.28364$

0.776684

0.891349

32.10695

0.501702

1.774616

0.28271

0.777399

0.891349

9.073615

1.231012

0.282535

0.777534

0.892475

1.803165

0.804453

$-0.28131$

0.778471

0.892556

1.803165

$-0.420481 .490313$

$-0.28214$

0.777837

2.694438

9.040958

4.795996

$-0.640132 .274548$

$-0.28143$

0.778381

0.892556

0.809361

$-0.42243$

1.628997

0.282031

0.777919

0.892556

55.76188

10.01987

86.03015

9.783116

0.394316

$-0.28161$

0.778245

0.892556

31.77465

$-0.7967$

405783

0.280495

0.779097

0.892556

$-0.28055$

0.779053

$-0.27976$

0.779661

0.279497

0.779864

$-0.27963$

0.779759

$-0.13361$

0.477796

$-0.31951 .145701$

$-0.27887$

0.780343

0.892711

6.249365

$-0.15654$

0.562137

$-0.27847$

0.780651

0.892711

0.877952

$-0.320391 .152517$

$-0.27799$

0.781017

0.892745

3.062101

0.769373

2.776994

0.277053

0.78174

0.892745

64.52546

0.399824

1.446959

0.27632

0.782302

0.892745

7.947788

$-0.26104$

0.944192

$-0.27647$

0.782187

0.893013

25.50404

$-0.295161 .072666$

$-0.27517$

0.783188

0.893084

6.307467

$-0.25286$

0.925471

$-0.27322$

0.784684

0.893222

147.6103

$-0.35412$

1.293788

$-0.27371$

0.784307

0.893768

1.100937

163908

0.599635

0.273346

0.784587

0.273422

0.784529

0.893849

4.218399

$-0.27417$

0.78395

0.893849

2.706011

0.716541

2.6108

0.274453

0.783737

1.688946

0.75696

2.778805

0.272405

0.785311

$-0.27108$

0.786332

0.894581

3.154757

$-0.39611 \quad 1.46126$

$-0.26951$

0.787536

0.894604

3.193218

21.41156

- 415231.540678

0.270241

0.786975

0.894604

1.750248 
Lactobacillus paracasei

Obesumbacterium proteus

Staphylococcus agnetis

Leptospira mayottensis

Tannerella sp. oral taxon HOT-286

Candidatus Kinetoplastibacterium blastoc

Pseudoalteromonas sp. NC201

Salinigranum rubrum

Corynebacterium simulans

Virgibacillus sp. SK37

Gammapapillomavirus 18

Butyrivibrio hungatei

Pedobacter sp. G11

Rhodococcus sp. NJ-530

Paenibacillus donghaensis

Mucilaginibacter sp. PAMC 26640

Caldanaerobacter subterraneus

Streptococcus sp. A12

Phoenicibacter massiliensis

Turicibacter sp. $\mathrm{H} 121$

Propionibacterium virus PHL179M00

Candidatus Protochlamydia naegleriophil

Sphingopyxis sp. LPB0140

Fischerella sp. NIES-4106

Gottschalkia acidurici

Bacillus filamentosus

Chryseobacterium carnipullorum

Methanosarcina thermophila

Psychromonas sp. CNPT3

Spiroplasma clarkii

Shewanella denitrificans

Candidatus Planktophila sulfonica

Capnocytophaga sputigena

Vibrio diabolicus

Propionibacterium virus Wizzo

Calothrix sp. NIES-2098

Lactococcus phage phi7

Cutibacterium acnes

Selenomonas sp. oral taxon 920

Candidatus Blochmannia floridanus

Propionibacterium virus Pirate

Spiroplasma culicicola

Acinetobacter sp. ADP1

Hungateiclostridium thermocellum

Blochmannia endosymbiont of Camponot

Chryseobacterium piperi

Plantactinospora sp. BB1

Pseudomonas sp. R3-18-08

Brucella sp. 141012304

Francisella sp. FDC440
44.0379

4.8616

33.24832

0.72582

263.3694

2.878667

1.072366

18.49469

10615.67

4.637859

1.398138

13.75336

28.74779

206.8558

30.57358

24.51211

1.567614

144.5661

8.176814

12.06865

10.90758

2.108153

3.987437

6.680703

19.77488

15.67375

37.84222

2.246237

7.610134

1.943258

1.578587

7.319722

143.0951

1.115032

7.384198

5.862124

8.135266

286485.5

76.84713

1.025915

7.281062

2.961405

31.78811

4.610452

0.757556

14.41359

42.74861

1.843586

0.361612

2.048926
$-0.26394$

0.408989

0.983441

1.522189

0.131531

0.493212

$-0.76991$

2.893021

$-0.15167$

0.566254

$0.596455 \quad 2.23685$

$-0.77759$

2.920229

$-0.16012$

0.601324

0.105379

0.395354

0.462657

1.74451

$-0.75635$

2.864845

$-0.33189$

1.261629

$-0.19407$

0.736201

0.244928

0.931203

$-0.17458$

0.671555

$-0.18446$

0.716055

$-0.55051$

2.13916

$-0.13957$

0.542072

$-0.27891$

1.094901

$-0.30747$

1.210395

$-0.44387 \quad 1.746578$

$\begin{array}{ll}-0.48067 & 1.910329\end{array}$

$0.341408 \quad 1.35773$

0.297946

1.183883

0.18347

0.728464

$-0.18633$

0.748459

0.161452

0.647631

0.608103

2.444522

$-0.30971$

1.250117

$0.51901 \quad 2.097725$

$\begin{array}{ll}-0.528 & 2.139019\end{array}$

$-0.28687 \quad 1.176628$

$-0.12850 .52612$

$-0.711552 .919539$

$0.346461 \quad 1.424371$

$0.46077 \quad 1.899682$

$-0.43539$

1.797851

0.126551

0.526615

$-0.124580 .517874$

0.69742 .920836

$0.417148 \quad 1.745266$

0.531424

2.223058

0.213935

0.900925

$0.383011 \quad 1.633051$

$-0.685162 .924909$

0.2230340 .951528

$-0.20343$

0.872543

$-0.63665 \quad 2.736179$

$-0.67462 \quad 2.933345$

0.4818152 .094983
$-0.26839$

0.788403

0.788172

0.789713

0.895756

0.268685

0.266683

0.790142

0.895756

$-0.26613$

$-0.26785$

0.788815

0.895774

0.266649

0.789739

$-0.26628$

0.790026

$-0.26627$

0.790028

0.895774

0.266542

0.789822

0.265207

0.79085

0.895774

$-0.26401$

0.791771

$-0.26306$

0.792502

$-0.2636$

0.263024

0.792086

0.792532

0.895774

$-0.25997$

0.79489

$-0.2576$

0.796715

$-0.25735$

0.796911

$-0.25747$

0.796815

0.895774

$-0.25474$

0.798924

$-0.25402$

0.79948

0.895774

$-0.25414$

0.799389

$-0.25162$

0.801337

0.251455

0.251668

0.801462

0.801298

0.251859

0.80115

0.8034

$-0.24895$

0.249297

0.248761

0.803131

$-0.24774$

0.803545

0.804332

0.247416

0.804587

0.80503

$-0.24684$

$-0.24423$

$-0.24372$

0.807049

0.807447

0.895774

0.243238

0.807821

0.242551

0.808353

$-0.24217$

0.808647

0.24031

0.81009

0.8099

$-0.24055$

0.811286

0.238767

0.811093

0.239051

0.811066

0.237462

0.812299

0.234537

0.814568

$-0.23425$

0.814792

0.234396

0.814678

$-0.23315$

0.815643

0.81601

0.818105

0.818103

0.896297

0.897061

0.897085

0.897085 
Streptococcus lutetiensis

Serinicoccus sp. JLT9

Weissella soli

Algoriphagus machipongonensis

Streptomyces sp. ICC1

Aliivibrio wodanis

Candidatus Rhodoluna planktonica

Blautia hansenii

Cronobacter turicensis

Flavobacteriaceae bacterium

Microbacterium paraoxydans

Butyrivibrio fibrisolvens

Thermocrinis ruber

Pseudomonas rhizosphaerae

Propionibacterium sp. oral taxon 193

Macrococcus sp. IME1552

Pseudomonas sp. SWI44

Desulfosporosinus orientis

Lutibacter sp. LPB0138

Lysinibacillus sphaericus

Pseudomonas rhodesiae

Helicobacter canadensis

Acinetobacter sp. WCHAc010052

Bacillus megaterium

Lactobacillus sanfranciscensis

Nitratifractor salsuginis

Propionibacterium virus PHL116M00

Desulfotomaculum reducens

Propionibacterium virus PHL041M10

Pseudomonas sp. FGI182

Psychrobacter sp. G

Exiguobacterium antarcticum

Leuconostoc virus LN03

Capnocytophaga stomatis

Shewanella halifaxensis

Ruegeria sp. NKC1-1

Kangiella geojedonensis

Catenovulum sp. CCB-QB4

Propionibacterium virus Solid

Propionibacterium virus P1001

Moritella viscosa

Gramella sp. MAR_2010_102

bacterium 2013Arg42i

Lactobacillus allii

Streptomyces sp. ICC4

Halomonas sp. GT

Kocuria palustris

Arthrospira platensis

Bacillus cohnii

Chryseobacterium sp. StRB126
13.99677

185.2868

2.405082

7.116857

630.5358

7.118535

11.95036

77.81062

4.097025

6.614913

470.0421

25.48771

0.695412

62.65575

1704.706

60.05624

18.18137

2.586871

13.95374

32.76446

84.80743

2.403863

13.31724

76.44473

1.098231

10.01978

8.321845

2.776209

13.36337

6.383251

52.66534

4.504519

1.156229

10.07013

6.742622

1.275535

1.857223

1.091619

23.17545

8.183354

6.866874

0.790887

1.030124

2.772486

738.5596

2.441604

1291.18

3.59212

14.26784

16.18551 $\begin{array}{lll}-0.31881 & 1.398007\end{array}$

$-0.09709$

0.469925

0.425862

2.058162

0.299638

1.316309

0

$-0.29805$

66418

$-0.1712$

1.330565

$-0.19748$

0.418519

0.763626

0.884234

0.313188

1.877028

1.412786

0.175241

0.793002

0.220978

0.999657

$-0.64254$

2.92601

0.135747

0.622545

$-0.144510 .66756$

$-0.192450 .887883$

$-0.332351 .533563$

$-0.35073$

1.623273

$-0.26697$

1.238237

0.151916

0.707788

$-0.12843$

0.598699

0.477955

2.241883

$-0.24432$

1.147848

$-0.13075$

0.614824

0.424318

2.001148

$-0.24321$

1.149198

0.3936411 .881618

$-0.339461 .62156$

0.393461

1.875295

$-0.36464$

1.750719

$-0.23301$

1.123484

$0.284238 \quad 1.373728$

$-0.59948 \quad 2.918921$

$-0.244111 .196946$

$-0.290541 .426919$

$-0.483892 .377224$

0.424896

2.088503

0.441199

2.197239

$-0.28652$

1.424342

0.352386

1.754854

0.279679

1.398343

$-0.58392$

2.923946

0.584855

2.920648

$-0.30651 \quad 1.539517$

0.1942880 .976288

$-0.44387$

2.233616

$-0.08944$

0.452367

$-0.32193$

1.626673

0.165401

0.839034

$-0.18035$

0.917903
$-0.22805$

$-0.22798$

0.228322

0.227635

$-0.22652$

$-0.224$

$-0.22419$

$-0.22333$

0.222969

0.221681

0.220984

0.221054

$-0.2196$

0.218051

$-0.21648$

$-0.21675$

$-0.21671$

$-0.21606$

$-0.21561$

0.214635

$-0.21452$

0.213194

$-0.21285$

$-0.21267$

0.212037

$-0.21163$

0.209203

$-0.20934$

0.209813

$-0.20828$

$-0.2074$

0.20691

$-0.20538$

$-0.20395$

$-0.20361$

$-0.20355$

0.203445

0.200797

$-0.20116$

0.200807

0.200007

$-0.1997$

0.200248

$-0.1991$

0.199007

$-0.19872$

$-0.19772$

$-0.1979$

0.197133

$-0.19648$
$0.81961 \quad 0.916672$

$0.819663 \quad 0.916672$

$0.819396 \quad 0.916672$

$0.81993 \quad 0.916689$

$0.820797 \quad 0.917376$

$0.822755 \quad 0.919$

$0.822607 \quad 0.919$

$0.823275 \quad 0.919298$

$0.82356 \quad 0.919334$

$0.824562 \quad 0.920171$

$0.825105 \quad 0.920212$

$0.82505 \quad 0.920212$

$0.826185 \quad 0.921135$

0.8273890 .922194

$0.828615 \quad 0.922713$

$0.828399 \quad 0.922713$

0.8284310 .922713

$0.828937 \quad 0.922789$

$0.829294 \quad 0.922903$

0.8300520 .923285

$0.830144 \quad 0.923285$

0.8311760 .924042

$0.831446 \quad 0.924042$

$0.831587 \quad 0.924042$

$0.832078 \quad 0.924305$

$\begin{array}{lll}0.832394 & 0.924374\end{array}$

0.8342890 .925631

0.8341810 .925631

0.8338140 .925631

$\begin{array}{lll}0.83501 & 0.926148\end{array}$

$0.835696 \quad 0.926627$

$\begin{array}{ll}0.83608 & 0.92677\end{array}$

$0.837277 \quad 0.927815$

$0.838395 \quad 0.928356$

0.8386550 .928356

$0.838704 \quad 0.928356$

$0.838787 \quad 0.928356$

$\begin{array}{lll}0.840857 & 0.929799\end{array}$

$\begin{array}{ll}0.840574 & 0.929799\end{array}$

$0.84085 \quad 0.929799$

$0.841475 \quad 0.929897$

$\begin{array}{ll}0.841713 & 0.929897\end{array}$

$\begin{array}{lll}0.841286 & 0.929897\end{array}$

$\begin{array}{ll}0.842188 & 0.929897\end{array}$

$\begin{array}{lll}0.842257 & 0.929897\end{array}$

$\begin{array}{lll}0.842479 & 0.929897\end{array}$

$0.843264 \quad 0.930198$

0.8431190 .930198

$0.843724 \quad 0.930424$

$0.844236 \quad 0.930706$ 
Anaerococcus prevotii

Flavobacterium gilvum

Pseudomonas tolaasii

Lachnoclostridium phytofermentans

Erysipelotrichaceae bacterium SG0102

Bacillus velezensis

Yersinia aleksiciae

Spirochaeta africana

Prevotella denticola

Chromobacterium violaceum

Methylomonas clara

Solitalea canadensis

Scardovia inopinata

Streptomyces sp. ADI95-16

Dickeya fangzhongdai

Neisseria subflava

Marinobacter sp. BSs20148

Pseudomonas sp. R4-34-07

Pseudomonas extremorientalis

Anabaena sp. WA102

Stenotrophomonas sp. G4

Brachybacterium saurashtrense

Propionibacterium virus P1.1

Methylorubrum zatmanii

Capnocytophaga leadbetteri

Oenococcus oeni

Selenomonas sp. oral taxon 126

Pseudomonas frederiksbergensis

Halobacteriovorax marinus

Tetragenococcus osmophilus

Sulfurospirillum barnesii

Sedimentisphaera salicampi

Propionibacterium phage PHL085N00

Monoglobus pectinilyticus

Lactobacillus kefiranofaciens

Clostridium isatidis

Streptococcus phage SM1

Capnocytophaga gingivalis

Crinalium epipsammum

Hydromonas sp. F02

Pseudomonas moraviensis

Rahnella aquatilis

Lactobacillus hokkaidonensis

Colwellia psychrerythraea

Leptolyngbya boryana

Desulfobacterium autotrophicum

Pseudothermotoga thermarum

Actinobacillus suis

Helicobacter sp. MIT 01-6242

Nostoc linckia

\begin{tabular}{|c|c|c|c|c|c|}
\hline & -0.08824 & & & & \\
\hline .56672 & -0.1646 & 0.840606 & -0.19581 & 0.844757 & 0.930758 \\
\hline 05798 & 0.134682 & 0.691975 & 0.194634 & 0.845679 & 0.931451 \\
\hline .26636 & 0.145948 & 0.752187 & 0.194031 & 0.846152 & 0.93146 \\
\hline .20582 & 0.216717 & 1.121677 & 0.193208 & 0.846796 & 0.93146 \\
\hline .91459 & -0.21169 & 1.096898 & -0.19299 & 0.846968 & 0.93146 \\
\hline 194355 & -0.40334 & 2.085631 & -0.19339 & 0.846652 & 0.93146 \\
\hline .75099 & 0.182361 & 0.941358 & 0.193721 & 0.846395 & 0.93146 \\
\hline 2.5609 & -0.11007 & 0.571543 & -0.19258 & 0.84729 & 0.931533 \\
\hline 1.3118 & -0.12202 & 0.634734 & -0.19224 & 0.847553 & 0.93154 \\
\hline 17.1235 & 0.195155 & 1.020371 & 0.191259 & 0.848323 & 0.932104 \\
\hline 16.56286 & -0.16225 & 0.849925 & -0.1909 & 0.848602 & 0.93213 \\
\hline 18723 & 0.17636 & 0.927915 & 0.190061 & 0.849262 & 0.932412 \\
\hline .35569 & -0.14915 & 0.785336 & -0.18992 & 0.849372 & 0.932412 \\
\hline 915444 & -0.55015 & 2.912243 & -0.18891 & 0.850164 & 719 \\
\hline 8.1413 & 0.113518 & 0.600824 & 0.188937 & 0.850142 & 0.932719 \\
\hline 098797 & 0.547528 & 2.919374 & 0.18755 & 0.851229 & 0.933607 \\
\hline 329235 & -0.54698 & 2.93447 & -0.1864 & 0.852133 & 0.934255 \\
\hline .44783 & 0.1386 & 0.744604 & 0.18614 & 0.852335 & 0.934255 \\
\hline 673199 & 0.541195 & 2.912577 & 0.185813 & 0.852591 & 4255 \\
\hline .36415 & 0.150087 & 0.810742 & 0.185123 & 0.853133 & 0.934567 \\
\hline 7.8028 & -0.09147 & 0.49714 & -0.184 & 0.854012 & 0.93 \\
\hline 14.51887 & -0.25228 & 1.373899 & -0.18363 & 0.854307 & 0.935009 \\
\hline 7.66466 & 0.327956 & 1.785991 & 0.183627 & 0.854306 & 0.935009 \\
\hline 203.3719 & -0.11957 & 0.656893 & -0.18202 & 0.855566 & 0.935542 \\
\hline 2.644431 & 0.348119 & 1.906718 & 0.182575 & 0.855131 & 0.935542 \\
\hline 66.94933 & -0.09213 & 0.505774 & -0.18215 & 0.855466 & 0.935542 \\
\hline 8.41803 & -0.09767 & 0.540283 & -0.18078 & 0.856543 & 0.936251 \\
\hline 523094 & -0.25299 & 1.401284 & -0.18054 & 0.856729 & 0.936251 \\
\hline 2.648207 & 0.292951 & 1.6362 & 0.179044 & 0.857903 & 0.937253 \\
\hline 276029 & 0.425083 & 2.382903 & 0.178389 & 0.858418 & 0.93735 \\
\hline 1.033499 & -0.52062 & 2.920343 & -0.17827 & 0.858508 & 0.93735 \\
\hline 6.76887 & 0.271876 & 1.544236 & 0.176058 & 0.860248 & 0.93773 \\
\hline 19.7919 & -0.22914 & 1.301705 & -0.17603 & 0.860271 & 0.93773 \\
\hline 5.093906 & -0.25675 & 1.449431 & -0.17714 & 0.859399 & 0.93773 \\
\hline 14.74392 & 0.153443 & 0.872519 & 0.175862 & 0.860402 & 0.93773 \\
\hline 3.478825 & -0.3594 & 2.033304 & -0.17676 & 0.8597 & 0.93773 \\
\hline 160.5525 & 0.104403 & 0.588244 & 0.177482 & 0.85913 & 0.93773 \\
\hline 9.861701 & -0.18085 & 1.036789 & -0.17444 & 0.861522 & 0.938669 \\
\hline 8.813671 & 0.221725 & 1.276631 & 0.173679 & 0.862117 & 0.939037 \\
\hline 107.8609 & -0.10798 & 0.629723 & -0.17147 & 0.863855 & 0.940367 \\
\hline 134.2072 & -0.106 & 0.617501 & -0.17166 & 0.863703 & 0.940367 \\
\hline 6.979329 & 0.189961 & 1.113812 & 0.17055 & 0.864577 & 0.940645 \\
\hline 1.522513 & -0.43686 & 2.562414 & -0.17049 & 0.864628 & 0.940645 \\
\hline 10.43849 & -0.16478 & 0.970581 & -0.16977 & 0.865189 & 0.940974 \\
\hline 6.812914 & -0.19305 & 1.139786 & -0.16937 & 0.865505 & 0.941036 \\
\hline 0.390751 & 0.493763 & 2.933976 & 0.168291 & 0.866354 & 0.941116 \\
\hline 3.975047 & 0.262264 & 1.556415 & 0.168505 & 0.866186 & 0.941116 \\
\hline 0.57728 & -0.49297 & 2.92834 & -0.16834 & 0.866313 & 0.941116 \\
\hline 16.8478 & 0.172095 & 1.029755 & 0.167122 & 0.867274 & 0.941323 \\
\hline
\end{tabular}


Propionibacterium virus Lauchelly

Bacillus pseudofirmus

Acinetobacter bereziniae

Thermoanaerobacterium xylanolyticum

Candidatus Nanopelagicus limnes

Photorhabdus asymbiotica

Mycobacterium sp. JLS

Glaciecola sp. 4H-3-7+YE-5

Clostridium aceticum

Sodalis endosymbiont of Henestaris halor

Shewanella pealeana

Hippea maritima

Gillisia sp. Hel1_33_143

Pseudomonas orientalis

Acinetobacter lactucae

Pseudomonas synxantha

Formosa sp. Hel1_33_131

Paraliobacillus sp. X-1125

Pseudoalteromonas aliena

Dialister sp. Marseille-P5638

Stenotrophomonas sp. LM091

Moraxella osloensis

Phaeobacter inhibens

Lactobacillus lindneri

Polaribacter sp. KT 15

Enterobacter ludwigii

Sulfitobacter sp. SK025

Dickeya zeae

Methanocorpusculum labreanum

Candidatus Izimaplasma sp. HR1

Pseudomonas cerasi

Solibacillus silvestris

Acetobacterium woodii

Leuconostoc suionicum

Marinomonas sp. MWYL1

Propionibacterium virus MrAK

Flavobacterium faecale

Endomicrobium proavitum

Clostridium sp. BNL1100

Kribbella flavida

Lactobacillus sp. BHWM-4

Rhizobium sp. S41

Candidatus Azobacteroides pseudotricho।

Bacillus muralis

Pantoea vagans

Nostoc carneum

Xenorhabdus doucetiae

Chryseobacterium arthrosphaerae

Enterococcus gallinarum

Pseudomonas protegens
16.06299

3.883875

39.15459

4.003095

1.029103

6.28622

86.93741

4.140759

7.235444

0.565501

2.788699

0.474415

5.46681

125.5154

11.05918

210.9767

5.259805

6.549651

2.033899

31.46664

331.0304

25029.71

36.12016

2.962506

2.387919

1.112387

22.88569

43.47832

0.850884

5.615662

1.618069

8.829065

5.68322

3.84558

4.586136

24.58941

12.02903

1.218442

8.888912

141.3567

0.913125

136.8303

8.041905

3.961639

237.129

0.566464

9.766922

23.37029

1.049875

203.0288
0.23661

$-0.21615$

0.129677

$-0.23373$

$-0.4803$

$-0.19985$

$-0.1179$

$-0.25676$

$-0.18729$

$-0.48087$

$-0.310$

0.476271

$-0.19788$

0.091445

$-0.1748$

$-0.09362$

$-0.26308$

0.210624

0.359065

$-0.17299$

$-0.08412$

0.090289

0.098556

0.273535

0.376222

$-0.45302$

$-0.11973$

0.07301

$-0.45162$

0.20087

$-0.38593$

0.149248

$-0.21519$

0.278174

$-0.20554$

0.298158

0.193894

$-0.43033$

0.154278

$-0.06679$

0.358248

0.172701

0.180408

$-0.19305$

0.097718

0.423409

0.142286

$-0.12787$

$-0.41271$

$-0.07656$ $\begin{array}{ll}.411593 & 0.167619\end{array}$

1.2938

0.777976

1.419669

2.920295

1.214499

0.720368

1.567909

1.132289

2.928624

1.904083

2.931254

1.217633

0.567789

1.087111

0.584276

1.660157

1.328879

2.27116

1.097557

0.535006

0.574916

0.634968

1.756924

2.415753

2.919253

0.771336

0.473339

2.922635

1.309604

2.527585

0.975959

1.432355

1.883285

1.392593

2.010763

1.306337

2.90551

1.05079

0.454052

2.492439

1.200504

1.249229

1.338516

0.676872

2.928512

1.000605

0.903144

2.91993

0.54685
$-0.16706$

0.166685

$-0.16463$

$-0.16447$

$-0.16455$

$-0.16367$

$-0.16376$

$-0.16541$

$-0.1642$

$-0.16328$

0.16248

$-0.16251$

0.161054

$-0.16079$

$-0.16023$

$-0.15847$

0.158497

0.158097

$-0.15761$

$-0.15723$

0.157048

0.155214

0.15569

0.155737

$-0.15518$

$-0.15523$

0.154245

$-0.15453$

0.153382

$-0.15269$

0.152924

$-0.15024$

0.147707

$-0.14759$

0.148281

0.148425

$-0.14811$

0.146821

$-0.1471$

0.143734

0.143857

0.144415

$-0.14423$

0.144366

0.144582

0.1422

$-0.14158$

$-0.14134$

$-0.14001$

0.8668830 .941323

0.8673210 .941323

0.8676180 .941364

0.8692320 .941972

0.8693610 .941972

0.8692960 .941972

0.8699910 .941972

0.8699220 .941972

0.8686250 .941972

0.8695780 .941972

0.8702970 .942024

0.8709280 .942145

$0.870902 \quad 0.942145$

0.8720510 .943021

0.8722560 .943021

0.8726970 .943218

0.8740870 .944159

0.8740650 .944159

0.874380 .944195

0.8747630 .944248

0.8750650 .944248

0.8752070 .944248

0.8766530 .944433

0.8762780 .944433

0.876240 .944433

0.8766770 .944433

0.8766420 .944433

$0.877416 \quad 0.94467$

$0.877195 \quad 0.94467$

0.8780970 .945123

0.8786440 .945152

0.8784580 .945152

0.8805790 .946954

0.8825740 .947796

0.8826640 .947796

0.8821210 .947796

0.8820070 .947796

0.8822570 .947796

$0.883273 \quad 0.94789$ 
Muricauda lutaonensis

Lactobacillus buchneri

Marinobacter sp. CP1

Acinetobacter Iwoffii

Dictyoglomus thermophilum

Fusobacterium hwasookii

Zobellia galactanivorans

Propionibacterium virus ATCC29399BT

Human polyomavirus 5

Human papillomavirus KC5

Mesoplasma syrphidae

Rhodococcus sp. S2-17

Propionibacterium virus PHL060L00

Staphylococcus virus 37

Staphylococcus piscifermentans

Acinetobacter sp. LoGeW2-3

Fictibacillus phosphorivorans

Brevundimonas diminuta

Chryseobacterium indologenes

Rhodoluna lacicola

Lactobacillus sp. CBA3606

Faecalibacterium prausnitzii

Propionibacterium phage PHLO30NOO

Leuconostoc mesenteroides

Aeromonas sp. CU5

Providencia heimbachae

Negativicoccus massiliensis

Fusobacterium necrophorum

Rhodococcus sp. 2G

Megasphaera stantonii

Simkania negevensis

Campylobacter ureolyticus

Murdochiella vaginalis

Tetragenococcus halophilus

Moritella yayanosii

Candidatus Planktophila vernalis

Psychrobacter sp. P2G3

Acinetobacter pittii

Flavobacterium johnsoniae

Spiroplasma monobiae

Serratia fonticola

Lactobacillus fermentum

Colwellia sp. PAMC 20917

Ignavibacterium album

Mycoplasma penetrans

Lactobacillus crispatus

Belliella baltica

Streptobacillus moniliformis

Carboxydocella thermautotrophica

Sedimentisphaera cyanobacteriorum
4.249086

3.884609

12.04787

582.7408

1.054165

34.01487

5.505683

3.113176

86.96373

3.313383

0.362718

162.7021

10.87973

0.624199

16.34997

27.16975

5.278481

361.8637

142.7746

10.15447

0.592266

954.8395

11.59803

166.4589

7.595709

4.350988

268.4872

37.80884

56.09804

10.20235

0.355545

94.99381

147.0778

29.40555

7.465574

0.554038

17.00337

290.2627

50.16558

0.536918

42.6784

57.3204

14.29563

8.46807

4.873769

49.08365

11.10341

14.17922

4.099295

0.807125
$-0.21609$

$-0.19608$

1.556949

$-0.139341 .011703$

0.08477

0.615461

$-0.30258$

2.209617

0.732503

1.200922

2.814264

0.381897

2.001111

2.582542

2.934765

0.395041

0.45054

$-0.235431 .767593$

0.39084

2.927116

0.105776

0.802688

$0.108926 \quad 0.82554$

0.169763

1.282482

0.062673

0.481407

0.080557

0.618017

0.096657

0.739182

0.378713

2.927806

$0.074428 \quad 0.574234$

$-0.30848$

2.405036

$-0.10685$

0.831386

$-0.16501 \quad 1.30609$

$-0.221391 .757308$

$-0.073980 .589926$

$-0.08892$

0.712537

0.156476

1.248104

0.117828

0.943083

0.364616

2.934985

$-0.09859$

0.800336

0.08632

0.701721

$-0.10863$

0.889739

$-0.135311 .108724$

0.352812 .928737

$-0.18251 \quad 1.526065$

0.061274

0.515608

0.07341

0.633867

$-0.336632 .929212$

$\begin{array}{ll}-0.06634 & 0.580421\end{array}$

0.091229

0.799751

$-0.12685$

1.121552

$-0.119081 .057211$

0.1651631 .480373

$-0.092780 .830786$

$-0.119871 .085481$

$-0.097030 .878633$

$-0.152291 .420695$

0.3176862 .923449
$-0.13879$

$-0.13896$

$-0.13773$

$-0.13773$

$-0.13694$

0.135319

0.13631

0.135701

$-0.13572$

0.135152

0.134607

$-0.13344$

$-0.13319$

0.133524

0.131777

0.131945

0.132371

0.130188

0.130348

0.130763

0.12935

0.129613

$-0.12826$

$-0.12852$

$-0.12634$

$-0.12598$

$-0.12541$

$-0.1248$

0.125371

0.124939

0.124231

$-0.12318$

0.123012

$-0.12209$

$-0.12204$

0.120465

$-0.11959$

0.118838

0.115814

$-0.11492$

$-0.11429$

0.114071

$-0.1131$

$-0.11264$

0.111568

$-0.11167$

$-0.11043$

$-0.11043$

$-0.10719$

0.108668
$0.889615 \quad 0.951328$

0.8894810 .951328

0.8904520 .951665

0.8904530 .951665

0.8910790 .952055

0.892360 .952166

0.8915760 .952166

0.8920580 .952166

0.8920460 .952166

0.8924920 .952166

0.8929220 .952346

0.8938430 .952699

0.8940390 .952699

0.8937790 .952699

0.8951610 .953057

0.8950280 .953057

0.8946910 .953057

0.8964180 .953558

0.8962910 .953558

0.8959630 .953558

$0.89708 \quad 0.953705$

0.8968730 .953705

$0.89794 \quad 0.954061$

0.8977390 .954061

0.8994630 .955303

0.8997470 .955303

0.9002020 .955303

0.9006840 .955303

$0.90023 \quad 0.955303$

0.9005720 .955303

$0.901132 \quad 0.9555$

0.9019640 .955966

0.9020980 .955966

0.9028250 .956223

0.9028650 .956223

0.9041150 .957267

0.9048040 .957718

$0.905404 \quad 0.958074$

0.90780 .960331

0.9085060 .960798

0.9090080 .960953

0.9091810 .960953

0.9099510 .961488

0.9103170 .961595

0.9111660 .961933

0.9110820 .961933

0.9120680 .962327

0.9120660 .962327

0.9146370 .962848

0.9134660 .962848 
Campylobacter cuniculorum

Roseovarius sp. TM1035

Pseudomonas sp. Os17

Seonamhaeicola sp. S2-3

Gammapapillomavirus 24

Propionibacterium virus PHL092M00

Serratia sp. P2ACOL2

Lawsonella clevelandensis

Bacillus pumilus

Enterococcus faecalis

Bifidobacterium pseudocatenulatum

Alphapapillomavirus 2

Acetobacter persici

Lactococcus phage jm3

Providencia rettgeri

Vibrio mediterranei

Mucilaginibacter paludis

Propionibacterium virus PA6

Bacillus sp. X1(2014)

Leptotrichia buccalis

Sulfurimonas denitrificans

[Eubacterium] sulci

Streptococcus sobrinus

Microbacterium sp. PM5

Bacillus flexus

Gammapapillomavirus 8

Erwinia billingiae

Leptotrichia sp. oral taxon 498

Kangiella profundi

Elizabethkingia meningoseptica

Candidatus Hamiltonella defensa

Enterobacter sp. E20

Paenibacillus sp. Y412MC10

Pectobacterium parmentieri

Legionella lansingensis

Pseudomonas sp. JY-Q

Dickeya solani

Erwinia sp. Ejp617

Chroococcidiopsis thermalis

Bacillus sp. Y1

Tobacco vein clearing virus

Desulfovibrio hydrothermalis

Paenibacillus larvae

Gloeomargarita lithophora

Macrococcus caseolyticus

Lactococcus virus CB14

Alkaliphilus metalliredigens

Desulfofarcimen acetoxidans

Flavobacterium crocinum

Thalassospira marina

$\begin{array}{rrrrrr}0.885101 & -0.26608 & 2.504438 & -0.10624 & 0.91539 & 0.962848 \\ 6.794911 & -0.1869 & 1.755975 & -0.10644 & 0.915234 & 0.962848 \\ 2.265337 & -0.30876 & 2.909028 & -0.10614 & 0.915473 & 0.962848 \\ 12.10836 & 0.112321 & 1.054727 & 0.106493 & 0.915191 & 0.962848 \\ 2.034387 & 0.316061 & 2.909216 & 0.108641 & 0.913487 & 0.962848 \\ 42.49899 & 0.140777 & 1.326084 & 0.10616 & 0.915455 & 0.962848 \\ 11.99349 & 0.144768 & 1.360613 & 0.106399 & 0.915266 & 0.962848 \\ 3777.258 & 0.055252 & 0.51088 & 0.108151 & 0.913876 & 0.962848 \\ 19.0946 & -0.08005 & 0.732915 & -0.10922 & 0.913026 & 0.962848 \\ 193.4141 & -0.07383 & 0.699005 & -0.10562 & 0.915885 & 0.962975 \\ 61.61994 & 0.09256 & 0.878862 & 0.105318 & 0.916124 & 0.962975 \\ 5.364853 & 0.260109 & 2.492115 & 0.104373 & 0.916873 & 0.963485 \\ 6.764757 & -0.1196 & 1.152773 & -0.10375 & 0.917365 & 0.963723 \\ 5.389523 & 0.183809 & 1.781654 & 0.103168 & 0.91783 & 0.963933 \\ 15.51662 & 0.090477 & 0.890147 & 0.101643 & 0.91904 & 0.964926 \\ 4.148335 & 0.148665 & 1.476134 & 0.100713 & 0.919779 & 0.965423 \\ 28.24242 & -0.06282 & 0.626098 & -0.10033 & 0.92008 & 0.965449 \\ 34.31993 & 0.134063 & 1.340459 & 0.100012 & 0.920334 & 0.965449 \\ 2.625535 & 0.170783 & 1.746559 & 0.097783 & 0.922105 & 0.966577 \\ 128.6797 & 0.055176 & 0.565019 & 0.097654 & 0.922207 & 0.966577 \\ 0.796231 & -0.25028 & 2.559646 & -0.09778 & 0.922106 & 0.966577 \\ 53.02975 & 0.079496 & 0.833458 & 0.09538 & 0.924013 & 0.967912 \\ 25.65919 & -0.08747 & 0.914378 & -0.09566 & 0.923794 & 0.967912 \\ 110.7517 & -0.04051 & 0.429303 & -0.09437 & 0.924816 & 0.968474 \\ 13.08825 & 0.098125 & 1.051542 & 0.093315 & 0.925653 & 0.968799 \\ 2.030112 & 0.253246 & 2.714064 & 0.093309 & 0.925658 & 0.968799 \\ 76.42011 & -0.05568 & 0.603736 & -0.09222 & 0.926523 & 0.969217 \\ 40.70789 & 0.064443 & 0.699439 & 0.092135 & 0.926591 & 0.969217 \\ 2.444275 & 0.173955 & 1.915843 & 0.090798 & 0.927653 & 0.96977 \\ 17.20995 & 0.068608 & 0.753056 & 0.091106 & 0.927409 & 0.96977 \\ 6.411892 & 0.150418 & 1.672396 & 0.089942 & 0.928334 & 0.970203 \\ 0.989605 & -0.25283 & 2.920536 & -0.08657 & 0.931014 & 0.971843 \\ 14.20202 & 0.069993 & 0.905032 & 0.077338 & 0.938355 & 0.973406 \\ 4.473891 & 0.105709 & 1.356389 & 0.077934 & 0.93788 & 0.973406 \\ 19.84171 & -0.05264 & 0.665923 & -0.07905 & 0.936993 & 0.973406 \\ 18.09541 & -0.04898 & 0.605513 & -0.08088 & 0.935535 & 0.973406\end{array}$


Gordonia rubripertincta

Enterobacter cloacae complex sp.

Deinococcus radiodurans

Enterobacter sp. Crenshaw

Thermosipho africanus

Acinetobacter calcoaceticus

Streptococcus ferus

Gemella morbillorum

Lactobacillus curvatus

Pseudomonas umsongensis

Thermococcus eurythermalis

Serratia sp. SSNIH1

Desulfocapsa sulfexigens

Bacillus sp. FJAT-18017

Propionibacterium virus PHL141N00

Corynebacterium flavescens

Staphylococcus phage phiRS7

Candidatus Baumannia cicadellinicola

Carnobacterium sp. 17-4

Sphingobacteriaceae bacterium GW460-1

Candidatus Methanomethylophilus alvus

Erysipelothrix rhusiopathiae

Brevibacterium aurantiacum

Thermus scotoductus

Pseudomonas mucidolens

Chlamydia sp. 2742-308

Fervidobacterium pennivorans

Marinobacter salinus

Propionibacterium virus P104A

Streptococcus sp. I-P16

Salinispora tropica

Lysinibacillus sp. 2017

Leptotrichia sp. oral taxon 847

Paenibacillus sp. BIHB4019

Runella sp. SP2

Bacillus jeotgali

Christensenella massiliensis

Gluconobacter albidus

Bacillus simplex

Yersinia pseudotuberculosis

Coraliomargarita akajimensis

Staphylococcus auricularis

Clostridium sporogenes

Acinetobacter defluvii

uncultured crAssphage

Salegentibacter salegens

Chryseolinea sp. KIS68-18

Calothrix sp. NIES-3974

Thermus brockianus

Colwellia sp. MT41
504.3071

18.51412

102.3164

0.705455

1.170518

38.83435

11.32435

258.0886

152.3367

43.90167

0.250255

1.341186

0.994507

8.397954

12.09156

2592.475

2.706225

3.020775

17.8398

25.04565

0.465657

27.59913

552.8338

36.53945

39.85925

0.440338

1.127874

10.81766

11.49125

68.90645

42.54851

4.303936

20.67392

8.082799

12.09928

7.049156

7.543923

6.184766

31.68897

0.966284

1.084423

248.0416

111.0922

30.48488

0.939002

5.685686

61.56131

6.176866

2.57482

2.96326 $\begin{array}{lll}-0.0415 & 0.515492\end{array}$

$\begin{array}{ll}-0.06929 & 0.88857\end{array}$

0.0519230 .639469

$-0.227412 .924975$

$-0.23412 .918652$

$\begin{array}{ll}-0.06737 & 0.837689\end{array}$

$-0.101711 .273638$

0.040471

0.536656

0.054344

0.720266

0.665041

2.937236

$-0.22108$

2.916245

$-0.188582 .464985$

$-0.078461 .049975$

$-0.120261 .672048$

0.0193490 .269495

$-0.1594$

2.214126

0.128808

1.770365

$-0.06386$

0.87297

$-0.04876$

0.679902

$-0.208382 .931118$

$-0.053030 .747818$

$-0.03505 \quad 0.49814$

$-0.04404$

0.645052

$\begin{array}{ll}-0.04171 & 0.607194\end{array}$

0.1997212 .932256

$-0.176752 .669442$

0.072621 .106928

$-0.125131 .982562$

$-0.048530 .768159$

0.0310340 .488229

$0.075207 \quad 1.184487$

$-0.05924$

0.920652

-0.07666 1.255353

$-0.058270 .954594$

0.0781041 .301255

$-0.08293$

1.38655

$-0.068351 .166677$

$-0.04582 \quad 0.77431$

$-0.175382 .920832$

$0.130845 \quad 2.202932$

$-0.03639$

0.618995

0.05218

0.947349

$-0.048190 .874583$

$0.161708 \quad 2.921305$

0.0715591 .293715

$-0.03375$

0.597517

$-0.08028$

1.450726

$-0.097351 .748187$

0.0932481 .679615

$-0.0805$

$0.935843 \quad 0.973406$

$-0.07798$

0.937842

0.973406

0.081196

0.935286

0.973406

$-0.07775$

0.93803

0.973406

$-0.08021$

0.936071

0.973406

$-0.08042$

0.9359

0.973406

$-0.07986$

0.93635

0.973406

0.075413

0.939886

0.97345

0.07545

0.939857

0.97345

0.076439

0.93907

0.97345

$-0.07527$

0.940003

0.97345

0.075296

0.939979

0.97345

$-0.0765$

$-0.07473$

0.939018

0.97345

$-0.07192$

0.94043

0.973616

0.071796

0.942664

0.974431

$-0.07199$

0.942764

0.974431

0.072758

0.942607

0.974431

$-0.07315$

0.941999

0.974431

$-0.07172$

0.941685

0.974431

$-0.07109$

0.942825

0.974431

$-0.07091$

$-0.07036$

$-0.06827$

0.943325

0.97454

$-0.0687$

0.943466

0.97454

0.943904

0.974715

0.945571

0.975736

0.068112

0.945231

0.975736

$-0.06621$

0.945697

0.975736

0.065605

0.947208

0.977018

$-0.06312$

0.947693

0.977241

$-0.06318$

0.949673

0.977897

0.063565

0.949627

0.977897

0.063493

0.949317

0.977897

$-0.06435$

0.949374

0.977897

$-0.06106$

0.948693

0.977897

$-0.06105$

0.951308

0.979041

0.060022

0.951322

0.979041

$-0.05981$

0.952138

0.979122

$-0.05858$

0.952305

0.979122

$-0.05918$

0.953285

0.979122

$-0.06004$

0.95281

0.979122

0.059396

0.95212

0.979122

$-0.05878$

0.952637

0.979122

0.05508

0.953125

0.979122

$-0.0551$ 
Megamonas hypermegale

Propionibacterium virus Procrass1

Listeria innocua

Leuconostoc kimchii

Thermosynechococcus sp. NK55a

Sphingobacterium sp. G1-14

Chania multitudinisentens

Enterococcus hirae

Photorhabdus thracensis

Pseudomonas mediterranea

Treponema primitia

Propionibacterium virus ATCC29399BC

Propionibacterium virus PHL114L00

Erysipelothrix larvae

Bacillus xiamenensis

Elizabethkingia ursingii

Propionibacterium virus PHL071N05

Vibrio parahaemolyticus

Glutamicibacter nicotianae

Acinetobacter larvae

Chamaesiphon minutus

Salinimonas sp. HMF8227

Streptococcus sp. I-G2

Acinetobacter nosocomialis

Lysinimonas sp. 2DFWR-13

Entomoplasma luminosum

Candidatus Pelagibacter sp. HIMB1321

Planococcus versutus

Halobacteriovorax sp. BALOs_7

Enterobacter sp. R4-368

Psychrobacter arcticus

Thermodesulfobacterium geofontis

Formosa sp. Hel1_31_208

Propionibacterium phage Enoki

Bifidobacterium kashiwanohense

Bacillus foraminis

Photorhabdus laumondii

Candidatus Nanopelagicus hibericus

Thermoanaerobacterales bacterium SK-G

Vibrio tasmaniensis

Thermovibrio ammonificans

Chryseobacterium joostei

Snodgrassella alvi

Winogradskyella sp. J14-2

Elizabethkingia bruuniana

Salinibacterium sp. CGMCC 1.16371

Salimicrobium jeotgali

Citrobacter freundii

Citrobacter braakii

Sediminispirochaeta smaragdinae
10.00933

5.121363

0.060391

1.059575

0.056996

0.954548

$-0.05302$

0.957718

0.979499

6.841867

$-0.07774$

1.471689

$-0.05282$

4.463516

$-0.14263$

2.708517

$-0.05266$

1.559248

0.110042

2.104081

0.052299

0.957872

0.979837

84.23243

0.0478

0.909961

0.05253

0.958004

0.979837

7.853463

0.06277

1.165091

7.107411

8.947296

$-0.06048$

1.126886

0.053875

0.95829

0.979837

25.74423

0.057399

1.116158

$-0.05367$

0.958106

0.979837

7.159209

0.749578

0.051426

0.957035

0.979837

22.92

0.056372

1.11708

$-0.05114$

0.957195

0.979837

18.72913

$-0.099742 .006895$

0.050464

0.958986

0.979837

9.853054

$-0.084191 .699557$

1.247034

0.058719

1.20285

0.959217

3.070417

0.104993

2.175528

$-0.04954$

0.048816

0.048261

14.32192

0.094264

2.043327

0.046133

43.19387

153.7385

0.082171

1.766276

0.046522

$-0.03106$

0.666947

$-0.04657$

0.018298

0.387889

0.047173

12.89374

0.058453

1.270395

0.046012

1.453427

0.1165692 .569491

4.406216

$-0.06559$

1.45237

0.045367

67.79932

0.030449

0.685054

48.41303

0.032127

0.731939

164.6009

0.022532

0.518102

2.186079

0.096556

2.267578

3.067152

$-0.08152$

1.904981

7.415671

0.060306

1.483905

3.620787

0.070975

1.753446

4.696087

$-0.05537$

1.367871

24.41654

0.035225

0.87636

0.858447

$-0.10994$

2.794306

3.777799

0.064003

1.667578

13.62034

0.053509

1.386832

$-0.04516$

0.044448

0.043893

0.043489

0.042581

0.04064

0.040477

$-0.04048$

0.040195

$-0.03934$

0.038381

0.038583

0.717967

0.037823

5.905202

0.027156

1.302195

$-0.03754$

5.018604

0.989982

$-0.04941$

1.373142

$-0.03598$

2.920643

0.035946

$-0.03941 \quad 1.111977$

$-0.03544$

2.026305

$-0.03297$

2.7981

$-0.03318$

0.855777

14.9251

31.13192

$-0.09283$

1.073414

0.031657

0.02532

0.815612

0.031044

2.942702

$-0.05532$

1.782663

$-0.03103$

$-0.03174$

3.216665

$-0.064052 .018172$

80.51657

0.014592

0.477036

0.03059

$-0.03009$

$-0.02889$

68.05547

$-0.02628$

0.909675

2.204031

$-0.07639$

2.655942

$-0.02876$

2.2645
$-0.0497$

$-0.0428$

0.959753

0.960363

0.960493

0.961066

0.961508

0.963204

0.962894

0.962855

0.962376

0.963301

0.963815

0.963982

0.964547

0.96499

0.965311

0.966035

0.965865

0.967583

0.967713

0.967714

0.967938

0.968617

0.969384

0.969223

0.969828

0.970055

0.971297

0.971325

0.971728

0.973695

0.973535

0.974746

0.975235

0.975246

0.974683

0.975597

0.975992

0.976952

0.977055

$-0.0276$

0.977985
.980234

0.980506 


\begin{tabular}{|c|c|c|c|c|c|c|}
\hline ulfitobacterium dichloroeliminans & 5.521767 & -0.04814 & 1.765828 & -0.02726 & 0.978251 & \\
\hline Pasteurellaceae bacterium NI1060 & 5.979164 & -0.03312 & 1.226123 & -0.02701 & 0.978449 & 0.98821 \\
\hline Pseudomonas sp. R11-23-07 & 2.401552 & 0.077449 & 2.901161 & 0.026696 & 0.978702 & 0.98821 \\
\hline Dokdonia sp. 4H-3-7-5 & 0.681048 & -0.07626 & 2.925448 & -0.02607 & 0.979202 & 0.98844 \\
\hline Legionella clemsonensis & 2.709335 & 0.038754 & 1.549587 & 0.025009 & 0.980048 & 0.989019 \\
\hline Streptococcus mutans & 369.9229 & 0.017072 & 0.70901 & 0.024078 & 0.98079 & 0.98922 \\
\hline Francisella sp. FSC1006 & 2.257032 & 0.046694 & 1.914827 & 0.024386 & 0.980545 & 0.98922 \\
\hline Thermincola potens & 4.358153 & 0.030761 & 1.366668 & 0.022508 & 0.982042 & 0.990208 \\
\hline Actinotignum schaalii & 168.0202 & -0.01098 & 0.500896 & -0.02192 & 0.982515 & 0.99041 \\
\hline Chryseobacterium glaciei & 19.53588 & 0.018052 & 0.900374 & 0.02005 & 0.984004 & 0.991319 \\
\hline Bifidobacterium adolescentis & 294.6065 & -0.01205 & 0.608302 & -0.0198 & 0.984201 & 0.991319 \\
\hline Xanthomonas fragariae & 25.85526 & 0.011126 & 0.563763 & 0.019735 & 0.984255 & 0.991319 \\
\hline Chlorobium phaeobacteroides & 1.330448 & 0.045796 & 2.358223 & 0.01942 & 0.984506 & 0.991319 \\
\hline Lactobac & 3.659717 & 0.025498 & 1.430279 & 0.017827 & 0.985777 & 0.992049 \\
\hline shanyuanii & 13.84625 & 0.015443 & 0.858446 & 0.017989 & 0.985648 & 0.992049 \\
\hline Mycopla & 2.775407 & 0.038025 & 2.407788 & 0.015792 & 0.9874 & 0.993407 \\
\hline Paenibacillus & 1.627957 & -0.03215 & 2.301946 & -0.01397 & 0.988857 & 0.994598 \\
\hline Mycobacteri & 58.7201 & -0.01865 & 1.368876 & -0.01362 & 0.989131 & 0.994599 \\
\hline Chryseobacte & 46.15918 & 0.010577 & 0.810652 & 0.013048 & 0.98959 & 0.994603 \\
\hline Shewanella li & 4.029667 & -0.01967 & 1.562397 & -0.01259 & 0.989955 & 0.994603 \\
\hline Helico & 4.971233 & -0.02799 & 2.172324 & -0.01289 & 0.989718 & 0.994603 \\
\hline Sodalis praecaptivus & 16.16688 & 0.012001 & 1.040765 & 0.011531 & 0.9908 & 0.994902 \\
\hline Propionibacterium virus PHL111M01 & 16.61314 & 0.019066 & 1.615726 & 0.0118 & 0.990585 & 0.994902 \\
\hline Marinobacter psycl & 0.578432 & 0.031684 & 2.927903 & 0.010821 & 0.991366 & 0.994929 \\
\hline Chryseobacter & 33.89231 & -0.00754 & 0.697766 & -0.01081 & 0.991373 & 0.994929 \\
\hline Gemella sp & 5.105433 & -0.02361 & 2.292164 & -0.0103 & 0.991782 & 0.995065 \\
\hline Bacillus wiedmannii & 2.820446 & -0.01911 & 2.232459 & -0.00856 & 0.993172 & 0.995909 \\
\hline Colwellia sp. PAMC 21821 & 2.701474 & -0.01581 & 1.777708 & -0.0089 & 0.992902 & 0.995909 \\
\hline Herbinix luporum & 11.96346 & 0.007722 & 1.136606 & 0.006794 & 0.994579 & 0.996497 \\
\hline Lactobacillus johnsonii & 26.82487 & -0.00492 & 0.711787 & -0.00691 & 0.994484 & 0.996497 \\
\hline Candidatus Pelagibacter sp. IMCC9063 & 0.963454 & 0.017549 & 2.485605 & 0.00706 & 0.994367 & 0.996497 \\
\hline Elizabethkingia anophelis & 75.84501 & -0.00337 & 0.575968 & -0.00585 & 0.995331 & 0.996703 \\
\hline Pseudomonas sp. TKP & 2.185557 & 0.015676 & 2.679826 & 0.00585 & 0.995333 & 0.996703 \\
\hline Anaerolineaceae bacterium oral taxon 43 & 6.583372 & -0.00576 & 1.372672 & -0.00419 & 0.996654 & 0.997476 \\
\hline Exiguobacterium sp. $\mathrm{MH} 3$ & 24.64131 & 0.005819 & 1.3251 & 0.004391 & 0.996496 & 0.997476 \\
\hline Propionibacterium virus PHL009M11 & 16.968 & -0.00942 & 2.481391 & -0.0038 & 0.996971 & 0.997514 \\
\hline Clostridium tyrobutyricum & 19.40266 & 0.00286 & 0.826942 & 0.003459 & 0.99724 & 0.997514 \\
\hline Aneurinibacillus sp. XH2 & 2.40513 & -0.00324 & 1.897905 & -0.00171 & 0.998639 & 0.998639 \\
\hline
\end{tabular}


baseMean log2FoldCr IfCSE stat pvalue padj

Arcobacter

$\begin{array}{lllllll}217.1963 & 1.918119 & 0.390478 & 4.912236 & 9.00 \mathrm{E}-07 & 0.000854\end{array}$

$\begin{array}{llllllll}\text { Methanosarcina } & 98.02084 & 1.755386 & 0.374929 & 4.68192 & 2.84 \mathrm{E}-06 & 0.000854\end{array}$

$\begin{array}{lllllllll}\text { Mycoplasma } & 254.5904 & 1.803441 & 0.389037 & 4.63565 & 3.56 \mathrm{E}-06 & 0.000854\end{array}$

$\begin{array}{llllllll}\text { Borrelia } & 80.47319 & 2.539091 & 0.533238 & 4.761648 & 1.92 \mathrm{E}-06 & 0.000854\end{array}$

$\begin{array}{llllllll}\text { Spiroplasma } & 163.5803 & 1.973828 & 0.455042 & 4.337681 & 1.44 \mathrm{E}-05 & 0.002765\end{array}$

$\begin{array}{lllllllll}\text { Carnobacterium } & 733.7274 & 2.671146 & 0.63576 & 4.201503 & 2.65 \mathrm{E}-05 & 0.004242\end{array}$

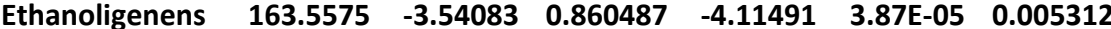

$\begin{array}{lllllllll}\text { Clostridium } & 1611.695 & 1.255679 & 0.310157 & 4.04853 & 5.15 E-05 & 0.006185\end{array}$

$\begin{array}{lllllllll}\text { Francisella } & 102.4188 & 1.631129 & 0.409995 & 3.978414 & 6.94 \mathrm{E}-05 & 0.0074\end{array}$

$\begin{array}{lllllllll}\text { Helicobacter } & 100.1122 & 1.387951 & 0.354711 & 3.912911 & 9.12 \mathrm{E}-05 & 0.008754\end{array}$

Lacinutrix

Aquimarina

Polaribacter

$\begin{array}{llllllll}49.17966 & 1.49295 & 0.385011 & 3.877684 & 0.000105 & 0.009203\end{array}$

$\begin{array}{lllllll}51.55159 & 1.812231 & 0.474958 & 3.815559 & 0.000136 & 0.01087\end{array}$

$\begin{array}{lllllll}145.6738 & 1.330483 & 0.358039 & 3.716032 & 0.000202 & 0.014945\end{array}$

$\begin{array}{lllllllll}\text { Candidatus Meth } & 10.2439 & -3.35361 & 0.913911 & -3.66951 & 0.000243 & 0.016664\end{array}$

Bacillus

$\begin{array}{llllllll}4037.308 & 1.514815 & 0.429665 & 3.525576 & 0.000423 & 0.027044\end{array}$

Phietavirus

$\begin{array}{lllllll}3547.438 & 2.889099 & 0.827362 & 3.491942 & 0.00048 & 0.027079\end{array}$

Ehrlichia

$\begin{array}{llllllll}25.47551 & 3.021406 & 0.864775 & 3.493865 & 0.000476 & 0.027079\end{array}$

$\begin{array}{llllllll}\text { Bacterioplanes } & 5.789919 & -2.72224 & 0.791907 & -3.43758 & 0.000587 & 0.030593\end{array}$

Brachyspira

Rickettsia

$\begin{array}{llllllll}95.83986 & 1.682075 & 0.492526 & 3.415198 & 0.000637 & 0.030593\end{array}$

$\begin{array}{llllllll}59.45872 & 1.709544 & 0.50009 & 3.418475 & 0.00063 & 0.030593\end{array}$

$\begin{array}{lllllll}41.11011 & -2.09786 & 0.63149 & -3.32208 & 0.000893 & 0.040846\end{array}$

$\begin{array}{lrrrrrrr}\text { Woeseia } & 15.14639 & -2.23886 & 0.678729 & -3.29861 & 0.000972 & 0.042399 \\ \text { Geoalkalibacter } & 27.14344 & -1.75151 & 0.54039 & -3.2412 & 0.00119 & 0.049682\end{array}$

$\begin{array}{lrrrrrrr}\text { Geoalkalibacter } & \mathbf{2 7 . 1 4 3 4 4} & -\mathbf{1 . 7 5 1 5 1} & \mathbf{0 . 5 4 0 3 9} & \mathbf{- 3 . 2 4 1 2} & \mathbf{0 . 0 0 1 1 9} & \mathbf{0 . 0 4 9 6 8 2} \\ & & \end{array}$

$\begin{array}{llllllll}\text { Winogradskyella } & 43.24964 & 1.427262 & 0.447153 & 3.191887 & 0.001413 & 0.054277\end{array}$

$\begin{array}{llllllll}\text { Candidatus Pelag } & 36.99456 & 1.763674 & 0.557002 & 3.166368 & 0.001544 & 0.056993\end{array}$

$\begin{array}{llllllll}\text { Methanococcus } & 29.3845 & 2.133272 & 0.705935 & 3.02191 & 0.002512 & 0.08931\end{array}$

$\begin{array}{llllllll}\text { Candidatus Nano| } & 20.20345 & -2.90617 & 0.966908 & -3.00563 & 0.00265 & 0.090868\end{array}$

$\begin{array}{llllllll}\text { Geminocystis } & 46.44007 & 1.315398 & 0.441439 & 2.979799 & 0.002884 & 0.092893\end{array}$

$\begin{array}{llllllll}\text { Thermoanaeroba } & 53.95976 & 1.424844 & 0.478483 & 2.977838 & 0.002903 & 0.092893\end{array}$

$\begin{array}{llllllll}\text { Borreliella } & 33.42886 & 2.500426 & 0.856497 & 2.919364 & 0.003507 & 0.102035\end{array}$

$\begin{array}{llllllll}\text { Thioflavicoccus } & 39.10327 & -1.23174 & 0.420697 & -2.92786 & 0.003413 & 0.102035\end{array}$

$\begin{array}{llllllll}\text { Phyllobacterium } & 24.61895 & -1.41056 & 0.48252 & -2.92333 & 0.003463 & 0.102035\end{array}$

$\begin{array}{llllllll}\text { Rhodomicrobium } 22.80382 & -1.54054 & 0.547925 & -2.81159 & 0.00493 & 0.139194\end{array}$

$\begin{array}{llllllll}\text { Azorhizobium } & 84.55738 & -0.88202 & 0.321928 & -2.7398 & 0.006148 & 0.143946\end{array}$

$\begin{array}{llllllll}\text { Sulfolobus } & 12.52057 & 3.172668 & 1.157597 & 2.740736 & 0.00613 & 0.143946\end{array}$

$\begin{array}{llllllll}\text { Pelodictyon } & 11.17924 & -1.8083 & 0.65483 & -2.76147 & 0.005754 & 0.143946\end{array}$

$\begin{array}{llllllll}\text { Olleya } & 20.03512 & 1.945045 & 0.697031 & 2.79047 & 0.005263 & 0.143946\end{array}$

$\begin{array}{llllllll}\text { Phreatobacter } & 101.1055 & -0.78457 & 0.284953 & -2.75335 & 0.005899 & 0.143946\end{array}$

$\begin{array}{lllllll}\text { Hyphomicrobium } & 109.5445 & -0.97642 & 0.351891 & -2.77478 & 0.005524 & 0.143946\end{array}$
Results with $p$ value $<0.05$ from the table on the left, organised by the condition and differe Genera more abundant in control condition

baseMean log2FoldCh IfcSE stat pvalue padj

$\begin{array}{lllllll}\text { Ethanoligenens } & 163.5575 & -3.54083 & 0.860487 & -4.11491 & 3.87 \mathrm{E}-05 & 0.005312\end{array}$ $\begin{array}{lllllll}\text { Candidatus Methylopum } \quad 10.2439 & -3.35361 & 0.913911 & -3.66951 & 0.000243 & 0.016664\end{array}$ $\begin{array}{lllllll}\text { Bacterioplanes } & 5.789919 & -2.72224 & 0.791907 & -3.43758 & 0.000587 & 0.030593\end{array}$ $\begin{array}{lllllll}\text { Woeseia } & 15.14639 & -2.23886 & 0.678729 & -3.29861 & 0.000972 & 0.042399\end{array}$ $\begin{array}{lllllll}\text { Libanicoccus } & 41.11011 & -2.09786 & 0.63149 & -3.32208 & 0.000893 & 0.040846\end{array}$ $\begin{array}{lllllll}\text { Geoalkalibacter } & 27.14344 & -1.75151 & 0.54039 & -3.2412 & 0.00119 & 0.049682\end{array}$

Genera more abundant in lesional skin

\begin{tabular}{lllllll} 
& baseMean log2FoldCh IfcSE & stat & \multicolumn{2}{l}{ pvalue padj } \\
Ehrlichia & 25.47551 & 3.021406 & 0.864775 & 3.493865 & 0.000476 & 0.027079
\end{tabular}

Phietavirus

Carnobacterium

Borrelia

Spiroplasma

Arcobacter

Aquimarina

Mycoplasma

Methanosarcina

Rickettsia

Brachyspira

Francisella

Bacillus

Lacinutrix

Helicobacter

Polaribacter

Clostridium $\begin{array}{lllllll}25.47551 & 3.021406 & 0.864775 & 3.493865 & 0.000476 & 0.027079\end{array}$ $\begin{array}{llllllll}733.7274 & 2.671146 & 0.63576 & 4.201503 & 2.65 \mathrm{E}-05 & 0.004242\end{array}$ $\begin{array}{llllllll}80.47319 & 2.539091 & 0.533238 & 4.761648 & 1.92 \mathrm{E}-06 & 0.000854\end{array}$ $\begin{array}{lllllll}163.5803 & 1.973828 & 0.455042 & 4.337681 & 1.44 \mathrm{E}-05 & 0.002765\end{array}$ $\begin{array}{llllllll}217.1963 & 1.918119 & 0.390478 & 4.912236 & 9.00 \mathrm{E}-07 & 0.000854\end{array}$ $\begin{array}{lllllll}51.55159 & 1.812231 & 0.474958 & 3.815559 & 0.000136 & 0.01087\end{array}$ $\begin{array}{lllllll}254.5904 & 1.803441 & 0.389037 & 4.63565 & 3.56 \mathrm{E}-06 & 0.000854\end{array}$ $\begin{array}{llllllll}98.02084 & 1.755386 & 0.374929 & 4.68192 & 2.84 \mathrm{E}-06 & 0.000854\end{array}$ $\begin{array}{lllllll}59.45872 & 1.709544 & 0.50009 & 3.418475 & 0.00063 & 0.030593\end{array}$ $\begin{array}{lllllll}95.83986 & 1.682075 & 0.492526 & 3.415198 & 0.000637 & 0.030593\end{array}$ $\begin{array}{llllllll}102.4188 & 1.631129 & 0.409995 & 3.978414 & 6.94 \mathrm{E}-05 & 0.0074\end{array}$ $\begin{array}{lllllll}4037.308 & 1.514815 & 0.429665 & 3.525576 & 0.000423 & 0.027044\end{array}$ $\begin{array}{lllllll}49.17966 & 1.49295 & 0.385011 & 3.877684 & 0.000105 & 0.009203\end{array}$ $\begin{array}{llllllll}100.1122 & 1.387951 & 0.354711 & 3.912911 & 9.12 \mathrm{E}-05 & 0.008754\end{array}$ $\begin{array}{lllllll}145.6738 & 1.330483 & 0.358039 & 3.716032 & 0.000202 & 0.014945\end{array}$ $\begin{array}{lllllll}1611.695 & 1.255679 & 0.310157 & 4.04853 & 5.15 \mathrm{E}-05 & 0.006185\end{array}$ 
$\begin{array}{llllll}81.04342 & 1.253418 & 0.453659 & 2.762906 & 0.005729 & 0.143946\end{array}$ $\begin{array}{llllllll}\text { Cloacibacillus } & 10.63198 & -2.06765 & 0.75993 & -2.72084 & 0.006512 & 0.148837\end{array}$

$\begin{array}{llllllll}\text { Acidobacterium } & 43.12249 & -1.46647 & 0.542088 & -2.70522 & 0.006826 & 0.152391\end{array}$

$\begin{array}{llllllll}\text { Archaeoglobus } & 9.770977 & -1.83289 & 0.682889 & -2.68402 & 0.007274 & 0.158713\end{array}$

$\begin{array}{llllllll}\text { Thiomonas } & 445.7656 & -1.054 & 0.396951 & -2.65523 & 0.007925 & 0.163264\end{array}$

$\begin{array}{llllllll}\text { Tenacibaculum } & 80.85238 & 0.948289 & 0.358488 & 2.645245 & 0.008163 & 0.163264\end{array}$

$\begin{array}{llllllll}\text { Bartonella } & 85.77432 & 0.856866 & 0.323589 & 2.648008 & 0.008097 & 0.163264\end{array}$

$\begin{array}{lllllllll}\text { Acidianus } & 11.92358 & 3.388747 & 1.273524 & 2.660921 & 0.007793 & 0.163264\end{array}$

$\begin{array}{lllllll}\text { Afipia } & 230.3713 & -0.95513 & 0.37484 & -2.5481 & 0.010831 & 0.169077\end{array}$

$\begin{array}{lllllll}\text { Moorella } & 20.29407 & -1.41409 & 0.554495 & -2.55023 & 0.010765 & 0.169077\end{array}$

$\begin{array}{llllllll}\text { Halobacillus } & 20.68037 & -1.63309 & 0.637375 & -2.56221 & 0.010401 & 0.169077\end{array}$

Steroidobacter $\quad \begin{array}{lllllll}35.70928 & -1.14688 & 0.449578 & -2.55101 & 0.010741 & 0.169077\end{array}$

$\begin{array}{llllllll}\text { Desulfomicrobiur } & 40.99859 & -1.10382 & 0.434034 & -2.54316 & 0.010985 & 0.169077\end{array}$

$\begin{array}{llllllll}\text { Aeromonas } & 630.9045 & -0.51952 & 0.20456 & -2.53967 & 0.011096 & 0.169077\end{array}$

$\begin{array}{llllllll}\text { Desulfovibrio } & 341.01 & -0.5945 & 0.227572 & -2.61235 & 0.008992 & 0.169077\end{array}$

$\begin{array}{llllllll}\text { Pseudoalteromor } & 180.2689 & 0.733389 & 0.281944 & 2.601184 & 0.00929 & 0.169077\end{array}$

$\begin{array}{llllllll}\text { Laribacter } & 57.86705 & -0.86796 & 0.340673 & -2.54779 & 0.010841 & 0.169077\end{array}$

$\begin{array}{llllllll}\text { Halomicrobium } & 8.741374 & -2.2648 & 0.88865 & -2.54859 & 0.010816 & 0.169077\end{array}$

$\begin{array}{llllllll}\text { Virgibacillus } & 96.14603 & 0.849969 & 0.331786 & 2.561803 & 0.010413 & 0.169077\end{array}$

$\begin{array}{llllllll}\text { Nostoc } & 204.0961 & 0.801683 & 0.315598 & 2.540205 & 0.011079 & 0.169077\end{array}$

$\begin{array}{llllllll}\text { Epibacterium } & 19.63941 & -1.52355 & 0.59004 & -2.58211 & 0.00982 & 0.169077\end{array}$

$\begin{array}{lllllll}\text { Cyanothece } & \quad 54.71629 & 0.714488 & 0.275874 & 2.589905 & 0.0096 & 0.169077\end{array}$

$\begin{array}{llllllll}\text { Brucella } & 41.4629 & -1.14585 & 0.4509 & -2.54126 & 0.011045 & 0.169077\end{array}$

$\begin{array}{lllllll}\text { Methanocaldocor } & 18.18896 & 2.375016 & 0.937671 & 2.532888 & 0.011313 & 0.169691\end{array}$

$\begin{array}{llllllll}\text { Hyphomonas } & 62.48402 & -0.83637 & 0.332232 & -2.51742 & 0.011822 & 0.172938\end{array}$

$\begin{array}{llllllll}\text { Halobiforma } & 15.8217 & -1.6813 & 0.668403 & -2.51541 & 0.01189 & 0.172938\end{array}$

$\begin{array}{lllllll}\text { Methanosphaera } & 12.95317 & 3.218215 & 1.292264 & 2.49037 & 0.012761 & 0.173906\end{array}$

$\begin{array}{llllllll}\text { Halogeometricur } & 7.303584 & -2.36144 & 0.944453 & -2.50033 & 0.012408 & 0.173906\end{array}$

$\begin{array}{llllllll}\text { Marinithermus } & 47.39235 & -1.11278 & 0.447335 & -2.48757 & 0.012862 & 0.173906\end{array}$

$\begin{array}{llllllll}\text { Pontimonas } & 27.66046 & -1.49431 & 0.598924 & -2.49499 & 0.012596 & 0.173906\end{array}$

Indioceanicola $\quad \begin{array}{lllllll}57.48856 & -0.78858 & 0.314937 & -2.50393 & 0.012282 & 0.173906\end{array}$

$\begin{array}{llllllll}\text { Sorangium } & 473.9773 & -0.81992 & 0.332045 & -2.46929 & 0.013538 & 0.175628\end{array}$

$\begin{array}{lllllllll}\text { Fischerella } & 49.86579 & 1.469589 & 0.594013 & 2.473999 & 0.013361 & 0.175628\end{array}$

$\begin{array}{llllllll}\text { Tolumonas } & 7.402814 & -1.87582 & 0.758835 & -2.47198 & 0.013437 & 0.175628\end{array}$

$\begin{array}{llllllll}\text { Rhodospirillum } & 112.4971 & -0.7312 & 0.296982 & -2.46211 & 0.013812 & 0.176795\end{array}$

$\begin{array}{lllllll}\text { Methylorubrum } & 574.0359 & -0.6609 & 0.270565 & -2.44266 & 0.014579 & 0.184162\end{array}$

$\begin{array}{llllllll}\text { Halothiobacillus } & 37.58879 & -0.94776 & 0.389469 & -2.43346 & 0.014955 & 0.186336\end{array}$

$\begin{array}{llllllll}\text { Thauera } & 733.7273 & -0.71709 & 0.295219 & -2.42902 & 0.01514 & 0.186336\end{array}$

$\begin{array}{lllllll}\text { Geobacter } & 312.5122 & -0.68723 & 0.283527 & -2.42386 & 0.015357 & 0.186612\end{array}$

$\begin{array}{lllllll}\text { Candidatus Prom } 34.15326 & -1.01379 & 0.420123 & -2.41307 & 0.015819 & 0.189824\end{array}$ 
Gemmatirosa $\quad \begin{array}{llllllll}124.4254 & -0.87057 & 0.364172 & -2.39055 & 0.016823 & 0.195132\end{array}$

$\begin{array}{llllllll}\text { Cystobacter } & 129.053 & -0.6539 & 0.274407 & -2.38297 & 0.017174 & 0.195132\end{array}$

$\begin{array}{llllllll}\text { Vogesella } & 71.62203 & -0.8434 & 0.353409 & -2.38647 & 0.017011 & 0.195132\end{array}$

$\begin{array}{llllllll}\text { Komagataeibacte } & 143.6393 & -0.58249 & 0.244901 & -2.37845 & 0.017385 & 0.195132\end{array}$

$\begin{array}{llllllll}\text { Wolbachia } & 22.09465 & 1.117979 & 0.471388 & 2.371673 & 0.017708 & 0.195396\end{array}$

$\begin{array}{llllllll}\text { Haloplanus } & 38.96758 & -1.17504 & 0.501371 & -2.34365 & 0.019096 & 0.20479\end{array}$

$\begin{array}{lllllllll}\text { Bradymonas } & 22.48771 & -1.22476 & 0.523035 & -2.34164 & 0.019199 & 0.20479\end{array}$

$\begin{array}{llllllll}\text { Micavibrio } & 10.49342 & -2.10025 & 0.893942 & -2.34943 & 0.018802 & 0.20479\end{array}$

$\begin{array}{llllllll}\text { Slackia } & 48.38209 & -1.5114 & 0.647105 & -2.33563 & 0.01951 & 0.205825\end{array}$

$\begin{array}{llllllll}\text { Ferrimonas } & 15.51851 & -1.24172 & 0.541744 & -2.29208 & 0.021901 & 0.20613\end{array}$

$\begin{array}{llllllll}\text { Thermaerobacter } & 62.1009 & -0.81671 & 0.356052 & -2.29381 & 0.021802 & 0.20613\end{array}$

$\begin{array}{llllllll}\text { Natronomonas } & 16.84829 & -1.43567 & 0.61741 & -2.3253 & 0.020056 & 0.20613\end{array}$

$\begin{array}{llllllll}\text { Sphaerobacter } & 55.41318 & -0.95517 & 0.416403 & -2.29386 & 0.021799 & 0.20613\end{array}$

$\begin{array}{llllllll}\text { Rubrobacter } & 158.1838 & -1.10424 & 0.481669 & -2.29253 & 0.021875 & 0.20613\end{array}$

$\begin{array}{llllllll}\text { Methyloceanibac } & 62.02076 & -0.82494 & 0.357686 & -2.30633 & 0.021092 & 0.20613\end{array}$

$\begin{array}{llllllll}\text { Archangium } & 106.2168 & -0.79982 & 0.348171 & -2.29722 & 0.021606 & 0.20613\end{array}$

$\begin{array}{lllllll}\text { Salinicola } & 64.5992 & -0.98068 & 0.425165 & -2.30659 & 0.021078 & 0.20613\end{array}$

$\begin{array}{llllllll}\text { Legionella } & 113.2075 & 0.694169 & 0.302236 & 2.296778 & 0.021631 & 0.20613\end{array}$

$\begin{array}{lllllllll}\text { Myroides } & 46.73626 & 1.076776 & 0.466717 & 2.307128 & 0.021048 & 0.20613\end{array}$

$\begin{array}{llllllll}\text { Pannonibacter } & 139.8627 & -0.69126 & 0.301074 & -2.29599 & 0.021677 & 0.20613\end{array}$

$\begin{array}{llllllll}\text { Phycisphaera } & 42.482 & -1.0042 & 0.44025 & -2.28098 & 0.022549 & 0.208148\end{array}$

$\begin{array}{llllllll}\text { Sedimenticola } & 14.07082 & -1.53717 & 0.673271 & -2.28314 & 0.022422 & 0.208148\end{array}$

$\begin{array}{llllllll}\text { Dokdonia } & 20.0812 & 1.427801 & 0.633963 & 2.252182 & 0.024311 & 0.212045\end{array}$

$\begin{array}{llllllll}\text { Bradyrhizobium } & 5158.38 & -0.70608 & 0.314527 & -2.24488 & 0.024776 & 0.212045\end{array}$

$\begin{array}{llllllll}\text { Gloeobacter } & 118.3552 & -0.80306 & 0.357112 & -2.24876 & 0.024528 & 0.212045\end{array}$

$\begin{array}{llllllll}\text { Methanobacteriu } & 22.60309 & 1.894599 & 0.848487 & 2.232915 & 0.025555 & 0.212045\end{array}$

$\begin{array}{llllllll}\text { Lacunisphaera } & 55.63255 & -1.20287 & 0.534934 & -2.24863 & 0.024536 & 0.212045\end{array}$

$\begin{array}{llllllll}\text { Porphyromonas } & 2149.603 & -1.35095 & 0.606198 & -2.22856 & 0.025843 & 0.212045\end{array}$

Granulibacter $\quad \begin{array}{lllllll}46.05282 & -0.73531 & 0.328054 & -2.24144 & 0.024997 & 0.212045\end{array}$

$\begin{array}{lllllll}\text { Orrella } & 110.94 & -0.80218 & 0.354933 & -2.26009 & 0.023816 & 0.212045\end{array}$

$\begin{array}{llllllll}\text { Candidatus Arthrı } & 26.48728 & 2.041317 & 0.912603 & 2.236807 & 0.025299 & 0.212045\end{array}$

$\begin{array}{lllllll}\text { Methyloversatilis } & 68.60885 & -0.80521 & 0.358745 & -2.24451 & 0.0248 & 0.212045\end{array}$

$\begin{array}{llllllll}\text { Syntrophobacter } & 12.67065 & -1.57621 & 0.703203 & -2.24147 & 0.024996 & 0.212045\end{array}$

$\begin{array}{lllllllll}\text { Anabaena } & 28.90293 & 1.545317 & 0.687725 & 2.246999 & 0.02464 & 0.212045\end{array}$

$\begin{array}{llllllll}\text { Marinobacterium } 22.36146 & -1.01973 & 0.457509 & -2.22887 & 0.025823 & 0.212045\end{array}$

$\begin{array}{llllllll}\text { Myxococcus } & 451.1725 & -0.6487 & 0.293734 & -2.20846 & 0.027213 & 0.214868\end{array}$

$\begin{array}{llllllll}\text { Methanobrevibac } & 59.5735 & 1.357094 & 0.611469 & 2.219397 & 0.02646 & 0.214868\end{array}$

$\begin{array}{llllllll}\text { Advenella } & 41.2729 & -0.75429 & 0.341752 & -2.20711 & 0.027306 & 0.214868\end{array}$ 
$\begin{array}{lllllll}72.60733 & -0.80741 & 0.365403 & -2.20963 & 0.027131 & 0.214868\end{array}$ $\begin{array}{llllllll}\text { Jonquetella } & 93.82533 & -1.97429 & 0.892681 & -2.21164 & 0.026992 & 0.214868\end{array}$

$\begin{array}{lllllllll}\text { Acidiferrobacter } & 28.99224 & -1.06207 & 0.483141 & -2.19827 & 0.02793 & 0.21799\end{array}$

$\begin{array}{llllllll}\text { Mesorhizobium } & 932.254 & -0.66653 & 0.306754 & -2.17286 & 0.029791 & 0.219356\end{array}$

$\begin{array}{llllllll}\text { Cellulophaga } & 46.20234 & 0.901237 & 0.415276 & 2.170213 & 0.029991 & 0.219356\end{array}$

$\begin{array}{llllllll}\text { Pseudorhodoplan } 65.24289 & -0.85057 & 0.393346 & -2.16239 & 0.030588 & 0.219356\end{array}$

$\begin{array}{llllllll}\text { Anaeromyxobactı } & 258.8735 & -0.72902 & 0.33354 & -2.18572 & 0.028836 & 0.219356\end{array}$

$\begin{array}{llllllll}\text { Alistipes } & 375.4841 & -1.22787 & 0.567936 & -2.162 & 0.030618 & 0.219356\end{array}$

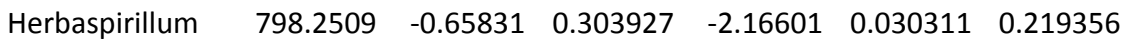

$\begin{array}{llllllll}\text { Ferroplasma } & 5.551802 & 3.120732 & 1.434183 & 2.175965 & 0.029558 & 0.219356\end{array}$

$\begin{array}{llllllll}\text { Nitratireductor } & 47.69866 & -0.82635 & 0.380426 & -2.17217 & 0.029843 & 0.219356\end{array}$

$\begin{array}{llllllll}\text { Cupriavidus } & 8028.509 & -0.76613 & 0.352057 & -2.17615 & 0.029544 & 0.219356\end{array}$

Gibbsiella $\quad \begin{array}{llllllll}29.11858 & -1.02172 & 0.471412 & -2.16736 & 0.030207 & 0.219356\end{array}$

$\begin{array}{lllllll}\text { Brenneria } & 31.349 & -0.9116 & 0.421625 & -2.16211 & 0.030609 & 0.219356\end{array}$

$\begin{array}{llllllll}\text { Filimonas } & 69.44225 & -0.80753 & 0.374392 & -2.15691 & 0.031013 & 0.220536\end{array}$

$\begin{array}{llllllll}\text { Candidatus Bloch } 26.26265 & 1.798287 & 0.834915 & 2.153856 & 0.031251 & 0.220598\end{array}$

$\begin{array}{llllllll}\text { Azoarcus } & 646.684 & -0.64346 & 0.299556 & -2.14805 & 0.03171 & 0.222199\end{array}$

$\begin{array}{lllllllll}\text { Sulfuriferula } & 16.82915 & -1.32655 & 0.618781 & -2.14381 & 0.032048 & 0.222941\end{array}$

$\begin{array}{llllllll}\text { Cyanobium } & 88.39987 & -0.87717 & 0.415904 & -2.10907 & 0.034939 & 0.223968\end{array}$

$\begin{array}{llllllll}\text { Nitrospira } & 75.57332 & -0.77595 & 0.363712 & -2.13341 & 0.032891 & 0.223968\end{array}$

$\begin{array}{llllllll}\text { Methylosinus } & 47.4255 & -1.01026 & 0.478837 & -2.10983 & 0.034873 & 0.223968\end{array}$

$\begin{array}{llllllll}\text { Methylobacillus } & 19.14536 & -1.4755 & 0.700711 & -2.10572 & 0.035228 & 0.223968\end{array}$

$\begin{array}{llllllll}\text { Candidatus Solibc } & 55.41394 & -0.79361 & 0.374087 & -2.12147 & 0.033882 & 0.223968\end{array}$

$\begin{array}{llllllll}\text { Ectothiorhodospi } & 27.76752 & -0.97788 & 0.46026 & -2.12462 & 0.033618 & 0.223968\end{array}$

$\begin{array}{lllllllll}\text { Formosa } & 30.51289 & 1.140292 & 0.539052 & 2.115366 & 0.034399 & 0.223968\end{array}$

$\begin{array}{llllllll}\text { Desulfurivibrio } & 12.58888 & -1.51005 & 0.711095 & -2.12355 & 0.033708 & 0.223968\end{array}$

$\begin{array}{llllllll}\text { Phaeobacter } & 150.1708 & -0.65263 & 0.305508 & -2.13623 & 0.032661 & 0.223968\end{array}$

$\begin{array}{llllllll}\text { Chlorobaculum } & 51.7495 & -0.74684 & 0.354557 & -2.10639 & 0.03517 & 0.223968\end{array}$

$\begin{array}{llllllll}\text { Chelativorans } & 52.82142 & -0.76361 & 0.35817 & -2.13198 & 0.033008 & 0.223968\end{array}$

$\begin{array}{llllllll}\text { Stackebrandtia } & 111.868 & -0.5748 & 0.272191 & -2.11176 & 0.034707 & 0.223968\end{array}$

$\begin{array}{llllllll}\text { Massilia } & 1680.355 & -0.60145 & 0.282348 & -2.13017 & 0.033158 & 0.223968\end{array}$

$\begin{array}{llllllll}\text { Pseudoflavitalea } & 80.35848 & -0.81824 & 0.390272 & -2.09659 & 0.03603 & 0.227559\end{array}$

$\begin{array}{llllllll}\text { Mycolicibacter } & 193.9833 & -0.67486 & 0.322552 & -2.09224 & 0.036417 & 0.228498\end{array}$

$\begin{array}{lllllll}\text { Pseudogulbenkial } & 82.14934 & -0.73953 & 0.355323 & -2.0813 & 0.037407 & 0.232491\end{array}$

$\begin{array}{llllllll}\text { Parvibaculum } & 40.20128 & -0.72275 & 0.347817 & -2.07795 & 0.037714 & 0.232491\end{array}$

$\begin{array}{lllllll}\text { Symbiobacterium } 62.55812 & -0.9495 & 0.45726 & -2.07649 & 0.037848 & 0.232491\end{array}$

$\begin{array}{llllllll}\text { Collimonas } & 497.5483 & -0.63201 & 0.305023 & -2.07201 & 0.038264 & 0.232491\end{array}$

$\begin{array}{llllllll}\text { Devosia } & 166.7052 & -0.64637 & 0.311736 & -2.07346 & 0.038129 & 0.232491\end{array}$

$\begin{array}{lllllll}\text { Desulfuromonas } & 70.57741 & -0.68232 & 0.330353 & -2.06542 & 0.038883 & 0.2333\end{array}$

$\begin{array}{llllllll}\text { Acidisphaera } & 49.36085 & -0.7916 & 0.382843 & -2.06769 & 0.038669 & 0.2333\end{array}$ 
$\begin{array}{llllllll}\text { Chondromyces } & 115.8496 & -0.7686 & 0.372774 & -2.06183 & 0.039224 & 0.233881\end{array}$ $\begin{array}{lllllll}125.6071 & -0.67288 & 0.327764 & -2.05295 & 0.040077 & 0.234598\end{array}$ $\begin{array}{llllllll}\text { Gluconacetobact } & 55.45683 & -0.71787 & 0.349444 & -2.05432 & 0.039945 & 0.234598\end{array}$ $\begin{array}{llllllll}\text { Devriesea } & 55.70677 & -0.94182 & 0.457918 & -2.05675 & 0.039711 & 0.234598\end{array}$ Sulfuricaulis $\quad \begin{array}{llllllll}34.18265 & -1.00417 & 0.492142 & -2.0404 & 0.04131 & 0.234663\end{array}$ $\begin{array}{lllllllll}\text { Flavisolibacter } & 47.31715 & -0.97915 & 0.478143 & -2.04781 & 0.040578 & 0.234663\end{array}$ $\begin{array}{llllllll}\text { Wenzhouxiangell. } 43.00072 & -0.89854 & 0.439565 & -2.04416 & 0.040937 & 0.234663\end{array}$

$\begin{array}{llllllll}\text { Clostridioides } & 424.9304 & 0.840729 & 0.410104 & 2.050038 & 0.040361 & 0.234663\end{array}$ $\begin{array}{llllllll}\text { Acetobacter } & 90.27825 & -0.42794 & 0.209659 & -2.04112 & 0.041239 & 0.234663\end{array}$

$\begin{array}{llllllll}\text { Pseudolabrys } & 103.1281 & -0.72501 & 0.356408 & -2.03421 & 0.041931 & 0.236785\end{array}$ $\begin{array}{llllllll}\text { Candidatus Accur } 91.22169 & -0.88756 & 0.436969 & -2.03118 & 0.042236 & 0.237116\end{array}$ $\begin{array}{llllllll}\text { Tistrella } & 99.16057 & -0.76096 & 0.377893 & -2.0137 & 0.044041 & 0.240089\end{array}$ $\begin{array}{llllllll}\text { Chelatococcus } & 160.2895 & -0.74279 & 0.36825 & -2.01708 & 0.043687 & 0.240089\end{array}$ $\begin{array}{llllllll}\text { Dokdonella } & 144.6886 & -0.68459 & 0.340002 & -2.01348 & 0.044064 & 0.240089\end{array}$

$\begin{array}{llllllll}\text { Leadbetterella } & 8.325436 & -1.77524 & 0.884525 & -2.007 & 0.04475 & 0.240089\end{array}$

$\begin{array}{llllllll}\text { Rubinisphaera } & 9.666133 & -1.56168 & 0.778819 & -2.00519 & 0.044943 & 0.240089\end{array}$ $\begin{array}{llllllll}\text { Ahniella } & 17.03069 & -1.10693 & 0.553925 & -1.99834 & 0.04568 & 0.240089\end{array}$ $\begin{array}{llllllll}\text { Chloroflexus } & 18.69939 & -1.17756 & 0.58883 & -1.99983 & 0.045518 & 0.240089\end{array}$ $\begin{array}{llllllll}\text { Paucibacter } & \quad 569.7574 & -0.77172 & 0.386337 & -1.99754 & 0.045767 & 0.240089\end{array}$

$\begin{array}{llllllll}\text { Burkholderia } & 18667.47 & -0.71704 & 0.356274 & -2.01261 & 0.044156 & 0.240089\end{array}$ $\begin{array}{llllllll}\text { Aliivibrio } & 38.31409 & 0.927995 & 0.461359 & 2.011438 & 0.044279 & 0.240089\end{array}$ $\begin{array}{llllllll}\text { Maricaulis } & 20.0503 & -0.87256 & 0.432159 & -2.01908 & 0.043479 & 0.240089\end{array}$ $\begin{array}{llllllll}\text { Vitreoscilla } & 121.5844 & -0.54446 & 0.271794 & -2.0032 & 0.045156 & 0.240089\end{array}$ $\begin{array}{llllllll}\text { Neorhizobium } & 142.5557 & -0.5686 & 0.285324 & -1.99283 & 0.04628 & 0.241462\end{array}$ $\begin{array}{llllllll}\text { Sagittula } & 65.33639 & -0.75065 & 0.378691 & -1.98223 & 0.047453 & 0.244577\end{array}$ $\begin{array}{llllllll}\text { Rubrivivax } & 611.6802 & -0.73353 & 0.369719 & -1.98403 & 0.047252 & 0.244577\end{array}$ $\begin{array}{lllllll}\text { Achromobacter } & 1689.016 & -0.62016 & 0.313125 & -1.98055 & 0.047641 & 0.244577\end{array}$ $\begin{array}{llllllll}\text { Methylocaldum } & 45.01062 & -0.77012 & 0.391451 & -1.96734 & 0.049144 & 0.245194\end{array}$ $\begin{array}{llllllll}\text { Elizabethkingia } & 113.2991 & 0.634719 & 0.322548 & 1.967828 & 0.049088 & 0.245194\end{array}$ $\begin{array}{llllllll}\text { Sulfurifustis } & 49.80448 & -0.68434 & 0.34808 & -1.96604 & 0.049294 & 0.245194\end{array}$ $\begin{array}{llllllll}\text { Mesoplasma } & 40.46939 & 1.22521 & 0.621594 & 1.971078 & 0.048715 & 0.245194\end{array}$ $\begin{array}{llllllll}\text { Thioalkalivibrio } & 156.9496 & -0.58012 & 0.293536 & -1.97633 & 0.048117 & 0.245194\end{array}$ $\begin{array}{llllllll}\text { Cedecea } & 40.74216 & -0.83808 & 0.424855 & -1.97263 & 0.048537 & 0.245194\end{array}$ $\begin{array}{llllllll}\text { Maribacter } & 46.62286 & 0.730065 & 0.371891 & 1.963114 & 0.049633 & 0.245606\end{array}$ $\begin{array}{llllllll}\text { Rhodoplanes } & 112.8927 & -0.69361 & 0.354448 & -1.95688 & 0.050361 & 0.247077\end{array}$ $\begin{array}{llllllll}\text { Curvibacter } & 139.6121 & -0.73863 & 0.37759 & -1.95617 & 0.050445 & 0.247077\end{array}$ $\begin{array}{llllllll}\text { Turneriella } & 8.96966 & -1.4888 & 0.762086 & -1.95358 & 0.050751 & 0.247312\end{array}$ $\begin{array}{llllllll}\text { Rhodopseudomo } & 778.3759 & -0.57641 & 0.296206 & -1.94598 & 0.051657 & 0.247955\end{array}$ $\begin{array}{llllllll}\text { Rhizobacter } & 453.5193 & -0.74037 & 0.379925 & -1.94873 & 0.051328 & 0.247955\end{array}$ $\begin{array}{llllllll}\text { Pandoraea } & 1478.395 & -0.67718 & 0.347918 & -1.94637 & 0.05161 & 0.247955\end{array}$ 


\begin{tabular}{|c|c|c|c|c|c|c|}
\hline & & & & & & \\
\hline chrobactrum & 170.5509 & -0.61067 & 0.314619 & -1.94099 & 0.05226 & 248363 \\
\hline seateles & L8.5792 & -0.70517 & 0.364454 & -1.93487 & 006 & \\
\hline nnobiu: & 23.26903 & 738 & 92 & 93033 & 3566 & \\
\hline canulicella & 3.84629 & -0.76294 & 0.39641 & -1.92462 & 0.054277 & 254175 \\
\hline mo & 316.8727 & 168 & 0.371791 & -1.91419 & 596 & \\
\hline deria & 15417.11 & -0.74867 & 0.390775 & -1.91585 & 0.055384 & .25456 \\
\hline elftia & 1555.15 & -0.70693 & 0.36838 & -1.91901 & 0.05 & \\
\hline roba & 37.15048 & 0.746871 & 90318 & 1.91 & 685 & \\
\hline ast & 63.76894 & -0.90388 & 0.47089 & -1.91952 & 1919 & \\
\hline acter & 76.81581 & 286 & 0.923065 & -1.90979 & 0.05616 & 1994 \\
\hline Dor & 21.89708 & 067 & 0.54 & -1.90862 & 0.056311 & \\
\hline zos & 778.9391 & 058 & 0.294 & -1.90406 & 0.056903 & 6463 \\
\hline us & 14.0 & & 0.8 & -1.8 & 309 & 987 \\
\hline er & 6.411926 & 117 & 0.8 & -1.8 & 707 & 987 \\
\hline jel & 0.4147 & -0.6 & 07 & -1.89736 & 0.057781 & 5987 \\
\hline uvi & 7.312 & -1.5 & 0.84 & -1.89682 & 0.05 & \\
\hline ich & 70.97009 & 0.92 & 0.48 & 1.891101 & 0.058611 & 8103 \\
\hline os & 198. & -0 & 0.2 & -1.8 & 246 & 456 \\
\hline $\mathrm{orc}$ & 1571.313 & 12 & 75 & -1.87921 & 0.06 & 3456 \\
\hline is & 42 & & 0.4 & -1.8 & 274 & 456 \\
\hline s & 125.6041 & & 0.2 & $-1.8 \varepsilon$ & 922 & 456 \\
\hline ta & 336. & 17 & & -1.8 & 163 & 456 \\
\hline riun & 392.6793 & 379 & 0.28 & -1.87627 & 0.0 & 456 \\
\hline ari & 46.31126 & 502 & 317 & -1.87987 & 126 & 456 \\
\hline erri & 27.95294 & 327 & 0.4 & -1.87946 & 0.060182 & 3456 \\
\hline oca & 313.4314 & -0.5 & 353 & -1.88018 & 083 & 3456 \\
\hline & 169. & 57 & 0.30 & -1.8 & 0.06 & 456 \\
\hline os & 481.2075 & 804 & 0.32 & -1.86681 & 0.061928 & 0.258456 \\
\hline Meli & 73.54086 & -0.67814 & 0.36346 & -1.86579 & 0.062071 & 0.258456 \\
\hline piri & 25.58719 & -0.7731 & 0.4 & -1.86435 & 0.062273 & 3456 \\
\hline robiur & 10.75552 & -1.50948 & 0.806675 & -1.87123 & 0.061313 & 0.258456 \\
\hline Cone & 151.7755 & -0.59587 & 0.321 & -1.85484 & 0.063618 & 0.262119 \\
\hline & 669.0389 & -0.75691 & 0.409044 & -1.85044 & 0.064251 & 0.263593 \\
\hline Sand & 143.1197 & -0.57835 & 0.313783 & -1.84316 & 0.065306 & 0.263666 \\
\hline & 96.75577 & -0.8395 & 0.45 & -1.84559 & 0.064951 & 0.263666 \\
\hline & 39.77691 & -0.73193 & 0.397199 & -1.84274 & 0.065367 & 0.263666 \\
\hline Acid & 110.6314 & -0.54375 & 0.294388 & -1.84705 & 0.064739 & 0.263666 \\
\hline & & & 0.400 & -1.83316 & 0.066778 & 0.26443 \\
\hline Viveispirillum & 61.17711 & -0.76183 & 0.415173 & -1.83496 & 0.066511 & 0.26443 \\
\hline
\end{tabular}


$\begin{array}{llllllll}\text { Mannheimia } & 24.58925 & -1.11724 & 0.608252 & -1.8368 & 0.06624 & 0.264436\end{array}$

$\begin{array}{llllllll}\text { Thermosediminib } & 5.90939 & -2.08347 & 1.150003 & -1.81171 & 0.070032 & 0.271234\end{array}$

$\begin{array}{llllllll}\text { Niabella } & 82.58798 & -0.70597 & 0.390114 & -1.80964 & 0.070351 & 0.271234\end{array}$

$\begin{array}{llllllll}\text { Agrobacterium } & 511.7324 & -0.52336 & 0.289021 & -1.81079 & 0.070174 & 0.271234\end{array}$

$\begin{array}{llllllll}\text { Aureimonas } & 67.04548 & -0.80759 & 0.444321 & -1.81759 & 0.069127 & 0.271234\end{array}$

$\begin{array}{lllllllll}\text { Vulgatibacter } & 68.08027 & -0.83881 & 0.462113 & -1.81516 & 0.069499 & 0.271234\end{array}$

$\begin{array}{lllllllll}\text { Lysobacter } & & 595.4976 & -0.55895 & 0.308274 & -1.81316 & 0.069807 & 0.271234\end{array}$

$\begin{array}{llllllll}\text { Polaromonas } & 1013.321 & -0.60771 & 0.337323 & -1.80156 & 0.071615 & 0.271239\end{array}$

$\begin{array}{llllllll}\text { Halobacterium } \quad 48.42077 & -0.70646 & 0.392646 & -1.79923 & 0.071983 & 0.271239\end{array}$

$\begin{array}{llllllll}\text { Rufibacter } & 47.95847 & -0.70709 & 0.392251 & -1.80265 & 0.071444 & 0.271239\end{array}$

$\begin{array}{llllllll}\text { Zymobacter } & & 19.72757 & -1.18671 & 0.656752 & -1.80694 & 0.070772 & 0.271239\end{array}$

$\begin{array}{llllllll}\text { Dietzia } & 9419.783 & -1.0078 & 0.560796 & -1.79709 & 0.072322 & 0.271239\end{array}$

$\begin{array}{llllllll}\text { Rivularia } & 13.85698 & 1.799637 & 1.002441 & 1.795256 & 0.072613 & 0.271239\end{array}$

$\begin{array}{llllllll}\text { Deferribacter } & 6.185401 & 2.550149 & 1.41592 & 1.801054 & 0.071694 & 0.271239\end{array}$

Limnochorda $\quad \begin{array}{lllllll}67.30247 & -0.64858 & 0.360927 & -1.79699 & 0.072338 & 0.271239\end{array}$

$\begin{array}{llllllll}\text { Candidatus Koribi } & 50.42796 & -0.80547 & 0.449321 & -1.79265 & 0.073029 & 0.27142\end{array}$

$\begin{array}{llllllll}\text { Actinomadura } & 91.658 & -0.56901 & 0.317944 & -1.78965 & 0.073509 & 0.27142\end{array}$

$\begin{array}{llllllll}\text { Nitrosospira } & 27.26096 & -0.86793 & 0.484903 & -1.7899 & 0.07347 & 0.27142\end{array}$

$\begin{array}{lllllllll}\text { Magnetospira } & 18.65446 & -0.90898 & 0.50869 & -1.78691 & 0.073951 & 0.272005\end{array}$

$\begin{array}{llllllll}\text { Planococcus } & 127.4786 & 0.601173 & 0.337331 & 1.782146 & 0.074725 & 0.273803\end{array}$

$\begin{array}{llllllll}\text { Rhodanobacter } & 127.6883 & -0.6433 & 0.361446 & -1.77978 & 0.075111 & 0.27417\end{array}$

$\begin{array}{llllllll}\text { Thiolapillus } & 11.5739 & -1.38529 & 0.785306 & -1.76401 & 0.07773 & 0.274342\end{array}$

$\begin{array}{llllllll}\text { Thermanaerovibr } & 9.907115 & -1.80291 & 1.015034 & -1.77621 & 0.075698 & 0.274342\end{array}$

$\begin{array}{llllllll}\text { Allochromatium } & 47.06659 & -0.70008 & 0.395884 & -1.76841 & 0.076992 & 0.274342\end{array}$

$\begin{array}{lllllll}\text { Serpentinomonas } & 262.5297 & -0.63823 & 0.361723 & -1.76441 & 0.077663 & 0.274342\end{array}$

$\begin{array}{llllllll}\text { Verminephrobact } 324.6197 & -0.74309 & 0.418675 & -1.77486 & 0.075921 & 0.274342\end{array}$

$\begin{array}{llllllll}\text { Gilliamella } & 16.32456 & 1.545782 & 0.872776 & 1.771109 & 0.076543 & 0.274342\end{array}$

$\begin{array}{llllllll}\text { Luteitalea } & 92.89886 & -0.55656 & 0.314459 & -1.7699 & 0.076743 & 0.274342\end{array}$

$\begin{array}{llllllll}\text { Acidovorax } & 6003.767 & -0.60607 & 0.343369 & -1.76508 & 0.07755 & 0.274342\end{array}$

$\begin{array}{llllllll}\text { Singulisphaera } & 49.37911 & -0.64 & 0.361387 & -1.77096 & 0.076568 & 0.274342\end{array}$

$\begin{array}{llllllll}\text { Rhizobium } & 1416.802 & -0.36211 & 0.20606 & -1.75732 & 0.078863 & 0.276309\end{array}$

$\begin{array}{llllllll}\text { Variovorax } & 3512.527 & -0.62281 & 0.354321 & -1.75776 & 0.078788 & 0.276309\end{array}$

$\begin{array}{llllllll}\text { Phenylobacteriun } & 187.5564 & -0.64792 & 0.371692 & -1.74317 & 0.081304 & 0.282796\end{array}$

$\begin{array}{lllllll}\text { Hungateiclostridii } & 41.18104 & 0.802613 & 0.460245 & 1.743882 & 0.08118 & 0.282796\end{array}$

$\begin{array}{llllllll}\text { Vibrio } & 420.333 & 0.401826 & 0.231315 & 1.737136 & 0.082363 & 0.285446\end{array}$

$\begin{array}{llllllll}\text { Acidimicrobium } & 25.16354 & -1.1917 & 0.687232 & -1.73405 & 0.082909 & 0.286304\end{array}$

$\begin{array}{llllllll}\text { Sulfuritalea } & 135.3515 & -0.79804 & 0.46169 & -1.72851 & 0.083897 & 0.287719\end{array}$

$\begin{array}{lllllll}\text { Denitrobacterium } & 11.47692 & -1.5346 & 0.887877 & -1.72839 & 0.083918 & 0.287719\end{array}$ 
$\begin{array}{llllllll}\text { Gordonibacter } \quad 153.9833 & -0.72076 & 0.419596 & -1.71774 & 0.085844 & 0.291288\end{array}$ $\begin{array}{llllllll}\text { Mitsuaria } & 819.3828 & -0.62757 & 0.364904 & -1.71982 & 0.085466 & 0.291288\end{array}$

$\begin{array}{llllllll}\text { Candidatus Kinetı } & 35.21797 & 1.172157 & 0.682438 & 1.717602 & 0.085869 & 0.291288\end{array}$

$\begin{array}{llllllll}\text { Thermobifida } & 57.5563 & -0.72651 & 0.424846 & -1.71006 & 0.087255 & 0.294947\end{array}$

$\begin{array}{llllllll}\text { Rhodoferax } & 1121.966 & -0.59781 & 0.350256 & -1.70678 & 0.087862 & 0.295958\end{array}$

$\begin{array}{llllllll}\text { Castellaniella } & 129.6143 & -0.64844 & 0.380375 & -1.70473 & 0.088246 & 0.296209\end{array}$

$\begin{array}{lllllll}\text { Alicycliphilus } & 1020.301 & -0.64593 & 0.379923 & -1.70017 & 0.0891 & 0.296911\end{array}$

$\begin{array}{llllllll}\text { Hydrogenophaga } & 1069.722 & -0.59624 & 0.350435 & -1.70143 & 0.088862 & 0.296911\end{array}$

$\begin{array}{llllllll}\text { Peptoclostridium } & 8.843755 & -2.08298 & 1.226246 & -1.69866 & 0.089382 & 0.296911\end{array}$

$\begin{array}{lllllllll}\text { Rhodobaca } & 13.22583 & -1.21724 & 0.71763 & -1.69619 & 0.089849 & 0.297432\end{array}$

$\begin{array}{lllllllll}\text { Thermus } & 153.6987 & -0.527 & 0.310997 & -1.69455 & 0.090161 & 0.297438\end{array}$

$\begin{array}{llllllll}\text { Marinomonas } & 30.17464 & 0.80757 & 0.479325 & 1.684808 & 0.092026 & 0.298462\end{array}$

$\begin{array}{lllllllll}\text { Sporosarcina } & 55.60376 & 0.657001 & 0.38975 & 1.685697 & 0.091854 & 0.298462\end{array}$

$\begin{array}{llllllll}\text { Gemmata } & 107.905 & -0.56589 & 0.335225 & -1.68808 & 0.091395 & 0.298462\end{array}$

$\begin{array}{llllllll}\text { Mariprofundus } & 7.834838 & -1.28739 & 0.761431 & -1.69074 & 0.090886 & 0.298462\end{array}$

$\begin{array}{lllllllll}\text { Sinorhizobium } & 490.7736 & -0.47417 & 0.281229 & -1.68605 & 0.091787 & 0.298462\end{array}$

$\begin{array}{llllllll}\text { Oligotropha } & 90.01068 & -0.77974 & 0.464765 & -1.67771 & 0.093404 & 0.301911\end{array}$

$\begin{array}{llllllll}\text { Alcanivorax } & 161.4023 & -0.48228 & 0.288127 & -1.67384 & 0.094162 & 0.302375\end{array}$

$\begin{array}{lllllll}\text { Magnetospirillum } 488.3525 & -0.6694 & 0.400321 & -1.67216 & 0.094492 & 0.302375\end{array}$

$\begin{array}{llllllll}\text { Blattabacterium } & 32.66303 & 1.094536 & 0.654257 & 1.672943 & 0.094338 & 0.302375\end{array}$

$\begin{array}{llllllll}\text { Alkalilimnicola } & 32.27428 & -0.63525 & 0.382143 & -1.66235 & 0.096443 & 0.303025\end{array}$

$\begin{array}{lllllll}\text { Haloarcula } & 29.28672 & -0.81902 & 0.49203 & -1.66457 & 0.095999 & 0.303025\end{array}$

$\begin{array}{lllllllll}\text { Thermosipho } & 12.52226 & 1.463608 & 0.877147 & 1.668601 & 0.095196 & 0.303025\end{array}$

$\begin{array}{llllllll}\text { Acaryochloris } & 10.85162 & -1.4515 & 0.873548 & -1.66162 & 0.096589 & 0.303025\end{array}$

$\begin{array}{llllllll}\text { Halotalea } & 41.1766 & -0.9798 & 0.587813 & -1.66685 & 0.095544 & 0.303025\end{array}$

$\begin{array}{llllllll}\text { Sneathia } & 16.14543 & -1.69359 & 1.016795 & -1.66562 & 0.09579 & 0.303025\end{array}$

$\begin{array}{llllllll}\text { Caulobacter } & 688.5792 & -0.53217 & 0.321342 & -1.65609 & 0.097704 & 0.30467\end{array}$

$\begin{array}{llllllll}\text { Tsukamurella } & 494.9575 & -0.64285 & 0.388224 & -1.65587 & 0.097748 & 0.30467\end{array}$

$\begin{array}{lllllll}\text { Candidatus Desul } & 9.400194 & -1.28019 & 0.774435 & -1.65306 & 0.098318 & 0.305455\end{array}$

$\begin{array}{llllllll}\text { Stappia } & 47.90579 & -0.73386 & 0.449393 & -1.63301 & 0.102467 & 0.306748\end{array}$

$\begin{array}{llllllll}\text { Frondihabitans } & 221.1655 & -0.46043 & 0.280624 & -1.64074 & 0.100851 & 0.306748\end{array}$

$\begin{array}{llllllll}\text { Streptacidiphilus } & 89.24745 & -0.56474 & 0.344035 & -1.64151 & 0.100692 & 0.306748\end{array}$

$\begin{array}{lllllllll}\text { Jeotgalibaca } & 19.98855 & -1.04047 & 0.634783 & -1.6391 & 0.101193 & 0.306748\end{array}$

$\begin{array}{llllllll}\text { Neisseria } & 2840.677 & -0.78107 & 0.47876 & -1.63144 & 0.102797 & 0.306748\end{array}$

$\begin{array}{lllllllll}\text { Roseiflexus } & & 33.92029 & -0.83716 & 0.507554 & -1.6494 & 0.099066 & 0.306748\end{array}$

$\begin{array}{llllllll}\text { Dyadobacter } & 35.46747 & -0.84485 & 0.516144 & -1.63685 & 0.101661 & 0.306748\end{array}$

$\begin{array}{llllllll}\text { Micromonospora } & 1364.13 & -0.41423 & 0.254208 & -1.6295 & 0.103208 & 0.306748\end{array}$

$\begin{array}{llllllll}\text { Desulfococcus } & 24.65118 & -0.82762 & 0.504174 & -1.64153 & 0.100687 & 0.306748\end{array}$

$\begin{array}{llllllll}\text { Comamonas } & 1259.088 & -0.54932 & 0.33533 & -1.63815 & 0.101389 & 0.306748\end{array}$

$\begin{array}{llllllll}\text { Aminomonas } & 10.3649 & -1.08235 & 0.663017 & -1.63246 & 0.102582 & 0.306748\end{array}$ 
$\begin{array}{llllll}28.6045 & -0.64389 & 0.394901 & -1.63051 & 0.102994 & 0.306748\end{array}$

Breoghania

$\begin{array}{lllllll}48.02763 & -0.90401 & 0.552102 & -1.63739 & 0.101548 & 0.306748\end{array}$

$\begin{array}{lllllllll}\text { Paucimonas } & 25.36404 & -0.68265 & 0.416786 & -1.63788 & 0.101446 & 0.306748\end{array}$

$\begin{array}{lllllllll}\text { Trichodesmium } & 13.84461 & 1.405047 & 0.863261 & 1.627604 & 0.103609 & 0.306989\end{array}$

$\begin{array}{llllllll}\text { Sideroxydans } & 36.10873 & -0.70009 & 0.431212 & -1.62354 & 0.104474 & 0.30756\end{array}$

$\begin{array}{lllllllll}\text { Sulfurimonas } & 11.0008 & 1.712359 & 1.055584 & 1.622191 & 0.104762 & 0.30756\end{array}$

$\begin{array}{llllllll}\text { Luteimonas } & 205.0794 & -0.56489 & 0.347741 & -1.62445 & 0.104279 & 0.30756\end{array}$

$\begin{array}{llllllll}\text { Simiduia } & 8.46266 & -1.38666 & 0.856424 & -1.61912 & 0.105421 & 0.308549\end{array}$

$\begin{array}{lllllllll}\text { Anderseniella } & 17.65278 & -0.79522 & 0.492859 & -1.61347 & 0.106642 & 0.31023\end{array}$

$\begin{array}{llllllll}\text { Methylovorus } & 30.46046 & -0.74115 & 0.459252 & -1.61381 & 0.106568 & 0.31023\end{array}$

$\begin{array}{llllllll}\text { Micropruina } & 154.3115 & -0.49598 & 0.308838 & -1.60594 & 0.108287 & 0.314065\end{array}$

$\begin{array}{llllllll}\text { Bacteroides } & 3025.104 & -0.90569 & 0.5646 & -1.60413 & 0.108685 & 0.314271\end{array}$

$\begin{array}{llllllll}\text { Liberibacter } & & 15.84691 & 1.269955 & 0.806556 & 1.574541 & 0.115362 & 0.315512\end{array}$

$\begin{array}{llllllll}\text { Eggerthella } & 68.18041 & -0.65566 & 0.410999 & -1.59529 & 0.110647 & 0.315512\end{array}$

$\begin{array}{llllllll}\text { Mycolicibacteriur } & 3265.406 & -0.58133 & 0.366721 & -1.5852 & 0.112921 & 0.315512\end{array}$

$\begin{array}{llllllll}\text { Aromatoleum } & 102.501 & -0.70461 & 0.447459 & -1.57468 & 0.11533 & 0.315512\end{array}$

$\begin{array}{llllllll}\text { Prauserella } & 85.67317 & -0.5622 & 0.356667 & -1.57626 & 0.114966 & 0.315512\end{array}$

$\begin{array}{llllllll}\text { Beutenbergia } & 99.82996 & -0.60261 & 0.377617 & -1.59582 & 0.11053 & 0.315512\end{array}$

$\begin{array}{llllllll}\text { Crenobacter } & 62.42948 & -0.65318 & 0.415436 & -1.57228 & 0.115885 & 0.315512\end{array}$

$\begin{array}{llllllll}\text { Desulfarculus } & 45.02333 & -0.82201 & 0.516318 & -1.59207 & 0.11137 & 0.315512\end{array}$

$\begin{array}{llllllll}\text { Plantibacter } & 252.2058 & -0.49035 & 0.309368 & -1.585 & 0.112967 & 0.315512\end{array}$

$\begin{array}{lllllll}\text { Simplicispira } & 284.2342 & -0.57333 & 0.360949 & -1.5884 & 0.112197 & 0.315512\end{array}$

$\begin{array}{llllllll}\text { Porphyrobacter } & 141.2203 & -0.50712 & 0.318279 & -1.59331 & 0.11109 & 0.315512\end{array}$

$\begin{array}{llllllll}\text { Solitalea } & 37.35774 & -1.28926 & 0.823244 & -1.56607 & 0.117331 & 0.315512\end{array}$

$\begin{array}{llllllll}\text { Janthinobacteriur } & 780.3968 & -0.48604 & 0.310323 & -1.56623 & 0.117295 & 0.315512\end{array}$

$\begin{array}{llllllll}\text { Thermobacillus } & 32.06261 & -0.78996 & 0.503164 & -1.56999 & 0.116417 & 0.315512\end{array}$

$\begin{array}{llllllll}\text { Kozakia } & 9.323654 & -1.06482 & 0.67927 & -1.5676 & 0.116975 & 0.315512\end{array}$

$\begin{array}{llllllll}\text { Mucinivorans } & 5.741704 & -1.95435 & 1.236171 & -1.58097 & 0.113885 & 0.315512\end{array}$

$\begin{array}{lllllll}\text { Parasaccharibact، } & 7.684368 & -1.35842 & 0.859887 & -1.57977 & 0.11416 & 0.315512\end{array}$

$\begin{array}{llllllll}\text { Methylococcus } & 53.71728 & -0.75524 & 0.481175 & -1.56958 & 0.116512 & 0.315512\end{array}$

$\begin{array}{lllllll}\text { Methylocystis } & 86.04393 & -0.47856 & 0.302916 & -1.57985 & 0.114141 & 0.315512\end{array}$

$\begin{array}{llllllll}\text { Microvirga } & 156.3065 & -0.5223 & 0.331669 & -1.57477 & 0.11531 & 0.315512\end{array}$

$\begin{array}{llllllll}\text { Streptomyces } & 8440.292 & -0.40591 & 0.257595 & -1.57575 & 0.115084 & 0.315512\end{array}$

$\begin{array}{llllllll}\text { Oscillatoria } & 34.32137 & 1.084893 & 0.683101 & 1.588189 & 0.112244 & 0.315512\end{array}$

$\begin{array}{llllllll}\text { Aquitalea } & 47.60303 & -0.63122 & 0.400339 & -1.57672 & 0.11486 & 0.315512\end{array}$

$\begin{array}{lllllll}\text { Caldicellulosirupt } & 42.85725 & 0.651303 & 0.408316 & 1.595097 & 0.110691 & 0.315512\end{array}$

$\begin{array}{llllllll}\text { Brevirhabdus } & 42.96909 & -0.67892 & 0.427887 & -1.58668 & 0.112585 & 0.315512\end{array}$

$\begin{array}{llllllll}\text { Opitutus } & 103.4462 & -0.5788 & 0.370168 & -1.56362 & 0.117907 & 0.316176\end{array}$

$\begin{array}{llllllll}\text { Spirosoma } & 130.3614 & -0.52494 & 0.337087 & -1.55728 & 0.119404 & 0.317529\end{array}$

$\begin{array}{llllllll}\text { Aminobacter } & 151.2694 & -0.54933 & 0.352594 & -1.55796 & 0.119242 & 0.317529\end{array}$ 


\begin{tabular}{|c|c|c|c|c|c|c|}
\hline & & & & & & \\
\hline avonifractor & 122.3365 & -0.93309 & 0.604231 & -1.54425 & 0.122527 & 318848 \\
\hline sinibacillus & 100.336 & 0.541454 & 095 & 2826 & .22873 & \\
\hline & 36.51 & 1.003283 & 999 & & 038 & \\
\hline dellovibrio & 1.92616 & -0.60652 & 0.393016 & -1.54324 & 0.122772 & 318848 \\
\hline onas & 3.62754 & -0.70739 & 0.457704 & & 2222 & 848 \\
\hline & 946591 & -1.48152 & 0.957383 & 54746 & 0.121752 & 318848 \\
\hline ranulosicoccus & 8.678677 & -1.09264 & 0.706538 & 647 & 21991 & \\
\hline & 226.2131 & -0.37532 & 0.24328 & & 22889 & 848 \\
\hline tium & 51.64636 & -0.53827 & 0.347987 & 682 & 0.121908 & \\
\hline er & 66.77889 & -0.49048 & 0.320572 & 002 & 126011 & 066 \\
\hline spo & 84.25941 & & 1396 & & 5407 & \\
\hline ium & 109.1227 & -0.60312 & 0.395065 & 663 & 26853 & 5485 \\
\hline & 23.1 & -0.91 & 327 & & 728 & 707 \\
\hline$n$ & 10.51623 & -1.1 & 261 & & 625 & 719 \\
\hline & 285.427 & -0.68625 & 5207 & 301 & 012 & 393 \\
\hline oe & 52.81 & -0.7 & 0.51 & & 337 & \\
\hline $\operatorname{arc}$ & 228.2962 & 0.802323 & 0.531983 & 1.5 & 151 & 3993 \\
\hline e & 12.26 & -1.0 & 0.7 & & 796 & 369 \\
\hline $\mathrm{p}$ & 76.7861 & -0.62402 & 0.415606 & & 233 & 588 \\
\hline & 69 & & 257 & & 968 & 674 \\
\hline an & 1006.324 & -0.60 & 014 & 867 & 396 & 674 \\
\hline le & 84.75 & -0.5 & 0.39 & & 582 & 944 \\
\hline ate & 18.62216 & $-0.9<$ & 0.63 & 534 & 453 & 632 \\
\hline Im & 417.6975 & 0.44 & 068 & 691 & 956 & 308 \\
\hline b & 12.09359 & 1.426539 & 0.963732 & 1.480223 & 814 & 808 \\
\hline ur & 61.44361 & -0.41927 & 0.283243 & 025 & 808 & 808 \\
\hline & 8.763 & -1.5 & 1.020 & & 507 & 808 \\
\hline & 23.77555 & -0.73611 & 0.49915 & 473 & 285 & 46204 \\
\hline & 30.88298 & -0.57713 & 0.393048 & -1.46834 & 0.142011 & 0.348671 \\
\hline & 529.3 & -0.59143 & 0.402472 & & 699 & 3671 \\
\hline Nias & 104.1593 & -0.61507 & 0.419745 & 534 & 2829 & 0.349786 \\
\hline Mic & 6.663545 & 3.388553 & 2.322399 & 075 & 545 & 0784 \\
\hline & .852549 & -1.73604 & 1.190277 & 852 & 0.144698 & 0.350784 \\
\hline Nonc & 213.2137 & -0.45146 & 0.308853 & -1.46174 & 0.143813 & 0.350784 \\
\hline & 531.8622 & -0.46941 & 0.321438 & 034 & 197 & 0784 \\
\hline rank & 495.9309 & -0.41858 & 0.289627 & 524 & 0.148391 & 0.357928 \\
\hline licyclobacillus & 93.74074 & -0.6014 & 0.41577 & -1.44647 & 0.148045 & 0.357928 \\
\hline & 86.34167 & -0.50018 & & & 0.148982 & 0.35845 \\
\hline hodovulum & 149.083 & -0.56047 & 0.390841 & -1.43401 & 0.151569 & 0.35927 \\
\hline
\end{tabular}


$\begin{array}{llllllll}\text { Planctomyces } & 136.9156 & -0.4096 & 0.28559 & -1.43423 & 0.151506 & 0.359274\end{array}$

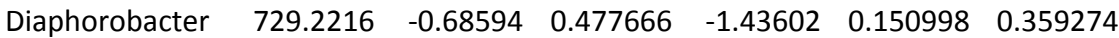

$\begin{array}{llllllll}\text { Beijerinckia } & 17.47694 & -0.89754 & 0.624575 & -1.43705 & 0.150704 & 0.359274\end{array}$

$\begin{array}{llllllll}\text { Hydrogenophilus } & 33.03657 & 0.897275 & 0.623695 & 1.438643 & 0.150252 & 0.359274\end{array}$

$\begin{array}{llllllll}\text { Tatumella } & 22.86477 & -0.96627 & 0.672007 & -1.43789 & 0.150466 & 0.359274\end{array}$

$\begin{array}{llllllll}\text { Kiritimatiella } & 23.25193 & -0.83481 & 0.582857 & -1.43228 & 0.152065 & 0.359563\end{array}$

$\begin{array}{llllllll}\text { Dermabacter } & 1539.663 & -0.67627 & 0.472976 & -1.42982 & 0.152768 & 0.360336\end{array}$

$\begin{array}{lllllllll}\text { Oceanisphaera } & 8.411693 & -1.41204 & 0.990287 & -1.42589 & 0.153901 & 0.361712\end{array}$

$\begin{array}{lllllll}\text { Streptosporangiu } & 202.8271 & -0.42024 & 0.294868 & -1.42518 & 0.154104 & 0.361712\end{array}$

$\begin{array}{llllllll}\text { Microcoleus } & 11.26762 & -1.47964 & 1.041496 & -1.42069 & 0.155408 & 0.363883\end{array}$

$\begin{array}{llllllll}\text { Chitinophaga } & 102.3824 & -0.38957 & 0.2747 & -1.41817 & 0.15614 & 0.364706\end{array}$

$\begin{array}{llllllll}\text { Zobellella } & 56.05209 & -0.69537 & 0.491608 & -1.41448 & 0.157221 & 0.36634\end{array}$

$\begin{array}{llllllll}\text { Ketogulonicigenil } & 47.94712 & -0.50846 & 0.361179 & -1.40778 & 0.159196 & 0.369731\end{array}$

$\begin{array}{llllllll}\text { Halorubrum } & 50.22931 & -0.64188 & 0.456229 & -1.40693 & 0.159447 & 0.369731\end{array}$

$\begin{array}{lllllllll}\text { Lentibacillus } & 9.182485 & -1.30728 & 0.930704 & -1.40461 & 0.160137 & 0.370437\end{array}$

$\begin{array}{llllllll}\text { Pelagibacterium } & 35.09947 & -0.60874 & 0.434429 & -1.40125 & 0.16114 & 0.37097\end{array}$

$\begin{array}{llllllll}\text { Trueperella } & 136.1428 & -0.46537 & 0.332038 & -1.40157 & 0.161045 & 0.37097\end{array}$

$\begin{array}{llllllll}\text { Lentzea } & 138.2869 & -0.40304 & 0.288127 & -1.39884 & 0.161862 & 0.371502\end{array}$

$\begin{array}{llllllll}\text { Actinoplanes } & 723.5694 & -0.43783 & 0.313206 & -1.39789 & 0.162145 & 0.371502\end{array}$

$\begin{array}{llllllll}\text { Thiocystis } & 39.71191 & -0.78858 & 0.56524 & -1.39512 & 0.16298 & 0.372525\end{array}$

$\begin{array}{llllllll}\text { Thiodictyon } & 61.75158 & -0.48906 & 0.355188 & -1.3769 & 0.168542 & 0.372746\end{array}$

$\begin{array}{llllllll}\text { Celeribacter } & 148.6785 & -0.44398 & 0.324 & -1.37032 & 0.170586 & 0.372746\end{array}$

$\begin{array}{llllllll}\text { Maritalea } & & 11.21589 & -1.20074 & 0.867834 & -1.3836 & 0.16648 & 0.372746\end{array}$

$\begin{array}{llllllll}\text { Haloferax } & 42.57577 & -0.52097 & 0.375955 & -1.38571 & 0.165834 & 0.372746\end{array}$

$\begin{array}{lllllllll}\text { Sutterella } & 29.87603 & -0.64539 & 0.468072 & -1.37883 & 0.167946 & 0.372746\end{array}$

$\begin{array}{llllllll}\text { Auritidibacter } & 169.7226 & -0.53984 & 0.394636 & -1.36795 & 0.171327 & 0.372746\end{array}$

$\begin{array}{lllllll}\text { Saccharomonosp' } 472.9596 & -0.47287 & 0.345885 & -1.36713 & 0.171586 & 0.372746\end{array}$

$\begin{array}{lllllllll}\text { Pseudovibrio } & 6.632825 & -1.04648 & 0.751115 & -1.39324 & 0.163547 & 0.372746\end{array}$

$\begin{array}{llllllll}\text { Marinobacter } & 171.5936 & -0.28829 & 0.210129 & -1.37195 & 0.17008 & 0.372746\end{array}$

$\begin{array}{llllllll}\text { Rhodothermus } & 57.40825 & -0.63381 & 0.463264 & -1.36814 & 0.171269 & 0.372746\end{array}$

$\begin{array}{lllllll}\text { Acidothermus } & 26.64245 & -0.8367 & 0.61206 & -1.36702 & 0.171618 & 0.372746\end{array}$

$\begin{array}{llllllll}\text { Amycolatopsis } & 922.209 & -0.4428 & 0.322974 & -1.37102 & 0.170369 & 0.372746\end{array}$

$\begin{array}{llllllll}\text { Halanaerobium } & 23.70768 & 0.801699 & 0.580957 & 1.379963 & 0.167598 & 0.372746\end{array}$

$\begin{array}{llllllll}\text { Chthonomonas } & 8.230239 & -1.29526 & 0.944555 & -1.37129 & 0.170285 & 0.372746\end{array}$

$\begin{array}{llllllll}\text { Nitrospirillum } & 123.5791 & -0.51749 & 0.375119 & -1.37954 & 0.167727 & 0.372746\end{array}$

$\begin{array}{lllllll}\text { Altererythrobact } \quad 369.8429 & -0.464 & 0.335008 & -1.38504 & 0.16604 & 0.372746\end{array}$

$\begin{array}{llllllll}\text { Blastomonas } & 66.55685 & -0.45304 & 0.32828 & -1.38003 & 0.167578 & 0.372746\end{array}$

$\begin{array}{llllllll}\text { Aquabacterium } & 976.754 & -0.66034 & 0.478858 & -1.379 & 0.167896 & 0.372746\end{array}$

$\begin{array}{llllllll}\text { Oxalobacter } & 7.643576 & -1.05555 & 0.772072 & -1.36716 & 0.171575 & 0.372746\end{array}$

$\begin{array}{llllllll}\text { Prosthecochloris } & 25.13602 & -0.78107 & 0.567701 & -1.37585 & 0.168867 & 0.372746\end{array}$ 
$\begin{array}{llllllll}\text { Ralstonia } & 105309.4 & -0.50182 & 0.362172 & -1.38557 & 0.165878 & 0.372746\end{array}$ $\begin{array}{llllllll}\text { Providencia } & 75.3085 & 0.555233 & 0.403466 & 1.376155 & 0.168773 & 0.372746\end{array}$

$\begin{array}{llllllll}\text { Immundisolibact } \quad 78.98035 & -0.46151 & 0.338888 & -1.36184 & 0.17325 & 0.37544\end{array}$

$\begin{array}{llllllll}\text { Caldilinea } & 16.0765 & -0.80183 & 0.59075 & -1.35731 & 0.174684 & 0.377695\end{array}$

$\begin{array}{lllllll}\text { Ottowia } & 378.2706 & -0.58603 & 0.433141 & -1.35298 & 0.176062 & 0.379819\end{array}$

$\begin{array}{llllllll}\text { Bernardetia } & 25.08347 & 0.964919 & 0.714157 & 1.35113 & 0.176654 & 0.380241\end{array}$

$\begin{array}{llllllll}\text { Kutzneria } & 158.8718 & -0.37561 & 0.278968 & -1.34641 & 0.17817 & 0.382647\end{array}$

$\begin{array}{llllllll}\text { Hartmannibacter } & 51.08944 & -0.53236 & 0.3969 & -1.34129 & 0.179825 & 0.384901\end{array}$

$\begin{array}{llllllll}\text { Azotobacter } & 188.164 & -0.48282 & 0.360127 & -1.34069 & 0.180021 & 0.384901\end{array}$

$\begin{array}{llllllll}\text { Beggiatoa } & 12.89407 & -1.23073 & 0.920487 & -1.33704 & 0.181208 & 0.386578\end{array}$

$\begin{array}{llllllll}\text { Verrucomicrobiur } & 81.38629 & -0.66227 & 0.496801 & -1.33307 & 0.182508 & 0.388487\end{array}$

$\begin{array}{llllllll}\text { Pigmentiphaga } & 143.9347 & -0.43484 & 0.326565 & -1.33156 & 0.183006 & 0.388685\end{array}$

$\begin{array}{llllllll}\text { Frischella } & 5.868071 & -1.48192 & 1.114979 & -1.3291 & 0.183816 & 0.389543\end{array}$

$\begin{array}{lllllll}\text { Chromohalobact } ~ & 29.32787 & -0.53564 & 0.403539 & -1.32735 & 0.184395 & 0.389754\end{array}$

$\begin{array}{llllllll}\text { Filomicrobium } & 13.35083 & -0.88554 & 0.668272 & -1.32512 & 0.185133 & 0.389754\end{array}$

$\begin{array}{lllllllll}\text { Photorhabdus } & 37.15836 & 0.568857 & 0.429073 & 1.325779 & 0.184913 & 0.389754\end{array}$

$\begin{array}{llllllll}\text { Pseudonocardia } & 849.9054 & -0.42417 & 0.320989 & -1.32144 & 0.186355 & 0.391468\end{array}$

$\begin{array}{llllllll}\text { Echinicola } & 25.28042 & -0.83364 & 0.634586 & -1.31368 & 0.188955 & 0.395881\end{array}$

$\begin{array}{llllllll}\text { Nitrobacter } & 131.9366 & -0.52222 & 0.39782 & -1.31271 & 0.189281 & 0.395881\end{array}$

$\begin{array}{llllllll}\text { Desulfatibacillum } & 22.8588 & -0.69062 & 0.527749 & -1.30861 & 0.190667 & 0.39705\end{array}$

$\begin{array}{llllllll}\text { Stanieria } & 38.11665 & 1.169934 & 0.893441 & 1.30947 & 0.190375 & 0.39705\end{array}$

$\begin{array}{llllllll}\text { Arachidicoccus } & 79.57458 & -0.41906 & 0.32115 & -1.30487 & 0.191936 & 0.397124\end{array}$

$\begin{array}{llllllll}\text { Jatrophihabitans } & 130.0573 & -0.54595 & 0.418339 & -1.30504 & 0.191878 & 0.397124\end{array}$

$\begin{array}{llllllll}\text { Dechloromonas } & 88.3344 & -0.33809 & 0.259103 & -1.30485 & 0.191943 & 0.397124\end{array}$

$\begin{array}{llllllll}\text { Geodermatophilı } 261.8148 & -0.6241 & 0.478743 & -1.30363 & 0.192361 & 0.397133\end{array}$

$\begin{array}{llllllll}\text { Ureaplasma } & 5.755253 & 2.081812 & 1.598911 & 1.302018 & 0.19291 & 0.397395\end{array}$

$\begin{array}{lllllll}\text { Methylomonas } & 48.40726 & -0.57857 & 0.444773 & -1.30083 & 0.193316 & 0.397395\end{array}$

$\begin{array}{llllllll}\text { Proteus } & 52.42165 & 0.71082 & 0.547446 & 1.298431 & 0.194139 & 0.398235\end{array}$

$\begin{array}{llllllll}\text { Neoasaia } & 24.61859 & -0.63053 & 0.48715 & -1.29432 & 0.195553 & 0.400006\end{array}$

$\begin{array}{llllllll}\text { Starkeya } & 88.57022 & -0.46138 & 0.356693 & -1.29351 & 0.195836 & 0.400006\end{array}$

$\begin{array}{llllllll}\text { Paludisphaera } & 75.95092 & -0.41076 & 0.317924 & -1.29202 & 0.19635 & 0.400204\end{array}$

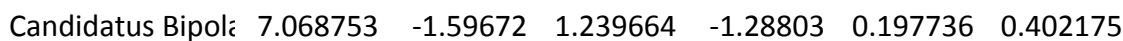

$\begin{array}{llllllll}\text { Ndongobacter } & 63.83242 & -0.99606 & 0.774841 & -1.2855 & 0.198618 & 0.403115\end{array}$

$\begin{array}{llllllll}\text { Chlamydia } & 29.03196 & 0.563719 & 0.439403 & 1.282921 & 0.19952 & 0.404091\end{array}$

$\begin{array}{lllllllll}\text { Campylobacter } \quad 609.3191 & 0.417422 & 0.326197 & 1.279665 & 0.200663 & 0.404698\end{array}$

$\begin{array}{llllllll}\text { Sulfuricella } & 38.77779 & -0.6387 & 0.499041 & -1.27985 & 0.200597 & 0.404698\end{array}$

$\begin{array}{llllllll}\text { Yangia } & 144.0293 & -0.30324 & 0.237485 & -1.27689 & 0.201641 & 0.404986\end{array}$

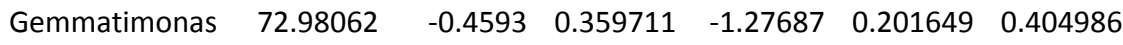

$\begin{array}{llllllll}\text { Sulfurospirillum } & 44.6875 & 0.598389 & 0.470158 & 1.272741 & 0.20311 & 0.407068\end{array}$

$\begin{array}{llllllll}\text { Escherichia } & 611.9084 & 0.447707 & 0.353591 & 1.266172 & 0.205451 & 0.410903\end{array}$ 
$\begin{array}{lllllll}10.73791 & -0.96279 & 0.765374 & -1.25793 & 0.208417 & 0.415967\end{array}$ Eikenella $\begin{array}{llllll}87.67366 & -0.61713 & 0.492173 & -1.25389 & 0.209883 & 0.418024\end{array}$

Marinitoga $\begin{array}{lllllll}6.712612 & 1.507236 & 1.203828 & 1.252035 & 0.210557 & 0.418498\end{array}$ $\begin{array}{llllllll}\text { Actinotignum } & 209.8055 & 0.652932 & 0.522231 & 1.250275 & 0.211199 & 0.418907\end{array}$ Nakamurella $\quad \begin{array}{lllllll}276.2023 & -0.47582 & 0.38162 & -1.24685 & 0.212453 & 0.420526\end{array}$ $\begin{array}{lllllllll}\text { Cnuibacter } & & 183.6917 & -0.66037 & 0.530509 & -1.24478 & 0.213213 & 0.421161\end{array}$ $\begin{array}{llllllll}\text { Actinomyces } & 10645.59 & -0.48317 & 0.389601 & -1.24018 & 0.214909 & 0.423032\end{array}$

$\begin{array}{lllllllll}\text { Fibrobacter } & 8.926343 & -1.10926 & 0.894696 & -1.23982 & 0.215041 & 0.423032\end{array}$ $\begin{array}{llllllll}\text { Staphylococcus } & 429774.2 & 1.270435 & 1.026193 & 1.238008 & 0.215713 & 0.423486\end{array}$ $\begin{array}{llllllll}\text { Candidatus Symb } & 34.23852 & -0.66055 & 0.534681 & -1.23541 & 0.216677 & 0.42451\end{array}$ $\begin{array}{llllllll}\text { Parvimonas } & 229.0009 & -0.5837 & 0.474016 & -1.2314 & 0.218175 & 0.426573\end{array}$ $\begin{array}{llllllll}\text { Auraticoccus } & 114.9347 & -0.44043 & 0.358386 & -1.22893 & 0.219099 & 0.42751\end{array}$ $\begin{array}{llllllll}\text { Erythrobacter } & 279.6508 & -0.33414 & 0.273276 & -1.22273 & 0.22143 & 0.428099\end{array}$ $\begin{array}{llllllll}\text { Gallionella } & 24.39283 & -0.62489 & 0.511231 & -1.22233 & 0.221584 & 0.428099\end{array}$ $\begin{array}{llllllll}\text { Salinibacter } & 26.28125 & -0.6639 & 0.543109 & -1.2224 & 0.221557 & 0.428099\end{array}$ $\begin{array}{llllllll}\text { Colwellia } & 74.08489 & 0.527461 & 0.430937 & 1.223987 & 0.220957 & 0.428099\end{array}$ $\begin{array}{lllllll}\text { Gemmobacter } & 34.26738 & -0.54597 & 0.446713 & -1.2222 & 0.22163 & 0.428099\end{array}$ $\begin{array}{llllllll}\text { Cellulomonas } \quad 588.2067 & -0.42808 & 0.350792 & -1.22032 & 0.222344 & 0.428615\end{array}$ Pimelobacter $\quad \begin{array}{lllllll}200.099 & -0.39731 & 0.326604 & -1.2165 & 0.223793 & 0.430545\end{array}$ $\begin{array}{llllllll}\text { Hoyosella } & 84.04082 & -0.55407 & 0.45667 & -1.21328 & 0.225021 & 0.431225\end{array}$ $\begin{array}{llllllll}\text { Sphingopyxis } & 623.7829 & -0.35867 & 0.295631 & -1.21322 & 0.225045 & 0.431225\end{array}$ $\begin{array}{llllllll}\text { Anabaenopsis } & 27.41316 & -0.8298 & 0.685416 & -1.21066 & 0.226027 & 0.431383\end{array}$ $\begin{array}{llllllll}\text { Pa6virus } & 1046.415 & 0.845544 & 0.697793 & 1.211741 & 0.225612 & 0.431383\end{array}$ $\begin{array}{llllllll}\text { Salinisphaera } & 29.32365 & -0.48852 & 0.405804 & -1.20384 & 0.228653 & 0.434974\end{array}$ $\begin{array}{llllllll}\text { Thermosynechoc } & 18.39869 & -0.84176 & 0.70023 & -1.20212 & 0.229316 & 0.434974\end{array}$ $\begin{array}{lllllll}\text { Novosphingobiun } & 461.5752 & -0.32559 & 0.2709 & -1.20189 & 0.229405 & 0.434974\end{array}$ $\begin{array}{llllllll}\text { Parabacteroides } & 257.5667 & -0.99179 & 0.825748 & -1.20108 & 0.229721 & 0.434974\end{array}$ $\begin{array}{llllllll}\text { Halorhodospira } & 33.38652 & -0.50276 & 0.419514 & -1.19844 & 0.230747 & 0.436058\end{array}$ $\begin{array}{llllllll}\text { Aeromicrobium } & 663.4701 & -0.3882 & 0.324455 & -1.19647 & 0.231513 & 0.436645\end{array}$ $\begin{array}{llllllll}\text { Coriobacterium } & 23.72219 & -0.89608 & 0.750855 & -1.19341 & 0.232707 & 0.437289\end{array}$ $\begin{array}{llllllll}\text { Dickeya } & 197.0842 & -0.26294 & 0.22035 & -1.19327 & 0.232765 & 0.437289\end{array}$ $\begin{array}{lllllllll}\text { Corallococcus } & 89.85311 & -0.49621 & 0.417706 & -1.18794 & 0.234859 & 0.438138\end{array}$ $\begin{array}{llllllll}\text { Mageeibacillus } & 31.94118 & -1.11771 & 0.941087 & -1.18768 & 0.234959 & 0.438138\end{array}$ $\begin{array}{llllllll}\text { Oceanobacillus } & 43.35809 & 0.518841 & 0.43693 & 1.187468 & 0.235043 & 0.438138\end{array}$ $\begin{array}{lllllllll}\text { Weissella } & 70.65298 & 0.560122 & 0.470397 & 1.190744 & 0.233754 & 0.438138\end{array}$ $\begin{array}{llllllll}\text { Microbacterium } & 3796.623 & -0.44043 & 0.372868 & -1.18119 & 0.237528 & 0.441057\end{array}$ $\begin{array}{llllllll}\text { Acidithiobacillus } & 43.30361 & -0.47027 & 0.397782 & -1.18223 & 0.237116 & 0.441057\end{array}$ $\begin{array}{llllllll}\text { Odoribacter } & 83.32391 & -0.97022 & 0.823537 & -1.17812 & 0.238751 & 0.442472\end{array}$ $\begin{array}{lllllll}\text { Dinoroseobacter } & 54.44958 & -0.56353 & 0.478816 & -1.17692 & 0.239227 & 0.442502\end{array}$ $\begin{array}{llllllll}\text { Kurthia } & 10.50537 & -1.06813 & 0.910028 & -1.17373 & 0.240501 & 0.444002\end{array}$ 
$\begin{array}{llllllll}\text { Candidatus Nitro } 37.98842 & -0.65846 & 0.568795 & -1.15763 & 0.247014 & 0.452675\end{array}$

$\begin{array}{llllllll}\text { Marinovum } & 45.16872 & -0.51073 & 0.441255 & -1.15746 & 0.247085 & 0.452675\end{array}$

$\begin{array}{llllllll}\text { Bibersteinia } & 7.847548 & -1.17555 & 1.019906 & -1.1526 & 0.249073 & 0.455448\end{array}$

$\begin{array}{llllllll}\text { Desulfallas } & \quad 5.870057 & -1.05314 & 0.919419 & -1.14544 & 0.252026 & 0.459695\end{array}$

$\begin{array}{lllllll}\text { Prevotella } & 3721.933 & -0.43524 & 0.381003 & -1.14234 & 0.253311 & 0.459695\end{array}$

$\begin{array}{llllllll}\text { Butyrivibrio } & 62.18187 & 0.707024 & 0.618521 & 1.143089 & 0.253002 & 0.459695\end{array}$

$\begin{array}{llllllll}\text { Melissococcus } & 9.275291 & 1.266554 & 1.107575 & 1.143539 & 0.252815 & 0.459695\end{array}$

$\begin{array}{llllllll}\text { Sulfitobacter } & 146.8013 & -0.33326 & 0.293227 & -1.13654 & 0.255732 & 0.463212\end{array}$

$\begin{array}{llllllll}\text { Mariniflexile } & 11.24007 & 1.072799 & 0.949849 & 1.129441 & 0.258712 & 0.463666\end{array}$

$\begin{array}{llllllll}\text { Fermentimonas } & 10.17569 & -1.29955 & 1.154133 & -1.126 & 0.260166 & 0.463666\end{array}$

$\begin{array}{llllllll}\text { Sinomonas } & 163.2584 & -0.37713 & 0.334352 & -1.12796 & 0.259339 & 0.463666\end{array}$

$\begin{array}{llllllll}\text { Algibacter } & \quad & 7.996602 & 1.065372 & 0.939453 & 1.134035 & 0.25678 & 0.463666\end{array}$

$\begin{array}{llllllll}\text { Microcella } & 84.50994 & -0.47461 & 0.422061 & -1.12451 & 0.260798 & 0.463666\end{array}$

$\begin{array}{llllllll}\text { Oceanithermus } & 52.28489 & -0.39003 & 0.345365 & -1.12932 & 0.258762 & 0.463666\end{array}$

$\begin{array}{llllllll}\text { Brevundimonas } & 1765.118 & -0.40132 & 0.356745 & -1.12495 & 0.260608 & 0.463666\end{array}$

Nitrosomonas $\quad \begin{array}{llllllll}152.2672 & -0.59563 & 0.529982 & -1.12388 & 0.261065 & 0.463666\end{array}$

$\begin{array}{llllllll}\text { Aquiflexum } & 9.042458 & -0.97465 & 0.86294 & -1.12946 & 0.258705 & 0.463666\end{array}$

$\begin{array}{lllllllll}\text { Arthrospira } & 9.736271 & -0.96431 & 0.858436 & -1.12334 & 0.261295 & 0.463666\end{array}$

$\begin{array}{lllllll}\text { Limnobaculum } & 6.563421 & -0.97328 & 0.859753 & -1.13205 & 0.257613 & 0.463666\end{array}$

$\begin{array}{llllllll}\text { Halopiger } & 18.37543 & -0.87832 & 0.783449 & -1.1211 & 0.262246 & 0.464495\end{array}$

$\begin{array}{lllllllll}\text { Xanthobacter } & 128.938 & -0.39601 & 0.353781 & -1.11937 & 0.262981 & 0.464938\end{array}$

$\begin{array}{llllllll}\text { Martelella } & 153.2839 & -0.43203 & 0.386912 & -1.11661 & 0.26416 & 0.466166\end{array}$

$\begin{array}{llllllll}\text { Halorhabdus } & 18.05047 & -0.65929 & 0.591639 & -1.11435 & 0.265128 & 0.467014\end{array}$

$\begin{array}{llllllll}\text { Haliangium } & 141.1498 & -0.35848 & 0.323236 & -1.10905 & 0.26741 & 0.468176\end{array}$

$\begin{array}{lllllll}\text { Polymorphum } & 76.53721 & -0.39864 & 0.359081 & -1.11017 & 0.266924 & 0.468176\end{array}$

$\begin{array}{llllllll}\text { Kineococcus } & 128.536 & -0.45932 & 0.414442 & -1.10829 & 0.267738 & 0.468176\end{array}$

$\begin{array}{llllllll}\text { Mixta } & 40.97447 & -0.56077 & 0.505369 & -1.10963 & 0.267159 & 0.468176\end{array}$

$\begin{array}{lllllllll}\text { Gordonia } & 4991.276 & -0.50974 & 0.462578 & -1.10196 & 0.270479 & 0.468995\end{array}$

Solimonas $\quad \begin{array}{llllllll}89.95242 & -0.45215 & 0.410465 & -1.10157 & 0.270649 & 0.468995\end{array}$

$\begin{array}{llllllll}\text { Hahella } & 14.50293 & -0.62195 & 0.562521 & -1.10565 & 0.26888 & 0.468995\end{array}$

$\begin{array}{llllllll}\text { Sanguibacter } & \quad 135.2697 & -0.37664 & 0.341741 & -1.10212 & 0.270412 & 0.468995\end{array}$

$\begin{array}{llllllll}\text { Kyrpidia } & 30.67351 & -0.56711 & 0.513467 & -1.10447 & 0.269391 & 0.468995\end{array}$

$\begin{array}{llllllll}\text { Candidatus Aquilı } & 7.834318 & -0.88224 & 0.803498 & -1.098 & 0.272205 & 0.470842\end{array}$

$\begin{array}{lllllll}\text { Methylomicrobiu } & 57.02666 & -0.56654 & 0.516697 & -1.09647 & 0.272875 & 0.471\end{array}$

$\begin{array}{llllllll}\text { Jonesia } & 45.76045 & -0.56133 & 0.512377 & -1.09554 & 0.273278 & 0.471\end{array}$

$\begin{array}{llllllll}\text { Aquaspirillum } & 30.63081 & -0.40384 & 0.369853 & -1.09189 & 0.274881 & 0.472914\end{array}$

$\begin{array}{llllllll}\text { Austwickia } & 66.81253 & -0.48941 & 0.451072 & -1.085 & 0.277924 & 0.473062\end{array}$

$\begin{array}{lllllll}\text { Haloterrigena } & 38.6949 & -0.49487 & 0.455446 & -1.08656 & 0.277232 & 0.473062\end{array}$ 
$\begin{array}{lllllll}\text { Isoptericola } & 274.6441 & -0.36 & 0.330738 & -1.08848 & 0.276384 & 0.473062\end{array}$ $\begin{array}{lllllll}\text { Roseibacterium } & 33.41435 & -0.53982 & 0.496976 & -1.0862 & 0.27739 & 0.473062\end{array}$

$\begin{array}{llllllll}\text { Candidatus Phaec } 14.06176 & -0.84366 & 0.77732 & -1.08534 & 0.277771 & 0.473062\end{array}$

$\begin{array}{llllllll}\text { Methylovulum } & 14.80906 & -1.10638 & 1.017237 & -1.08763 & 0.276759 & 0.473062\end{array}$

$\begin{array}{llllllll}\text { Haemophilus } & 1386.94 & 0.479103 & 0.443677 & 1.079845 & 0.280211 & 0.476111\end{array}$

$\begin{array}{llllllll}\text { Cellvibrio } & 52.97656 & -0.51945 & 0.482367 & -1.07688 & 0.281534 & 0.476573\end{array}$

$\begin{array}{llllllll}\text { Tabrizicola } & 39.34514 & -0.44504 & 0.413642 & -1.0759 & 0.281972 & 0.476573\end{array}$

$\begin{array}{llllllll}\text { Piscirickettsia } & 8.951355 & 1.068564 & 0.991544 & 1.077677 & 0.281178 & 0.476573\end{array}$

$\begin{array}{llllllll}\text { Halopenitus } & 8.769877 & -0.95943 & 0.892805 & -1.07463 & 0.282541 & 0.476695\end{array}$

$\begin{array}{llllllll}\text { Sphingorhabdus } & 71.34105 & -0.38594 & 0.360122 & -1.0717 & 0.283853 & 0.478068\end{array}$

$\begin{array}{llllllll}\text { Nocardia } & 1642.144 & -0.34795 & 0.325227 & -1.06988 & 0.284675 & 0.478613\end{array}$

$\begin{array}{llllllll}\text { Agrococcus } & 269.5979 & -0.41208 & 0.386416 & -1.06641 & 0.286239 & 0.480401\end{array}$

$\begin{array}{llllllll}\text { Cryobacterium } & 257.9756 & -0.34281 & 0.322136 & -1.06419 & 0.287242 & 0.481243\end{array}$

$\begin{array}{llllllll}\text { Tumebacillus } & 34.79467 & -0.40574 & 0.3821 & -1.06188 & 0.288292 & 0.482161\end{array}$

$\begin{array}{llllllll}\text { Candidatus Thiog } & 34.22475 & 0.768652 & 0.727354 & 1.056778 & 0.290613 & 0.485197\end{array}$

$\begin{array}{llllllll}\text { Saccharothrix } & 150.7478 & -0.4058 & 0.387572 & -1.04703 & 0.295084 & 0.491807\end{array}$

$\begin{array}{llllllll}\text { Weeksella } & 10.83434 & -1.23595 & 1.181915 & -1.04572 & 0.295692 & 0.49182\end{array}$

$\begin{array}{llllllll}\text { Finegoldia } & 7857.462 & -0.43297 & 0.41441 & -1.0448 & 0.296117 & 0.49182\end{array}$

$\begin{array}{lllllll}\text { Haliscomenobact } 30.32501 & 0.567626 & 0.545191 & 1.041151 & 0.297806 & 0.493771\end{array}$

$\begin{array}{llllllll}\text { Methylocella } & 34.73078 & -0.48036 & 0.46286 & -1.0378 & 0.299362 & 0.495496\end{array}$

$\begin{array}{llllllll}\text { Pusillimonas } & 18.80486 & -0.62793 & 0.611699 & -1.02654 & 0.304637 & 0.498647\end{array}$

$\begin{array}{llllllll}\text { Neomicrococcus } & 115.0831 & -0.55542 & 0.539402 & -1.02969 & 0.303156 & 0.498647\end{array}$

$\begin{array}{llllllll}\text { Streptococcus } & 35319.43 & -0.47231 & 0.458376 & -1.03039 & 0.302827 & 0.498647\end{array}$

$\begin{array}{llllllll}\text { Zunongwangia } & 8.669293 & 1.068098 & 1.038017 & 1.028979 & 0.303489 & 0.498647\end{array}$

$\begin{array}{llllllll}\text { Pelobacter } & 76.13951 & -0.31693 & 0.307864 & -1.02946 & 0.303265 & 0.498647\end{array}$

$\begin{array}{llllllll}\text { Scytonema } & 32.16726 & 0.516316 & 0.503242 & 1.025979 & 0.304902 & 0.498647\end{array}$

$\begin{array}{llllllll}\text { Blautia } & 148.9723 & -0.421 & 0.410205 & -1.02631 & 0.304745 & 0.498647\end{array}$

$\begin{array}{llllllll}\text { Betapapillomavirı } 754.4662 & -1.12054 & 1.094835 & -1.02348 & 0.306082 & 0.498996\end{array}$

Actinobacillus $\quad \begin{array}{lllllll}102.3218 & -0.61399 & 0.600639 & -1.02222 & 0.306675 & 0.498996\end{array}$

$\begin{array}{llllllll}\text { Geosporobacter } & 18.56622 & -0.95058 & 0.929755 & -1.0224 & 0.30659 & 0.498996\end{array}$

$\begin{array}{lllllll}\text { Pseudarcicella } & 11.48293 & -0.82693 & 0.81044 & -1.02035 & 0.307564 & 0.499596\end{array}$

$\begin{array}{llllllll}\text { Halorientalis } & 17.15971 & -0.60578 & 0.594586 & -1.01883 & 0.308282 & 0.499917\end{array}$

$\begin{array}{llllllll}\text { Eubacterium } & 220.0555 & -0.45592 & 0.448135 & -1.01737 & 0.308977 & 0.500199\end{array}$

$\begin{array}{llllllll}\text { Agromyces } & 303.8156 & -0.37492 & 0.371331 & -1.00966 & 0.312657 & 0.502838\end{array}$

$\begin{array}{llllllll}\text { Syntrophomonas } & 10.04712 & 1.238292 & 1.226557 & 1.009568 & 0.312702 & 0.502838\end{array}$

$\begin{array}{lllllll}\text { Stigmatella } & 63.58261 & -0.3439 & 0.339624 & -1.01258 & 0.311259 & 0.502838\end{array}$

$\begin{array}{llllllll}\text { Georgenia } & 132.6235 & -0.35711 & 0.353127 & -1.01127 & 0.311886 & 0.502838\end{array}$

$\begin{array}{llllllll}\text { Alkaliphilus } & 33.64461 & 0.619234 & 0.615273 & 1.006439 & 0.314205 & 0.504409\end{array}$

$\begin{array}{lllllll}\text { Aurantimicrobiun } 83.56232 & -0.49216 & 0.490045 & -1.00432 & 0.315227 & 0.505205\end{array}$

$\begin{array}{llllllll}\text { Cronobacter } & 142.5909 & -0.34813 & 0.347038 & -1.00316 & 0.315785 & 0.505256\end{array}$ 
$\begin{array}{llllll}15831.4 & -0.29922 & 0.29969 & -0.99842 & 0.318074 & 0.508071\end{array}$ $\begin{array}{lllllll}\text { Dermacoccus } & 3257.387 & 0.572915 & 0.577245 & 0.992498 & 0.320954 & 0.510126\end{array}$ $\begin{array}{llllllll}\text { Shimwellia } & 750.2851 & -1.26069 & 1.269447 & -0.9931 & 0.320659 & 0.510126\end{array}$ Anaerostipes $\quad \begin{array}{llllllll}230.4103 & -0.7092 & 0.714174 & -0.99303 & 0.320695 & 0.510126\end{array}$ $\begin{array}{llllllll}\text { Pelagibaca } & 50.4034 & -0.41121 & 0.416205 & -0.98799 & 0.323157 & 0.512778\end{array}$ $\begin{array}{llllllll}\text { Nitrosococcus } & 24.622 & -0.37006 & 0.375405 & -0.98577 & 0.324247 & 0.513355\end{array}$ $\begin{array}{llllllll}\text { Fibrella } & 52.46655 & -0.50463 & 0.512283 & -0.98507 & 0.32459 & 0.513355\end{array}$

$\begin{array}{llllllll}\text { Methanoculleus } & 20.94618 & -0.4016 & 0.409121 & -0.98162 & 0.326288 & 0.514102\end{array}$ $\begin{array}{llllllll}\text { Tannerella } & 378.8161 & -0.45869 & 0.466951 & -0.98232 & 0.325945 & 0.514102\end{array}$

$\begin{array}{llllllll}\text { Thalassospira } & 33.08575 & -0.37075 & 0.377988 & -0.98085 & 0.326669 & 0.514102\end{array}$ $\begin{array}{llllllll}\text { Arthrobacter } & 2228.952 & -0.37183 & 0.379673 & -0.97934 & 0.327411 & 0.514427\end{array}$ $\begin{array}{lllllll}\text { Kordia } & 7.183607 & 1.255569 & 1.293962 & 0.970329 & 0.331882 & 0.51975\end{array}$ $\begin{array}{llllllll}\text { Negativicoccus } & 258.7426 & -0.56689 & 0.583591 & -0.97139 & 0.331355 & 0.51975\end{array}$ $\begin{array}{lllllll}\text { Pseudarthrobact } \epsilon 47.2038 & -0.36482 & 0.376589 & -0.96875 & 0.33267 & 0.520136\end{array}$ $\begin{array}{llllllll}\text { Pirellula } & 10.40979 & -0.62103 & 0.644041 & -0.96426 & 0.334913 & 0.522791\end{array}$ $\begin{array}{llllllll}\text { Pectobacterium } & 163.0272 & -0.34919 & 0.363226 & -0.96135 & 0.336378 & 0.524225\end{array}$ $\begin{array}{lllllll}\text { Cellulosimicrobiu } & 253.5076 & -0.39058 & 0.407612 & -0.9582 & 0.33796 & 0.525838\end{array}$ $\begin{array}{llllllll}\text { Yersinia } & 180.966 & -0.32171 & 0.338545 & -0.95028 & 0.34197 & 0.526553\end{array}$ $\begin{array}{llllllll}\text { Roseobacter } & 37.647 & -0.52127 & 0.54887 & -0.94971 & 0.342259 & 0.526553\end{array}$ $\begin{array}{lllllllll}\text { Treponema } & 134.3863 & 0.255412 & 0.268706 & 0.950527 & 0.341845 & 0.526553\end{array}$ $\begin{array}{llllllll}\text { Macrococcus } & 140.7256 & 0.488804 & 0.513354 & 0.952178 & 0.341007 & 0.526553\end{array}$ $\begin{array}{lllllll}\text { Candidatus Sulcia } & 6.945142 & 1.170841 & 1.230281 & 0.951685 & 0.341257 & 0.526553\end{array}$ $\begin{array}{llllllll}\text { Collinsella } & 390.5017 & -0.62272 & 0.652259 & -0.95471 & 0.339724 & 0.526553\end{array}$ $\begin{array}{llllllll}\text { Parageobacillus } & 7.148003 & -1.17138 & 1.227163 & -0.95455 & 0.339807 & 0.526553\end{array}$ $\begin{array}{llllllll}\text { Azospira } & 147.0426 & -0.43978 & 0.463726 & -0.94836 & 0.342947 & 0.526767\end{array}$ Dehalogenimona: $14.10235 \quad-0.62252 \quad 0.657524 \quad-0.94676 \quad 0.343762 \quad 0.527175$ $\begin{array}{llllllll}\text { Ruminococcus } & 163.0935 & -0.46764 & 0.496927 & -0.94106 & 0.346672 & 0.53079\end{array}$ $\begin{array}{llllllll}\text { Proteiniphilum } & 12.47909 & -0.75612 & 0.81061 & -0.93278 & 0.350934 & 0.536459\end{array}$ $\begin{array}{llllllll}\text { Thermotoga } & 12.01835 & -0.82509 & 0.885652 & -0.93162 & 0.351535 & 0.536524\end{array}$ $\begin{array}{llllllll}\text { Tepidanaerobact } & 5.675524 & -1.00936 & 1.090861 & -0.92529 & 0.354815 & 0.53896\end{array}$ $\begin{array}{lllllll}\text { Listeria } & 114.893 & 0.2957 & 0.319038 & 0.926847 & 0.354006 & 0.53896\end{array}$ $\begin{array}{llllllll}\text { Alphabaculovirus } & 8.738047 & 1.016321 & 1.097224 & 0.926266 & 0.354308 & 0.53896\end{array}$ $\begin{array}{llllllll}\text { Desulfosporosinu } & 40.20555 & 0.464564 & 0.5052 & 0.919564 & 0.3578 & 0.542636\end{array}$ $\begin{array}{lllllll}\text { Xylella } & 25.66693 & -0.41608 & 0.45439 & -0.91569 & 0.35983 & 0.544853\end{array}$ $\begin{array}{llllllll}\text { Actinopolymorph } & 80.3425 & -0.31378 & 0.343482 & -0.91352 & 0.360968 & 0.54571\end{array}$ $\begin{array}{lllllll}\text { Rathayibacter } & 109.6523 & -0.2623 & 0.287464 & -0.91245 & 0.361533 & 0.54571\end{array}$ $\begin{array}{llllllll}\text { Intrasporangium } & 155.5045 & -0.34563 & 0.381074 & -0.907 & 0.364407 & 0.548328\end{array}$ $\begin{array}{llllllll}\text { Actinoalloteichus } & 537.4014 & 0.37725 & 0.415934 & 0.906994 & 0.36441 & 0.548328\end{array}$ $\begin{array}{llllllll}\text { Paraoerskovia } & 70.05303 & -0.34863 & 0.385401 & -0.9046 & 0.365678 & 0.549375\end{array}$ $\begin{array}{llllllll}\text { Methanocella } & 6.735621 & -0.90588 & 1.005722 & -0.90073 & 0.367733 & 0.550739\end{array}$ 
$\begin{array}{lllllll}\text { Acetoanaerobiur } & 15.75858 & -0.56801 & 0.630357 & -0.9011 & 0.367537 & 0.550739\end{array}$ $\begin{array}{lllllll}\text { Propionimicrobiu } & 158.5119 & -0.45057 & 0.501782 & -0.89793 & 0.36922 & 0.552105\end{array}$

$\begin{array}{llllllll}\text { Leifsonia } & 433.1803 & -0.29625 & 0.332597 & -0.89071 & 0.373083 & 0.557013\end{array}$

$\begin{array}{lllllllll}\text { Octadecabacter } & 25.90776 & -0.41764 & 0.470665 & -0.88734 & 0.374897 & 0.557986\end{array}$

$\begin{array}{lllllll}\text { Mycetocola } & 74.68093 & -0.33136 & 0.373115 & -0.88809 & 0.37449 & 0.557986\end{array}$

$\begin{array}{llllllll}\text { Bifidobacterium } & 2948.14 & 0.389246 & 0.439899 & 0.884854 & 0.376236 & 0.559112\end{array}$

$\begin{array}{llllllll}\text { Paraphotobacteri } & 7.910704 & 0.86645 & 0.989904 & 0.875287 & 0.381418 & 0.565064\end{array}$

$\begin{array}{lllllllll}\text { Trichormus } & 13.21995 & 0.953617 & 1.089425 & 0.875339 & 0.381389 & 0.565064\end{array}$

$\begin{array}{lllllllll}\text { Acholeplasma } & 36.76557 & 0.443849 & 0.509723 & 0.870765 & 0.383883 & 0.565893\end{array}$

$\begin{array}{llllllll}\text { Kangiella } & 18.28151 & -0.5211 & 0.599031 & -0.86991 & 0.384352 & 0.565893\end{array}$

$\begin{array}{llllllll}\text { Lacimicrobium } & 17.76524 & -0.62371 & 0.716446 & -0.87056 & 0.383997 & 0.565893\end{array}$

$\begin{array}{llllllll}\text { Gammaretrovirus } & 14.57035 & 1.476307 & 1.699136 & 0.868858 & 0.384925 & 0.565893\end{array}$

$\begin{array}{llllllll}\text { Persicobacter } & 8.545405 & -0.6335 & 0.728851 & -0.86917 & 0.384755 & 0.565893\end{array}$

$\begin{array}{lllllll}\text { Acidipropionibact } 307.4582 & -0.31163 & 0.362985 & -0.85853 & 0.390601 & 0.571244\end{array}$

$\begin{array}{llllllll}\text { Leuconostoc } & 422.1705 & 0.426194 & 0.498389 & 0.855144 & 0.392472 & 0.571244\end{array}$

$\begin{array}{llllllll}\text { Cobetia } & 32.60278 & -0.55568 & 0.64903 & -0.85618 & 0.3919 & 0.571244\end{array}$

$\begin{array}{lllllll}\text { Methylobacteriur } & 1649.991 & -0.26016 & 0.302446 & -0.86018 & 0.389687 & 0.571244\end{array}$

$\begin{array}{llllllll}\text { Candidatus Proto } & 10.9626 & -0.8198 & 0.959189 & -0.85468 & 0.39273 & 0.571244\end{array}$

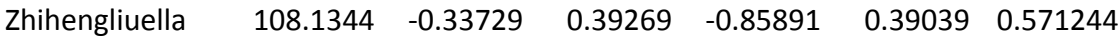

$\begin{array}{lllllllll}\text { Cycloclasticus } & 11.41057 & 0.896203 & 1.048181 & 0.855008 & 0.392547 & 0.571244\end{array}$

$\begin{array}{llllllll}\text { Geitlerinema } & 26.5096 & -0.50044 & 0.586636 & -0.85307 & 0.393619 & 0.57167\end{array}$

$\begin{array}{lllllll}\text { Zymomonas } & 13.42532 & -0.57371 & 0.673433 & -0.85191 & 0.394263 & 0.571741\end{array}$

$\begin{array}{llllllll}\text { Gallaecimonas } & 11.52632 & -0.70825 & 0.832755 & -0.85049 & 0.395055 & 0.572026\end{array}$

$\begin{array}{llllllll}\text { Veillonella } & 2782.758 & -0.38423 & 0.455832 & -0.84291 & 0.399277 & 0.577064\end{array}$

$\begin{array}{lllllllll}\text { llyobacter } & \quad & 7.571755 & 1.039274 & 1.234159 & 0.842091 & 0.399737 & 0.577064\end{array}$

$\begin{array}{llllllll}\text { Gallibacterium } & 9.327232 & -0.99529 & 1.184386 & -0.84034 & 0.400717 & 0.57761\end{array}$

$\begin{array}{llllllll}\text { Deinococcus } & 2101.254 & 0.407504 & 0.486541 & 0.837553 & 0.402282 & 0.578833\end{array}$

$\begin{array}{llllllll}\text { Faecalibacterium } & 1095.896 & -0.47437 & 0.56697 & -0.83668 & 0.402771 & 0.578833\end{array}$

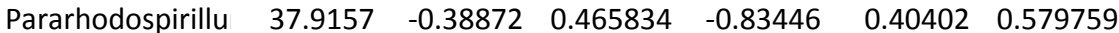

$\begin{array}{llllllll}\text { Halomonas } & 523.7645 & -0.20138 & 0.243339 & -0.82757 & 0.407915 & 0.583622\end{array}$

$\begin{array}{llllllll}\text { Chryseobacteriun } & 2141.554 & 0.330101 & 0.398892 & 0.827546 & 0.407928 & 0.583622\end{array}$

$\begin{array}{llllllll}\text { Cohaesibacter } & 15.29227 & -0.48437 & 0.587995 & -0.82376 & 0.410077 & 0.585607\end{array}$

$\begin{array}{llllllll}\text { Ereboglobus } & 27.98436 & -0.52163 & 0.633852 & -0.82295 & 0.410535 & 0.585607\end{array}$

$\begin{array}{llllllll}\text { Microbulbifer } & 81.08462 & -0.37172 & 0.453116 & -0.82036 & 0.412009 & 0.586838\end{array}$

$\begin{array}{lllllllll}\text { Entomoplasma } & 14.10536 & 0.738208 & 0.904497 & 0.816154 & 0.414412 & 0.587486\end{array}$

$\begin{array}{llllllll}\text { Megasphaera } & 56.8097 & -0.35156 & 0.431283 & -0.81515 & 0.414984 & 0.587486\end{array}$

$\begin{array}{lllllllll}\text { Isosphaera } & 10.52665 & -0.53664 & 0.656787 & -0.81707 & 0.413887 & 0.587486\end{array}$

$\begin{array}{llllllll}\text { Auricoccus } & 34.2614 & 0.60262 & 0.737799 & 0.816781 & 0.414054 & 0.587486\end{array}$

$\begin{array}{llllllll}\text { Marmoricola } & 274.3748 & -0.26218 & 0.322004 & -0.81421 & 0.415524 & 0.587486\end{array}$

$\begin{array}{llllllll}\text { Saccharopolyspor } & 147.8914 & -0.26094 & 0.32135 & -0.812 & 0.416791 & 0.58841\end{array}$ 


\begin{tabular}{|c|c|c|c|c|c|c|}
\hline & & & & & & \\
\hline reutzia & 146.2697 & -0.54868 & 0.681482 & -0.80513 & 0.420744 & 0.59225 \\
\hline & 37.1072 & -0.33265 & 0.414621 & 0.80231 & 22377 & \\
\hline & 890794 & 998 & 992 & 998 & 723 & \\
\hline tus & 30.9628 & -0.24554 & 0.309849 & -0.79244 & 0.428104 & 0.597484 \\
\hline occus & 17.4222 & -0.46653 & 0.588841 & -0.79228 & 197 & 7484 \\
\hline & 3741.624 & -0.39631 & 0.500184 & -0.79233 & 0.428167 & 0.597484 \\
\hline loorea & 12.57012 & 0.735869 & 0.927952 & 0.793003 & 27776 & 0.59 \\
\hline & 86.41 & 4402 & 43708 & .78709 & 123 & 0.600258 \\
\hline pacter & 98.80177 & -0.2159 & 0.274421 & -0.78674 & 435 & 0.600258 \\
\hline bact & 39.9623 & -0.58412 & 556 & -0.78347 & 351 & 0.602051 \\
\hline op & 2291.206 & 382 & 0.477827 & 3234 & 17 & 105 \\
\hline eni & 677.8101 & 0.155933 & 0.199626 & 0.781126 & 729 & 0.602221 \\
\hline & 203 & & & & & 999 \\
\hline ra & 7.276666 & 196 & 0.9 & 558 & & 999 \\
\hline & 146 & 521 & 001 & 401 & & 0.605414 \\
\hline & 6.39 & 323 & 08 & & & 0.60 \\
\hline isi & 27.13388 & 5977 & 0.47 & 291 & 514 & 0.612742 \\
\hline & 8.38 & 593 & 1.0 & 593 & & 262 \\
\hline n & 299.5 & 381 & $0.3 c$ & 859 & 99 & 0.61 \\
\hline & & & & 563 & 69 & 5262 \\
\hline lac & .574 & -0. & 0.6 & 194 & 209 & 3242 \\
\hline $\mathrm{n}$ & 19 & 0.29 & 0.3 & 985 & & 0.618966 \\
\hline efl & 101 & 422 & 0.5 & 501 & 266 & 0.62 \\
\hline angic & 6.350177 & 0.73 & 1.00 & 468 & & 0.62916 \\
\hline 1or & 23004.98 & 0.382632 & 0.52 & 417 & 695 & 0.62916 \\
\hline on & 66.24576 & -0.22772 & 0.31 & -0.73222 & 033 & 0.630087 \\
\hline & 88.2 & 598 & 0.33 & 372 & 174 & 0.631947 \\
\hline าa & 5.676205 & 5556 & 1.038099 & -0.72783 & 719 & 0.631947 \\
\hline Noca & 983.8462 & -0.27219 & 0.3747 & -0.72641 & 7587 & 0.632231 \\
\hline tyto & 538.5 & -0.3 & 584 & 267 & 883 & 0.63 \\
\hline naerobius & 7.423102 & 0.994849 & 1.379389 & 0.721225 & 0771 & 0.634748 \\
\hline saia & 10.09087 & -0.50248 & 0.699069 & -0.71878 & 275 & 0.635882 \\
\hline & 35.62079 & 539 & 0.469802 & .7139 & 47529 & 0.639046 \\
\hline Atop & 131.7167 & -0.39357 & 0.553067 & -0.71161 & 0.476708 & 0.63939 \\
\hline & 1217.0 & 3074 & 0.432145 & 133 & 879 & 0.63939 \\
\hline actor & 7.392528 & -0.73203 & 1.03383 & -0.70807 & 0.478901 & 0.640313 \\
\hline Euze & 81.26483 & -0.2281 & 0.32204 & -0.70831 & 0.478751 & 0.640313 \\
\hline & 58.92 & 696 & & 0.704412 & & 0.641568 \\
\hline Segniliparus & 88.75068 & -0.35514 & 0.503719 & -0.70504 & 0.480786 & 0.64156 \\
\hline
\end{tabular}


$\begin{array}{lllllll}\text { Plantactinospora } & 183.9187 & -0.23529 & 0.334614 & -0.70316 & 0.481955 & 0.641715\end{array}$ $\begin{array}{llllllll}\text { Methanofollis } & 13.60672 & -0.48463 & 0.698224 & -0.69409 & 0.487625 & 0.648365\end{array}$ $\begin{array}{llllllll}\text { Sphingobium } & 1263.024 & -0.24837 & 0.358946 & -0.69194 & 0.488973 & 0.649259\end{array}$ $\begin{array}{llllllll}\text { Glaesserella } & 26.74016 & -0.4528 & 0.657406 & -0.68877 & 0.490967 & 0.651006\end{array}$ $\begin{array}{llllllll}\text { Leptotrichia } & 329.1814 & -0.29882 & 0.436757 & -0.68419 & 0.493858 & 0.653936\end{array}$ $\begin{array}{llllllll}\text { Algoriphagus } & 11.25202 & -0.49349 & 0.724151 & -0.68148 & 0.495569 & 0.655298\end{array}$ $\begin{array}{lllllll}\text { Marinilactibacillu } & 10.0694 & -0.58546 & 0.861604 & -0.6795 & 0.496819 & 0.656047\end{array}$

$\begin{array}{llllllll}\text { Candidatus Phyto } & 16.76813 & 0.717421 & 1.064612 & 0.673881 & 0.500387 & 0.659851\end{array}$

$\begin{array}{lllllllll}\text { Nodularia } & 5.538512 & -0.83025 & 1.253931 & -0.66212 & 0.507894 & 0.661917\end{array}$

$\begin{array}{llllllll}\text { Calothrix } & 186.1979 & 0.291976 & 0.440866 & 0.662279 & 0.507792 & 0.661917\end{array}$

$\begin{array}{llllllll}\text { Kribbella } & 119.3126 & -0.23018 & 0.345894 & -0.66547 & 0.505749 & 0.661917\end{array}$

$\begin{array}{llllllll}\text { Enterobacter } & 634.0888 & -0.18036 & 0.272569 & -0.66171 & 0.508159 & 0.661917\end{array}$

$\begin{array}{llllllll}\text { Faecalibaculum } & 6.337424 & -0.89056 & 1.332944 & -0.66812 & 0.504058 & 0.661917\end{array}$

$\begin{array}{lllllll}\text { Glutamicibacter } & 426.4653 & -0.23435 & 0.351525 & -0.66666 & 0.504991 & 0.661917\end{array}$

$\begin{array}{llllllll}\text { Pediococcus } & 42.24056 & 0.310563 & 0.463812 & 0.669589 & 0.50312 & 0.661917\end{array}$

$\begin{array}{lllllllll}\text { Halobacteroides } & 6.344781 & 0.86237 & 1.303117 & 0.661775 & 0.508116 & 0.661917\end{array}$

$\begin{array}{llllllll}\text { Luteipulveratus } & 142.8837 & -0.22773 & 0.34398 & -0.66203 & 0.507952 & 0.661917\end{array}$

$\begin{array}{llllllll}\text { Pasteurella } & 440.2585 & -0.29146 & 0.444129 & -0.65626 & 0.511659 & 0.665573\end{array}$

$\begin{array}{llllllll}\text { Natrinema } & 25.49342 & -0.39538 & 0.605665 & -0.6528 & 0.513887 & 0.667566\end{array}$

$\begin{array}{llllllll}\text { Rothia } & 9131.302 & -0.37459 & 0.580298 & -0.64552 & 0.518591 & 0.66915\end{array}$

$\begin{array}{lllllllll}\text { Acidihalobacter } & 82.25801 & -0.22572 & 0.34863 & -0.64744 & 0.517348 & 0.66915\end{array}$

$\begin{array}{llllllll}\text { Brochothrix } & 211.4917 & 0.544407 & 0.84224 & 0.646379 & 0.518034 & 0.66915\end{array}$

$\begin{array}{llllllll}\text { Terriglobus } & 127.0671 & -0.26157 & 0.404545 & -0.64657 & 0.517911 & 0.66915\end{array}$

$\begin{array}{llllllll}\text { Janibacter } & 810.1264 & -0.31171 & 0.479982 & -0.64943 & 0.516062 & 0.66915\end{array}$

$\begin{array}{llllllll}\text { Saccharospirillum } & 16.50144 & -0.36486 & 0.569085 & -0.64113 & 0.521441 & 0.671923\end{array}$

$\begin{array}{llllllll}\text { Muricauda } & 12.3436 & -0.50863 & 0.796307 & -0.63873 & 0.522999 & 0.672127\end{array}$

$\begin{array}{llllllll}\text { Chondrocystis } & 20.48813 & -0.45916 & 0.718543 & -0.63901 & 0.522813 & 0.672127\end{array}$

$\begin{array}{llllllll}\text { Spirochaeta } & 31.46233 & -0.23629 & 0.374483 & -0.63097 & 0.528057 & 0.675922\end{array}$

$\begin{array}{llllllll}\text { Phytobacter } & 13.09555 & -0.62994 & 0.997499 & -0.63152 & 0.527702 & 0.675922\end{array}$

$\begin{array}{llllllll}\text { Methylotenera } & 22.57596 & -0.36298 & 0.575272 & -0.63096 & 0.528064 & 0.675922\end{array}$

$\begin{array}{llllllll}\text { Gryllotalpicola } & 88.04589 & -0.22091 & 0.350909 & -0.62954 & 0.528993 & 0.67621\end{array}$

$\begin{array}{llllllll}\text { Humibacter } & 228.9883 & -0.32464 & 0.520318 & -0.62392 & 0.532681 & 0.679116\end{array}$

$\begin{array}{llllllll}\text { Modestobacter } & 237.013 & -0.27836 & 0.446084 & -0.62401 & 0.532624 & 0.679116\end{array}$

$\begin{array}{lllllll}\text { Psychromicrobiur } & 62.70074 & -0.38599 & 0.622061 & -0.6205 & 0.53493 & 0.681078\end{array}$

$\begin{array}{llllllll}\text { Kibdelosporangiu } & 161.7652 & -0.24823 & 0.401181 & -0.61876 & 0.536076 & 0.681357\end{array}$

$\begin{array}{llllllll}\text { Thalassococcus } & 48.98035 & -0.24914 & 0.40358 & -0.61731 & 0.537028 & 0.681357\end{array}$

$\begin{array}{llllllll}\text { Citromicrobium } & 39.87432 & -0.27499 & 0.445734 & -0.61693 & 0.537279 & 0.681357\end{array}$

$\begin{array}{llllllll}\text { Sep1virus } & 78.94591 & -1.05085 & 1.709352 & -0.61477 & 0.538709 & 0.682269\end{array}$

$\begin{array}{llllllll}\text { Olsenella } & 339.7671 & -0.26194 & 0.42688 & -0.61363 & 0.539463 & 0.682325\end{array}$

$\begin{array}{llllllll}\text { Halobacteriovora } & 16.16218 & -0.50106 & 0.824521 & -0.6077 & 0.543384 & 0.68638\end{array}$ 
$\begin{array}{lllllll}12.63505 & 0.590119 & 0.981253 & 0.601394 & 0.547578 & 0.688056\end{array}$

Thioclava $\quad \begin{array}{llllllll}50.33392 & -0.29043 & 0.490568 & -0.59202 & 0.553835 & 0.695009\end{array}$

$\begin{array}{llllllll}\text { Belliella } & 9.648409 & -0.58475 & 0.990545 & -0.59034 & 0.554966 & 0.695518\end{array}$

$\begin{array}{llllllll}\text { Mobiluncus } & 437.5561 & -0.34485 & 0.587914 & -0.58657 & 0.557496 & 0.697778\end{array}$

$\begin{array}{llllllll}\text { Draconibacteriun } & 9.365857 & 0.526685 & 0.909285 & 0.579231 & 0.562434 & 0.703042\end{array}$

$\begin{array}{lllllll}\text { Pseudopropionibi } 273.6351 & -0.26779 & 0.465794 & -0.57492 & 0.565345 & 0.705762\end{array}$

$\begin{array}{llllllll}\text { Sphingomonas } & 2155.901 & -0.17745 & 0.312944 & -0.56702 & 0.570698 & 0.711519\end{array}$

$\begin{array}{llllllll}\text { Jeotgalicoccus } & 11.02021 & -0.47896 & 0.872261 & -0.5491 & 0.582937 & 0.71978\end{array}$

$\begin{array}{llllllll}\text { Actinosynnema } & 218.2281 & -0.19776 & 0.359289 & -0.55042 & 0.58203 & 0.71978\end{array}$

$\begin{array}{llllllll}\text { Desulfobacteriur } & 7.418919 & -0.59837 & 1.088569 & -0.54968 & 0.582538 & 0.71978\end{array}$

$\begin{array}{llllllll}\text { Idiomarina } & 24.91039 & -0.36302 & 0.658944 & -0.55091 & 0.581693 & 0.71978\end{array}$

$\begin{array}{llllllll}\text { Clavibacter } & 397.5848 & -0.20542 & 0.373844 & -0.54949 & 0.582668 & 0.71978\end{array}$

$\begin{array}{llllllll}\text { Flammeovirga } & 18.98843 & 0.550634 & 0.993414 & 0.554284 & 0.579384 & 0.71978\end{array}$

$\begin{array}{llllllll}\text { Meiothermus } & 71.23934 & -0.24797 & 0.45205 & -0.54854 & 0.583322 & 0.71978\end{array}$

$\begin{array}{llllllll}\text { Miniimonas } & 139.4614 & -0.22453 & 0.407138 & -0.55148 & 0.581302 & 0.71978\end{array}$

$\begin{array}{llllllll}\text { Flavivirga } & 8.205913 & 0.60049 & 1.101383 & 0.545215 & 0.585606 & 0.721671\end{array}$

$\begin{array}{llllllll}\text { Chroococcidiopsi: } & 10.7473 & -0.61229 & 1.131567 & -0.5411 & 0.588437 & 0.72423\end{array}$

$\begin{array}{llllllll}\text { Methanococcoidt } & 8.473109 & -0.50936 & 0.943925 & -0.53962 & 0.589459 & 0.724559\end{array}$

$\begin{array}{llllllll}\text { Molluscipoxvirus } & 1745.656 & -1.54452 & 2.885459 & -0.53528 & 0.592458 & 0.727314\end{array}$

$\begin{array}{llllllll}\text { Desulfobacca } & 8.278969 & -0.4578 & 0.865982 & -0.52864 & 0.597052 & 0.732018\end{array}$

$\begin{array}{llllllll}\text { Anaerococcus } & 2616.476 & -0.21002 & 0.404675 & -0.519 & 0.603763 & 0.739302\end{array}$

$\begin{array}{llllllll}\text { Erwinia } & 301.2503 & -0.22788 & 0.444417 & -0.51276 & 0.608118 & 0.740996\end{array}$

$\begin{array}{llllllll}\text { Allokutzneria } & 149.7368 & -0.18198 & 0.355018 & -0.5126 & 0.608234 & 0.740996\end{array}$

$\begin{array}{llllllll}\text { Oscillibacter } & 177.5452 & -0.35235 & 0.684596 & -0.51469 & 0.606771 & 0.740996\end{array}$

$\begin{array}{lllllllll}\text { Pseudoclostridiur } & 6.631289 & 0.657312 & 1.28102 & 0.513116 & 0.60787 & 0.740996\end{array}$

$\begin{array}{llllllll}\text { Turicibacter } & 12.69701 & 0.584642 & 1.144287 & 0.510922 & 0.609406 & 0.741482\end{array}$

$\begin{array}{lllllllll}\text { Tetragenococcus } & 45.30757 & 0.310957 & 0.611881 & 0.508198 & 0.611314 & 0.742863\end{array}$

$\begin{array}{llllllll}\text { Ezakiella } & 864.1796 & -0.27554 & 0.546593 & -0.50411 & 0.614185 & 0.745407\end{array}$

$\begin{array}{llllllll}\text { Rhodococcus } & 6728.821 & -0.21444 & 0.429217 & -0.49962 & 0.617344 & 0.746411\end{array}$

$\begin{array}{llllllll}\text { Fastidiosipila } & 167.8877 & 0.285927 & 0.571772 & 0.500073 & 0.617024 & 0.746411\end{array}$

$\begin{array}{llllllll}\text { Methylophaga } & 7.013605 & -0.47024 & 0.938854 & -0.50087 & 0.616465 & 0.746411\end{array}$

$\begin{array}{lllllllll}\text { Psychrobacter } & 580.6341 & 0.264958 & 0.534085 & 0.496098 & 0.619825 & 0.748468\end{array}$

$\begin{array}{llllllll}\text { Klebsiella } & 1059.336 & 0.202264 & 0.411714 & 0.491273 & 0.623233 & 0.749755\end{array}$

$\begin{array}{lllllllll}\text { Murdochiella } & 168.2988 & -0.34534 & 0.702839 & -0.49135 & 0.623177 & 0.749755\end{array}$

$\begin{array}{lllllllll}\text { Dehalococcoides } & 20.38504 & -0.44084 & 0.896403 & -0.49178 & 0.622872 & 0.749755\end{array}$

$\begin{array}{llllllll}\text { Alkalitalea } & 10.32683 & 0.549704 & 1.125875 & 0.488246 & 0.625375 & 0.75045\end{array}$

$\begin{array}{llllllll}\text { P68virus } & 13.06186 & -0.72329 & 1.479289 & -0.48894 & 0.624882 & 0.75045\end{array}$ 
$\begin{array}{llllllll}\text { Exiguobacterium } & 233.8615 & 0.279164 & 0.573552 & 0.486728 & 0.626451 & 0.750803\end{array}$ $\begin{array}{llllllll}\text { Acidaminococcus } & 31.64573 & 0.308593 & 0.645186 & 0.478301 & 0.632436 & 0.756155\end{array}$

$\begin{array}{llllllll}\text { Gramella } & 55.86711 & 0.147152 & 0.308419 & 0.477115 & 0.63328 & 0.756155\end{array}$

$\begin{array}{llllllll}\text { Hafnia } & 24.99889 & -0.30041 & 0.628538 & -0.47795 & 0.632689 & 0.756155\end{array}$

$\begin{array}{lllllll}\text { Candidatus Fukat } & 12.2009 & -0.47438 & 1.000333 & -0.47422 & 0.635342 & 0.757675\end{array}$

$\begin{array}{lllllllll}\text { Enterococcus } & 460.5717 & 0.149689 & 0.32406 & 0.461918 & 0.64414 & 0.767214\end{array}$

$\begin{array}{llllllll}\text { Renibacterium } \quad 65.75292 & -0.22762 & 0.497046 & -0.45795 & 0.646988 & 0.769651\end{array}$

$\begin{array}{llllllll}\text { Halostagnicola } & 10.61844 & -0.47846 & 1.049402 & -0.45594 & 0.648435 & 0.769736\end{array}$

$\begin{array}{llllllll}\text { Biseptimavirus } & 43.62423 & 1.129679 & 2.47943 & 0.455621 & 0.648663 & 0.769736\end{array}$

$\begin{array}{lllllllll}\text { Tessaracoccus } & 1155.667 & 0.207571 & 0.457131 & 0.454074 & 0.649776 & 0.770105\end{array}$

$\begin{array}{llllllll}\text { Kingella } & 16.58097 & -0.38876 & 0.858309 & -0.45294 & 0.650594 & 0.770124\end{array}$

$\begin{array}{llllllll}\text { Natrialba } & & 7.106001 & -0.39704 & 0.885059 & -0.4486 & 0.65372 & 0.772871\end{array}$

$\begin{array}{lllllllll}\text { Lactobacillus } \quad 2203.113 & 0.155599 & 0.354456 & 0.438979 & 0.660676 & 0.780135\end{array}$

$\begin{array}{llllllll}\text { Acetobacterium } & 15.81532 & -0.32713 & 0.750746 & -0.43573 & 0.66303 & 0.781952\end{array}$

$\begin{array}{llllllll}\text { Catenulispora } & 114.5841 & -0.17888 & 0.415685 & -0.43032 & 0.666965 & 0.785627\end{array}$

$\begin{array}{lllllllll}\text { Candidatus Plank } & 88.33599 & -0.18579 & 0.44165 & -0.42068 & 0.673987 & 0.792926\end{array}$

$\begin{array}{llllllll}\text { Labrenzia } & 73.37176 & -0.1706 & 0.409966 & -0.41613 & 0.677312 & 0.795862\end{array}$

$\begin{array}{llllllll}\text { Leptolyngbya } & 43.34417 & -0.12104 & 0.296056 & -0.40885 & 0.682652 & 0.801156\end{array}$

$\begin{array}{llllllll}\text { Antarctobacter } & 18.88443 & -0.20579 & 0.511192 & -0.40258 & 0.687258 & 0.805577\end{array}$

$\begin{array}{llllllll}\text { Thiohalobacter } & 34.17658 & -0.17306 & 0.43832 & -0.39483 & 0.692971 & 0.810295\end{array}$

$\begin{array}{llllllll}\text { Desulfotomaculuı } 29.49225 & -0.17082 & 0.431491 & -0.39588 & 0.692194 & 0.810295\end{array}$

$\begin{array}{lllllll}\text { Salegentibacter } & 16.45196 & 0.232511 & 0.604223 & 0.38481 & 0.700379 & 0.81796\end{array}$

$\begin{array}{llllllll}\text { Chloracidobacteri } & 13.18805 & -0.29873 & 0.778656 & -0.38365 & 0.701237 & 0.817968\end{array}$

$\begin{array}{llllllll}\text { Filifactor } & 76.38004 & -0.21662 & 0.573736 & -0.37756 & 0.70576 & 0.818618\end{array}$

$\begin{array}{llllllll}\text { Alphapolyomavirı } & 118.7539 & -0.78293 & 2.059822 & -0.3801 & 0.703874 & 0.818618\end{array}$

$\begin{array}{lllllll}\text { Cyclobacterium } & 25.13699 & 0.207324 & 0.549703 & 0.377156 & 0.706058 & 0.818618\end{array}$

$\begin{array}{lllllll}\text { Desulfurispirillum } 8.196643 & -0.37918 & 0.995646 & -0.38083 & 0.703327 & 0.818618\end{array}$

$\begin{array}{llllllll}\text { Friedmanniella } & 424.2821 & -0.12814 & 0.339481 & -0.37744 & 0.705843 & 0.818618\end{array}$

$\begin{array}{llllllll}\text { Muribaculum } & 15.04121 & -0.34684 & 0.927038 & -0.37414 & 0.7083 & 0.820227\end{array}$

$\begin{array}{llllllll}\text { Salinigranum } & 15.32474 & -0.18537 & 0.498619 & -0.37177 & 0.710062 & 0.820288\end{array}$

$\begin{array}{llllllll}\text { Morganella } & 14.72113 & -0.27698 & 0.74425 & -0.37215 & 0.709778 & 0.820288\end{array}$

$\begin{array}{llllllll}\text { Sphingobacteriun } & 199.9506 & 0.125864 & 0.341518 & 0.368541 & 0.71247 & 0.821093\end{array}$

$\begin{array}{llllllll}\text { Gammapapillom } \bar{c} & 210.4195 & -0.41389 & 1.122741 & -0.36864 & 0.712397 & 0.821093\end{array}$

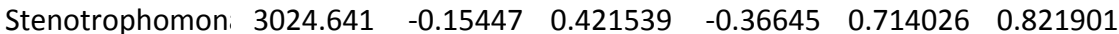

$\begin{array}{llllllll}\text { Pantoea } & 554.3169 & 0.119326 & 0.327668 & 0.364168 & 0.715732 & 0.822878\end{array}$

$\begin{array}{llllllll}\text { Mucilaginibacter } & 197.7755 & 0.117957 & 0.331535 & 0.35579 & 0.721998 & 0.829088\end{array}$

$\begin{array}{llllllll}\text { Corynebacterium } & 294379.3 & -0.13213 & 0.372857 & -0.35438 & 0.723055 & 0.82931\end{array}$

$\begin{array}{llllllll}\text { Ignavibacterium } & 7.368368 & -0.33968 & 0.972929 & -0.34913 & 0.726994 & 0.831424\end{array}$

$\begin{array}{llllllll}\text { Snodgrassella } & 27.49234 & 0.257444 & 0.738809 & 0.348458 & 0.727496 & 0.831424\end{array}$

$\begin{array}{llllllll}\text { Phoenicibacter } & 8.152893 & 0.359413 & 1.029797 & 0.349013 & 0.727079 & 0.831424\end{array}$ 
$\begin{array}{llllllll}\text { Serinicoccus } & 181.2867 & -0.13701 & 0.404917 & -0.33836 & 0.735089 & 0.837113\end{array}$

$\begin{array}{lllllllll}\text { Phycicoccus } & 192.6474 & -0.13862 & 0.411584 & -0.33679 & 0.736272 & 0.837466\end{array}$

$\begin{array}{llllllll}\text { Marivivens } & \quad 5.90437 & 0.421129 & 1.259896 & 0.334257 & 0.738186 & 0.838649\end{array}$

$\begin{array}{lllllllll}\text { Chryseolinea } & 59.37665 & -0.15303 & 0.469086 & -0.32623 & 0.744251 & 0.84454\end{array}$

$\begin{array}{llllllll}\text { Shinella } & 180.0277 & -0.12101 & 0.373622 & -0.32388 & 0.746026 & 0.844557\end{array}$

$\begin{array}{lllllllll}\text { Microcystis } & 12.88276 & -0.21586 & 0.664655 & -0.32478 & 0.74535 & 0.844557\end{array}$

$\begin{array}{llllllll}\text { Psychromonas } & 21.70296 & 0.211152 & 0.6645 & 0.317761 & 0.750666 & 0.846815\end{array}$

$\begin{array}{llllllll}\text { Alphapapillomavi } & 39.19101 & 0.456153 & 1.43308 & 0.318303 & 0.750255 & 0.846815\end{array}$

$\begin{array}{llllllll}\text { Arenibacter } & 5.715612 & -0.31352 & 0.981801 & -0.31934 & 0.749472 & 0.846815\end{array}$

$\begin{array}{llllllll}\text { Blastococcus } & 167.8706 & 0.116628 & 0.368913 & 0.316139 & 0.751897 & 0.847208\end{array}$

Roseovarius $\quad \begin{array}{llllllll}31.67813 & -0.20231 & 0.643159 & -0.31456 & 0.753096 & 0.847564\end{array}$

$\begin{array}{llllllll}\text { Lelliottia } & 141.1207 & 0.164792 & 0.527686 & 0.312291 & 0.754819 & 0.848485\end{array}$

$\begin{array}{llllllll}\text { Gottschalkia } & 30.95062 & 0.232761 & 0.74805 & 0.311157 & 0.755682 & 0.848485\end{array}$

$\begin{array}{lllllllll}\text { Brevibacillus } & 61.80745 & 0.09217 & 0.298519 & 0.308756 & 0.757507 & 0.848684\end{array}$

$\begin{array}{llllllll}\text { Cellulosilyticum } & 13.05912 & 0.26978 & 0.874212 & 0.308598 & 0.757627 & 0.848684\end{array}$

$\begin{array}{llllllll}\text { Pedobacter } & 172.8255 & -0.09795 & 0.324629 & -0.30172 & 0.762868 & 0.853558\end{array}$

$\begin{array}{llllllll}\text { Taylorella } & 10.76885 & -0.28824 & 0.96688 & -0.29811 & 0.76562 & 0.854645\end{array}$

$\begin{array}{llllllll}\text { Brachybacterium } & 2207.381 & -0.14491 & 0.485665 & -0.29838 & 0.765413 & 0.854645\end{array}$

$\begin{array}{llllllll}\text { Micrococcus } & 48492.58 & -0.16479 & 0.55658 & -0.29608 & 0.767166 & 0.855377\end{array}$

$\begin{array}{llllllll}\text { Christensenella } & 79.0214 & -0.13776 & 0.471051 & -0.29244 & 0.769949 & 0.857483\end{array}$

$\begin{array}{lllllllll}\text { Capnocytophaga } & 815.5575 & 0.098173 & 0.343396 & 0.285887 & 0.774964 & 0.861571\end{array}$

$\begin{array}{llllllll}\text { Monoglobus } & 16.7079 & 0.34351 & 1.204027 & 0.285301 & 0.775413 & 0.861571\end{array}$

$\begin{array}{llllllll}\text { Leclercia } & 127.728 & 0.164602 & 0.600707 & 0.274014 & 0.784074 & 0.870186\end{array}$

$\begin{array}{llllllll}\text { Fusobacterium } & 734.3253 & 0.080611 & 0.298978 & 0.269623 & 0.78745 & 0.872924\end{array}$

$\begin{array}{llllllll}\text { Salinimonas } & 11.04942 & -0.24467 & 0.920126 & -0.26591 & 0.790309 & 0.875083\end{array}$

$\begin{array}{llllllll}\text { Rhodoluna } & 41.85134 & 0.155931 & 0.599548 & 0.26008 & 0.794802 & 0.879044\end{array}$

$\begin{array}{llllllll}\text { Akkermansia } & 93.33959 & -0.14313 & 0.56379 & -0.25386 & 0.799601 & 0.879361\end{array}$

$\begin{array}{lllllllll}\text { Barnesiella } & 42.16963 & 0.171734 & 0.676475 & 0.253867 & 0.799598 & 0.879361\end{array}$

$\begin{array}{lllllllll}\text { Cytophaga } & & 19.47716 & 0.187933 & 0.731175 & 0.257029 & 0.797157 & 0.879361\end{array}$

$\begin{array}{llllllll}\text { Acinetobacter } & 4649.62 & 0.099933 & 0.393784 & 0.253776 & 0.799669 & 0.879361\end{array}$

$\begin{array}{llllllll}\text { Erysipelothrix } & 66.74323 & -0.14281 & 0.560328 & -0.25487 & 0.798823 & 0.879361\end{array}$

$\begin{array}{llllllll}\text { Cohnella } & 25.80082 & -0.14808 & 0.591226 & -0.25045 & 0.802236 & 0.881174\end{array}$

$\begin{array}{llllllll}\text { Intestinimonas } & 50.27408 & -0.20614 & 0.859722 & -0.23978 & 0.810502 & 0.888221\end{array}$

$\begin{array}{llllllll}\text { Riemerella } & 18.06455 & -0.17441 & 0.725041 & -0.24055 & 0.8099 & 0.888221\end{array}$

$\begin{array}{lllllllll}\text { Novibacillus } & \quad 6.579413 & 0.281534 & 1.186024 & 0.237377 & 0.812365 & 0.888877\end{array}$

$\begin{array}{lllllllll}\text { Shewanella } & 359.9426 & 0.071389 & 0.301706 & 0.236619 & 0.812952 & 0.888877\end{array}$

$\begin{array}{llllllll}\text { Cloacibacterium } & 67.01921 & 0.08806 & 0.375715 & 0.234381 & 0.814689 & 0.889763\end{array}$

$\begin{array}{llllllll}\text { Cryptobacterium } & 23.6838 & -0.2124 & 0.940873 & -0.22575 & 0.821395 & 0.894036\end{array}$ 
$\begin{array}{llllllll}\text { Streptobacillus } & 14.08066 & -0.19076 & 0.837703 & -0.22772 & 0.819866 & 0.894036\end{array}$ $\begin{array}{llllllll}\text { Jannaschia } & 16.29609 & 0.160488 & 0.708206 & 0.226612 & 0.820726 & 0.894036\end{array}$

$\begin{array}{lllllllll}\text { Dialister } & \quad 71.28216 & 0.137492 & 0.619758 & 0.221848 & 0.824432 & 0.896325\end{array}$

$\begin{array}{llllllll}\text { Mogibacterium } & 193.5118 & 0.093275 & 0.440865 & 0.211573 & 0.83244 & 0.902986\end{array}$

$\begin{array}{llllllll}\text { Buttiauxella } & 11.50891 & -0.23969 & 1.130681 & -0.21199 & 0.832118 & 0.902986\end{array}$

$\begin{array}{llllllll}\text { Anaerotignum } & 7.365365 & -0.22891 & 1.089816 & -0.21005 & 0.833631 & 0.903258\end{array}$

$\begin{array}{lllllll}\text { Ornithobacteriun } & 48.38132 & -0.2211 & 1.07283 & -0.20609 & 0.836723 & 0.905586\end{array}$

$\begin{array}{llllllll}\text { Microterricola } & 194.9217 & -0.07454 & 0.364533 & -0.20449 & 0.83797 & 0.905914\end{array}$

$\begin{array}{llllllll}\text { Faecalitalea } & 88.18678 & -0.17738 & 0.877267 & -0.2022 & 0.839763 & 0.906831\end{array}$

$\begin{array}{llllllll}\text { Sodalis } & 41.37469 & 0.065857 & 0.32819 & 0.200667 & 0.840959 & 0.907101\end{array}$

$\begin{array}{llllllll}\text { Marivirga } & 8.825377 & -0.17559 & 0.886863 & -0.19799 & 0.843053 & 0.90834\end{array}$

$\begin{array}{lllllll}\text { Herbinix } & 11.02209 & 0.204297 & 1.06208 & 0.192356 & 0.847464 & 0.912069\end{array}$

$\begin{array}{llllllll}\text { Lachnoanaerobac } 43.57429 & -0.11067 & 0.587693 & -0.18831 & 0.850634 & 0.913432\end{array}$

Salmonella $\quad \begin{array}{llllllll}301.5728 & 0.065847 & 0.349203 & 0.188563 & 0.850436 & 0.913432\end{array}$

$\begin{array}{llllllll}\text { Geobacillus } & 92.1644 & -0.05031 & 0.272806 & -0.18441 & 0.85369 & 0.91569\end{array}$

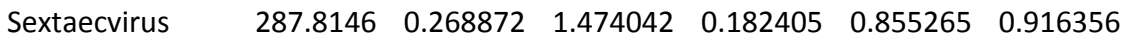

$\begin{array}{llllllll}\text { Halomicronema } & 9.179456 & 0.180506 & 1.008861 & 0.178921 & 0.858 & 0.918261\end{array}$

$\begin{array}{llllllll}\text { Ruminiclostridiun } & 5.725996 & 0.199628 & 1.142672 & 0.174703 & 0.861313 & 0.92078\end{array}$

$\begin{array}{llllllll}\text { Rhizorhabdus } & 103.2171 & -0.08497 & 0.496824 & -0.17104 & 0.864196 & 0.922332\end{array}$

$\begin{array}{llllllll}\text { Paracoccus } \quad 5125.444 & -0.08272 & 0.485433 & -0.17041 & 0.864686 & 0.922332\end{array}$

$\begin{array}{llllllll}\text { Candidatus Azobc } 7.148957 & 0.191551 & 1.141659 & 0.167783 & 0.866754 & 0.922838\end{array}$

$\begin{array}{llllllll}\text { Actinopolyspora } & 99.74314 & -0.07062 & 0.421931 & -0.16736 & 0.867083 & 0.922838\end{array}$

$\begin{array}{llllllll}\text { Anoxybacillus } & 54.62685 & -0.06652 & 0.401497 & -0.16569 & 0.8684 & 0.923216\end{array}$

$\begin{array}{llllllll}\text { Sphaerochaeta } & 13.33856 & -0.09176 & 0.591021 & -0.15526 & 0.876617 & 0.930921\end{array}$

$\begin{array}{llllllll}\text { Desulfitobacteriu } & 37.41351 & 0.073401 & 0.485211 & 0.151277 & 0.879757 & 0.932804\end{array}$

$\begin{array}{lllllllll}\text { Gillisia } & \quad 5.661673 & 0.172511 & 1.145907 & 0.150546 & 0.880334 & 0.932804\end{array}$

$\begin{array}{llllllll}\text { Aerococcus } & 273.3131 & -0.05318 & 0.365258 & -0.14558 & 0.884251 & 0.935922\end{array}$

$\begin{array}{llllllll}\text { Alloactinosynnem } & 123.4809 & -0.05927 & 0.414607 & -0.14295 & 0.886328 & 0.937087\end{array}$

$\begin{array}{llllllll}\text { Parascardovia } & 84.30919 & -0.07856 & 0.555528 & -0.14141 & 0.887548 & 0.937336\end{array}$

$\begin{array}{llllllll}\text { Petrimonas } & 13.9172 & 0.11361 & 0.810445 & 0.140182 & 0.888516 & 0.937336\end{array}$

$\begin{array}{llllllll}\text { Mordavella } & 22.50616 & -0.14703 & 1.082366 & -0.13584 & 0.891948 & 0.939924\end{array}$

$\begin{array}{llllllll}\text { Lachnoclostridiun } 311.2429 & -0.06804 & 0.506291 & -0.13439 & 0.893094 & 0.940099\end{array}$

$\begin{array}{llllllll}\text { Nonlabens } & 54.65511 & -0.04203 & 0.326122 & -0.12888 & 0.897451 & 0.942618\end{array}$

$\begin{array}{llllllll}\text { Curtobacterium } & 587.4296 & 0.060294 & 0.465022 & 0.129657 & 0.896837 & 0.942618\end{array}$

$\begin{array}{llllllll}\text { Fervidobacterium } & 5.80828 & 0.132385 & 1.066399 & 0.124142 & 0.901203 & 0.945524\end{array}$

$\begin{array}{lllllll}\text { Hymenobacter } & 508.4689 & 0.043689 & 0.363464 & 0.120203 & 0.904323 & 0.947762\end{array}$

$\begin{array}{llllllll}\text { Runella } & 40.38872 & 0.037248 & 0.320376 & 0.116263 & 0.907444 & 0.949996\end{array}$

$\begin{array}{lllllllll}\text { Seonamhaeicola } & 10.08098 & 0.109999 & 0.957617 & 0.114868 & 0.90855 & 0.950118\end{array}$

$\begin{array}{llllllll}\text { Moritella } & 19.80048 & -0.09883 & 0.888361 & -0.11125 & 0.911416 & 0.952077\end{array}$

$\begin{array}{llllllll}\text { Lysinimonas } & 140.6076 & 0.048328 & 0.441011 & 0.109585 & 0.912739 & 0.952423\end{array}$ 
$\begin{array}{lllllll}\text { Thermococcus } & 49.11227 & 0.025849 & 0.239888 & 0.107755 & 0.91419 & 0.952902\end{array}$ $\begin{array}{llllllll}\text { Megamonas } & 8.542522 & -0.10062 & 0.973786 & -0.10333 & 0.9177 & 0.955523\end{array}$

$\begin{array}{llllllll}\text { Serratia } & 976.2495 & -0.03919 & 0.396958 & -0.09873 & 0.921353 & 0.958287\end{array}$

$\begin{array}{llllllll}\text { Coxiella } & 16.25107 & 0.06173 & 0.659765 & 0.093564 & 0.925456 & 0.959937\end{array}$

$\begin{array}{llllllll}\text { Scardovia } & 18.22912 & -0.08486 & 0.912259 & -0.09302 & 0.925888 & 0.959937\end{array}$

$\begin{array}{llllllll}\text { Edwardsiella } & 67.18008 & -0.02679 & 0.288227 & -0.09296 & 0.925939 & 0.959937\end{array}$

$\begin{array}{llllllll}\text { Cutibacterium } & 543142.7 & 0.08156 & 0.9501 & 0.085844 & 0.931591 & 0.964178\end{array}$

$\begin{array}{lllllllll}\text { Microvirgula } & 67.83889 & -0.03984 & 0.467186 & -0.08528 & 0.932039 & 0.964178\end{array}$

$\begin{array}{llllllll}\text { Solibacillus } & 28.12367 & 0.041633 & 0.502904 & 0.082786 & 0.934022 & 0.96519\end{array}$

$\begin{array}{llllllll}\text { Xanthomonas } & 1716.342 & -0.02622 & 0.334165 & -0.07845 & 0.937471 & 0.967712\end{array}$

$\begin{array}{llllllll}\text { Photobacterium } & 51.11582 & 0.036632 & 0.476311 & 0.076907 & 0.938698 & 0.967937\end{array}$

$\begin{array}{llllllll}\text { Gemella } & 274.6567 & 0.035251 & 0.478331 & 0.073695 & 0.941253 & 0.968673\end{array}$

Fictibacillus $\quad \begin{array}{llllllll}14.88886 & -0.04647 & 0.632503 & -0.07347 & 0.941429 & 0.968673\end{array}$

$\begin{array}{llllllll}\text { Roseburia } & 126.5463 & -0.0599 & 0.847176 & -0.07071 & 0.943629 & 0.969897\end{array}$

$\begin{array}{llllllll}\text { Raoultella } & \quad 119.2911 & -0.02446 & 0.376771 & -0.06492 & 0.948236 & 0.970939\end{array}$

$\begin{array}{lllllllll}\text { Paludibacter } & \quad 8.683974 & 0.055581 & 0.899152 & 0.061814 & 0.950711 & 0.970939\end{array}$

Gluconobacter $\quad 40.21495 \quad-0.03159 \quad 0.502501 \quad-0.06286 \quad 0.949879 \quad 0.970939$

$\begin{array}{llllllll}\text { Zobellia } & 8.63567 & -0.07398 & 1.168496 & -0.06331 & 0.94952 & 0.970939\end{array}$

$\begin{array}{lllllll}\text { Halocynthiibacter } & 7.021786 & 0.064925 & 0.967157 & 0.06713 & 0.946478 & 0.970939\end{array}$

$\begin{array}{llllllll}\text { Sulfurovum } & 7.240974 & -0.07321 & 1.102263 & -0.06642 & 0.947047 & 0.970939\end{array}$

$\begin{array}{llllllll}\text { Lactococcus } & 1835.985 & -0.02953 & 0.520516 & -0.05672 & 0.954765 & 0.974043\end{array}$

$\begin{array}{llllllll}\text { Dehalobacterium } & 7.142204 & -0.06001 & 1.129791 & -0.05311 & 0.957642 & 0.975941\end{array}$

$\begin{array}{llllllll}\text { Oleispira } & 5.66908 & -0.05727 & 1.255843 & -0.0456 & 0.963628 & 0.981\end{array}$

$\begin{array}{lllllll}\text { Halohasta } & 7.121839 & -0.05619 & 1.273591 & -0.04412 & 0.964809 & 0.981162\end{array}$

$\begin{array}{llllllll}\text { Sk1virus } & 134.2668 & -0.04463 & 1.134425 & -0.03934 & 0.96862 & 0.982954\end{array}$

$\begin{array}{llllllll}\text { Citrobacter } & 242.9684 & -0.01116 & 0.278163 & -0.04011 & 0.968005 & 0.982954\end{array}$

$\begin{array}{llllllll}\text { Thermodesulfobil } 8.934138 & -0.03278 & 1.05081 & -0.03119 & 0.975115 & 0.988221\end{array}$

$\begin{array}{llllllll}\text { Candidatus Hamil } 5.984679 & -0.04664 & 1.541798 & -0.03025 & 0.975868 & 0.988221\end{array}$

$\begin{array}{llllllll}\text { Vagococcus } & 23.48341 & 0.009934 & 0.374488 & 0.026526 & 0.978838 & 0.990183\end{array}$

$\begin{array}{llllllll}\text { Salinibacterium } & 74.72791 & -0.01031 & 0.430995 & -0.02392 & 0.980919 & 0.991244\end{array}$

$\begin{array}{lllllll}\text { Ornithinimicrobic } 203.9469 & -0.00688 & 0.3769 & -0.01827 & 0.985426 & 0.993636\end{array}$

$\begin{array}{llllllll}\text { Simonsiella } & 20.87036 & 0.017377 & 1.039255 & 0.016721 & 0.986659 & 0.993636\end{array}$

$\begin{array}{lllllllll}\text { Leminorella } & 14.01301 & 0.013207 & 0.898298 & 0.014702 & 0.98827 & 0.993636\end{array}$

$\begin{array}{llllllll}\text { Kocuria } & 5240.436 & 0.006116 & 0.441098 & 0.013865 & 0.988938 & 0.993636\end{array}$

$\begin{array}{lllllllll}\text { Aeribacillus } & \quad 5.756274 & 0.022148 & 1.278965 & 0.017317 & 0.986184 & 0.993636\end{array}$

$\begin{array}{llllllll}\text { Paeniclostridium } & 30.18852 & 0.008735 & 0.663487 & 0.013165 & 0.989496 & 0.993636\end{array}$

$\begin{array}{llllllll}\text { Peptoniphilus } & 122.5153 & -0.00156 & 0.582951 & -0.00267 & 0.997871 & 0.998952\end{array}$

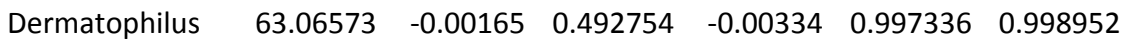

Selenomonas $\quad \begin{array}{lllllll}325.1897 & -0.0011 & 0.420815 & -0.00262 & 0.997911 & 0.998952\end{array}$

$\begin{array}{lllllll}\text { Histophilus } & 10.27419 & 0.000498 & 0.779429 & 0.000639 & 0.99949 & 0.99949\end{array}$ 


\begin{tabular}{|c|c|c|c|c|c|}
\hline Desulfotalea & 2.611218 & -2.21453 & 1.359795 & -1.62858 & $0.103403 \mathrm{NA}$ \\
\hline Siansivirga & 3.580563 & -0.91679 & 1.314442 & -0.69747 & $0.485508 \mathrm{NA}$ \\
\hline lagellimonas & 0.645972 & -0.58839 & 2.83367 & -0.20764 & $0.835509 \mathrm{NA}$ \\
\hline Salinicoccus & 4.061659 & 0.129068 & 1.339403 & 0.096362 & $0.923233 \mathrm{NA}$ \\
\hline Croceibacter & 3.621466 & 0.098628 & 1.315044 & 0.075 & $0.940215 \mathrm{NA}$ \\
\hline Dichelobacter & 3.132802 & -0.75804 & 1.18705 & -0.6386 & $0.523086 \mathrm{NA}$ \\
\hline Ketobacter & 3.81681 & -1.90244 & 1.092272 & -1.74173 & 0.081556 NA \\
\hline Candidatus Punic & 3.327694 & -1.57498 & 1.210731 & -1.30085 & $0.193311 \mathrm{NA}$ \\
\hline Desulfomonile & 2.513746 & -1.34245 & 1.256377 & -1.06851 & $0.285289 \mathrm{NA}$ \\
\hline Rummeliibacillus & 4.872283 & -1.72578 & 1.23344 & -1.39916 & 0.161765 NA \\
\hline Thermodesulfovil & 4.895903 & -1.94979 & 1.188247 & -1.6409 & $0.100819 \mathrm{NA}$ \\
\hline Cylindrospermur & 2.885639 & -0.31975 & 1.399797 & -0.22843 & $0.819315 \mathrm{NA}$ \\
\hline Endomicrobium & 0.417085 & -0.22422 & 2.93411 & -0.07642 & $0.939087 \mathrm{NA}$ \\
\hline Thermincola & 4.149451 & -0.77139 & 1.235582 & -0.62431 & $0.532425 \mathrm{NA}$ \\
\hline Paenisporosarcin & 4.307222 & -0.43294 & 1.247179 & -0.34714 & $0.728487 \mathrm{NA}$ \\
\hline Sedimentisphaer: & 4.837113 & -1.01498 & 1.04081 & -0.97518 & $0.329471 \mathrm{NA}$ \\
\hline Oleiphilus & 4.250232 & -2.82849 & 1.039074 & -2.72213 & 0.006486 NA \\
\hline Thermacetogeniu & 5.33496 & -0.84704 & 1.269179 & -0.66739 & $0.504522 \mathrm{NA}$ \\
\hline Candidatus Meth & 0.826114 & -0.89683 & 2.733641 & -0.32807 & 0.742858 NA \\
\hline Desulfocapsa & 0.994337 & -0.3666 & 2.161217 & -0.16963 & $0.865304 \mathrm{NA}$ \\
\hline Aureitalea & 2.181744 & -0.90027 & 1.483632 & -0.6068 & $0.543981 \mathrm{NA}$ \\
\hline Methylacidiphilur & 0.844368 & -0.53543 & 2.209687 & -0.24231 & $0.808538 \mathrm{NA}$ \\
\hline Carboxydocella & 3.148266 & -0.38371 & 1.326537 & -0.28925 & $0.772387 \mathrm{NA}$ \\
\hline Chamaesiphon & 1.686086 & -0.22117 & 2.055974 & -0.10757 & $0.914335 \mathrm{NA}$ \\
\hline Coraliomargarita & 1.323245 & -1.07371 & 2.531103 & -0.42421 & $0.671415 \mathrm{NA}$ \\
\hline Cyanobacterium & 4.931494 & 0.201095 & 1.272077 & 0.158084 & $0.87439 \mathrm{NA}$ \\
\hline Dictyoglomus & 5.151687 & -0.16576 & 1.040288 & -0.15934 & $0.8734 \mathrm{NA}$ \\
\hline Paraliobacillus & 4.662725 & -0.0821 & 1.207282 & -0.06801 & $0.94578 \mathrm{NA}$ \\
\hline Desulfofarcimen & 3.644227 & 0.607124 & 1.225073 & 0.495582 & $0.620189 \mathrm{NA}$ \\
\hline Thermoclostridiul & 3.585443 & -0.05291 & 1.398885 & -0.03782 & 0.969829 NA \\
\hline Lmd1virus & 2.830689 & 1.956676 & 2.539755 & 0.770419 & $0.441051 \mathrm{NA}$ \\
\hline Halothece & 4.972815 & -0.13693 & 1.268765 & -0.10793 & $0.914055 \mathrm{NA}$ \\
\hline Planctopirus & 3.78307 & -1.31094 & 1.118814 & -1.17173 & $0.241307 \mathrm{NA}$ \\
\hline Candidatus Endol & 2.971932 & 2.077706 & 1.829685 & 1.135553 & $0.256144 \mathrm{NA}$ \\
\hline Mahella & 4.298156 & -1.48732 & 1.480857 & -1.00436 & $0.315203 \mathrm{NA}$ \\
\hline Acidilobus & 1.595189 & -2.85097 & 1.754158 & -1.62526 & $0.104107 \mathrm{NA}$ \\
\hline Agarivorans & 2.205438 & -0.87527 & 1.491249 & -0.58694 & $0.557244 \mathrm{NA}$ \\
\hline Gloeomargarita & 5.373066 & 0.377311 & 1.262561 & 0.298846 & $0.765057 \mathrm{NA}$ \\
\hline Terribacillus & 5.190434 & -0.48831 & 1.179182 & -0.41411 & 0.678792 NA \\
\hline Methanomassiliic & 1.565467 & 0.781253 & 1.902448 & 0.410657 & $0.681324 \mathrm{NA}$ \\
\hline
\end{tabular}


$\begin{array}{llllll}\text { Melioribacter } & 1.786114 & -0.88032 & 1.607553 & -0.54762 & 0.583956 \text { NA }\end{array}$ $\begin{array}{llllll}\text { Methanohalophil } 2.608096 & -0.58062 & 1.298872 & -0.44702 & 0.654861 \text { NA }\end{array}$

$\begin{array}{lllllll}\text { Candidatus Atelor } 3.075229 & 1.035048 & 1.724447 & 0.60022 & 0.548359 & \text { NA }\end{array}$

$\begin{array}{lllllll}\text { Agarilytica } & 4.825281 & 1.002669 & 1.627649 & 0.616022 & 0.53788 \text { NA }\end{array}$

$\begin{array}{llllll}\text { Thermovibrio } & 1.181055 & -0.8181 & 2.126752 & -0.38467 & 0.700482 \\ & \text { NA }\end{array}$

Amphibacillus $\quad \begin{array}{llllll}3.70092 & -1.08335 & 1.309776 & -0.82712 & 0.408167 & \text { NA }\end{array}$

$\begin{array}{lllllll}\text { Wigglesworthia } & 3.923184 & 1.919854 & 1.482544 & 1.294972 & 0.19533\end{array}$

$\begin{array}{lllllll}\text { Oblitimonas } & 3.524699 & -1.0742 & 1.170779 & -0.91751 & 0.358876 & \text { NA }\end{array}$

Halodesulfurarch $4.916714 \quad-0.25809 \quad 1.273575 \quad-0.20265 \quad 0.839407$ NA

$\begin{array}{lllllll}\text { Haematospirillum } & 2.558847 & -1.54177 & 1.37724 & -1.11946 & 0.262943 & \text { NA }\end{array}$

$\begin{array}{lllllll}\text { Paraglaciecola } & 2.856745 & -1.01759 & 1.717243 & -0.59257 & 0.553468 \text { NA }\end{array}$

$\begin{array}{lllllll}\text { Parachlamydia } & 1.363381 & -0.58868 & 2.106818 & -0.27942 & 0.779926 \\ \text { NA }\end{array}$

$\begin{array}{lllllll}\text { Candidatus Desul } & 1.524333 & 0.749205 & 2.483396 & 0.301685 & 0.762892 & \text { NA }\end{array}$

$\begin{array}{llllll}\text { Natronobacteriur } 3.108549 & -1.90161 & 1.440649 & -1.31997 & 0.186845 \text { NA }\end{array}$

$\begin{array}{lllllll}\text { Thermanaeromor } & 2.046366 & -1.64098 & 2.019566 & -0.81254 & 0.416481 & \text { NA }\end{array}$

$\begin{array}{lllllll}\text { Calditerrivibrio } & 1.636352 & 1.618683 & 2.03935 & 0.793725 & 0.427356 & \mathrm{NA}\end{array}$

$\begin{array}{lllllll}\text { Pleurocapsa } & 1.643127 & -1.43892 & 1.758207 & -0.8184 & 0.413129 & \text { NA }\end{array}$

$\begin{array}{lllllll}\text { Hippea } & 0.257865 & -0.33681 & 2.938557 & -0.11462 & 0.908749 \text { NA }\end{array}$

Hydrogenobaculı $2.456086 \quad-0.7844 \quad 1.89565 \quad-0.41379 \quad 0.679028$ NA

$\begin{array}{lllllll}\text { Denitrovibrio } & 1.009245 & -0.82955 & 1.800105 & -0.46083 & 0.644919 & \text { NA }\end{array}$

$\begin{array}{lllllll}\text { Pragia } & 2.370053 & -1.94907 & 1.373525 & -1.41903 & 0.15589 \text { NA }\end{array}$

$\begin{array}{lllllll}\text { Nitrosopumilus } & 3.649158 & 1.094559 & 1.524571 & 0.717945 & 0.472791 \mathrm{NA}\end{array}$

$\begin{array}{lllllll}\text { Thalassotalea } & 1.194545 & -0.95815 & 1.940334 & -0.49381 & 0.621444 \text { NA }\end{array}$

$\begin{array}{lllllll}\text { Kayvirus } & 4.52068 & -0.62993 & 2.900921 & -0.21715 & 0.828092 \\ & 4.911501 & -0.97427 & 1.118571 & -0.87099 & 0.383759\end{array}$

$\begin{array}{lllllll}\text { Ammonifex } & 4.911501 & -0.97427 & 1.118571 & -0.87099 & 0.383759 & \text { NA }\end{array}$

$\begin{array}{lllllll}\text { Sfi21dt1virus } & 1.637426 & -2.15298 & 2.920828 & -0.73711 & 0.461054 \text { NA }\end{array}$

$\begin{array}{lllllll}\text { Catenovulum } & 1.282561 & 0.196026 & 1.918088 & 0.102199 & 0.918599 & \text { NA }\end{array}$

$\begin{array}{lllllll}\text { Candidatus Cyclol } 3.685985 & -0.57538 & 1.237177 & -0.46507 & 0.641878 \text { NA }\end{array}$

$\begin{array}{llllll}\text { Magnetococcus } & 2.446447 & -2.26536 & 1.7094 & -1.32524 & 0.185092 \\ \text { NA }\end{array}$

$\begin{array}{lllllll}\text { Basilea } & 5.133195 & -0.28426 & 1.366503 & -0.20802 & 0.835213 & \text { NA }\end{array}$

$\begin{array}{lllllll}\text { Pseudohongiella } & 2.902304 & -2.98745 & 1.463412 & -2.04143 & 0.041208 \text { NA }\end{array}$

$\begin{array}{llllllll}\text { Parapoxvirus } & 1.704017 & -2.92874 & 2.877159 & -1.01793 & 0.308712 \text { NA }\end{array}$

$\begin{array}{lllllll}\text { Thermoproteus } & 4.798012 & 0.116242 & 1.205312 & 0.096441 & 0.92317 \text { NA }\end{array}$

$\begin{array}{llllll}\text { Halothermothrix } & 4.665893 & 1.633116 & 1.40471 & 1.1626 & 0.244992 \\ \text { NA }\end{array}$

$\begin{array}{lllllll}\text { Thermocrinis } & 2.585131 & -1.81828 & 1.706193 & -1.0657 & 0.286561 & \text { NA }\end{array}$

$\begin{array}{llllll}\text { Candidatus Babel } & 1.857017 & 3.445747 & 2.115426 & 1.628867 & 0.103341\end{array}$

$\begin{array}{llllll}\text { Thermosulfidibac } & 1.858818 & -1.27568 & 1.735512 & -0.73505 & 0.462311\end{array}$

$\begin{array}{lllllll}\text { Hydromonas } & 5.136116 & -0.26195 & 1.1266 & -0.23251 & 0.816141 & \text { NA }\end{array}$

$\begin{array}{lllllll}\text { Candidatus Parac } & 0.758415 & 0.569212 & 2.443197 & 0.232979 & 0.815778 \text { NA }\end{array}$

$\begin{array}{lllllll}\text { Simkania } & 0.476166 & 0.240925 & 2.933749 & 0.082122 & 0.93455 & \text { NA }\end{array}$ 


\begin{tabular}{|c|c|c|c|c|c|}
\hline Saprospira & 1.083494 & -1.13306 & 2.143336 & -0.52864 & $0.597054 \mathrm{NA}$ \\
\hline Oligella & 5.083567 & -1.07627 & 1.018473 & -1.05674 & $0.290628 \mathrm{NA}$ \\
\hline Tamlana & 2.762415 & 2.549008 & 2.11805 & 1.20347 & $0.228795 \mathrm{NA}$ \\
\hline Sulfurihydrogenik & 4.426557 & -0.02991 & 1.270094 & -0.02355 & $0.981213 \mathrm{NA}$ \\
\hline Planktothrix & 3.847081 & -1.69403 & 1.489746 & -1.13713 & $0.255485 \mathrm{NA}$ \\
\hline Desulfobacula & 2.319451 & -1.06372 & 1.268059 & -0.83886 & $0.401548 \mathrm{NA}$ \\
\hline Solendovirus & 1.452513 & 0.315152 & 2.515631 & 0.125278 & $0.900304 \mathrm{NA}$ \\
\hline Wizardvirus & 1.372713 & 0.891227 & 2.276272 & 0.391529 & $0.695406 \mathrm{NA}$ \\
\hline Defluviitoga & 1.516111 & 2.438297 & 2.197059 & 1.109801 & $0.267085 \mathrm{NA}$ \\
\hline Salinispira & 1.968419 & -1.69725 & 1.579627 & -1.07446 & $0.282615 \mathrm{NA}$ \\
\hline Commensalibact $\epsilon$ & 2.243253 & -0.39988 & 1.758836 & -0.22736 & 0.820146 NA \\
\hline Methanoregula & 2.235204 & -1.01328 & 1.276301 & -0.79392 & $0.427241 \mathrm{NA}$ \\
\hline Acetohalobium & 2.98098 & 0.260505 & 1.497023 & 0.174016 & $0.861853 \mathrm{NA}$ \\
\hline Psychroflexus & 2.940878 & 0.047627 & 1.417476 & 0.0336 & $0.973196 \mathrm{NA}$ \\
\hline Lit1virus & 1.698766 & -2.92936 & 2.326829 & -1.25895 & $0.208049 \mathrm{NA}$ \\
\hline Raphidiopsis & 1.049961 & -1.66214 & 2.390752 & -0.69524 & $0.486906 \mathrm{NA}$ \\
\hline Saccharophagus & 4.175013 & -3.11082 & 1.360285 & -2.28689 & $0.022203 \mathrm{NA}$ \\
\hline Oenococcus & 3.901926 & 0.10544 & 1.124451 & 0.093771 & $0.925291 \mathrm{NA}$ \\
\hline Congregibacter & 3.965501 & -1.57191 & 1.491435 & -1.05396 & $0.291903 \mathrm{NA}$ \\
\hline Endozoicomonas & 4.15818 & -2.77069 & 1.279101 & -2.16612 & $0.030302 \mathrm{NA}$ \\
\hline Chloroherpeton & 2.512989 & -0.48613 & 1.520235 & -0.31977 & $0.749141 \mathrm{NA}$ \\
\hline Zhongshania & 4.443025 & -0.87042 & 1.073336 & -0.81095 & $0.417393 \mathrm{NA}$ \\
\hline Neorickettsia & 1.154605 & -0.24678 & 2.128899 & -0.11592 & $0.907716 \mathrm{NA}$ \\
\hline Deltapolyomavirı & 4.210992 & 1.192614 & 2.900304 & 0.411203 & $0.680924 \mathrm{NA}$ \\
\hline Halanaeroarchaeı & 4.857746 & -1.64278 & 1.00746 & -1.63062 & $0.102971 \mathrm{NA}$ \\
\hline Gilvibacter & 1.387162 & -1.99666 & 1.632719 & -1.22291 & $0.221365 \mathrm{NA}$ \\
\hline Orientia & 4.273248 & 3.62009 & 1.87117 & 1.934666 & $0.053031 \mathrm{NA}$ \\
\hline Bacteriovorax & 3.459514 & -1.39538 & 1.365524 & -1.02186 & $0.306846 \mathrm{NA}$ \\
\hline Candidatus Profft & 1.431663 & -2.68531 & 1.973716 & -1.36053 & $0.173661 \mathrm{NA}$ \\
\hline Hydrogenovibrio & 0.63812 & -0.312 & 2.541549 & -0.12276 & 0.902299 NA \\
\hline Nitrososphaera & 0.196212 & -0.03114 & 2.941412 & -0.01059 & 0.991552 NA \\
\hline Aequorivita & 2.760397 & 0.618717 & 1.606852 & 0.385049 & $0.700201 \mathrm{NA}$ \\
\hline Lawsonia & 3.910299 & -1.06136 & 1.256995 & -0.84436 & $0.398467 \mathrm{NA}$ \\
\hline Tatlockia & 0.612046 & -1.47423 & 2.435679 & -0.60527 & $0.545002 \mathrm{NA}$ \\
\hline Spongiibacter & 2.005018 & -1.66827 & 1.488842 & -1.12052 & $0.262493 \mathrm{NA}$ \\
\hline Gloeocapsa & 3.60652 & -0.64549 & 1.323433 & -0.48774 & $0.625735 \mathrm{NA}$ \\
\hline Basfia & 4.983455 & -2.10282 & 1.578561 & -1.33211 & $0.182824 \mathrm{NA}$ \\
\hline Pyrococcus & 2.614694 & 0.187292 & 1.602976 & 0.11684 & $0.906987 \mathrm{NA}$ \\
\hline Grimontia & 2.900798 & -1.92496 & 1.348015 & -1.428 & $0.153292 \mathrm{NA}$ \\
\hline Wolinella & 1.258121 & -0.63316 & 2.103251 & -0.30104 & $0.763384 \mathrm{NA}$ \\
\hline
\end{tabular}


$\begin{array}{llllll}\text { Methanothermok } & 0.934738 & 2.233904 & 2.895538 & 0.771499 & 0.440411\end{array}$

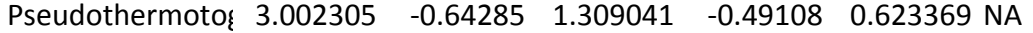
$\begin{array}{lllllll}\text { Carboxydothermı } & 1.20622 & -0.93421 & 1.929477 & -0.48418 & 0.628261 \\ \text { NA }\end{array}$ $\begin{array}{lllllll}\text { Desulfurobacteril } & 2.817621 & -1.85636 & 1.440599 & -1.2886 & 0.197537 & \mathrm{NA}\end{array}$ Synechocystis $\quad 4.424027 \quad-0.40069 \quad 1.089879 \quad-0.36765 \quad 0.713137$ NA $\begin{array}{lllllll}\text { Candidatus Nucle } & 1.845164 & -3.05958 & 1.898938 & -1.61121 & 0.107134 \text { NA }\end{array}$

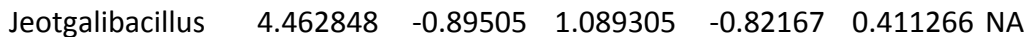

$\begin{array}{lllllll}\text { Candidatus Izima| } 4.358608 & -0.15751 & 1.194858 & -0.13182 & 0.895126 \text { NA }\end{array}$

$\begin{array}{lllllll}\text { Methanothrix } & 4.742449 & -1.49386 & 1.140488 & -1.30985 & 0.190248 \text { NA }\end{array}$

Flexistipes $\quad \begin{array}{lllllll}1.739196 & -1.15682 & 1.894659 & -0.61057 & 0.541486 \text { NA }\end{array}$ $\begin{array}{lllllll}\text { Desulfohalobium } & 2.991325 & -0.54897 & 1.30758 & -0.41983 & 0.674607 & \text { NA }\end{array}$ $\begin{array}{llllll}\text { Reinekea } & 4.298798 & -1.50135 & 1.21841 & -1.23222 & 0.217866 \text { NA }\end{array}$ $\begin{array}{llllll}\text { Candidatus Hoagl } & 0.724128 & 2.162588 & 2.931862 & 0.737616 & 0.460748 \text { NA }\end{array}$ $\begin{array}{llllll}\text { Caldanaerobacteı } & 1.649431 & -0.44676 & 1.907636 & -0.2342 & 0.814833\end{array}$ $\begin{array}{lllllll}\text { Candidatus Nitro؛ } 4.561572 & 0.855682 & 1.42139 & 0.602004 & 0.547172 & \text { NA }\end{array}$ $\begin{array}{lllllll}\text { Candidatus Cardiı } 4.565864 & 0.063609 & 1.447725 & 0.043937 & 0.964954 \text { NA }\end{array}$ $\begin{array}{lllllll}\text { Acetomicrobium } & 0.651316 & 0.12473 & 2.262397 & 0.055132 & 0.956033 & \mathrm{NA}\end{array}$ $\begin{array}{lllllll}\text { Dactylococcopsis } & 1.57836 & -2.00956 & 2.07007 & -0.97077 & 0.331663 & \text { NA }\end{array}$ Thermodesulfoba $3.408609 \quad-0.56863 \quad 1.509413 \quad-0.37673 \quad 0.706378$ NA

$\begin{array}{lllllll}\text { Gynuella } & 4.176185 & -1.76911 & 1.200058 & -1.47419 & 0.140431 & \text { NA }\end{array}$ $\begin{array}{lllllll}\text { Desulfurella } & 4.763829 & 1.442353 & 1.620528 & 0.890051 & 0.373438 \text { NA }\end{array}$ $\begin{array}{lllllll}\text { Ichthyobacterium } & 1.427874 & 0.081119 & 2.112167 & 0.038406 & 0.969364\end{array}$ $\begin{array}{lllllll}\text { Salinivirga } & 3.792853 & -2.09376 & 1.261455 & -1.6598 & 0.096955 & \text { NA }\end{array}$ Candidatus Ruthii $1.035186 \quad-2.224592 .495553 \quad-0.89142 \quad 0.372704$ NA $\begin{array}{lllllll}\text { Rhodopirellula } & 5.193066 & -2.05106 & 0.925595 & -2.21593 & 0.026696 \text { NA }\end{array}$ $\begin{array}{lllllll}\text { Avibacterium } & 2.945168 & -2.36155 & 1.404194 & -1.68179 & 0.09261 \mathrm{NA}\end{array}$ $\begin{array}{llllll}\text { Thermofilum } & 1.249172 & -2.06125 & 1.948704 & -1.05776 & 0.290167 \\ \text { NA }\end{array}$ $\begin{array}{lllllll}\text { Paenalcaligenes } & 3.050735 & -0.85583 & 1.219297 & -0.7019 & 0.48274 \text { NA }\end{array}$ $\begin{array}{llllll}\text { Hirschia } & 1.13381 & -2.37041 & 1.800435 & -1.31658 & 0.18798 \\ & 3.963459 & -2.01659 & 1.268799 & -1.58937 & 0.111978 \mathrm{NA}\end{array}$ $\begin{array}{lllllll}\text { Euzebyella } & 3.963459 & -2.01659 & 1.268799 & -1.58937 & 0.111978 \text { NA }\end{array}$ $\begin{array}{lllllll}\text { Dehalobacter } & 4.844935 & -1.46874 & 1.21543 & -1.20841 & 0.226888 & \text { NA }\end{array}$ $\begin{array}{llllll}\text { Methanocorpuscl } & 0.689023 & -1.63305 & 2.92807 & -0.55772 & 0.577034 \text { NA }\end{array}$ $\begin{array}{lllllll}\text { Owenweeksia } & 3.448505 & -1.36175 & 1.285713 & -1.05914 & 0.289538 \mathrm{NA}\end{array}$ $\begin{array}{lllllll}\text { Laceyella } & 4.151092 & -2.02395 & 1.366132 & -1.48152 & 0.138469 & \text { NA }\end{array}$ $\begin{array}{lllllll}\text { Fluoribacter } & 3.115192 & -3.10114 & 1.674 & -1.85253 & 0.06395 & \text { NA }\end{array}$ $\begin{array}{lllllll}\text { Lonsdalea } & 4.389266 & -0.36159 & 1.244251 & -0.29061 & 0.771353 & \text { NA }\end{array}$

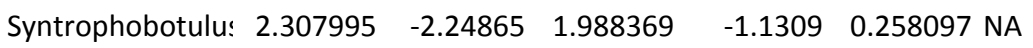
Sphaerospermop: $4.846578 \quad 1.765513 \quad 1.401364 \quad 1.259853 \quad 0.207722$ NA Candidatus Kuent $1.528945 \quad-1.20697 \quad 1.912059-0.63124 \quad 0.527885$ NA $\begin{array}{lllllll}\text { Thermobaculum } & 4.821718 & -1.78723 & 1.068958 & -1.67194 & 0.094537 \text { NA }\end{array}$ 


$\begin{array}{lrrrrr}\text { Parvularcula } & 5.172922 & -3.12849 & 1.015269 & -3.08144 & 0.00206 \mathrm{NA} \\ \text { Syntrophus } & 3.935811 & -0.97585 & 1.162479 & -0.83945 & 0.401215 \mathrm{NA} \\ \text { Metakosakonia } & 0.541399 & -0.01106 & 2.931421 & -0.00377 & 0.996991 \mathrm{NA} \\ \text { Thioploca } & 3.790406 & 1.817136 & 1.695309 & 1.071861 & 0.283782 \mathrm{NA} \\ \text { Caldisphaera } & 1.704541 & 4.765525 & 2.327063 & 2.047871 & 0.040573 \mathrm{NA} \\ \text { Kosmotoga } & 0.93418 & -0.43175 & 2.172654 & -0.19872 & 0.842482 \mathrm{NA} \\ \text { Desulfobacter } & 2.256403 & -1.32813 & 1.276044 & -1.04082 & 0.29796 \mathrm{NA} \\ \text { Arcticibacterium } & 4.351829 & -0.22112 & 1.151083 & -0.1921 & 0.847666 \mathrm{NA} \\ \text { Caldithrix } & 2.985447 & -1.80473 & 1.254675 & -1.4384 & 0.15032 \mathrm{NA} \\ \text { Sediminicola } & 4.707105 & -1.59821 & 1.251326 & -1.27721 & 0.201527 \mathrm{NA} \\ \text { Pseudanabaena } & 5.050413 & -1.26639 & 0.956425 & -1.32409 & 0.185473 \mathrm{NA} \\ \text { Sediminispirochar } & 1.849669 & -0.31129 & 1.604927 & -0.19396 & 0.846206 \mathrm{NA} \\ \text { Brevefilum } & 2.000186 & -0.37049 & 1.880108 & -0.19706 & 0.84378 \mathrm{NA} \\ \text { Obesumbacteriur } & 4.764622 & 0.3432 & 1.420224 & 0.241652 & 0.80905 \mathrm{NA} \\ \text { Pajaroellobacter } & 1.542387 & -2.79927 & 1.921348 & -1.45693 & 0.145136 \mathrm{NA} \\ \text { Salimicrobium } & 0.662108 & -0.24424 & 2.828849 & -0.08634 & 0.931196 \mathrm{NA} \\ \text { Syntrophothermı } & 1.592439 & -1.52292 & 1.885653 & -0.80764 & 0.4193 \mathrm{NA}\end{array}$


ence in fold change

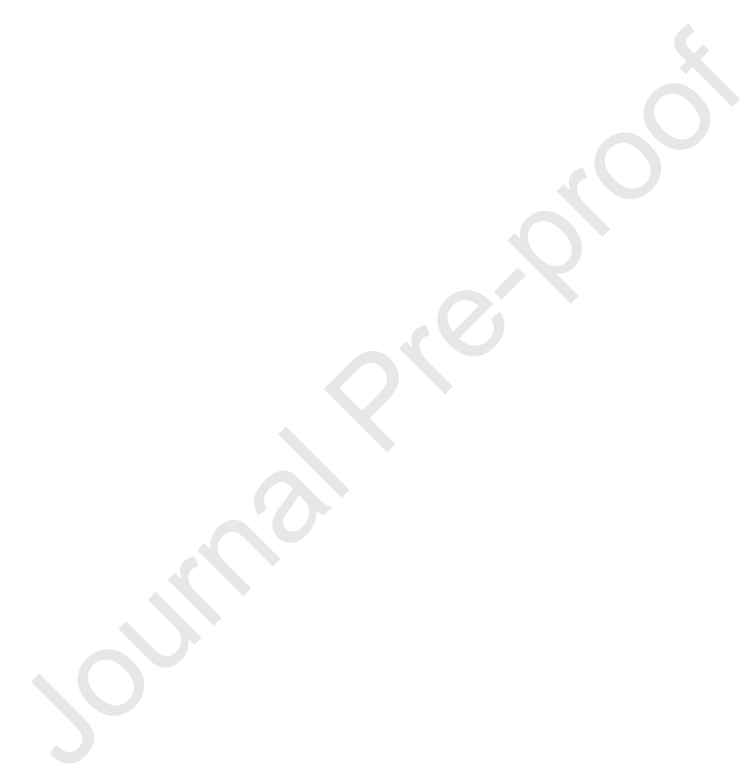


Table S3A: DESeq2 results comparing heathy appearing skin versus lymphoma-affected skin before removal of reads mapping tchomo sapiens additionä $\mathrm{fr} O \mathrm{O}$ of

\begin{tabular}{|c|c|c|c|c|c|c|c|}
\hline & Staphylococcus argenteus & 306.9312 & 2.798081 & 0.7019 & 3.98644 & $6.711-05$ & 0.02034 \\
\hline More abundant in controls & Streptomyces sp. SM17 & 11.13063 & -23.3284 & & & & \\
\hline & Bordetella pertussis & 175.7464 & -27.0922 & 2.535417 & -10.6855 & $1.19 \mathrm{E}-26$ & $2.17 \mathrm{E}-23$ \\
\hline & Streptomyces sp. PVA 94-07 & 13.5985 & -23.6034 & 2.506801 & -9.41573 & $4.70 \mathrm{E}-21$ & $5.70 E-18$ \\
\hline & Methylobacterium oryzae & 5.936652 & -22.4632 & 2.409511 & -9.32273 & $1.13 \mathrm{E}-20$ & $1.03 E-17$ \\
\hline & Serratia sp. LS-1 & 5.682808 & -22.4188 & 2.451911 & -9.14341 & $6.05 E-20$ & $4.40 E-17$ \\
\hline & Burkholderia mallei & 15.64886 & -23.794 & 2.921648 & -8.14405 & $3.82 \mathrm{E}-16$ & $2.32 E-13$ \\
\hline & Enterobacteriaceae bacterium ENNIH2 & 15.55911 & -21.9864 & 2.921651 & -7.52535 & $5.26 \mathrm{E}-14$ & $2.73 E-11$ \\
\hline & Achromobacter ruhlandii & 93.17166 & -8.3322 & 1.779652 & -4.68193 & $2.84 \mathrm{E}-06$ & 0.001149 \\
\hline & Pseudomonas S S. A214 & 13.08098 & -5.67961 & 1.421667 & -399504 & $\begin{array}{l}6.47 E_{-0}-5 \\
\end{array}$ & 0.02034 \\
\hline & Pseudomonas sp. St29 & 12.09194 & -5.43832 & 1358924 & -400193 & 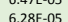 & 0.02034 \\
\hline
\end{tabular}

\begin{tabular}{|c|c|c|c|c|c|c|c|}
\hline & & & & & stat & & padj \\
\hline \multirow[t]{10}{*}{ More abundant in controls } & Bordetella pertussis & 168.646922 & -27.0590291 & 2.531905 & $5-10.6872$ & $1.17 \mathrm{E}-26$ & $4.25 E-23$ \\
\hline & Streptomyces sp. PVA 94-07 & 13.5808162 & -23.62261963 & 2.511117 & 7-9.40722 & $5.09 E-21$ & $9.27 \mathrm{E}-18$ \\
\hline & Methylobacterium oryzae & 5.71572359 & -22.42307046 & 2.413935 & $5-9.28901$ & $1.56 \mathrm{E}-20$ & $1.89 \mathrm{E}-17$ \\
\hline & Serratia sp. LS-1 & 5.55394539 & -22.40099735 & 2.452874 & -9.13255 & $6.69 \mathrm{E}-20$ & $6.08 E-17$ \\
\hline & Enterobacteriaceae bacterium ENNIH2 & 15.2269105 & -23.77994882 & 2.921655 & -8.13921 & 3.98E-16 & $2.72 E-13$ \\
\hline & Burkholderia mallei & 14.7909703 & -23.73784285 & 2.921668 & $3-8.12476$ & 4.48E-16 & $2.72 \mathrm{E}-13$ \\
\hline & Streptomyces sp. SM17 & 7.98191474 & -20.97608447 & 2.849455 & $5-7.36144$ & $1.82 \mathrm{E}-13$ & $9.46 E-11$ \\
\hline & Achromobacter ruhlandii & 89.1918983 & -8.227766811 & 1.777764 & $4-4.62815$ & 3.69E-06 & 0.001678 \\
\hline & Pseudomonas sp. St29 & 11.4377574 & -5.352938714 & 1.356342 & -3.9466 & $7.93 \mathrm{E}-05$ & 0.032042 \\
\hline & Pseudomonas sp. A214 & 12.1683667 & -5.499267147 & 1.426912 & -3.85396 & 0.0001162 & 0.042281 \\
\hline More abundant in lesions & Staphylococcus argenteus & 87.1459201 & 0.410660923 & 0.52976 & 50.77518 & 0.4382318 & 0.683432 \\
\hline
\end{tabular}




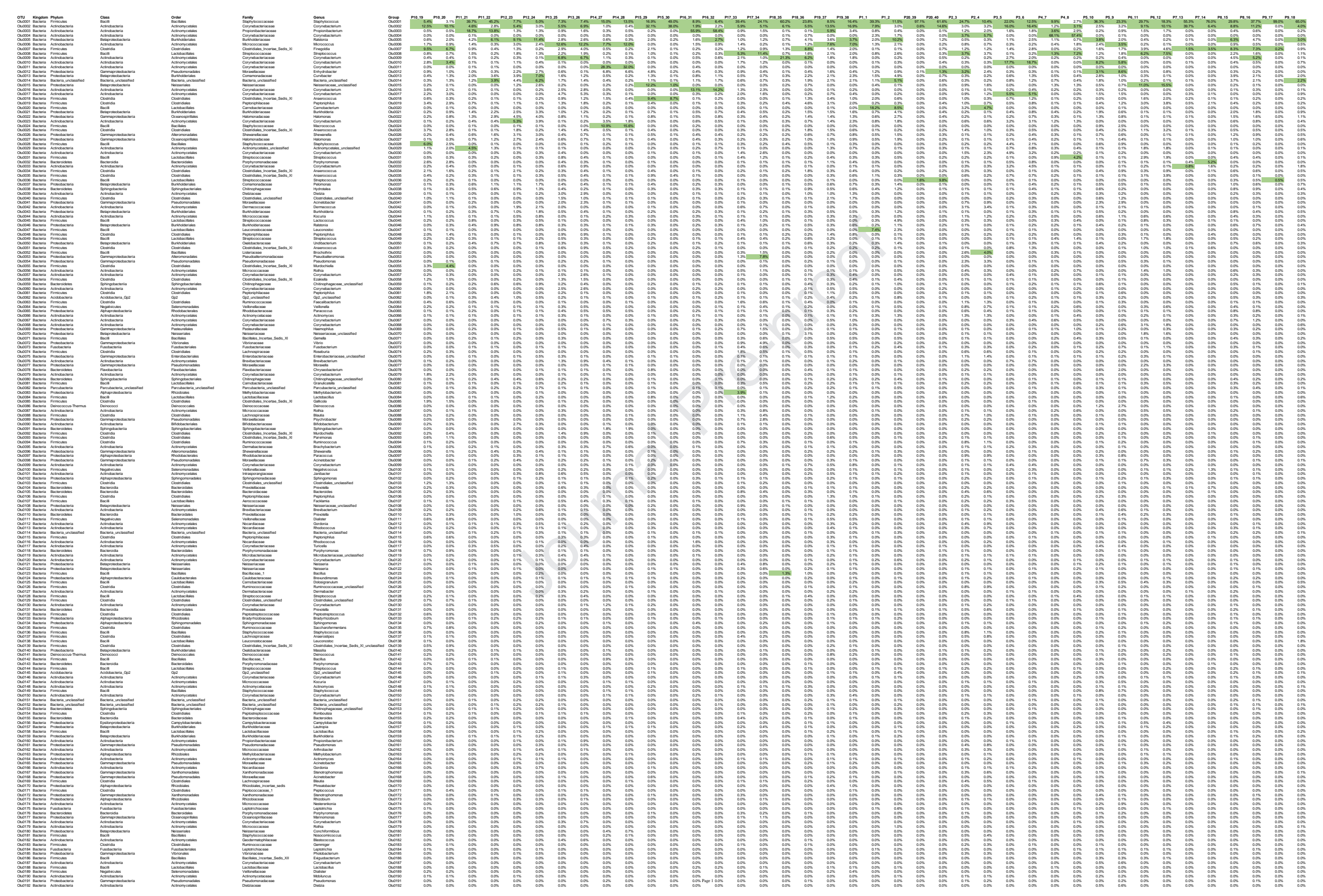




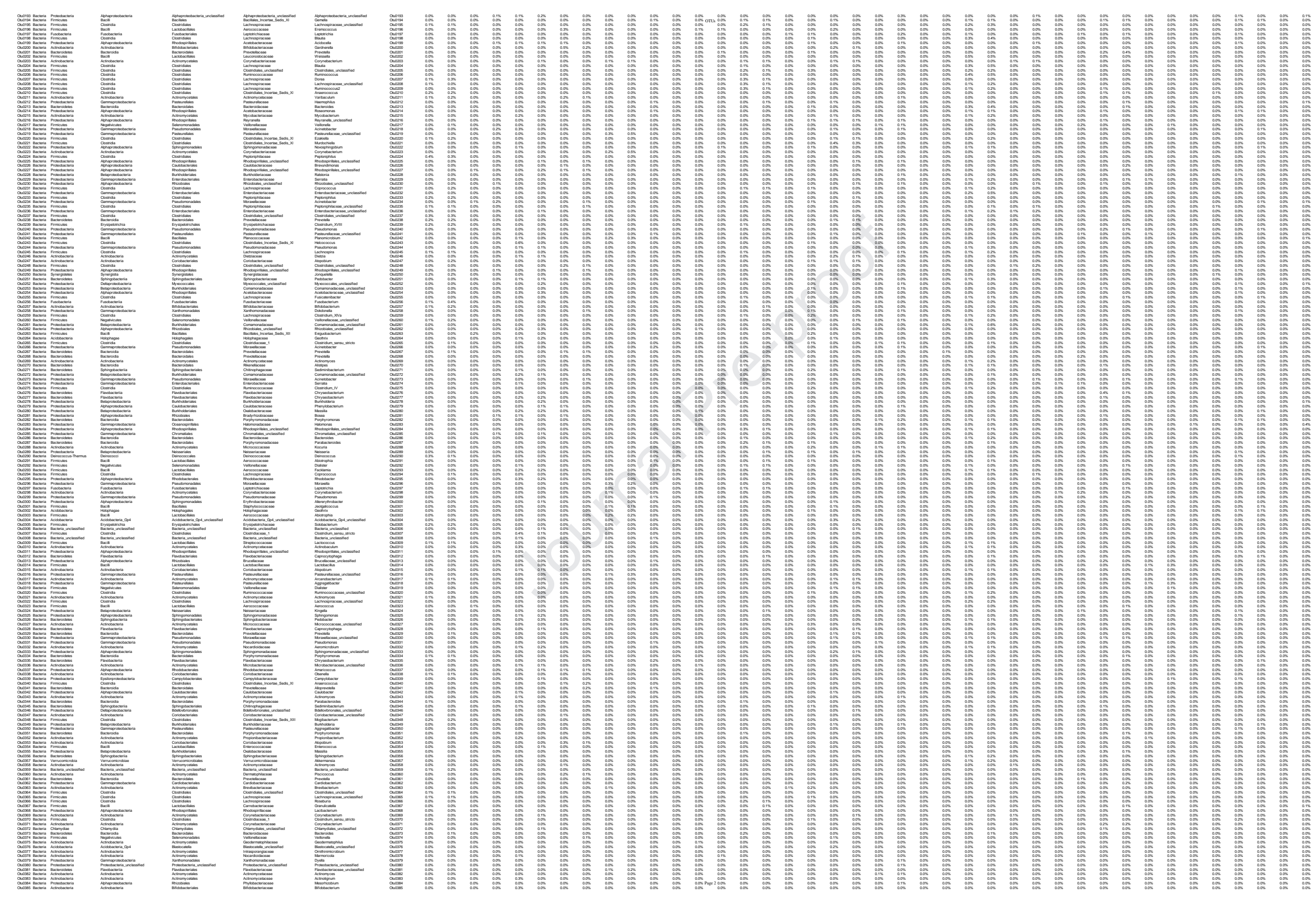




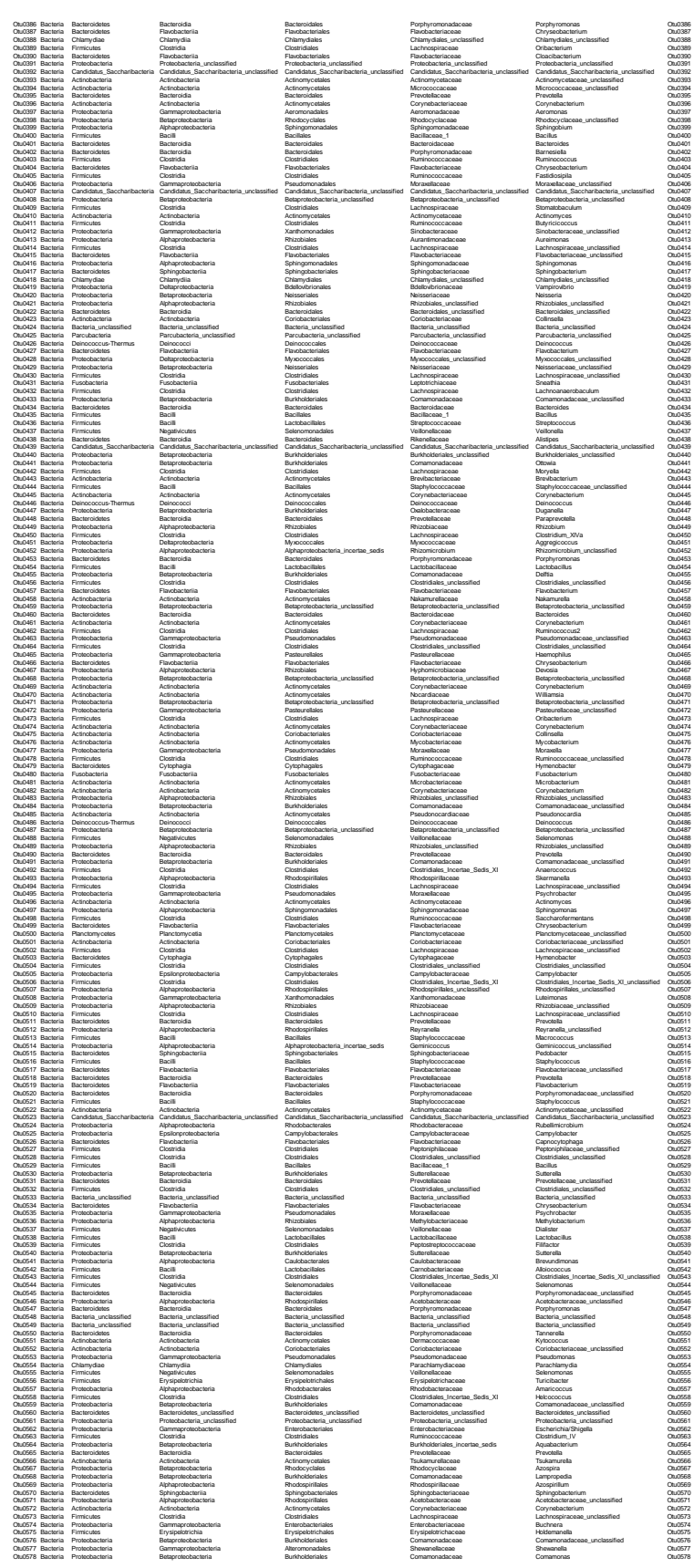




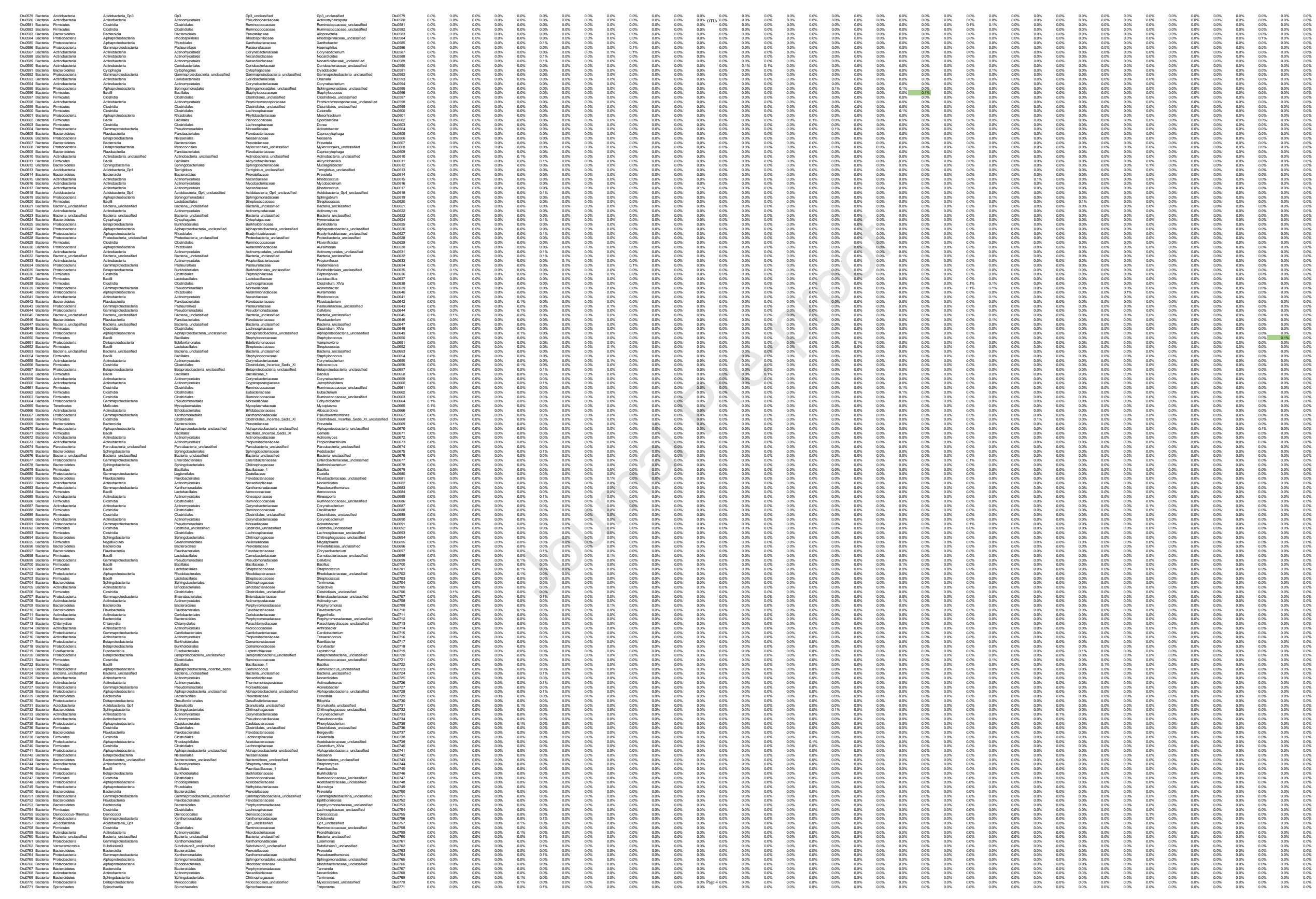




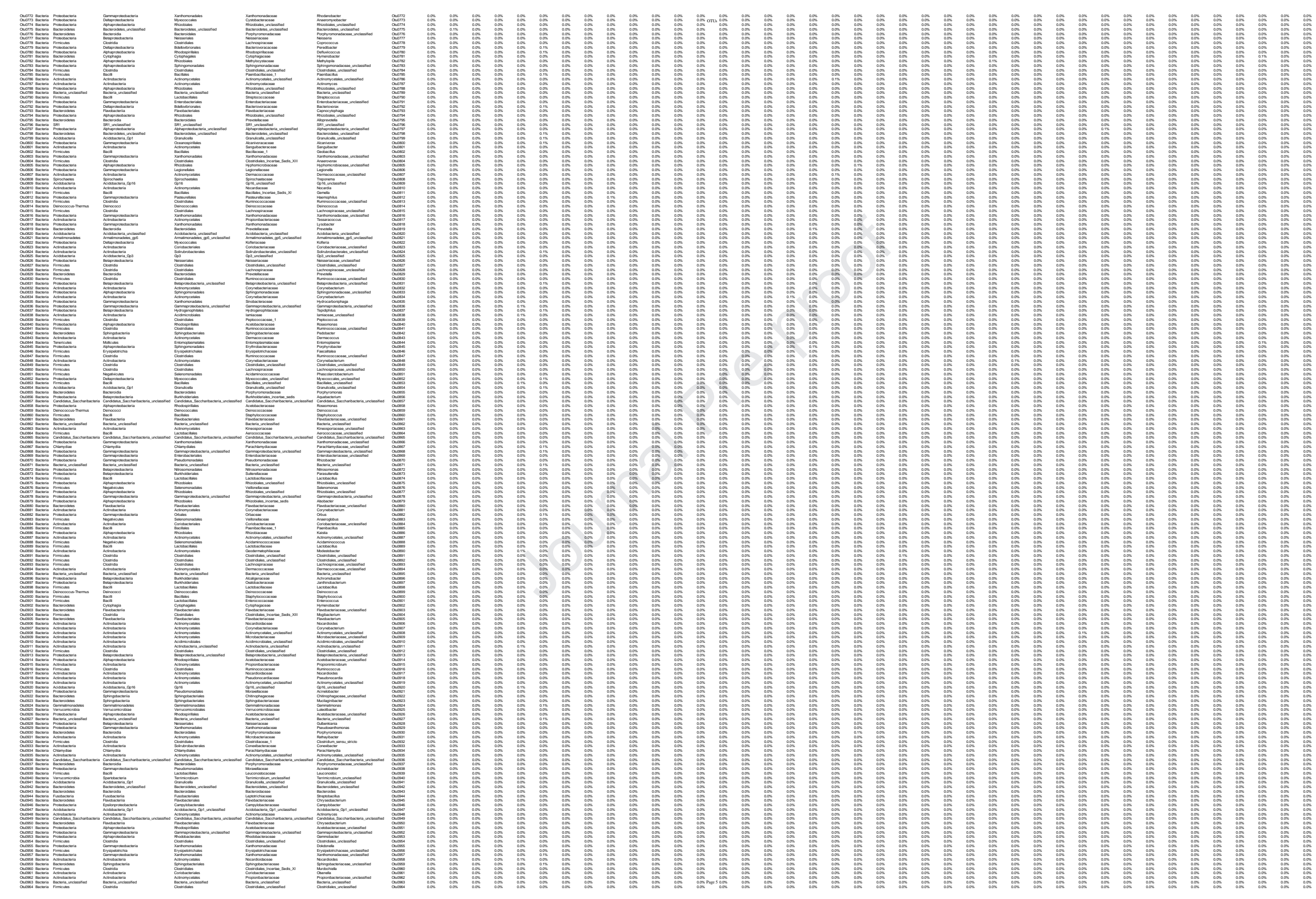



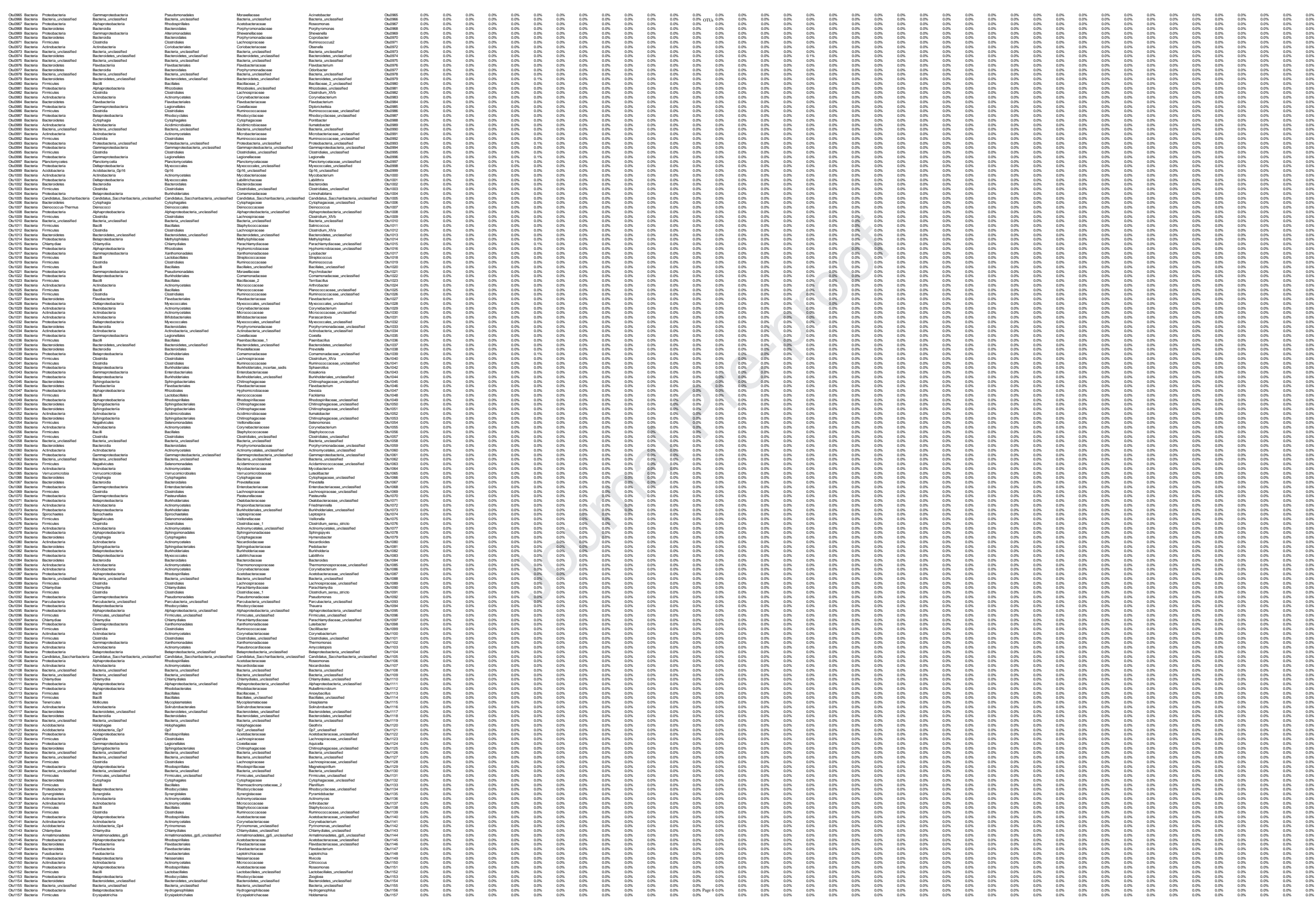


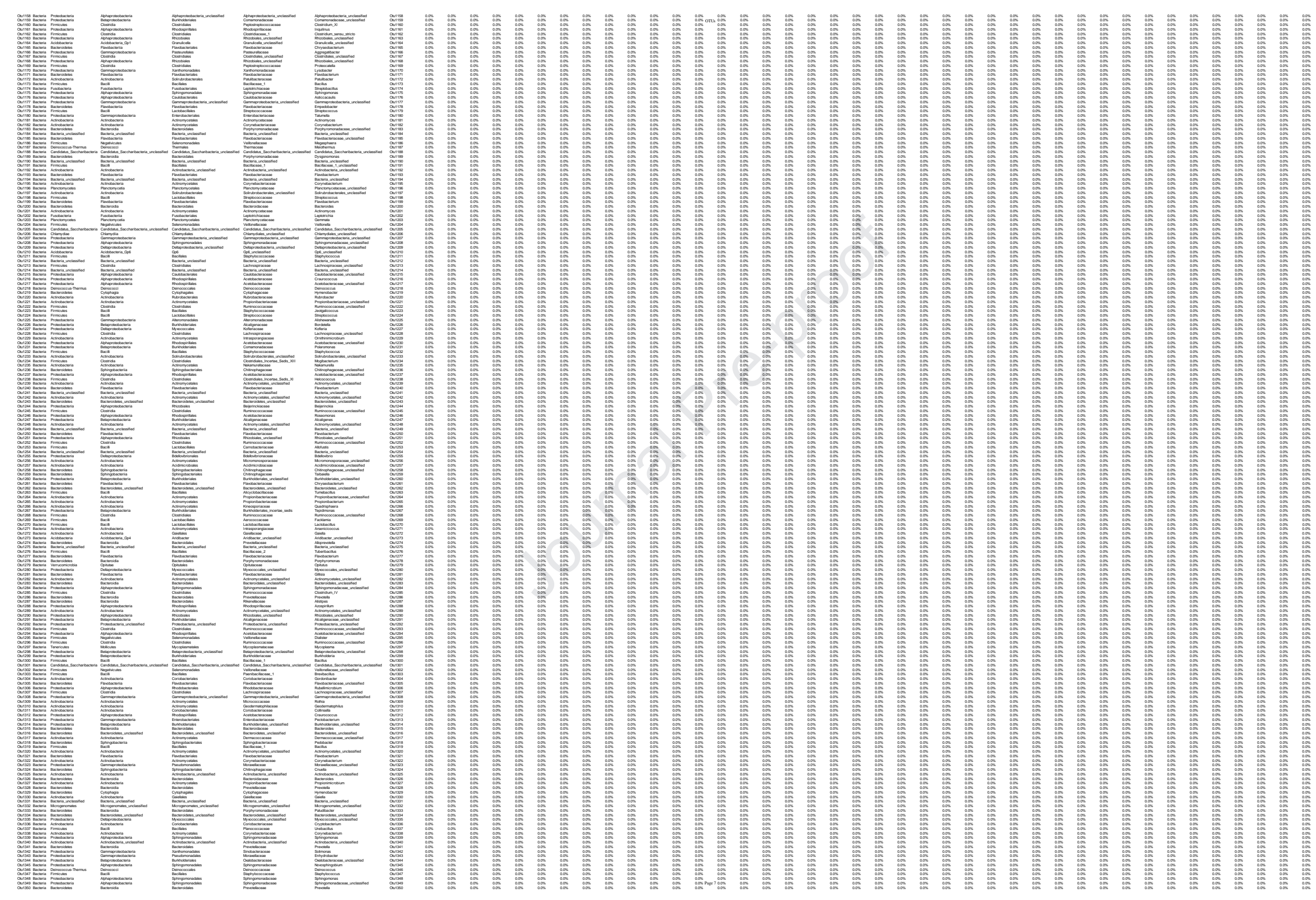



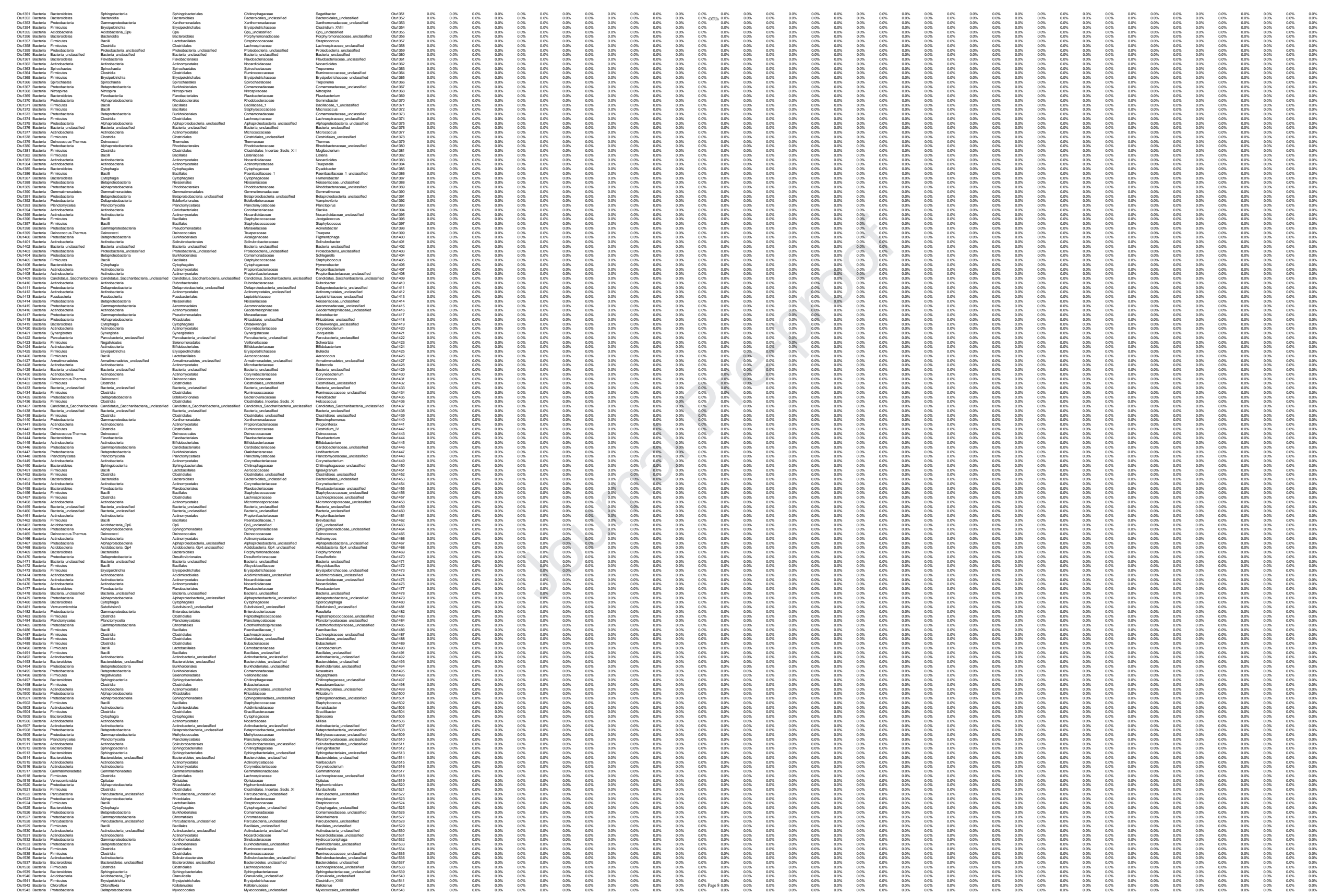

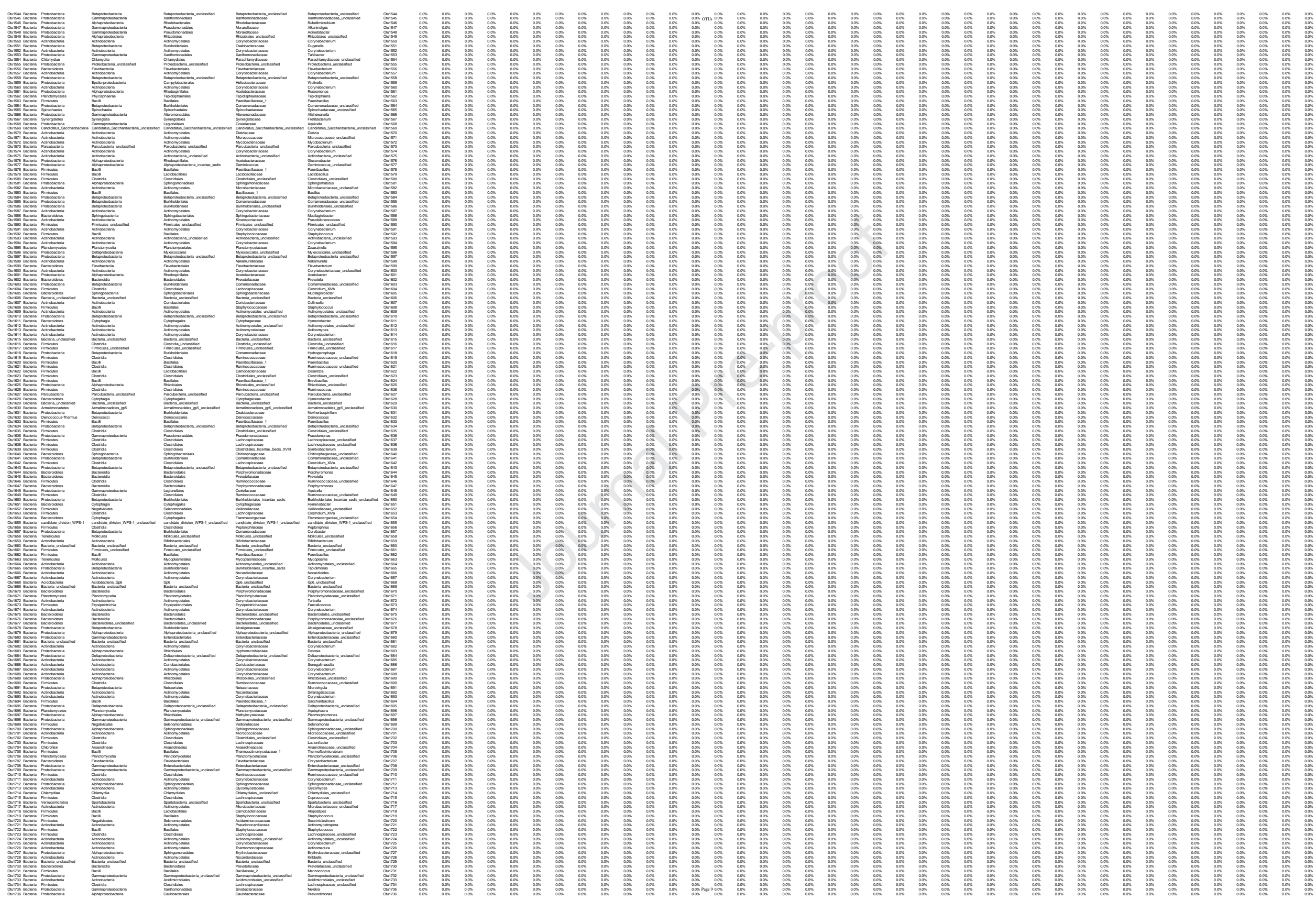


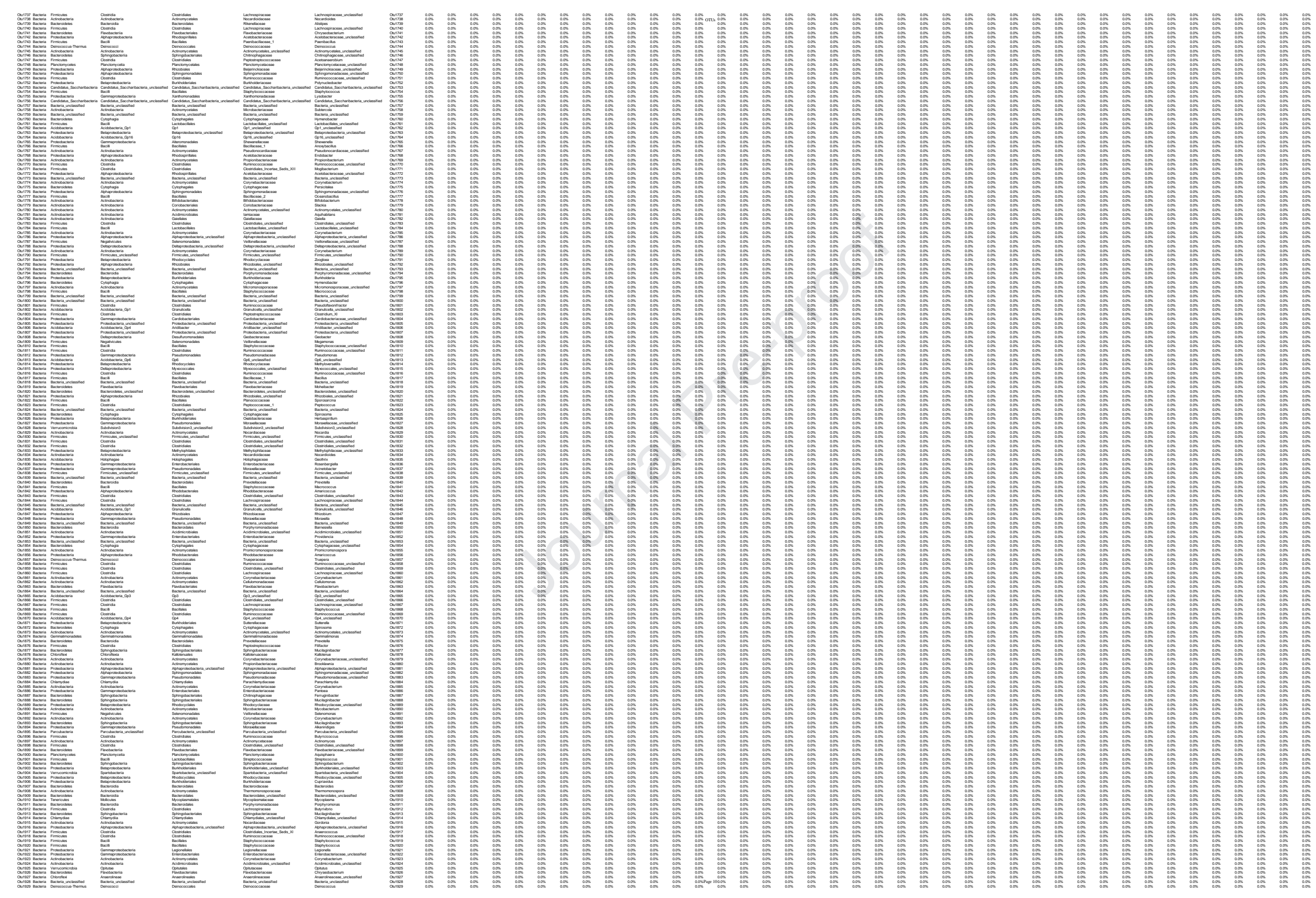



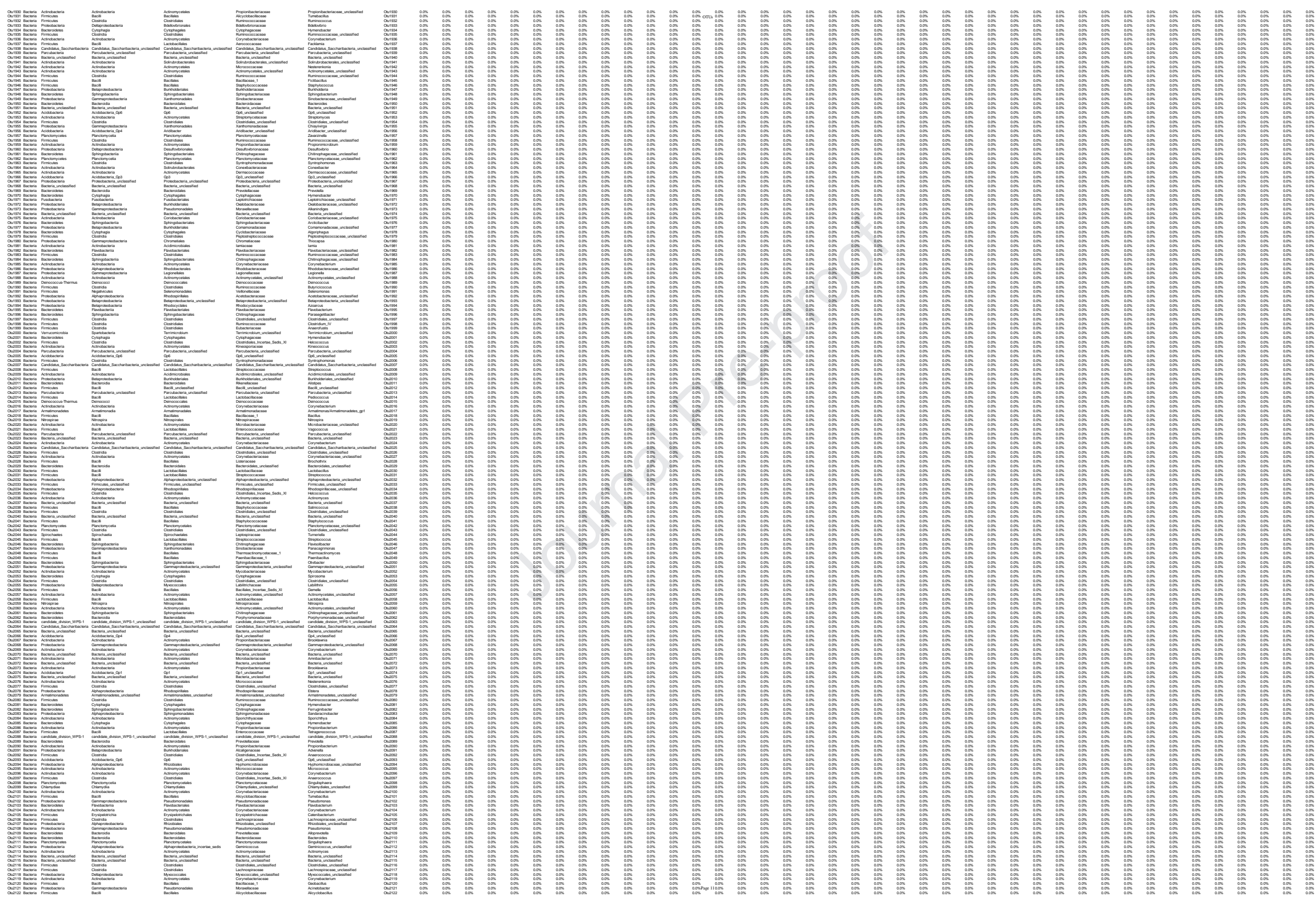


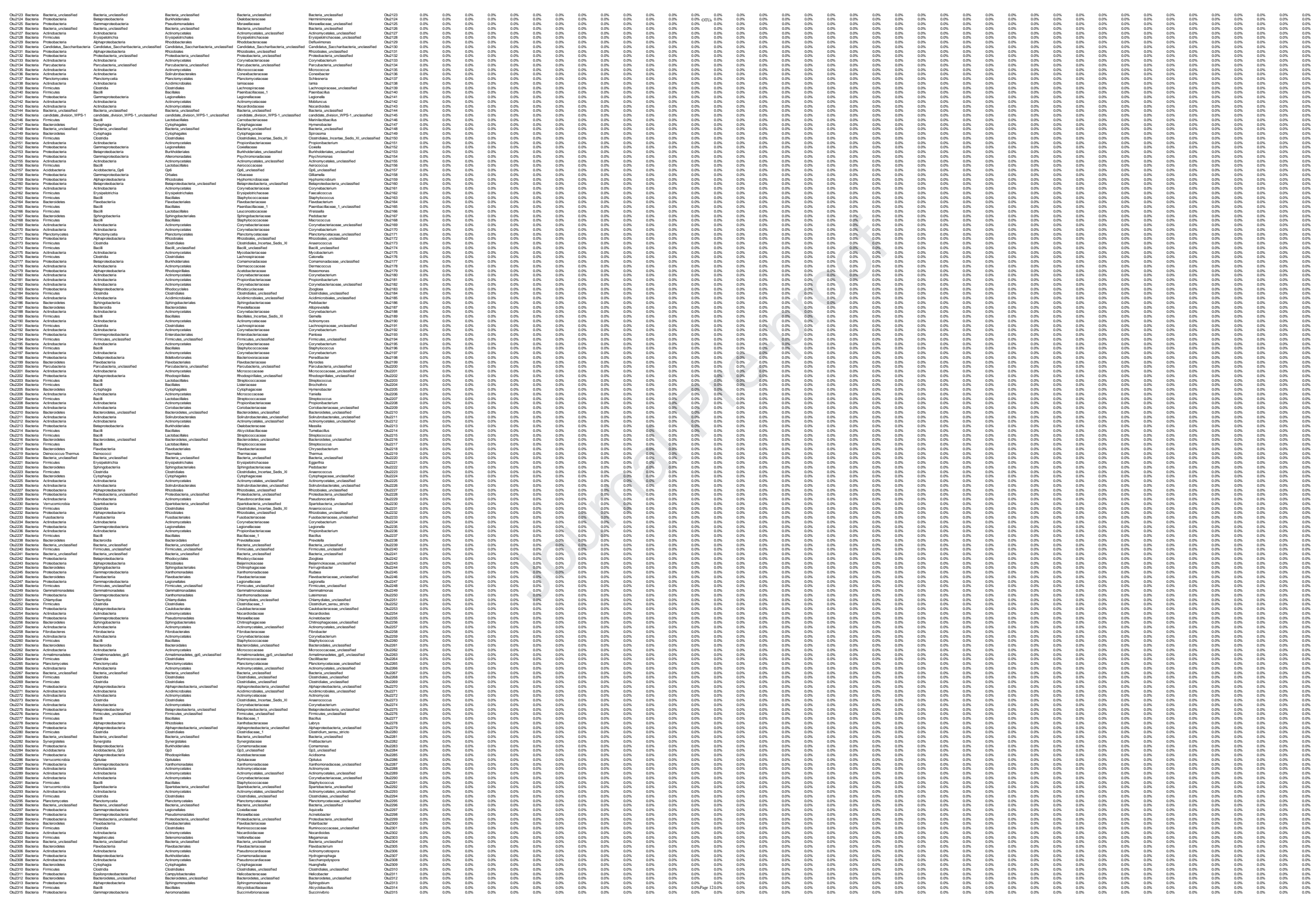



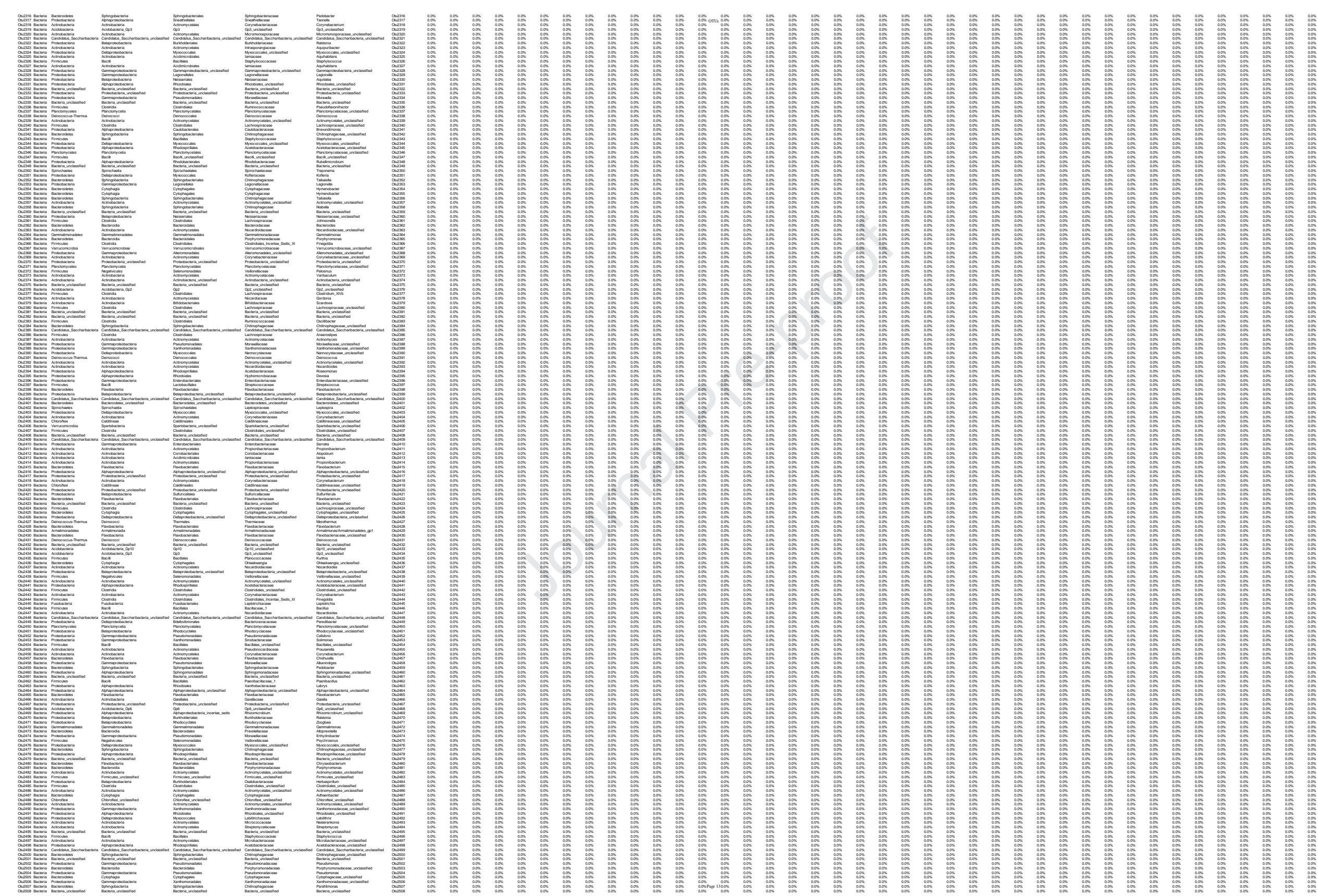

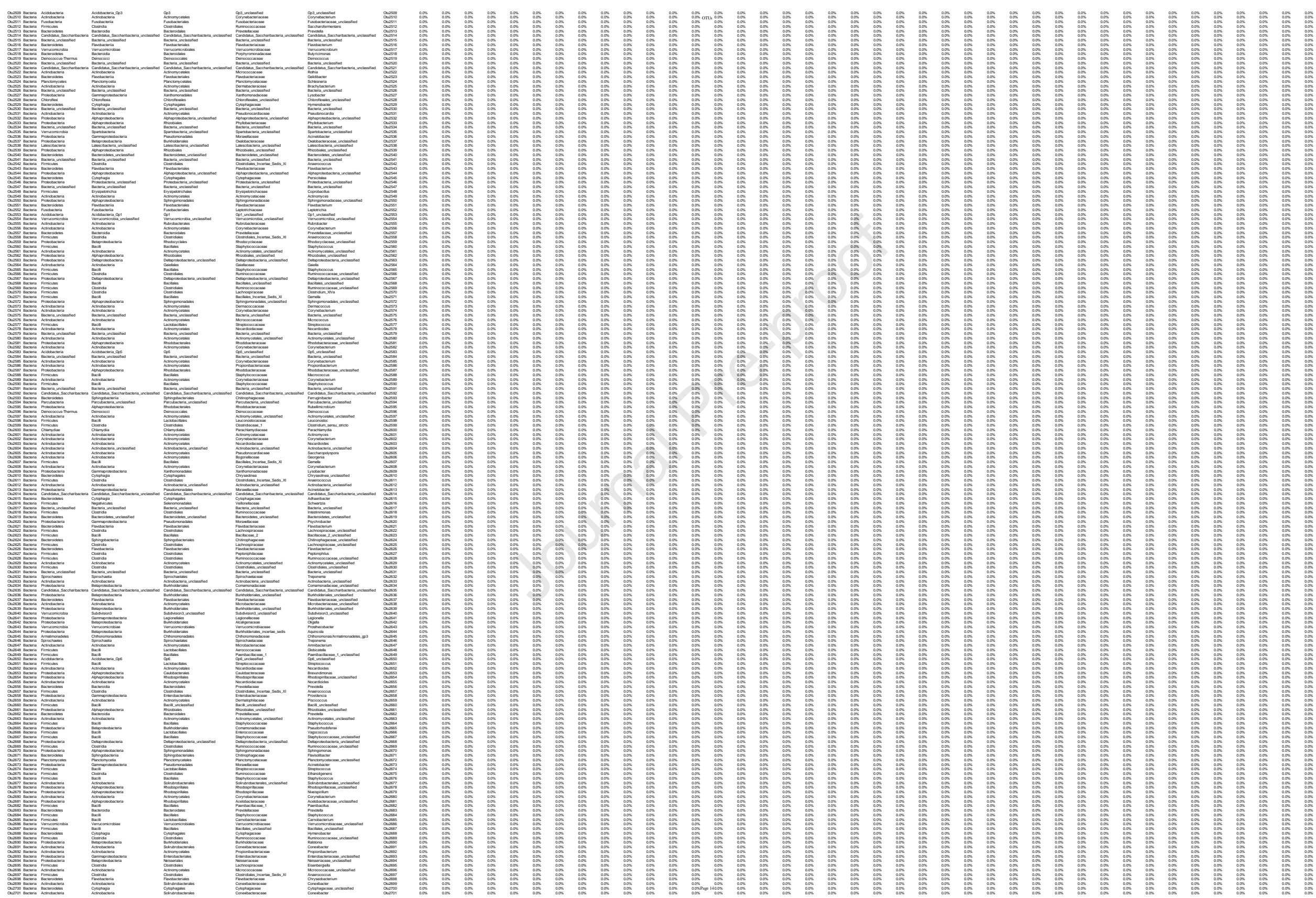


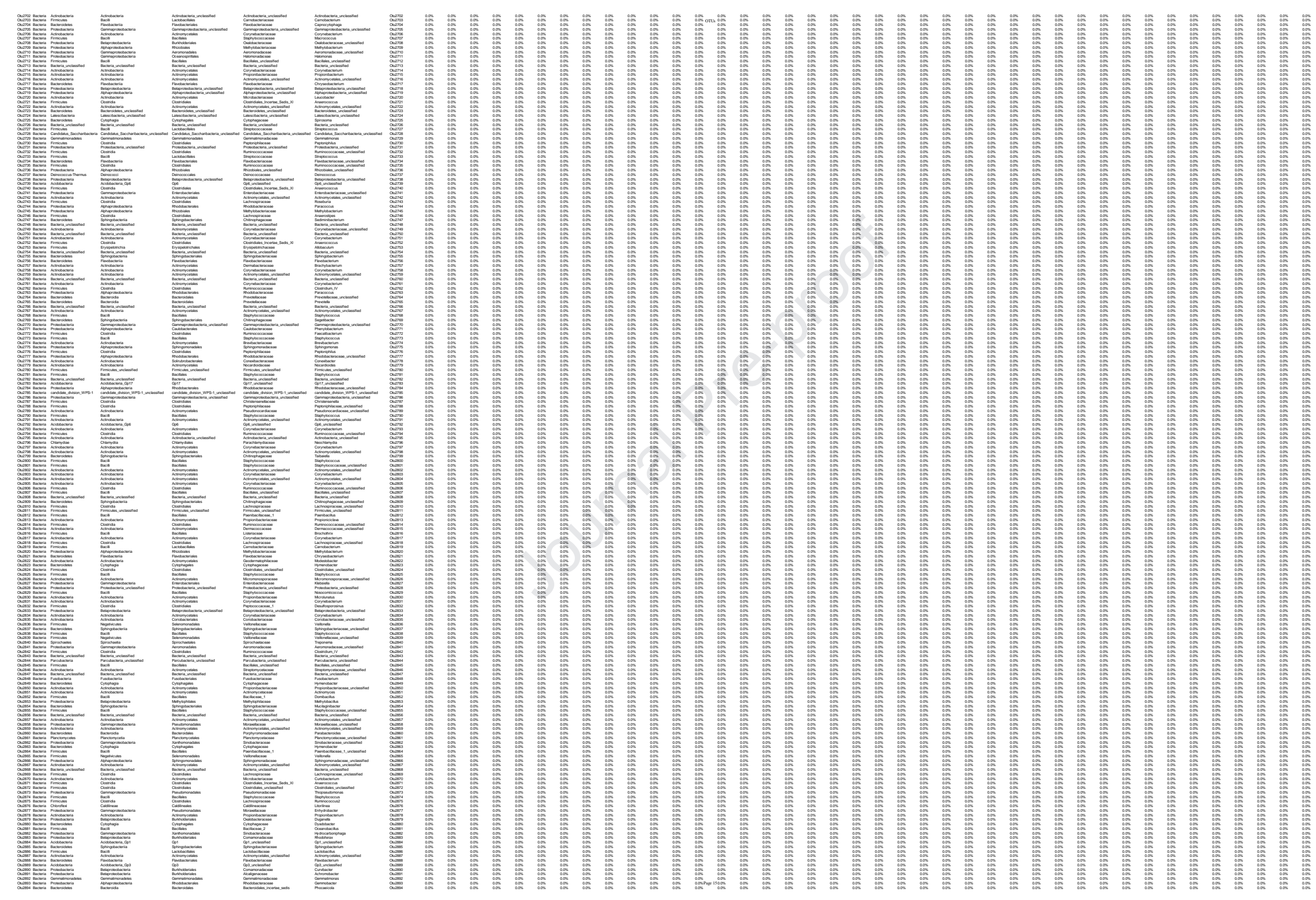




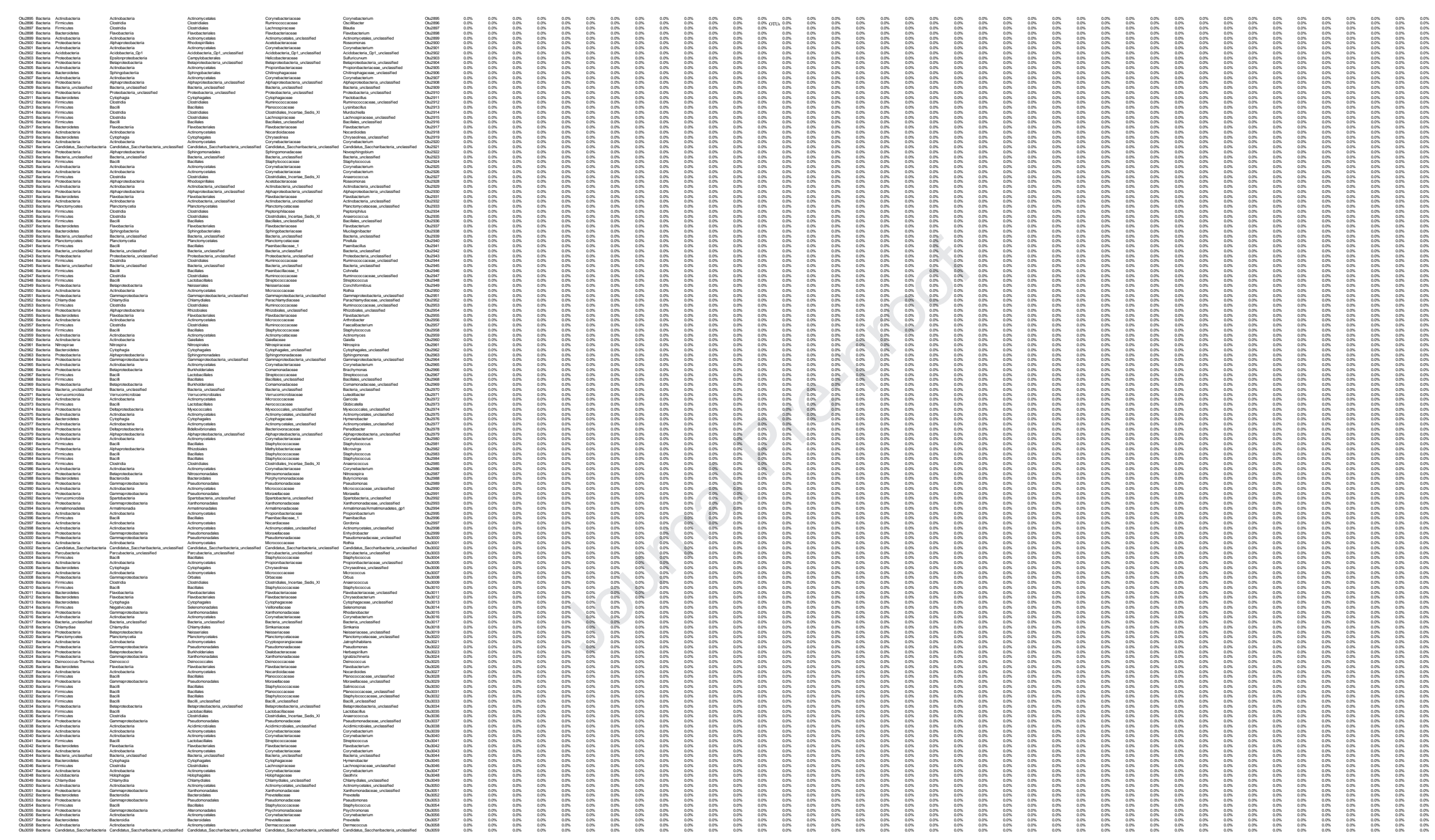




\begin{tabular}{|c|c|c|c|}
\hline Sample & TA & TG & AAATRIX-COUNT \\
\hline P10_19 & $47.0 \%$ & $52.9 \%$ & $0.1 \%$ \\
\hline P10_20 & $55.3 \%$ & $44.7 \%$ & $0.0 \%$ \\
\hline P11_21 & $98.6 \%$ & $1.3 \%$ & $0.1 \%$ \\
\hline P11_22 & $99.3 \%$ & $0.6 \%$ & $0.1 \%$ \\
\hline P12_23 & $0.7 \%$ & $99.1 \%$ & $0.2 \%$ \\
\hline P12_24 & $1.8 \%$ & $98.1 \%$ & $0.2 \%$ \\
\hline P13_25 & $4.6 \%$ & $95.3 \%$ & $0.1 \%$ \\
\hline P13_26 & $96.1 \%$ & $3.9 \%$ & $0.0 \%$ \\
\hline P14_27 & $34.4 \%$ & $65.6 \%$ & $0.0 \%$ \\
\hline P14_28 & $20.0 \%$ & $79.9 \%$ & $0.0 \%$ \\
\hline P15_29 & $99.3 \%$ & $0.7 \%$ & $0.0 \%$ \\
\hline P15_30 & $99.9 \%$ & $0.1 \%$ & $0.0 \%$ \\
\hline P16_31 & $0.1 \%$ & $8.3 \%$ & $91.6 \%$ \\
\hline P16_32 & $96.3 \%$ & $3.6 \%$ & $0.0 \%$ \\
\hline P17_33 & $0.3 \%$ & $6.2 \%$ & $93.5 \%$ \\
\hline P17_34 & $0.1 \%$ & $8.2 \%$ & $91.6 \%$ \\
\hline P18_35 & $0.4 \%$ & $99.2 \%$ & $0.4 \%$ \\
\hline P18_36 & $3.1 \%$ & $92.4 \%$ & $4.5 \%$ \\
\hline P19_37 & $0.5 \%$ & $7.7 \%$ & $91.8 \%$ \\
\hline P19_38 & $0.2 \%$ & $4.9 \%$ & $94.9 \%$ \\
\hline P1_1 & $99.9 \%$ & $0.1 \%$ & $0.0 \%$ \\
\hline P1_2 & $97.8 \%$ & $2.2 \%$ & $0.1 \%$ \\
\hline P20_39 & $0.0 \%$ & $99.3 \%$ & $0.6 \%$ \\
\hline P20_40 & $0.2 \%$ & $97.3 \%$ & $2.5 \%$ \\
\hline P2_3 & $91.8 \%$ & $8.2 \%$ & $0.0 \%$ \\
\hline $\mathrm{P} 2 \_4$ & $75.6 \%$ & $24.4 \%$ & $0.0 \%$ \\
\hline P3_5 & $1.7 \%$ & $17.3 \%$ & $81.1 \%$ \\
\hline P3_6 & $3.7 \%$ & $15.3 \%$ & $80.9 \%$ \\
\hline P4_7 & $3.0 \%$ & $1.2 \%$ & $95.9 \%$ \\
\hline P4_8 & $97.8 \%$ & $1.1 \%$ & $1.1 \%$ \\
\hline P5_10 & $94.0 \%$ & $5.9 \%$ & $0.1 \%$ \\
\hline P5_9 & $37.8 \%$ & $12.3 \%$ & $49.9 \%$ \\
\hline P6_11 & $98.1 \%$ & $1.9 \%$ & $0.0 \%$ \\
\hline P6_12 & $99.4 \%$ & $0.6 \%$ & $0.0 \%$ \\
\hline P7_13 & $99.0 \%$ & $1.0 \%$ & $0.0 \%$ \\
\hline P7_14 & $99.8 \%$ & $0.2 \%$ & $0.0 \%$ \\
\hline P8_15 & $0.3 \%$ & $7.0 \%$ & $92.7 \%$ \\
\hline P8_16 & $0.4 \%$ & $6.0 \%$ & $93.5 \%$ \\
\hline P9_17 & $2.9 \%$ & $97.1 \%$ & $0.0 \%$ \\
\hline P9_18 & $94.9 \%$ & $5.1 \%$ & $0.0 \%$ \\
\hline
\end{tabular}

Page 1 
Journal Pre-proof

\begin{tabular}{|c|c|c|c|}
\hline Bacterial species & Bacterial genus & $\begin{array}{l}\text { Descriptions of } \\
\text { human disease }\end{array}$ & $\begin{array}{l}\text { Descriptions of human } \\
\text { cutaneous disease }\end{array}$ \\
\hline Streptomyces spp. & Actinobacteria & None & None \\
\hline $\begin{array}{l}\text { Streptomyces sp. } \\
\text { SM17 }\end{array}$ & Actinobacteria & None & None \\
\hline $\begin{array}{l}\text { Streptomyces sp. } \\
\text { PVA 94-07 }\end{array}$ & Actinobacteria & None & None \\
\hline Bordetella pertussis & Bordetella & $\begin{array}{l}\text { Causative agent of } \\
\text { pertussis (whooping } \\
\text { cough) }\end{array}$ & None \\
\hline $\begin{array}{l}\text { Methylobacterium } \\
\text { oryzae }\end{array}$ & Methylobacterium & None & None \\
\hline Serratia spp. & Serratia & $\begin{array}{l}\text { Serratia spp. are } \\
\text { opportunistic } \\
\text { pathogens; } \\
\text { Nosocomial } \\
\text { infections, infections } \\
\text { in } \\
\text { immunocompromised } \\
\text { patients (e.g. } \\
\text { arthritis) }\end{array}$ & $\begin{array}{l}\text { Nosocomial skin and soft } \\
\text { tissue infections, skin } \\
\text { infections in } \\
\text { immunocompromised patients }\end{array}$ \\
\hline Serratia sp. LS-1 & Serratia & None & None \\
\hline Burkholderia mallei & Burkholderia & $\begin{array}{l}\text { None (causative } \\
\text { agent of glanders in } \\
\text { animals, e.g. horses) }\end{array}$ & None \\
\hline $\begin{array}{l}\text { Enterobacteriaceae } \\
\text { bacterium }\end{array}$ & $\begin{array}{l}\text { Unclassified } \\
\text { Enterobacteriaceae }\end{array}$ & None & None \\
\hline $\begin{array}{l}\text { Achromobacter } \\
\text { ruhlandii }\end{array}$ & Achromobacter & None & None \\
\hline Pseudomonas spp. & Pseudomonas & $\begin{array}{l}\text { Pseudomonas spp. } \\
\text { are opportunistic } \\
\text { pathogens; } \\
\text { Nosocomial } \\
\text { infections; infections } \\
\text { in } \\
\text { immunocompromised } \\
\text { patients }\end{array}$ & $\begin{array}{l}\text { Nosocomial respiratory tract, } \\
\text { skin and wound infections, } \\
\text { septic in } \\
\text { immunocompromised patients }\end{array}$ \\
\hline $\begin{array}{l}\text { Pseudomonas sp. } \\
\text { A214 }\end{array}$ & Pseudomonas & None & None \\
\hline $\begin{array}{l}\text { Pseudomonas sp. } \\
\text { st29 }\end{array}$ & Pseudomonas & $\begin{array}{l}\text { Reduced IgG-binding } \\
\text { in pediatric IBD } \\
\text { patients }\end{array}$ & None \\
\hline
\end{tabular}

